\title{
MAKING THE INVISIBLE Visible: Visualization of Music AND LyRics For DeAF AND Hard of HeARing Audiences
}

\author{
by \\ David W. Fourney \\ Master of Science, University of Saskatchewan, 2008 \\ Post-Graduate Diploma, University of Saskatchewan, 2004 \\ Bachelor of Science, University of Saskatchewan, 2001 \\ Bachelor of Arts Honours, University of Saskatchewan, 1993
}

A dissertation
presented to Ryerson University

in partial fulfillment of the

requirements for the degree of

Doctor of Philosophy

in the Program of

Mechanical and Industrial Engineering

Toronto, Ontario, Canada, 2015

(C) David W. Fourney 2015 



\section{AUTHOR'S DECLARATION FOR ELECTRONIC SUBMISSION OF A DISSERTATION}

I hereby declare that I am the sole author of this dissertation. This is a true copy of the dissertation, including any required final revisions, as accepted by my examiners.

I authorize Ryerson University to lend this dissertation to other institutions or individuals for the purpose of scholarly research.

I further authorize Ryerson University to reproduce this dissertation by photocopying or by other means, in total or in part, at the request of other institutions or individuals for the purpose of scholarly research.

I understand that my dissertation may be made electronically available to the public. 

Making the Invisible Visible:

Visualization of Music and Lyrics for Deaf and Hard of Hearing Audiences

Doctor of Philosophy 2015

David W. Fourney

Mechanical and Industrial Engineering

Ryerson University

\begin{abstract}
Music is a major art form present in all cultures. It is nearly ubiquitous in Western culture. As a shared social experience, music is a shared knowledge space. Some music is so well recognized that people may refer to it by name (e.g., Beethoven's 5th symphony, the Star Wars theme). Signing Deaf communities have indigenous forms of lyrical music. While these may not be melodic in the Western expectation, they still conform to the idea of music as metrical lyrics accompanied by instrumentation.

Music is a system made up of several elements and attributes containing discrete information chunks that can be perceived by a human listener. Music communicates complex emotional signals and, in some cultures, has specific conventions designed to manipulate emotions. However, deaf, deafened, and hard of hearing people are unable to fully access the richness of the aural musical experience.

This dissertation explores ways to make information that is presented in an auditory modality accessible to those who cannot fully perceive it due to hearing loss or environmental barriers; making the invisible visible. A multi-disciplinary, multi-method project, this research examines the design of visualizations intended to present different kinds of otherwise invisible music information in a visible, entertaining form.
\end{abstract}

Ninety-four people, 66 women and 28 men, who identified themselves as Hard of Hearing (46), Deaf (38), Cochlear Implantees (8), or Deafened (2), participated in one of four experiments. Eight of the 94 participated in a fifth experiment. Research questions included determining the fundamen- 
tal properties of music that visualizations must present, determining what emotional responses users might have to visualizations of music information, and determining whether a music experience similar to that of hearing audiences can be experienced via visualizations of music information.

Among several findings, this project learned that many deaf, deafened and hard of hearing people are active consumers of music; lyrics represent a key piece of music information hard of hearing people require of any visualization; different visualizations might be better suited to different musical genres or emotional moods; and the set of music visualizations tested convey some types of emotional information but do not obviously communicate the emotional component of the music. 


\section{Acknowledgements}

This Dissertation was a challenging project. It required quickly gaining a deep understanding of multiple subjects, such as music theory, before I could tackle the research questions themselves. This work, would not have been possible without the support and caring of several people.

Special thanks to my supervisor, Dr. Filippo Salustri, for providing me with an excellent atmosphere for doing research. I have been fortunate to have his invaluable guidance, caring, and patience. Dr. Salustri willingly assumed responsibility for the heavy lifting and took the weight off of my shoulders during the low-points of my studies. Without Fil's support, which continued throughout his sabbatical, this work would never have been completed.

Thank you to the other members of my Examination Committee: Dr. W. Patrick Neumann, Dr. M. Wahab M. Ismail, and Dr. Kathryn Woodcock of Ryerson University and Dr. Richard Ladner of the University of Washington for agreeing to examine this Dissertation.

I am indebted to Dr. Benjamin Bahan of Gallaudet University for a discussion that helped me to shape some of my design ideas.

I owe my gratitude to Joe Monkhouse, my Research Assistant, for his time, energy, and attention to detail in transcribing interviews and reviewing focus group data. He also assisted with coding and statistical analyses. This work would have never gotten finished without his help.

I would like to acknowledge and thank Dr. Deborah Fels and the students of the Inclusive Media and Design Centre (previously Centre for Learning Technologies), especially Mike P., Jorge, Quoc, and Hong Tai, for making available many resources. Special thanks also to Dr. Frank Russo and the students of his Science of Music, Auditory Research and Technology (SMART) lab, specifically Arla, for their thoughts on my work.

Thanks also to the Canadian Hard of Hearing Association (CHHA) for making available to me some space at their conference venue to conduct interviews.

Many thanks to my research participants. Without them there would have been no way to do my project.

This research benefitted from funding provided by the Toronto Rehabilitation Institute, Standards Council of Canada (SCC), Natural Sciences and Engineering Research Council (NSERC), and Social Sciences and Humanities Research Council (SSHRC). In particular, without the support of a (partial) Toronto Rehab Scholarship in Rehabilitation-related Research for Students with Disabilities, I would not have been able to move to Toronto to begin my studies leading to this Dissertation.

I thank my family for their support and my wife for her love and patience. Colleen's dedication and support helped me to focus on my work in the good times as well as the most stressful times.

Finally, I am forever grateful for the substantial financial support of my late father, the Rev. Dr. Lloyd Fourney, without which the last two years of my studies would have been impossible. 

Dedication

in memory of my dad 



\section{Contents}

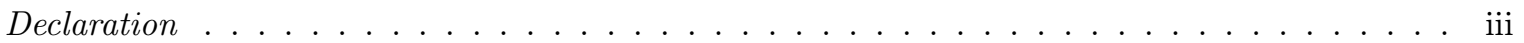

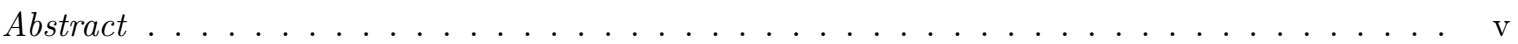

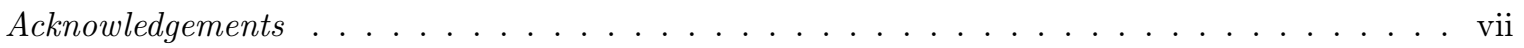

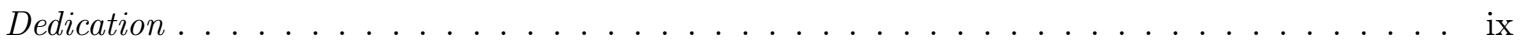

List of Tables . . . . . . . . . . . . . . . . . . . . . . xvii

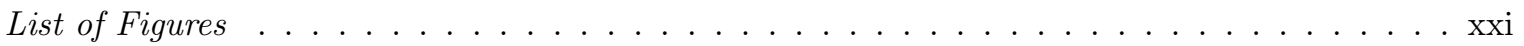

1 Introduction $\quad 1$

1.1 Universal Usability . . . . . . . . . . . . . . . . . . . . . . . . 3

1.1.1 Usability and Accessibility . . . . . . . . . . . . . . . . . 4

1.2 Research Objectives . . . . . . . . . . . . . . . . . . . . . 8

1.3 Dissertation Organization $\ldots \ldots \ldots \ldots \ldots \ldots \ldots \ldots$

2 Background $\quad 11$

2.1 The Audience . . . . . . . . . . . . . . . . . . . . . . . . . 11

2.1 .1 Disability . . . . . . . . . . . . . . . . . . 13

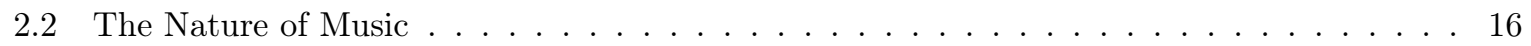

2.3 The Nature of Emotion $\ldots \ldots \ldots \ldots \ldots \ldots$

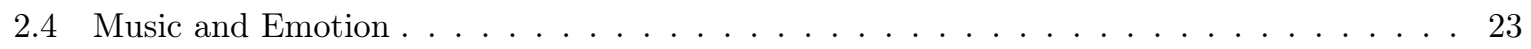

2.4.1 Emotion Conventions in Music . . . . . . . . . . . . . . . . . . . 24

2.4 .2 Emotion Conventions in Music in Film . . . . . . . . . . . . . . . . . 25

2.5 Sensory Modalities . . . . . . . . . . . . . . . . . . . . . . 29

2.5 .1 Auditory . . . . . . . . . . . . . . . . . . . . . . 29

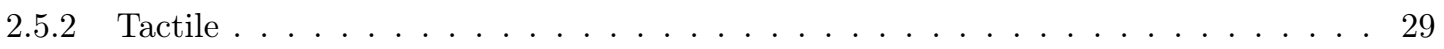

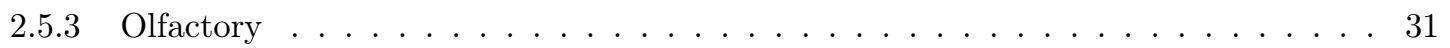

2.5 .4 Visual . . . . . . . . . . . . . . . . . . . . . . 32

2.5.5 Sensory Substitution or Sensory Supplementation . . . . . . . . . . . . . 33

2.6 Theories of Information Visualization \& Sonification . . . . . . . . . . . . . . . 33

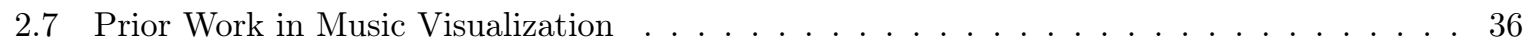

2.7.1 Relevant Existing Visualization Solutions _ . . . . . . . . . . . . . . 37

2.7 .2 Textual Visualizations . . . . . . . . . . . . . . . . . . . . . . . . 42 
2.7.3 Visualizations and Emotion . . . . . . . . . . . . . . . . . . 48

2.7.4 Why Music Visualizations? . . . . . . . . . . . . . . . . . . . . . 49

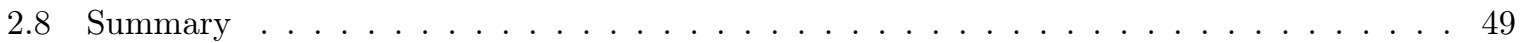

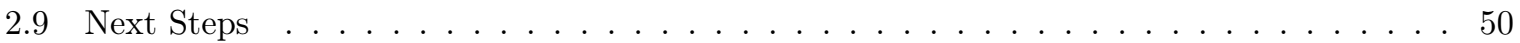

3 Methodology $\quad 51$

3.1 Design Research Methodology . . . . . . . . . . . . . . . . . . . . . . 52

3.1.1 DRM Stage 0: Criteria Definition / Research Clarification . . . . . . . . . . . . . 52

3.1 .2 DRM Stage 1: Descriptive Study I . . . . . . . . . . . . . . . . . . 52

3.1 .3 DRM Stage 2: Prescriptive Study . . . . . . . . . . . . . . . . . . 52

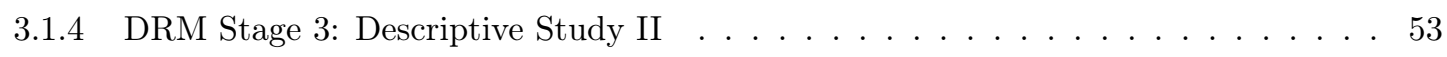

3.2 Grounded Theory . . . . . . . . . . . . . . . . . . . . . 53

3.2 .1 The Basics . . . . . . . . . . . . . . . . . . . . . . . 53

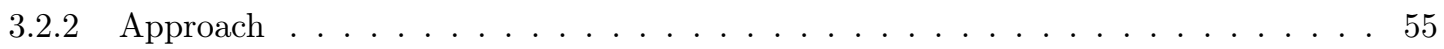

3.2 .3 Software Support . . . . . . . . . . . . . . . . . . . . . . 56

3.2.4 When Should Grounded Theory be Used? . . . . . . . . . . . . . . . . . . . . 57

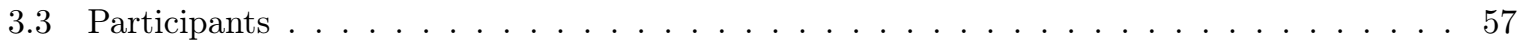

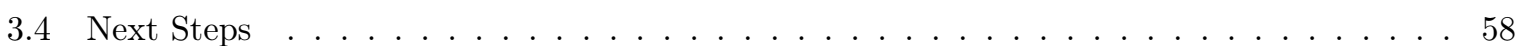

4 Need for the Work $\quad 61$

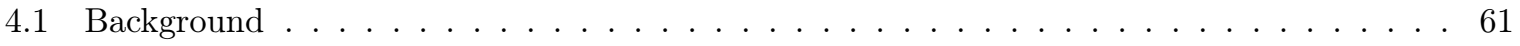

4.1.1 Music in the Primary and Secondary Schools . . . . . . . . . . . . . . . 61

4.1.2 The Importance of Music to D/HH Preteens and Adolescents . . . . . . . . . . . . 62

4.1 .3 The Music of the Deaf . . . . . . . . . . . . . . . . . . 63

4.1 .4 Music and the Hard of Hearing . . . . . . . . . . . . . . . . . . . . 65

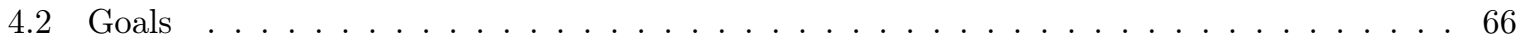

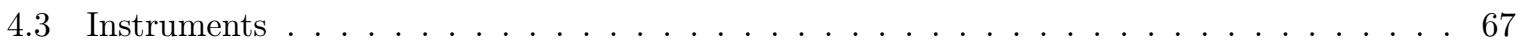

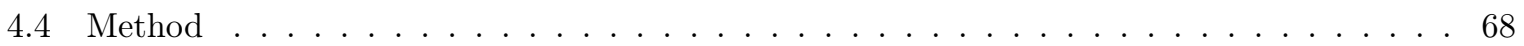

$4.4 .1 \quad$ Pre-experiment Questionnaire . . . . . . . . . . . . . . . . 68

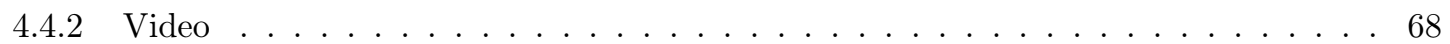

$4.4 .3 \quad$ Post-experiment Questionnaire . . . . . . . . . . . . . . . . . . . . 69

4.4 .4 Music Questionnaire . . . . . . . . . . . . . . . . . . . . . 69

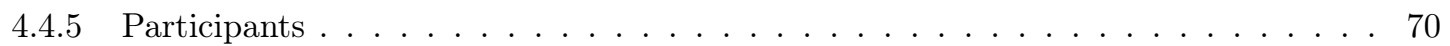

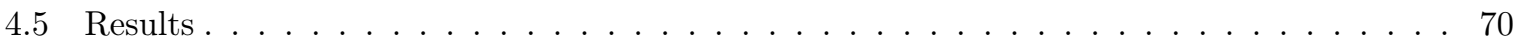

4.5.1 Goal 1 - Are efforts to improve accessibility desired? . . . . . . . . . . . . . . . 70

4.5.2 Goal 2 - What information is needed? . . . . . . . . . . . . . 75

4.6 Discussion . . . . . . . . . . . . . . . . . . . . . . . . . . 79

4.6.1 Goal 1 - Are efforts to improve accessibility desired? . . . . . . . . . . . . . . 79

4.6.2 Goal 2 - What information is needed? . . . . . . . . . . . . . . 80

4.6 .3 Limitations of the Design . . . . . . . . . . . . . . . . . . . 82 


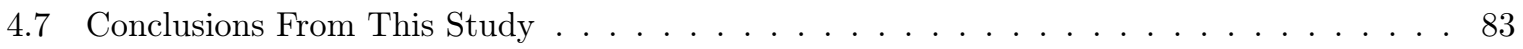

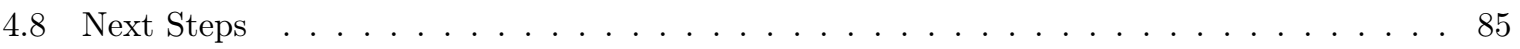

$\begin{array}{llr}5 & \text { Focus Group I } & \mathbf{8 7}\end{array}$

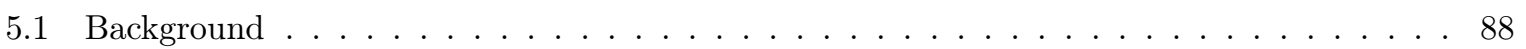

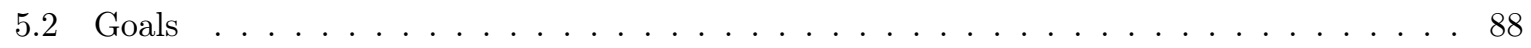

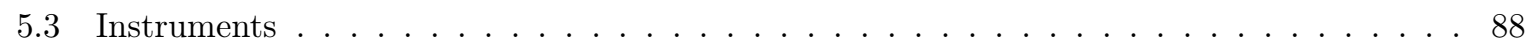

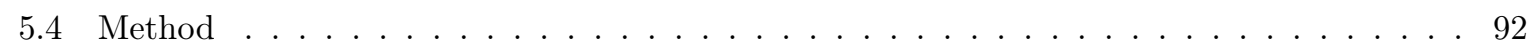

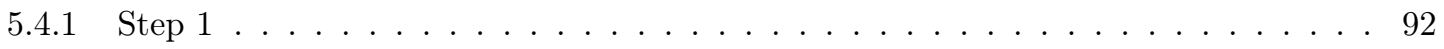

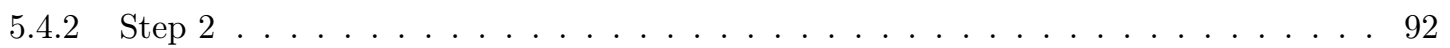

5.4 .3 Visualizations . . . . . . . . . . . . . . . . . . . . 93

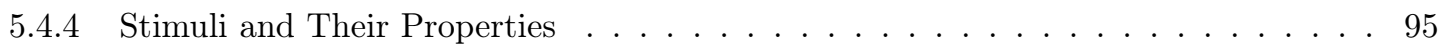

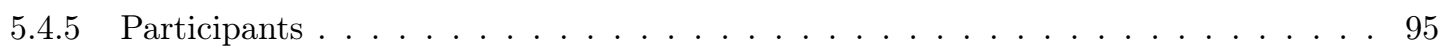

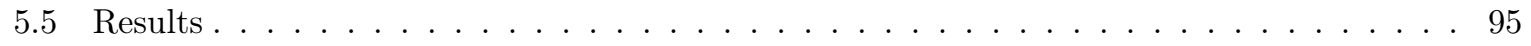

5.5.1 Goal 1 - Are efforts to improve accessibility desired? . . . . . . . . . . . . . 96

5.5.2 Goal 2 - How do viewers respond to specific visualizations? . . . . . . . . . . 96

5.6 Discussion . . . . . . . . . . . . . . . . . . . . . . . . . . 100

5.6.1 Goal 1 - Are efforts to improve accessibility desired? . . . . . . . . . . . . . . . 100

5.6.2 Goal 2 - How do viewers respond to specific visualizations? . . . . . . . . . . . . 101

5.6 .3 Limitations of the Design . . . . . . . . . . . . . . . . . . . 103

5.7 Conclusions From This Study . . . . . . . . . . . . . . . . . . . . . 104

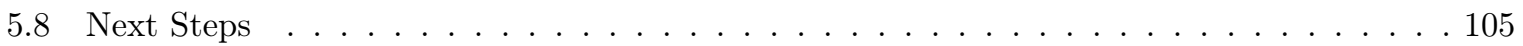

6 Focus Group II $\quad 107$

6.1 Goals . . . . . . . . . . . . . . . . . . . . . . . 107

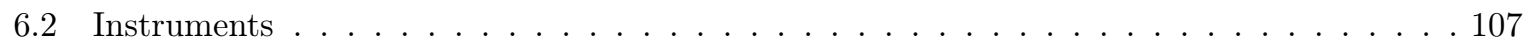

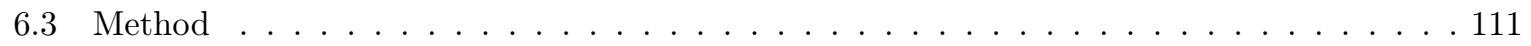

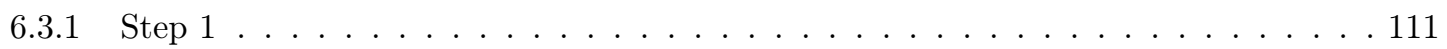

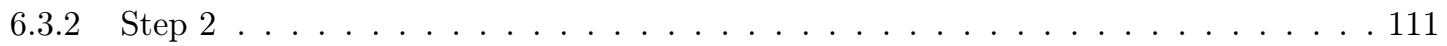

6.3 .3 Visualizations . . . . . . . . . . . . . . . . . . . . . . . . . . 112

6.3.4 Stimuli and their Properties . . . . . . . . . . . . . . . . . . . 113

6.3 .5 Questionnaires . . . . . . . . . . . . . . . . . . . 114

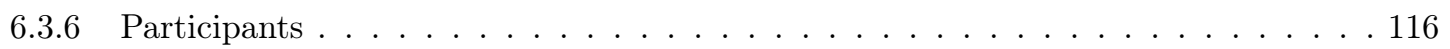

6.4 Results . . . . . . . . . . . . . . . . . . . . . . . . 116

6.4.1 Goal 1 - Desire for Improved Accessibility . . . . . . . . . . . . . . . . . . . 116

6.4 .2 Goal 2 - Music Information Preferences . . . . . . . . . . . . . . . . . . 118

6.4 .3 Goal 3 - Level of Enjoyability . . . . . . . . . . . . . . . . . . . . . . . 119

6.4.4 Goal 4-Conveyance of Emotion . . . . . . . . . . . . . . . . . . 121

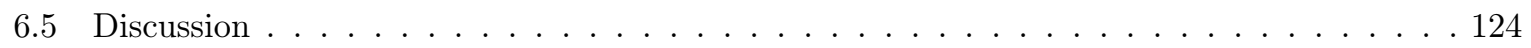

6.5.1 Goal 1 - Are efforts to improve accessibility desired? . . . . . . . . . . . . . . . 124 
6.5.2 Goal 2 - How do viewers respond to specific visualizations? . . . . . . . . . . . 125

6.5.3 Goal 3 - Do participants enjoy visualizations? . . . . . . . . . . . . . . . 126

6.5.4 Goal 4 - Can participants detect emotions through visualizations? . . . . . . . . . 127

6.5 .5 Limitations of the Design . . . . . . . . . . . . . . . . . 128

6.6 Conclusions From This Study ～. . . . . . . . . . . . . . . . . . . . . 129

6.7 Next Steps . . . . . . . . . . . . . . . . . . . . . . 130

7 Survey of Users' Music Needs 131

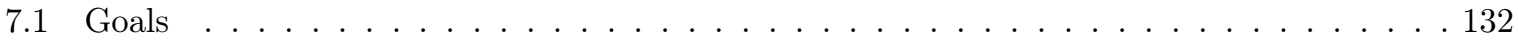

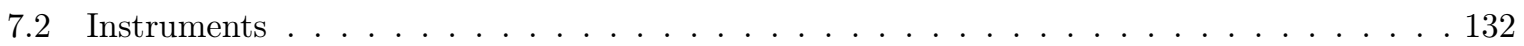

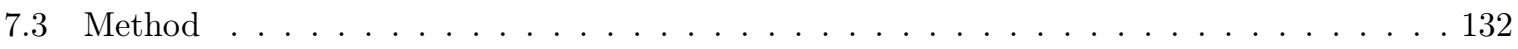

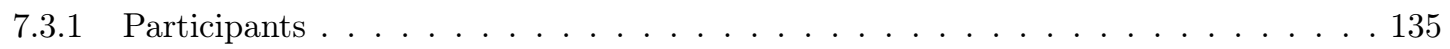

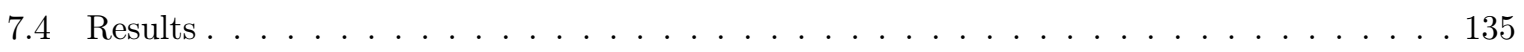

7.4.1 Goal 1 - Are efforts to improve accessibility desired? . . . . . . . . . . . . . 135

7.4.2 Goal 2 - What music information is wanted for access? . . . . . . . . . . . . 139

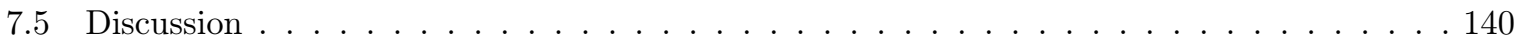

7.5.1 Goal 1 - Are efforts to improve accessibility desired? . . . . . . . . . . . . . 140

7.5.2 Goal 2 - What music information is wanted for access? . . . . . . . . . . . . . 142

7.5 .3 Limitations of the Design . . . . . . . . . . . . . . . . . . . . 142

7.6 Conclusions From This Study . . . . . . . . . . . . . . . . . 143

7.7 Next Steps . . . . . . . . . . . . . . . . . . . . . 143

8 One-on-One Interviews $\quad 145$

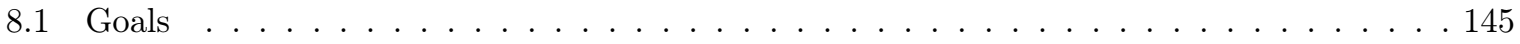

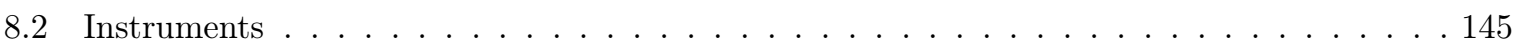

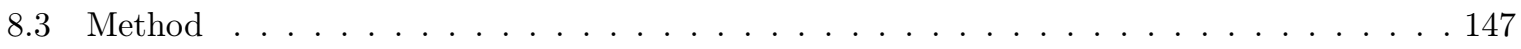

8.3 .1 Grounded Theory . . . . . . . . . . . . . . . . . . . . . 149

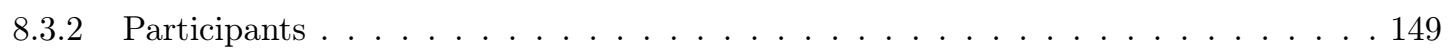

8.4 Results . . . . . . . . . . . . . . . . . . . . . . . . . . . 149

8.4.1 Goal 1 - Visualization Responses . . . . . . . . . . . . . . . . . . . 150

8.4.2 Goal 2 - Solutions Used to Access Music . . . . . . . . . . . . . . . . . . . 151

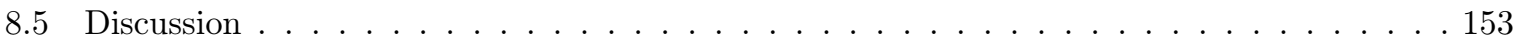

8.5.1 Goal 1 - How do viewers respond to specific visualization properties? . . . . . . 153

8.5.2 Goal 2 - What tools or activities do HH people use to access music? . . . . . . . . 154

8.5 .3 Limitations of the Design . . . . . . . . . . . . . . . . . 155

8.6 Conclusions From This Study . . . . . . . . . . . . . . . . 156

9 Discussion and Conclusion $\quad 159$

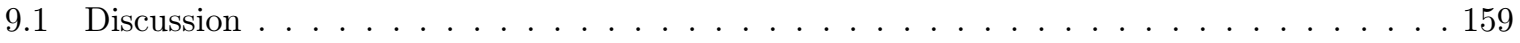

9.1 .1 The Issue of Lyrics . . . . . . . . . . . . . . . . . . . . . . 163

9.2 Some Design Guidelines and Constraints . . . . . . . . . . . . . . . . 165 


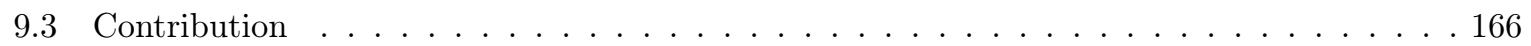

9.4 Future Directions . . . . . . . . . . . . . . . . . . . . . . 167

9.4 .1 Example Future Study . . . . . . . . . . . . . . . . . . . . . . 168

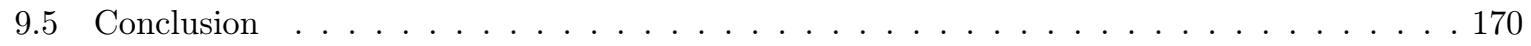

$\begin{array}{ll}\text { Appendices } & 171\end{array}$

$\begin{array}{ll}\text { A Web-based Survey } & 173\end{array}$

B Focus Group I 243

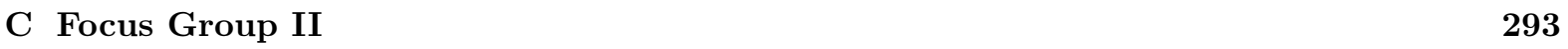

$\begin{array}{ll}\text { D Paper Survey } & 325\end{array}$

E One-on-One Interviews $\quad 343$

E.1 Interview Coding . . . . . . . . . . . . . . . . . . . . . . 355

E.2 Interview Transcripts . . . . . . . . . . . . . . . . . . . . . . 358

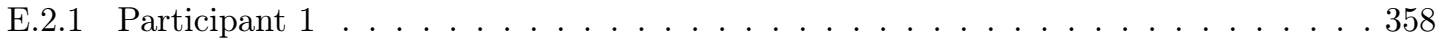

E.2.2 Participant $2 \ldots \ldots \ldots \ldots \ldots \ldots$

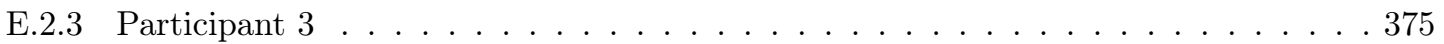

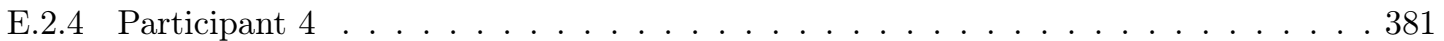

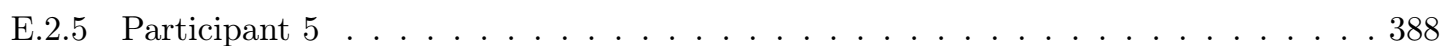

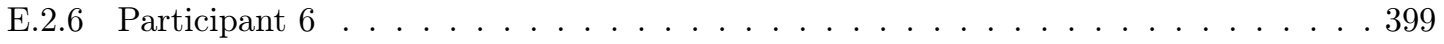

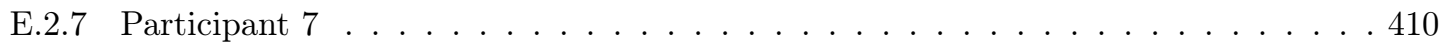

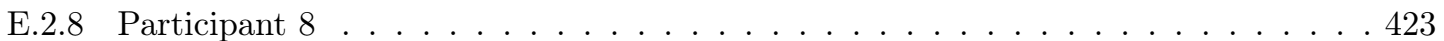

$\begin{array}{ll}\text { References } & 449\end{array}$

$\begin{array}{ll}\text { Acronyms } & 451\end{array}$ 



\section{List of Tables}

2.1 Medical, functional, legal, and cultural definitions of "Deaf" and "Hard of Hearing". . . . . 15

4.1 How often do you listen to music? (by hearing identity) . . . . . . . . . . . . 71

4.2 What statement describes you best? (by hearing identity) . . . . . . . . . . . 71

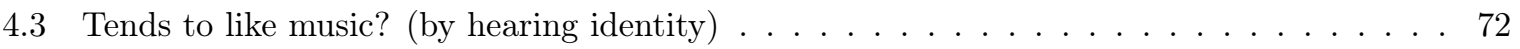

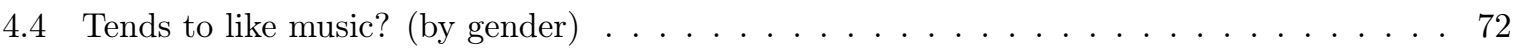

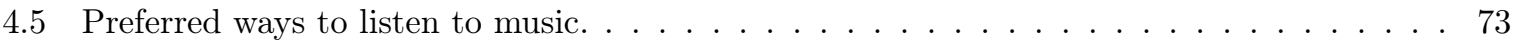

4.6 Music experience in school (by hearing identity) . . . . . . . . . . . . . . . . . . 74

4.7 Music experience in school (by tendency to like music) . . . . . . . . . . . . . . 74

4.8 Preferred forms for representing music information. . . . . . . . . . . . . 76

4.9 Chi-square test results of participant's preferences for representing music information by gen-

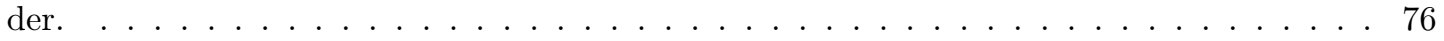

4.10 Frequency results representation of lyrics by gender. . . . . . . . . . . . . 77

4.11 Frequency results representation of beat by gender. . . . . . . . . . . . . 77

4.12 Frequency results representation of artist information by gender. . . . . . . . . . . . 77

4.13 Frequency results representation of song title by gender. . . . . . . . . . . . . 78

5.1 List of songs. . . . . . . . . . . . . . . . . . . . . . . . . . . . 92

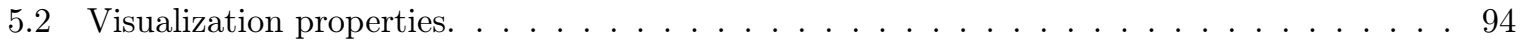

5.3 How often do you listen to music? (by hearing identity) $\ldots \ldots \ldots \ldots$

5.4 What statement describes you best? (by hearing identity) . . . . . . . . . . . . 96

5.5 Music experience in school (by hearing identity) . . . . . . . . . . . . . . . 97

5.6 Participant visualization preferences (\%) for different types of music information. . . . . . 98

5.7 Preferred visualization style (by hearing identity) . . . . . . . . . . . . . . . . . 99

5.8 Visualization style giving "best sense" of the music (by hearing identity) . . . . . . . . . . 99

5.9 Visualization style giving "best sense" of the music (by hearing identity) . . . . . . . . . . 99

6.1 List of songs. . . . . . . . . . . . . . . . . . . . . . . . . . 111

6.2 Visualization properties. . . . . . . . . . . . . . . . . . . . . . . 114

6.3 How often do you listen to music? (by hearing identity) . . . . . . . . . . . . 116

6.4 What statement describes you best? (by hearing identity) . . . . . . . . . . . 117 
6.5 Music experience in school (by hearing identity) . . . . . . . . . . . . . . 117

6.6 Participant visualization preferences for different types of music information. . . . . . . 118

6.7 Visualization style giving "best sense" of the music (by hearing identity) . . . . . . . . . 119

6.8 Which style did you enjoy most? (by hearing identity) . . . . . . . . . . . . . . 119

6.9 Which style did you enjoy most? (by highest completed education) . . . . . . . . . . 120

6.10 Reported enjoyment of twelfth visualization by highest level of education. . . . . . . . . 120

6.11 Reported enjoyment of twelfth visualization by most enjoyed visualization. . . . . . . . . . 121

6.12 Participant's enjoyment rating frequency by visualization. . . . . . . . . . . . . . . 122

6.13 Average rating of PAD (Pleasure, Arousal. Dominance) dimensions by the intended emo-

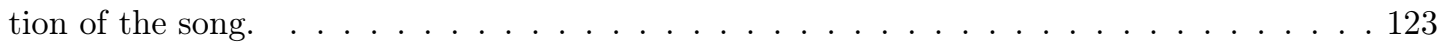

6.14 Average rating of PAD (Pleasure, Arousal. Dominance) dimensions by visualization. . . . 123

6.15 Average rating of emotion by the intended emotion of the song. . . . . . . . . . . . . 124

6.16 Average rating of emotion by visualization. . . . . . . . . . . . . . . 125

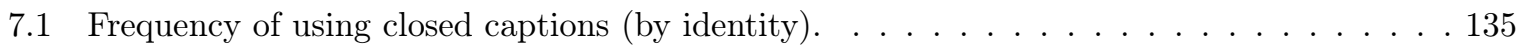

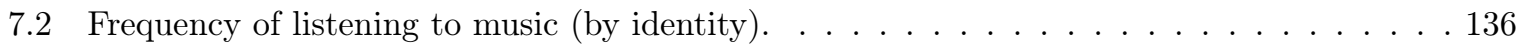

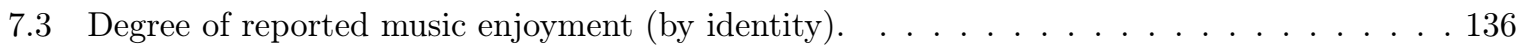

7.4 What statement describes you best? (by frequency of listening to music) . . . . . . . . 137

7.5 Technology used when listening to music (by identity) . . . . . . . . . . . . 137

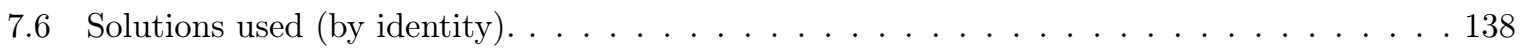

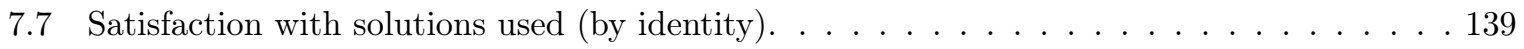

7.8 Preferred music information from computer (by identity) . . . . . . . . . . . . . . 140

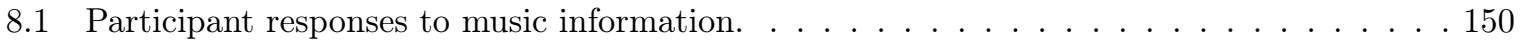

9.1 How often do you listen to music? (by hearing identity) . . . . . . . . . . . . . 161

9.2 What statement describes you best? (by hearing identity) . . . . . . . . . . . . . 162

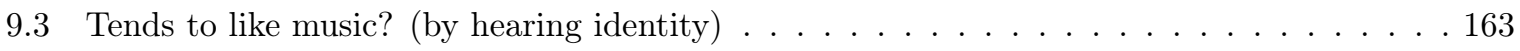

A.1 Web based survey Music Questionnaire data Part 1. . . . . . . . . . . . . . . . . 227

A.2 Web based survey Music Questionnaire data Part 2. . . . . . . . . . . . . . . 229

A.3 Web based survey Music Questionnaire data Part 3. . . . . . . . . . . . . . . . 230

A.4 Web based survey Music Questionnaire data Part 4. . . . . . . . . . . . . . . 233

A.5 Web based survey Music Questionnaire data Part 5. . . . . . . . . . . . . . . . . 234

A.6 Web based survey Music Questionnaire data Part 6. . . . . . . . . . . . . . . 236

A.7 Web based survey Music Questionnaire data Part 7. . . . . . . . . . . . . . . 237

A.8 Web based survey Music Questionnaire data Part 8. . . . . . . . . . . . . . . . . . 239

A.9 Web based survey Music Questionnaire data Part $9 . \ldots \ldots$. . . . . . . . . . . 241

B.1 Focus Group I demographics. . . . . . . . . . . . . . . . . . . . 268

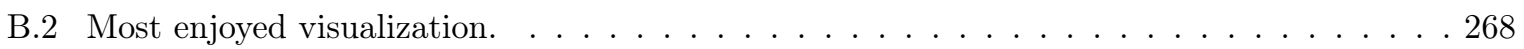


B.3 Participant's selection of visualizations best showing instrument information. . . . . . . 269

B.4 Participant's selection of visualizations best showing percussion information. . . . . . . . 269

B.5 Participant's selection of visualizations best showing melody information. . . . . . . . . 270

B.6 Participant's selection of visualizations best showing rhythm information. . . . . . . . . 270

B.7 Participant's post-test written responses on most / least enjoyable part of the focus group. 271

B.8 Participant's response to Block 1 Song 1 visualization. . . . . . . . . . . . . . . . . 272

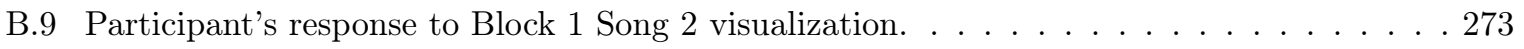

B.10 Participant's response to Block 1 Song 3 visualization. . . . . . . . . . . . . . . . . . 274

B.11 Participant's response to Block 1 Song 4 visualization. . . . . . . . . . . . . . . . 275

B.12 Participant's response to Block 1 Song 5 visualization. . . . . . . . . . . . . . . 276

B.13 Participant's response to Block 1 Song 6 visualization. . . . . . . . . . . . . . . . 277

B.14 Participant's response to Block 1 Song 7 visualization. . . . . . . . . . . . . . . 278

B.15 Participant's response to Block 2 Song 1 visualization. . . . . . . . . . . . . . . . . 279

B.16 Participant's response to Block 2 Song 2 visualization. . . . . . . . . . . . . . . . 280

B.17 Participant's response to Block 2 Song 3 visualization. . . . . . . . . . . . . . . . . . 281

B.18 Participant's response to Block 2 Song 4 visualization. . . . . . . . . . . . . . . . 282

B.19 Participant's response to Block 2 Song 5 visualization. . . . . . . . . . . . . . . . 283

B.20 Participant's response to Block 2 Song 6 visualization. . . . . . . . . . . . . . . . 285

B.21 Participant's response to Block 3 Song 1 visualization. . . . . . . . . . . . . 286

B.22 Participant's response to Block 3 Song 2 visualization. . . . . . . . . . . . . . . . . 287

B.23 Participant's response to Block 3 Song 5 visualization. . . . . . . . . . . . . . . . . 288

B.24 Participant's response to Block 4 Song 1 visualization. . . . . . . . . . . . . . . . . . 289

B.25 Participant's response to Block 4 Song 4 visualization. . . . . . . . . . . . . . . . . 290

B.26 Participant's response to Block 4 Song 7 visualization. . . . . . . . . . . . . . . . 291

C.1 PreTest responses. . . . . . . . . . . . . . . . . . . . . . . . . . 314

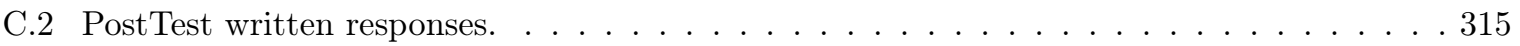

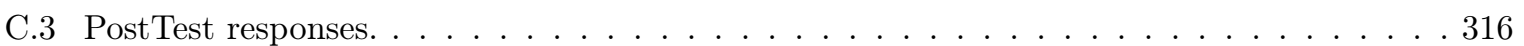

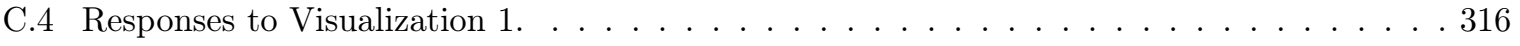

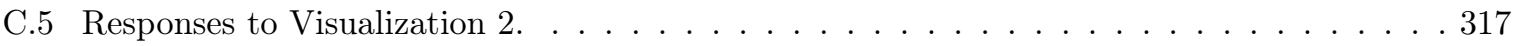

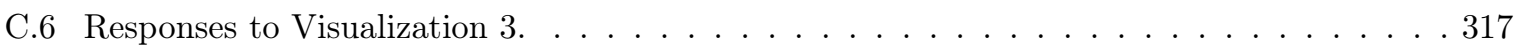

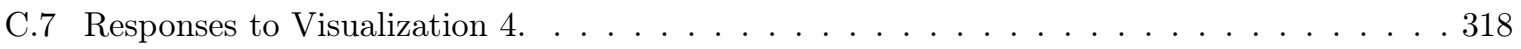

C.8 Responses to Visualization 5. . . . . . . . . . . . . . . . . . . . . . . . . 319

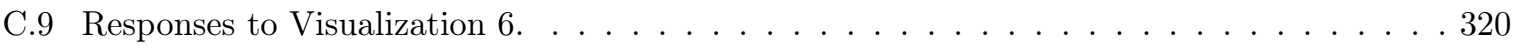

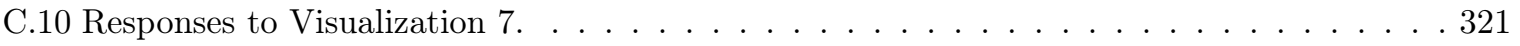

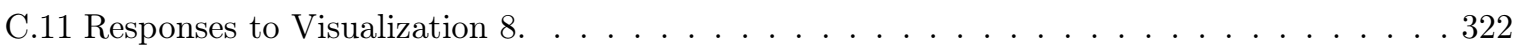

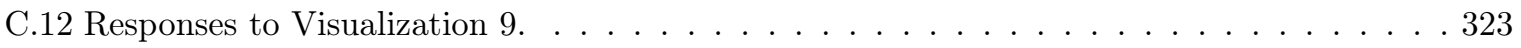

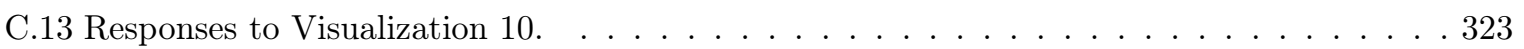

C.14 Responses to Visualization $11 . \ldots \ldots \ldots \ldots \ldots \ldots$

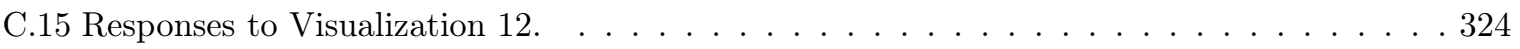


D.1 Paper survey demographic responses. . . . . . . . . . . . . . . . . . 328

D.2 What technologies do you use to listen to music? . . . . . . . . . . . . . . . . 329

D.3 What are the most important pieces of information you would want a computer to help you

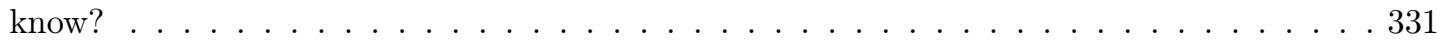

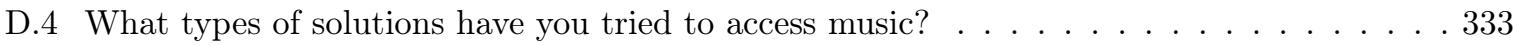

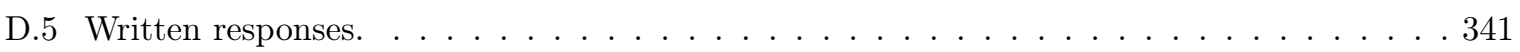

E.1 Codes used in data analysis. . . . . . . . . . . . . . . . . . . . . . . . . . . . . . . 


\section{List of Figures}

2.1 The human ear (Tierney, Zissman, \& Eddington, 1994) . . . . . . . . . . . . . . . 20

2.2 Intertitle from "All on Account of a Transfer" (Williams, 1913) . . . . . . . . . . . . 26

2.3 Deaf students taking a music lesson at the Alberta School for the Deaf in 1969 . . . . . . 30

2.4 Focus of perception model. Adapted from Lübbers (2005) . . . . . . . . . . . . . 36

2.5 A music visualization created by the Atari Video Music (Green, 2014) . . . . . . . . . . 38

2.6 Magnetosphere music visualization (Griffiths, 2008) . . . . . . . . . . . . . . . 40

2.7 Music Animation Machine Piano Roll. . . . . . . . . . . . . . . . . . . . . . 41

2.8 Music Animation Machine Part Motion. . . . . . . . . . . . . . . . . . . . . . . . . 41

2.9 Music Animation Machine Tonality Compass. . . . . . . . . . . . . . . . . . . . . . . 41

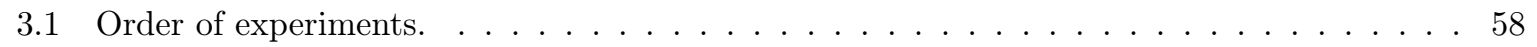

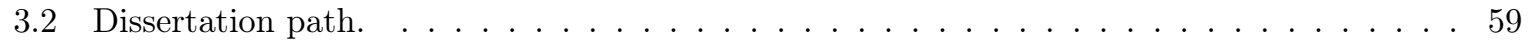

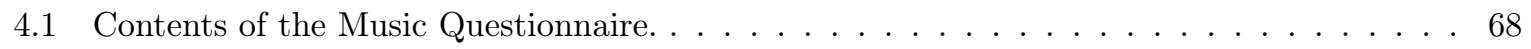

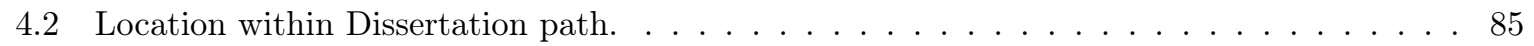

5.1 Focus Group Pre-study Questionnaire. . . . . . . . . . . . . . . . . . . . 89

5.2 Focus Group Post-visualization Questionnaire. . . . . . . . . . . . . . . . . . 90

5.3 Focus Group Post-study Questionnaire. ． . . . . . . . . . . . . . . . . 91

5.4 The Motion Pixels of Music $(\mathrm{MPM})$ visualization. . . . . . . . . . . . . . . 94

5.5 Location within Dissertation path. . . . . . . . . . . . . . . 105

6.1 Focus Group Pre-study Questionnaire . . . . . . . . . . . . . . . . . . 108

6.2 Focus Group Post-visualization Questionnaire. . . . . . . . . . . . . . . . . . . 109

6.3 Focus Group Post-study Questionnaire. . . . . . . . . . . . . . . . . . . . 110

6.4 The Radar visualization. . . . . . . . . . . . . . . . . . . . . . . . 113

6.5 Self-Assessment Manikin overbearingness scale (Bradley \& Lang, 1994). . . . . . . . . . . 115

6.6 Location within Dissertation path. . . . . . . . . . . . . . . . . . . 129

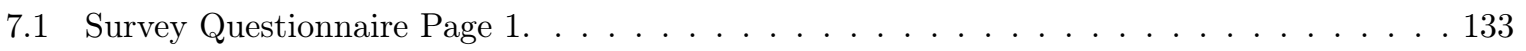

7.2 Survey Questionnaire Page $2 \ldots \ldots \ldots \ldots \ldots$

7.3 Location within Dissertation path. . . . . . . . . . . . . . . . 143 


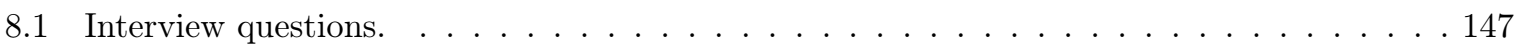

8.2 Focus Group Post-study Questionnaire. . . . . . . . . . . . . . . . . . . . . 148

8.3 The music visualizations: (a) Part Motion, (b) Bars, and (c) Radar. . . . . . . . . . . . . 149

8.4 Location within Dissertation path. . . . . . . . . . . . . . . . . 156

9.1 FLOE Project Video Player showing transcript view of lyrics. . . . . . . . . . . . . 169 


\section{Chapter 1}

\section{Introduction}

In Western culture, the presence of music in people's lives is nearly ubiquitous - music is heard in clubs, restaurants, malls, elevators, etc. People may carry music on them wherever they go using their phones or other music players (e.g., iPods). According to the International Federation of the Phonographic Industry (IFPI), in 2008, the worldwide music industry generated nearly USD \$18B (International Federation of the Phonographic Industry (IFPI), 2009) in music sales.

In addition to being an important medium for the conveyance of cultural information, such as shared experience and knowledge (e.g., Rose, 1994), music is entertaining and enjoyable for its own sake. As Hamm, Nettl, and Byrnside state, "we would not have music if it did not do something important for us" (1975, p. 71). This "something important" is "not done by any other activity or any other facet of life." (1975, p. 71).

Humans invented music "as a form of social communication, a tool to pass information about the group's shared mental state to a number of individuals at once." (Loersch \& Arbuckle, 2013).

Traditionally music is a social experience. People sing hymns to affirm their commitment to their church, or national anthems to demonstrate their love of country. People talk to their friends about the music they listen to. People go to concerts to hear music with other people. In this sense, music does not simply entertain, it is a form of connection (Jacobs, 2013, August 5).

Many people repeatedly report music evoking emotional responses and memory flashbacks (Janata, Tomic, \& Rakowski, 2007), relaxation (Knight \& Rickard, 2001), and other experiences which make music pleasurable (Kohut \& Levarie, 1950). As Alfred Newman once said, "There is nothing in the world that will make people cry or make them laugh as quickly as music." (Jones, 1937, p. C9).

However, music is not only enjoyed on its own. For example, television programs and films are made up of information and content in auditory as well as visual modalities. Combining them in creative ways provides the entertainment value of the show. Once viewed, it is left up to the viewer to capture and then decode this information to surmise the intent of the program's creators. In the same way, emotional content contained in the program or film is also encoded, transmitted, and decoded (Fourney \& Fels, 2008). Some of the important emotional information is encoded through the music used. Music accompanying the opening credits might be used to 'set the mood' of the program or film. 
Music used as background can shape the audience's emotional response to the scene and can affect the audience's interpretation of those emotions.

While there can be redundancy with visual effects through facial expression, body language, or special effects, so that the emotional impact is reinforced, the same music can be used to oppose those visual effects such as to show irony or comedy. Such music content can be so powerful that it may carry long-term cultural significance (Fourney \& Fels, 2008). For example, many North American adults closely associate the main shark theme from the movie "Jaws", a simple alternating pattern of the notes $\mathrm{E}$ and $\mathrm{F}$, with images of an impending shark attack and the anticipation of doom, even if the music is not being used in the context of sharks (Berardinelli, 2002; Matessino, 1999, Sept. 24).

The experience of entertainment and enjoyment is produced for hearing audiences which occupy the larger portion of society. For Deaf, Deafened, and Hard of Hearing (D/HH) people this experience of entertainment and enjoyment is limited. While it is true that the deaf community creates its own music (Fourney \& Fels, 2009), people who are deaf experience barriers when attempting to access the music of the larger hearing culture. They do not have access to this shared experience because it is not provided in a modality that is more appropriate to this population of consumers. Existing strategies for deaf people to access this auditory information include putting one's hands on speakers or setting the volume loud enough to create a moderate tactile effect from the air pressure or floor vibration. People who are hard of hearing experience different barriers when attempting to access the music of the larger hearing culture. Their residual hearing may not allow the individual to hear the full range of tones or perceive sound in stereo. Existing strategies for hard of hearing people to access this auditory information include amplification, making the music loud enough to hear but losing the clarity of the sound, or selecting specific genre based on their accessibility (e.g., lack of lyrics).

Music is often described as an aural experience; it has to be heard to be enjoyed. Until the development of the first practical sound recording and reproduction device, Edison's phonograph, in the late nineteenth century, the experience of music was primarily live. Live performance provides an opportunity for an experience that goes beyond the auditory. It is an emerging social experience.

Within live performance the three phases of music, its creation, transmission, and reception, are couched in a larger social space. The music is on the one hand part of the social space and feeding the social experience, while on the other hand (simultaneously) the reason for the social experience. People come together to perform the music and/or to experience the performance of the music. Within the shared experience people talk about the music and individually as well as collectively respond to the music.

In the live environment exists a chance for more senses to be stimulated. The music experience might be felt, seen, tasted, or smelled. Tactile and visual components of live music that can enhance or complement the overall music experience include feeling vibrations such as the drum beat, or watching the movements of the performers (band members, instrumentalists, vocalists) or dance accompaniment. The sense of taste and smell are impacted by the presence of food and drink in the concert space. The phonograph's ability to record and reproduce the aural portion of such live performances removed this additional social, tactile, olfactory, and visual information from the experience and changed the focus of musical entertainment. Without the visual, tactile, social and other experi- 
ences of live performance to provide any non-auditory information, music is mostly an auditory experience. When (artificially) focused only on this aural component, music becomes mostly invisible to those who cannot hear it. This aural focus excludes $\mathrm{D} / \mathrm{HH}$ people from participation.

Music videos have returned some of the visual, or visible, elements of live performances but they are not intended to increase the accessibility or reprise the social experience of the music. Music videos may be more oriented to marketing the product or boosting the "image" of the artist (Straw, 1988) than visually conveying the artist's own interpretation of the music.

For me, as a hard of hearing person, one of the challenges of this research is to derive an understanding of the concept of music. The literature regarding music or sound typically assumes that the reader can hear. Few authors make an attempt to explain music to those who cannot hear at all, cannot hear in stereo, or do not hear very well. By "music", this Dissertation does not mean music as presented on paper (i.e., a musical score), or held in memory, or words, or dance. Music is a process of expression by a performer, transmission via some medium, and reception / interpretation by a listener. While this Dissertation will tend to focus on that which is received or heard, all three elements of the music experience (performance, transmission, and reception) will be touched upon.

One possible solution to creating access to this cultural information and entertainment medium is making the invisible properties of music visual, and therefore visible. For any prototype visualization of music to be successful, it needs to convey the sense of music, emotional content, and overall entertainment. The lyrics alone are not enough (Fourney \& Fels, 2009). Ultimately, the experience should be similar to that enjoyed through sound except that the means of enjoyment would be different. If the song is intended to be soothing, it should be visually perceived as such.

Of the greatest interest to me is whether meaningful and entertaining music visualization is feasible to meet the needs of D/HH music enthusiasts, and the extent to which it can be accomplished automatically without human intervention.

\subsection{Universal Usability}

The information and communications products and services design philosophy that ensures products are usable for every person is commonly referred to as universal usability, design for all, universal access, and universal design. Research in universal usability is needed to understand the challenges of technology variety, user diversity, and gaps in user knowledge (Shneiderman, 2000).

Research in visualizing music for $\mathrm{D} / \mathrm{HH}$ consumers, in particular, has to understand the gaps in user knowledge. Since many D/HH people already access music in some manner, understanding what information can already be perceived and what further information is still needed is a challenge. Any music visualization solution needs to be at least as usable and as accessible as the current solution, it cannot add new barriers.

In addition, there is great user diversity. People who are $\mathrm{D} / \mathrm{HH}$ access music with differing levels of success and enjoyment (Fourney \& Fels, 2009). When listening to music, they encounter various barriers such as incomprehensible lyrics, too low volume, and flat tone. Some report no longer enjoy- 
ing music as they once did prior to acquiring hearing loss (Fourney \& Fels, 2009; Sacks, 2007). Others report seeing no point in music because they have never been able to hear it (Fourney \& Fels, 2009). Thus, for D/HH audiences, music brings issues of usability and issues of accessibility. While one may be able to access the music to some degree, the information it contains may not be entirely usable. The concepts and practice of usability and accessibility may help to better understand these challenges and some of the roles they play in music visualization solutions and point to ways to address them.

\subsubsection{Usability and Accessibility}

Although closely related, the concepts of usability and accessibility are distinct. It is possible to create a usable product that is not accessible. Similarly, it is possible to create an accessible product that is not usable. To avoid either scenario, it is necessary to understand both concepts and their relationship to solution design. This section will define and discuss these concepts.

International standards in the area of software ergonomics that include definitions of usability and accessibility have been developed by International Organization for Standardization (ISO). The definition of usability in ISO 9241-11 is "the extent to which a product can be used by specified users to achieve specified goals with effectiveness, efficiency and satisfaction in a specified context of use" (International Organization for Standardization [ISO], 1998, p. 2). The standard describes how to identify the information required when specifying or evaluating usability in terms of measures of user performance and satisfaction, and provides guidance on how to describe the context of use of the product and the measures of usability in an explicit way. It also includes an explanation of how the usability of a product can be specified and evaluated as part of a quality system (ISO, 1998).

The three terms effectiveness, efficiency, and satisfaction refer respectively to how well the product does the job for the user, how easily ${ }^{1}$ the job is done for and by the user, and the user's freedom from discomfort as well as attitudes (positive or negative) towards the use of the system. In this context, the notions of effectiveness and efficiency are highly coupled. By definition, usability focuses on "specific users" and thus may ignore or exclude other users and issues of accessibility because these users are not specified as part of the user group. An example is music visualizations that are designed to entertain those who can already hear the music but do not support those who cannot also hear the music.

Usability problems impact all users but to different extents; a given usability issue may impact one user more than another. A usability issue becomes an accessibility issue when it has a disproportionate effect on people with disabilities (G. C. Vanderheiden, personal communication, November 29, 2007). When accessibility is separated from usability, it is often perceived as a "special case" of usability where special designs are needed to fit the requirements of users with specific types of disability. This view suggests that specific users with specific disabilities should be required to purchase a significantly different (which may also translate into increased cost) version of common consumer products

\footnotetext{
${ }^{1}$ In this context, "easy" refers to the "[r]esources expended in relation to the accuracy and completeness with which users achieve goals." (ISO, 1998, p. 2)
} 
which have been specially designed to meet their needs. This is not practical because each user group is so small that no product can be provided at reasonable cost to the consumer (or producer) (Vanderheiden, 1990). In addition, this approach does not meet the needs of the majority of consumers with disabilities because it excludes the widest possible range of users - specific designs for specific groups of users with disabilities by definition miss other specific groups of users with disabilities (Fourney, 2007).

Sometimes this "special case" perspective can lead designers to believe that everything needs to be designed to be used by everyone. This is based on a misperception that everything needs to be designed so that it is accessible to every possible person with a disability. It may be "impractical if not impossible - to design everything so that it is accessible by everyone regardless of their limitations" (Vanderheiden, 1990, p. 388) and it may be "unreasonable to design everything to be usable by everyone" (Vanderheiden, 1990, p. 390). One solution is to take a middle ground: Products should be adaptable and compatible with assistive technologies. Design with the widest range of users in mind from the beginning, identifying which, if any, cannot be included. The goal of inclusive design is to keep the group of excluded users as small as possible.

Some "special case" designs do exist. For example Braille, a system for representing text tactilely, was specifically designed for the blind. While it was not designed to reinvent the writing system for all possible users, Braille can be read by those who are trained in its use and have the ability to tactilely distinguish the characters.

ISO 9241-171 is an international standard that provides requirements and recommendations for the design of systems and software that will enable users with disabilities greater accessibility to computer systems. Instead of treating accessibility separately from usability, it combines them. The ISO 9241171 definition of accessibility is "usability of a product, service, environment or facility by people with the widest range of capabilities" (International Organization for Standardization [ISO], 2008, p. 2). Accessibility then focuses on the widest range of users recognizing that different users have different needs and may require different accommodations.

There are three views of how to create accessibility. Each view has a different focus: the user, the product, or the interaction between the user and the product (Carter \& Fourney, 2004). A focus on the user forces the user to work around the accessibility of the product and corresponds to the biomedical and functional-rehabilitative models of disability; that is, that a disability is an "illness" to be "treated" or "fixed." The user seems to bear the "blame" for any problems. In this view, the "barrier" is perceived to somehow be an attribute of the user not the system or environment (e.g., Vitense, Jacko, and Emery (2002) refer to, "individuals with barriers limiting one or more channels of perception, such as a visual impairment", p. 76). In this view, meeting the needs of users and universal access is often seen as a burden rather than as a design goal. Accessibility is created by modifying the user. An example would be requiring a right-handed person to use a mouse designed for the lefthanded in their right hand. The issue is not that the right-handed person cannot use their left hand, but that it is awkward to do so, slow, and possibly uncomfortable. An extreme example would be the surgical modification of the user's body such as changing them so they can hear. The issue addressed here is not whether the $\mathrm{D} / \mathrm{HH}$ person cannot hear, but that the information is not entirely accessible 
to them.

A focus on the product forces the product / system to resolve accessibility problems. It shifts the focus onto the product / system and away from the user. The ISO 9241-171 definition of "accessibility" quoted above has the advantage of focusing the "problem" of accessibility on the usability of the system and away from the user, however, it continues to see human-computer interaction as a twoparty affair and still assigns blame to one of them (i.e., the product / system). Accessibility can be viewed as needing expensive or impractical changes to the system. Sometimes less expensive and more practical solutions are available. For example, Vanderheiden presents the case of users with disabilities not being able to get into an elevator before the door closes. The proposed solution was to simply extend the timer on the door. However, because many building codes require elevators to visit each floor in some set frequency, this solution required installing several more elevators. The eventual (and significantly cheaper) solution was to reprogram the elevator's controlling computer to announce its arrival before the door opened. Similarly, viewing music as the source of the entire problem and expecting music to resolve its usability and accessibility problems is impractical. This would require a complete re-engineering and reinvention of music (which is not an engineered product) to design accessibility for all possible audiences into it (Vanderheiden, 1990).

A focus on the interaction between the user and the product recognizes that the problem of accessibility is neither the "fault" of the user nor the system. The focus is on, "... removing barriers that prevent people with disabilities from participating in substantial life activities..." (Bergman \& Johnson, 1995, p. 88). There is a third party involved, the "barrier" - an interface which handicaps the interaction between the user and the system. This interaction can be described as a negotiation among parties where each party brings their own "terms" into the negotiation and a working partnership is sought (Carter \& Fourney, 2004). Parties can only communicate if they have some shared context, which may include various types of knowledge and abilities. Anything missing in the shared context of the interaction is a barrier to the communication between the user and the product (Carter \& Fourney, 2004). Applying this in the context of music would seem to indicate a better approach than focusing on the user or the product. Assisting the interaction between the music and the $\mathrm{D} / \mathrm{HH}$ listener to find new paths to the information the listener cannot otherwise access is a potential source for solutions.

One of the goals of this research is to create a means to increase the shared context available to $\mathrm{D} / \mathrm{HH}$ music consumers. While it is not practical to reinvent music and choose a design where all the properties of music are accessible to all people, it may be reasonable to find a way to communicate a sufficient quantity of the properties of music to increase the communicative effect. At the same time, any solution to this problem will be a "special case" design because it focuses on the needs of a specific cultural / disabled population.

The Principles of Universal Design provide some guidance on how to go about implementing inclusive or accessible designs that can be applied to this research. As proposed by the Center for Universal Design, the Principles of Universal Design are (Story, Mueller, \& Mace, 1998):

1. Equitable use. The design is useful and marketable to people with diverse abilities. It is appeal- 
ing to all users and provides the same or equivalent means of use for all users.

2. Flexibility in use. The design accommodates a wide range of individual preferences and abilities. It allows for choice in methods of use such as left- or right- handed use and adapts to the user's pace.

3. Simple and intuitive use. The use of the design is easy to understand, regardless of the user's experience, knowledge, language skills, or current concentration level. It is intuitive, minimizes complexity, and provides effective prompting and feedback.

4. Perceptible information. The design communicates necessary information effectively to the user, regardless of ambient conditions or the user's sensory abilities. It presents information in multiple redundant modalities and maximizes its legibility in each mode.

5. Tolerance for error. The design minimizes hazards and the adverse consequences of accidental or unintended actions. It is fail-safe and provides warnings of hazards and errors.

6. Low physical effort. The design can be used efficiently and comfortably and with a minimum of fatigue. It minimizes sustained physical effort and enables the user to maintain a neutral body position.

7. Size and space for approach and use. The design provides appropriate size and space for approach, reach, manipulation, and use regardless of the user's body size, posture, or mobility. It provides a clear line of sight and comfortable reach for any standing or seated user.

Principle Four of the Principles of Universal Design, "Perceptible Information", is most relevant to this research. This principle requires the design to communicate necessary information effectively to the user, regardless of ambient conditions or the user's sensory abilities. In particular, it encourages using different modalities (i.e., auditory, visual, tactile) to redundantly present essential information. For example, instead of presenting information only in an auditory manner, this principle would encourage visual presentation as well. It is this principle that guides the need for visual representations of auditory information, such as music, to support D/HH people.

Other relevant principles are Principles Three and Five. Principle Three, "Simple and intuitive use", implies that the user interface of any visualization solution should be obvious. In the case of music visualization, this principle could also be interpreted to mean that the visualization itself should be simple and intuitive, even without sound. Principle Five, "Tolerance for error", essentially means any solution should be robust and the resulting visualization should not cause harm (e.g., improper red-flash can trigger seizures).

Also relevant are Principles One and Two. Principle One, "Equitable use" suggests that, while a music visualization solution would, by definition, be inaccessible to people who are blind, it should not preclude other users. The solution should still enable users to listen to the music. According to this principle, any resulting visualization should also be appealing. Further, its user interface should allow for full use via keyboard. Principle Two, "Flexibility in use", implies that any solution should be 
adaptable; designed with as many user options as necessary. For example, if a user wants to not hear any music, this should be possible. Other options might be colour choice, size on screen, or amount of information presented.

The less relevant principles are Principles Six and Seven. Principle Six, "Low physical effort", is specific to issues of physical ergonomics such as work effort. Principle Seven, "Size and space for approach and use", is specific to issues of physical ergonomics such as body shape and size. Neither is relevant because music visualization for $\mathrm{D} / \mathrm{HH}$ audiences is an issue more related to cognitive ergonomics such as perception. We have no control over any of the physical environment in which the visualization is used.

\section{$1.2 \quad$ Research Objectives}

Since, as noted earlier, of the greatest interest to me is whether meaningful and entertaining music visualization is feasible to meet the needs of $\mathrm{D} / \mathrm{HH}$ music enthusiasts, and the extent to which it can be accomplished automatically without human intervention, the goals of this Dissertation are to:

(a) define the needs of $\mathrm{D} / \mathrm{HH}$ music enthusiasts; and

(b) evaluate the feasibility of using music visualization as a substitute for auditory musical experience.

While it is not practical to reinvent music and choose a design where all the properties of music are accessible to all people, it may be reasonable to find a way to communicate a sufficient quantity of the invisible properties of music in a visible way to increase the communicative effect. As a result, one of the areas of exploration in this Dissertation is the nature of music to identify its properties and its uses in hearing as well as D/HH cultures.

Thus, at a high level the main research questions are:

1. What are the fundamental properties of music that a visualization must present and that produces an entertaining and meaningful experience for $\mathrm{D} / \mathrm{HH}$ people?

This will require an exploration of the atomic components of music and how these elements of music influence the entertainment of its listeners. This research question is consistent with Principle Four of the Principles of Universal Design, "Perceptible Information", because of the principle's requirement for communication of information.

2. What are the emotional experiences and interpretations of users from visualizations? Logically, just as aural music is interpretable by the listener, a visualization of music should be interpretable by the viewer. Ideally, if listeners can construct an emotional experience from the music information they hear, viewers of music visualizations should be able to construct some emotional experience from the information that they see. What kind of emotional experience viewers might have is currently unknown. Whether that emotional experience is the same as that of the hearing listener or even the same as that intended by the music creator is beyond 
the scope of this work. This research question is consistent with several of the Principles of Universal Design such as Principle Three, "Simple and intuitive use", which implies that the user interface of any visualization solution should be obvious.

\section{Is it possible to create a $\mathrm{D} / \mathrm{HH}$ music experience similar to hearing audiences?}

While the previous question addresses what information $\mathrm{D} / \mathrm{HH}$ users might receive via visualizations of music, this question addresses the issue of equity. Should D/HH people even expect a "similar" experience? If so, what is a "similar" experience? Of the Principles of Universal Design, Principle One "Equitable use", is particularly relevant here because it implies that the music itself, not just the visualization, should be equitable across users regardless of ability.

\subsection{Dissertation Organization}

This Dissertation is organized into the following chapters:

Chapter 1 provides introductory material, motivation and research objectives for the Dissertation.

Chapter 2 on page 11 discusses further background information on the research questions, including the nature of music, and develops an approach to visualization.

Chapter 3 on page 51 provides a high level description of the method used for the series of studies completed for this Dissertation and describes the Design Research Methodology (DRM) approach to design.

Chapters 4 to 8 on pages 61 through 157 presents each of the studies completed for this Dissertation.

Chapter 9 on page 159 presents an overall summary discussion of the results, discusses the conclusions and contributions of this Dissertation, and suggests future work.

Technical material is provided in Appendices A to E on pages 173 to 434, as needed, to allow the main Dissertation to present a solution in a manner that can be understood by a general audience. A listing of abbreviations specific to this Dissertation is provided at the end of this document on page 451. 



\section{Chapter 2}

\section{Background}

Before the research objectives of this Dissertation can be further explored, it is necessary to better understand the existing knowledge on music, the $\mathrm{D} / \mathrm{HH}$ communities, and the various ways different types of music information have been presented. The purpose of this chapter is to provide background information to these areas, show how past research relates to the current research questions, and explore open issues where the current research questions may provide more information.

\subsection{The Audience}

Hearing loss is considered one of the most common forms of disability around the world. In North America, hearing loss is the third most chronic disability among older adults and the fastest growing hidden disability.

Although such statistics are difficult to gather and likely suffer from under-reporting, it is estimated that:

- $10 \%$ of the global population has a mild or worse hearing loss (World Health Organization et al., 2001) and 360 million people (i.e., $5.3 \%$ of the population) are thought to have a moderate (40dB) or worse hearing loss (World Health Organization [WHO], 2013).

- Between 1 in 8 and 1 in 22 Canadians are D/HH. That is, there are approximately 310,000 d/Deaf ${ }^{1}$ and deafened Canadians and possibly 2.8 million Hard of Hearing (HH) Canadians (Canadian Association of the Deaf [CAD], 2007, May 26). A Canadian Hearing Society survey found about $25 \%$ of adult Canadians report having some hearing loss (Canadian Hearing Society [CHS], 2002), while an analysis of responses to the Canada Community Health Survey found about $4.02 \%$ of respondents were thought to have hearing problems (Woodcock \& Pole, 2007).

\footnotetext{
${ }^{1}$ As needed, this Dissertation will follow the established convention of using the upper- or lower-case letter d/D to classify the status of d/Deaf people. "Deaf" people are those who identify themselves as members of the signing Deaf community and regard themselves as culturally Deaf, whereas "deaf" people are those who do not sign and regard themselves as having a significant hearing loss (Woodward, 1972).
} 
- About 1 in 20 Americans are D/HH. That is, about 1 million Americans are functionally deaf and nearly 10 million are $\mathrm{HH}$. More than half of all D/HH Americans are 65 years or older and less than $4 \%$ are under 18 years of age (Mitchell, 2006).

- $16 \%$ of adult Europeans or about 71 million European adults aged 18 to 80 years have a hearing loss greater than 25dB (Hearit, 2006)

There are three types of hearing loss: conductive, sensorineural, and mixed. In a conductive hearing loss, sound is blocked or cannot get into the ear as the result of excess earwax, colds, injuries to the ear (e.g., loss of pinnae), etc. A sensorineural hearing loss occurs where sounds can get into and travel through the ear, but a person cannot perceive the sound as a result of an issue with the cochlea or auditory nerve. A mixed hearing loss occurs where a person has both conductive and sensorineural loss.

A hearing loss can be bilateral (i.e., in both ears) or unilateral (i.e., one ear). Typically, people with a bilateral hearing loss have an unequal hearing loss and will describe one ear as their "better" ear (e.g., a person with a bilateral hearing loss can have some hearing in one ear and no hearing in the other, or hear in both ears to different degrees). Unilateral hearing loss, also called "single-sided deafness" occurs where a person has a hearing loss in only one ear.

While people can experience a hearing loss in specific frequencies, typically a person experiences a loss of a range of frequencies. High frequency hearing loss is the most common and occurs especially among seniors. Human speech is transmitted in the high frequencies. Although vowel sounds are below this range, certain consonant sounds have primary frequencies above $3000 \mathrm{~Hz}$. While there is variation among individuals, persons with high frequency hearing loss can have difficulty hearing the distinction between English sibilants such as the ' $f$ ', ' $s$ ', ' $t h$ ', ' $c h$ ' and ' $s h$ ' sounds and may not distinguish unvoiced consonants like ' $k$ ' and ' $t$ '. Words that begin or end with these sounds can be difficult to hear. The result is that phrases such as "chase the cat" and "shake the sack" sound alike (S. Fox, 2015).

Low frequency or "reverse slope" hearing loss occurs where a person cannot hear sounds in frequencies below $2000 \mathrm{~Hz}$. Since speech information resides in high and mid-range frequencies, a low frequency hearing loss is "symptom free" and harder to identify.

There are many causes of hearing loss. For example (WHO, 2013):

1. genetics;

2. illness (e.g., certain infectious diseases, chronic ear infections, etc.);

3. particular drugs are known to be "ototoxic" (e.g., antibiotics such as gentamicin, loop diuretics such as furosemide, platinum-based chemotherapy agents such as cisplatin, large doses of nonsteroidal anti-inflammatory drugs (NSAIDS) such as acetylsalicylic acid (i.e., aspirin) (Wikipedia, 2015c));

4. age-related (e.g., presbycusis, calcification of bones, etc.) hearing loss can start as early as 18 years of age; 
5. noise-induced hearing loss can be caused by one-time exposure to an intense "impulse" sound (e.g., explosion, firearm), or continuous exposure to loud sounds over an extended period of time (e.g., noise generated in a woodworking shop, iPods, etc.);

6. injury to the ear or head ${ }^{2}$ (Woodcock \& Aguayo, 2000);

7. etc.

While some of these causes (e.g., noise induced hearing loss) can result in hearing losses in specific narrow frequency ranges, other causes can have wider impacts on hearing.

It should also be noted that a hearing loss can be sudden (a noticeable permanent loss) or progressive (variable increasing levels of permanent hearing loss experienced slowly, possibly imperceptibly, over years or decades). This can impact a person's experience of their hearing loss, their ability to cope, and their overall attitude towards it (Woodcock \& Aguayo, 2000).

The number of people with hearing loss is likely to grow for two reasons:

- First, as average lifespans gradually increase and the number of people over 60 gets larger, the number of people with age-related hearing loss will grow. According to United Nations global population projections, almost 1 in 10 people are over 60 years old and by 2050, it is estimated that 1 in 5 people will be over 60 . Globally, people aged over 60 are expected to outnumber children aged $0-14$ by 2050. In developed countries this is expected to occur much sooner, for example, in Canada it is estimated that by 2015 the number of older adults will exceed the number of children (UN, 2011). A recent survey shows $46 \%$ of Canadians aged 45 to 87 have a hearing loss (CHS, 2002).

- Second, as people increasingly expose themselves to loud noise, the number of people with noiseinduced hearing loss will likely grow. Continuous exposure to loud noise can damage the structure of hair cells in the cochlea, resulting in hearing loss and tinnitus. In Ontario, the current maximum noise exposure is $85 \mathrm{~dB}$ per 8 hour work day (Ministry of Labour, 2007). In the United States, the current recommended maximum noise exposure is $85 \mathrm{~dB}$ per 8 hour work day or less than 15 minutes of 100dB exposure per work day (National Institute for Occupational Safety and Health [NIOSH], 1998). Given that a 5th generation iPod Touch has a 40 hour battery life and is capable of volumes as high as $115 \mathrm{~dB}$, music can be played longer and louder.

\subsubsection{Disability}

Populations that are perceived to be "disabled" can be defined / described in multiple ways.

Definitions that focus on the individual as the unit of analysis tend to centre on either a bio-medical model with emphasis on the attributes of the individual, or on a functional-rehabilitative model with emphasis on the promotion or restoration of fuller functioning in the individual. Such definitions frame disability with reference to the individual and in terms of individual deficits. Most of the models in

\footnotetext{
${ }^{2}$ In addition to possible traumatic brain injury, head injuries can damage the structures in the ear and cause a sudden hearing loss.
} 
this category assume a "norm," below which a person should fall if she or he is identified as a person with a disability. Medical definitions have the view that the aetiology of disability resides in the individual as a consequence of events such as disease, accident, war, genetic structure, birth trauma, or other acute causes and that by identifying the aetiology involved, disability can be "treated", "cured", or "prevented". The functional-rehabilitative approach has the view that emphasizes the actions or activities that an individual can perform (with or without assistance) as a result of a bio-medical condition (Hong Kong Equal Opportunities Commission, 1999).

Definitions that focus on the society as the unit of analysis tend to centre either on an environmental model with attention directed to ecological barriers (social, economic, political, institutional and legal) which can result in disability, or a human rights model with emphasis on the rights to which all people, including those with disabilities, are entitled. Such definitions focus not on the individual but on the social, economic, political, institutional and legal conditions that can result in disability. Environmental definitions recognize that a physical, sensory or intellectual disability will not limit many individuals as much as being denied an education, the right to employment, or the right to marry and have a family of their own. Human rights approaches to disability are premised on the recognition of a set of fundamental rights to which all people are entitled regardless of one's individual characteristics, consequently reducing the need for a specific definition of disability (Hong Kong Equal Opportunities Commission, 1999).

While a medical model view of disability ignores the imperfection and deficiencies of the environment (United Nations Commission for Social Development on Disability, 1993), definitions with a focus on society recognize that, "disability has too long been viewed as a problem of the individual and not the relationship between an individual and his/her environment" (Disabled Peoples' International [DPI], 1993). Thus, unlike definitions that focus on an individual's deficits, definitions that focus on society require that the structural conditions in a society that result in disability be addressed and ameliorated.

Just because one is perceived to have a disability in one society does not necessarily mean one is also perceived to have a disability (or even the same disability) in another. For example, the island of Martha's Vineyard once had a strain of hereditary deafness such that a large number of residents were born deaf. As a result, most people on the island knew at least one person who was deaf and a large number of islanders were fluent in the local Sign Language. At a time when mainland United States did not allow deaf persons to hold office, much less vote, there was little social differentiation based on hearing status on Martha's Vineyard (Groce, 1985).

Table 2.1 summarizes some of the ways $\mathrm{D} / \mathrm{HH}$ populations are described / defined by others as well as themselves.

The difference between those who describe themselves as Deaf and those who describe themselves as Hard of Hearing is probably best captured in the cultural definition; the one group is a linguistic minority with their own language, while the other is a distinct subculture within a spoken language community. Not well understood from the ideas presented in Table 2.1 are the concerns of these populations with respect to music.

In addition, there is significant mixing among the populations of people with hearing loss. For 


\begin{tabular}{lll}
\hline \multicolumn{1}{c}{ Definition } & \multicolumn{1}{c}{ Hard of Hearing } & \multicolumn{1}{c}{ Deaf } \\
\hline $\begin{array}{l}\text { Medical } / \\
\text { Audiological }\end{array}$ & $\begin{array}{l}\text { A person with a severe or better hear- } \\
\text { ing loss (HL). }\end{array}$ & $\begin{array}{l}\text { A person with a severe to profound } \\
\text { hearing loss (85+ dB). }\end{array}$ \\
\hline Functional & $\begin{array}{l}\text { A person who gets meaningful in- } \\
\text { formation from sound (possibly only } \\
\text { when it is amplified). }\end{array}$ & $\begin{array}{l}\text { A person who typically cannot get } \\
\text { meaningful information from sound } \\
\text { (even if it is amplified). }\end{array}$ \\
\hline Legal & $\begin{array}{l}\text { A person with a HL who prefers to } \\
\text { use an oral language as their primary } \\
\text { form of communication. }\end{array}$ & $\begin{array}{l}\text { A person with a HL who prefers to } \\
\text { use a sign language as their primary } \\
\text { form of communication. }\end{array}$ \\
\hline Cultural & $\begin{array}{l}\text { A person who identifies themselves } \\
\text { with the Hard of Hearing commu- }\end{array}$ & $\begin{array}{l}\text { A person who identifies themselves } \\
\text { with the Deaf community (such a per- } \\
\text { nity or as a member of a social group } \\
\text { of HH people. }\end{array}$ \\
& & \\
\hline
\end{tabular}

Table 2.1: Medical, functional, legal, and cultural definitions of "Deaf" and "Hard of Hearing".

example, some people who would fit the medical definition of a deaf person are functionally hard of hearing. Some HH people are members of the signing Deaf community. This mixing can confound research results that rely on poorly defined group differences. From a design perspective it is vital to understand that these are distinct groups with very specific user needs.

\section{Deaf}

People who are deaf are not able to hear and/or unable to make meaning of anything they might perceive as sound.

Hearing is a specialization of the sense of touch. The two senses share a common genetic basis (Frenzel et al., 2012). Both deal with force. Both rely on mechanosensation. Physiologically, touch and hearing both convert mechanical stimuli into neuronal signals: cutaneous mechanoreceptors are responsible for touch while inner ear hair cells are responsible for hearing. Ultimately, a common physicochemical basis likely determines how channel proteins sense force (Kung, 2005).

Since hearing, is a specialization of the sense of touch, people who are deaf can feel sound travelling through the ear canal (e.g., as a tickling sensation as the hairs in the canal are moved) but may have no associated meaning to the sensation. People who are born deaf are very sensitive to vibration and can feel loud noise as the sound wave vibrates their body.

Thus people who are born deaf tend to have a limited music experience in the auditory sense. The full range of musical sounds in recorded music is simply not accessible to them. Presumably, this would mean that people who are born deaf have a limited psychological reality to auditory music. Chapter 4 will explore some reasons why this may not be so. 


\section{Hard of Hearing}

People who are born hard of hearing $(\mathrm{HH})$ tend to have some limited ability to hear music. Depending on the degree of hearing loss, whether the loss is mostly high frequency or low frequency, and if the hearing loss is bilateral (i.e., both ears) or one-sided, HH people experience different barriers when attempting to access the music of the larger hearing culture.

The full range of musical sounds in recorded music is somewhat accessible to this population. Since their residual hearing may not allow the individual to hear the full range of tones or perceive sound in stereo, for many $\mathrm{D} / \mathrm{HH}$ music consumers different parts of music are missing / muffled. In fact many $\mathrm{D} / \mathrm{HH}$ people may choose to listen to music that fits within their ability to hear it either with or without amplification. This may mean that, whatever music experience a D/HH person has could be skewed since amplifying any sound can distort it. (Chapter 4 will explore this population more fully.)

\section{Deafened}

People who are born hearing and later experience a loss of their hearing may refer to themselves as deafened. Because a hearing person would potentially have a life experience with music and potentially develop a psychological reality of music, the experience of hearing loss does not mean that the knowledge of music is lost only that the ability to hear it is lost. The memory of music remains. The ability to form new memories such as by experiencing new songs is lost. Chapter 4 will explore this population more fully.

\subsection{The Nature of Music}

Music has different meanings to different people and cultures, including D/HH culture, and is expressed in different ways. The literature regarding performed music or sound typically assumes that the reader can hear. Few authors make an attempt to explain music to those who cannot hear or do not hear very well. As a result, many of the definitions of music and its properties use auditory-based terminology. This section will attempt to provide definitions of the various aspects of music and explain the principle components in sound-independent ways.

Various dictionaries have very different definitions of music.

Webster's International Dictionary defines music as (Gove et al., 2002, p. 1490):

- "the science or art of incorporating pleasing, expressive, or intelligible combinations of vocal or instrumental tones into a composition having definite structure and continuity"

- "vocal or instrumental sounds having rhythm, melody, or harmony"

- "an agreeable sound"

The Random House Dictionary of the English Language defines music as (Flexner \& Hauck, 1987, p. 1268): 
- "an art of sound in time that expresses ideas and emotions in significant forms through the elements of rhythm, melody, harmony, and color."

- "the tones or sounds employed, occurring in single line (melody) or multiple lines (harmony), and sounded or to be sounded by one or more voices or instruments, or both."

- "any sweet, pleasing, or harmonious sounds or sound: the music of the waves."

The third edition of the American Heritage Dictionary of the English Language defines music as (Editors of the American Heritage Dictionaries, 1992, p. 1190):

- "The art of arranging sounds in time so as to produce a continuous, unified, and evocative composition, as through melody, harmony, rhythm, and timbre."

- "Vocal or instrumental sounds possessing a degree of melody, harmony, or rhythm."

- "An aesthetically pleasing or harmonious sound or combination of sounds: the music of the wind."

The 1913 edition of Webster's notes, "Not all sounds are tones. Sounds may be unmusical and yet please the ear. Music deals with tones, and with no other sounds." (Porter, 1913, p. 956).

In summary, music involves an ordering of tones. It has an aesthetic quality that is pleasing to those who can hear it. It is a science and an art. The definition from Random House suggests that music "expresses ideas and emotions", thus music is a means of communication.

Levitin notes that, when listening to music, we hear multiple attributes of music at once. He lists tone, pitch, timbre, loudness, tempo, rhythm, contour, spatial location, and reverberation as the "fundamental building blocks" (Levitin, 2007, p. 14) of performed music. The first eight of these perceptual attributes can be varied independently (Levitin \& Tirovolas, 2009). All nine are the primitives upon which all other properties of music are built.

- A tone is a discrete musical sound. A note is something written on a score. While both terms appear to refer to the same thing, the former is heard while the latter is seen (Levitin, 2007).

- Pitch is the psychological construct people use when thinking about tones. While it is related to the actual frequency of the sound wave of a tone, it is not equivalent because while frequency is an objective, scientific concept, pitch is subjective. When we double or halve a frequency, we end up with a note that sounds remarkably similar to the one we started out with. This relationship, a frequency ratio of 2:1 or 1:2, is called the "octave." (Levitin, 2007, p. 29) For example, if the A above middle $\mathrm{C}$ is $440 \mathrm{~Hz}$, the $\mathrm{A}$ an octave above that is $880 \mathrm{~Hz}$.

Pitch is what gives listeners the sense of a tone's relative position in the musical scale (higher or lower). "Pitch is one of the primary means by which musical emotion is conveyed" (Levitin, 2007, p. 26). For example, a high note can convey excitement, a low note can convey sadness, and a series of notes creates a more powerful, nuanced melody.

Pitch is so important that the brain represents it directly; unlike almost any other musical attribute. The auditory cortex has a "tonotopic" map, with low to high tones stretched out across 
the cortical surface. In this sense, the brain contains a "map" of different pitches, and different areas of the brain respond to different pitches (Levitin, 2007, p. 27).

- Timbre (sometimes called "colour") is that quality that allows a listener to easily distinguish between the sounds of different instruments even when they play "the same note, for the same length of time, at the same loudness" (Schmidt-Jones, 2013). "A listener can hear not only the difference between an oboe and a flute, but also the difference between two different oboes." (SchmidtJones, 2013). Font faces provide a non-sound analogy to timbre. The same character can be represented in different fonts (e.g., Times Roman, Arial, etc.), a 'B' is still a 'B', but the personality of the font can be easily perceived. With experience, one can name different font faces. The definition of music from Random House suggests that music has "color". There is no mention of whether this is a reference to a form of synesthesia (e.g., see Cytowic, 2002) or to timbre. Schmidt-Jones' (2013) description of timbre as a property of music implies that music has qualities such as loudness and length, as well as pitch. It also raises questions about what is important to the listener (e.g., Can the listener distinguish between two different oboes? Does the listener want to distinguish between two different oboes?).

- Loudness is a psychological construct that relates to the physical amplitude of the tone (Levitin, 2007). The intensity level of a sound can be objectively measured and expressed in the deciBel (dB) scale. Conversely, the loudness of a sound is subjective and varies from person to person. Sounds with equal intensities but different frequencies are perceived by the same person to have unequal loudness. The non-SI unit phon is used to indicate an individual's perception of loudness

- Tempo is the overall pace or speed of the piece (Levitin, 2007).

- Rhythm is the way that the durations of a group of notes fit together into larger units (Levitin, 2007). For example, visually, rhythm appears in a musical score using different notes (e.g., whole, quarter, etc.) to denote their individual durations. Grouping them together and grouping these groups is what leads to rhythm. For example, Ballroom and Latin dance are visual expressions of rhythm, the dancer's feet and body act out the rhythm.

- Contour describes the overall shape of the music, the up and down of the melodies. It describes whether a note goes up or down, not the amount of change (Levitin, 2007). It is the most identifying attribute of music (Dowling, 1999). Visually, contour might be thought of as curves the written notes make on a piece of sheet music.

- Spatial location is the source of the sound in three dimensional space. Human stereo hearing is very sensitive to position in the left-right plane and somewhat sensitive in the forward-back plane. Humans are not very sensitive to location in the up-down plane (Levitin, 2007). People try to mimic spatial location through the use of stereo headsets and surround sound entertainment systems. 
- Reverberation (or "reverb") is the sense of how far the source of the sound is (or appears to be) combined with the size of the room the music is in. Reverberation, "has an underappreciated role in communicating emotion and creating an overall pleasing sound" (Levitin, 2007, p. 16). As sound bounces off objects and surfaces around the listener, it builds up the experience. It is for this reason that poorly designed concert halls might reduce a sound such that the listener cannot hear anything or cannot perceive a stereo effect (Sacks, 2007).

For example, when clapping one's hands in a small room full of furniture and heavy curtains, the sound dies away almost immediately and the room is said to be acoustically "dead", but when clapping one's hands in a large room with hard walls, the sound bounces back and forth off the walls several times before the sound dies away and the room is described as "lively." Thus, musicians sound better in a "live" room than a "dead" one (Powell, 2010, p. 231).

People try to mimic reverberation through the use of stereo headsets and surround sound entertainment systems. Schroeder describes proper reverberation as "colourless", it does not sound like "rain on a tin roof" (Schroeder, 1992, p. 42), and free of unwanted flutter, a pistol shot sounds like a single decaying shot, not like a machine gun burst.

A visual analogy of good reverberation would be a circle, bubble or other icon, slowly, smoothly, and evenly shrinking or fading out. A visual analogy to this experience of a sense of space can be experienced in rooms that have large mirrors on opposite walls (Shepard \& Levitin, 2002).

Reverberation should not be confused with Echo. Although one may enjoy the effect of sounds lasting longer because they are bouncing around the room, the goal is that all the bounced sound from each note overlaps so that it reaches one's ears as a single, long note. If the walls of the room are too far apart, the time between bounces will be too long and the experience will not be a single, extended note. Instead one will hear the note, then a gap, then the note again. This effect is "echo." Concert hall designers live in the hope that their designs will give the audience lots of pleasant reverberation, but no echoes (Powell, 2010, p. 231).

According to Levitin, these attributes are separable; varying one will not alter the others. Although the production of music can be broken down into these attributes, how the perceptual and cognitive system processes the signals representing these attributes to be meaningful is complex and influenced by cultural factors. The brain organizes these musical attributes into higher level concepts such as metre, key, harmony, and melody (Dowling, 1999; Levitin, 2007; Weinberger, 1999):

- Metre, in which tones are grouped with one another across time, is created through a combination of loudness and rhythm cues.

- Key is a culturally defined hierarchy of importance between tones in a musical piece.

- Harmony, in its most basic form, is a parallel melody to the primary one. It is the relationship between the pitches of different tones. 


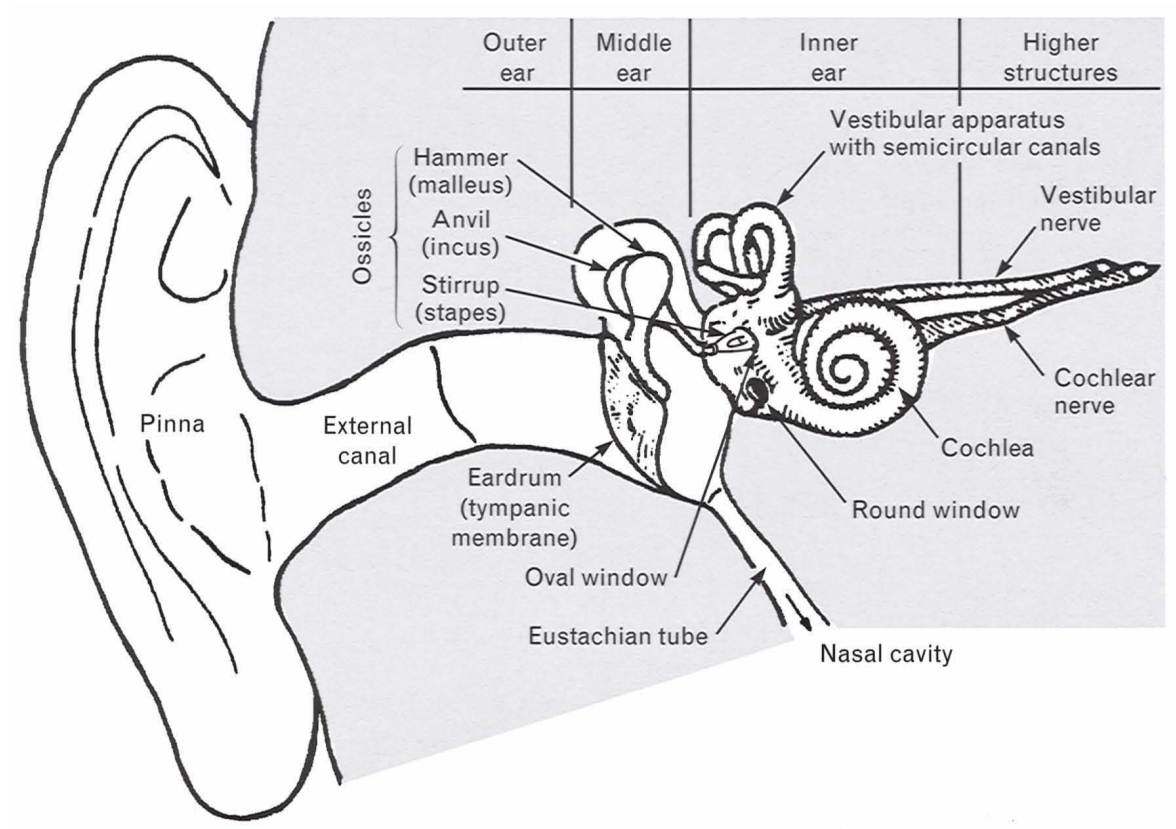

Figure 2.1: The human ear (Tierney et al., 1994).

- Melody is the main theme of a musical piece. While the basic notion of melody changes with each genre, it is essentially, "the piece you sing along with" (Levitin, 2007, p. 16). "Melodies are defined by the pattern or relation of successive pitches across time" (Levitin, 2007, p. 27). Note, melodies are based on certain subsets of tones (i.e., their "key"), not all available tones in an octave.

\section{Human Perception of Music}

Auditorially, sounds, such as music, are perceived via the auditory pathway which consists of the outer, middle, and inner ears, the auditory nerve, nuclei at each level of the brain stem, and several areas of the auditory cortex (Weinberger, 1999). A detailed discussion of the full auditory pathway is beyond the scope of this paper. What follows is a brief discussion of the sense of hearing (Hawkins, 2014).

As shown in Figure 2.1, the outer ear consists of the pinna and external canal (i.e., ear canal), ending at the tympanic membrane (i.e., the eardrum). The pinna, the visible portion of the ear, collects sounds and directs them into the ear canal to the tympanic membrane. The ear canal is a passage that concentrates and amplifies the air vibrations to the tympanic membrane. The tympanic membrane converts the vibrations of the air into mechanical vibrations.

The middle ear is an air-filled cavity located behind the tympanic membrane. The air comes from the back of the pharynx through a Eustachian tube. The middle ear contains three bones: the malleus (or hammer), incus (or anvil), and stapes (or stirrup). These are arranged so that movement of the tympanic membrane causes movement of the malleus, which causes movement of the incus, which 
causes movement of the stapes. The stapes ends at the oval window converting the mechanical vibration into vibration of liquid.

The inner ear, or cochlea, is the organ of hearing. As the footplate of the stapes presses into the oval window of the cochlea, the fluid inside of the latter is moved, flowing against the receptor cells (i.e., hair cells). As these receptors fire, they stimulate the spiral ganglion, which sends information through the auditory portion of the eighth cranial nerve to the brain. The inner ear also contains the vestibular system, which is beyond the scope of this paper.

Once the perceptual system processes the external sound data, the cognitive system is required to make it meaningful. Music derives from three human cognitive abilities: theory of mind, recursion, and abstract representation (Levitin \& Tirovolas, 2009). Theory of mind refers to the human ability to attribute mental state to oneself and others. Cultural experience (through performance, education, observation and/or training) and innate abilities (such as a sense of rhythm) contribute to the theory of mind states for music. Recursion is the ability to repeat themes and create variations on themes. Music is thematic and often repetitious, as the human mind is exposed to this over time, recursive elements are established in the cognitive system. Abstract representation is the ability to construct meaning and interpretation from various signals that enter the brain through the perceptual systems. Combined with cultural experiences, learning, recursion properties and practice, the human mind can draw connections between perceived auditory signals and emotion, entertainment, and other interpretations of music.

Research in the cognitive mechanisms relating to perception provides evidence for auditory analogues to visual perception. For example, the size constancy of vision and the loudness constancy of audition are analogous. Size constancy is based on the idea that while objects on the retina expand and contract as the object moves closer and farther away, objects in the real world are of constant size. One can perceive an object, as it is, independently of its distance. Similarly, as the amplitude of a sound changes, its source may be perceived as closer or farther away. Accompanying changes in timbre (i.e., the musician's changing effort will influence the higher-frequency components of the sound), help the listener determine whether the change in amplitude is due to changing distance or a simple decrease in amplitude (Shepard \& Levitin, 2002).

Another example can be seen in perceptual completion. When visually presented with a shape interrupted with white spaces, the closure effect automatically completes the shape in one's mind. A similar effect can be seen auditorially, when a sinusoidal sound is replaced by bursts of white noise, the effect on the listener is a smoothly sweeping sinusoidal sound covered up by noise bursts. There is a similar effect when music is used in place of sinusoidal sound (Shepard \& Levitin, 2002). Understanding how these and other cognitive and perceptual systems contribute to musical processing may help in developing visualizations of music.

The result of combining the higher order properties of music (e.g., metre), which themselves resulted from the combination of the primitives of music, has an aesthetically pleasing effect. (Levitin, 2007) does not mention the aesthetics as a noteworthy property of music, however, I suggest that it is important because it affects the entertainment value of music. The dictionary definitions also define music as having "an agreeable sound" (Gove et al., 2002, p. 1490). Put simply, we perceive cer- 
tain note progressions as "pleasant", while others we perceive as "unpleasant" or "wrong". In the same way, certain groups of notes (i.e., chords) sound more pleasant than others. As highlighted in the song "Four Chords" by The Axis of Awesome, it is for this reason that formulaic instrumentation (Percino, Klimek, \& Thurner, 2014) and the so-called "pop-punk chord progression"3 is seen in many contemporary pop and rock songs (Murphy, 2014; Wikipedia, 2015b). This suggests a property of music is agreeableness:

- Agreeableness is the aesthetic qualities of music that make it enjoyable and pleasing.

Another property that is often assumed when discussing music is the notion of binaural hearing that can take advantage of stereo sound. Assuming a person has two working ears, binaural hearing is ability to use the information provided by the differences between one's two ears (Colburn, ShinnCunningham, Kidd, \& Durlach, 2006). In particular, binaural hearing provides the listener with localization of sound (i.e., the subjective sense of the location of a sound source based on interaural temporal and intensity differences) and summation for enhanced loudness (i.e., the subjective experience of loudness of a sound presented simultaneously to both ears is approximately double that presented to one ear) (Welsh, Welsh, Rosen, \& Dragonette, 2004).

Sacks remarks that, until one experiences single-sided deafness, "We tend to take our stereo world for granted" (Sacks, 2007, p. 146). He argues that people who cannot hear in stereo miss the qualities of "rotundity", "spaciousness, voluminousness, richness, [and] resonance" (Sacks, 2007, p. 144). Each of these terms refer to the way that sound can fill a space; its fullness. For example, "richness" refers to sound being full and mellow in tone. From these properties, a listener can sense the depth and distance of music. Without them, music becomes "flat". The ability to hear in stereo provides another avenue of emotional content through the fullness of music and effects such as reverberation.

There are numerous properties and qualities of music, some of which rely on combinations of two or more lower level or primitive properties (e.g., metre). What is less obvious is which properties are most important to the overall experience and enjoyment of music and whether there is a set of primitive properties that can be used to describe all other properties. Identifying a set of important primitive properties would provide a strong foundation for and the necessary building blocks to develop an approach to visualizing music that focuses on these properties. Determining those primitives which can be used to describe the other properties also creates the possibility that the visualizations of such primitive properties could be combined to create higher level properties.

Composers use these properties in creative ways to create music that fits the desired mood they want to express in the hopes the audience interprets the same mood. The effect that music has on people can be multidimensional. People listening to music experience emotion, entertainment, pleasure, or a welcome distraction.

\footnotetext{
${ }^{3}$ This progression is specifically a cadence of the tonic, dominant, submediant, and subdominant cords, or I-V-vi-IV in Roman Numeral notation. For example, in the key of $\mathrm{C}$ major, this would be: $\mathrm{C}-\mathrm{G}-\mathrm{Am}-\mathrm{F}$.
} 


\subsection{The Nature of Emotion}

Various psychological models exist for describing emotions. They include, the PAD (pleasure-displeasure, arousal-nonarousal, and dominance-submissiveness) model (Mehrabian, 1996), and the models of basic emotions (Ekman, 1999; Plutchik, 1980; Sauter, Eisner, Ekman, \& Scott, 2010).

The PAD model of emotion is a dimensional model that attempts to decompose emotions into three orthogonal underlying dimensions: Pleasure (or valence), the degree of pleasantness of the emotional experience; Arousal, the level of activation of the emotion; and Dominance, the level of attention or rejection of the emotion (Mehrabian, 1996; J. A. Russell \& Mehrabian, 1977). Each element has opposing dimensions (pleasure / displeasure, arousal / sleep, and dominance / submission) and forms one axis with opposing end points of a three-axes model. Emotional responses can be plotted along each of these axes. This model is complex to use as it requires the dissection of emotional responses into three separate categories. While commonly used in marketing research to understand the role of dominance-submissiveness in consumer behaviour (Yani-de Soriano \& Foxall, 2006), the PAD model has been used in HCI research (e.g., Lottridge, 2008).

The psychological models of basic emotion proposed by Plutchik (1980) and Ekman (1999) suggest that emotions are analogs to colours on a colour wheel, such that there are "primary emotions" from which all other emotions are derived. They suggest that there exists a set of five to eight primitive emotions. Plutchik's eight emotion model includes acceptance, anger, anticipation, disgust, joy, fear, sadness, and surprise (1980). The five emotion model of Ekman and Friesen, which is based on crosscultural facial expressions research, includes anger, happiness, sadness, fear, and surprise (1986). In a review of a dozen different lists of basic emotions and their biological bases, Ortony and Turner (1990) found, "that there is no coherent nontrivial notion of basic emotions" (1990, p. 315); however, they suggested that there are "basic elements" (1990, p. 329), which are not themselves emotions, out of which different emotions are built. H. Smith and Schneider also found no support for the universality of these models of emotion or the emotion wheel theory and further argues that Ekman's model is, "impossible to replicate. . without introducing pretest training and forced-choice formats" (H. Smith \& Schneider, 2009, p. 583). Regardless of their controversy, these models have been used to develop a model for formulating emotive captions that combine graphics and text to represent emotion and sound effects and test them with D/HH audiences (e.g., Lee, Fels, \& Udo, 2007).

\subsection{Music and Emotion}

Emotion is one important outcome of music; it affects people's feelings. For example, Toland describes, "the excitement, the rush, the indescribable thrill that moves me to turn up the volume, sing along with the melody or tell everyone in the room to shut the hell up so I can listen." Others use music as a background experience to cover up (or as a distraction from) the hum of the refrigerator (Toland, 2001), mask tinnitus (Fourney \& Fels, 2009), or, in the case of early silent movies, drown out the noise of clanking projectors (Cavalcanti, 1939). Still others draw on music for entertainment, as a form of escape, or to fill time. 
Csíkszentmihályi (1990) has found that the performance of music can create for the performer a state of mind which he calls "flow". Flow is, "the state of mind we enter when completely involved in making music, doing sports, or whenever we are focused on any challenging and enjoyable activity" (Csíkszentmihályi, 2004, p. 341).

For D/HH people, the emotional element of music may be unknown to them. Darrow explored the ability of deaf people to perceive emotion in music and reported that, "When the instructions for the music listening task were given to the Deaf participants, many were amused at the idea of determining the emotion of the music. Asking them to emotionally interpret music was obviously a novel concept to them." (Darrow, 2006, p. 11) Suggesting some D/HH people are not aware that there is an emotional component to music, while others are. In addition, for some D/HH people, the emotional experience may include the frustration of inaccessible information in the music.

\subsubsection{Emotion Conventions in Music}

Although it is well-known that listeners make associations between music and emotion, it is not known how much of this experience is innate and how much is learned through cultural interpretation (Darrow, 2006). While culture may play a role in judgments of emotion in music, compositional dimensions of music, such as tempo, rhythm, texture, timbre, and pitch, also influence the listener's perception of emotion in music and may serve as universal cues for the emotional assessment of music (Darrow, 2006).

Certain emotional associations with music may have been culturally conventionalized. For example in Western music, Darrow (2006) suggests:

- major mode is associated with happy, minor mode with sad,

- loud music is associated with animated or happy, soft music with delicate or peaceful,

- even-valued rhythms are seen as serious, uneven-valued rhythms as playful or happy, and

- staccato articulation is seen as lively or energetic, legato articulation is seen as peaceful or gentle.

The approach of looking for music conventions has been the basis of work by various authors. For example, Cooke analyzed elements of musical expression to establish a lexicon of the specific emotions these elements were able to convey. He attempted to test this theory empirically and took as his material Western tonal music composed since the 15th century. He identified several musical patterns that were commonly used to express specific emotions in this set of music (Cooke, 1959). However, efforts to substantiate his theory empirically have been unsuccessful (Balkwill \& Thompson, 1999).

Kivy (1980) also theorized that certain melodic patterns are conventionally associated with specifiable expressive predicates, such as "is sad," "is angry," or "is joyful". However, he acknowledged that some links between music and emotion may be "natural." Conventions that Kivy identified as being typical of Western classical music included falling melodic lines signifying sadness, leaping melodic motion signifying joy, and slow tempo with measured rhythm signifying dignity. 
Western listeners are able to perceive emotional expressiveness in the music of an unfamiliar tonal system and evidence suggests that this ability is based on psychophysical elements such as tempo, timbre, and complexity rather than musical experience, knowledge, or enculturation (Balkwill \& Thompson, 1999).

These associations are important because they provide some direction in determining the presence of emotion in music and eventually expressing emotion through music visualization. With respect to determining the presence of emotion in music, an automated analysis of the musical piece could be used to find these attributes and identify them for translation into a visualization (e.g., Kim et al., 2010).

With respect to visualizing the emotion of music, it might be possible to map these types of associations to equivalents in the visual domain. For example, the loudness of the music could be translated into quickness of the animation. If loud music is animated or happy, then a more animated faster moving visualization might be able to communicate happiness. Conversely, if soft music is perceived as quieter, then a slower, more subtle animation might be able to communicate sadness. Another possibility is to express staccato articulation through a series of rapid flashes while legato articulation could be expressed through flashes with much longer durations.

These musical conventions and other psychophysical elements are used in film and television entertainment to provide the emotional atmosphere. It may be useful to briefly examine how music is used in film entertainment and how it reflects the use of these emotional associations to enhance the visual experience.

\subsubsection{Emotion Conventions in Music in Film}

While the earliest films did not have a soundtrack, they were by no means "silent". Live sound accompaniment (e.g., live piano) was used from the beginning to give viewers a sense of being part of the scene. In the early 20th century, films were accompanied by phonographs. As films got longer, film showmen would employ barkers to provide commentary and attempt synchronized speech. With the opening of the first cinemas, intertitles, printed dialogue or narration shown between scenes, were introduced to explain the action and dialogue while music would be used as an accompaniment (Cavalcanti, 1939). Figure 2.2 shows an intertitle from the comedy short All on Account of a Transfer (Williams, 1913).

With the advent of commercial films, which were housed in permanent theatres, a more complicated and elaborate sound process where the music played an increasingly larger role in the show was introduced. The competition among cinema owners meant that one piano became three; the three pianos would give way to a salon orchestra, which in turn gave way to a symphony orchestra (Cavalcanti, 1939).

In these early days, music was used for two reasons: to hide the noise of the projectors and to provide an emotional atmosphere (Cavalcanti, 1939). From the beginning, it was understood that the sound affects the scene. In modern film and television, sound is edited last because it is the final opportunity the director has to fully shape the scene (Handzo, 1985). 


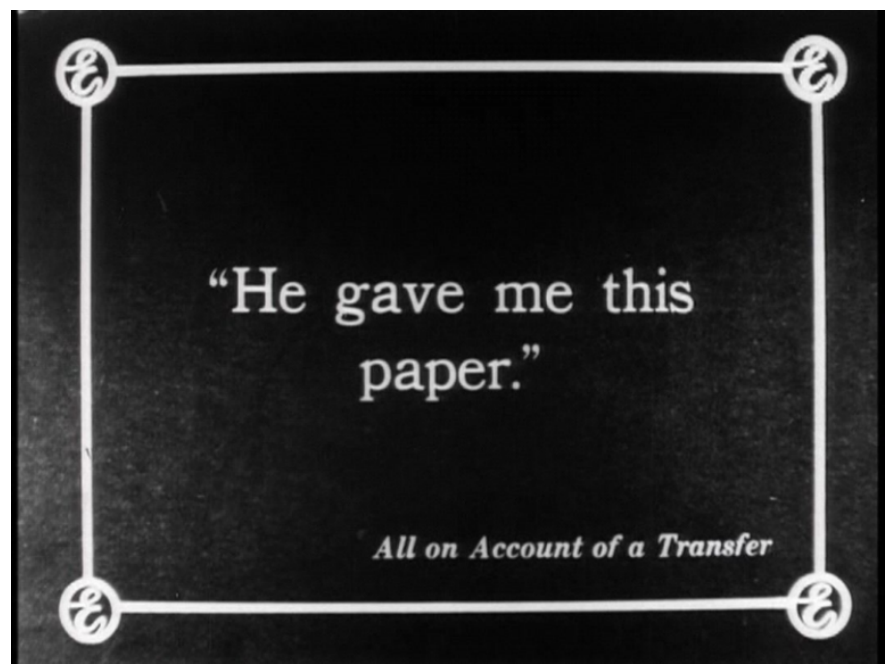

Figure 2.2: Intertitle from "All on Account of a Transfer" (Williams, 1913).

When sound was first introduced in film, a system of leitmotifs was used; certain themes would be associated with certain characters as they appeared on the screen. Throughout the film, the orchestra would move from one short theme to the next (Cavalcanti, 1939). These pieces of music would be relatively well-known to the audience (e.g., portions of Pique Dame, Londonderry Air, the Mass in B Minor, etc.) (Cavalcanti, 1939). They were known by musicians to elicit specific moods responses (no one says how it was determined what mood category a piece belonged to). Either large film companies would prepare and publish a complete musical score for distribution with the films or the musicians would keep multiple sheet music on their stands, switching from one to the next as needed (Cavalcanti, 1939). These themes might also come from a published "mood music catalogue" that provided scores for specific moods (e.g., terror, joyful, etc.) Wierzbicki (2009). In essence, someone watching the same movie in two different theatres might have had similar emotional experiences, but heard different "sound tracks" to achieve it.

With the introduction of the cinema organ came the ability to render effects. The organ's console would have several stops which might be labelled "train", "chains", "crockery", "horse", "siren", "side drum", "bass drum", "cymbals", "piano", "airplane", "child crying", etc. (Cavalcanti, 1939). This allowed the creation of live sound effects in the theatre as well as live music, further enhancing the emotional effect of the movie experience.

The first "talkie" film using pre-recorded sound technology (called Vitaphone) was The Jazz Singer which opened in 1927. Vitaphone, which used 16-inch phonograph records, was developed in 1925. By the time The Jazz Singer opened, more than 130 American theatres were equipped with Vitaphone devices.

The sound came directly from the phonograph player or, by microphone, through a public-address system. The first public showing of a movie using the first sound-on-film technology, Movietone (also developed in 1925), was a newsreel in 1927. Movietone used a variable-density optical track on the 
same strip of film. There was no separate sound system required as the projector came with its own speakers. Vitaphone was owned by Warner Brothers, Movietone was owned by Fox. Another soundon-film technology developed by General Electric (and RCA), Photophone (patented in 1925), was used by everyone else (Wierzbicki, 2009). The sound-on-film formats of Movietone and Photophone became the dominant technologies. This technology is the basis of modern film sound (i.e., Dolby).

Once the technology existed to integrate sound into the film itself, there was an immediate interest in speech synchronized with the moving picture. Soon after, the first "musical" films, movies based on Broadway musical shows, were introduced. This gave way to "performed-music" movies with personalities such as Canadian Deanna Durbin. Instead of the music being the sole focus of the film, the action of the story would continue while the performer sang. Combining music and drama had the effect of dramatic excitement, a new third element, "in which both music and images are enhanced, and suspense, humour, sentiment, acquire almost sensational valency." (Cavalcanti, 1939, p. 33).

As a result of combining music and drama, two different kinds of film and television music emerged: diegetic, and non-diegetic. Diegetic, or source, music occurs where the source of the sound is presented within the scene (e.g., song on a radio, singer in bar, etc.). Non-diegetic (also called "extra-diegetic") music has no visible source in the scene; the music seems to come from nowhere and is not heard by the characters in the scene (e.g., mood music) (Kassabian, 2001). While commonly used in Europe, non-diegetic music did not enter the North American film industry until the 1950's (Wierzbicki, 2009).

After World War II the presence of music in film became, as Jerry Goldsmith describes, saved for the "right moments and make them count", rather than used to fill the screen with as much sound as possible (Champlin, 1967). With the commercial success of the title song of High Noon in 1952, there was a phase where movie music production became more commercialized with the popular focus of getting soundtracks onto the charts and creating a commercial sensation (Wierzbicki, 2009). The "art" of moviemaking was no longer the sole motivation for film technology development. While it is not as common to see whole soundtrack albums hit the charts, there is still emphasis in marketing specific songs either when artists are also in the movie (e.g., Preacher's Wife) or a popular artist records parts of the soundtrack (e.g., Titanic, Frozen).

A musicians' strike in 1958 and the temporary loss of music in films meant the orchestral score became less common and film music branched out into so many different forms (e.g., rock, jazz, blues, pop, synthesized, etc.), that the "classical" genre typical of previous films was no longer the only genre. Current film music cannot be described as a single genre (Wierzbicki, 2009).

Further, with the added competition from television, studios focused on the development of new sound technologies, such as surround sound, to bring in audiences (Wierzbicki, 2009). The development of surround sound changed how audiences experienced music and other sound effects in the theatre. It opened up a whole new world of possibilities for sound use and design that allowed for greater realism because, if you can hear in stereo, it creates the illusion of being in the middle of the events in the film. The advent of surround sound also motivated greater quality control (e.g., through THX certification) to ensure clearer sound in all theatres.

In modern film and television, music is used to provide "stock characterizations", musical archetypes that are based on cultural stereotypes, and instrumentation (Gorbman, 1987, p. 83) of the characters 
and scenes. For example, Kalinak (1982) describes conventions used in films in the forties and fifties to illustrate stock characterizations of "the fallen woman" and "the virtuous wife". Where the former gets dotted rhythms and jazz or blues style saxophones, the latter is presented with violins and flutes that sweep upwards melodically using even rhythms and simple harmonic language.

Gorbman (1987) describes Hollywood's use of codified harmonies, melodic patterns, and rhythms in cinema for establishing setting. For example, the notion of "Indian territory" would be set by using a 4/4 allegretto drumbeat with the first beat emphatically accented. Turn-of-the-century Vienna might be set using a Strauss-like waltz. Rome and Paris are set using accordions. Similarly, a comedic character might be introduced by woodwinds or xylophones with occasional wrong-sounding notes.

The impact of sound on the attention of audience members has some fairly well-known conventions and strategies. Audience members tend to give different levels of conscious attention to the music at different times according the prominence of the music compared with other sounds and visuals. Kassabian (2001) explores an "Attention Continuum" (p. 52) to describe this phenomenon. In descending order:

- Theme songs. The audience will give the highest degree of attention to the theme song. They may have familiarity with it from film trailers, advertisements, other films, etc. Theme songs are used not just with the opening credits and opening scenes but also used as leitmotifs later in the film (e.g., James Bond).

- Music presented as the only sound in a scene. Where there is no dialogue or other sounds in a scene competing for auditory attention, the music gets all of the auditory attention.

- Music presented with little audio/visual competition. Sounds are in low profile compared to the music.

- The scene is memorable but the music is not. Scenes with a great deal of visual activity with sound effects and music but little or no dialogue tend to have forgettable music. The music is usually presented to provide "a sense of pace and unity without commanding much attention" (p. $54)$.

- Music presented as background to dialogue. Depending on the importance of the dialogue in the film, background music can be simple, subtle, and soft. Its role is only to affect mood. The audience will give little attention to such music.

Other than these fairly basic strategies, the integration of music in film or television tends to follow a fairly intuitive process rather than programmatic. Holden (1989) states that in film music, "what's good is what works" (H19). However, one possible dilemma of this approach when examining how to convey this mantra using the visual domain is that it is not necessarily true that "what works" musically will translate into an equivalent visual expression directly. 


\subsection{Sensory Modalities}

There are four major sensory modalities that can be used to present music.

\subsubsection{Auditory}

According to Loeffler, Western music uses a 12-tone octave tuning system (i.e., a "perfect octave"). An interval between two musical pitches is half or double the note's frequency; hence, the ratio between octaves is 2:1. For example one octave above A4 $(440 \mathrm{~Hz})$ is $880 \mathrm{~Hz}$ while one octave below is $220 \mathrm{~Hz}$. Octaves with a distance greater than 1 are calculated as $2^{\mathrm{n}}$, where $\mathrm{n}$ denotes the octave. Perfect octaves are further split into 12 semi-tones, where each semi-tone contains 100 cents; thus, each octave contains 1200 cents (Loeffler, 2006).

Notes produced by instruments are not a single pure tone frequency, but rather "a mixture of sinusoids of different frequencies with different weights that are perceived to be equivalent to single frequency" (Loeffler, 2006). For this reason, although humans can differentiate auditory pitches separated by 5 to 6 cents and musically untrained adults can reliably recognize differences of 25 cents, a human's ability to differentiate pitches of a note can be off by as much as \pm 12 cents (Geringer \& Worthy, 1999; Loeffler, 2006; Peretz \& Hyde, 2003).

The auditory modality would appear to be an obvious way to present music since music is naturally presented as sound. This modality is not useful to us because this is where the problem of presenting music to $\mathrm{D} / \mathrm{HH}$ audiences lies.

\subsubsection{Tactile}

The development of technologies using the tactile modality to present music to $\mathrm{D} / \mathrm{HH}$ people has a very long history. Here are a few examples:

In December 1896, McKendrick reported to the Philosophical Society of Glasgow an experiment in electricity where he ran wires from a phonograph to two platinum plates dipped in a beaker containing a weak salt solution. Putting one's fingers into the salt solution while the phonograph was playing would stimulate the nerves such that one could feel rhythm, time, tone duration and intensity, each distinctive note, and each chord of music played by the phonograph via electric resistance, but no sensation of pitch. He reported to the Society a study involving four deaf persons, one of whom became deaf at eleven years old. She declared that what she felt was music. The others were able to appreciate the rhythm, bobbing their heads up and down, keeping time with the sensations in their fingertips (McKendrick, 1897).

The following year, in a report to the Royal Society of Edinburgh, McKendrick reported repeating this experiment using a rubber lined trough for the saline solution and having deaf people put their hands into the trough. Experimenting with the deaf, he found they were startled by the effect. Deaf participants reported a pleasure sensation and felt they had a better appreciation for why people coveted music (see McPherson, 1897). 


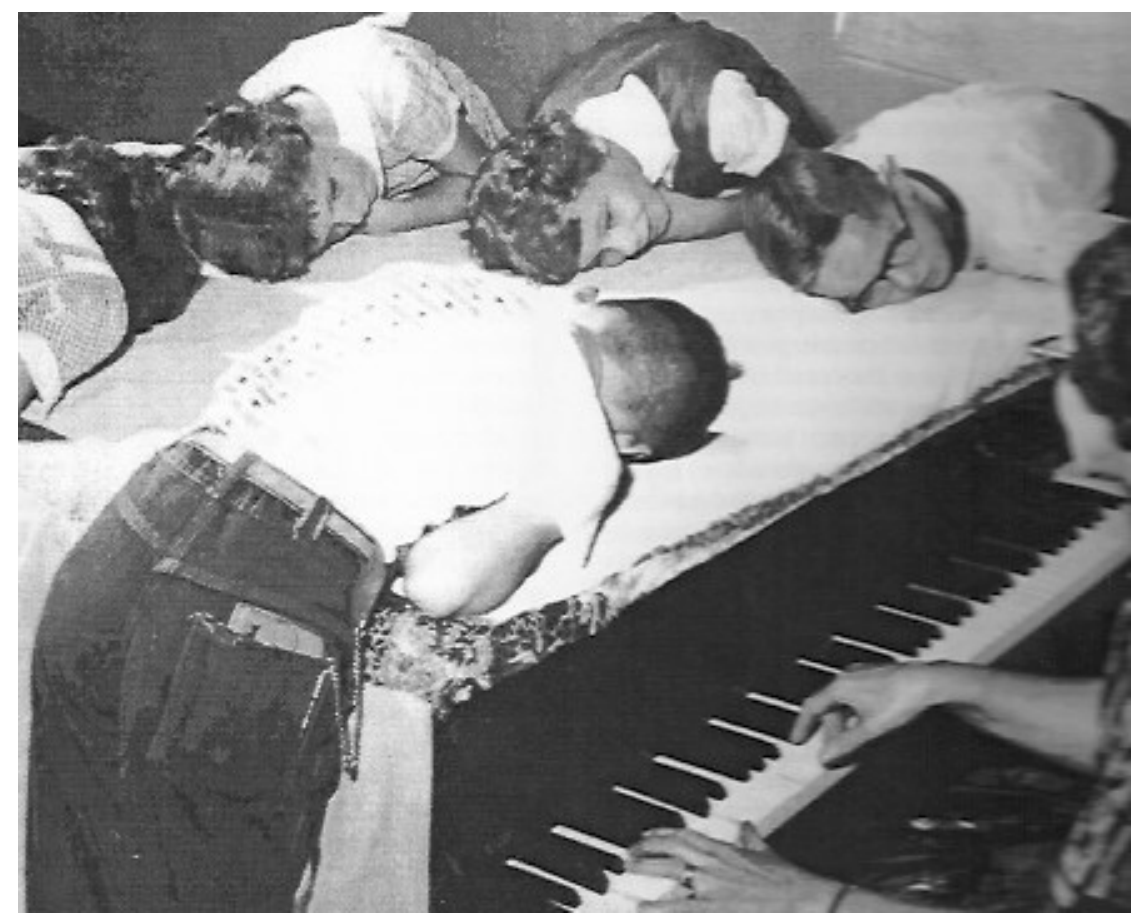

Figure 2.3: Deaf students taking a music lesson at the Alberta School for the Deaf in 1969.

As illustrated in Figure 2.3, many schools for the deaf developed music programs where students were presented with music in some vibrotactile manner, such as lying on a large piano.

In 1925, Gault began reporting a series of experiments where he and his colleagues exposed deaf participants to a device he called a "Teletactor", a hand-held machine with a series of five reeds each of which were tuned to different sets of frequencies, that had been connected to a phonograph. While most often used in experiments involving the tactile presentation of speech, music experiments were also conducted (Gault, 1927).

In the 1980s, Darrow used a SOMATRON, a vibrotactile couch, to present music to deaf schoolaged children. Deaf students were able to discern pitch changes and identify rhythms (Darrow, 1992; Darrow \& Goll, 1989).

More recently, Karam et al. (2010) developed the Emoti-chair. This chair presents a high resolution audio-tactile version of music to the back as a means of presenting music to $\mathrm{D} / \mathrm{HH}$ consumers. The system uses eight separate audio-tactile channels to deliver sound to the body, and provides an opportunity to experience a broad range of musical elements as physical vibrations (Karam et al., 2010). However, it is not a perfect system. According to Branje, the just-noticeable difference of human skin is 200 cents, with a maximum of $1000 \mathrm{~Hz}$; however, auditory music contains frequencies above $1000 \mathrm{~Hz}$. This means Emoti-Chair users are missing 19,000Hz of the music (Branje, Maksimouski, Karam, Fels, \& Russo, 2010). While Emoti-Chair users are able to distinguish differences between frequencies as low as 200 cents and consistently distinguish frequency differences below 400 cents (one- 
third of an octave), this is still less than the auditory system, which distinguishes differences as low as 6 cents (Loeffler, 2006).

\subsubsection{Olfactory}

The presentation of music information via olfaction, the sense of smell, has three challenges: metrics, technology, and perception.

The first challenge is one of metrics, as Alexander Graham Bell asked a century ago,

Did you ever try to measure a smell? Can you tell whether one smell is just twice as strong as another. Can you measure the difference between one kind of smell and another. It is very obvious that we have very many different kinds of smells, all the way from the odor of violets and roses up to asafetida. But until you can measure their likenesses and differences you can have no science of odor (Bell, 1914, p. 652).

Where sounds can be measured in frequencies, and notes can be measured in semi-tones or frequency differences (and in the same manner we can describe colours in terms of frequencies and hues), we have no such equivalent for odours. Without such an equivalent, one cannot map auditory information to olfactory presentation. While there is ongoing work in this area (e.g., Secundo, Snitz, \& Sobel, 2014), our understanding of the science of odour is still not sufficiently mature.

The second challenge is one of technology. Quite simply, while there exist a variety of machines that can pump out various types of scent to provide olfactory cues (e.g., single scent olfactory icons), there is currently no machine that can rapidly release multiple scents to present information without overwhelming the environment (e.g., the breathing space). One can rapidly present multiple colours or multiple tones one at a time. Colours and tones are more transient than scent, which can linger and thus cause adaptation (Sarter, 2006; Sato, Ohtsu, Bannai, \& Okada, 2009).

The third challenge is one of perception. Consider some of the requirements an odour presentation system would demand on a human user. A system might:

- present multiple scents at once,

- rely on location to aid information presentation, and/or

- rely on different qualities or quantities of scent to present information.

But humans tend to discern one scent at a time, whichever is strongest. Humans lack the ability to smell "stereo scent" in the same way many humans can process stereo sound or stereo vision. While the human sense of smell is still very acute, recognizing thousands of different smells and detecting odours even in infinitesimal quantities, it is feeble, even clumsy, compared to that of many animals. As de Lespinois points out:

[A] dog can sort through a myriad of scents simultaneously. So if we smell a stew cooking, dogs smell each ingredient of the stew, and the cook. Dogs can move one nostril at a time 
permitting them to perceive the direction of a scent. Once they pick up the scent, it dissolves on their wet nose. These liquified molecules are then pushed further back into the snout. The dog devotes 40 times more of its brain power to smell than we do, using about 60 square inches of scent receptors called the epithelium. That's about the size of an 8.5 by 11 inch piece of paper. If you compared our scent receptor area, you would find it's a little smaller than a postage stamp (de Lespinois \& Doe, 2010).

Humans are capable of detecting certain substances in dilutions of less than one part in several billion parts of air. Humans have about five or six million olfactory receptors in our nose. We may not be able to match the olfactory feats of bloodhounds (whose nose has 300 million olfactory receptors), but humans can, for example, "track" a trail of invisible human footprints across clean blotting paper using our sense of smell (K. Fox, n.d.).

Olfactory receptors are not solely confined to the nose, but are in fact found throughout the body in various organs. Recent research has found that olfactory receptors can even be found in the skin (Busse et al., 2014). The presentation of information via combinations of the tactile and olfactory modalities may be a potential future direction for olfaction-based information presentation.

Thus, the identification and examination of possible uses, benefits and limitations of the olfactory channel remains one of the important research challenges in the area of multimodal information presentation (Sarter, 2006). But without a means of reliable odour measurement, production, and perception, the use of olfaction to present complex information is not yet within our grasp.

\subsubsection{Visual}

Human visual resolution is stronger than human auditory resolution. Humans can detect very small changes in visual illumination, but only a few different ranges in sound volume. Spatially, humans can detect a one degree change in the direction of a three dimensional sound whereas the central visual field can detect differences as small as two seconds. Similarly, very large amounts of information can be presented on a screen while the transient nature of sound requires their discrete presentation (Kramer, 1994).

For D/HH people, the use of the visual modality to present information takes advantage of several observed strengths. In particular, Deaf adults tend to show greater visual attention. For example, the visual system of deaf adults may monitor their peripheral visual field more than hearing adults (Bavelier, Dye, \& Hauser, 2006).

This information path also takes advantage of mature technology people already have. Screens have become ubiquitous in our society. Everyone seems to carry a screen of some sort. Using a visual modality supports a cheap technology nearly everyone already has and avoids the need to purchase new untested technologies. Screens are also very portable, avoiding the need for people to purchase large bulky technology. 


\subsubsection{Sensory Substitution or Sensory Supplementation}

Presenting information in multiple forms, (i.e. visually, auditorially, tactilely, etc.) falls into two major categories: sensory substitution and sensory supplements. Sensory substitution is the use of one sense to receive information normally received by another sense. Sensory supplements augment the perceptual information of the user with additional, possibly redundant, information using another sense. Cross-modal displays, where information that is designed to be presented to one sensory modality (i.e., audio, visual, and tactile) is displayed using an alternative modality, is the area of research in human-computer interaction that is involved in sensory substitution and supplementation (Karam \& Fels, 2008).

Cross-model displays range from presenting visual or audio information using haptic displays to presenting audio signals using visualizations. Typically, these displays are designed to support redundant presentation of information in applications such as games. For example, first person shooter games use a combination of music (e.g., to evoke heightened alertness) and sound effects (e.g., gunshots, footsteps) to present information (i.e., someone nearby is shooting at you) in addition to the visual display (e.g., explosions, gore).

Sensory substitution systems designed for D/HH users include tactile displays such as the "emotichair" (Karam \& Fels, 2008) and the "artificial ear" (Richardson \& Frost, 1977). Other examples include signalling systems which are designed to visually present environmental sounds. For example, a ringing telephone might flash a lamp. The characteristics of the ringing tone (e.g., one long ring followed by one short ring) are represented by the flash pattern of the lamp to further indicate that it is the telephone and not some other environmental sound source such as a doorbell.

Music visualization is yet another example of a cross-modal display because it presents audio signals that comprise music as visualizations. However, music visualization also involves the translation of meaning that is more than the sum of the information parts so that a direct translation of the basic music elements (e.g., frequency into waveform display) may not be appropriate or useful.

The kind of information processed by a sensory substitution device is often metamodal, it can be accessed through any sensory modality (Martin \& Le Corre, 2015). However, something is always lost in translation because the information presented in the alternative modality cannot fully capture / replace what the primary modality would have perceived (Tang, Fourney, Huang, \& Carter, 2008).

When used to substitute one modality for the other, to support users who are blind or deaf, there is a need to identify what characteristics of the input modality are necessary to present using the alternative display (Karam \& Fels, 2008) and what characteristics will be lost / missing as a result of these choices. For these reasons supplementing a person's existing sensory capabilities is always preferred over sensory substitution.

\subsection{Theories of Information Visualization \& Sonification}

Based on the notion that a visual presentation is easier to use than a textual description (Shneiderman, 1996), information visualization, the study of the visual representation of information, is a strongly 
interdisciplinary field. There are several different genres of visualization. For example, scientific visualization emphasizes realistic renderings of volumes and surfaces. It takes complex information, such as magnetic resonance imaging (MRI) data and displays it in a manner that is comprehensible. Data visualization, on the other hand, attempts to represent data in an abstract form such as a mind map and can be more artistic.

The human vision system is able to scan, recognize, and recall images rapidly. It can detect changes in size, colour, shape, movement, or texture. With this perceptual system, a human can point to a single pixel in a multi-pixel display (Shneiderman, 1996). For these reasons, the visual modality provides a higher bandwidth for information presentation than the other senses.

There is no clear generally accepted definition of visualization; however, several models have been proposed (Kosara, 2007). Generally, visualizations draw on the following three criteria:

- Based on non-visual data. The visualization is not image processing or photography, it is based on a data set that comes from outside the program.

- Produces an image. One or more images are produced from the data, and these visual representations are the primary means of communicating the data.

- Result is readable and recognizable. The resulting transformation should enable the viewer to understand the underlying data. This could mean the viewer requires training and practice to fully interpret the visualization (e.g., in the case of medical imaging).

As Kosara notes, "a transformation of data into a visual shape does not imply readability" (Kosara, 2007, p. 632), in particular music visualizations, while data-based, are not readable. When approaching a visualization, the viewer needs to realize that data is being visualized, understand what is being visualized and then understand how the display is to be read (Holmquist, 2004).

Shneiderman (1996) presents a mantra for how viewers approach information visualization: overview first, zoom and filter, then details on demand. He argues these tasks need to be supported in visualization interfaces.

The overview enables the viewer to gain a sense of the dataset; to form a mental model (Shneiderman, 1996). This does not imply the overviewed data is the entire dataset, only that the viewer acquires an awareness of some aspect of some data rapidly and, where possible, pre-attentively (Spence, 2007). After getting an overview, the viewer may focus in on areas of interest, filter out uninteresting items, and finally select an item or group of items getting details on these items when needed (Shneiderman, 1996).

Although Shneiderman (1996) suggests the overview must always precede the details, Spence (2007) suggests that certain use cases may be exceptions. It is entirely possible that the viewer might start by viewing one object in the dataset, the details, and then want to look at objects with related attributes. For example, a person shopping for a new house in an unfamiliar area may choose to look at one house just to get a sense of what is available as a starting point for forming a mental model (Spence, 2007). 
An example information visualization technique is the so-called "fisheye". The fisheye view is a method of distortion that allows the viewer to focus on specific data within the context of nearby and global data. Fisheyes are characterized by an in-place magnification of the area of interest encompassed by a continuous transition to the demagnification of the surrounding context (Gutwin \& Skopik, 2003).

The research presented in this Dissertation is interested in determining ways to convert sound (i.e., music) into visual information. The reverse process, Information sonification, uses sound to present information. Understanding how data is mapped into sound may help in arriving at ideas for how to convert sound into visual information.

Information sonification (or auralization) draws on the field of visualization but presents data via sound. There are three types of sonification: Iconic Sonification, Direct Conversion Audification, and Musical Sonification. In Iconic Sonification, data is mapped to sounds associated with the phenomena. For example, when sonifying weather information, one might use the sound of rain to indicate a high probability of rain. Direct Conversion Audification uses a direct mapping of the data to sound. The user then listens for patterns that are represented in the data. For example electromagnetic wave data can be mapped directly to sound waves creating a sonification of the earth's magnetic field. In Musical Sonification, data is mapped to sound in a musical way. For example, the University of California, Berkeley church bells have been used as the basic element of a sonification of solar wind density data (Center for Science Education [CSE], 2010).

The use of sonification takes advantage of certain perceptual strengths of human stereo hearing. For example (Kramer et al., 1997):

- Human hearing is sensitive to changes in sounds over time. A person can discriminate between periodic and aperiodic events and can detect small changes in the frequency of continuous signals. In the case of human vision, such fast-changing or transient data might be blurred or completely missed.

- Human stereo hearing allows the listener to be oriented in space in any direction. The direction of the source of the sound can be detected even if the source cannot be seen. By comparison, vision requires the viewer to be oriented in a particular direction to see the visual information.

- Human hearing also provides auditory event memory. Highly notable musical patterns can be easily recognized and recalled even when subjected to radical transformations. For example, the same song at a higher or lower octave can still be recognized.

These and other psychoacoustic skills assume the listener has stereo hearing (hearing in both ears), good auditory discrimination, and intact high and low frequency hearing.

An example information sonification technique is Lübbers' "focus of perception" approach to music information retrieval. In discussing ways to aurally explore a large music collection, (Lübbers, 2005) draws on the "fisheye" method of information visualization to present a circular aura of "similar" songs. The user is presented monaurally multiple pieces at once. Depending on the distance of the song and the angle of its placement from the user's perspective, certain songs may be played louder than the 


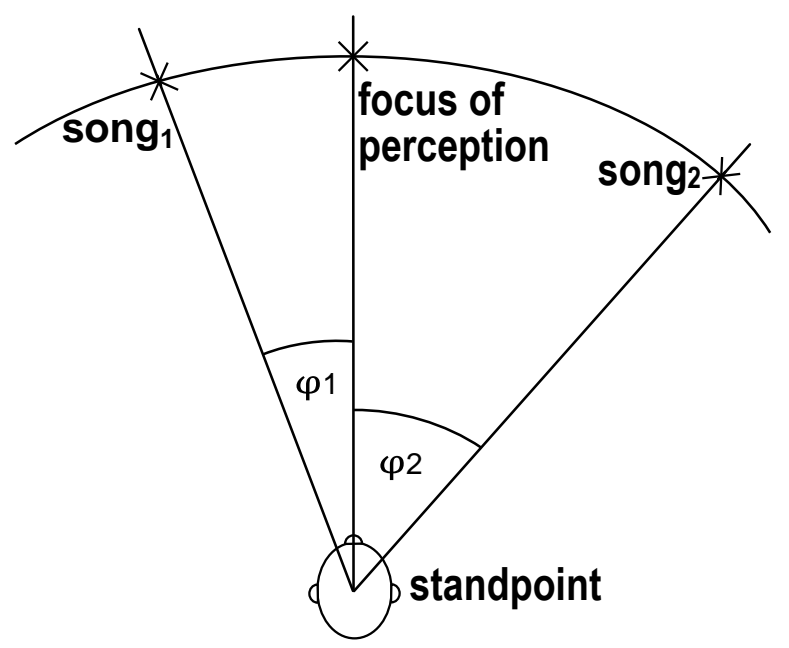

Figure 2.4: Focus of perception model. Adapted from Lübbers (2005).

others and songs outside the aura will not be played at all. As the user changes their focus, they can zoom in on specific songs and select the one they want or move their aura to other songs. As shown in Figure 2.4, although $s g_{1}$ and song $_{2}$ are the same distance to the listener's standpoint, song $_{1}$ is played back louder since $|\varphi 1|$ is significantly lower than $|\varphi 2|$. This "focus of perception" approach is an aural equivalent of Shneiderman's mantra, overview first, zoom and filter, then details on demand.

\subsection{Prior Work in Music Visualization}

The visual representation of music has a long history. For example, Common Music Notation, the system of lines and notes used in Western countries to aid the performance of a musical composition, traces its origins to the Middle Ages (Isaacson, 2005). Many of these approaches act as supplements to the aural experience and are not intended to be used independently without sound accompaniment.

Most of these approaches to visualization require large amounts of human intervention and creativity. Such intervention limits what is available to audiences that require a visual presentation of music and take time to develop. Human intervention means that time and other resources are spent to develop the presentation. The presentation of the information may not be the piece that the audience is interested in being shown. The interpretation of information will be idiosyncratic to the person making the interpretation.

Music can be made visual through dance, paintings and other visual arts. For example, the 1940 Disney film, Fantasia, provides an animated visual rendition of music where various degrees of abstract and literal art are used to communicate the emotion, entertainment, and other properties of the music. In Fantasia, during a performance of Modest Mussorgsky's Night on Bald Mountain, Chernabog summons fire and lava as a crescendo of timpani erupts. Such visual expressions of music are often one-time events for specific musical compositions. They require large amounts of human resources 
to produce a single result; something that would not be feasible for all television, film and music productions.

A second example is the work of Nancy Herman. Starting in 1974, Herman developed a paintingstyle that links musical notes to colours and painted several well known musical compositions (Herman, 2009); however, each painting took "several weeks or even months to complete" (Mitroo, Herman, \& Badler, 1979). Later, she attempted to visualize songs using fabric. The resulting works were not shown with musical accompaniment (N. Herman, personal communication, June 27, 2009). More recently, she used paint software to create static drawings (Herman, 2009) and videos in colour sequences that move in time to the music, as well as exploring the use of Flash to create "visual music" as "if the colors were attached to keys on a musical keyboard" (Herman, n.d.). All of her work involves human intervention. ${ }^{4}$

Closed captioned music videos also require human intervention. A single music video can be properly captioned "within a few days" and costs money (D. Russell, 1989). While they used to be quite rare, captioned music videos have become more common. Comments by the online survey participants mentioned above showed that providing captions for lyrics alone is not enough, there is a need to know why the music is being used, not just its contents.

A recent trend is the development of thousands of YouTube videos of lyrics for songs. Again, using human intervention, the videos are hand crafted by volunteers. Often incorporating visual elements such as pictures or graphics related to the song or the artist, there is no standard for these videos. Many are difficult to read as a result of being too visually busy, having poor font choices, and other design issues.

Thus, creating visualizations of music information by hand requires the expense of resources such as human effort, time, and money. Further, such visualizations restrict the amount of visual content available to $\mathrm{D} / \mathrm{HH}$ audiences to a limited library that may not interest all members of the audience. There is a need for a way to visualize music that reduces the expense and expands the available selection.

Before creating new visualizations for $\mathrm{D} / \mathrm{HH}$ audiences, it is important to understand what has already been achieved in music visualizations so far.

\subsubsection{Relevant Existing Visualization Solutions}

For the purposes of this research, visualizing music means using software to generate a visual representation of specific music properties. The intent is to reduce the amount of human intervention and still enable users to visualize music in a way that is understandable, meaningful, and entertaining.

Perhaps the simplest visualization of music available is probably that which can be produced by software that mimics an oscilloscope. Visually, pitch is depicted on an oscilloscope as the spacing between the peaks or valleys of the sound wave (i.e., the wavelength), the smaller the space, the higher the frequency or pitch. Amplitude appears as the height of the peaks and depth of the valleys of the

\footnotetext{
${ }^{4}$ It is interesting to note that Frank Zappa often did the reverse. He would "draw music", create visual art (sketches, collages, etc.) of the music, before writing or playing it (Oregon Public Broadcasting, 2012).
} 


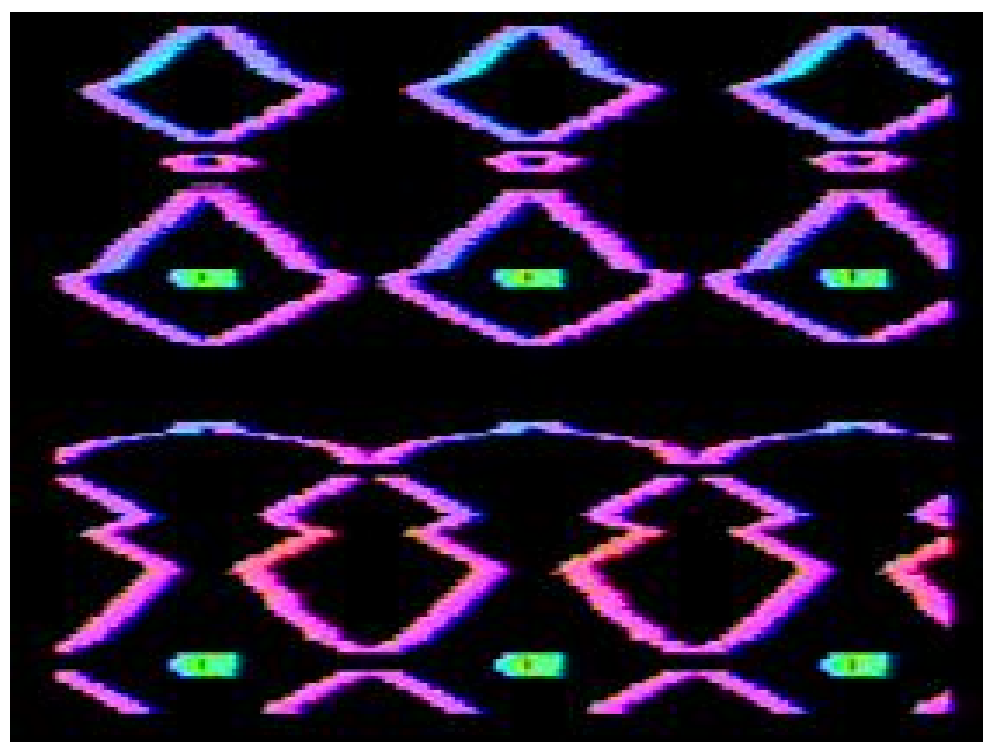

Figure 2.5: A music visualization created by the Atari Video Music (Green, 2014).

sound wave. The smaller the wave, the softer the sound.

Early research in electronic music visualization focused on entertainment. One of the first attempts to electronically visualize music was the Atari Video Music, first released in 1976. A console designed to be connected to a record player and television set, the Video Music had twelve buttons and five knobs that enabled users to custom create the video effect (Wikipedia, 2014a). When placed in "Auto All" mode, the Video Music would react automatically to the music in real time (Brown, 1978). The resulting displays on the television were the 'psychedelic' precursors to software-based music player visualizations. Colour was used to indicate the source channel (each of the left and right speaker had its own colour). The system was able to display 16 colours each of which would be used to show a specific frequency range (e.g., sounds in the $0-120 \mathrm{~Hz}$ range used colour 16, while sounds in the range $1200-1440 \mathrm{~Hz}$ used colour 7 , no information was provided about why those specific colours were selected and assigned to each number). As seen in Figure 2.5, the music was presented in various visual arrangements in the form of diamond-shaped objects that were either solid, rings, or holes. Holes were created by having objects "subtracted" from one another (Brown, 1978; Wikipedia, 2014a). No user studies were ever conducted on this system. This console was on the market for about a year. In 1993, it returned to the market as the Atari Jaguar's built-in Virtual Light Machine (Wolf \& Perron, 2003).

Mitroo et al. (1979) proposed using software to synthesize visualizations of music in real time. Based on Herman's early work, the underlying hypothesis was that the spectral colours could be related to each other in the same way as musical tones to generate images of notes, chords, and chord progressions. The system was limited by the resolution of the display $(240 \times 320)$ and the available colours (4096). Although Herman's real paintings have been either square or rectangular, this system used a circular format (e.g., a chord progression would be displayed in concentric rings about the centre). Unlike the Atari device which could automatically generate visualizations from a sound 
source, the system itself was not entirely "real time" since the tones were manually coded as numeric values. This resulted in a graphic presented on a raster graphics display described as a "musical painting" (Mitroo et al., 1979). Unfortunately, this work was never user tested.

The idea of using colour to represent notes is still used and seems to have staying power (e.g., Malinowski, n.d.). However, the use of colour presents several challenges since, just as music is not culturally universal, there is no absolute set of cultural meanings for a given colour. Most colours can have positive or negative connotations and these vary not only among cultures but also among individuals. For example, in Western cultures black can represent formality, sophistication and sexuality, and it can also be a symbol of mourning. An example of differences between cultures is the meaning of white. In Western cultures, white is associated with weddings and purity yet, in Asia, it is associated with funerals and death. Colours can also have different meanings to different individuals. For example, a number presented in red may be meaningless to one individual, while it can mean debt to an accountant. The meaning and perception of colours are open to individual interpretation and may be misconstrued when used to represent music information and emotions without any additional cues.

Around November 1986, Dalgarno developed a system which converted music into a coloured visual form displayed on a screen using a microcomputer. This system was accompanied by a "tactile sensation device" for people who were D/HH (Dalgarno, 1989).

There are numerous music visualization styles that can be seen in common computer music players such as Windows Media Player, iTunes, and WinAmp. Those players that show visualizations of digital audio files such as WAV or MP3 usually respond to beat and/or frequency information that can be extracted from the digital audio file using readily available detection algorithms (e.g., Puckette \& Apel, 1998). Developers of these visualizations admit that the selection of visual parameters (e.g., colour, shape, motion) that change in association with audio input (e.g., frequency, amplitude, timbre) tends to be arbitrary (Lipscomb \& Kim, 2004). However, there has been little research to assess whether these visualizations provide sufficient information and entertainment for $\mathrm{D} / \mathrm{HH}$ users.

For example, the iTunes media player application provides visualizations based on waveform analysis. Figure 2.6 depicts the default visualizer used in iTunes 8, Magnetosphere. This music visualizer included moving streams of light that wrap around black, planet-like orbs. Based on an analogy of magnetism, the Magnetosphere visualization is a simulation of interacting charged particles, shown as balls of light; particles move about based on the strength of their magnetic charges. The magnetic charges change as a direct result of audio input (Hodgin, n.d.). For example, with increased amplitude, the visualization becomes more elaborate and fills more of the screen while differences in frequencies are depicted by the size of the charged particles. How the audio input impacts the visualization is not fully documented. While the result is visually pleasing, it is not very informative and can mislead the viewer. For example, without sound, this visually exciting visualization can lead viewers to believe the song is emotionally exciting even though it may be emotionally sad.

Currently, however, most music visualization research focuses on music analysis and music education. Isaacson (2005) presents a review of seven different approaches used to visualize music for analysis such as spectrograms and tonal diagrams. While such visualizations can reveal sophisticated information about a musical work, they are useful and meaningful to those who are concerned about mu- 


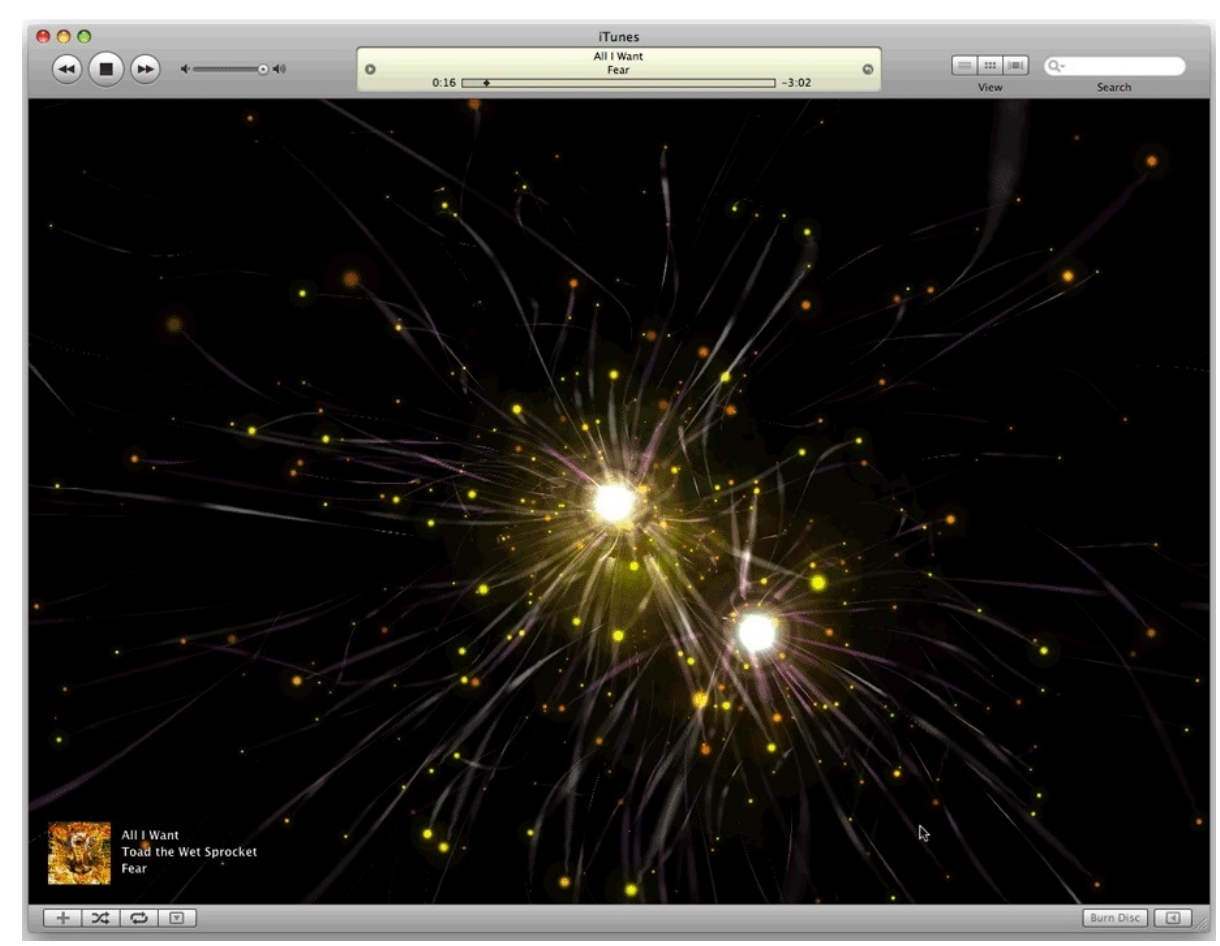

Figure 2.6: Magnetosphere music visualization (Griffiths, 2008).

sical structure and meaning and they require musical training to understand. Real-time visualizations using design metaphors based on how musicians mentally visualize sound have also been developed to train musicians via visual feedback. Such systems improve the training of musicians because they allow the analysis of one's own sound quality (Ferguson, Vande Moere, \& Cabrera, 2005). They may also provide interpretable metaphors for visual displays.

A concrete example of this style of analysis which I present as a fairly representative example is the Music Animation Machine (MAM), which provides various visualizations of real-time music data based on MIDI file input. Three of them are: piano roll, part motion (balls), and tonal compass (Malinowski, n.d.).

- The piano roll display, shown in Figure 2.7, conveys information about the music's structure through bars of colour representing the notes. These bars scroll across the screen as the music plays. Their position on the screen indicates their pitch (i.e., higher notes are higher on the screen, lower notes are lower) and their timing in relation to each other. Different colours denote different instruments or voices, thematic material, or tonality. In addition to a "now line", each note lights up at the exact moment it sounds (Malinowski, n.d.).

- In the part motion or balls display, notes are represented by circles, the size of which corresponds to the length of the note. As shown in Figure 2.8, the notes of each part (a part is a unique track / channel combination) are connected sequentially by lines. When the note is sound- 


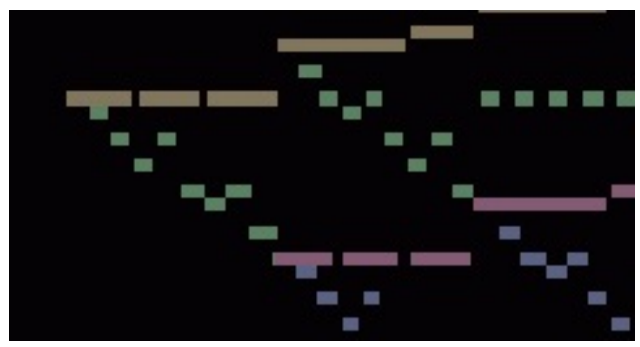

Figure 2.7: Music Animation Machine Piano Roll.

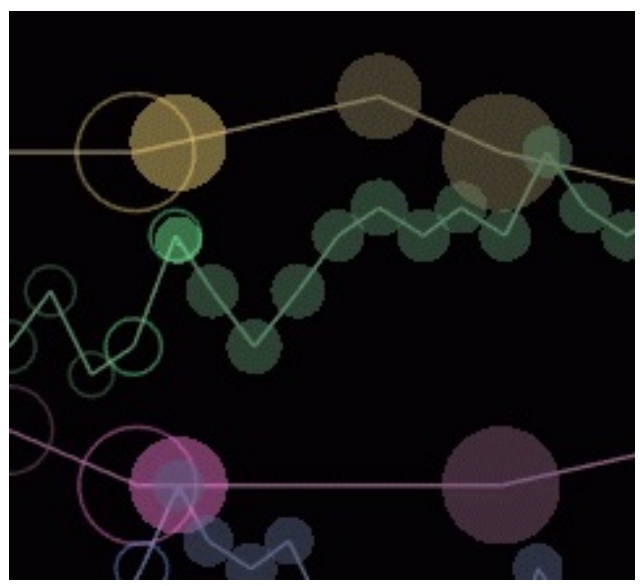

Figure 2.8: Music Animation Machine Part Motion.

ing, the core of each ball detaches from its outline and moves toward the next note in the part. As it moves, the ball shrinks (Malinowski, n.d.).

- The tonality compass display, shown in Figure 2.9, arranges the pitches around a circle based on the circle-of-fifths model. Each pitch is denoted as a circle. The size of the circles show the "weight" of the pitch (weight is based on the dynamic level of the pitch). This visualization is particularly useful for showing chords and tonality (Malinowski, n.d.).

Each of the MAM's different ways of viewing music is a different form of an equalizer. While some of its visualizations (e.g., the part motion or balls display) may prove visually interesting, such mu-

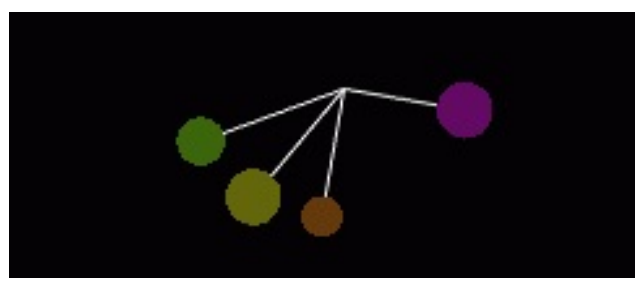

Figure 2.9: Music Animation Machine Tonality Compass. 
sic analysis-oriented visualizations require training, practice, and/or sound to fully understand. The MAM works as a music visualizer for musicologists, but it cannot stand alone; it requires sound to be fully understood (Fourney \& Fels, 2009; Malinowski, n.d.).

The focus of the MAM is on specific quantifiable qualities of music such as beat and frequency. Beyond certain primitive elements of music, even with these primitives summed to form a full visualization, it does not appear to represent emotion in a way that the music equivalent can. This is different from visualizations such as those provided by iTunes which are designed for their strong aesthetic appeal and do not seem to be concerned with the relationship to the music properties.

Musician training applications often employ real-time visualizations using design metaphors based on how musicians mentally visualize sound. These applications have been developed to train musicians via visual feedback and represent an improvement over manual systems because they allow the analysis of a musician's own sound quality (Ferguson et al., 2005).

Similar music visualizations have been developed to assist D/HH users to learn a musical instrument. For example, a computer-aided music learning system has been developed to assist D/HH junior high school students to play a melody on a flute. The system used a visualization of animated music notation to present real-time feedback to students. After 16 weeks of training, results showed students supported by the software had better music-learning performance than students who were not. Further, these students reported having a stronger interest in practising or learning music and put more effort into learning the instrument compared to traditionally instructed students (Yang, Lay, Liou, Tsao, \& Lin, 2007). However, as will be discussed, using music visualizations in this way may not be culturally appropriate in all Deaf communities.

Research in the use of avatars to display music information provides another direction of music visualization. For example, Taylor, Torres, and Boulanger created a system that allows musicians to interact with a life-sized virtual character in an immersive virtual environment using a wall-sized display. The system responds to the musician in real-time. Using a MIDI keyboard, the musician can either perform specific musical gestures triggering pre-programmed responses or perform spontaneously and observe the system's response. This system is also designed to model emotional information. Using a microphone, the musician can sing to the system which maps these vocal pitches to a model of Western tonal music theory to infer emotional meaning. The virtual character then makes specific movements based on this emotional inference (Taylor, Torres, \& Boulanger, 2005). However, this animation was not user tested so it is unclear whether the resulting animation communicated an appropriate emotional effect.

\subsubsection{Textual Visualizations}

Textual visualizations of lyrics have been explored since the early 20th century and the advent of photography and the "magic lantern".

The magic lantern slide projector could project glass slides that contained illustrated songs along with advertisements and public service announcements. Illustrated songs were a popular form of entertainment in the nickelodeon theatre that featured projected slides accompanied by live music (pi- 
ano and vocals). Most illustrated songs used popular melodies or tunes that were easy to sing along with. Illustrated songs would culminate with audiences singing along to the chorus at the end of the song (Altman, 2001; CineWiki, 2009; Wikipedia, 2014d).

The slides served to "illustrate" the lyrics, making them come alive for the audience. A full set of slides for one song consisted of a title slide, fourteen or more narrative slides, and a chorus slide. The title slide, in addition to displaying the name of the song, depicted the cover of the sheet music (the main purpose of illustrated songs was to sell sheet music). The narrative slides, usually four slides for each verse and three for the chorus, corresponded to lines of the song and often used visual puns for comedic effect. The chorus slide was also the artistic signature of the slide maker, often containing information such as the slide maker's address (Altman, 2001).

The magic lantern predated the existence of film. When film became popular, exhibitors simply projected the film reels from the same magic lantern projector. Despite the popularity of the illustrated song in the early 1900's, by 1913 illustrated songs had ceased production and disappeared from many theatres (Altman, 2001).

During the so-called "silent era" of films (1894-1929), direct cueing, inserting song lyrics into the intertitles of silent films, was occasionally used as early as 1917 and became common practice by the mid-1920's, the "golden age" of silent film. However, as it is thought that a total of $70 \%$ of American silent films are believed to be completely lost (Pierce, 2013), direct cueing may have occurred even earlier and many unknown examples could exist prior to the mid-teens of the twentieth century. For example, in John Ford's 1917 film Bucking Broadway the lyrics of the song "Home, Sweet Home" are shown on the intertitles as the film shows cowboys clearly singing the song. This cued the audience to expect to hear the song and required the theatre's musician(s) to play the song (Kalinak, 2007).

Various textual visualizations have been developed to present music information to $\mathrm{D} / \mathrm{HH}$ people. Often these visualizations were developed to support using music and / or singing as a means to teach oral speech skills.

In the 1970's, the National Technical Institute for the Deaf (NTID) had a program to teach D/HH college students to sing. The lyrics would be presented using a simple overhead transparency and the (hearing) instructor would use a pointer to direct attention to the words of a song as they were played from a tape recorder (Murray, 1978).

Murray developed a "balloon captioning technique" to present lyrics (1978; 1979). Using a prism as a type of "fisheye" lens and the lyrics set in type, Murray would photograph the lyrics onto high contrast film with the prism overlaid on the lyrics according to the timing of the song. Once the soundtrack was added, the resulting $16 \mathrm{~mm}$ film could then be played with a soundtrack and synchronized lyrics. A three-minute song would produce a film of 4,320 frames. The visual result was a wave or bubble moving across the caption showing the viewer exactly which words are being spoken or sung. Unlike a bouncing ball visualization, which can distract the attention of $\mathrm{D} / \mathrm{HH}$ viewers, this approach focuses attention on individual words while simultaneously making visible any time progression within individual words (Murray, 1979). Don Arioli, an animator with the National Film Board of Canada, had developed a similar technique where lettering ballooned in synchronization with the audio. However, Arioli's approach involved hand painted cells (three cells for each letter of every word) (Murray, 
1978).

In the late 1960s and early 1970s, the first closed captioned and teletext broadcasts were delivered (Graziplene, 2000). Closed captioning is a technique for translating dialogue into text. A television set with a built-in decoder is able to display the translated text on the screen to match the dialogue of a show (Abrahamian, 2003). At that time, the display of text on the screen as closed captions or teletext was restricted by the limitations of the television display technologies.

The North American captioning system, called Line 21 captioning, was invented around 1971. Within the analog NTSC television picture, 21 of the 525 lines are used for the Vertical Blanking Interval (VBI). Line 21 is the last line of the VBI before the picture begins. Captions are encoded into line 21 and then decoded for the television viewer through a captioning decoder (Strauss, 2006). Each VBI line can carry data at a rate of 9600 baud or less (S. Smith, 1987).

NTSC captioning, as per the EIA/CEA-608 standard, uses a fixed bandwidth of $480 \mathrm{bit} / \mathrm{s}$ per line 21 field for a maximum of 32 characters per line per caption (maximum four captions) for a 30 frame broadcast. It supports a monospaced font. The character set was largely based on ASCII with the exception of a few special characters, most notably an eighth note which is used to denote the presence of music (Wikipedia, 2014b). Captions could only be modified using italics (or slanted), all caps or mixed case. Traditionally NTSC captions are presented as white text over a black background, however colour is supported. With the advent of Digital television, ATSC broadcasts have many more features. Field (2000) provides a description of the technical standards for North American television.

Captions can be presented in one of three modes: Roll-up, Paint-on, or Pop-on (Canadian Association of Broadcasters [CAB], 2012a).

- With "pop-on captions", a phrase or sentence appears on the screen all at once, remains on screen for a few seconds, and then either disappears or is replaced by another full caption. The captions are timed to synchronize with the program and placed on the screen to help identify the speaker. Pop-on captions are used for captioning prerecorded programing.

- "Roll-up captions" (or scrolling captions) roll onto and off the screen in a continuous motion. Usually two or three lines of text appear at one time. Each new line appears below the last new line pushing the other lines on the screen up. The top, or oldest, line of text disappears as each new line is added to the bottom. Roll-up captions are used for all live captioning and can also be used for prerecorded captioning.

- "Paint-on" captions can appear similar to pop-on captions, but each word is added one at a time. It is most often seen during captioned television advertisements with very short lead in times because the delay necessary to broadcast a pop-on caption in advance of the initial spoken audio is too short.

Pop-on captions are often considered the "gold standard" for captioning. Studies show that consumers prefer this type of captions to scrolling captions because they are perceived as easier to read (CONNECTUS Consulting Inc., 2008). 
The equivalent of NTSC closed captions used in countries that use PAL (e.g., Europe, Australia, etc.) is based on Teletext. Features such as colour, multiple fonts, different font sizes, mixed case lettering and animations are commonly used. To access the captions, viewers use a teletext enabled television (or equivalent set-top box) and, while watching the desired television channel, access a specific page of the teletext system (e.g., in Australia this is page 801). Historically, teletext was used to provide text versions of news stories, stock market figures, etc. (see Wikipedia, 2015a), but this service is no longer available. Currently, the only page of teletext is the captions (Aussie Deaf Kids, n.d.; Media Access, n.d.). It is an acceptable broadcast engineering practice when translating NTSC captions to teletext for PAL compatible countries to substitute the eighth note character used for denoting music for a number sign (\#) because of its similarity to the musical sharp sign ( $\sharp$ ) (Wikipedia, 2014b).

Jordan, Albright, Branner, and Sullivan (2003) published an extensive report on the state of closed captioning in North America. They surveyed 107 D/HH captioning consumers and interviewed 17 industry representatives regarding their satisfaction and knowledge of closed captioning, and carried out a content analysis of existing captioning for quality and consistency. One of the important sound elements that was identified by $\mathrm{D} / \mathrm{HH}$ people was the desire to have music captioned.

Presently, music is typically identified through the listing of the song title and / or the appearance of a music note icon. Not only does this provide little information about the properties and meaning of the music, but it also has little meaning to people who have limited experience or interest in music.

In Canada, the current (voluntary) English language captioning standard states the following for the display of music $(\mathrm{CAB}, 2012 \mathrm{a})$ :

- Discourages the use of colour.

- Discourages the use of paint-on captions to show lyrics as they are sung.

- Requires the use of pop-on captions for lengthy lyric passages even if the rest of the program is captioned using roll-on captions.

- Allows lyrics to roll-up only when "unusually fast" or when there are a few lines of singing mixed with conversation.

- Requires the use of a music note at the beginning and end of each lyrical phrase, regardless of using roll-on or pop-on presentation, to indicate that the lines are being sung and not spoken.

- Recommends writing out lyrics for all songs only if they are discernible.

- Recommends using a descriptive parenthetical description of the music where lyrics are not discernible (e.g., "(60's rock instrumental)" vs. "(Music)").

The equivalent french language standard is largely the same except that it requires the captioning of lyrics only if the song is in French, otherwise the captions need only indicate the language of the song in parentheses (Canadian Association of Broadcasters [CAB], 2012b).

In fact, lyrics are rarely displayed in captioning. One reason for this is that music used to elicit an emotional response to a television audience rarely has lyrics. For example, a scene intended by its 
creators to be scary, and accompanied by mood music to create that effect for the hearing audience is likely to be captioned by:

- a single eighth note;

- some information on the music (e.g., "ominous") bracketed in eighth notes;

- the name of the song (e.g., "Jaws Theme") bracketed in eighth notes;

- a descriptive name of the song (e.g., "shark theme") bracketed in eighth notes; or

- artist information as well as the name of the song (e.g., "John Williams' Jaws Theme") bracketed in eighth notes.

All of which are highly unlikely to elicit a fear response.

Harkins (1995) carried out a study on user attitudes toward closed captioning of non-speech information (NSI) in North America. NSI describes those aspects of the soundtrack, other than spoken word, that convey information about plot, humour, mood, or the meaning of a spoken passage. Deaf $(n=106)$ and hard of hearing $(n=83)$ consumers $(93 \%$ of whom owned a captioning decoder at home) were asked to review a series of videos and rate them for preference. With respect to music, Harkins explored six ways to depict its presence:

Description (e.g., "( soft, sad background music )", "( scary music )");

Music icon where an eighth note would appear in the top-right corner of the screen;

Music icon and capital letters where lyrics would appear in capitalized text bracketed by one eighth note at the beginning and end of each caption block;

Music icon and upper/lower case letters where lyrics would appear in mixed case text bracketed by one eighth note at the beginning and end of each caption block;

Paint-on lyrics where words would appear as they are sung using the paint-on approach of captions; and

No description where no additional information would be shown (Harkins, 1995).

In scenes with background music, $83 \%$ of participants preferred description while in scenes with singing, $82 \%$ of participants choose styles with the music icons. In their concluding recommendations Harkins (1995). recommended that:

1. background music be indicated especially if it contributes to the plot or mood,

2. a description of the music should be given where possible, and

3. music note icons should surround lyrics. 
They provided no recommendation on the use of mixed case lettering. The paint-on style of presenting the lyrics, a very new technology at the time, was rejected as only $18 \%$ of participants expressed any preference for it.

Participants clearly expressed a desire to have more of the background sound information such as music and sound effects provided, but tended to reject new approaches to presenting NSI. In addition to rejecting the use of paint-on captions to present lyrics, Harkins (1995) also found that using other new features to present NSI, such as colour and flashing text, were also not acceptable to participants.

Music visualizations for $\mathrm{D} / \mathrm{HH}$ users have begun to explore how to communicate emotional information through the lyrics. For example, EnACT, a software tool for creating animated text captions, enables users to mark emotions in lyrics and then render those marks into animated text for display as captions (Vy, Mori, Fourney, \& Fels, 2008). However, lyrics are typically sung in a way that can communicate information about the music, not just the emotional content.

A second example is a system developed by Diaz-Marino, Carpendale, and Greenberg (2005). It uses kinetic or moving text to communicate how the lyrics are being sung not just what is being sung. Traditional karaoke displays assume that the singer already knows and can hear the tune, there is no information provided on the musical properties of the song. Diaz-Marino et al. (2005) added visualizations of vocal properties such as pitch and force as well as information about beat and timing to a karaoke style display. This system was not user tested so it is unclear whether their visualization strategy worked to communicate the emotions contained in the lyrics.

Fels, Lee, Branje, and Hornburg (2005) used graphics, colour, icons, animation and text to present music information in television programming. Icons were used to depict music. For example, a violin icon would move across the screen to show a change in music pitch or tempo. Text was used to depict lyrics. Emoticons (e.g., smiley face) were used to show the emotions. Colour was used to show emotion and intensity. However, a usability study found that $\mathrm{D} / \mathrm{HH}$ audiences did not like the use of colour and deaf audiences generally did not accept this use of graphics and icons.

Silverman and Fels (2002) created graphic captions using a comic book approach. They used speech bubbles and icons with descriptions to depict background sounds and transitions in the video short "Il Menu," an amusing spoof on opera that contained dialogue and music. For example, a piano keys icon was used to indicate music. It was marked by a red " $\mathrm{x}$ " to indicate that the music had suddenly stopped. A usability study found that D/HH viewers thought that the approach increased their understanding of the content and heightened their sense of themes, depth, and feeling but they had difficulty with the use of comic conventions because they associated these conventions with children's content.

Canadian Network for Inclusive Cultural Exchange [CNICE] (2005) describes how an animation was added to the opening scene of the TV series "Rocket Science". The scene begins with the "CC" or closed caption symbol morphing into a musical note which then moves and weaves through the show's opening animated sequence. This animation was intended to both let the user know about the music and give a sense of the music itself. However, this animation was not user tested so it is unclear whether the animated notes created the desired enjoyment effect.

Other text based visualizations of music information display information about the song and artist 
but not the lyrics. For example, the radio text (RT) function specified within the Radio Data System (RDS) of International Electrotechnical Commission (IEC) 62106 allows a radio station to transmit a 64-character free-form string that can be in sync with the station's programming (e.g., the title and artist of the currently playing song). This information can then be displayed to the listener via a screen attached to the radio.

Outside of the captioning research discussed above, there has been little research to assess whether music visualizations provide sufficient information and entertainment for $\mathrm{D} / \mathrm{HH}$ users. The properties of music that tend to be provided in visualizations are lyrics, tonality, harmony, loudness, pitch, and timing. There is limited work in visualizing the emotional content of music. While these sound elements are creatively combined by musicians to express emotions and provide entertainment, it is unclear whether using visual equivalents of these sound elements and their combination can produce similar or equivalent effects. Chapters 5 and 6 will present studies designed to try to answer these questions.

\subsubsection{Visualizations and Emotion}

Information visualization is generally understood to be a part of human-centred computing. As such, visualization techniques usually need to be evaluated in user studies. They are designed for immediate understanding, not emotional response (Kosara, 2007).

Visualizations impact emotion when designed with a view for aesthetics. For example, the sublime, that which inspires awe, grandeur, and evokes a deep emotional and/or intellectual response, is an aesthetic criterion that can be used to assess visualizations. Works of art generally possess a sublime quality. Visualizations, with their focus on usability and immediate understanding, should be antisublime (Sack, 2011). As Kosara (2007) states,

The sublime subsumes the two criteria of readability and recognizability, since for a work of art to be sublime, it cannot be easily readable (or user friendly). It must present enough of an enigma to keep an audience interested without being easy to solve. The opposite is obviously true for a tool that is designed to aid in data analysis. (p. 633).

Aesthetics cannot be ignored. Research suggests that, when tasked with gleaning information from visualizations, users approach aesthetic visualizations more thoroughly and with greater patience (Cawthon \& Moere, 2007).

However, "there is a lack of holistic empirical studies to characterize what visual properties make users think a graph is pretty or visually appealing" (Chen, 2005, p. 15). While music and its impact on emotions is a domain under active research, the emotional impact of information visualization is less understood.

Further, "there is no single theory of aesthetics" (Filonik \& Baur, 2009, p. 580). Each domain, whether art, psychology, industrial design, etc., approaches aesthetics in very different ways. Everyone has a subjective view of aesthetics as a result of their personal, social, and cultural background. 


\subsubsection{Why Music Visualizations?}

As mentioned earlier, creating visualizations of music information by hand raises several issues. Such visualizations:

- require the expense of resources such as human effort, time, and money; and

- restrict the amount of visual content available to $\mathrm{D} / \mathrm{HH}$ audiences to a limited library that may not interest all members of the audience.

On the other hand, automated visualizations have several advantages. Using these visualizations $\mathrm{D} / \mathrm{HH}$ audiences are

- able to access the music information on their own terms;

- not controlled by the musical preferences of others; and

- not dependent on the work of others.

The visualization of music might be one (of possibly many) tools that can be used to make a particular type of information, the music and its patterns, more widely available to the $\mathrm{D} / \mathrm{HH}$. It promotes the independence of the $\mathrm{D} / \mathrm{HH}$ music consumer.

\subsection{Summary}

The purpose of this chapter was to provide background information to these areas of the $\mathrm{D} / \mathrm{HH}$ communities, music, and the various ways different types of music information have been presented; show how past research relates to the current research questions; and explore open issues where the current research questions may provide more information.

In North America, hearing loss is the third most chronic disability among older adults and the fastest growing hidden disability. While the difference between those who describe themselves as Deaf and those who describe themselves as Hard of Hearing is largely cultural, both groups have concerns with respect to music that is not well understood.

The literature regarding that nature of music typically assumes that the reader can hear. Few authors make any attempt to explain music to those who cannot hear or do not hear very well. Music is made up of nine atomic primitives (i.e., tone, pitch, timbre, loudness, tempo, rhythm, contour, spatial location, and reverberation) each of which can be varied without altering the others. The brain organizes these nine musical attributes into four higher level concepts (i.e., metre, key, harmony, and melody). Other properties exist (e.g., agreeableness) which result from combining these atomic primitives and higher order properties in an aesthetically pleasing manner. Composers use these properties in creative ways to create music that fits the desired mood they want to express in the hopes the audience interprets the same mood. 
Music affects people's feelings. While culture may play a role in judgments of emotion in music, Western listeners are able to perceive emotional expressiveness in the music of an unfamiliar tonal system and evidence suggests that this ability is based on psychophysical elements such as tempo, timbre, and complexity rather than musical experience, knowledge, or enculturation. Nonetheless, musical conventions (e.g., leitmotifs, codified rhythms, etc.) and other psychophysical elements are used in film and television entertainment to provide the emotional atmosphere.

Of the four major sensory modalities that can be used to present music, the auditory modality is not useful because this is where the problem of presenting music to $\mathrm{D} / \mathrm{HH}$ audiences lies; the tactile modality is an active research area for music information presentation but involves large, expensive, non-portable equipment; and the olfactory modality has ongoing challenges which must be addressed before its use for complex information presentation is possible. The visual modality takes advantage of mature, cheap, potentially portable technology and a complex range of human visual capabilities.

There is a long history of prior art in music information visualization. Creating visualizations of music information by hand requires the expense of resources such as human effort, time, and money. Issues relating to design and ease of interpretation as well as the limited library of what has been made available make this approach infeasible. Using software to generate visual representations of specific music properties seems to be a more feasible approach because it both reduces the expenditure of resources and increases the range of music available to audiences.

Recall that one of the research questions explored by this Dissertation is: What are the fundamental properties of music that a visualization must present and that produces an entertaining and meaningful experience for $\mathrm{D} / \mathrm{HH}$ people? It is clear from the literature reviewed above that music is a highly complex information space. This raises several questions about whether the focus of visualizations should be on specific atomic music primitives (e.g., tones) or higher level concepts (e.g., melody, key), both, or something else entirely.

The literature suggests that music is a highly complex container of shared information and knowledge presented through sound. For hearing people, music is not just a cultural element, it impacts their lives. What is not clear in the literature is whether music impacts $\mathrm{D} / \mathrm{HH}$ communities in similar ways.

Recall another of the research questions explored in this Dissertation is what, if any, emotional experiences and interpretations users might get from visualizations. It is clear from the literature that music communicates complex emotional signals and that it has specific conventions designed to manipulate emotions. What is not clear in the literature is whether this can be translated into other modalities.

\subsection{Next Steps}

The next chapter provides a description of the methodological approach of this Dissertation as well as a high level description of the series of studies completed for this Dissertation. 


\section{Chapter 3}

\section{Methodology}

Given the wide range of research areas discussed in the previous chapter, various methods and tools were combined for this Dissertation. Primarily, the methodological focus was on user-centred or participatory research design tools such as focus groups, interviews, and surveys. Other methods, such

as Grounded Theory (GT), were embedded as needed. While not generally used during the execution of the experiments reported in this Dissertation, the Design Research Methodology (DRM) (Blessing \& Chakrabarti, 2009) was used to organize the reporting of the experiments and the results.

DRM is primarily developed for executing design research (Blessing \& Chakrabarti, 2009). However, it provides a useful organizing principle for research through design. An iterative approach composed of four stages, individual research projects using DRM often focus on one or two stages only repeating them until some goal is reached. This application of the scientific method to design is the only methodology of its kind in engineering design.

As shown by the breadth of the literature review in the previous chapter, there is so little research involving music and the $\mathrm{D} / \mathrm{HH}$ population that a research methodology well suited for areas where there is very little existing knowledge or research is needed. GT is one such research methodology. Unlike positivist research, which starts with a hypothesis, a study using GT may begin with a question, or even just a collection of qualitative data. Data is collected, coded, reviewed, refined (as "categories"), and eventually becomes the basis for constructing new theory. Typically, it requires the researcher to review what literature may exist, collect data, analyze it towards the development of a theory, adjust and collect more data, further define concepts and theories, and then perform a literature search based on the information collected to compare any emergent theory with extant literature (Pace, 2004). This is then used to build further research.

These methodologies will be discussed in more detail below. 


\subsection{Design Research Methodology}

The main aims of the DRM are to $a$ ) help researchers in identifying research areas and projects, $b$ ) help researchers in selecting suitable research methods to address the issues, and $c$ ) quantify the benefits and problems with a particular design intervention. DRM pieces together the various types of research to encourage a reflection on the research approach applied, and to provide pointers to methods in other disciplines that are useful for addressing the variety of aspects involved in engineering design.

In sum, the DRM's four main stages traverse from understanding the problem space and measuring the "as is" situation to implementing a design or intervention and measuring its impact. The four main iterative stages are:

\subsubsection{DRM Stage 0: Criteria Definition / Research Clarification}

The first DRM stage, Criteria Definition / Research Clarification, identifies the goals that the research is expected to fulfill and the focus of the research project. The problems, questions and hypotheses of the research and the relevant disciplines and areas to be reviewed are determined. Since its primary role is to develop an initial representation of the existing situation and of the desired situation, in this stage designers make explicit the current understanding and beliefs.

The resulting preliminary understanding and overall research plan become the focus of the next DRM stage.

\subsubsection{DRM Stage 1: Descriptive Study I}

The second DRM stage, Descriptive Study I (DS-I), aims to provide a basis for the development of modifications (so-called "supports") to improve design and often results in a reference model or theory (i.e., the Initial Reference Model(s)).

In essence, the DS-I stage is about measuring a baseline, or determining the "as is" state of a situation before some kind of intervention is applied.

The role of the DS-I stage is to obtain a better understanding of the existing situation by identifying and clarifying in more detail the factors that influence the current situation, identifying and clarifying the way in which these factors influence the situation, and suggesting the factors that might be suitable to address in the next DRM stage, as these are likely to lead to an improvement of the existing situation. These goals are met through a literature review of relevant empirical studies or conducting empirical studies.

\subsubsection{DRM Stage 2: Prescriptive Study}

The third DRM stage, Prescriptive Study (PS), attempts to develop an impact model or theory, based on the Initial Reference Model(s) or new understanding created in the Descriptive Study stages. The Impact Model / Theory describes the expected improved situation should some modification occur. 
In essence, the PS stage is about changing the "as is" situation through the application of an intervention. This is where designed things are created and exposed to users.

Having developed an Impact Model describing the desired, improved situation expected as a consequence of the modification(s), the designer selects some part of the Impact Model to address and determines the related Success and Measurable Success Criteria which will be used to evaluate the modification. The resulting Outline Evaluation Plan is used in the next DRM stage.

\subsubsection{DRM Stage 3: Descriptive Study II}

The fourth DRM stage, Descriptive Study II (DS-II), is a process of verification and validation to identify whether the modification can be used in the situation for which it is intended. It attempts to determine whether the modification does address the factors it is supposed to address (i.e., is it usable) as well as whether the modification contributes to success or has any implications / side effects (i.e., is it useful). New understanding derived from this stage can lead to a new Reference Model / Theory to be applied in a further PS.

In essence, DS-II is about repeating the measurements from DS-I to see the changes caused by the intervention introduced in PS. Also in this stage, the differences between baseline and new situations is analyzed to determine the degree of success of the intervention.

The role of DS-II is to identify whether the modification can be used in the situation for which it is intended. This stage evaluates the modification to determine whether it addresses the factors it is supposed to address and whether it contributes to success, addressing usefulness, implications and side effects.

\subsection{Grounded Theory}

Grounded Theory (GT) will be seen in Chapter 8. GT is a qualitative research methodology that was developed by Barney Glaser and Anselm Strauss in the 1960's for research involving human participants (Glaser \& Strauss, 1967). It was originally intended for use by sociologists, but has since been applied to a variety of fields (e.g., social work, health studies, psychology, management, etc.) and has been adapted accordingly (Goulding, 1999). Since its first publication, both authors have diverged. Currently, there are at least two schools of GT, (i.e., Glaserian and Straussian). The focus of this section will be the original method.

\subsubsection{The Basics}

GT has three basic elements: concepts, categories and hypotheses.

Concepts are the basic units of analysis. It is from the conceptualisation of data, rather than the data itself, that theory is developed. Concepts describe the way a researcher labels like events. As Corbin and Strauss (1990, p. 7) state: 
Theories can't be built with actual incidents or activities as observed or reported; that is, from "raw data." The incidents, events, happenings are taken as, or analyzed as, potential indicators of phenomena, which are thereby given conceptual labels. If a respondent says to the researcher, "Each day I spread my activities over the morning, resting between shaving and bathing," then the researcher might label this phenomenon as "pacing." As the researcher encounters other incidents, and when after comparison to the first, they appear to resemble the same phenomena, then these, too, can be labelled as "pacing." Only by comparing incidents and naming like phenomena with the same term can the theorist accumulate the basic units for theory.

Categories are a kind of concept container. Although higher in level and more abstract than the concepts they represent, categories are generated through the same analytic process of making comparisons to highlight similarities and differences that is used to produce concepts. As explained by Corbin and Strauss (1990, p. 7):

Categories are the "cornerstones" of developing theory. They provide the means by which the theory can be integrated. We can show how the grouping of concepts forms categories by continuing with the example presented above. In addition to the concept of "pacing," the analyst might generate the concepts of "self-medicating," "resting," and "watching one's diet." While coding, the analyst may note that, although these concepts are different in form, they seem to represent activities directed toward a similar process: keeping an illness under control. They could be grouped under a more abstract heading, the category: "Self Strategies for Controlling Illness."

Conceptual categories can then be explored in other comparison groups, which may support the categorical concept or suggest modifications to make it more generalizable. GT strives toward verification through the process of category saturation, staying in the field until no further evidence emerges (Goulding, 1999).

Hypotheses (or "propositions" or "theories") indicate generalized relationships between a category and its concepts and between discrete categories. They provide a collection of explanations that explain the subject of the research. It should be noted that GT is explicitly emergent; it does not test a hypothesis. Instead GT sets out to find what theory accounts for the "research situation" as it is. Thus, generating hypotheses from the data requires only enough data to suggest the hypothesis, not to prove it.

New theory is developed from the contrast between "the daily realities (what is actually going on) of substantive areas" (Glaser \& Strauss, 1967, p. 239) and how those who participate in these "daily realities" (i.e., the "actors") interpret them.

In this sense, GT is an interpretive process. It looks for, "the actual production of meanings and concepts used by social actors in real settings" (Gephart, 2004, p. 457).

The GT process of generation and development of concepts, categories and hypotheses is an iterative process. Theory is not generated a priori and then subsequently tested, rather, as Corbin and Strauss (1990, p. 23) describe, it is, 
... inductively derived from the study of the phenomenon it represents. That is, discovered, developed, and provisionally verified through systematic data collection and analysis of data pertaining to that phenomenon. Therefore, data collection, analysis, and theory should stand in reciprocal relationship with each other. One does not begin with a theory, [and] then prove it. Rather, one begins with an area of study and what is relevant to that area is allowed to emerge.

Further, theory generation does not require many cases. Just one case could be used to generate conceptual categories and then a few more cases could be used to confirm them (Glaser \& Strauss, 1967). The idea, "is not to provide a perfect description of an area, but to develop a theory that accounts for much of the relevant behavior" (Glaser \& Strauss, 1967, p. 30).

Therefore, GT is an iterative emergent process which observes behaviour and events and then labels them in a manner consistent with the meaning/interpretation of these events by the actors. The GT methodology results in a set of concepts organized by categories and summarized by hypotheses.

\subsubsection{Approach}

The GT methodology, as described by Glaser and Strauss (1967), is built upon two key concepts: "constant comparison", and "theoretical sampling". Both concepts violate longstanding positivist assumptions about how the research process should work (Suddaby, 2006).

- In constant comparison, data are collected and analyzed simultaneously. This contradicts the myth of a clean separation between data collection and analysis.

- In theoretical sampling, decisions about which data should be collected next are determined by the theory that is being constructed. This violates the positivist ideal of hypothesis testing in that the direction of new data collection is determined, not by known hypotheses, but by ongoing interpretation of data and emerging conceptual categories.

GT research may be based on single or multiple sources of data such as interviews, observations, focus groups, life histories, and introspective accounts of experiences. The use of multiple data sources, known as "triangulation", is preferred since it enhances construct validity and reliability (Pandit, 1996). At the same time, researchers avoid being too structured in their methods of collecting information.

For example, an interview is not conducted using a prescribed formal schedule of questions since this would defeat the objective: to attain first hand information from the point of view of the informant. However, informants usually want some sense of the nature of the research and what information is sought. Thus, a totally unstructured interview would cause confusion and incoherence, and result in meaningless data, while a totally structured interview may be just an extension of the researcher's expectations. A balanced approach which allows the informant to feel comfortable enough to expand on their experiences, without the researcher telling them what to say is most desired (Goulding, 1999). 
Careful selection of interviewees can be guided by the notion of theoretical sampling. In GT, the sampling of informants is directed by the emerging theory. A researcher will initially go to the most obvious places or most likely informants for information. As concepts are identified and the theory starts to develop, further individuals, situations and places can be incorporated to further strengthen the findings (Glaser, 1978; Goulding, 1999).

It is important to note that the theoretical sampling of GT is very different from the statistical (or random) sampling commonly used in quantitative studies. Theoretical sampling aims to maximize opportunities for exploring emerging concepts. Statistical sampling aims to be representative of the population under study (Pace, 2004).

As data are collected, they are analyzed using open coding, axial coding, and selective coding. This is then tied together through memoing.

Open coding is the process of breaking down the data into distinct units of meaning. This process is associated with early concept development.

For example, an interview would be fully transcribed, the text would be analyzed line by line in an attempt to identify key words or phrases which connect the informant's account to the experience under investigation (Goulding, 1999).

Axial (or Theoretical) coding is a process which identifies the dynamic interrelationships of concepts (Goulding, 1999). This process is associated with identifying categories.

Selective coding is the process of delimiting coding to only those concepts that relate to the core category (Pace, 2004). If a core category is identified during a study, it may become its main theme. In selective coding, data collection and analysis becomes more focused, resulting in a theory with a smaller set of higher-level concepts (Glaser \& Strauss, 1967).

Memoing is a process of tying together pieces of data to general concepts. Memos are short documents that a researcher writes to themselves as they proceed through the analysis of a body of data. It is a sense-making tool that records the researcher's insights and ideas when they strike (Miles \& Huberman, 1994). Such memos can be used to help reorient the researcher at a later date (Goulding, 1999).

\subsubsection{Software Support}

GT is a labour-intensive methodology. Various software tools have been developed to support it. For example, NVivo (2012), which integrates well with SPSS (IBM Corp., 2013), handles various types of data sources including documents and transcripts. In particular, it links audio and video to transcripts very well. It can help with creating links among data (Richards, 2014).

However the use of computer software to code qualitative data in GT has been criticized. For example, Goulding (1999) argued that "an over-reliance on computer-aided analysis minimizes the personal experiences of the researcher, the process, and the situational factors which serve to add depth, rather than detract from the emerging developments." 


\subsubsection{When Should Grounded Theory be Used?}

According to Glaser, "Grounded Theory becomes an answer where other methodologies did not work well enough, especially in the sensitive dependent variable fields within the health science and business and management." (Glaser, 1995, p. 15).

According to Stern, "... the strongest case for the use of grounded theory is in investigations of relatively uncharted water, or to gain a fresh perspective in a familiar situation." (Stern, 1995, p. 30).

According to Strauss and Corbin, "If someone wanted to know whether one drug is more effective than another, then a double blind clinical trial would be more appropriate than grounded theory study. However, if someone wanted to know what it was like to be a participant in a drug study, then he or she might sensibly engage in a grounded theory project or some other type of qualitative study." (Corbin \& Strauss, 1990, p. 40).

This methodology generates substantive as well as formal theories, in either a well codified set of properties or running theoretical discussions; it is a theory generator (Glaser \& Strauss, 1967). As such, GT is most appropriate to situations where phenomenon is poorly understood and theory may not exist. Note, however that GT is not "atheoretical".

According to Goulding (1999), there is a common belief that GT ignores or avoids extant theory until the end of the analytical process. He argues that while it is true that GT was developed at a time when sociology had too great an emphasis on the verification of existing theory, in fact, existing theory has an important role in sensitizing the researcher to the conceptual significance of emerging concepts and categories (Goulding, 1999). According to Glaser, knowledge and theory are inextricably interlinked and should be used as if they were another informant (1978). Without this grounding in extant knowledge, pattern recognition would be limited to the obvious and the superficial, depriving the analyst of the conceptual leverage from which to develop theory (Glaser, 1978). Thus, GT requires an understanding of related theory and empirical work to enhance theoretical sensitivity (Goulding, 1999).

\subsection{Participants}

This Dissertation involved several different kinds of studies including focus groups, interviews, online and paper surveys, and usability studies. Altogether, 8 different music visualization styles were tested.

In total there were more than 100 unique participants across the studies described in Chapters 4 to 7. The study discussed in Chapter 8 drew on participants from the study in Chapter 7. All population groups were represented (Hard of Hearing, Deaf, Deafened, and Cochlear Implant users). Participants ranged from 18 to over 65 years of age. Generally, there were more women participants than men (about 70\%:30\%). 


\subsection{Next Steps}

The next five chapters each describe one of the five experiments conducted for this project. Figure 3.1 shows the order of the experiments. Note that the paper survey and interviews were conducted at the same time.

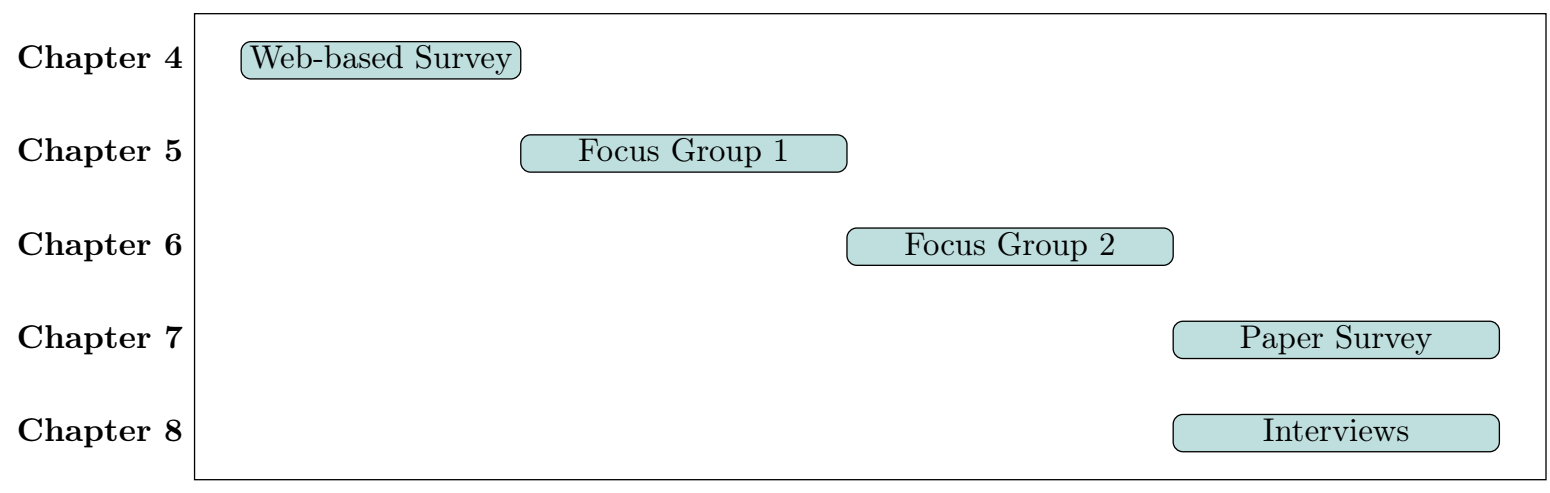

Figure 3.1: Order of experiments.

As mentioned earlier, the DRM provides a useful organizing principle for research through design. While it dies not attempt to follow the DRM as a method, this Dissertation will use the DRM as a means of tying the various elements of this project together. As seen in Figure 3.2 on the facing page, the DRM occurs within this Dissertation as a set of loops combined into one larger loop. Each of the four stages of an "inner loop" DRM cycle is marked by numbers in boxes. For the Dissertation as a whole, the work in each chapter represents part of the DRM and its iterative approach:

- The first DRM stage, Criteria Definition / Research Clarification was largely completed through the background provided in Chapter 2.

- Given the limited literature on the issue of music and the D/HH communities, Chapter 4 describes a web-based survey of music attitudes that was attached to a larger experiment of animated lyric captions. The results of this study shows a need for the current project and represents part of the scoping required by DRM Stage 0.

- A focus group conducted to evaluate various music visualizations as part of the DS-I (Descriptive Study I) stage of the DRM, providing a baseline of critical parameters in a situation into which some design intervention will be eventually introduced, is described in Chapter 5. These visualizations were either publicly available or prototypes designed and developed at Ryerson University.

- Chapter 6 contributes to DRM Stage 2 (Prescriptive Study) as various music visualizations explored in Chapter 5 were discarded and new prototypes were designed and developed at Ryerson. Chapter 6 describes a focus group conducted to evaluate this revised set of music visualizations. 


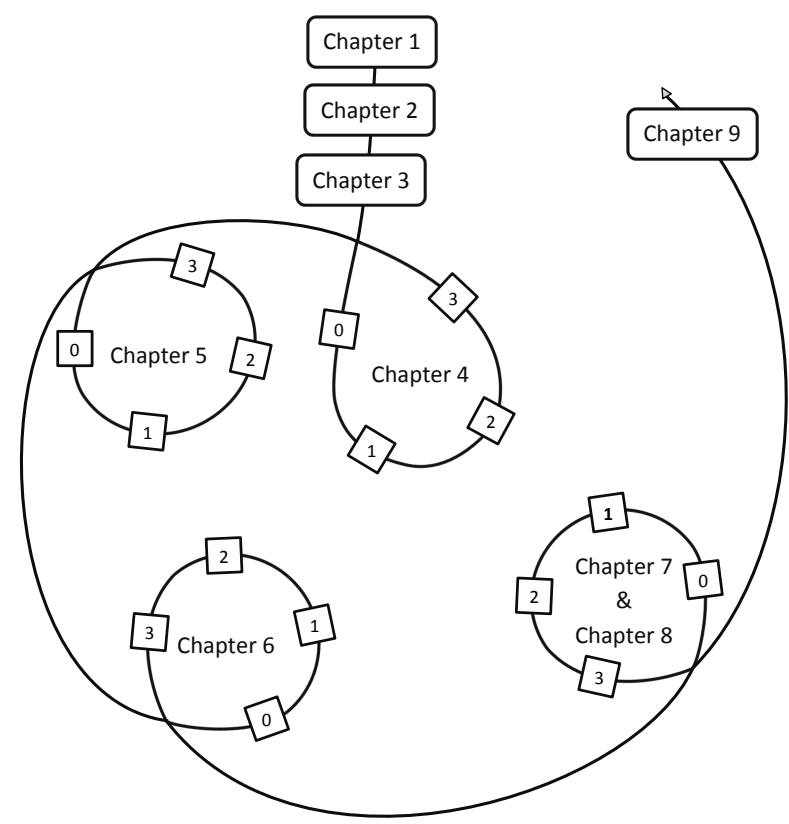

Figure 3.2: Dissertation path.

- Chapter 7 opens with a recognized need to refine the scoping of DRM Stage 0 discussed in Chapter 4. One of the ongoing issues was that the focus groups were more skewed in the number of $\mathrm{d} /$ Deaf participants or issues that might be perceived as specific to the $\mathrm{d} /$ Deaf and it was not clear if $\mathrm{HH}$ people had specific needs unique to their community. A paper survey was conducted at a conference for $\mathrm{HH}$ people to get more information from members of the $\mathrm{HH}$ community. Chapter 7 describes the results of this survey. In addition to returning to DRM Stage 0, this process also revisited DRM Stage 1, DS-I.

- Finally, since there was a need to get a better sense of an individual's response rather than the response of a focus group, one-on-one interviews were conducted among HH people. Chapter 8 describes these interviews and discusses their findings. This process further revisited DS-I and contributed to the PS stage.

As Figure 3.2 shows, a series of smaller DRM loops is also seen in this Dissertation. The experiments within each of Chapters 4,5 and 6 show the full DRM cycle because they introduce an intervention, the exposure of the participants to some prototype, and measure key parameters in their appreciation of music. The experiments in Chapters 7 and 8, when combined, also show the full cycle, with Chapter 7 representing the scoping Stage 0 and Chapter 8 continuing the loop. 



\section{Chapter 4}

\section{Need for the Work}

For hearing people, music is not just a cultural element. As McGraw (2008, Nov. 12) states, "music is so important. It's that thread that ties all your life together. ... It weaves you through, even some of the dark times in your life." While this may be true for hearing people, it is not clear that the D/HH appreciate music in the same way, if at all. The success of individuals such as Sean Forbes, a deaf rapper, suggests that some level of appreciation does occur (Maler, 2013).

Hearing people repeatedly report music evoking emotional responses and memory flashbacks (Janata et al., 2007), relaxation (Knight \& Rickard, 2001), and other experiences which make music pleasurable (Kohut \& Levarie, 1950). As a result, music is an important medium for conveying cultural information such as shared experience and knowledge (e.g., Loersch \& Arbuckle, 2013; Rose, 1994).

When $\mathrm{D} / \mathrm{HH}$ people do not have access to the music of the larger hearing culture, access to this shared experience - a shared knowledge space - may be lost. What is not clear is whether the $\mathrm{D} / \mathrm{HH}$ consumer even wants access to this information. This chapter explores the questions of:

- Do D/HH people appreciate music, and are efforts to create music information that is accessible to the $\mathrm{D} / \mathrm{HH}$ wanted?

- If the $\mathrm{D} / \mathrm{HH}$ want accessible music information, what types of information would be considered useful and how should it be presented?

First, this chapter will present a review of the literature on the music experiences of the $\mathrm{D} / \mathrm{HH}$. Then it will describe an online study conducted to assess the desirability of having access to music by $\mathrm{D} / \mathrm{HH}$ participants. Finally it will report the results of that study.

\subsection{Background}

\subsubsection{Music in the Primary and Secondary Schools}

In Western Culture, music holds such value that it is routinely included as part of a standardized curriculum for formal early years, elementary and secondary education (e.g., Ministry of Education and 
Training, 1998) even though controversy exists on whether such music programs are properly supported (Coalition for Music Education in Canada [CMEC], 2005).

Music programs have been provided to D/HH students in North America for over a century (Solomon, 1980). The first article in the American Annals of the Deaf and Dumb (the precursor to the American Annals of the Deaf) to advocate for music education for $\mathrm{D} / \mathrm{HH}$ children was published in 1848 (Darrow \& Heller, 1985). The first known use of music in the education of the $\mathrm{D} / \mathrm{HH}$ was a drum used in a military training class at the New York School for the Deaf in 1877 (Burns, 1965).

Where programs are provided, it is not always true that D/HH students are fully involved. Darrow and Gfeller (1991) reported that $23 \%$ of mainstreamed D/HH students in school systems that they surveyed did not receive any music education while more than half (52\%) attended regular music classes. More recently, Fix (2008) found that, of 27 oral schools for the deaf surveyed, $22(81 \%)$ had music programs while Kestner (2001) found that of 24 schools for the deaf surveyed (12 oral, 12 state), $67 \%$ of the oral schools and $42 \%$ of the state schools had music programs.

The reasons for providing such programs are varied. Little is said about exposure to music for its own sake. Instead, studies suggest that music education can benefit children who are $\mathrm{D} / \mathrm{HH}$ through improvements in auditory, speech, and social skills as well as cognitive and emotional development (Fix, 2008). However, it has been noted that research relating to music education and $\mathrm{D} / \mathrm{HH}$ children has been written by hearing authors (Darrow, 1993), a population which has historically taken a paternalistic attitude towards the deaf (Padden \& Humphries, 1988), suggesting that,

this situation calls into question the importance of music in the lives of deaf individuals, and underscores the notion that the hearing are speaking for the deaf, perhaps erroneously, regarding this importance and the value of music in educational programs for the deaf (Darrow, 1993, p. 94).

The preceding review shows clearly that while music has been used extensively in primary and secondary education, little is yet known about how best to use music in these settings. Access to these programs by $\mathrm{D} / \mathrm{HH}$ students is limited and the focus of these programs seems centred on the interests of hearing educators rather than the $\mathrm{D} / \mathrm{HH}$ people they serve.

\subsubsection{The Importance of Music to D/HH Preteens and Adolescents}

Regardless of whether a music program is available to them in their schools, for D/HH students in mainstreamed programs, music knowledge affects their day-to-day interactions with other hearing students because they are often the only D/HH student in their mainstream setting (the classroom and possibly the entire school). Oliva (2004) uses the term "solitaires" to refer to D/HH students who are alone in mainstream settings. It is estimated that approximately $15 \%$ of all $\mathrm{D} / \mathrm{HH}$ students in the United States are solitaires while up to $40 \%$ of all D/HH students in the United States have few, if any, same-age peers who are also D/HH (Oliva, 2004). Thus, shared cultural information such as knowledge about a new artist is probably more important to a D/HH student's social life with hearing peers than classroom knowledge about music. 
A similar phenomenon may be seen among deaf students attending a school for the deaf. For example, Medugno (2005) tells of the day his preteen deaf daughter, who attended a school for the deaf, wanted a Britney Spears CD. She wanted to fit in with the larger culture of similarly aged peers who are also fans of the same artist.

Music videos are very popular among teenagers. Captioned music videos are now very common. In a randomly chosen hour of thirteen current music videos spanning 2006 to 2009 and broadcast on a dedicated music video channel, only four $(30 \%)$ were not captioned. Captioned music videos started appearing in May of 1989 when American funk metal band "Living Color" decided to caption its videos. Soon, Cyndi Lauper and other artists followed suit. However, captioning was only made available when artists demanded it (D. Russell, 1989). By 1991, captioned pop music videos on dedicated cable channels (e.g., MTV) were still very rare. In Canada, YTV's Hit List, a music video television program which first aired in 1991 (Wikipedia, 2014c) was closely watched by D/HH people, because it was among the earliest such programs to provide closed captions (using roll-up captions) for every music video it featured. Perhaps unintentionally, programs like Hit List ensured that D/HH teenagers and young adults, at least in Canada, had access to important social knowledge which, until recently, was not otherwise easily available to them.

Although it is now more common for televised music videos to be captioned, unfortunately, the number of music videos being televised is steadily declining in favour of videos hosted on the Internet (typically without any captioning) and viewed on demand. For example, in 2010 MuchMusic, a Canadian cable channel originally established for the broadcast of music videos, attempted to reduce the percentage of music video programming that the station showed from 50 to 25 per cent on the basis that, "music videos no longer distinguish the service as they are readily available through other sources." (Canadian Radio-television and Telecommunications Commission [CRTC], 2010).

These examples suggest that there is music from the larger hearing culture that similarly-aged $\mathrm{D} / \mathrm{HH}$ people want to access either to fit in with their hearing peers or for other reasons. While $\mathrm{D} / \mathrm{HH}$ youth have greater access to music through greater availability of captioned music videos, it is unclear whether the lyrics alone (i.e., the content of the captions) are enough, whether there is enough information available to them to feel sufficiently informed about something their hearing peers enjoy, or whether they gain any entertainment from the experience.

The preceding review shows that anecdotal evidence exists that music is used extensively among $\mathrm{D} / \mathrm{HH}$ youth, however, outside of captioning, little is yet known about how best to support these users.

\subsubsection{The Music of the Deaf}

Any study of music and $\mathrm{D} / \mathrm{HH}$ people must be grounded in the context of $\mathrm{D} / \mathrm{HH}$ people and the role music plays in that context. This section will explore how music is created and transmitted to $\mathrm{D} / \mathrm{HH}$ people.

Every culture is thought to have its own body of music, "there is no culture known to man, no single civilization of the past, that does not have its own body of music" (Hamm et al., 1975, p. 71).

Darrow (1993) posits that the Deaf culture "may be the one exception" (p. 95). Although Deaf cul- 
ture, as described by Padden and Humphries (1988), is part of the larger society, it remains relatively unknown to most of the hearing population within which it resides. Ethnocentrism, the tendency to judge other cultures in terms of one's own, can result from such a lack of knowledge. Such ethnocentrism may lead hearing researchers such as Darrow to not recognize the body music extant in the Deaf community.

To understand how music is created within the Deaf community, one must realize that d/Deaf musicians create music for one of three audiences: $a$ ) other Deaf members of the community, $b$ ) d/Deaf and hearing combined audiences, and $c$ ) hearing audiences alone. The last may not be "Deaf music" at all but rather music created by a person who happens to be deaf solely within the norms and expectations of the larger hearing society.

\section{Music for Deaf Audiences}

Common forms of representing music for d/Deaf audiences include sign singing and dance. Sign singing, can occur in two major forms: translated songs or percussion signing (Bahan, 2006).

In translated songs, the lyrics of a song are translated or adapted into a signed language and then performed for an audience. A hearing interpreter may adapt the lyrics of an oral language song into a sign language or a Deaf performing artist might express an oral language song through a sign language. Such songs may involve ritual such as singing a national anthem (Bahan, 2006). While an interpreter might attempt to map each word directly to a sign without changing word order, a performer / artist may or may not use an exact translation of the original oral song. The artist may adapt the pace so that the song is visually pleasing (Bahan, 2006). Through sign alteration and manipulation of the signing space, the artist might express pitch, timbre, and phrasing (Maler, 2013). The artist may even change the lyrics to show playfulness and creativity with the signed language (Padden \& Humphries, 1988).

An example of translation by d/Deaf performing artists can be found with the Deaf Performing Artists Network (D-PAN) which exists to, "create media designed specifically to serve deaf audiences through the use of American Sign Language (ASL)". This work has been widely accepted within the Deaf community for the idea and the imaginative use of new media. An example is the use of graphic effects in the D-PAN video for Christina Aguilera's "Beautiful" (D-PAN [dpanvideos], 2008). During the chorus, the sign CAPTION is used to bring words onto the screen (to interpret the line "Words can't bring me down") while the sign GO-AWAY is used to "swat" these negative words away (to interpret the line "Don't you bring me down today") (Norman, 2008).

Percussion signing (sometimes called "Deaf Rap") is a form of musical expression arranged to certain beats. There are at least three types, "1, 2, 1, 2", "1, 2-1, 2, 3", and a combination of these two (Bahan, 2006). Deaf Rap is often accompanied by beats on a "Deaf Rap Drum", typically a large bass drum (Deaf Culture Centre, n.d.). The role of the drummer is to mark the cadence. For example, the Gallaudet University school fight song uses a "1, 2 - 1, 2, 3" beat (Padden \& Humphries, 1988).

In another type of percussion signing song, although its origins are unknown, a leader stands in front of a group next to a large board or sign with a list of words, and leads the group through the list 
as they sing the song together (e.g., an example is transcribed in Padden \& Humphries, 1988, p. 77).

Percussion signing is popular in deaf organizations and schools for the deaf for signed performances (Deaf Culture Centre, n.d.). However, it has been suggested that the tradition of percussion signing has been dying out (Bahan, 2006; Padden \& Humphries, 1988). However, my experience of percussion signing during a cultural festival, Crider's (2009) observations of percussion signing at various protests, and Tipton's (1997) documentation of hymns that are performed in ASL with a drum accompaniment, all suggest that the use of drums during sign performances is still very common.

\section{Music for Hearing Audiences}

Many deafened artists compose or perform music for the enjoyment of hearing audiences. Their deafness is seen as secondary to their musical expression rather than an integral part of their identity. Examples include Ludwig van Beethoven and Evelyn Glennie (Judkins, 1997).

\section{Music for Both Audiences}

Some deaf composers and performers actively include hearing and deaf elements into their performances making it accessible to deaf and hearing audiences alike. For example, "Beethoven's Nightmare" is an all-Deaf rock band, while Sean Forbes is one of many deaf rappers. Their musical performances include lyrics simultaneously sung in English and ASL.

Deaf people produce music for the enjoyment of deaf signers, or deaf, hearing, and hard of hearing non-signers. Deaf performers are creating music, but little is being asked of what deaf people think of music, whether they enjoy it, and what value they attach to it.

\subsubsection{Music and the Hard of Hearing}

As discussed in Chapter 2, a HH person can be described as, "a person who has a hearing loss and whose usual means of communication is a spoken language" (Canadian Hard of Hearing Association [CHHA], 2006). This definition includes a broad spectrum of hearing loss, from those who are latedeafened to those deaf in childhood and educated orally. However, generally speaking, sign language based access to information such as music is not appropriate for $\mathrm{HH}$ persons since, by definition, most do not sign.

Several authors have noted the presence of a HH culture (e.g., Ford, 1992; Israelite, Ower, \& Goldstein, 2002) or identity (e.g., Laszlo, 1994). Like the deaf community, HH identity is taught to new members and becomes self-perpetuating. It is not a single custom or tradition but shaped by life experience. The social experiences of $\mathrm{HH}$ children and the definitions of the world they will form as adults are very different from that of those whose hearing loss occurs when they are seniors (McKellin, 1994).

There is scant scholarly literature that makes it clear whether HH people actively enjoy music. MarkeTrak is a consumer survey that measures attitudes towards Hearing Aids (HAs) by asking for what activities a given consumer wears their HAs (Kochkin, 1990). Of the eight surveys conducted since 1989, only the last four included "Listening to music" as an item (Kochkin, 2000). One could 
suppose that, if HH people are thought to not enjoy music, consumer research in HAs would not include questions regarding music.

Culturally, $\mathrm{HH}$ people actively try to make use of whatever residual hearing they have (Laszlo, 1994) and many HH people use HAs to support their residual hearing (Turton \& Smith, 2013). However, hearing aids are known to distort music (Chasin, 2003; Chasin \& Hockley, 2014). This raises the question if $\mathrm{HH}$ people actively enjoy music, and hearing aids negatively interfere with their enjoyment of music, would they stop listening to music or stop using HAs?

It has been observed that consumer satisfaction of HAs has dropped over the past ten years leading to a high incidence of them being left "in the drawer" (Kochkin, 2000, p. 38). However, one consumer satisfaction surveys reports $77 \%$ of respondents are satisfied ( $9 \%$ are dissatisfied) with using their HAs while listening to music (Kochkin, 2005).

Technologies such as captioned music videos or lifestyle choices such as specific genres of music (e.g., songs with deep bass or drums and few vocals) may be among the few available options for HH music consumers who cannot hear music without HAs and/or are not satisfied with the currently available hearing instruments and/or need additional information to fully enjoy the music experience.

While it appears that $\mathrm{D} / \mathrm{HH}$ people are doing a great deal of musical composition and performance, there seems to be little known about what D/HH people think about music and how they are consuming music. Are these consumers consuming music as sound in a manner similar to hearing audiences or are they consuming music in some other manner?

Usually survey research intended to reflect the lives of $\mathrm{D} / \mathrm{HH}$ people focuses on the opinions of others (e.g., teachers, audiologists, social workers, parents, etc.) rather than the opinions of those being discussed (Lane, 1992). The research presented in this chapter will achieve some understanding of the experience(s) of music by $\mathrm{D} / \mathrm{HH}$ adults in their own words.

This chapter presents the results of an online study aimed at collecting initial attitudinal and experiential data on music appreciation from $\mathrm{D} / \mathrm{HH}$ people. In addition, it reports on people's experience with school programs and the influence that this has had on their attitudes towards music.

\subsection{Goals}

The goal of the study presented in this chapter is to answer the following questions:

1. Are efforts to create music information that is accessible to the $\mathrm{D} / \mathrm{HH}$ wanted?

2. If so,

(a) what types of information would be considered useful?

(b) what might be the desired form for presenting it? 


\subsection{Instruments}

The study reported in this chapter was part of a larger four-part study that evaluated the usability of music captioned via animated (or "kinetic") text (Mori \& Fels, 2009; Vy et al., 2008). This chapter describes and discusses the results of the fourth part, the Music Questionnaire. Appendix A shows instructions given and questions asked of all participants during the entire web-based survey. The remainder of this section will show only the questions asked in the Music Questionnaire.

\section{Music Questionnaire}

Because we used the lyrics of a song in this study, we are interested in your perspective and experience with music.

1. How often do you listen to music?

(a) All the time, (b) Frequently, (c) Sometimes, (d) Rarely, (e) Never

2. Which of the following statements describes you best?

(a) I really enjoy listening to music.

(b) I like listening to music.

(c) I do not like or dislike music.

(d) I do not like listening to music.

(e) I do not see the point of music.

3. How do you most often listen to music (check all that apply)?

(a) On radio, (b) On mobile music player, (c) Stereo at home, (d) Internet, (e) Television

4. If information about music from film, television, and radio could be displayed, what would be useful to have and what form would be best?

\begin{tabular}{|l|l|l|l|l|l|}
\cline { 2 - 6 } \multicolumn{1}{c|}{} & Text & Graphics & Animation & Icons & Don't care \\
\hline Lyrics / Words & & & & & \\
\hline Music Notes & & & & & \\
\hline Instruments & & & & & \\
\hline Beat / Rhythm & & & & & \\
\hline Speed of music playing & & & & & \\
\hline $\begin{array}{l}\text { Emotion conveyed } \\
\text { through the music }\end{array}$ & & & & & \\
\hline Composer / Singer's names & & & & & \\
\hline Title of song & & & & & \\
\hline
\end{tabular}

Other suggestions, please specify: 
5. What is your experience of music in elementary or secondary school?

(a) Very positive. (b) Somewhat positive. (c) Somewhat negative. (d) Very negative.

(e) Did not have any / Did not take it. (f) Do not remember.

6. We would greatly appreciate any additional comments.

Figure 4.1: Contents of the Music Questionnaire.

In addition to the last question asking for "additional comments", participants were also provided space to give comments in response to any of the other five questions.

\subsection{Method}

The study reported in this chapter was part of a larger study that evaluated the usability of music captioned via animated text (Mori \& Fels, 2009; Vy et al., 2008). The larger study consisted of four parts: a) completing a Pre-experiment Questionnaire, b) watching a Video, $c$ ) completing a Post-experiment Questionnaire, and $d$ ) completing a Music Questionnaire. This chapter describes and discusses the results of the fourth part, the Music Questionnaire. To provide context, the remainder of this section will describe the method of the entire study and conclude with a summary description of the participants.

\subsubsection{Pre-experiment Questionnaire}

In the first part of the larger study, participants were asked to visit a webpage using their own computer and preferred browser. After consenting to participate, participants were asked five demographic and pre-experiment questions to determine their hearing identity (hearing, hard of hearing, or deaf), gender, age range, highest level of education completed, and frequency of using captions when watching television.

\subsubsection{Video}

In the second part, participants were asked to watch one of two different prototype videos with animated lyrics.

One of the prototype videos, Forgive $M e$, had lead and background singers that required differentiation on screen. The lead singer's lyrics were displayed in a light blue 32 point (pt) font and placed in the upper left area of the screen. The background singers' lyrics were indicated in a yellow $22 \mathrm{pt}$ font and placed in the lower right area of the screen. It was possible that the lyrics of the lead and background singers could appear simultaneously on the screen. All lyrics were presented against a black background and timed to appear on the screen as they were sung in the song. The other prototype 
video, Family Snapshot, had one singer whose lyrics were presented in a white 22 pt font against a black background and were timed to appear as they were sung in the song.

The video presented to the participant was selected randomly by a randomizer embedded in the webpage. The lyrics were animated to indicate various emotions (e.g., happy, angry). No sound accompanied the lyrics. To not bias participants who may or may not be knowledgeable or interested in music, participants were only told that they would be watching a video. This avoided referring to the animated lyrics as "songs."

\subsubsection{Post-experiment Questionnaire}

After participants viewed one of these prototype videos, they moved to the third part where they were asked to complete a seventeen-item questionnaire about their understanding of the video and their opinion of the various caption elements (e.g., colour, movement, and position on screen). At the end of this questionnaire, participants were told that the video used lyrics from a musical recording.

\subsubsection{Music Questionnaire}

In the fourth part, participants were asked to share their "attitude and experience with music" and provide their opinions on specific topics. As shown in Section 4.3 on page 67 , using forced-choice questions, this six-item Music Questionnaire asked how frequently participants listened to music, the extent they enjoyed music, what technologies they used to listen to music (e.g., radio, MP3 player, etc.), what kinds of music information they were interested in, and their experience of music, if any, in their elementary and secondary schools.

These questions were asked for several reasons. Asking how often a participant listens to music provides information on whether the participant is a consumer of music and the degree which they believe they engage with music. The question on the extent a person enjoyed music was asked to determine the respondent's general attitude toward music.

Participants were asked to identify the technologies they use when listening to music to learn several things about them such as what technologies they prefer to use (since some technologies are more useful depending on a person's degree of hearing loss), whether they are consumers of music presenting technologies, and whether certain technologies are more likely to be used by members of the $\mathrm{D} / \mathrm{HH}$ communities than others.

The question asking what kind of music information participants would think is most useful as well as how this information might be represented visually used a table where each row was a type of information (lyrics, notes, instruments, beat, music speed, emotion, artist information, and song title) and each column was a type of representation (text, graphics, animation, icons, don't care). Participants were able to select, via checkboxes, as many responses as they wished.

The fifth question asked participants whether experience of music in school was positive or negative. This item is intended to gauge whether a negative / positive school music experience may have had impact on later music enjoyment / engagement as well as provide respondents with an opportunity to consider their early experiences with music. 
Each question was followed with an opportunity to provide further comments in an open-ended form.

The sixth question was open-ended and asked for any final comments the participant wanted to express.

\subsubsection{Participants}

The survey was online for one week. There were $14 \mathrm{HH}$ (3 males, 11 females) and 28 deaf (10 males, 18 females) participants. All of the participants but three reported watching captions "All the time" when watching television (one $\mathrm{HH}$ respondent reported occasionally watching captions, one deaf and one $\mathrm{HH}$ respondent reported never watching captions). Participants ranged in age from 19 to over 65 (median 45-54). The median age range for HH participants was 55-64 while that of deaf participants was 45-54. Of the two prototype videos, 23 participants saw Forgive Me (8 HH, 15 deaf) while 19 viewed Family Snapshot (6 HH, 13 deaf).

\subsection{Results}

All raw data is presented in Appendix A.

\subsubsection{Goal 1 - Are efforts to improve accessibility desired?}

Table 4.1 on the facing page shows the contingency table of hearing identity and how much the participant listens to music.

\section{Selected comments:}

- "I do not often know what the lyrics are, but I love the beats and rhythm."

- "I do love music, even though I know I am getting but a shadow of the real thing."

- "I am full deaf."

- "I can listen to music by the beat and sound but can't hear the words. It would be good to include captions in music videos on youtube, etc..."

- "music helps me to relax."

- "I LOVE music. I have been in band since the 7th grade and continue to play and enjoy musical instruments."

- "I do lot of reading song lyric and think of a way to translate/ express into ASL"

- "On my walkman etc., I find classical music very relaxing" 


\begin{tabular}{|c|c|c|c|c|c|c|c|c|}
\hline & & & \multicolumn{5}{|c|}{ Frequency of listening to music } & Total \\
\hline & & & $\begin{array}{l}\text { All the time } \\
\text { (daily) }\end{array}$ & $\begin{array}{l}\text { Frequently } \\
\text { (weekly) }\end{array}$ & Sometimes & Rarely & Never & \\
\hline \multirow[t]{3}{*}{ Identity } & $\mathrm{HH}$ & Count & $\begin{array}{r}3 \\
21.4 \%\end{array}$ & $\begin{array}{r}4 \\
28.6 \%\end{array}$ & $\begin{array}{r}3 \\
21.4 \%\end{array}$ & $\begin{array}{r}3 \\
21.4 \%\end{array}$ & $\begin{array}{r}1 \\
7.1 \%\end{array}$ & $\begin{array}{r}14 \\
100.0 \%\end{array}$ \\
\hline & Deaf & Count & 4 & 6 & 8 & 4 & 6 & 28 \\
\hline & & $\%$ & $14.3 \%$ & $21.4 \%$ & $28.6 \%$ & $14.3 \%$ & $21.4 \%$ & $100.0 \%$ \\
\hline Total & & $\begin{array}{r}\text { Count } \\
\%\end{array}$ & $\begin{array}{r}7 \\
16.7 \%\end{array}$ & $\begin{array}{r}10 \\
23.8 \%\end{array}$ & $\begin{array}{r}11 \\
26.2 \%\end{array}$ & $\begin{array}{r}7 \\
16.7 \%\end{array}$ & $\begin{array}{r}7 \\
16.7 \%\end{array}$ & $\begin{array}{r}42 \\
100.0 \%\end{array}$ \\
\hline
\end{tabular}

Table 4.1: How often do you listen to music? (by hearing identity)

\begin{tabular}{|c|c|c|c|c|c|c|c|}
\hline & & \multicolumn{5}{|c|}{ Statement that describes you best } & \multirow[t]{2}{*}{ Total } \\
\hline & & $\begin{array}{c}\text { I really enjoy } \\
\text { listening to } \\
\text { music. }\end{array}$ & $\begin{array}{l}\text { I like } \\
\text { listening to } \\
\text { music. }\end{array}$ & $\begin{array}{c}\text { I do not } \\
\text { like or dislike } \\
\text { music. }\end{array}$ & $\begin{array}{c}\text { I do not like } \\
\text { listening to } \\
\text { music. }\end{array}$ & $\begin{array}{l}\text { I do not see } \\
\text { the point of } \\
\text { music. }\end{array}$ & \\
\hline \multirow[t]{2}{*}{$\mathrm{HH}$} & Count & 5 & 6 & 0 & 1 & 1 & 13 \\
\hline & $\%$ & $38.5 \%$ & $46.2 \%$ & $0.0 \%$ & $7.7 \%$ & $7.7 \%$ & $100.0 \%$ \\
\hline \multirow{2}{*}{ Deaf } & Count & 8 & 9 & 7 & 1 & 2 & 27 \\
\hline & $\%$ & $29.6 \%$ & $33.3 \%$ & $25.9 \%$ & $3.7 \%$ & $7.4 \%$ & $100.0 \%$ \\
\hline \multirow[t]{2}{*}{ Total } & Count & 13 & 15 & 7 & 2 & 3 & 40 \\
\hline & $\%$ & $32.5 \%$ & $37.5 \%$ & $17.5 \%$ & $5.0 \%$ & $7.5 \%$ & $100.0 \%$ \\
\hline
\end{tabular}

Table 4.2: What statement describes you best? (by hearing identity)

As shown in Table 4.2, of the 40 participants who responded to the question "What statement describes you best?", most D/HH participants responded that they either really enjoy (13 or 33\%) or like (15 or $38 \%$ ) music. Specifically, 17 deaf participants (42\% of overall, $63 \%$ of the deaf) reported that they like or enjoy listening to music. Conversely, 3 participants (8\%), 2 deaf and $1 \mathrm{HH}$, reported that they "do not see the point of music".

\section{Selected comments:}

- "I used to love listening to music. Wish they would add captioning to all music videos on DVD all the time."

- "I am deaf."

- "A day without music is like a day without sunshine to me!"

- "no comment. I can't hear. :)"

Table 4.3 reviews the data in Table 4.2 in terms of respondents who really enjoy or like music. There is no statistically significant relationship between whether one likes music and their hearing identity. 


\begin{tabular}{rrrrrr}
\hline & & & \multicolumn{2}{c}{ Likes music } & Total \\
\hline \multirow{3}{*}{ Identity } & $\mathrm{HH}$ & & No & Yes & \\
\cline { 3 - 5 } & & Count & 2 & 11 & 13 \\
& \multirow{2}{*}{ Deaf } & \% within Identity & $15.4 \%$ & $84.6 \%$ & $100.0 \%$ \\
& & \% within Identity & $38.5 \%$ & $61.5 \%$ & $100.0 \%$ \\
& & Count & 12 & 27 & 39 \\
& & \% within Identity & $30.8 \%$ & $69.2 \%$ & $100.0 \%$ \\
\hline
\end{tabular}

Table 4.3: Tends to like music? (by hearing identity)

\begin{tabular}{rrrrrr}
\hline & & \multicolumn{3}{c}{ Likes music } & Total \\
\hline \multirow{3}{*}{ Gender } & \multirow{3}{*}{ Male } & & \multicolumn{2}{c}{ No } & Yes \\
\cline { 3 - 5 } & & Count & 7 & 4 & 11 \\
& Female within Gender & $63.6 \%$ & $36.4 \%$ & $100.0 \%$ \\
\multirow{4}{*}{ Total } & Count & 5 & 23 & 28 \\
& & $\%$ within Gender & $17.9 \%$ & $82.1 \%$ & $100.0 \%$ \\
& & Count & 12 & 27 & 39 \\
& & $\%$ within Gender & $30.8 \%$ & $69.2 \%$ & $100.0 \%$ \\
\hline
\end{tabular}

Table 4.4: Tends to like music? (by gender)

Table 4.4 reviews the data in Table 4.2 in terms of respondents who really enjoy or like music according to their gender. A chi-square test for independence was conducted to determine if there is any relationship between the participant's gender and their report of how much they liked music.

$H_{0}$ : Gender and the Participant's report of how much they liked music are independent.

$H_{a}$ : Gender and the Participant's report of how much they liked music are not independent.

There is a statistically significant relationship between whether one likes music and their gender such that women were more likely to report that they liked music, $\mathcal{X}^{2}(1, \mathrm{~N}=39)=7.770, p=0.005$. Since the $p$-value is less than the significance level (0.05), we cannot accept the null hypothesis.

Table 4.5 on the facing page shows the different ways each group listened to music (participants could select more than one response to this question). Five of the 42 participants left the item blank.

\section{Selected comments:}

- "Music tunes out my tinnitus."

- "loud at airshows and motorsports I love."

- "Like the feeling of vibration"

- "I borrow quite a lot of musical CD's and DVD's from the library." 


\begin{tabular}{lcccccc}
\hline \# of responses (\%) & Radio & Mobile Player & Stereo & Computer & Television & Total \\
\hline Hard of Hearing & $7(50.0)$ & $4(28.6)$ & $8(57.1)$ & $3(21.4)$ & $6(42.9)$ & 28 \\
Deaf & $6(21.4)$ & $4(14.3)$ & $10(35.7)$ & $7(25.0)$ & $21(75.0)$ & 48 \\
Total & $13(31.0)$ & $8(19.0)$ & $18(42.9)$ & $10(23.8)$ & $27(64.3)$ & 76 \\
\hline
\end{tabular}

Table 4.5: Preferred ways to listen to music.

- "never"

- "Now that I have problems with background noise, I don't listen to music as often as I used to."

- "Radio is usually just as background in the car, when I really don't care about lyrics"

- "Concerts."

- "Because Television is visually - great for all Deaf and Hard of Hearing People."

- "CDs in the vehicle"

- "Wish they include captioning on Internet!!!"

- "also with the vehicle with my music on with DVD or tapes"

- "ON Radio in the car on the way to work and traveling in general, and at home on my mp3 player and iPod"

- "I like it loud music on the internet or on tv, anything that I can have"

Table 4.6 on the next page shows the contingency table of hearing identity and experience of music in elementary or secondary school.

\section{Selected comments:}

- "I was told to stop singing. I sensed that my music teacher really hated me as she gave so much body language!"

- "We learned to read music and sang a great deal."

- "rhythm class was fun sometimes"

- "HaHa! should deafer take music course at school uh?"

- "bands parade and cheerleaders sport events."

- "I did not take any music classes because I was not able to hear the notes or the sound of music. Although I wear two hearing aids and I can hear well but I can't listen to the radio because all i can hear is mumbling." 


\begin{tabular}{|c|c|c|c|c|c|c|c|c|c|}
\hline & & & \multicolumn{6}{|c|}{ Experience of music in elementary or secondary school } & Total \\
\hline \multirow{5}{*}{ Id } & \multirow{3}{*}{$\mathrm{HH}$} & \multirow[b]{2}{*}{ Count } & $\begin{array}{l}\text { Very } \\
\text { pos. }\end{array}$ & $\begin{array}{c}\text { Somewhat } \\
\text { pos. }\end{array}$ & $\begin{array}{c}\text { Somewhat } \\
\text { neg. }\end{array}$ & $\begin{array}{l}\text { Very } \\
\text { neg. }\end{array}$ & $\begin{array}{c}\text { No class / } \\
\text { Did not } \\
\text { take }\end{array}$ & $\begin{array}{l}\text { Do not } \\
\text { recall }\end{array}$ & \\
\hline & & & 1 & 8 & 1 & 1 & 2 & 1 & 14 \\
\hline & & $\%$ & $7.1 \%$ & $57.1 \%$ & $7.1 \%$ & $7.1 \%$ & $14.3 \%$ & $7.1 \%$ & $100.0 \%$ \\
\hline & \multirow[t]{2}{*}{ Deaf } & Count & 4 & 9 & 5 & 1 & 6 & 2 & 27 \\
\hline & & $\%$ & $14.8 \%$ & $33.3 \%$ & $18.5 \%$ & $3.7 \%$ & $22.2 \%$ & $7.4 \%$ & $100.0 \%$ \\
\hline \multirow[t]{2}{*}{ Total } & & Count & 5 & 17 & 6 & 2 & 8 & 3 & 41 \\
\hline & & $\%$ & $12.2 \%$ & $41.5 \%$ & $14.6 \%$ & $4.9 \%$ & $19.5 \%$ & $7.3 \%$ & $100.0 \%$ \\
\hline
\end{tabular}

Table 4.6: Music experience in school (by hearing identity).

\begin{tabular}{|c|c|c|c|c|c|}
\hline & & & \multicolumn{2}{|c|}{ Likes music } & Total \\
\hline & & & No & Yes & \\
\hline \multirow{15}{*}{ Experience of music in school } & Very positive. & Count & 1 & 2 & 3 \\
\hline & & $\%$ & $33.3 \%$ & $66.7 \%$ & $100.0 \%$ \\
\hline & Somewhat posi- & Count & 4 & 13 & 17 \\
\hline & tive. & $\%$ & $23.5 \%$ & $765 \%$ & $1000 \%$ \\
\hline & Somewhat neg- & Count & 2 & 4 & 6 \\
\hline & & & & & \\
\hline & & $\%$ & $33.3 \%$ & $66.7 \%$ & $100.0 \%$ \\
\hline & Very negative. & Count & 0 & 2 & 2 \\
\hline & & $\%$ & $0.0 \%$ & $100.0 \%$ & $100.0 \%$ \\
\hline & Did not have & Count & 3 & 4 & 7 \\
\hline & $\begin{array}{l}\text { any / Did not take } \\
\text { it. }\end{array}$ & & & & \\
\hline & & $\%$ & $42.9 \%$ & $57.1 \%$ & $100.0 \%$ \\
\hline & Do not remem- & Count & 1 & 2 & 3 \\
\hline & ber. & & & & \\
\hline & & $\%$ & $33.3 \%$ & $66.7 \%$ & $100.0 \%$ \\
\hline \multirow[t]{2}{*}{ Total } & & Count & 11 & 27 & 38 \\
\hline & & $\%$ & $28.9 \%$ & $71.1 \%$ & $100.0 \%$ \\
\hline
\end{tabular}

Table 4.7: Music experience in school (by tendency to like music).

- "As a hard of hearing person, it was very difficult and awkward for me to participate in mandatory music classes. I wasn't able to hear the words or follow what was going on."

- "They used American Sign Language and facial [expressions] while they sang."

A chi-square test for independence was conducted to determine if there is any relationship between the participant's reports of how frequently they listened to music and the video that they had watched before answering the Music Questionnaire.

$H_{0}$ : Participant's report of frequency of listening to music and the video they saw during the survey 
are independent.

$H_{a}$ : Participant's report of frequency of listening to music and the video they saw during the survey are not independent.

There is a significant difference in participant's reports of how frequently they listened to music and the video that they had watched before answering the Music Questionnaire, $\mathcal{X}^{2}(4, \mathrm{~N}=42)=$ $10.273, p=0.036$. Since the $p$-value is less than the significance level $(0.05)$, we cannot accept the null hypothesis. Participants were more likely to report listening to music "All the time" if they saw Family Snapshot, while they were more likely to respond "Frequently" if they saw Forgive Me.

\subsubsection{Goal 2 - What information is needed?}

As shown in Section 4.3 on page 67, the Music Questionnaire asked participants what kind of music information they would think is most useful and how they thought this information might be best visually represented. Participants were provided with eight types of music information (lyrics, notes, instruments, beat, music speed, emotion, artist information, and song title) and five categories for representing music information (text, graphics, animation, icons and don't care).

With respect to the five categories for representing music information, an initial analysis of the results suggested that the participants were confused with the difference between the terms "icon" and "graphic", and seemed to think of those two concepts as similar. Thus, the categories "icon" and a "graphic" were combined. Table 4.8 on the next page shows the frequency of participant's different preferences for representing music information.

\section{Selected comments:}

- "A question - In this case, what is the difference between a graphic and an icon?"

- "I like when songs are captioned in text in italic writing with the music notes. However, I wish there was sometimes a little more info on the beats and rhythm."

- "Not sure"

- "most deafers don't care on music name/songs"

- "Deaf people like loud bass than high pitch because it is feeling vibration."

- "I am not knowledgeable about music, just find it relaxing."

A contingency table analysis was carried out to examine whether there is any relationship between how participants wanted music information presented and the type of music information. The result is shown in Table 4.8. A chi-square test for independence was conducted to determine if there is any relationship between the participant's preferred form of representing music information and the type of music information to be represented. 


\begin{tabular}{lccccc}
\hline \# of responses (\%) & Text & $\begin{array}{c}\text { Graphics } \\
\text { / icons }\end{array}$ & Animation & Don't care & Blank \\
\hline Lyrics & $32(76.2)$ & $0(0)$ & $3(7.1)$ & $3(7.1)$ & $4(9.5)$ \\
Notes & $6(14.3)$ & $9(21.4)$ & $5(11.9)$ & $12(28.6)$ & $10(23.8)$ \\
Instruments & $9(21.4)$ & $16(38.1)$ & $3(7.1)$ & $9(21.4)$ & $5(11.9)$ \\
Beat & $7(16.7)$ & $10(23.8)$ & $16(38.1)$ & $4(9.5)$ & $5(11.9)$ \\
Speed & $7(16.7)$ & $9(21.4)$ & $11(26.2)$ & $5(11.9)$ & $10(23.8)$ \\
Emotion & $9(21.4)$ & $8(19.0)$ & $10(23.8)$ & $8(19.0)$ & $7(16.7)$ \\
Singer & $27(64.3)$ & $3(7.1)$ & $1(2.4)$ & $6(14.3)$ & $5(11.9)$ \\
Title & $30(71.4)$ & $4(9.5)$ & $2(4.8)$ & $4(9.5)$ & $2(4.8)$ \\
\hline
\end{tabular}

Table 4.8: Preferred forms for representing music information.

\begin{tabular}{lcccc}
\hline & $d f$ & $N$ & $\mathcal{X}^{2}$ & $p$ \\
\hline Representation of lyrics & 2 & 38 & 8.17 & 0.017 \\
Representation of beat & 3 & 37 & 13.844 & 0.003 \\
Representation of artist information & 3 & 37 & 7.925 & 0.048 \\
Representation of song title & 3 & 40 & 15.089 & 0.002 \\
\hline
\end{tabular}

Table 4.9: Chi-square test results of participant's preferences for representing music information by gender.

$H_{0}$ : Participant's preferred form of representing music information and the type of music information to be represented are independent.

$H_{a}$ : Participant's preferred form of representing music information and the type of music information to be represented are not independent.

There is a statistically significant relationship between participant's preferred form of representing music information and the type of music information to be represented, $\mathcal{X}^{2}(21, \mathrm{~N}=40)=120.24, p$ $<0.001$. Since the $p$-value is less than the significance level (0.05), we cannot accept the null hypothesis.

A contingency table analysis was carried out to examine whether there is any relationship between gender and question responses for all questions.

$H_{0}:$ Gender and voting question responses are independent.

$H_{a}:$ Gender and voting question responses are not independent.

Table 4.9 shows there are four significant chi-square results for gender and representation of lyrics, representation of beat/rhythm, representation of artist information, and representation of song title. Tables 4.10 to 4.13 show the response frequencies resulting from these chi-squared analyzes. Since the $p$-values shown in Table 4.9 are less than the significance level (0.05), we cannot accept the null hypotheses. 


\begin{tabular}{|c|c|c|c|c|c|c|}
\hline & & & \multicolumn{3}{|c|}{ Representation of Lyrics } & Total \\
\hline \multirow{4}{*}{ Gender } & \multirow{3}{*}{ Male } & & Text & Animation & Don't care & \\
\hline & & Count & 7 & 1 & 3 & 11 \\
\hline & & $\%$ within Gender & $63.6 \%$ & $9.1 \%$ & $27.3 \%$ & $100.0 \%$ \\
\hline & \multirow{4}{*}{ Female } & Count & 25 & 2 & 0 & 27 \\
\hline \multirow{3}{*}{ Total } & & $\%$ within Gender & $92.6 \%$ & $7.4 \%$ & $0.0 \%$ & $100.0 \%$ \\
\hline & & Count & 32 & 3 & 3 & 38 \\
\hline & & $\%$ within Gender & $84.2 \%$ & $7.9 \%$ & $7.9 \%$ & $100.0 \%$ \\
\hline
\end{tabular}

Table 4.10: Frequency results representation of lyrics by gender.

\begin{tabular}{|c|c|c|c|c|c|c|c|}
\hline & & & \multicolumn{4}{|c|}{ Representation of Beat / Rhythm } & Total \\
\hline \multirow{4}{*}{ Gender } & \multirow{3}{*}{ Male } & & Text & $\begin{array}{c}\text { Graphics } \\
\text { / icons }\end{array}$ & Animation & Don't care & \\
\hline & & Count & 0 & 4 & 3 & 4 & 11 \\
\hline & & $\%$ within Gender & $0.0 \%$ & $36.4 \%$ & $27.3 \%$ & $36.4 \%$ & $100.0 \%$ \\
\hline & Female & Count & 7 & 6 & 13 & 0 & 26 \\
\hline \multirow{3}{*}{ Total } & & $\%$ within Gender & $26.9 \%$ & $23.1 \%$ & $50.0 \%$ & $0.0 \%$ & $100.0 \%$ \\
\hline & & Count & 7 & 10 & 16 & 4 & 37 \\
\hline & & $\%$ within Gender & $18.9 \%$ & $27.0 \%$ & $43.2 \%$ & $10.8 \%$ & $100.0 \%$ \\
\hline
\end{tabular}

Table 4.11: Frequency results representation of beat by gender.

\begin{tabular}{|c|c|c|c|c|c|c|c|}
\hline & & & \multicolumn{4}{|c|}{ Representation of Artist Information } & Total \\
\hline & & & Text & $\begin{array}{c}\text { Graphics } \\
\text { / icons }\end{array}$ & Animation & Don't care & \\
\hline \multirow[t]{4}{*}{ Gender } & Male & Count & 5 & 1 & 1 & 4 & 11 \\
\hline & & $\%$ within Gender & $45.5 \%$ & $9.1 \%$ & $9.1 \%$ & $36.4 \%$ & $100.0 \%$ \\
\hline & Female & Count & 22 & 2 & 0 & 2 & 26 \\
\hline & & $\%$ within Gender & $84.6 \%$ & $7.7 \%$ & $0.0 \%$ & $7.7 \%$ & $100.0 \%$ \\
\hline \multirow[t]{2}{*}{ Total } & & Count & 27 & 3 & 1 & 6 & 37 \\
\hline & & $\%$ within Gender & $73.0 \%$ & $8.1 \%$ & $2.7 \%$ & $16.2 \%$ & $100.0 \%$ \\
\hline
\end{tabular}

Table 4.12: Frequency results representation of artist information by gender. 


\begin{tabular}{|c|c|c|c|c|c|c|c|}
\hline & & & \multicolumn{4}{|c|}{ Representation of Song Title } & Total \\
\hline & & & Text & $\begin{array}{l}\text { Graphics } \\
\text { / icons }\end{array}$ & Animation & Don't care & \\
\hline \multirow[t]{4}{*}{ Gender } & Male & Count & 4 & 2 & 1 & 4 & 11 \\
\hline & & $\%$ within Gender & $36.4 \%$ & $18.2 \%$ & $9.1 \%$ & $36.4 \%$ & $100.0 \%$ \\
\hline & Female & Count & 26 & 2 & 1 & 0 & 29 \\
\hline & & $\%$ within Gender & $89.7 \%$ & $6.9 \%$ & $3.4 \%$ & $0.0 \%$ & $100.0 \%$ \\
\hline \multirow[t]{2}{*}{ Total } & & Count & 30 & 4 & 2 & 4 & 40 \\
\hline & & $\%$ within Gender & $75.0 \%$ & $10.0 \%$ & $5.0 \%$ & $10.0 \%$ & $100.0 \%$ \\
\hline
\end{tabular}

Table 4.13: Frequency results representation of song title by gender.

A chi-square test for independence was conducted to determine if there is any relationship between the participant's reports of how they wanted beat information represented and the video they had just seen.

$H_{0}$ : Participant's reports of how they wanted beat information represented and the video they had just seen are independent.

$H_{a}$ : Participant's reports of how they wanted beat information represented and the video they had just seen are not independent.

There is a statistically significant relationship in participants' reports of how they wanted beat information represented and the video they had just seen, $\mathcal{X}^{2}(3, \mathrm{~N}=37)=8.556, p=0.036$. Since the $p$-value is less than the significance level (0.05), we cannot accept the null hypothesis. Viewers of Family Snapshot were more likely to indicate a desire for text or graphics whereas viewers of Forgive Me expressed a preference for animation.

A chi-square test for independence was conducted to determine if there is any relationship between the participant's reports of how they wanted speed of the music represented and the video they had just seen.

$H_{0}$ : Participant's reports of how they wanted speed of the music represented and the video they had just seen are independent.

$H_{a}$ : Participant's reports of how they wanted speed of the music represented and the video they had just seen are not independent.

There is a statistically significant relationship in participants' reports of how they wanted speed of the music represented and the video they had just seen, $\mathcal{X}^{2}(3, \mathrm{~N}=32)=8.718, p=0.033$. Viewers of Forgive Me were more likely to indicate a desire for text or graphics whereas viewers of Family Snapshot expressed a preference for animation. 


\subsection{Discussion}

\subsubsection{Goal 1 - Are efforts to improve accessibility desired?}

To determine whether efforts to create music information that is accessible to the $\mathrm{D} / \mathrm{HH}$ are wanted, there is a need to understand whether $\mathrm{D} / \mathrm{HH}$ people are active consumers of music.

There was no significant difference in participants' frequency of listening to music based on their hearing identity. Table 4.1 on page 71 shows the frequency of hearing identity and how much the participant listens to music. Regardless of hearing identity, 35 of the $42 \mathrm{D} / \mathrm{HH}$ participants (83\%) who responded to this item reported listening to music. However, their comments suggest their reasons for doing so may not always be related to entertainment. As one deaf participant commented, "music helps me to relax." Since D/HH people do use music to manage the effects of tinnitus (e.g., Tyler, 2006), we can expect to see reports of this use of music by this population. One HH participant commented, "Music tunes out my tinnitus."

As shown in Table 4.2 on page 71 , of the 40 participants who responded to the question "What statement describes you best?", most D/HH participants responded that they either really enjoy (13 or $33 \%$ ) or like (15 or $38 \%$ ) music. Specifically, 17 deaf participants ( $42 \%$ of overall, $63 \%$ of the deaf) reported that they like or enjoy listening to music. Conversely, 3 participants (8\%), 2 deaf and $1 \mathrm{HH}$, stated that they "do not see the point of music". As one deaf participant noted, "I am deaf." Darrow (1993) found that $46.8 \%$ of respondents who identified with Deaf culture considered music "Not at all" important yet, as Stewart notes, "many [deaf] people do love music, even if they can't hear much of it" (1990, as cited in Darrow, 1993).

As shown in, Table 4.5 on page 73 , when participants were asked how they most often listened to music, responses were quite varied. Interestingly, four deaf participants ( $14 \%$ of 37 respondents) reported using mobile music players such as iPods, Walkmans, etc. to listen to music. This suggests a market exists for mobile players among the $\mathrm{D} / \mathrm{HH}$. Music players that support video might prove very useful for D/HH users who want visual information as well as auditory music.

Some of the comments deaf participants provided to the question of how they most often listened to music went beyond the question. One pointed out, "loud at airshows and motorsports I love." While another said, "Like the feeling of vibration." For the deaf, "hearing" is simply another sense of touch (Glennie, n.d.).

As mentioned in Section 4.1.1 on page 61 , many deaf children do not have access to any music education programs and some choose not to take music classes. This experience could impact a person's use of music. Table 4.6 on page 74 shows the majority of $\mathrm{D} / \mathrm{HH}$ participants took some sort of music education and, in general, they report having had a positive experience. According to Table 4.7 on page 74, even if they had a negative school experience, participants still tended to report enjoying music. Comments provided by participants help to explore their experiences.

One deaf participant in this study commented that, "I did not take any music classes because I was not able to hear the notes or the sound of music."

A deaf person who had a somewhat positive experience reported that "rhythm class was fun some- 
times." Similarly, one of the youngest deaf participants reported a very positive experience, saying "They used American Sign Language and facial [expressions] while they sang."

One participant's very negative experience led to the comment that, "As a hard of hearing person, it was very difficult and awkward for me to participate in mandatory music classes. I wasn't able to hear the words or follow what was going on." Similarly, a deaf participant who found their music program very negative said, "I was told to stop singing. I sensed that my music teacher really hated me as she gave so much body language!"

There were a number of gender differences identified in the results. For example, D/HH women reported a higher frequency of listening to music with $45 \%$ of women reporting a daily or weekly frequency compared to $31 \%$ of men. Conversely, $38 \%$ of men reported "Never" listening to music compared to $7 \%$ of women. Women were also more likely to report that they liked music. While it is possible that these results may be an important gender difference, they may be an artifact of the more than twice as many female participants than male participants in this study.

\subsubsection{Goal 2 - What information is needed?}

As mentioned in Chapter 1, Principle Four of the Principles of Universal Design, "Perceptible Information", requires the design to communicate necessary information effectively to the user, regardless of ambient conditions or the user's sensory abilities and encourages using different modalities (i.e., auditory, visual, tactile) to redundantly present essential information. Determining what types of music information would be considered useful would be supportive of the Principles of Universal Design.

To help determine what types of music information would be considered useful and what might be the desired form for presenting it, participants were asked about how they would like to see music displayed and the kinds of information they would want to see.

Table 4.8 shows the majority of participants had a strong preference for text to be used for lyrics (32 of 38 participants or $84 \%$ ), song title (30 of 40 participants or $75 \%$ ) and artist information (27 of 37 participants or $73 \%$ ). It is very likely that this response is a reflection of the fact that this information is normally available in text and participants seem to accept this and want this information presented as text.

While the majority of $\mathrm{D} / \mathrm{HH}$ participants indicated a preference for using text to represent lyrics, three of the 25 deaf participants $(12 \%)$ specifically suggested animation to represent lyrics while another three responded "don't care". This suggests a preference among deaf participants for a culturally appropriate non-text representation of lyrics. In their comments, one deaf participant suggested using a sign language video to represent lyrics while another suggested including a sign translation in the corner of the screen. These ideas require caution since, as mentioned in Chapter 2, most HH people do not sign.

Another interpretation for the expressed preference for animation may be that this response is an effect of having just seen lyrics containing animated text earlier in the study. However, several participants completed the open-ended question by commenting directly on the captions they saw. Many specifically complained about the use of colour and animated effects in the captions. 
Three of 37 participants selected icons/graphics (8\%), and one selected animation (3\%) to present the artist information. No one made any suggestions of what an animated or graphic display of this information might contain. For example, a picture of the artist, album artwork, or some type of logo could be used. The remaining participants (6 of 37) selected "Don't care" (16\%). Five participants did not respond to this item.

Four of 40 participants selected icons/graphics (15\%) and two deaf participants selected animation (5\%) to represent song title. Again, no suggestions were made. One participant comments, "most deafers don't care on music name/songs" The remaining four participants selected "Don't care" (10\%). Two participants left the item blank.

The majority of participants (16 of 37 or $43 \%$ ) wanted beat/rhythm to be represented by animation. Ten participants $(27 \%)$ wanted it represented by graphics/icons, seven (19\%) wanted it represented using text, and four participants (11\%), three of whom had seen the Forgive Me video, did not care how it was represented. Five participants of the total $(12 \%)$ did not answer this question. One HH participant commented, "I wish there was sometimes a little more info on the beats and rhythm." Thus, this music information is desired, preferably in some non-textual form.

If we examine the descriptive information, there are some noteworthy trends. Twelve of 32 participants (38\%) selected "Don't care" for how music notes should be represented. Ten (24\%) participants left the item blank. Six of 32 participants preferred text (19\%), and 9 of 32 preferred icons/graphics $(28 \%)$. Given that the majority of participants (22) either gave no response or indicated they "Don't care" how music notes should be represented, there seems to be less interest in note information than other forms of music information. However, as one HH participant commented, "I like when songs are captioned in text, in italic writing with the music notes." The traditional representation of music in closed captioning is text with a music note icon. Italics are often used to indicate an off-screen singer.

The majority of $\mathrm{D} / \mathrm{HH}$ participants indicated a desire for instrument information. However, the 37 responding participants were split on whether to use text (9 of 37 or $24 \%$ ), graphics/icons (16 or $43 \%$ ), or animations (3 or $8 \%$ ) with the remainder selecting "Don't care" (9 or $24 \%$ ). More study is needed to understand how much instrument information $\mathrm{D} / \mathrm{HH}$ consumers want.

In terms of how they wanted speed of the music represented, 11 of 32 participants selected animation (34\%), 9 selected graphics/icons (28\%), 7 selected text (22\%), and 5 (three of whom had seen the Forgive Me video) selected "Don't care" (16\%). This was also the one item that was the most ignored since more participants (24\% of all participants) left it blank than any other item. Where a response was provided, animation slightly edged out all the others. This result suggests that either speed of the music may be a less important type of music information to provide, or this item was poorly understood.

Animation was also the preferred approach for representing emotion. Ten of 35 participants selected animation to show emotion (29\%), 9 expressed an interest in using text to show emotion (26\%), and 8 participants selected graphics/icons (23\%) while another 8 selected "Don't care" (23\%). The remaining participants left the item blank.

Several gender differences were noted. Tables 4.10 to 4.13 show the response frequencies resulting from these chi-squared analyzes presented in Table 4.9. Women showed a strong preference for the 
use of text to represent lyrics, artist information, and song title as well as a strong preference for the use of animation for the representation of rhythm and beat information. It is possible this result is a reflection of the large number of female participants, but it is reported here because it may be an important gender difference.

As reflected from their comments, for a few of the participants, this study led to some very deep thinking. A participant who used to actively compose music and play the piano prior to her hearing loss stated, "I deeply miss that experience."

Perhaps a comment by one deaf participant is most illustrative of the concerns and experiences surrounding music raised by participants,

I wish very much I could understand music. I wish I had a person who could talk to me about music and explain and answer my questions. Some voices are literally thrilling to listen to. I wish I could identify the instruments. I wish I knew what people talk about when they discuss music. I wish I could sing, or play an instrument myself. I like to watch captioned movies that talk about composers and their works. I have met people who are able to tell which CONDUCTOR is playing a piece! [I have also met] many who can identify who is singing a piece on the radio, or what the music is etc. I would love to see more TV programs that have captions to tell, who it is, what they are playing etc etc and more history about the piece.

\subsubsection{Limitations of the Design}

The analysis of the data suggests at least five limitations of this study, three of which are related to questionnaire design.

First, the questionnaire design was not able to investigate combinations of different methods to represent information. For example, animation and text could be combined to present a song title in a sign language as well as text.

Second, there may be some mix up in the participant's understanding of "animation". Since the study presents animated captions, a selection of "Animation" may in fact be a preference for animated captions or equally a preference for some other type of moving graphic (e.g., a sign video). Thus, both possible definitions were taken into account when interpreting the data.

A third limitation of this design is that while asking how to represent music information, the Music Questionnaire did not also ask for some ranking of the importance of each type of information. As a result this must be extrapolated from the responses. For example, it can be suggested that the information is important when a preference is specified and it can be suggested that there is no importance when a participant responds, "Don't care" or ignores the item and leaves it blank. However, any conclusion that is more specific cannot be established based on this data.

Alternatively, given the wording of question 4 in the Music Questionnaire (i.e., "If information about music from film, television, and radio could be displayed, what would be useful to have and what form would be best?"), if a participant responded "Don't care" or ignored the item and left it blank, it may be interpreted as the question suggests as not "useful". 
Also, there are few if any examples of meaningful graphics used in music or animation, etc., so it may be hard for $\mathrm{D} / \mathrm{HH}$ consumers to imagine the possibilities for representing music information visually.

The small sample size with an unequal number of men and women is another limitation. The chisquare test for independence is sensitive to sample size as well as small expected frequencies in one or more of the cells in the table. For these reasons caution must be taken in interpreting chi-square if one or more cells are less than 5. However, according to Frankfort-Nachmias and Leon-Guerrero, "There is no hard-and-fast rule regarding the size of expected frequencies." (2011, p. 350). Most researchers limit the use of chi-square to tables that either a) have no expected frequencies values below 5 or $b$ ) have no more than 20 percent of the expected frequencies values below 5 (Frankfort-Nachmias \& Leon-Guerrero, 2011).

Finally, the complexity of the larger experiment within which this survey was performed may have confounded the results. Recall that the Music Questionnaire was a small subset of the larger study. Participants completed a demographics survey, then watched one of two videos which was randomly assigned, and then completed a series of post test questions based on the video they watched all before completing the Music Questionnaire. There may have been a presentation effect with respect to the video presented. There was a relationship in participants' reports of how they wanted beat information represented and the video they had just seen where viewers of Family Snapshot were more likely to indicate a desire for text or graphics whereas viewers of Forgive Me expressed a preference for animation. There was also a relationship in participants' reports of how they wanted speed of the music represented and the video they had just seen where viewers of Forgive Me were more likely to indicate a desire for text or graphics whereas viewers of Family Snapshot expressed a preference for animation.

\subsection{Conclusions From This Study}

Recall the research questions explored in this chapter were:

1. Are efforts to create music information that is accessible to the $\mathrm{D} / \mathrm{HH}$ wanted?

2. If so, what types of information would be considered useful and what might be the desired form for presenting this music information?

In answer to the question of whether efforts to create accessible music information are wanted, the responses of $\mathrm{D} / \mathrm{HH}$ participants strongly support the notion that they actively seek music and enjoy the experience. Few reported having no interest in music. Some participants found the study very interesting and reported a desire to see more. At the same time, reflecting the poverty of using captions alone to visualize music, one participant stated, "As a Deaf person, I do not particularly care about music being conveyed through captions."

However, consistent with Principle One of the Principles of Universal Design, "Equitable use", visualizations of music cannot exclude the auditory experience. Many of the $\mathrm{D} / \mathrm{HH}$ participants in this 
study reported listening to music, not just having an interest in the lyrics. Yet, as one deafened participant said, "I find most music a screeching mishmash of Chinese-sounding (no offense, please) incomprehensibilities." There is a need to find that balance, which differs for each individual, between noise and entertainment.

In answer to the question as to what types of information would be considered useful and what might be the desired form for presenting this music information, there is also great individual difference. Participants in this study showed a clear interest in lyrics, song information, instruments, and rhythm / beat. There appeared to be less interest in notes or the speed of music. There may be only limited interest in information about the emotions being expressed. These findings need further investigation to best meet the needs of $\mathrm{D} / \mathrm{HH}$ consumers and may indicate a need to combine different forms of representation to visualize specific music information.

The responses and comments provided by participants make clear that $\mathrm{D} / \mathrm{HH}$ adults do not feel that their needs are met by current music captioning approaches. $\mathrm{D} / \mathrm{HH}$ captioning consumers do not want to just see a musical note or the title of a song. As one deaf participant said, "It's annoying when a song that is TWENTY YEARS OLD - just for an example, isn't fully captioned. How hard can it be to look up lyrics THAT OLD?" That said, captions of the lyrics alone, are not enough, as this study shows, other information must be included. For example, the comments of at least one participant suggested that the reason why the piece is being used is just as important as the musical information. Describing the use of music in television programs and movies, the participant wanted to know, "more history about the piece."

As mentioned earlier, usually survey research intended to reflect the lives of $\mathrm{D} / \mathrm{HH}$ people focus on the opinions of others (e.g., teachers, audiologists, social workers, parents, etc.) rather than the opinions of those being discussed. In particular, the hearing seem to be speaking for the deaf with regards to the importance and value of music to the Deaf community (e.g., Darrow, 1993). The responses of the participants of the larger study included comments such as "such an interesting survey" and "Thanks for your study and consideration." show that D/HH adults want to be asked what they think about music and how they consume / access it. Past research showed an ulterior motive, whether acknowledged or not, in understanding the way to present music to D/HH children. Researchers continue to focus on children and the "benefits" of music education to speech and oral language skill development. Little discussion occurs regarding access to music for its own sake.

This study joins the ranks of the very few (e.g., Brokaw, 1997; Darrow, 1993) which have explored the thoughts, feelings, and experiences of D/HH adults regarding music. While there has been recent attention on the issue of music and the D/HH communities by D/HH authors (e.g., Crider, 2009), more attention is needed to understand the needs of each population with respect to access to music information.

It is interesting that participants did not mention existing music visualizations used by music playing software such as Windows Media Player. Such visualizations employ an abstract art approach which may or may not provide any meaningful information to a $\mathrm{D} / \mathrm{HH}$ consumer. Future work could determine their usability for $\mathrm{D} / \mathrm{HH}$ music consumers and explore the role, if any, such visualizations may play for $\mathrm{D} / \mathrm{HH}$ music consumers. Chapters 5,6 and 8 describe experiments that present such vi- 


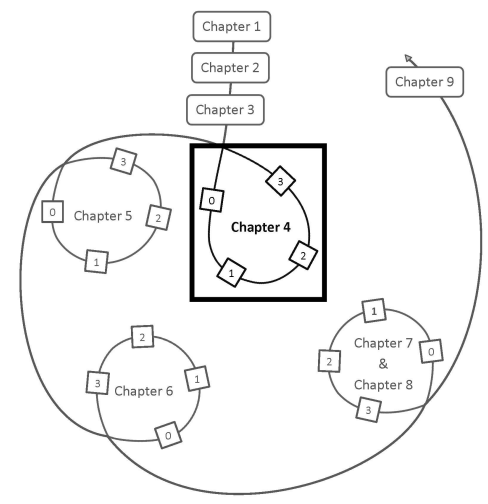

Figure 4.2: Location within Dissertation path.

sualizations.

Further work to better understand the needs and expectations of $\mathrm{D} / \mathrm{HH}$ music consumers is needed. Future work could take the shape of further surveys specific to their experiences of music or focus groups that explore different music visualization possibilities as well as consumer opinions.

\subsection{Next Steps}

The work reported in this chapter fills in some of the gaps created by the lack of literature on the issue of music and the $\mathrm{D} / \mathrm{HH}$ communities. In particular it shows that members of the $\mathrm{D} / \mathrm{HH}$ communities actively seek out and enjoy music. It shows the need for the current project and represents part of the scoping required by DRM Stage 0 .

As shown in Figure 4.2, the four parts of the experiment discussed in this chapter represent the full DRM cycle because it introduced an intervention, the exposure of the participants to the videos, and measured key parameters in their appreciation of music.

The next chapter describes a focus group conducted to evaluate various music visualizations. The visualizations were selected to be consistent with the scoping work reported in this chapter. As such, the focus group work qualifies as a DRM Stage 1 (Descriptive Study I) activity, which provides a baseline of critical parameters in a situation into which some design intervention will be eventually introduced. 



\section{Chapter 5}

\section{Focus Group I}

Music content can be so powerful that it may carry long-term cultural significance (Fourney \& Fels, 2008). For example, many North American adults closely associate the main shark theme from the movie "Jaws", a simple alternating pattern of the notes E and F (Matessino, 1999, Sept. 24), with images of an attacking shark and the anticipation of doom, even if the music is not being used in the context of sharks.

This chapter considers one possible solution to creating access for D/HH audiences to this cultural information and entertainment medium: making music visual. For any prototype visualization of music to be successful, it needs to convey the sense of music, its rhythm, melody, emotional content, and overall entertainment. Ultimately, the experience should be similar to that enjoyed through sound except that the means of enjoyment would be different. If the song is intended to be soothing, or invigorating, or melancholic, or joyful, then it should be perceived as such.

As previously discussed in Section 2.5.4 on page 32, the visual modality has several strengths that make it ideal for exploring the presentation of music information:

- Human visual resolution is stronger than human auditory resolution (Kramer, 1994).

- Very large amounts of information can be presented on a screen while the transient nature of sound requires their discrete presentation (Kramer, 1994).

- The visual system of deaf adults may monitor their peripheral visual field more than hearing adults (Bavelier et al., 2006).

- Screens represent a mature, cheap, portable, nearly ubiquitous technology.

In this chapter, different options for music visualization are explored towards determining what music information could be visualized and appropriately convey the experience and meaningfulness of music. This chapter presents the results of a focus group with $\mathrm{D} / \mathrm{HH}$ users who viewed these various options and provided their expectations for visualized music.

Portions of this chapter appear in Fourney and Fels (2009). 


\section{$5.1 \quad$ Background}

As mentioned in Chapter 2, music is a combination of rhythm, pitch, timing, timbre, and texture. Its performance can range from a strictly organized composition to improvisations. Each culture has its own definition of music and methods of expression. Music has elements which listeners find pleasing and enjoyable.

Music is often perceived as primarily an aural experience especially for recorded music. However, there are tactile and visual components of music that can enhance or complement the overall music experience such as feeling the drum beat or watching the movements of the performers (band members, instrumentalists, vocalists) or dance accompaniment.

Live performance is particularly exemplary of the use of visual and tactile enhancements. For example, live performances often involve dancing, or transmission of vibration through the floor from the percussion instruments. Recording and reproducing such live performances removes this additional information from the experience and changes the focus of musical entertainment to one that is exclusively aural. This focus excludes D/HH people from participation. Music videos have returned some of the visual elements of live performances but they are not intended to increase the accessibility of the music. Music videos appear to be more oriented to marketing the product or boosting the "image" of the artist (Straw, 1988) than visually conveying the artist's own interpretation of the music.

The lyrics in combination with the instruments, rhythm, melody, harmony, volume and tempo together provide an emotional effect. In addition, while music can act at an emotional level in humans, "to move people" (Toland, 2001), it can also be consumed in the background as a more pleasant auditory stimuli and more positive effect on their emotional level than what is currently in people's environment (e.g., car noise, the neighbour's lawn mower). One of the issues then is how the receptive experience of music information can be made more accessible to $\mathrm{D} / \mathrm{HH}$ people in a way that creates enjoyable experiences.

The next section presents the different visualization options used in the present study and outlines their properties.

\subsection{Goals}

The goal of the study presented in this chapter is to answer the following questions:

1. Are efforts to improve accessibility desired?

2. How do viewers respond to specific visualizations?

\subsection{Instruments}

Appendix B shows the instructions given and questions asked of all participants during the entire focus group as well as a full transcript. The remainder of this section will show only the questionnaires used to collect data during the focus group. 


\section{Music Visualization: Pre-study questionnaire}

This purpose of this questionnaire is to gather information about you and your experience with music and closed captioning. There are eight questions and it should take you about five minutes to complete this questionnaire. Thank you in advance for your time and assistance.

1. Do you identify yourself as: (please check one)
Hearing
Hard of hearing
Deaf
D Deafened
- Cochlear implant

2. Are you
Male
口 Female

3. Please indicate your age
ㅁ $19-24$
口 $25-34$
口 $35-44$
口 45- 54
口 55- 64
$65+$

4. What is your highest level of education?
- No formal education
口 Elementary school
- High School
口 Technical college
$\square$ University
口 Graduate school

5. How often do you use closed captioning when watching television?
a Always
- Sometimes
$\square$ Never

6. How often do you listen to music?
All the time (daily).
Frequently (weekly)
a Sometimes
Rarely
D Never

7. Which of the following statements best describes you?
I really enjoy listening to music.
a I like listening to music.
I do not like or dislike music.
I I do not like listening to music
I do not see the point of music.

8. What is your experience of music in elementary or secondary school?
Very positive.
- Somewhat positive
$\square$ Somewhat negative.
Very negative.
Did not have any / Did not take it.
$\square$ Do not remember.

Figure 5.1: Focus Group Pre-study Questionnaire. 


\section{Music Questions}

1. Was the music visualization enjoyable (circle one)?

$\begin{array}{lll}\text { Not enjoyable at Not that enjoyable Neutral } & \begin{array}{l}\text { Somewhat } \\ \text { enjoyable }\end{array} & \text { Enjoyable }\end{array}$

2. What did the visualization mean to you?

3. On a scale from 1 to 7 , was the music

Positive

1

2

3

4

5

6

Negative

7

4. In your opinion, what was the energy level of that music (circle one)

Sleepy

1

2

3

4

5

6

Awake

7

5. Identify which emotion you thought was expressed and rate its strength from 1 to 7 (circle one)

$$
\text { Weak }
$$

Strong

$\begin{array}{llllllll}\text { Happy } & 1 & 2 & 3 & 4 & 5 & 6 & 7 \\ \text { Sad } & 1 & 2 & 3 & 4 & 5 & 6 & 7 \\ \text { Angry } & 1 & 2 & 3 & 4 & 5 & 6 & 7 \\ \text { Fear } & 1 & 2 & 3 & 4 & 5 & 6 & 7\end{array}$

6. What did you like best of the visualization style?

7. What did you like least of the visualization style?

90

Figure 5.2: Focus Group Post-visualization Questionnaire. 
Music Visualization Post-Study Questionnaire

This purpose of this questionnaire is to gather information about you and your opinions about the visual representations that you just saw. There five questions and it should take you about ten minutes to complete this questionnaire. Thank you in advance for your time and assistance.

1. Which style did you enjoy most?

2. Which style gave you the best sense of what was happening in the music

3. Which style gave you the best sense of:

\begin{tabular}{|l|l|l|l|l|l|l|l|l|}
\hline & $\begin{array}{c}\text { Color } \\
\text { Going Out }\end{array}$ & $\begin{array}{c}\text { Color } \\
\text { Going In }\end{array}$ & $\begin{array}{c}\text { Lots of } \\
\text { Fans }\end{array}$ & $\begin{array}{c}\text { A Few } \\
\text { Fans }\end{array}$ & Psychedelic & $\begin{array}{c}\text { Flashing } \\
\text { Sun }\end{array}$ & $\begin{array}{c}\text { Moving } \\
\text { Shapes }\end{array}$ & None \\
\hline Drums/percussion & & & & & & & & \\
\hline $\begin{array}{l}\text { Instruments } \\
\text { playing }\end{array}$ & & & & & & & & \\
\hline Melody & & & & & & & & \\
\hline Rhythm & & & & & & & & \\
\hline
\end{tabular}

4. What was the most enjoyable about your experience with music visualization

5. What was the least enjoyable about your experience with music visualization

Figure 5.3: Focus Group Post-study Questionnaire. 


\begin{tabular}{ll}
\hline Songs & Artist or Composer \\
\hline Happy & Beatles \\
$\begin{array}{l}\text { Day Tripper } \\
\text { Rockin' Robin }\end{array}$ & Leon René \\
Anger & \\
Basket Case & Green Day \\
Purple Haze & Jimi Hendrix \\
Fear & \\
Anywhere & $?$ \\
Vampire Hunters & Wojciech Kilar \\
Sad & \\
16 Candles & The Crests \\
Carrickfergus / Carrighfergus & Loreena McKennitt \\
\hline
\end{tabular}

Table 5.1: List of songs.

\subsection{Method}

\subsubsection{Step 1}

In advance of the focus group, instrumental-only versions of 15 popular songs thirty seconds long were assessed by a group of four hearing people for the overall emotion each clip represented (happiness, sadness, anger, and fear). If these informants all agreed on the emotion the song represented, it was included in the set of songs to be presented to the focus group.

\subsubsection{Step 2}

Five different displays (iTunes, MPM, MAM Piano Roll display, MAM Part Motion display, and MAM Tonal Compass display) created from three different visualization tools (Music Animation Machine, iTunes, and Motion Pixels of Music) were used to create a total of 18 prototype visualizations of eight different popular music tracks. The list of songs used is presented in Table 5.1. The resulting prototypes were presented to $\mathrm{D} / \mathrm{HH}$ participants in a focus group setting.

To capture the individual experience as well as the larger group comments multiple tools were used to collect participant's responses to the prototypes. After each prototype was shown, participants were asked for their opinions about the visualization using a discussion format as well as the Post-visualization Questionnaire shown in Figure 5.2. Participants also completed a pre-study questionnaire to gather information about their music experiences as well as a post-study questionnaire to summarize their opinions of the music visualization experience.

Since different D/HH people have different levels of hearing and different abilities to process whatever sound they can hear, the songs were presented without sound to control for variation of hearing 
levels.

An ASL interpreter and a stenographer, to live caption the proceedings, were present to facilitate the participation of the $\mathrm{D} / \mathrm{HH}$ participants.

\subsubsection{Visualizations}

\section{Music Animation Machine}

The first visualization tool was the Music Animation Machine (MAM). As described in Chapter 2, it provides various visualizations of real-time music data based on MIDI file input. MAM was used to produce three different displays: Piano Roll, Part Motion (Balls), and Tonal Compass (Malinowski, n.d.).

\section{Magnetosphere}

As described in Chapter 2, the default visualizer of the iTunes media player application provides visualizations based on waveform analysis of the audio input. Since the visual parameters of such visualizations tend to be completely arbitrary (Lipscomb \& Kim, 2004).

\section{Motion Pixels of Music}

Recognizing that the visualizations provided by the above two visualizers do not present all the properties of music provided in other visualizations (i.e., lyrics, tonality, harmony, loudness, pitch, and timing), the Motion Pixels of Music (MPM) visualization was specifically developed for this study. The MPM provides information on tonality, harmony, pitch and timing as well as showing the instrumentation present, and beat / rhythm information. The visualization uses real-time music data based on MIDI file input. It can individually display all sixteen MIDI channels, where available. While lyrics can be accommodated in this tool, no lyrics were used in this study.

This visualization has two ways of depicting note duration: decay the animation outward or decay the animation inward. In the outward mode, shown in Figure 5.4, the note appears to continue to move out toward the edge of the MPM. In the inward mode the note appears to fall back to its origin.

Tempo or beat stimuli is represented using a cluster of circles that use a wave effect. Four of the circles in the cluster represent the cymbals (top most), high hat (leftmost), snare drum (centre circle), bass drum (largest circle) as common percussion instruments, and the fifth circle represents any fifth percussive instrument (e.g., tambourine, kettle drum, etc.).

Table 5.2 shows the common types of musical information each of these visualizations can display. Some visualizations are unique in displaying specific information (e.g., Magnetosphere displays amplitude, MPM displays instrumentation). It is not clear if any of these five visualizations (iTunes, MPM, MAM Piano Roll display, MAM Part Motion display, and MAM Tonal Compass display) were explicitly designed to communicate emotions. 


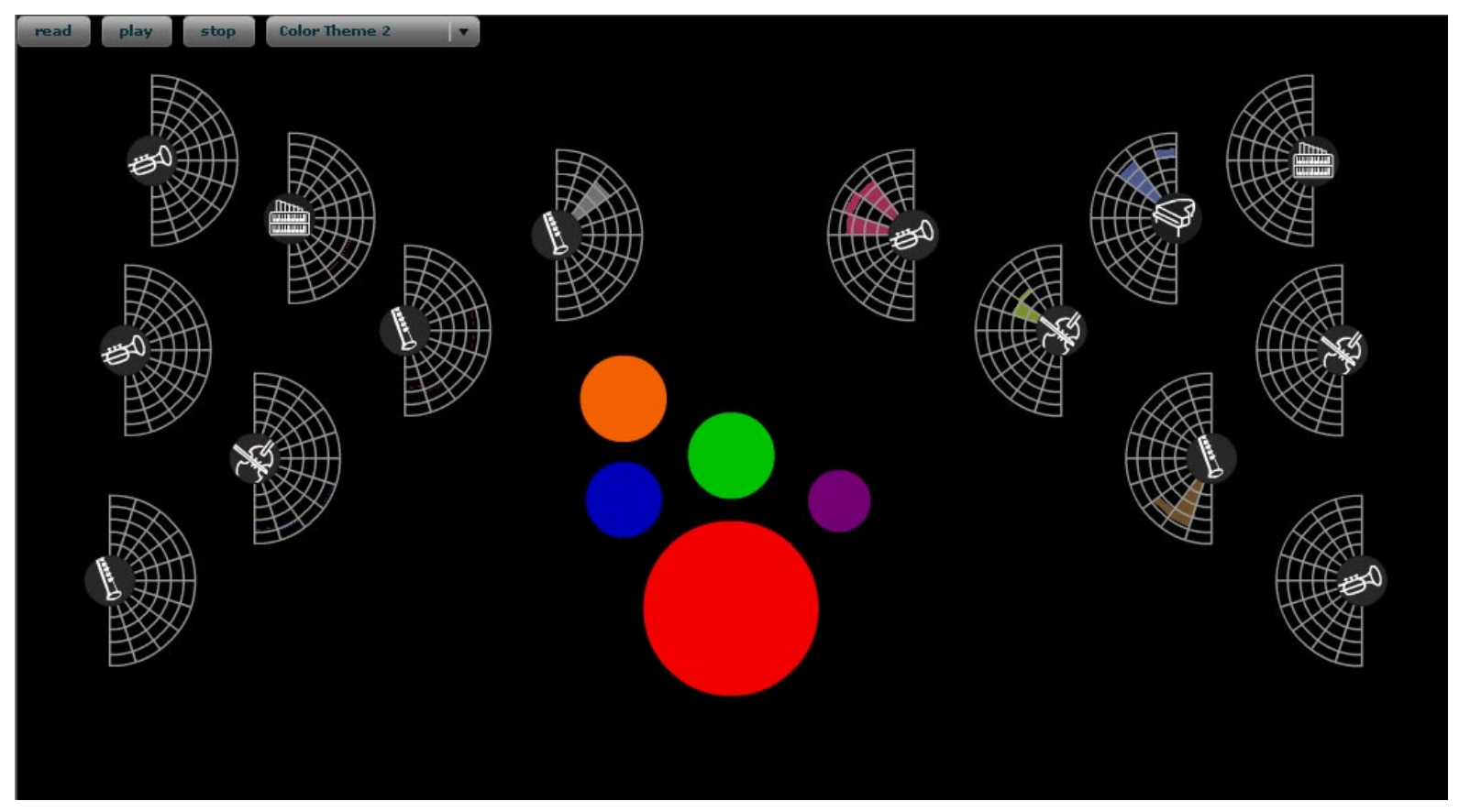

Figure 5.4: The Motion Pixels of Music (MPM) visualization.

\begin{tabular}{rcccccccc}
\hline Visualization & $\begin{array}{c}\text { Change in } \\
\text { Pitch }\end{array}$ & Timbre & Tempo & Tonality & $\begin{array}{c}\text { Change in } \\
\text { Frequency }\end{array}$ & $\begin{array}{c}\text { Duration } \\
\text { of Notes }\end{array}$ & Parts & Beat \\
\hline iTunes & & & & & $\mathrm{X}$ & $\mathrm{X}$ & & \\
MPM & $\mathrm{X}$ & $\mathrm{X}$ & $\mathrm{X}$ & $\mathrm{X}$ & & $\mathrm{X}$ & $\mathrm{X}$ & $\mathrm{X}$ \\
Piano Roll & $\mathrm{X}$ & $\mathrm{X}$ & $\mathrm{X}$ & & $\mathrm{X}$ & $\mathrm{X}$ & $\mathrm{X}$ & \\
Part Motion & $\mathrm{X}$ & & $\mathrm{X}$ & $\mathrm{X}$ & $\mathrm{X}$ & $\mathrm{X}$ & $\mathrm{X}$ & $\mathrm{X}$ \\
T. Compass & & & & $\mathrm{X}$ & & & & \\
\hline
\end{tabular}

Table 5.2: Visualization properties. 


\subsubsection{Stimuli and Their Properties}

Instrumental-only versions of eight popular songs representing four different emotions (i.e., two songs each representing one of happiness, sadness, anger, and fear) were randomly presented, without sound, for thirty seconds in either one or two of the above visualization techniques (i.e., iTunes, MPM, MAM Piano Roll display, MAM Part Motion display, and MAM Tonal Compass display). These specific visualization tools were selected because they were representative of the variation (e.g., using pitch, timbre and tempo in Piano Roll, tonality in Tonal Compass, frequency and amplitude for iTunes, and tonality, harmony, pitch, tempo, timbre and beat for MPM) that is available according to the literature.

Since the MPM visualization relies on MIDI information, it can only display the instruments coded into the MIDI file. Showing all sixteen channels when only a few have any information could impact the usability of the visualization. As a result, depending on which song was playing, the MPM visualization would show either a small number of MIDI channels (e.g., four or eight) or a very large number of MIDI channels (e.g., all sixteen). This variation was used to elicit responses on the amount of information that can be tolerated by viewers.

Songs were played either once or twice (i.e., each emotion had between two and four presentations). When a song was presented in two ways, one was always a MPM presentation. To help ensure they were not influenced by predisposition / familiarity with certain applications, the title bar of the application was always hidden from participants' view. After each presentation, participants completed a 7-item questionnaire about the emotional impact (ratings of discrete emotions such as fear, anger, happy and sad, and arousal/valence), level of enjoyment, and opinions of the visualization they had just seen. After all 18 visualizations were shown, participants then completed the post-study questionnaire and participated in a follow-up discussion.

\subsubsection{Participants}

The focus group had 10 participants: five deaf (three male, two female), one deafened (female) and four HH (female). One deaf male also identified himself as a Cochlear Implant (CI) user. Participants ranged in age from 25 to over 65 (4 were 25-34, 1 was 35-44, 2 were 45-54, 2 were 55-64, and 1 was $65+)$.

Nine participants indicated always using closed captions when watching television. One indicated only using captions "sometimes".

Three participants reported high school or some technical college as their highest level of formal education. The remaining seven were university graduates, three reported completing graduate school.

\subsection{Results}

All raw data is presented in Appendix B. 


\subsubsection{Goal 1 - Are efforts to improve accessibility desired?}

Table 5.3 shows the contingency table of hearing identity and how much the participant listens to music. Table 5.4, shows participant's responses to the question "What statement describes you best?"

Table 5.5 on the facing page shows the contingency table of hearing identity and experience of music in elementary or secondary school. When asked for a description of their music experience during elementary or high school, the majority indicated a somewhat positive experience. Three participants indicated a very positive experience while one deaf male indicated having a very negative experience.

\subsubsection{Goal 2 - How do viewers respond to specific visualizations?}

Participants were asked which visualization style gave them the "best" sense of drums / percussion, instruments playing, melody, and rhythm. The results of this question are shown in Table 5.6 on page 98.

Participants were asked which visualization style they enjoyed the most. The results of this question are shown in Table 5.7.

\begin{tabular}{|c|c|c|c|c|c|c|c|}
\hline & & & \multicolumn{4}{|c|}{ Frequency of listening to music } & \multirow[t]{2}{*}{ Total } \\
\hline & & & $\begin{array}{l}\text { All the time } \\
\text { (daily) }\end{array}$ & $\begin{array}{l}\text { Frequently } \\
\text { (weekly) }\end{array}$ & Sometimes & Rarely & \\
\hline \multirow[t]{6}{*}{ Identity } & $\mathrm{HH}$ & Count & 0 & 2 & 1 & 1 & 4 \\
\hline & & $\%$ within Identity & $0.0 \%$ & $50.0 \%$ & $25.0 \%$ & $25.0 \%$ & $100.0 \%$ \\
\hline & Deaf & Count & 2 & 1 & 1 & 1 & 5 \\
\hline & & $\%$ within Identity & $40.0 \%$ & $20.0 \%$ & $20.0 \%$ & $20.0 \%$ & $100.0 \%$ \\
\hline & Deafened & Count & 0 & 0 & 1 & 0 & 1 \\
\hline & & $\%$ within Identity & $0.0 \%$ & $0.0 \%$ & $100.0 \%$ & $0.0 \%$ & $100.0 \%$ \\
\hline \multirow[t]{2}{*}{ Total } & & Count & 2 & 3 & 3 & 2 & 10 \\
\hline & & $\%$ within Identity & $20.0 \%$ & $30.0 \%$ & $30.0 \%$ & $20.0 \%$ & $100.0 \%$ \\
\hline
\end{tabular}

Table 5.3: How often do you listen to music? (by hearing identity)

\begin{tabular}{|c|c|c|c|c|c|c|}
\hline & & & \multicolumn{3}{|c|}{ Statement that describes you best } & Total \\
\hline \multirow{6}{*}{ Identity } & \multirow[b]{2}{*}{$\mathrm{HH}$} & & $\begin{array}{c}\text { I really enjoy } \\
\text { listening to } \\
\text { music. }\end{array}$ & $\begin{array}{l}\text { I like } \\
\text { listening to } \\
\text { music. }\end{array}$ & $\begin{array}{l}\text { I do not } \\
\text { like or dislike } \\
\text { music. }\end{array}$ & \\
\hline & & Count & 1 & 2 & $\overline{1}$ & 4 \\
\hline & \multirow{3}{*}{ Deaf } & $\%$ within Identity & $25.0 \%$ & $50.0 \%$ & $25.0 \%$ & $100.0 \%$ \\
\hline & & Count & 3 & 2 & 0 & 5 \\
\hline & & $\%$ within Identity & $60.0 \%$ & $40.0 \%$ & $0.0 \%$ & $100.0 \%$ \\
\hline & \multirow{4}{*}{ Deafened } & Count & 1 & 0 & 0 & 1 \\
\hline \multirow{3}{*}{ Total } & & $\%$ within Identity & $100.0 \%$ & $0.0 \%$ & $0.0 \%$ & $100.0 \%$ \\
\hline & & Count & 5 & 4 & 1 & 10 \\
\hline & & $\%$ within Identity & $50.0 \%$ & $40.0 \%$ & $10.0 \%$ & $100.0 \%$ \\
\hline
\end{tabular}

Table 5.4: What statement describes you best? (by hearing identity) 


\begin{tabular}{|c|c|c|c|c|c|c|}
\hline & & & $\begin{array}{r}\mathrm{E} \\
\text { eleme }\end{array}$ & $\begin{array}{l}\text { perience of musi } \\
\text { ary or secondary }\end{array}$ & $\begin{array}{l}\text { in } \\
\text { school }\end{array}$ & Total \\
\hline \multirow{5}{*}{ Identity } & \multirow{3}{*}{$\mathrm{HH}$} & & Very pos. & Somewhat pos. & Very neg. & \\
\hline & & Count & 2 & 2 & 0 & 4 \\
\hline & & $\%$ within Identity & $50.0 \%$ & $50.0 \%$ & $0.0 \%$ & $100.0 \%$ \\
\hline & \multirow[t]{2}{*}{ Deaf } & Count & 0 & 4 & 1 & 5 \\
\hline & & $\%$ within Identity & $0.0 \%$ & $80.0 \%$ & $20.0 \%$ & $100.0 \%$ \\
\hline \multirow{4}{*}{ Total } & \multirow[t]{4}{*}{ Deafened } & Count & 1 & 0 & 0 & 1 \\
\hline & & $\%$ within Identity & $100.0 \%$ & $0.0 \%$ & $0.0 \%$ & $100.0 \%$ \\
\hline & & Count & 3 & 6 & 1 & 10 \\
\hline & & $\%$ within Identity & $30.0 \%$ & $60.0 \%$ & $10.0 \%$ & $100.0 \%$ \\
\hline
\end{tabular}

Table 5.5: Music experience in school (by hearing identity).

Participants were asked which visualization style gave them "the best sense of what was happening in the music". The results of this question are shown in Table 5.8.

Table 5.9 on page 99 shows the contingency table of most enjoyed visualization and the visualization participants selected as giving the best sense of the music.

$H_{0}$ : Participant's most enjoyed visualization and the visualization selected as giving the best sense of the music are independent.

$H_{a}$ : Participant's most enjoyed visualization and the visualization selected as giving the best sense of the music are not independent.

There was a statistically significant association between a participant's enjoyment of a particular visualization and their choice of which one gave the best sense of music $\mathcal{X}^{2}(6, \mathrm{~N}=10)=16.786, \mathrm{p}=$ 0.01 . Since the $p$-value is less than the significance level (0.05), we cannot accept the null hypothesis. Participants who indicated the iTunes visualization in the one category would also choose it in the other

At the end of the focus group, participants were asked What Was Most Enjoyable?

\section{Selected comments "What Was Most Enjoyable?":}

- "Feeling positive of the visualized beats"

- "colors and instrument with melody"

- "looking at the changing colors"

- "some of them are good"

- "lots of color"

- "nothing really - need to connect music" 


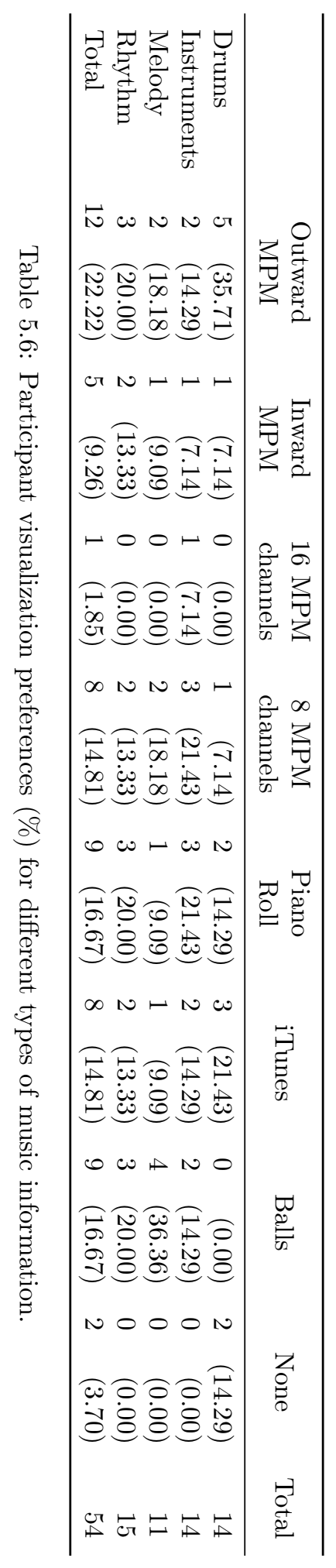




\begin{tabular}{|c|c|c|c|c|c|c|}
\hline & & & \multicolumn{3}{|c|}{ Most enjoyed visualization } & \multirow[t]{2}{*}{ Total } \\
\hline & & & Unknown & Balls & iTunes & \\
\hline \multirow[t]{6}{*}{ Identity } & $\mathrm{HH}$ & Count & 0 & 2 & $\overline{2}$ & 4 \\
\hline & & $\%$ within Identity & $0.0 \%$ & $50.0 \%$ & $50.0 \%$ & $100.0 \%$ \\
\hline & Deaf & Count & 1 & 0 & 4 & 5 \\
\hline & & $\%$ within Identity & $20.0 \%$ & $0.0 \%$ & $80.0 \%$ & $100.0 \%$ \\
\hline & Deafened & Count & 0 & 0 & 1 & 1 \\
\hline & & $\%$ within Identity & $0.0 \%$ & $0.0 \%$ & $100.0 \%$ & $100.0 \%$ \\
\hline \multirow[t]{2}{*}{ Total } & & Count & 1 & 2 & 7 & 10 \\
\hline & & $\%$ within Identity & $10.0 \%$ & $20.0 \%$ & $70.0 \%$ & $100.0 \%$ \\
\hline
\end{tabular}

Table 5.7: Preferred visualization style (by hearing identity).

\begin{tabular}{|c|c|c|c|c|c|c|c|}
\hline & & & \multicolumn{4}{|c|}{$\begin{array}{l}\text { Visualization that gave the best sense } \\
\text { of what was happening in the music }\end{array}$} & \multirow[t]{2}{*}{ Total } \\
\hline & & & No response & Balls & iTunes & Compass & \\
\hline \multirow[t]{6}{*}{ Identity } & $\mathrm{HH}$ & Count & 0 & 1 & 2 & 1 & 4 \\
\hline & & $\%$ within Identity & $0.0 \%$ & $25.0 \%$ & $50.0 \%$ & $25.0 \%$ & $100.0 \%$ \\
\hline & Deaf & Count & 1 & 0 & 4 & 0 & 5 \\
\hline & & $\%$ within Identity & $20.0 \%$ & $0.0 \%$ & $80.0 \%$ & $0.0 \%$ & $100.0 \%$ \\
\hline & Deafened & Count & 0 & 1 & 0 & 0 & 1 \\
\hline & & $\%$ within Identity & $0.0 \%$ & $100.0 \%$ & $0.0 \%$ & $0.0 \%$ & $100.0 \%$ \\
\hline \multirow[t]{2}{*}{ Total } & & Count & 1 & 2 & 6 & 1 & 10 \\
\hline & & $\%$ within Identity & $10.0 \%$ & $20.0 \%$ & $60.0 \%$ & $10.0 \%$ & $100.0 \%$ \\
\hline
\end{tabular}

Table 5.8: Visualization style giving "best sense" of the music (by hearing identity).

\begin{tabular}{|c|c|c|c|c|c|c|c|}
\hline & & & \multicolumn{4}{|c|}{$\begin{array}{l}\text { Visualization that gave the best sense } \\
\text { of what was happening in the music }\end{array}$} & \multirow[t]{2}{*}{ Total } \\
\hline & & & No response & Balls & iTunes & Compass & \\
\hline \multirow[t]{6}{*}{ Most enjoyed visualization } & Unknown & Count & 1 & 0 & 0 & 0 & 1 \\
\hline & & $\%$ & $100.0 \%$ & $0.0 \%$ & $0.0 \%$ & $0.0 \%$ & $100.0 \%$ \\
\hline & Balls & Count & 0 & 1 & 0 & 1 & 2 \\
\hline & & $\%$ & $0.0 \%$ & $50.0 \%$ & $0.0 \%$ & $50.0 \%$ & $100.0 \%$ \\
\hline & iTunes & Count & 0 & 1 & 6 & 0 & 7 \\
\hline & & $\%$ & $0.0 \%$ & $14.3 \%$ & $85.7 \%$ & $0.0 \%$ & $100.0 \%$ \\
\hline \multirow[t]{2}{*}{ Total } & & Count & 1 & 2 & 6 & 1 & 10 \\
\hline & & $\%$ & $10.0 \%$ & $20.0 \%$ & $60.0 \%$ & $10.0 \%$ & $100.0 \%$ \\
\hline
\end{tabular}

Table 5.9: Visualization style giving "best sense" of the music (by hearing identity). 
- "interesting concept - had never thought about presenting music in this way before"

At the end of the focus group, participants were asked What Was Least Enjoyable?

\section{Selected comments "What Was Least Enjoyable?":}

- "Not enough time to "digest" and write what we saw"

- "lots of fans"

- "no accompanying sounds / vibrations"

- "I feel that music was not really conveyed well"

- "dull color"

- "repetition and discomfort with opposing designs (hurts my eyes)"

- "couldn't really connect with any of the formats that were presented"

\subsection{Discussion}

\subsubsection{Goal 1 - Are efforts to improve accessibility desired?}

To determine whether efforts to create music information that is accessible to the $\mathrm{D} / \mathrm{HH}$ are wanted, there is a need to understand whether $\mathrm{D} / \mathrm{HH}$ people are active consumers of music. Participants of this focus group responded even more favourably to music than was shown in Chapter 4.

For example, Table 5.3 on page 96 shows the contingency table of hearing identity and how much the participant listens to music. Three deaf participants reported listening to music "weekly" or "daily". Two reported listening to music "rarely". The remaining participants reported listening to music "sometimes".

Similarly, Table 5.4 on page 96 shows participant's responses to the question "What statement describes you best?" While one HH participant reported neutral feelings towards music enjoyment, all other participants either "liked listening to music" (2 deaf, $2 \mathrm{HH}$ ) or "really enjoyed listening to music" (1 HH, 3 deaf, 1 deafened). No one responded "I do not like listening to music." or "I do not see the point of music."

Given that the recruiting materials for this focus group specifically mentioned music, it is likely that the participants self-selected on this item. In other words, it is unlikely, given the recruiting materials, that a person who might respond "I do not like listening to music." or "I do not see the point of music." would have attended this focus group.

Thus, similar to Chapter 4, in answer to the question of whether efforts to create accessible music information are wanted, the responses of $\mathrm{D} / \mathrm{HH}$ participants strongly support the notion that they actively seek music and enjoy the experience. No one reported having no interest in music. Some participants found the study very interesting and reported a desire to see more. 
The next section presents and discusses the responses to the different visualizations from the participants of the focus group.

\subsubsection{Goal 2 - How do viewers respond to specific visualizations?}

As mentioned in Chapter 1, Principle Three, "Simple and intuitive use", implies that the user interface of any visualization solution should be obvious. In the case of music visualization, this principle would suggest that the visualization itself should be simple and intuitive, even without sound. Similarly, Principle Four of the Principles of Universal Design, "Perceptible Information", requires the design to communicate necessary information effectively to the user. Determining what types of music information would be considered useful and whether specific visualization styles communicated such information in a intuitive manner would be supportive of the Principles of Universal Design.

As shown in Table 5.6 on page 98, when participants were asked which visualization style gave them the "best" sense of drums / percussion, instruments playing, melody, or rhythm, their responses were mixed.

Participants found that MPM (5) gave the best sense of percussion in the outward fans view. The iTunes (3) visualization was also preferred for percussion. Two participants selected "none".

Participants found that the Piano Roll (3) visualization gave the best sense of instruments playing while the Part Motion (4) visualization gave the best sense of the melody.

Participants were split on the best visualization for rhythm choosing MPM in the outward fans configuration (3), Piano Roll (3), and Part Motion (3).

There was a considerable quantity of rich qualitative data collected during the focus group. These results did not always agree with the data presented in Table 5.6.

The summary discussion provided the best overview of the participants' experience and enjoyment levels for all of the different displays. When asked at the end of the focus group session which visualization they enjoyed most, all but two participants said they preferred the iTunes visualization. Two HH female participants preferred the MAM Part Motion visualization as their most enjoyed. One participant did not make any selection.

Two (1 HH female, 1 deafened female) participants indicated that the Part Motion visualization gave them the best sense of what was happening in the music, but they enjoyed the iTunes visualization most. One HH female participant indicated that the MAM Tonal Compass visualization gave them the best sense of what was happening in the music, but preferred the Part Motion visualization overall. The remaining six participants selected the iTunes visualization as providing the best sense of music. One participant did not make any selection.

Results for the other visualizations were not surprising; for example, Malinowski (n.d.) suggested that the MAM Piano Roll display, "is no more interesting to deaf people than it is to hearing people when they watch it with the sound turned off". The results of the focus group bear this out, the Piano Roll was essentially meaningless without the sound or some explanation of what was happening. This suggests that such visualizations are deficient when unaccompanied by sound and that certain aspects of the perception of sound are not represented in the MAM visualizations (Malinowski, n.d.). 
The MAM Tonal Compass view was equally uninteresting. Participants could not relate to what the visualization was trying to show.

The MPM visualization, which was considered more informative and more clearly indicated how the song might sound, was described by participants as "boring" and too visually busy / taxing. Participants openly complained about the visualization when presented with a white background. Using a black background made the colours appear sharper; however, there was no improvement in music understanding or entertainment. This suggests that there is a need to make this visualization more interesting, perhaps through visual design. It also suggests a need to completely rethink the approach.

Participants wanted to see the "more vibrant" visualizations. As one participant said, the iTunes visualization "was visually appealing and uplifting." However, this visualization was the least meaningful and only presents information based on a waveform analysis. The result suggests that $\mathrm{D} / \mathrm{HH}$ viewers were not so interested in how accurately the visualization represents the music, but how entertaining the resulting representation appears. This result was not surprising since non-D/HH music consumers listen to music for entertainment, not analysis.

It is interesting that the emotional connection with visualizations, particularly the iTunes visualization, is that of wanting an "uplifting" experience regardless of the emotional intention of the music. Interpreting these types of statements as people expressing the desire to only consume uplifting or positive experiences from music visualizations would be presumptuous and likely inaccurate. However, is the expectation of music visualization to be a moving experience which may sometimes be uplifting or may also be sad and just as moving or emotional? Further research is required to better understand people's expectation of a musical experience.

The fact that, according to Table 5.7, two participants found the MAM Part Motion view enjoyable also suggests some direction for future work. While users want to be entertained, simple representations that communicate a sense of what is happening in the music might still prove useful, especially if combined with entertaining views.

Participants also found that they, "could not really connect" to the different visualizations. They could not see how music was represented. As one pointed out, the "music was not conveyed well." This further suggests that something was missing in the visual representation. Beyond the need to identify the properties of music which people find entertaining, there is a need to capture that part of music which people internalize. The hope is that a future visual representation would have a response such as, "this is how I would imagine music to look."

Two female participants (1 HH, 1 deaf) commented that the visualizations were presented without sound suggesting the presence of sound would have made some difference. Given that participants had difficulty connecting music to the visualization, using sound as an accompaniment to the visualization would provide additional information to those who can use it. Instead of seeing visualization as a replacement to auditory music, it may be better to perceive visualization as a supplement to existing auditory information. Future research could present music visualizations with auditory music.

Participants were also trying to find meaning in the colours used in the various visualizations. Given that it is difficult to find consensus on the meaning of specific colours, colour was only used as an aesthetic element rather than an informational one. Finding ways to use colour in music visualiza- 
tion that is meaningful on an individual level would seem to be a useful research direction.

One participant commented that they, "never thought of presenting music this way before." When asked what was the most enjoyable experience, a participant pointed to, "the fact that this is being experimented on." Comments like these imply that there is a sense within the D/HH communities that finding a way to display information about popular music is desired.

\subsubsection{Limitations of the Design}

The analysis of the data suggests at least four limitations of this study, one of which is related to questionnaire design.

The first limitation of this design is that while asking how to represent music information, the Post-study Questionnaire did not also ask for some ranking of the importance of each type of information. As a result this must be extrapolated from the responses and comments. For example, it can be suggested that the information is important when a preference is specified and it can be suggested that there is no importance when a participant ignores the item and leaves it blank. However, any conclusion that is more specific cannot be established based on this data.

Second, the wording of question 2 and question 3 in the Post-study Questionnaire (i.e., "Which style gave you the best sense of what was happening in the music?" and "Which style gave you the best sense of percussion / instruments playing / melody / rhythm?"), raises some issues around how the participant might interpret the phrase "best sense". While the design of the questionnaire was intended to leave this phrase open to interpretation, the fact that a participant may have no psychological reality of certain music information might mean they are unable to assess what visualization gave them the "best sense" of something because they would not know how to understand that concept.

Third, the small sample size with an unequal number of men and women, while appropriate for a focus group, limits the statistical power of the results. The chi-square test for independence is sensitive to sample size as well as small expected frequencies in one or more of the cells in the table. For these reasons caution must be taken in interpreting chi-square if one or more cells are less than 5 . However, according to Frankfort-Nachmias and Leon-Guerrero, "There is no hard-and-fast rule regarding the size of expected frequencies." (2011, p. 350). Most researchers limit the use of chi-square to tables that either a) have no expected frequencies values below 5 or b) have no more than 20 percent of the expected frequencies values below 5 (Frankfort-Nachmias \& Leon-Guerrero, 2011).

Finally, as mentioned earlier, given that the recruiting materials for this focus group specifically mentioned music, it is likely that the participants are self-selected. In other words, it is unlikely, given the recruiting materials, that a $\mathrm{D} / \mathrm{HH}$ community member who might not like music or have any understanding of music would have attended this focus group. This is a weakness of the interpretability of the data because it does not allow generalization of the results over all possible consumers, only those who have any interest in music. 


\subsection{Conclusions From This Study}

The focus group found that music was not conveyed well by the visualizations tested. Among the various observations:

- The iTunes visualization was enjoyed the most and was selected most often as giving the best sense of what was happening;

- While the Part Motion visualization presented the "best sense of what's happening" for several participants, they still preferred the iTunes visualization for overall enjoyment;

- Participants could not relate the Tonal Compass and Piano Roll visualizations to the music;

- Motion Pictures of Music (a visualization created in-house) was boring and too visually busy; and

- Visualizations of music need to be aesthetically enjoyable and not too visually busy.

This study explored several techniques for visualizing music with the goal of presenting music to $\mathrm{D} / \mathrm{HH}$ consumers. Visualizations that focus entirely on music notes and timing, such as a piano roll, were found lacking. Designs that were thought to provide maximum information with, hopefully, little necessary training, like MPM, were found to be boring. Music consumers who are interested in being entertained are not necessarily interested in large amounts of information. In terms of music, accuracy of the visualization takes second stage to entertainment.

Based on the verbal comments of the participants, there was just too many visualizations shown in the focus group. In particular, presenting four different views of MPM over the span of 18 different presentations of music was too much for the participants to take in. The MPM visualization also had the issue of forcing viewers to attend to multiple spaces at the same time rather than look at one core area of information.

Participants also seemed at times confused as to which visualization was being referred to in the questionnaires. The questionnaires were also too difficult to follow for people for whom English is not their first language, a common issue among Deaf signers. These issues suggest a need to completely redesign how some questions are asked or response choices are presented.

As shown in Figure 5.5 on the facing page, this focus group contained a full DRM loop because it introduced an intervention, the exposure of the participants to the videos and measured specific parameters in their experience of these visualizations. For the Dissertation as a whole, the work in this chapter represents part of the DS-I stage of the DRM. It qualifies as a DRM Stage 1 (Descriptive Study I) activity, which provides a baseline of critical parameters in a situation into which some design intervention will be eventually introduced. 


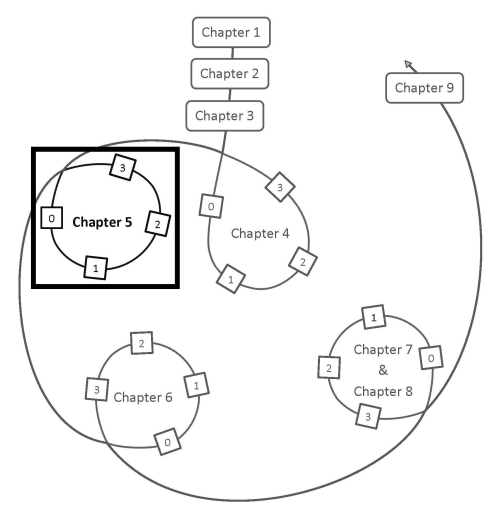

Figure 5.5: Location within Dissertation path.

\subsection{Next Steps}

These results raised several questions about visualization design to further explore. Based on these results, the MPM visualization and some of the displays provided by MAM must be discarded. It appears that the Part Motion visualization and iTune's Magnetosphere visualization need to be further explored. 



\section{Chapter 6}

\section{Focus Group II}

The focus group described in the previous chapter found that music was not conveyed well by the visualizations tested and that visualizations of music need to be aesthetically enjoyable and not too visually busy.

Since there were too many visualizations shown in the previous focus group and participants also seemed at times confused as to which visualization was being referred to in the questionnaires there is a need to reduce the number of visualizations and redesign how some questions were asked or response choices were presented.

Thus, in an attempt to see if music can be conveyed better and the overall methodology can be improved, a second focus group was held to explore the top two visualizations from the earlier focus group as well as a newly developed visualization, to be described in Section 6.3.3 on page 112 .

\subsection{Goals}

The goal of the study presented in this chapter is to answer the following questions:

1. Are efforts to improve accessibility desired?

2. How do viewers respond to visualizations of music information?

3. Do participants enjoy visualizations?

4. Are participants able to detect the intended emotion of a song solely through visualizations of music information?

\subsection{Instruments}

Appendix $\mathrm{C}$ shows the instructions given and questions asked of all participants during the entire focus group as well as a full transcript. The remainder of this section will show only the questionnaires used to collect data during the focus group. 


\section{Music Visualization Pre-study Questionnaire}

The purpose of this questionnaire is to gather information about you and your experience with music and closed captioning. There are eight questions and it should take you about five minutes to complete this questionnaire. Thank you in advance for your time and assistance.

1. Do you identify yourself as: (please check one)
- Hearing
- Hard of hearing
$\square$ Deaf
D Deafened
$\square$ Cochlear implant

\section{Please indicate your gender}

$\square$ Male
$\square \quad$ Female

3. Please indicate your age
口 $19-24$
$25-34$
口 $35-44$
口 45- 54
口 55- 64
$65+$

\section{What is your highest level of education?}
No formal education
- Elementary school
- High School
$\square$ Technical college
$\square$ University
a Masters / Ph.D.

5. How often do you use closed captioning when watching television?
$\square$ Always
口 Sometimes
口 Never

6. How often do you listen to music?
7. Which of the following statements best describes you?
- I really enjoy listening to music.
I like listening to music.
I do not like or dislike music.
I I do not like listening to music
I do not see the point of music.

8. What is your experience of music in elementary or secondary school?

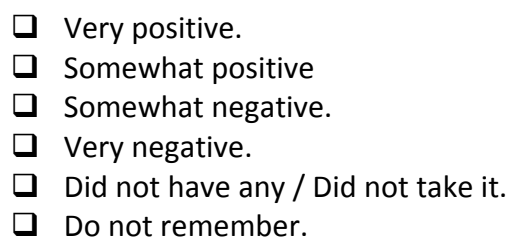

Figure 6.1: Focus Group Pre-study Questionnaire. 
Music Questions

1. Was the music visualization enjoyable (circle one)?

Not enjoyable at all Not that enjoyable Neutral Somewhat enjoyable Enjoyable

2. What did the visualization mean to you?

3. Was the music (mark one for each):

a)

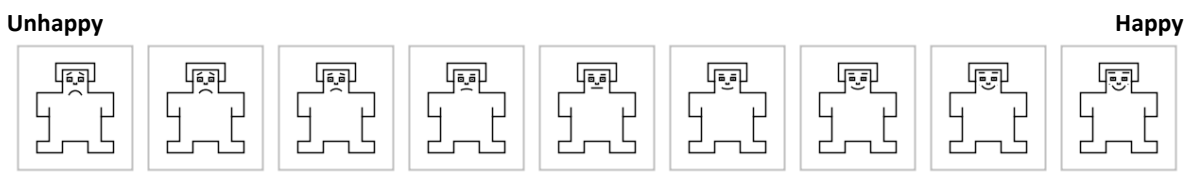

b)

Calm

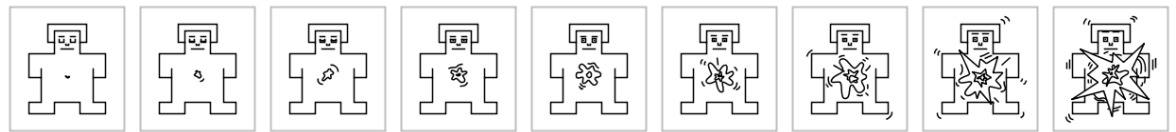

Subtle

c)
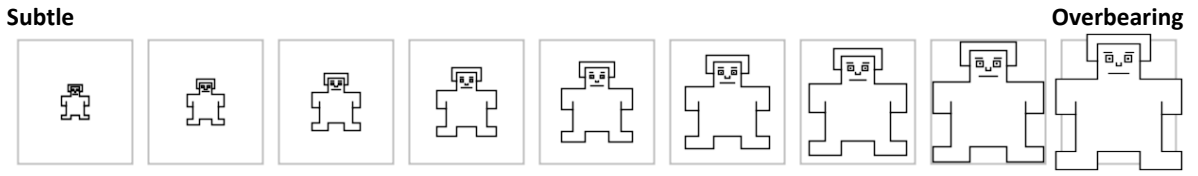

4. Identify which emotion you thought was expressed and rate its strength from $\mathbf{1}$ to $\mathbf{7}$ (circle one):

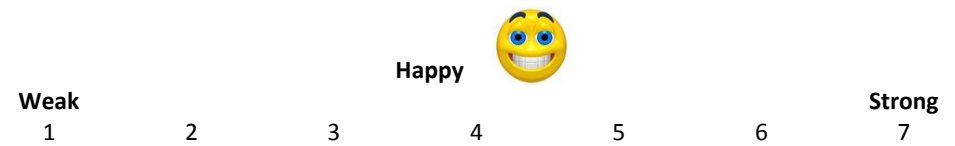

1

2

3

6

7

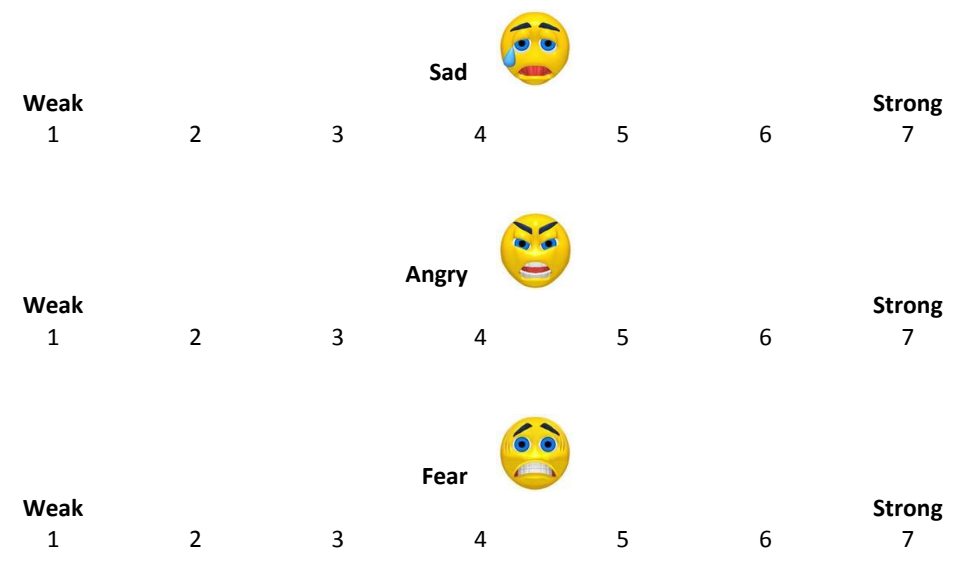

Figure 6.2: Focus Group Post-visualization Questionnaire. 
Music Visualization Post-Study Questionnaire

The purpose of this questionnaire is to gather information about you and your opinions about the visual representations that you just saw. There five questions and it should take you about ten minutes to complete this questionnaire. Thank you in advance for your time and assistance.

1. Which style did you enjoy most?

2. Which style gave you the best sense of what was happening in the music?

3. Which style gave you the best sense of:

\begin{tabular}{|l|c|c|c|}
\hline & Moving Circles & Starscape & \\
\hline $\begin{array}{l}\text { Drums/ } \\
\text { Percussion }\end{array}$ & Radar \\
\hline $\begin{array}{l}\text { Instruments } \\
\text { playing }\end{array}$ & & & \\
\hline Melody & & & \\
\hline Rhythm & & & \\
\hline
\end{tabular}

4. What was most enjoyable about your experience with music visualization?

5. What was least enjoyable about your experience with music visualization?

Figure 6.3: Focus Group Post-study Questionnaire. 


\begin{tabular}{lll}
\hline Songs & Visualization & Artist or Composer \\
\hline Happy & & \\
$\begin{array}{l}\text { Day Tripper } \\
\text { Schön ist es auf der Welt zu sein } \\
\text { Aqalera do Brasil }\end{array}$ & $\begin{array}{l}\text { Radar } \\
\text { Balls } \\
\text { iTunes }\end{array}$ & $\begin{array}{l}\text { Beatles } \\
\text { Roy Black \& Anita Hegerland } \\
\text { Ary Barroso }\end{array}$ \\
$\begin{array}{l}\text { Anger } \\
\text { Four Horsemen }\end{array}$ & \\
$\begin{array}{l}\text { Duality (after introduction) } \\
\text { The Beautiful People (middle section) }\end{array}$ & Balls & Metallica \\
& Radar & Slipknot \\
Fear & iTunes & Marilyn Manson \\
One Winged Angel (Final Fantasy VII) & & \\
Demons On The Prey (Doom Level 7) & iTunes & Nobuo Uematsu \\
Vampire Hunters (Bram Stoker's Dracula) & Balls & $\begin{array}{l}\text { Robert Prince } \\
\text { Radar }\end{array}$ \\
Wad & & \\
On That Day 5 Years Ago (Final Fantasy VII) & Radar & Nobuo Uematsu \\
Main Theme of Final Fantasy VII & iTunes & Nobuo Uematsu \\
At Last & Balls & Etta James \\
\hline
\end{tabular}

Table 6.1: List of songs.

\subsection{Method}

\subsubsection{Step 1}

In advance of the focus group, 30-second snippets of 20 popular songs (instrumental-only versions) were assessed by a group of four hearing people for the overall emotion each clip represented (happiness, sadness, anger, and fear). If these informants all agreed on the emotion the song represented, it was included in the set of twelve songs to be presented to the focus group.

\subsubsection{Step 2}

Using three different displays (Part Motion, Magnetosphere, and Radar) created from three different visualization tools (Music Animation Machine, iTunes, and Radar), prototype visualizations of 12 different popular music tracks representing four different emotions (happy, sad, angry, fear) were presented to D/HH participants in a focus group setting. This set of emotions was selected because they represent the "basic emotions" (Ekman \& Friesen, 1986). These specific visualizations were selected because they were representative of the variation (using pitch, timbre and tempo in Part Motion, frequency and amplitude for iTunes, and pitch, tempo, timbre and beat for Radar) that is available according to the literature.

The list of songs used is presented in Table 6.1.

To capture the individual experience as well as the larger group comments multiple tools were 
used to collect participant's responses to the prototypes. After each prototype was shown, participants were asked for their opinions about the visualization using a discussion format as well as the Post-visualization Questionnaire shown in Figure 6.2 on page 109. The Post-visualization Questionnaire asked participants about the effectiveness of the visualization in conveying emotions, representing music and whether the visual effects were enjoyable. Participants also completed a pre-study questionnaire to gather information about their music experiences as well as a post-study questionnaire to summarize their opinions of the music visualization experience.

Since different D/HH people have different levels of hearing and different abilities to process whatever sound they can hear, the songs were presented without sound to control for variation of hearing levels.

An ASL interpreter and a stenographer, to live caption the proceedings, were present to facilitate the participation of the $\mathrm{D} / \mathrm{HH}$ participants.

\subsubsection{Visualizations}

\section{Music Animation Machine}

The Music Animation Machine (MAM) described in Chapter 2 was used to produce the Part Motion (Balls) visualization.

\section{iTunes}

As noted in Chapter 2, the default visualizer of the iTunes media player application, Magnetosphere, provides visualizations based on waveform analysis of the audio input.

\section{Radar}

Recognizing that the above visualizations do not all present the properties of music, the Radar visualization, as shown in Figure 6.4, was developed by me and colleagues working at Ryerson's Inclusive Media and Design Centre ${ }^{1}$. Radar consists of a black circle with lines that span its radius called "blades". While the size of each blade is static, other properties are used to communicate information about the music. The colour of the blade identifies the instrument (or instrument family) being played. The intensity of the colour shows the volume of the note; the louder the note, the more solid the blade appears.

New blades move from the " 3 o'clock" position either clockwise or counter-clockwise to show the difference in pitch between instruments in the same family. This provides a sense of the history of the music so far. For example, as the pitch rises higher, the blade rotates counter-clockwise. A new pitch is considered different from the previous pitch, thus the blade starts at the " 3 o'clock" position and rotates counter-clockwise relative to the previous pitch. The speed of a blade's rotation is related to the instrument's own pitch; the higher the pitch, the faster it moves. The longer the note is held, the longer the blade is on screen.

\footnotetext{
${ }^{1}$ previously Centre for Learning Technologies
} 


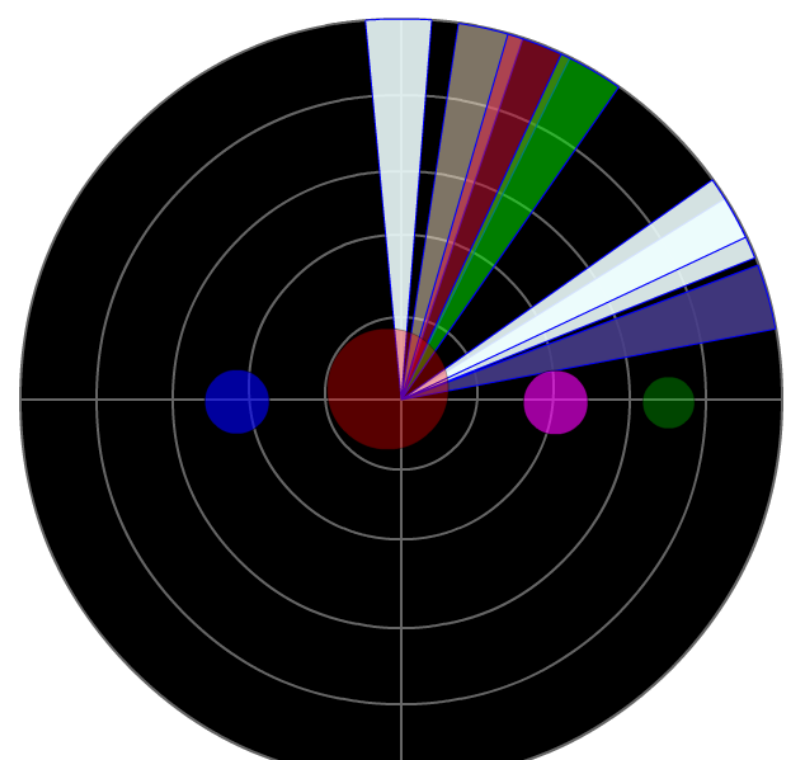

Figure 6.4: The Radar visualization.

Tempo or beat stimuli is represented using a cluster of circles. The large red circle in the middle represents the main source of the beat (e.g., bass drum). Other circles are added as needed to represent other percussion instruments.

\subsubsection{Stimuli and their Properties}

Instrumental-only versions of twelve popular songs representing four different emotions (i.e., three songs for each of: happy, sad, angry and fear) were randomly presented, without sound, for thirty seconds in each of the above visualization techniques (i.e., iTunes, MAM Part Motion display, and Radar). This set of emotions was selected because they represent the "basic emotions" (Ekman \& Friesen, 1986). These specific visualizations were selected because they were representative of the variation (using pitch, timbre and tempo in Part Motion, frequency and amplitude for iTunes, and pitch, tempo, timbre and beat for Radar) that is available according to the literature.

Table 6.2 shows the common types of musical information each of these visualizations can display. Some visualizations are unique in displaying specific information (e.g., Magnetosphere displays amplitude). It is not clear if any of these three visualizations (iTunes, MAM Part Motion display, and Radar) were explicitly designed to communicate emotions.

Each of the four emotions had three presentations using the three music visualization styles. To help ensure they were not influenced by predisposition / familiarity with certain applications, the title bar of the application was always hidden from participants' view. The presentation screen was always turned off while setting up a visualization to show. 


\begin{tabular}{rccccccc}
\hline Visualization & $\begin{array}{c}\text { Change in } \\
\text { Pitch }\end{array}$ & Timbre & Tempo & $\begin{array}{c}\text { Change in } \\
\text { Frequency }\end{array}$ & $\begin{array}{c}\text { Duration } \\
\text { of Notes }\end{array}$ & Parts & Beat \\
\hline iTunes & & & & $\mathrm{X}$ & $\mathrm{X}$ & & \\
Balls & $\mathrm{X}$ & & $\mathrm{X}$ & $\mathrm{X}$ & $\mathrm{X}$ & $\mathrm{X}$ & $\mathrm{X}$ \\
Radar & $\mathrm{X}$ & $\mathrm{X}$ & $\mathrm{X}$ & $\mathrm{X}$ & $\mathrm{X}$ & & $\mathrm{X}$ \\
\hline
\end{tabular}

Table 6.2: Visualization properties.

\subsubsection{Questionnaires}

Both the Post-visualization Questionnaire presented after each visualization was played and the Poststudy Questionnaire were changed from the previous focus group.

\section{Post-visualization Questionnaire}

After each presentation, participants participated in a follow-up discussion and completed the 9-item Post-visualization Questionnaire. This questionnaire (shown in Figure 6.3), which users know as "Music Questions", asked participants about the visualization presentation they just saw. It includes questions on whether the visualization was enjoyable; what, if any, meaning they felt it had; the degree they felt the music was happy / excited / overbearing; and the degree they thought the visualization (and thus the music) expressed happy / sad / angry / fear emotions.

As mentioned in Section 2.3, the Pleasure / Arousal / Dominance (PAD) model of emotion is a dimensional model that attempts to decompose emotions into three orthogonal underlying dimensions: a) Pleasure (or valence), the degree of pleasantness of the emotional experience; $b$ ) Arousal, the level of activation of the emotion; and c) Dominance, the level of attention or rejection of the emotion (Mehrabian, 1996; J. A. Russell \& Mehrabian, 1977). Each element has opposing dimensions (pleasure / displeasure, arousal / sleep, and dominance / submission) and forms one axis with opposing end points of a three-axes model. Emotional responses can be plotted along each of these axes. This model is complex to use as it requires the dissection of emotional responses into three separate categories.

Given the complexity of the PAD model, the Self-Assessment Manikin (SAM), a non-verbal pictorial assessment technique, was designed for assisting people in identifying which of the dimensions fit their emotional state. It visually represents Mehrabian's three PAD dimensions (Lang, 1985), and has been shown to better track personal response to an affective stimulus (Bradley \& Lang, 1994; Winton, Putnam, \& Krauss, 1984). Figure 6.5 shows the SAM overbearingness scale, which is used to measure the PAD dimension of Dominance.

SAM depicts each PAD dimension with a graphic character arrayed along a continuous nine-point scale. For pleasure, SAM ranges from a smiling, happy figure to a frowning, unhappy figure; for arousal, SAM ranges from sleepy with eyes closed to excited with eyes open. The dominance scale shows SAM ranging from a very small figure representing a feeling of being controlled or submissive to a very large figure representing in-control or a powerful feeling. 

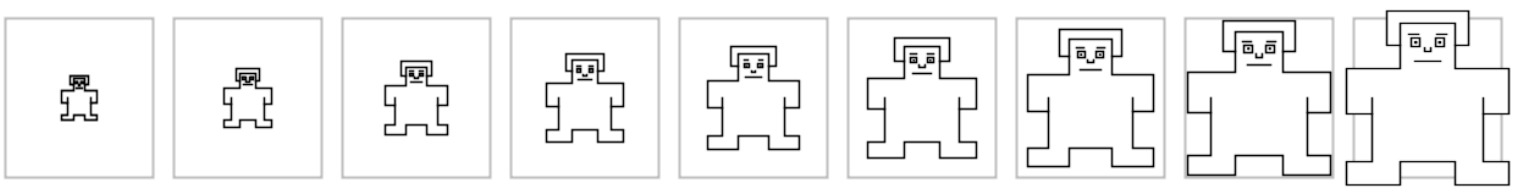

Figure 6.5: Self-Assessment Manikin overbearingness scale (Bradley \& Lang, 1994).

As a pictorial tool, SAM may be easier to administer than other text-based scales because it is less affected by the language barriers experienced by D/HH people and others (e.g., non-English speaking people). In addition, participants can complete ratings on the SAM scales in less than 15 seconds, which reduces fatigue.

While using SAM provides a simple mechanism for participants to evaluate their perception of the emotion of a song, no baseline PAD measures of the selected songs was developed for comparison with a hearing person's experience. While a baseline might allow one to define an objectively correct emotion for a specified song which can then be compared to participant responses, how to ensure such a metric is accurate is problematic.

Participants also completed a 7 -point scale rating the emotional impact of the visualization. There were four scales each representing happy, sad, angry, and fear. This set of emotions was selected because they represent the "basic emotions" (Ekman \& Friesen, 1986). Participants rated ("1-weak" to "7-strong") the emotions presented by the visualization. Emoticons expressing each of these emotions were added to assist the participant.

\section{Post-study Questionnaire}

After all 12 visualizations were shown, participants participated in a follow-up discussion and then completed the Post-study Questionnaire.

In the previous focus group, the question in the post-study questionnaire regarding which visualization style gave the best sense of drums, instruments playing, melody, and rhythm was entirely presented in text. It also had an option for "none".

In this focus group, the Post-study Questionnaire, as shown in Figure 6.3 on page 110 was changed so that the question regarding which visualization style gave the best sense of drums, instruments playing, melody, and rhythm used colour pictures of the visualizations rather than text descriptions. Further, this question was now forced choice and did not provide for a "none" option. Since this questionnaire was paper-based, the researchers believed that participants would write something on the questionnaire if they felt that none of the choices provided the desired music information.

This questionnaire also had several open-ended items for the collection of qualitative data in addition to the focus group discussions. These questions were the same as those used in the previous focus group. 


\begin{tabular}{|c|c|c|c|c|c|c|}
\hline & & & Frequency & f listening to & music & Total \\
\hline \multirow{6}{*}{ Identity } & \multirow{3}{*}{$\mathrm{HH}$} & \multirow{3}{*}{$\begin{array}{r}\text { Count } \\
\% \text { within Identity }\end{array}$} & $\begin{array}{l}\text { All the time } \\
\text { (daily) }\end{array}$ & $\begin{array}{l}\text { Frequently } \\
\text { (weekly) }\end{array}$ & Rarely & \\
\hline & & & 1 & 1 & 0 & 2 \\
\hline & & & $50.0 \%$ & $50.0 \%$ & $0.0 \%$ & $100.0 \%$ \\
\hline & \multirow[t]{2}{*}{ Deaf } & Count & 0 & 1 & 1 & 2 \\
\hline & & $\%$ within Identity & $0.0 \%$ & $50.0 \%$ & $50.0 \%$ & $100.0 \%$ \\
\hline & \multirow[t]{2}{*}{$\mathrm{CI}$} & Count & 1 & 0 & 0 & 1 \\
\hline \multirow{3}{*}{ Total } & & $\%$ within Identity & $100.0 \%$ & $0.0 \%$ & $0.0 \%$ & $100.0 \%$ \\
\hline & & Count & 2 & 2 & 1 & 5 \\
\hline & & $\%$ within Identity & $40.0 \%$ & $40.0 \%$ & $20.0 \%$ & $100.0 \%$ \\
\hline
\end{tabular}

Table 6.3: How often do you listen to music? (by hearing identity)

\subsubsection{Participants}

This focus group had six participants: 3 deaf ( 3 female), 1 cochlear implantee (1 female), 2 hard of hearing ( 1 male, 1 female). Participants ranged in age from 19 to 55 (2 were 19-24, 2 were 25-34, 2 were 45-54).

All six participants indicated always using closed captions when watching television.

Three participants reported high school or some technical college as their highest level of formal education. Two reported being university graduates. One participant did not answer this question.

\subsection{Results}

All raw data is presented in Appendix C.

\subsubsection{Goal 1 - Desire for Improved Accessibility}

As seen in Table 6.3, one deaf participant and one $\mathrm{HH}$ participant each reported listening to music "weekly", while one HH participant and one CI-using participant reported listening to music "daily". One deaf participant reported listening to music "rarely". The remaining deaf participant did not answer this question.

As shown in Table 6.4 on the facing page, half the group reported that they "really enjoy listening to music" (1 HH, 1 deaf, 1 CI user) while the other half reported they "liked listening to music" (2 deaf, $1 \mathrm{HH})$.

When asked for a description of their music experience during elementary or high school, as shown in Table 6.5, three participants indicated a somewhat positive experience. One indicated that they did not have any music classes. One could not recall their experience. One participant did not answer this question.

For the most part, contingency table analysis did not show any significant trends among these responses. 


\begin{tabular}{|c|c|c|c|c|c|}
\hline & & & \multicolumn{2}{|c|}{ Statement that describes you best } & \multirow[t]{2}{*}{ Total } \\
\hline \multirow{6}{*}{ Identity } & \multirow{3}{*}{$\mathrm{HH}$} & \multirow[b]{2}{*}{ Count } & $\begin{array}{l}\text { I really enjoy } \\
\text { listening to music. }\end{array}$ & $\begin{array}{c}\text { I like } \\
\text { listening to music. }\end{array}$ & \\
\hline & & & 1 & $\overline{1}$ & 2 \\
\hline & & $\%$ within Identity & $50.0 \%$ & $50.0 \%$ & $100.0 \%$ \\
\hline & Deaf & Count & 1 & 2 & 3 \\
\hline & & $\%$ within Identity & $33.3 \%$ & $66.7 \%$ & $100.0 \%$ \\
\hline & $\mathrm{CI}$ & Count & 1 & 0 & 1 \\
\hline \multirow{3}{*}{ Total } & & $\%$ within Identity & $100.0 \%$ & $0.0 \%$ & $100.0 \%$ \\
\hline & & Count & 3 & 3 & 6 \\
\hline & & $\%$ within Identity & $50.0 \%$ & $50.0 \%$ & $100.0 \%$ \\
\hline
\end{tabular}

Table 6.4: What statement describes you best? (by hearing identity)

\begin{tabular}{|c|c|c|c|c|c|c|}
\hline & & & elem & $\begin{array}{l}\text { Experience of music in } \\
\text { entary or secondary } \mathrm{s}\end{array}$ & hool & Total \\
\hline \multirow{5}{*}{ Identity } & \multirow{3}{*}{$\mathrm{HH}$} & & $\begin{array}{l}\text { Somewhat } \\
\text { positive }\end{array}$ & $\begin{array}{c}\text { Did not have any / } \\
\text { did not take it }\end{array}$ & $\begin{array}{l}\text { Do not } \\
\text { remember }\end{array}$ & \\
\hline & & Count & 2 & 0 & 0 & 2 \\
\hline & & $\%$ within Identity & $100.0 \%$ & $0.0 \%$ & $0.0 \%$ & $100.0 \%$ \\
\hline & \multirow[t]{2}{*}{ Deaf } & Count & 0 & 1 & 1 & 2 \\
\hline & & $\%$ within Identity & $0.0 \%$ & $50.0 \%$ & $50.0 \%$ & $100.0 \%$ \\
\hline \multirow{4}{*}{ Total } & \multirow[t]{4}{*}{ CI } & Count & 1 & 0 & 0 & 1 \\
\hline & & $\%$ within Identity & $100.0 \%$ & $0.0 \%$ & $0.0 \%$ & $100.0 \%$ \\
\hline & & Count & 3 & 1 & 1 & 5 \\
\hline & & $\%$ within Identity & $60.0 \%$ & $20.0 \%$ & $20.0 \%$ & $100.0 \%$ \\
\hline
\end{tabular}

Table 6.5: Music experience in school (by hearing identity). 


\begin{tabular}{|c|c|c|c|c|c|c|c|}
\hline & & Balls & & iTunes & & Radar & Total \\
\hline Drums / percussion & 2 & $28.57 \%$ & 1 & $14.29 \%$ & 4 & $57.14 \%$ & 7 \\
\hline Instruments playing & 4 & $57.14 \%$ & 2 & $28.57 \%$ & 1 & $14.29 \%$ & 7 \\
\hline Melody & 4 & $50.00 \%$ & 3 & $37.50 \%$ & 1 & $12.50 \%$ & 8 \\
\hline Rhythm & 3 & $33.33 \%$ & 3 & $33.33 \%$ & 3 & $33.33 \%$ & 9 \\
\hline
\end{tabular}

Table 6.6: Participant visualization preferences for different types of music information.

\subsubsection{Goal 2-Music Information Preferences}

Participants were asked which visualization style gave the best sense of drums / percussion, instruments playing, melody, and rhythm. The results of this question are shown in Table 6.6. Note that some participants provided more than one answer.

Participants stated that Radar was best for percussion information, while Part Motion was best for showing information about the instruments and melody. Participants chose all three for information on rhythm.

There was a considerable quantity of rich qualitative data collected during the focus group. These results did not always agree with the data presented in Table 6.6. For example, participants comments on the different visualizations varied widely:

- iTunes

- "gave me a more positive feel"

- [did not like] "excess movement"

- "just a jumble of lights, no connection to the music, very distracting"

- Radar

- "Not my favourite format. Could be any piece of music. Don't like the background. Makes me think/feel that I'm watching a target / target practice"

- "I feel like this visualization requires too much to be explained in the sense that I don't understand what all the circles mean, versus the 'lines' spreading."

- "lots of colour, brightness, beat"

- Part Motion

- "A parade of nice and beautiful dancing lights"

- "I prefer this format. I like that the format helps you anticipate in the piece, re: what's coming next as well as showing a discernible history of what's already happened."

- "it's picking upon different things each time I see this type of visualization"

Table 6.7 shows, participants' responses when asked which visualization gave the best sense of what was happening in the music. The majority chose the iTunes and Radar visualizations. 


\begin{tabular}{|c|c|c|c|c|c|c|c|}
\hline & & & \multicolumn{4}{|c|}{$\begin{array}{l}\text { Visualization that gave the best sense } \\
\text { of what was happening in the music }\end{array}$} & \multirow[t]{2}{*}{ Total } \\
\hline & & & Balls & iTunes & Radar & None & \\
\hline \multirow{6}{*}{ Identity } & $\mathrm{HH}$ & Count & 1 & 1 & 0 & $\overline{0}$ & 2 \\
\hline & & $\%$ within Identity & $50.0 \%$ & $50.0 \%$ & $0.0 \%$ & $0.0 \%$ & $100.0 \%$ \\
\hline & Deaf & Count & 0 & 1 & 2 & 0 & 3 \\
\hline & & $\%$ within Identity & $0.0 \%$ & $33.3 \%$ & $66.7 \%$ & $0.0 \%$ & $100.0 \%$ \\
\hline & CI & Count & 0 & 0 & 0 & 1 & 1 \\
\hline & & $\%$ within Identity & $0.0 \%$ & $0.0 \%$ & $0.0 \%$ & $100.0 \%$ & $100.0 \%$ \\
\hline \multirow[t]{2}{*}{ Total } & & Count & 1 & 2 & 2 & 1 & 6 \\
\hline & & $\%$ within Identity & $16.7 \%$ & $33.3 \%$ & $33.3 \%$ & $16.7 \%$ & $100.0 \%$ \\
\hline
\end{tabular}

Table 6.7: Visualization style giving "best sense" of the music (by hearing identity).

\begin{tabular}{|c|c|c|c|c|c|c|}
\hline & & & \multicolumn{3}{|c|}{ Most enjoyed visualization } & Total \\
\hline \multirow{6}{*}{ Identity } & \multirow[b]{2}{*}{$\mathrm{HH}$} & \multirow[b]{2}{*}{ Count } & Balls & iTunes & Radar & \\
\hline & & & 1 & 1 & 0 & 2 \\
\hline & \multirow{3}{*}{ Deaf } & $\%$ within Identity & $50.0 \%$ & $50.0 \%$ & $0.0 \%$ & $100.0 \%$ \\
\hline & & Count & 0 & 1 & 2 & 3 \\
\hline & & $\%$ within Identity & $0.0 \%$ & $33.3 \%$ & $66.7 \%$ & $100.0 \%$ \\
\hline & \multirow[t]{2}{*}{$\mathrm{CI}$} & Count & 1 & 0 & 0 & 1 \\
\hline \multirow{3}{*}{ Total } & & $\%$ within Identity & $100.0 \%$ & $0.0 \%$ & $0.0 \%$ & $100.0 \%$ \\
\hline & & Count & 2 & 2 & 2 & 6 \\
\hline & & $\%$ within Identity & $33.3 \%$ & $33.3 \%$ & $33.3 \%$ & $100.0 \%$ \\
\hline
\end{tabular}

Table 6.8: Which style did you enjoy most? (by hearing identity)

\subsubsection{Goal 3 - Level of Enjoyability}

The summary discussion, a transcript of which is given in full in Appendix C, provided the best overview of the participants' experience and enjoyment levels for all of the different displays. When asked at the end of the focus group session which visualization they enjoyed most, as seen in Table 6.8, participants were equally split, two each, across the three visualizations. The majority of the deaf participants (2) preferred Radar, while one preferred the iTunes visualization. The HH participants were split between the Part Motion and iTunes visualizations, while the participant with a CI most preferred Part Motion.

Table 6.8 shows the participants' most enjoyed visualization by hearing identity.

The contingency table found in Table 6.9 shows participants' most enjoyed visualization by highest level of education. A chi-square test for independence was conducted to determine if there is any relationship between participants' highest level of education and their reported most enjoyed visualization.

$H_{0}$ : Participants' highest level of education and their reported most enjoyed visualization are independent. 


\begin{tabular}{rrrrrrr}
\hline & & \multicolumn{2}{c}{ Most enjoyed visualization } & Total \\
\hline \multirow{3}{*}{ Highest level of education } & & Balls & iTunes & Radar \\
\cline { 3 - 6 } & High School & Count & 0 & 0 & 1 & 1 \\
& & $\%$ & $0.0 \%$ & $0.0 \%$ & $100.0 \%$ & $100.0 \%$ \\
& Technical College & Count & 0 & 2 & 0 & 2 \\
& & $\%$ & $0.0 \%$ & $100.0 \%$ & $0.0 \%$ & $100.0 \%$ \\
& University & Count & 2 & 0 & 0 & 2 \\
& & $\%$ & $100.0 \%$ & $0.0 \%$ & $0.0 \%$ & $100.0 \%$ \\
& & Count & 2 & 2 & 1 & 5 \\
& & $\%$ & $40.0 \%$ & $40.0 \%$ & $20.0 \%$ & $100.0 \%$ \\
\hline
\end{tabular}

Table 6.9: Which style did you enjoy most? (by highest completed education)

\begin{tabular}{|c|c|c|c|c|c|c|}
\hline & & & \multicolumn{3}{|c|}{ Enjoyment of Visualization 12} & \multirow[t]{2}{*}{ Total } \\
\hline & & & $\begin{array}{l}\text { Not that } \\
\text { enjoyable }\end{array}$ & Neutral & $\begin{array}{l}\text { Somewhat } \\
\text { enjoyable }\end{array}$ & \\
\hline \multirow[t]{6}{*}{ Highest level of education } & High School & Count & 1 & 0 & 0 & 1 \\
\hline & & $\%$ & $100.0 \%$ & $0.0 \%$ & $0.0 \%$ & $100.0 \%$ \\
\hline & Technical College & Count & 0 & 2 & 0 & 2 \\
\hline & & $\%$ & $0.0 \%$ & $100.0 \%$ & $0.0 \%$ & $100.0 \%$ \\
\hline & University & Count & 0 & 0 & 2 & 2 \\
\hline & & $\%$ & $0.0 \%$ & $0.0 \%$ & $100.0 \%$ & $100.0 \%$ \\
\hline \multirow[t]{2}{*}{ Total } & & Count & 1 & 2 & 2 & 5 \\
\hline & & $\%$ & $20.0 \%$ & $40.0 \%$ & $40.0 \%$ & $100.0 \%$ \\
\hline
\end{tabular}

Table 6.10: Reported enjoyment of twelfth visualization by highest level of education.

$H_{a}$ : Participants' highest level of education and their reported most enjoyed visualization are not independent.

A statistically significant association was found between participants' highest level of education and the visualization they most enjoyed such that high school completers chose Radar, college completers chose iTunes, and university completers chose Part Motion, $\mathcal{X}^{2}(4, \mathrm{~N}=5)=10, p=0.04$. Since the $p$-value is less than the significance level (0.05), we cannot accept the null hypothesis.

A contingency table analysis did not show any significant trends among responses to the different songs except for the very last one shown in Table 6.10 and Table 6.11.

$H_{0}$ : Participants' highest level of education and their reported enjoyment of any one visualization are independent.

$H_{a}$ : Participants' highest level of education and their reported enjoyment of any one visualization are not independent.

A statistically significant association was found between the participant's highest level of education and their enjoyment of the last (12th) visualization of the focus group such that high school com- 


\begin{tabular}{|c|c|c|c|c|c|c|}
\hline & & & \multicolumn{3}{|c|}{ Enjoyment of Visualization 12} & Total \\
\hline \multirow{7}{*}{ Most enjoyed visualization } & \multirow{3}{*}{ Balls } & \multirow{3}{*}{$\begin{array}{r}\text { Count } \\
\%\end{array}$} & $\begin{array}{l}\text { Not that } \\
\text { enjoyable }\end{array}$ & Neutral & $\begin{array}{c}\text { Somewhat } \\
\text { enjoyable }\end{array}$ & \\
\hline & & & 0 & 0 & 2 & \\
\hline & & & $0.0 \%$ & $0.0 \%$ & $100.0 \%$ & $100.0 \%$ \\
\hline & \multirow{2}{*}{ iTunes } & Count & 0 & 2 & 0 & \\
\hline & & $\%$ & $0.0 \%$ & $100.0 \%$ & $0.0 \%$ & $100.0 \%$ \\
\hline & \multirow{2}{*}{ Radar } & Count & 1 & 0 & 0 & \\
\hline & & $\%$ & $100.0 \%$ & $0.0 \%$ & $0.0 \%$ & $100.0 \%$ \\
\hline \multirow{2}{*}{\multicolumn{2}{|c|}{ Total }} & Count & 1 & 2 & 2 & \\
\hline & & $\%$ & $20.0 \%$ & $40.0 \%$ & $40.0 \%$ & $100.0 \%$ \\
\hline
\end{tabular}

Table 6.11: Reported enjoyment of twelfth visualization by most enjoyed visualization.

pleters found it "Not that enjoyable", college completers were "Neutral", and university completers found it "Somewhat enjoyable", $\mathcal{X}^{2}(4, \mathrm{~N}=5)=10, p=0.04$. Since the $p$-value is less than the significance level (0.05), we cannot accept the null hypothesis.

$H_{0}$ : Participants' reported most enjoyed visualization and their reported enjoyment of any one visualization are independent.

$H_{a}$ : Participants' reported most enjoyed visualization and their reported enjoyment of any one visualization are not independent.

A statistically significant association was found between the participant's most enjoyed visualization and their enjoyment of the last (12th) visualization of the focus group (which was a Part Motion display) such that those who most preferred Part Motion found it "Not that enjoyable", those who most enjoyed iTunes were "Neutral", and those who most enjoyed Radar found it "Somewhat enjoyable", $\mathcal{X}^{2}(4, \mathrm{~N}=5)=10, p=0.04$. Since the $p$-value is less than the significance level (0.05), we cannot accept the null hypothesis.

Table 6.12 on the following page shows the participants' level of enjoyability across the different types of visualizations.

\subsubsection{Goal 4 - Conveyance of Emotion}

\section{Levels of Emotion}

Analyses of variance (ANOVAs) comparing participants responses to the questions on the degree they felt the music was happy / excited / overbearing revealed no significant differences on the demographic variables. Kruskal-Wallis $\mathrm{H}$ tests also showed no statistically significant differences. Table 6.13 on page 123 shows participants' average rating (between 1 and 9) of PAD (Pleasure, Arousal. Dominance) dimensions by the intended emotion of the song. Table 6.14 on page 123 shows this same information by type of visualization. 


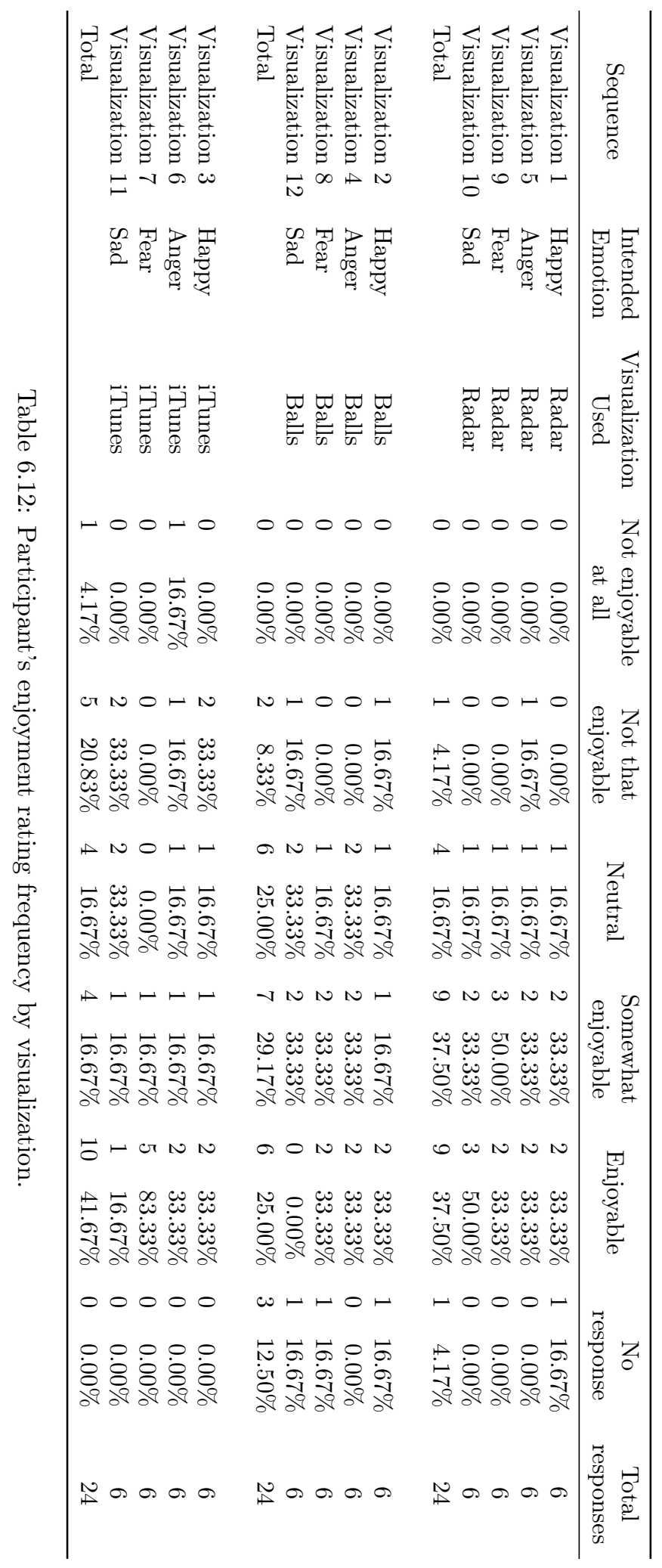




\begin{tabular}{|c|c|c|c|c|c|c|c|}
\hline \multirow[b]{2}{*}{ Sequence } & \multirow[b]{2}{*}{$\begin{array}{l}\text { Intended } \\
\text { Emotion }\end{array}$} & \multicolumn{2}{|c|}{ Pleasure } & \multicolumn{2}{|c|}{ Arousal } & \multicolumn{2}{|c|}{ Dominance } \\
\hline & & $\begin{array}{l}\text { Unhappy } \\
\bar{x}\end{array}$ & $\begin{array}{l}\text { / Happy } \\
\sigma\end{array}$ & $\begin{array}{l}\text { Calm } \\
\bar{x}\end{array}$ & $\begin{array}{c}\text { Excited } \\
\sigma\end{array}$ & $\begin{array}{l}\text { Subtle / } \\
\bar{x}\end{array}$ & $\begin{array}{c}\text { Overbearing } \\
\sigma\end{array}$ \\
\hline Visualization 1 & Happy & 7.5 & 1.12 & 5.8 & 1.77 & 5.0 & 1.10 \\
\hline Visualization 2 & Happy & 5.4 & 1.20 & 2.2 & 1.07 & 3.6 & 0.80 \\
\hline Visualization 3 & Happy & 5.3 & 2.05 & 4.0 & 1.15 & 3.8 & 1.60 \\
\hline Visualization 4 & Anger & 4.4 & 1.36 & 4.6 & 2.06 & 4.4 & 1.20 \\
\hline Visualization 5 & Anger & 4.4 & 2.06 & 6.3 & 2.05 & 6.2 & 1.72 \\
\hline Visualization 6 & Anger & 4.8 & 1.83 & 4.4 & 1.85 & 4.6 & 2.06 \\
\hline Visualization 7 & Fear & 7.0 & 1.26 & 7.2 & 0.40 & 5.6 & 1.36 \\
\hline Visualization 8 & Fear & 5.1 & 2.56 & 6.2 & 1.80 & 4.8 & 1.47 \\
\hline Visualization 9 & Fear & 5.0 & 1.26 & 4.8 & 1.47 & 4.0 & 1.87 \\
\hline Visualization 10 & Sad & 3.0 & 1.79 & 2.2 & 0.75 & 2.9 & 0.89 \\
\hline Visualization 11 & Sad & 5.0 & 2.10 & 3.8 & 1.92 & 4.8 & 2.14 \\
\hline Visualization 12 & Sad & 3.8 & 1.09 & 3.5 & 1.12 & 3.5 & 2.06 \\
\hline
\end{tabular}

Note. Scores ranged from 1 (unhappy, calm, subtle) to 9 (happy, excited, overbearing).

Table 6.13: Average rating of PAD (Pleasure, Arousal. Dominance) dimensions by the intended emotion of the song.

\begin{tabular}{lccccccc}
\hline \multirow{2}{*}{ Sequence } & & \multicolumn{2}{c}{ Pleasure } & \multicolumn{3}{c}{ Arousal } & \multicolumn{2}{c}{ Dominance } \\
\cline { 3 - 8 } & Visualization & \multicolumn{2}{c}{ Unhappy $/$ Happy } & \multicolumn{2}{c}{ Calm $/$ Excited } & \multicolumn{2}{c}{ Subtle / Overbearing } \\
& Used & $\bar{x}$ & $\sigma$ & $\bar{x}$ & $\sigma$ & $\bar{x}$ & $\sigma$ \\
\cline { 3 - 8 } Visualization 1 & Radar & 7.5 & 1.12 & 5.8 & 1.77 & 5.0 & 1.10 \\
Visualization 5 & Radar & 4.4 & 2.06 & 6.3 & 2.05 & 6.2 & 1.72 \\
Visualization 9 & Radar & 5.0 & 1.26 & 4.8 & 1.47 & 4.0 & 1.87 \\
Visualization 10 & Radar & 3.0 & 1.79 & 2.2 & 0.75 & 2.9 & 0.89 \\
& & & & & & & \\
Visualization 2 & Balls & 5.4 & 1.20 & 2.2 & 1.07 & 3.6 & 0.80 \\
Visualization 4 & Balls & 4.4 & 1.36 & 4.6 & 2.06 & 4.4 & 1.20 \\
Visualization 8 & Balls & 5.1 & 2.56 & 6.2 & 1.80 & 4.8 & 1.47 \\
Visualization 12 & Balls & 3.8 & 1.09 & 3.5 & 1.12 & 3.5 & 2.06 \\
& & & & & & & \\
Visualization 3 & iTunes & 5.3 & 2.05 & 4.0 & 1.15 & 3.8 & 1.60 \\
Visualization 6 & iTunes & 4.8 & 1.83 & 4.4 & 1.85 & 4.6 & 2.06 \\
Visualization 7 & iTunes & 7.0 & 1.26 & 7.2 & 0.40 & 5.6 & 1.36 \\
Visualization 11 & iTunes & 5.0 & 2.10 & 3.8 & 1.92 & 4.8 & 2.14 \\
\hline
\end{tabular}

Note. Scores ranged from 1 (unhappy, calm, subtle) to 9 (happy, excited, overbearing).

Table 6.14: Average rating of PAD (Pleasure, Arousal. Dominance) dimensions by visualization. 


\begin{tabular}{llllcccccc}
\hline Sequence & Intended & \multicolumn{2}{c}{ Happy } & \multicolumn{2}{c}{ Sad } & \multicolumn{2}{c}{ Anger } & \multicolumn{3}{c}{ Fear } \\
& Emotion & $\bar{x}$ & $\sigma$ & $\bar{x}$ & $\sigma$ & $\bar{x}$ & $\sigma$ & $\bar{x}$ & $\sigma$ \\
\hline Visualization 1 & Happy & 5.2 & 1.21 & 1.6 & 1.22 & 1.5 & 0.87 & 1.0 & 0.00 \\
Visualization 2 & Happy & 2.8 & 1.07 & 4.0 & 1.58 & 1.3 & 0.47 & 1.3 & 0.47 \\
Visualization 3 & Happy & 3.8 & 2.14 & 3.0 & 0.89 & 1.7 & 0.94 & 2.7 & 1.25 \\
& & & & & & & & & \\
Visualization 4 & Anger & 3.1 & 1.09 & 2.0 & 1.22 & 2.8 & 2.05 & 1.7 & 0.47 \\
Visualization 5 & Anger & 3.5 & 1.12 & 1.8 & 1.30 & 2.7 & 2.36 & 4.3 & 1.92 \\
Visualization 6 & Anger & 3.0 & 1.79 & 2.3 & 1.30 & 4.0 & 1.87 & 3.7 & 2.05 \\
& & & & & & & & & \\
Visualization 7 & Fear & 4.8 & 1.34 & 1.8 & 1.30 & 1.7 & 0.94 & 2.3 & 1.89 \\
Visualization 8 & Fear & 3.8 & 1.83 & 2.0 & 1.73 & 3.2 & 2.05 & 2.2 & 2.49 \\
Visualization 9 & Fear & 4.2 & 1.17 & 1.8 & 1.30 & 2.7 & 2.36 & 3.5 & 2.06 \\
& & & & & & & & & \\
Visualization 10 & Sad & 3.6 & 2.06 & 4.0 & 2.19 & 1.7 & 0.94 & 1.7 & 0.94 \\
Visualization 11 & Sad & 5.0 & 2.00 & 2.3 & 1.64 & 1.7 & 0.94 & 1.7 & 0.94 \\
Visualization 12 & Sad & 2.7 & 1.25 & 2.8 & 1.48 & 2.0 & 1.00 & 2.0 & 1.00 \\
\hline Note. Scores ranged from 1 (weak) & to 7 (strong). & & & & \\
\hline
\end{tabular}

Table 6.15: Average rating of emotion by the intended emotion of the song.

\section{Level of Discrete Emotion Ratings}

ANOVAs comparing participants responses to the questions on the degree they felt the visualization (and thus the music) tried to express happy / sad / angry / fear emotions revealed no significant differences on the demographic variables. Kruskal-Wallis H tests also showed no statistically significant differences. Table 6.15 summarizes the average discrete emotion ratings for each type of song. Participants were asked to rate each emotion they thought was expressed by the song from "1-weak" to "7-strong".

Finally, Table 6.16 on the facing page reorders the results shown in Table 6.15 to summarize the average scores (from 1 to 7 ) for each visualization by type of visualization.

\subsection{Discussion}

\subsubsection{Goal 1 - Are efforts to improve accessibility desired?}

To determine whether efforts to create music information that is accessible to the $\mathrm{D} / \mathrm{HH}$ are wanted, there is a need to understand whether $\mathrm{D} / \mathrm{HH}$ people are active consumers of music. Participants of this focus group responded favorably to music.

For example, Table 6.3 on page 116 shows the contingency table of hearing identity and how much the participant listens to music. The majority of the group (80\%) reported listening to music "weekly" or "daily". One deaf person reported listening to music "rarely".

Similarly, Table 6.4 on page 117 shows participant's responses to the question "What statement de- 


\begin{tabular}{lccccccccc}
\hline \multirow{2}{*}{ Sequence } & Visualization & \multicolumn{3}{c}{ Happy } & \multicolumn{3}{c}{ Sad } & \multicolumn{3}{c}{ Anger } & \multicolumn{2}{c}{ Fear } \\
& Used & $\bar{x}$ & $\sigma$ & $\bar{x}$ & $\sigma$ & $\bar{x}$ & $\sigma$ & $\bar{x}$ & $\sigma$ \\
\hline Visualization 1 & Radar & 5.2 & 1.21 & 1.6 & 1.22 & 1.5 & 0.87 & 1.0 & 0.00 \\
Visualization 5 & Radar & 3.5 & 1.12 & 1.8 & 1.30 & 2.7 & 2.36 & 4.3 & 1.92 \\
Visualization 9 & Radar & 4.2 & 1.17 & 1.8 & 1.30 & 2.7 & 2.36 & 3.5 & 2.06 \\
Visualization 10 & Radar & 3.6 & 2.06 & 4.0 & 2.19 & 1.7 & 0.94 & 1.7 & 0.94 \\
& & & & & & & & & \\
Visualization 2 & Balls & 2.8 & 1.07 & 4.0 & 1.58 & 1.3 & 0.47 & 1.3 & 0.47 \\
Visualization 4 & Balls & 3.1 & 1.09 & 2.0 & 1.22 & 2.8 & 2.05 & 1.7 & 0.47 \\
Visualization 8 & Balls & 3.8 & 1.83 & 2.0 & 1.73 & 3.2 & 2.05 & 2.2 & 2.49 \\
Visualization 12 & Balls & 2.7 & 1.25 & 2.8 & 1.48 & 2.0 & 1.00 & 2.0 & 1.00 \\
& & & & & & & & & \\
Visualization 3 & iTunes & 3.8 & 2.14 & 3.0 & 0.89 & 1.7 & 0.94 & 2.7 & 1.25 \\
Visualization 6 & iTunes & 3.0 & 1.79 & 2.3 & 1.30 & 4.0 & 1.87 & 3.7 & 2.05 \\
Visualization 7 & iTunes & 4.8 & 1.34 & 1.8 & 1.30 & 1.7 & 0.94 & 2.3 & 1.89 \\
Visualization 11 & iTunes & 5.0 & 2.00 & 2.3 & 1.64 & 1.7 & 0.94 & 1.7 & 0.94 \\
\hline \multicolumn{1}{l}{ Note. Scores ranged from 1 (weak) to 7 (strong). } \\
\hline
\end{tabular}

Table 6.16: Average rating of emotion by visualization.

scribes you best?" This group split 50\%/50\% on whether they "liked listening to music" (2 deaf, $1 \mathrm{HH}$ ) or "really enjoy listening to music" (1 HH, 1 deaf, $1 \mathrm{CI}$ ). No one responded "I do not like listening to music." or "I do not see the point of music." The deaf participant who reported rarely listening to music, also described themselves as liking music.

Given that the recruiting materials for this focus group specifically mentioned music, it is likely that the participants self-selected on this item. In other words, it is unlikely, given the recruiting materials, that a person who might respond "I do not like listening to music." or "I do not see the point of music." would have attended this focus group.

Thus, similar to Chapter 4 and Chapter 5, in answer to the question of whether efforts to create accessible music information are wanted, the responses of D/HH participants strongly support the notion that they actively seek music and enjoy the experience. No one reported having no interest in music. Some participants found the study very interesting and reported a desire to see more.

\subsubsection{Goal 2 - How do viewers respond to specific visualizations?}

As mentioned in Chapter 1, of the Principles of Universal Design, Principle Three, "Simple and intuitive use", implies that the user interface of any visualization solution should be obvious. In the case of music visualization, this principle would suggest that the visualization itself should be simple and intuitive, even without sound. Similarly, Principle Four, "Perceptible Information", requires the design to communicate necessary information effectively to the user. Determining what types of music information would be considered useful and whether specific visualization styles communicated such information in a intuitive manner would support the Principles of Universal Design.

As shown in Table 6.6 on page 118, when participants were asked which visualization style gave 
them the "best" sense of drums / percussion, instruments playing, melody, or rhythm, their responses were mixed.

Participants found that Radar (4) gave the best sense of percussion. The Part Motion (2) visualization was also preferred for percussion. Participants found that the Part Motion visualization gave the best sense of instruments playing (4) as well as the best sense of the melody (4). Participants were split on the best visualization for rhythm choosing all three (iTunes (3), Radar (3) and Part Motion $(3))$.

As Table 6.7 shows, when asked which visualization gave the best sense of what was happening in the music, one $\mathrm{HH}$ participant chose the Part Motion visualization while the other chose iTunes. The majority of the deaf participants (2) preferred Radar, while one preferred the iTunes visualization. One participant did not select a preference. Unlike the focus group described in the previous chapter, there was no statistically significant association between a participant's enjoyment of a particular visualization and their choice of which one gave the best sense of music.

\subsubsection{Goal 3 - Do participants enjoy visualizations?}

As mentioned in Chapter 1, Principle One of the Principles of Universal Design, "Equitable use" suggests that a music visualization should be appealing. Determining whether specific visualization styles were enjoyable would support the Principles of Universal Design.

When asked at the end of the focus group session which visualization they enjoyed most, as seen in Table 6.8, participants were equally split, two each, across the three visualizations. In fact no one group expressed a preference: HH participants were split between Part Motion and iTunes, the majority of deaf participants expressed a preference for Radar, and he sole CI-using participant expressed a preference for Part Motion.

The statistically significant contingency table analysis finding an association between participants' highest level of education and the visualization they most enjoyed shown in Table 6.9 is likely an artifact of the small sample size. The chi-square test for independence is sensitive to sample size as well as small expected frequencies in one or more of the cells in the table. In this case more than 20 percent of the expected frequencies values were below five.

Recall that the contingency table analysis of the twelfth visualization shown in Table 6.10 and Table 6.11 found statistically significant associations between the participant's

- highest level of education and their enjoyment of the twelfth visualization; and

- most enjoyed visualization and their enjoyment of the twelfth visualization.

This statistically significant result is likely also an artifact of the small sample size as some participants did not provide responses to these questions at the time. Note also that the result of the chisquare test for independence in both cases were the same. While we would expect that participants might begin to favour specific visualization types over others as one neared the end of the focus group, as the contingency table in Table 6.11 shows, the unexpected statistically significant result with Table 6.10 (which occurred with no other visualization) must raise some suspicion when analyzing the 
results of Table 6.11. It is entirely possible that this finding is valid, but it is also possible that other factors are influencing the result.

At the conclusion of the focus group, participants expressed very positive feelings about the study. Participants reported most enjoying watching the visualizations and being stimulated by the experience.

Participants reported that they least enjoyed not hearing the sound, feeling confused, and not feeling that they could connect what they were seeing to specific information (e.g., whether certain colours meant certain sounds, instruments, etc.).

\subsubsection{Goal 4-Can participants detect emotions through visualizations?}

As mentioned above, Principle Three of the Principles of Universal Design, "Simple and intuitive use", suggests that the visualization itself should be simple and intuitive, even without sound. Similarly, Principle Four, "Perceptible Information", requires the design to communicate necessary information effectively to the user. Determining whether information relating to emotion is conveyed by specific visualization styles in a intuitive manner would support the Principles of Universal Design.

Table 6.13 on page 123 summarizes the PAD (Pleasure, Arousal, Dominance) emotion ratings of each song for each visualization by intended emotion (rated from 1 to 9 ). While there exists no baseline to compare these results against a hearing person's experience:

- Happy songs tended to rate towards the Happy side of the Pleasure scale $(\bar{x}=6.07)$, the Calm side of the Arousal scale $(\bar{x}=4.0)$ and the Subtle side of the Dominance scale $(\bar{x}=4.13)$.

- Anger songs tended to rate towards the Happy side of the Pleasure scale $(\bar{x}=4.53)$, the Excited side of the Arousal scale $(\bar{x}=5.1)$ and the Overbearing side of the Dominance scale $(\bar{x}=5.07)$.

- Fear songs tended to rate towards the Happy side of the Pleasure scale $(\bar{x}=5.7)$, the Excited side of the Arousal scale $(\bar{x}=6.07)$ and the Subtle side of the Dominance scale $(\bar{x}=4.8)$.

- Sad songs tended to rate towards the Unhappy side of the Pleasure scale $(\bar{x}=3.93)$, the Calm side of the Arousal scale $(\bar{x}=3.17)$ and the Subtle side of the Dominance scale $(\bar{x}=3.73)$.

These results suggest that participants were able to interpret the intended emotion of the song using the PAD scale.

Table 6.15 on page 124 summarizes the average discrete emotion ratings of each song (from "1weak" to "7-strong") for each visualization by intended emotion:

- As shown in the first section of Table 6.15, participants tended to score happy songs as happy. Participants comments tended to identify the songs as happy. When asked what the visualization meant to them, responses included: "Good, happy, vibrant", and "Catchy music. Good beat."

- As shown in the second part of Table 6.15, participants tended to score angry songs as happy, fearful, or angry, depending on the visualization used. Participants comments did not trend toward the anger of the songs. Participants comments tended to identify the songs as aggressive 
and fearful. Responses included: "I felt like the person was on a stage, running from something or in a ballet.", and "Sort of fight/argue, group branch off, hang out one group, tough times."

- As shown in the third part of Table 6.15, participants tended to score fearful songs as happy. Participants comments tended to identify the fearful songs as slow and calm. Responses included: "Booming sounds with different volumes going up and down, almost like rhythms", and "Not sure."

- As shown in the fourth part of Table 6.15, participants tended to score sad songs as sad except in the iTunes visualization. Responses included: "seems slow relaxing music."

These results suggest that while participants were able to interpret the intended emotion of the song using the PAD scale, they were not necessarily able to accurately denote the intended emotion of the song using the discrete emotion ratings scale. However, their comments would suggest that the visualization was able to convey some interpretable sense of the emotion.

\subsubsection{Limitations of the Design}

The analysis of the data suggests at least four limitations of this study, two of which are related to questionnaire design.

The first limitation of this design is that while asking how to represent music information, the Post-study Questionnaire did not also ask for some ranking of the importance of each type of information. As a result this must be extrapolated from the responses and comments. For example, it can be suggested that the information is important when a preference is specified and it can be suggested that there is no importance when a participant ignores the item and leaves it blank. However, any conclusion that is more specific cannot be established based on this data.

Second, the wording of question 2 and question 3 in the Post-study Questionnaire (i.e., "Which style gave you the best sense of what was happening in the music?" and "Which style gave you the best sense of percussion / instruments playing / melody / rhythm?"), raises some issues around how the participant might interpret the phrase "best sense". While the design of the questionnaire was intended to leave this phrase open to interpretation, the fact that a participant may have no psychological reality of certain music information might mean they are unable to assess what visualization gave them the "best sense" of something because they would not know how to understand that concept.

Third, the small sample size with an unequal number of men and women, while appropriate for a focus group, limits the statistical power of the results. The chi-square test for independence is sensitive to sample size as well as small expected frequencies in one or more of the cells in the table. For these reasons caution must be taken in interpreting chi-square if one or more cells are less than 5 . However, according to Frankfort-Nachmias and Leon-Guerrero, "There is no hard-and-fast rule regarding the size of expected frequencies." (2011, p. 350). Most researchers limit the use of chi-square to tables that either $a$ ) have no expected frequencies values below 5 or $b$ ) have no more than 20 percent of the expected frequencies values below 5 (Frankfort-Nachmias \& Leon-Guerrero, 2011).

The small sample size also impacted the ANOVAs run on the data collected for this study since small sample sizes increase the difficulty of detecting assumption violations. Even if none of the test 
assumptions are violated, a one-way ANOVA with small sample sizes may not have sufficient power to detect any significant difference among the samples, even if the means are in fact different.

Finally, given that the recruiting materials for this focus group specifically mentioned music, it is likely that the participants are self-selected. In other words, it is unlikely, given the recruiting materials, that $\mathrm{D} / \mathrm{HH}$ community members who might not like music or have any understanding of music would have attended this focus group. This is a weakness of the interpretability of the data because it does not allow generalization of the results over all possible consumers, only those who have any interest in music.

\subsection{Conclusions From This Study}

In conclusion, among other issues, in this focus group we observed that the iTunes visualization was still very positively responded to, but no one visualization was enjoyed the most. Unlike the study in Chapter 5, the Part Motion visualization did not give the "best sense of what's happening". The Radar visualization seemed to receive a good response, with comments like, "looks like people dancing."

The participants enjoyed the visualizations and were able to interpret an emotional response from the music information the visualization conveyed.

While music was conveyed better in this focus group, it still needs more work. One participant's comment is particularly instructive in this regard, "I think that care has to be taken when selecting type of visualization to use with each style of music."

As shown in Figure 6.6, the work in this chapter represents the full DRM cycle because it introduced an intervention, the exposure of the participants to the videos, and measured specific parameters in their experience of these visualizations. In the larger scope of the Dissertation, this chapter, qualifies as contributing to a DRM Stage 2 (Prescriptive Study) activity because it evaluated a revised set of visualizations and added to our understanding of the situation into which some design interven-

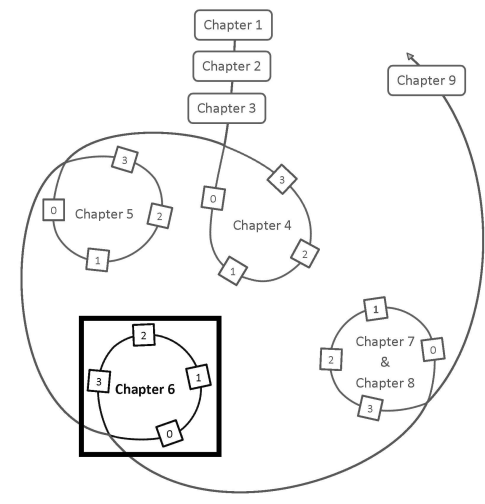

Figure 6.6: Location within Dissertation path. 
tion could be eventually introduced.

\subsection{Next Steps}

Having explored how $\mathrm{D} / \mathrm{HH}$ people respond to this set of visualizations, there remain several open questions. One such question is whether the $\mathrm{D} / \mathrm{HH}$ communities want some technological means to support music listening. Further, if there is a need or expressed desire to represent music in a way that is more accessible for $\mathrm{D} / \mathrm{HH}$ people, what information or types of information should be the focus of this technology?

The next chapter will revisit the DRM Stage 1 (Descriptive Study I). It describes a paper survey that was conducted to get more information from members of the $\mathrm{HH}$ community on these and other open issues. 


\section{Chapter 7}

\section{Survey of Users' Music Needs}

The work reported in the preceding chapters have clarified some aspects of the D/HH community's needs with regards to music. However, there exist several questions regarding the hard of hearing (HH) population that need to be better understood. For example:

- Do HH people actively listen to and enjoy music?

- Do HH music consumers report issues using their Assistive Listening Devices (ALDs) to listen to music?

- Do HH people make specific choices about the music they listen to as a result of their hearing loss?

- What, if anything, do HH music consumers feel they need to better support their experience?

- Is there a need or expressed desire to represent music in a way that is more accessible for $\mathrm{HH}$ people?

In the survey reported in Chapter 4, the majority of $\mathrm{HH}$ respondents reported enjoying music, but there were some comments that music was often experienced as noise rather than as meaningful and pleasant.

HH people actively try to make use of whatever residual hearing they have (Laszlo, 1994), unfortunately, HAs are known to distort music (Chasin, 2003). This distortion is a result of HA design. Many HAs have a limiter just after the microphone that is usually set somewhere between $88 \mathrm{~dB}$ and $100 \mathrm{~dB}$. This design decision is based on the logic that since the loudest components of shouted speech are in the range of $85 \mathrm{~dB}$ to $90 \mathrm{~dB}$ sound pressure level (SPL), any sound above that is considered noise and should be limited. The result is that sounds louder than 90dB (e.g., rock music can exceed 100dB SPL) will be cut off.

However, as noted in Chapter 4, few reports of HA satisfaction address this issue. The MarkeTrak survey, a consumer survey that measures attitudes towards HAs by asking for what activities a given 
consumer wears them, was conducted in 1989, 1990, 1991, 1994, 1997, 2000, 2004, and 2008. "Listening to music" was only added as a survey item in 1997 (Kochkin, 2000).

The most recent MarkeTrak survey shows that $77 \%$ of responding consumers indicated that "Listening to music" was an important need (they assigned scores of 3 or 4 on a four-point scale ranging from "Very important" to "Not at all important") while $36 \%$ indicated that that "Listening to music" was a critical need (they assigned assigned a 4 for "Very important" to this listening situation). This same survey found that $78 \%$ of respondents indicated some degree of satisfaction (17\% "Somewhat Satisfied", $41 \%$ "Satisfied", $20 \%$ "Very Satisfied"), $8 \%$ indicated some degree of dissatisfaction (4\% "Somewhat Dissatisfied", $2 \%$ "Dissatisfied", $2 \%$ "Very Dissatisfied"), and $14 \%$ responded "Neutral" (Kochkin, 2010).

No one has asked HH music consumers about how they choose the music they listen to. It is entirely possible that whether their HA supports a satisfactory listening experience could determine the range of music genre $\mathrm{HH}$ consumers enjoy. On the other hand, lifestyle choices such as choosing specific genres of music (e.g., songs with deep bass or drums and few vocals) may be among the few available options for $\mathrm{HH}$ music consumers who cannot hear music without HAs and/or are not satisfied with the currently available hearing instruments and/or need additional information to fully enjoy the music experience.

Whether HH music consumers feel they need some additional support to access music is not well understood. This chapter will describe a survey conducted with $\mathrm{HH}$ music consumers to determine if there is a desire for added support and, if so, how best to support their needs. Portions of this chapter appear in Fourney (2012).

\section{$7.1 \quad$ Goals}

The goal of the study presented in this chapter is to answer the following questions:

1. Are efforts to improve accessibility desired?

2. What music information is wanted for access?

\subsection{Instruments}

Figure 7.1 and Figure 7.2 shows the questionnaire used to collect data during the survey.

\subsection{Method}

The study was based on a paper survey. The 14-item survey used open and closed questions to collect responses from $\mathrm{HH}$ people on their attitudes towards music, the technologies they use when listening to music, what experiences (good and bad) they have while trying to listen to music and the various strategies they use to improve this experience. The survey also asked what, if anything, participants would want a computer to do to help them better understand or enjoy their music experience. 


\section{Music Visualization Questionnaire}

The purpose of this questionnaire is to gather information about you and your experience with music. There are fourteen questions and it should take you about five to ten minutes to complete this questionnaire. Thank you in advance for your time and assistance.

1. Do you identify yourself as: (please check one)
$\square$ Hearing
$\square$ Deaf
Hard of hearing
$\square$ Deafened
Cochlear implant

2. Please indicate your gender: (please check one)

a Male

Female

3. Please indicate your age: (please check one)
口 $18-24$
口 $35-44$
$55-64$
口 $25-34$
口 $45-54$
$65+$

4. What is your highest level of education completed? (please check one)
No formal education
$\square$ High School
$\square$ University
Elementary school
$\square$ Community college
Graduate school (e.g., Master's)

5. How often do you use closed captioning when watching television? (please check one)
$\square$ Always
$\square$ Sometimes
○ Never

6. How often do you listen to music? (please check one)
$\square$ Every day
$\square$ Weekly
$\square$ Sometimes
Rarely
Never

7. What technology do you use to listen to music? (check all that apply)

$\square$ Radio $\square$ Music player $\square$ Home stereo $\square$ Music video $\square$ Other

8. Which of the following statements best describes you? (check all that apply)

I really enjoy listening to music

I do not like listening to music

I like listening to music

I do not see the point of music

I do not like nor dislike music

9. If you wanted to use a computer to help you to better understand music, what would be the three most important pieces of information you would want to know? (please circle your three choices)

The name of the song

Artist information (e.g., band name)

Lyrics

Instrument currently being played

Beat

Other

Figure 7.1: Survey Questionnaire Page 1. 
10. What types of solutions have you tried to access music: (check all that apply).

$\square$ Turn volume up higher

$\square$ Place hands/body close to speakers

$\square$ Use a balloon

$\square$ Set stereo equalizer

$\square$ Adjust the balance, bass, or treble settings

$\square$ Other $\square$ Use ear phones

Only listen to music with a strong beat

$\square$ Use music playing software with visualizations (e.g., iTunes, Windows Media Player)

$\square$ Wear hearing aids / Cl / FM system

11. Are you satisfied with this solution(s)? (please check one)

$\square$ Satisfied

Somewhat satisfied

$\square$ Neutral

$\square$ Somewhat dissatisfied

Dissatisfied

12. Why or why not:

13. Please comment on any specific frustrations that you have had with trying to access music and what would improve your experience with music. (Use extra paper if needed.)

14. Please comment on what positive experiences you have had with music. (Use extra paper if needed.)

Figure 7.2: Survey Questionnaire Page 2. 


\subsubsection{Participants}

The paper survey was conducted at a conference for HH people. Of the 150 surveys distributed, 36 participants $(25 \mathrm{f}, 11 \mathrm{~m})$ aged $18-65+$ completed it. All respondents identified themselves as having a hearing loss with $26(72.2 \%)$ indicating they were HH, 7 (19.4\%) said they have a CI, $2(5.6 \%)$ identified themselves as deaf, and $1(2.8 \%)$ identified themselves as deafened.

As shown in Table 7.1, the majority of the respondents (25 or $69.4 \%$ ) indicated that they always watch television with the captioning decoder turned on. In addition, a number of them ( 8 or $22.2 \%$ ) indicated that they sometimes use closed captions while watching television. Only three respondents indicated never using captions. This suggests that most HH people use captions where available.

\begin{tabular}{|c|c|c|c|c|c|c|}
\hline & & & \multicolumn{3}{|c|}{$\begin{array}{c}\text { Frequency of watching television } \\
\text { with CC }\end{array}$} & \multirow[t]{2}{*}{ Total } \\
\hline & & & Always & Sometimes & Never & \\
\hline \multirow{8}{*}{ Identity } & $\mathrm{HH}$ & Count & 16 & 7 & 3 & 26 \\
\hline & & $\%$ within Identity & $61.5 \%$ & $26.9 \%$ & $11.5 \%$ & $100.0 \%$ \\
\hline & Deaf & Count & 1 & 1 & 0 & 2 \\
\hline & & $\%$ within Identity & $50.0 \%$ & $50.0 \%$ & $0.0 \%$ & $100.0 \%$ \\
\hline & Deafened & Count & 1 & 0 & 0 & 1 \\
\hline & & $\%$ within Identity & $100.0 \%$ & $0.0 \%$ & $0.0 \%$ & $100.0 \%$ \\
\hline & CI & Count & 7 & 0 & 0 & 7 \\
\hline & & $\%$ within Identity & $100.0 \%$ & $0.0 \%$ & $0.0 \%$ & $100.0 \%$ \\
\hline \multirow[t]{2}{*}{ Total } & & Count & 25 & 8 & 3 & 36 \\
\hline & & $\%$ within Identity & $69.4 \%$ & $22.2 \%$ & $8.3 \%$ & $100.0 \%$ \\
\hline
\end{tabular}

Table 7.1: Frequency of using closed captions (by identity).

\subsection{Results}

All raw data is presented in Appendix D.

\subsubsection{Goal 1 - Are efforts to improve accessibility desired?}

Table 7.2 is a contingency table of respondent's frequency of listening to music by identity.

$H_{0}$ : Respondent's report of how much they liked music and the frequency they listen to music are independent.

$H_{a}$ : Respondent's report of how much they liked music and the frequency they listen to music are not independent.

There was a statistically significant association between the frequency with which respondents listened to music and the degree they reported enjoying music $\mathcal{X}^{2}(12, \mathrm{~N}=36)=64.83, \mathrm{p}<.001$. Since the $p$-value is less than the significance level (0.05), we cannot accept the null hypothesis. 


\begin{tabular}{|c|c|c|c|c|c|c|c|c|}
\hline & & & \multicolumn{5}{|c|}{ Frequency of listening to music } & \multirow[t]{2}{*}{ Total } \\
\hline & & & Everyday & Weekly & Sometimes & Rarely & Never & \\
\hline \multirow{8}{*}{ Identity } & $\mathrm{HH}$ & Count & 15 & 4 & 4 & 2 & $\overline{1}$ & 26 \\
\hline & & $\%$ & $57.7 \%$ & $15.4 \%$ & $15.4 \%$ & $7.7 \%$ & $3.8 \%$ & $100.0 \%$ \\
\hline & Deaf & Count & 0 & 1 & 0 & 1 & 0 & 2 \\
\hline & & $\%$ & $0.0 \%$ & $50.0 \%$ & $0.0 \%$ & $50.0 \%$ & $0.0 \%$ & $100.0 \%$ \\
\hline & Deafened & Count & 0 & 1 & 0 & 0 & 0 & 1 \\
\hline & & $\%$ & $0.0 \%$ & $100.0 \%$ & $0.0 \%$ & $0.0 \%$ & $0.0 \%$ & $100.0 \%$ \\
\hline & $\mathrm{CI}$ & Count & 1 & 2 & 4 & 0 & 0 & 7 \\
\hline & & $\%$ & $14.3 \%$ & $28.6 \%$ & $57.1 \%$ & $0.0 \%$ & $0.0 \%$ & $100.0 \%$ \\
\hline \multirow[t]{2}{*}{ Total } & & Count & 16 & 8 & 8 & 3 & 1 & 36 \\
\hline & & $\%$ & $44.4 \%$ & $22.2 \%$ & $22.2 \%$ & $8.3 \%$ & $2.8 \%$ & $100.0 \%$ \\
\hline
\end{tabular}

Table 7.2: Frequency of listening to music (by identity).

Table 7.3 is a contingency table of respondent's reported degree music enjoyment by identity.

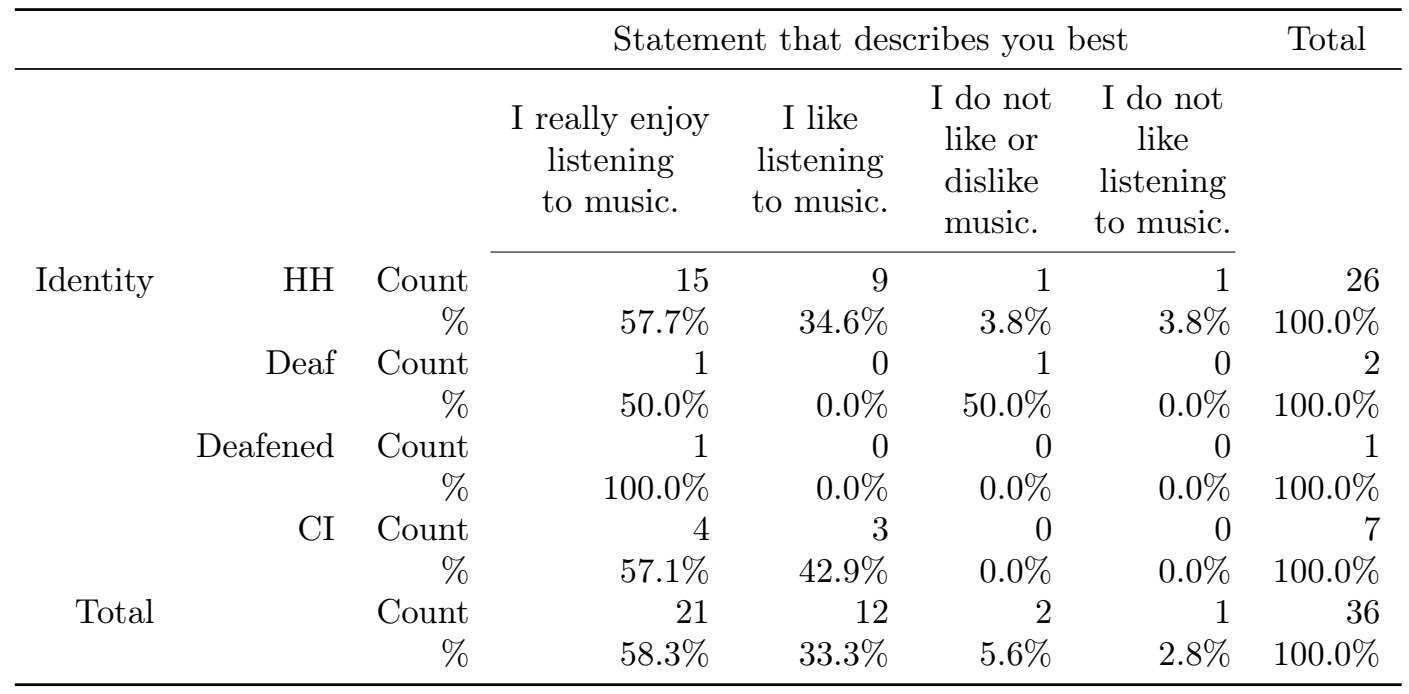

Table 7.3: Degree of reported music enjoyment (by identity).

Table 7.4 on the facing page is a contingency table of respondent's reported degree of music enjoyment by their reported frequency of listening to music. Table 7.5 is a contingency table of the technologies respondents reported they used when listening to music by identity.

\section{Selected comments:}

- "Wireless headphones \& stereo"

- "laptop, car"

- "Computer and car" 


\begin{tabular}{|c|c|c|c|c|c|c|c|}
\hline & & & \multicolumn{4}{|c|}{ Statement that describes you best } & Total \\
\hline & & & $\begin{array}{c}\text { I really enjoy } \\
\text { listening } \\
\text { to music. }\end{array}$ & $\begin{array}{c}\text { I like } \\
\text { listening } \\
\text { to music. }\end{array}$ & $\begin{array}{l}\text { I do not } \\
\text { like or } \\
\text { dislike } \\
\text { music. }\end{array}$ & $\begin{array}{l}\text { I do not } \\
\text { like } \\
\text { listening } \\
\text { to music. }\end{array}$ & \\
\hline \multirow{10}{*}{$\begin{array}{l}\text { Frequency } \\
\text { of listening } \\
\text { to music }\end{array}$} & Everyday & Count & 13 & 3 & 0 & 0 & 16 \\
\hline & & $\%$ & $81.3 \%$ & $18.8 \%$ & $0.0 \%$ & $0.0 \%$ & $100.0 \%$ \\
\hline & Weekly & Count & 5 & 3 & 0 & 0 & 8 \\
\hline & & $\%$ & $62.5 \%$ & $37.5 \%$ & $0.0 \%$ & $0.0 \%$ & $100.0 \%$ \\
\hline & Sometimes & Count & 3 & 5 & 0 & 0 & 8 \\
\hline & & $\%$ & $37.5 \%$ & $62.5 \%$ & $0.0 \%$ & $0.0 \%$ & $100.0 \%$ \\
\hline & Rarely & Count & 0 & 1 & 2 & 0 & 3 \\
\hline & & $\%$ & $0.0 \%$ & $33.3 \%$ & $66.7 \%$ & $0.0 \%$ & $100.0 \%$ \\
\hline & Never & Count & 0 & 0 & 0 & 1 & 1 \\
\hline & & $\%$ & $0.0 \%$ & $0.0 \%$ & $0.0 \%$ & $100.0 \%$ & $100.0 \%$ \\
\hline Total & & Count & 21 & 12 & 2 & 1 & 36 \\
\hline
\end{tabular}

Table 7.4: What statement describes you best? (by frequency of listening to music)

\begin{tabular}{|c|c|c|c|c|c|c|c|c|c|c|c|}
\hline \multicolumn{11}{|c|}{ Technology used when listening to music } & \multirow[t]{2}{*}{ Total } \\
\hline & & Radio & $\mathrm{Mu}$ & c Player & & tereo & & ic Video & & Other & \\
\hline $\mathrm{HH}$ & 15 & $32.61 \%$ & 11 & $23.91 \%$ & 10 & $21.74 \%$ & 3 & $6.52 \%$ & 7 & $15.22 \%$ & 46 \\
\hline CI & 1 & $12.50 \%$ & & & 3 & $37.50 \%$ & 1 & $12.50 \%$ & 3 & $37.50 \%$ & 8 \\
\hline Deaf & 2 & $100.00 \%$ & & & & & & & & & 2 \\
\hline Deafened & & & & & & & & & 1 & 100 & 1 \\
\hline Total & 18 & $31.58 \%$ & 11 & $19.30 \%$ & 13 & $22.81 \%$ & 4 & $7.02 \%$ & 11 & $19.30 \%$ & 57 \\
\hline
\end{tabular}

Table 7.5: Technology used when listening to music (by identity).

- "Computer w/ CD's \& ALD device"

- "iPod"

- "iPod computer"

- "It varies"

- "laptop"

Table 7.6 on the next page is a contingency table of the solutions respondents reported they have tried when listening to music by identity.

$H_{0}$ : Respondent's report of how much they liked music and their reported degree of satisfaction with their music listening solutions are independent. 


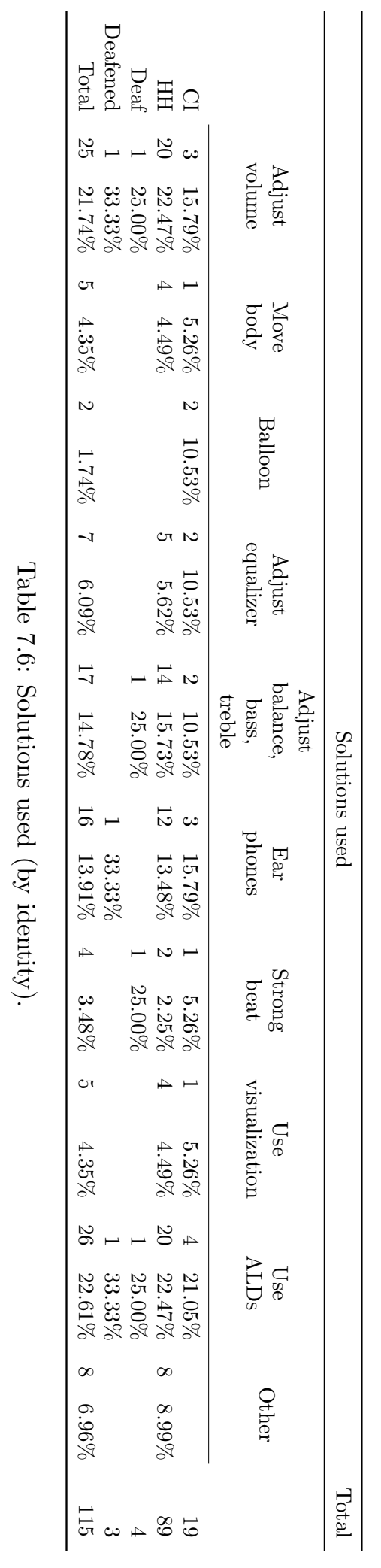


$H_{a}$ : Respondent's report of how much they liked music and their reported degree of satisfaction with their music listening solutions are not independent.

There was a statistically significant association between the degree of satisfaction with their music listening solutions and the degree respondents reported enjoying music $\mathcal{X}^{2}(8, \mathrm{~N}=35)=15.924, \mathrm{p}=$ .043. Since the $p$-value is less than the significance level (0.05), we cannot accept the null hypothesis.

$H_{0}$ : Respondent's report of how much they listen to music and their reported degree of satisfaction with their music listening solutions are independent.

$H_{a}$ : Respondent's report of how much they listen to music and their reported degree of satisfaction with their music listening solutions are not independent.

Similarly, there was a statistically significant association between the degree of satisfaction with their music listening solutions and the frequency with which respondents listened to music $\mathcal{X}^{2}(12, \mathrm{~N}$ $=35)=27.9, \mathrm{p}=.006$. Since the $p$-value is less than the significance level (0.05), we cannot accept the null hypothesis.

Table 7.7 is a contingency table of the satisfaction with the solutions respondents reported they have tried when listening to music by identity.

\begin{tabular}{|c|c|c|c|c|c|c|c|c|}
\hline & & & \multicolumn{5}{|c|}{ Satisfaction with solutions used } & \multirow[t]{2}{*}{ Total } \\
\hline & & & Satisfied & $\begin{array}{l}\text { Somewhat } \\
\text { satisfied }\end{array}$ & Neutral & $\begin{array}{l}\text { Somewhat } \\
\text { dissatisfied }\end{array}$ & Dissatisfied & \\
\hline \multirow[t]{8}{*}{ Identity } & $\mathrm{HH}$ & Count & 2 & 12 & 4 & 3 & $\overline{4}$ & 25 \\
\hline & & $\%$ & $8.0 \%$ & $48.0 \%$ & $16.0 \%$ & $12.0 \%$ & $16.0 \%$ & $100.0 \%$ \\
\hline & Deaf & Count & 0 & 1 & 0 & 0 & 1 & 2 \\
\hline & & $\%$ & $0.0 \%$ & $50.0 \%$ & $0.0 \%$ & $0.0 \%$ & $50.0 \%$ & $100.0 \%$ \\
\hline & Deafened & Count & 0 & 1 & 0 & 0 & 0 & 1 \\
\hline & & $\%$ & $0.0 \%$ & $100.0 \%$ & $0.0 \%$ & $0.0 \%$ & $0.0 \%$ & $100.0 \%$ \\
\hline & $\mathrm{CI}$ & Count & 0 & 2 & 1 & 3 & 1 & 7 \\
\hline & & $\%$ & $0.0 \%$ & $28.6 \%$ & $14.3 \%$ & $42.9 \%$ & $14.3 \%$ & $100.0 \%$ \\
\hline \multirow[t]{2}{*}{ Total } & & Count & 2 & 16 & 5 & 6 & 6 & 35 \\
\hline & & $\%$ & $5.7 \%$ & $45.7 \%$ & $14.3 \%$ & $17.1 \%$ & $17.1 \%$ & $100.0 \%$ \\
\hline
\end{tabular}

Table 7.7: Satisfaction with solutions used (by identity).

\subsubsection{Goal 2 - What music information is wanted for access?}

Table 7.8 is a contingency table of the types of music information respondents would like a computer to provide them when listening to music by identity.

\section{Selected comments:}

- ['Lyrics' was indicated as "1 2 3" - first, second, and third most important?] 
- ['Lyrics' circled and "\#1" put beside it. 'Name of the song' circled and "2" put beside it. A "3" was put beside 'Instrument']

- "if the music is produced and played at a very high quality, then the only other thing I want is the lyrics."

- "I prefer classical (or semiclassical music)"

- ["Important, also" written beside 'Instrument']

- "captioning"

- ["It depends" with arrows pointing to both 'Artist' and 'Beat' options]

- "not very computer literate yet"

- [“\#1 choice" written next to 'Lyrics']

- ["words comprehension" written next to 'Lyrics']

\begin{tabular}{rrrrrrrrr}
\hline & \multicolumn{6}{c}{ Computer supported music information } & Total \\
\hline & & Song Name & Artist & Lyrics & Instruments & Beat & Other & \\
\cline { 3 - 8 } HH & Count & 16 & 12 & 21 & 8 & 6 & 3 & 66 \\
& $\%$ & $24.24 \%$ & $18.18 \%$ & $31.82 \%$ & $12.12 \%$ & $9.09 \%$ & $4.55 \%$ & $100.00 \%$ \\
Deaf & Count & 2 & 0 & 2 & 1 & 2 & 0 & 7 \\
& $\%$ & $28.57 \%$ & $0.00 \%$ & $28.57 \%$ & $14.29 \%$ & $28.57 \%$ & $0.00 \%$ & $100.00 \%$ \\
Deafened & Count & 0 & 1 & 1 & 1 & 0 & 0 & 3 \\
& $\%$ & $0.00 \%$ & $33.33 \%$ & $33.33 \%$ & $33.33 \%$ & $0.00 \%$ & $0.00 \%$ & $100.00 \%$ \\
CI & Count & 5 & 5 & 6 & 1 & 2 & 0 & 19 \\
& $\%$ & $26.32 \%$ & $26.32 \%$ & $31.58 \%$ & $5.26 \%$ & $10.53 \%$ & $0.00 \%$ & $100.00 \%$ \\
Total & Count & 23 & 18 & 30 & 11 & 10 & 3 & 95 \\
& $\%$ & $24.21 \%$ & $18.95 \%$ & $31.58 \%$ & $11.58 \%$ & $10.53 \%$ & $3.16 \%$ & $100.00 \%$ \\
\hline
\end{tabular}

Table 7.8: Preferred music information from computer (by identity).

\subsection{Discussion}

\subsubsection{Goal 1 - Are efforts to improve accessibility desired?}

To determine whether efforts to create music information that is accessible to the $\mathrm{D} / \mathrm{HH}$ are wanted, there is a need to understand whether $\mathrm{D} / \mathrm{HH}$ people are active consumers of music. Respondents of this survey responded even more favourably to music than was shown in Chapter 4.

As shown in Table 7.3, nearly all respondents indicated that they "really enjoy" (21 or 58.3\%) or "like" (12 or $33.3 \%$ ) listening to music. Respondents could indicate a neutral response ("I do not like nor dislike music") to which one added the comment, "Can't always hear it properly." 
As shown in Table 7.2, 16 (44.4\%) respondents indicated listening to music every day. One $\mathrm{HH}$ person wrote that they listen to music not just everyday, but "usually all day."

Table 7.4 is a contingency table of the degree respondents reported enjoying music by their reports of their frequency of listening to music. The statistically significant relationship between the frequency with which respondents listened to music and the degree they reported enjoying music would be expected since one would not expect those who report not enjoying music to also frequently listen to it.

Respondents were asked to list the technologies they use when listening to music. As seen in Table 7.5, there was a range of responses: 18 use the radio, 13 use a home stereo, 12 use a music player such as an iPod, 5 use a computer, and 4 watch music videos (e.g., on a television with captioning).

Respondents were asked to identify the types of solutions they have used to try to access music. Table 7.6 shows there was a range of responses: 26 wear their HAs / CI / FM system (3 indicated they use special cables to directly connect their ALD to the stereo/computer playing the music), 25 increase the volume, 18 adjust the settings (e.g., balance, treble, bass, etc.), 16 use earphones / headset, 5 move closer to the speakers, 5 use music playing software with visualizations, 4 only listen to music with a strong beat, 2 have used a balloon (for the vibrotactile response created when the lowfrequency sounds are near the resonances of the balloon (Chasin, 2010)), 2 stated they try to memorize the lyrics, 1 indicated trying to cut down as much background noise in their environment as possible, and 1 stated that they have tried putting scotch tape over the microphones of their HA (this may reduce the microphone's sensitivity by $5-10 \mathrm{~dB}$ and thus fool the HA into accepting louder input (Chasin, 2010).

When asked if they were generally satisfied with the solutions they have tried, as Table 7.7 shows, of 35 respondents who answered this question, 18 (51.4\%) indicated they were either satisfied ( 2 or $5.6 \%$ ) or somewhat satisfied (16 or $44.4 \%$ ) while 12 were evenly split between somewhat dissatisfied or dissatisfied (6 each or 16.7\%). The statistically significant association between the degree of satisfaction with their music listening solutions and the degree respondents reported enjoying music suggests that the more satisfied respondents are with their music listening solutions, the more they enjoyed music. Similarly, the statistically significant association between the degree of satisfaction with their music listening solutions and the frequency with which respondents listened to music would be expected since one would expect that the more satisfied respondents are with their music listening solutions, the more they listen to music.

While these results seem like there may not be very many issues for HH music consumers, Table D.5 on page 341 shows in detail that many of the respondents still reported frustration with accessing music with and without HAs. For example, one respondent who has a CI and indicated that they "really liked" music also commented that she "used to" like music and, "I would love to like listening to music again. I am happy I can still get the beat of the song but would love to know what song it is." Another commented, "It just does not sound good. I cannot make sense of a song. Old songs are not always recognizable." A HA user said, "Losing more interest. Familiar music does not now sound the same."

The survey identified significant issues with HAs. Often respondents commented that they can only pick up the beat or some of the music, but not necessarily the lyrics. One CI user said, "Wearing my Cl, it's either the beat / music or words, but never the clarification of both being blended together. 
I heard better when I used to wear a hearing aid." Another respondent said, "All my life I have been HH. I have always listened to music but never for the most part understood words made out through music. I just listen to beats etc."

Many commented on the special efforts they take to listen to music such as only listening to music they enjoyed before their hearing loss (and thus hopefully remembering how it sounds). Some make the effort to memorize lyrics.

\subsubsection{Goal 2 - What music information is wanted for access?}

Respondents were asked to indicate what information, if any, they would most want a computer to help them with. Responses are shown in Table 7.8 on page 140. With respect to music information, the survey showed a strong desire (30 responses) for access to lyrics. When asked about access to any other music information (e.g., beat, instrumentation, etc.), song name had 23 responses, artist information had 18, instrument information had 11, and beat had 10. Respondents were asked to indicate three choices from a list, some indicated lyrics to be their first, second, and third choice. While respondents were not asked to rank their choices, in the case of lyrics some specifically noted it was their first choice.

\subsubsection{Limitations of the Design}

The analysis of the data suggests at least three limitations of this study, one of which is related to questionnaire design.

The first limitation of this design is that while asking how to represent music information (i.e., question \#9), the survey did not also ask for some ranking of the importance of each type of information. As mentioned earlier, many respondents imposed ranking onto the question (e.g., by noting 'lyrics' as their most desired / important choice). This raises some challenges. For example, when analyzing the responses of those who did not add any further ranking, or comment, the importance of their response is unknown.

The timing of this survey was also a limitation. Given that it was administered in parallel with the interviews described in the next chapter, there was no opportunity to use an initial analysis of the results to assist in the design of the interviews.

Finally, the setting of this survey was not entirely ideal. The survey was administered in the midst of a national conference for HH people. The conference theme, "Good Vibrations" was oriented to the experience of music. It was advertised as including performances by three different musical groups, including a well-known Canadian country and folk singer who, among various honours, has earned three Juno Awards. Given that the conference theme and events specifically focused on music, it is likely that the participants of the survey are self-selected. In other words, it is unlikely, given the nature of the conference, that a $\mathrm{HH}$ community member who might not like music would have attended this conference. This is a weakness of the interpretability of the data because it does not allow generalization of the results over all possible consumers, only those who have any interest in music. 


\subsection{Conclusions From This Study}

Music is not fully accessible to HH people. The sometimes long written comments respondents provided in this survey show the impact is significant: The music experience is frustrating, the lyrics are difficult (if not impossible) to understand, the full entertainment experience is lacking.

This survey of HH people shows that there is a significant desire for access to lyrics. How to present a meaningful presentation of lyrics has not been sufficiently researched. For example, captioned videos have been used to explore whether animating the captioned lyrics can help express emotional content. However, the captions were confusing; and not all viewers liked the animation or understood its role (Vy et al., 2008). Future research in the area of captioning music lyrics is needed.

As shown in Figure 7.3, the work in this chapter represents a partial DRM cycle because it does not introduce or examine a specific intervention. At the beginning of this chapter, there was a recognized need to refine the scoping of DRM Stage 0 discussed in Chapter 4. In particular, it remained unclear if $\mathrm{HH}$ people had specific needs unique to their community. As shown above, this survey found that indeed they do.

In the larger scope of the Dissertation, this chapter, qualifies as contributing to a DRM Stage 1 (DS I) activity because it added to our understanding of the situation into which some design intervention could be eventually introduced.

\subsection{Next Steps}

To get a better sense of an individual's response, several people who had completed the survey described in this chapter were asked to participate in one-on-one interviews. The next chapter continues to revisit DRM Stage 1 (Descriptive Study I). It describes these interviews and discusses their findings.

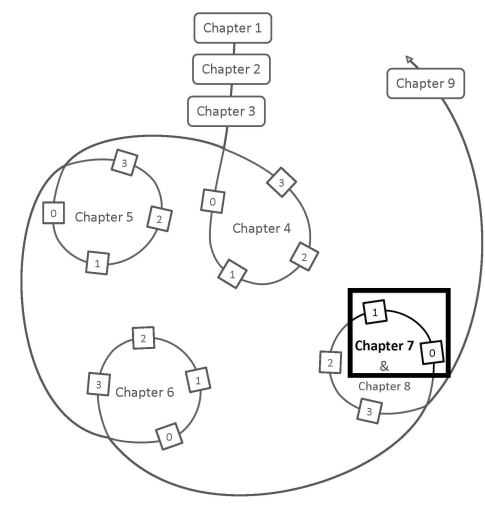

Figure 7.3: Location within Dissertation path. 



\section{Chapter 8}

\section{One-on-One Interviews}

Over the several experiments discussed in the previous chapters, groups of people were either surveyed or interviewed through focus groups about music and the various prototypes and approaches that had been developed or collected for this project to visualize music and lyrics. Interviews are often used to help interpret data collected in surveys and other group methods (American Statistical Association [ASA], 1997). Volunteers from among those who had completed the survey discussed in the previous chapter were asked to participate in one-on-one interviews.

Portions of this chapter appear in (Fourney, 2012).

\subsection{Goals}

The goal of the study presented in this chapter is to answer the following questions:

1. How do viewers respond to specific properties (e.g., colour, speed) of particular visualizations?

2. What tools or activities do HH people currently use to access music?

Note that Goal 1 is not looking at whether specific colours mean specific things, but rather whether any colour says anything or means anything to the viewer.

\subsection{Instruments}

Appendix E shows the forms used and questions asked of all participants during the interviews as well as full transcripts. The remainder of this section will show only the directed questions of the interviews and the questionnaires used to collect additional data.

\section{Interview Questions}

1. Please tell me a little about yourself. Were you born deaf/hard of hearing or did you experience a hearing loss later in your life? 
2. If you experienced a hearing loss, how old were you?

(a) What memories do you have of music prior to your hearing loss?

3. Is there a difference in your experience of music today compared to the past? Do the same recordings still sound the same? What, if anything is missing today?

4. Do you think your music preferences are shaped by what you can or cannot hear? How does your hearing influence your choices of music you want to listen to?

5. When you listen to music, what strategies or solutions do you use to help you enjoy, understand, etc. the experience?

(a) How well does strategy X work for you?

(b) Why do you use it?

(c) Does it work all of the time, some of the time?

6. (optional) Do you wear hearing aids while listening to music? How well does this work for you?

7. (optional) When you want to listen to music, what do you do (e.g., alone in a quiet room, concerts, etc.)?

8. How do you listen to music (e.g., Radio, Music player, Home stereo, Music videos, etc.)?

(a) How well does strategy X work for you?

(b) Why do you use it?

(c) Does it work all of the time, some of the time?

9. What specific frustrations have you experienced when trying to listen to music?

(a) What do you think would improve your experience with music?

10. If you wanted to use a computer to help you to better understand music, what would be the important pieces of information you would really want to know? (e.g., Song title, Artist name, Lyrics, etc.)

Now I am going to show you some short videos of music visualizations that have been developed at Ryerson and elsewhere. I will not play the sound. As you watch it please keep in mind how you would interpret this.

A Does this video say anything to you?

(a) What worked for you?

(b) What did not work for you? 
B What did you think of the colours? Do the colours have any meaning for you?

(a) YES: what did they mean to you?

(b) NO: did you want the colours to have any meaning for you?

C What did you think of the speed of the visualization?

(a) Was it too fast / slow?

(b) What do you think should happen to the visualization if the song is fast / slow?

Questions $A, B$, and $C$ are repeated for the remaining two visualizations.

Figure 8.1: Interview questions.

Questions B and C focus on colour and speed to spark a broader discussion. Sometimes to get a person to spontaneously talk about problems with a product requires some prompting. Since colour and speed are items that most everyone has an immediate and intuitive feel for and can probably respond easily to, these topics are good "ice-breakers" for an interview. Thus, Questions B and C were intended to prompt the participant to discuss these items and highlight other issues that they see.

This study used the Post-study Questionnaire already described in Chapter 6 and reproduced in Figure 8.2 on the following page for reference.

\subsection{Method}

During the interviews, participants were asked for more detailed information about their hearing loss, their experiences with music, and the strategies they use to access music. In addition, participants were presented with three different visualizations of the same song (participants were not told it was the same song) in randomized order with the audio muted (i.e., sound off) and asked, using a combination of closed survey tools and open interview questions, for their response.

The song used was a MIDI version of the third movement of Johann Sebastian Bach's 4th Brandenburg Concerto (BWV 1049). As the presto movement, this five minutes long piece has a variety of quickly played notes and tends to fully exercise the visualizations used in this study.

The three visualizations used were: Part Motion (Balls), Bars, and Radar. The Part Motion and Radar visualizations were used because they seemed to have success in earlier studies (e.g., the study described in Chapter 6 found that Part Motion gave the best sense of what was happening). The Bars visualization was added to balance the study and explore a new model of presentation.

The Part Motion visualization, as shown in Figure 8.3(a), was described in Chapter 2.

The Bars visualization, as shown in Figure 8.3(b), was based on the Classic Spectrum Analyser Plugin for Winamp (Lynch, 2007, 2009). In this visualization, notes are presented in a bar graph format. Each of the 32 bars rise and fall according to the note's onset, intensity (the louder the note, the higher the bar), and length (the longer the note, the longer the bar continues to be seen). Like 
Music Visualization Post-Study Questionnaire

The purpose of this questionnaire is to gather information about you and your opinions about the visual representations that you just saw. There five questions and it should take you about ten minutes to complete this questionnaire. Thank you in advance for your time and assistance.

1. Which style did you enjoy most?

2. Which style gave you the best sense of what was happening in the music?

3. Which style gave you the best sense of:

\begin{tabular}{|l|c|c|c|}
\hline & Moving Circles & Starscape & \\
\hline $\begin{array}{l}\text { Drums/ } \\
\text { Percussion }\end{array}$ & Radar \\
\hline $\begin{array}{l}\text { Instruments } \\
\text { playing }\end{array}$ & & & \\
\hline Melody & & & \\
\hline Rhythm & & & \\
\hline
\end{tabular}

4. What was most enjoyable about your experience with music visualization?

5. What was least enjoyable about your experience with music visualization?

Figure 8.2: Focus Group Post-study Questionnaire. 


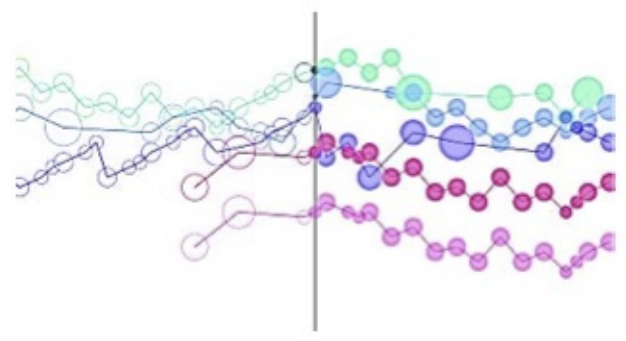

(a)

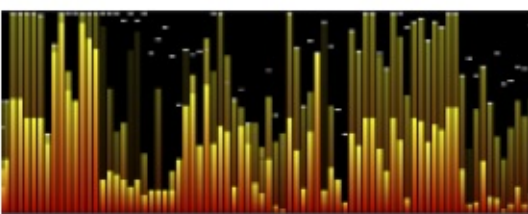

(b)

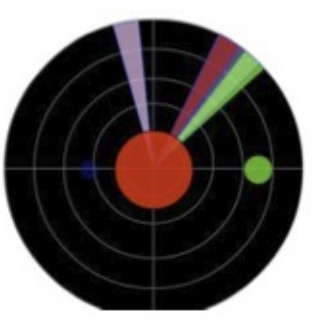

(c)

Figure 8.3: The music visualizations: (a) Part Motion, (b) Bars, and (c) Radar.

the Radar visualization, the longer the note is held, the longer the bar is on screen. While the Classic Spectrum Analyzer uses a fast Fourier transform (FFT) calculation, there is an emphasis function applied to this data to make the result more visually interesting. The result is that this visualization tends to be biased for treble sounds. The plug-in supports several display modes, the "Fire" display was used for this study.

The Radar visualization, as shown in Figure 8.3(c), was described in Chapter 6.

Each interview was conducted face-to-face in a quiet room for approximately 30 minutes. All interviews were video-recorded and later transcribed.

\subsubsection{Grounded Theory}

The study described in this chapter draws on Grounded Theory (GT) methodology as described in Chapter 3. In particular, the interviews conducted were transcribed and thematically coded for further analysis.

\subsubsection{Participants}

The one-on-one interviews involved eight participants ( 6 female, 2 male) aged 18 - $65+$ who all identified themselves as $\mathrm{HH}$ (in this case, this is a cultural label, audiologically some participants were deaf persons who did not sign, and some were CI users). Three participants were born HH. Five participants were born hearing and acquired their hearing loss later in life.

\subsection{Results}

All raw data is presented in Appendix E.

In terms of their musical experiences, several participants indicated a reliance on past memory of music to help them enjoy and understand music. Two could no longer hear music at all and were interested in this research as a new way to experience music. As with the survey described in Chapter 7, 


\begin{tabular}{|c|c|c|c|c|c|c|c|c|c|}
\hline \multirow[b]{3}{*}{ Best sense of percussion / drums } & \multicolumn{8}{|c|}{ Visualization } & \multirow{3}{*}{$\begin{array}{r}\text { Total } \\
\quad 9\end{array}$} \\
\hline & \multicolumn{2}{|c|}{ Part Motion } & \multicolumn{2}{|r|}{ Bars } & \multicolumn{2}{|c|}{ Radar } & \multicolumn{2}{|c|}{ None } & \\
\hline & 3 & $33.33 \%$ & 1 & $11.11 \%$ & 3 & $33.33 \%$ & 2 & $22.22 \%$ & \\
\hline Best sense of Instruments playing & 3 & $33.33 \%$ & 3 & $33.33 \%$ & 2 & $22.22 \%$ & 1 & $11.11 \%$ & 9 \\
\hline Best sense of Melody & 5 & $62.50 \%$ & 0 & $0.00 \%$ & 2 & $25.00 \%$ & 1 & $12.50 \%$ & 8 \\
\hline Best sense of Rhythm & 1 & $12.50 \%$ & 4 & $50.00 \%$ & 2 & $25.00 \%$ & 1 & $12.50 \%$ & 8 \\
\hline $\begin{array}{l}\text { Best sense of what was } \\
\text { happening in the music }\end{array}$ & 4 & $57.14 \%$ & 1 & $14.29 \%$ & 2 & $28.57 \%$ & 0 & $0.00 \%$ & 7 \\
\hline Enjoyed most & 4 & $44.44 \%$ & 3 & $33.33 \%$ & 2 & $22.22 \%$ & 0 & $0.00 \%$ & 9 \\
\hline Total & 20 & & 12 & & 13 & & 5 & & 50 \\
\hline
\end{tabular}

Note. Some participants made more than one choice.

Table 8.1: Participant responses to music information.

all but one participant indicated that they really want access to lyrics.

When asked what they felt was missing in their experience of music, 6 reported missing the lyrics, 2 reported not hearing (or being able to identify) the entire song, 2 could not always detect the beat / rhythm, 2 reported missing the lower (bass) pitch ranges, and 1 reported missing the higher pitch ranges. As one participant reported, "Music is now just noise."

The interviews had two major areas of discussion: a) the participants' experiences with music, both past and present; and b) the participants' responses to the visualizations. The remainder of this section will first discuss specific results that only pertain to the participants' responses to the visualizations. This section will then discuss the participants' comments and descriptions regarding the solutions and strategies they have tried or currently use when attempting to listen to music.

The codes used for the data analysis of the interview transcripts are listed in Appendix E.

\subsubsection{Goal 1 - Visualization Responses}

\section{Part Motion (Balls) Visualization}

As shown in Table 8.1, participants responded well to the Part Motion visualization indicating that it was the best choice for understanding what instruments were playing and the melody. Participants really enjoyed this visualization and indicated it was the one that gave them the best sense of the music.

One participant found that "The colour wasn't vibrant enough" and that there was not enough colour in this visualization, while another said that, "the way it works it doesn't need to be colourized."

When first presented with this visualization, all but one participant was not quite certain how to read it. For example, "The circles I mean at first they didn't really mean anything to me." As they continued to watch it, nearly all of them reported seeing music in the visualization (e.g., "the colours ... may represent the different tones of the music"). The participants tended to recognize that the colours represented the different instruments.

All of the participants reported that the speed of the visualization was "good", neither too fast nor too slow. 


\section{Bars Visualization}

Table 8.1 further shows that the Bars visualization was not perceived as highly as the Part Motion. Participants indicated that this visualization provided the best sense of the rhythm.

Participants liked the colours used. They were described as "hot" and "dancing together." One participant interpreted the redness of the visualization as showing, "how loud and deep the sound is." However, another participant reported that the blending of yellows and reds looked "monotone" and that the colours provided "no meaning to me whatsoever."

Some participants quickly saw the music in the visualization and openly mentioned it without prompting. For example, one said that she thought she saw the song's bridge. Six of the participants reported seeing music in this visualization. Three thought they were seeing a "rock" song.

In contrast to the participants' experience of the speed of the Part Motion visualization as "good", all of the participants described the speed of the Bars visualization as "fast". Several participants were bothered by the busyness of the visualization:

- "I find too many actions going on at once."

- "it was very fast, like extremely fast."

- "It's just a mixture. It's like a jumble."

\section{Radar Visualization}

Participants responded most poorly to the Radar visualization. It had several colour palate issues, especially for the older participants.

Participants did not ascribe any meaning to the colours or could not determine what the colours were intended to represent. Older participants mentioned that the colours were not bright enough. One participant did recognize that the central red circle represented the main beat of the song. Another found this same feature "very annoying."

Six participants reported seeing music in the visualization, especially a beat. As one participant said, "I swear there is a drum playing in there." Similar to the Part Motion visualization, participants described the speed of the Radar visualization as "good" or "moderate", neither too fast nor too slow.

While some participants described this visualization as "confusing and annoying", there were a few that recognized that the up and down movement of the blades showed different tones or pitches.

\subsubsection{Goal 2 - Solutions Used to Access Music}

As with the survey presented in the previous chapter, participants discussed the solutions they use when trying to listen to music. A thematic analysis showed their comments fell along several themes.

\section{Environment}

Participants discussed how they manipulate their environments, including themselves, to support music listening. Moving to face the source of sound and/or moving closer to the source of sound was a 
common option.

Several participants described how they manipulated the settings of their stereos for music playing. Two mentioned manipulating the equalizer, balance, and other settings to get a better (for them) sound. Four mentioned turning up the volume.

Two mentioned the need for a quiet space. For example, "If I am in a quiet room and I can get away from any background noises and stuff, that makes a big difference." One common statement was, "If I am alone I will turn the volume up." Participants described needing to be alone to enjoy music and frequently mentioned that the way the HH person listens to music annoys hearing family members (e.g., its too loud).

Live concert environments were also mentioned. Two participants stated that they get more enjoyment from music in live performance venues than when listening to recordings, "if I go to a live concert, if it is a small venue, I feel like I have a satisfactory experience listening to music." This may be because concert environments provide more than a purely auditory experience, "I also just love the energy of the people, the performers themselves."

\section{Technology}

Participants frequently discussed using different technology to support listening to music. This included discussions of their HAs, CIs, and other ALDs. For example, one participant reported directly connecting their music player to their CI. Four participants reported wearing large headphones to hear music. Two of these people reported wearing the headphones over their ALDs.

Several participants reported issues with their ALDs:

- One participant said that they had a better experience listening to music with their old HA ("I [could] hear the clarity of the words and the music combined very well") than with their current CI. This person found that they cannot hear music at all with their CI ("But when I was fitted with the $\mathrm{Cl}$, I couldn't do that anymore. And I mourn the loss of music").

- Several participants reported that their HA was not meeting their full needs, "[with HAs] I am doing very well, functioning very well with conversation, but music is just gone." One participant noted that when wearing HAs, she heard too many sounds that were not the music, "I find that with the hearing aids on I am hearing things that are actually distracting me from the music."

A few participants reported removing their ALDs to listen to music. One reported that he would remove his HA to wear earbuds when listening to music. This person reported that the earbuds gave him a better experience.

\section{Screens}

Several participants reported the use of a screen when listening to music. For example, one participant reported using online videos to watch performers sing songs, "YouTube allows me to visualize and see her sing." By watching the person sing the song, this HH viewer got more information about the words being used because they could lip-read the performer. 
Four participants reported using captions. However, there were several comments that this worked best where the captions were in coordination / synchronization with the song. Captioning lag was noted as an issue when understanding a song.

\section{Familiarity}

As mentioned in the previous chapter, $\mathrm{HH}$ people report making an effort to memorize the lyrics of music they enjoy. Interview participants reported taking extra efforts to learn a song and become fully familiar with it.

Five participants reported a preference for listening to familiar music. Regarding "new" songs, participants either reported not bothering with them because they did not know the song or listening to it multiple times to hopefully eventually understand and work out the melody and/or words.

Listening to lyrics and following lyrics was a frequent area of discussion. According to one participant, "When it comes to music with lyrics, I will often download an entire album, look up all the lyrics, learn them, and this allows me to actually hear the song better when I am listening to them." Four participants spoke of getting the lyrics either through album information (e.g., album notes, J-card, CD booklet, etc.) or online. By memorizing or following the lyrics, the HH listener can anticipate what they will hear as they listen to the song.

Although one participant stated that she plays music in the background while doing other work, another participant noted that there was a specific mental effort for them when listening to music, "Background music doesn't really do it for me, so I have to be paying attention to the music to enjoy it." This is not surprising because the variability of hearing across the HH population means that different people will have different experiences and different uses of music. Hearing people report similar uses of music (e.g., Beentjes, Koolstra, \& van der Voort, 1996).

\section{Behaviour of Others}

Some participants noted that their understanding of the song being played or information about the song being played is based on the behaviour of the people around them. Two participants noted that family members or friends will often tell them the name of a song that is being played. Another participant stated that, when at a bar, they know what is playing by lipreading strangers as they sing / mouth the lyrics.

\subsection{Discussion}

\subsubsection{Goal 1 - How do viewers respond to specific visualization proper- ties?}

As mentioned in Chapter 1, of the Principles of Universal Design, Principle Three, "Simple and intuitive use", implies that the user interface of any visualization solution should be obvious. In the case of music visualization, this principle would suggest that the visualization itself should be simple and 
intuitive, even without sound. Similarly, Principle Four, "Perceptible Information", requires the design to communicate necessary information effectively to the user. Determining what types of music information would be considered useful and whether specific visualization styles effectively communicated such information in a intuitive manner would support the Principles of Universal Design.

Participants stated that each visualization suggested a different genre. For some participants:

- The Part Motion visualization was described as a "country" song.

- The Bars visualization was described as a "very fast and very loud" song.

- The Radar visualization was described as a "happy, bouncy song."

Note that these visualizations all represented the same song. This observation suggests several things. First, that participants actively tried to apply meaning to what they were seeing. Second, that when designing music visualizations, different visualizations could be used with different music genres (rather than seeking a specific design for all music types) as a means of assisting viewers and providing more information.

Helmholtz theorized that perception is an active process involving memory and other internal processes (Helmholtz, 1867/1925). Similarly, (Gregory, 1998) theorized perceptions as predictive hypotheses, based on knowledge stored from the past. In trying to understand the images, the participants tended to compare what they were watching to their past experiences, prior knowledge, or to other images. For example, several described the Bars visualization as looking like a "nighttime cityscape" or "wall of fire." The Part Motion visualization was described as "bicycles" and "people dancing." The Radar visualization was described as an old television "test pattern" and "a clown."

Having a psychological reality of music assisted participants when interpreting the visualizations. When specifically prompted with a question like, "did you see music in that visualization?", the responses were mixed. Participants with more music experience and music training appeared to respond better to the visualizations than those who did not. This suggests that for $\mathrm{D} / \mathrm{HH}$ adults, any form of music information presentation (including visualization) may need frequent, repeated exposure and training, especially for those with little musical experience. This should be expected since no one develops an understanding of music without a lifetime of exposure. Hearing children are repeatedly exposed to the music of their family's culture(s) from a very young age (in the womb). Starting with very simple musical tunes and moving to more complex songs throughout their childhood, hearing people carry that musical knowledge into adulthood. Hearing adults develop their understanding of music over a lifetime.

\subsubsection{Goal 2 - What tools or activities do HH people use to access music?}

Participants were asked if they make specific choices about the music they listen to as a result of their hearing loss. All of them, including those who had been hearing for some period in their lives, responded that they had never considered the question. Of the six who provided an answer to this question, only one said "No." Those who had lost their hearing all reported listening to music that they 
knew from before their hearing loss and relying on this past memory of specific music pieces to help them fill the gaps they cannot hear anymore.

The responses of the on the topic of the technologies participants use to listen to music support literature that indicates issues with ALDs (e.g., Chasin, 2003; He \& Limb, 2014). No one ALD supports the needs of $\mathrm{HH}$ music consumers. There is a substantive technology gap remaining. The inadequacies of the devices impacts understanding both the music and the lyrics. From participants' descriptions of the work they do to become familiar with music, there is evidence that $\mathrm{HH}$ music consumers take exhausting extra efforts to enjoy the music they can understand.

\subsubsection{Limitations of the Design}

The analysis of the data suggests at least four limitations of this study, two of which are related to questionnaire design.

The first limitation of this design is that while asking how to represent music information, the Post-study Questionnaire did not also ask for some ranking of the importance of each type of information. As a result this must be extrapolated from the responses and comments. For example, it can be suggested that the information is important when a preference is specified and it can be suggested that there is no importance when a participant ignores the item and leaves it blank. However, any conclusion that is more specific cannot be established based on this data.

Second, the wording of question 2 and question 3 in the Post-study Questionnaire (i.e., "Which style gave you the best sense of what was happening in the music?" and "Which style gave you the best sense of percussion / instruments playing / melody / rhythm?"), raises some issues around how the participant might interpret the phrase "best sense". While the design of the questionnaire was intended to leave this phrase open to interpretation, the fact that a participant may have no psychological reality of certain music information might mean they are unable to assess what visualization gave them the "best sense" of something because they would not know how to understand that concept.

Third, the small sample size with an unequal number of men and women, while appropriate for a series of interviews, limits the statistical power of quantitative results. The chi-square test for independence is sensitive to sample size as well as small expected frequencies in one or more of the cells in the table. In most cases, researchers limit the use of chi-square to tables that either a) have no expected frequencies values below 5 or $b$ ) have no more than 20 percent of the expected frequencies values below 5 (Frankfort-Nachmias \& Leon-Guerrero, 2011). However, in the case of this study, the interest lay more in the qualitative results.

This study was intended as a usability study. The goal was not validation of any specific designs but to gain further insight. Nielsen and Landauer suggest that collecting data from a single test user can elicit a third of all there is to know about the usability of a specific design. Each successive participant will repeat some of the insights of previous participants. As this implies, the best results come from testing small numbers of users (e.g., $N=5$, 8, etc.) (Nielsen \& Landauer, 1993). The caveat is that these participants must be comparable users. When groups of users with sufficiently different needs and behaviours are involved (i.e., different populations), there is a need to test each group sep- 
arately since each group may raise a different set of usability issues. Thus, the small sample size is appropriate given the purpose of the study.

Finally, the setting for this set of interviews was not entirely ideal. The interviews were conducted in the midst of a national conference for HH people. The conference theme, "Good Vibrations" was oriented to the experience of music. It was advertised as including performances by three different musical groups, including a well-known Canadian country and folk singer who, among various honours, has earned three Juno Awards. Given that the conference theme and events specifically focused on music, it is unlikely that the interview participants would not somehow report liking music. In other words, it is unlikely, given the nature of the conference, that a $\mathrm{HH}$ community member who might not like music would have attended this conference (and thus be available to volunteer to participate in an interview). This is a weakness of the interpretability of the data because it does not allow generalization of the results over all possible HH consumers, only those who have any interest in music.

\subsection{Conclusions From This Study}

Music is not fully accessible to $\mathrm{D} / \mathrm{HH}$ audiences. The impact is significant: The music experience is frustrating because the lyrics are difficult to understand, the emotional content is not fully communicated, and the full entertainment experience is lacking.

Like the survey of HH people discussed in the previous chapter, these interviews show that there is a significant desire for access to lyrics. This is clearly exemplified in the extra efforts taken to understand and / or memorize the lyrics. Given the effort that HH music consumers are willing to take to get access to some part of a piece of music, these interviews raise questions like: Why do we want to experience music? What benefits does music provide?

As shown in Figure 8.4, the work in this chapter represents the full DRM cycle because it introduced an intervention, the exposure of the participants to the videos. In the larger scope of the Disser-

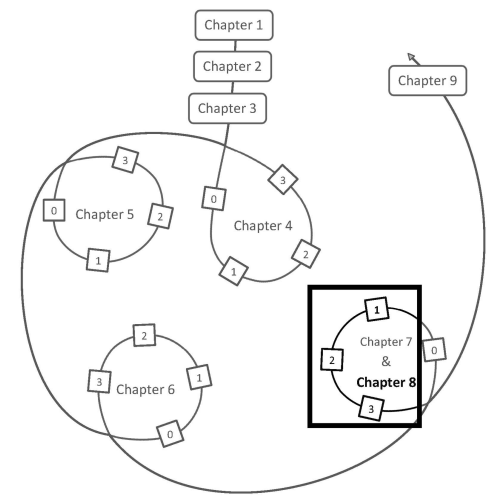

Figure 8.4: Location within Dissertation path. 
tation, the work in this chapter and Chapter 7 revisited the DRM's Stage 1 (Descriptive Study I) and contributed to the Stage 2 (Prescriptive Study) stage. The next chapter will conclude this Dissertation. 



\section{Chapter 9}

\section{Discussion and Conclusion}

This chapter reviews the problem investigated in this Dissertation and synthesizes the results of my work providing an overview of conclusions drawn from the reported work. The chapter describes future directions of this research and potential future applications. Finally, this chapter ends with the conclusion of this Dissertation and a statement of its contribution.

\subsection{Discussion}

At a high level, the main research questions of this Dissertation are:

\section{What are the fundamental properties of music that a visualization must present and that produces an entertaining and meaningful experience for $\mathrm{D} / \mathrm{HH}$ people?}

This question required an exploration of the invisible components of music and how these elements of music influence the entertainment of its listeners. As was clear from the literature reviewed in Chapter 2, music is a highly complex information space involving multiple atomic components.

This raised several questions about whether the focus of visualizations should be on making visible specific atomic music primitives (e.g., tones) or higher level concepts (e.g., melody, key), both, or something else entirely. As was shown in the focus group described in Chapter 5, visualizations of tonality, harmony, pitch change, timing, instrumentation present, and beat / rhythm information were too complex and not appreciated. Participants strongly preferred aesthetic and entertainment elements over information elements. In contrast, as the Chapter 8 interviews showed, some knowledge of how to interpret the visualization is needed or the information is not able to be fully appreciated.

While this question remains partially answered, especially for deaf respondents, the results of the studies in Chapter 5 and Chapter 8 suggest that the Part Motion display seems to provide some understanding that can be associated with the idea of "music". For HH respondents, the results of Chapter 7 and Chapter 8 shows lyrics represent a key piece of music information they require of a visualization. The issue of lyrics will be further discussed below. 


\section{What are the emotional experiences and interpretations of users from visualizations?}

Logically, just as aural music (the invisible) is interpretable by the listener, a visualization of music (the visible) should be interpretable by the viewer. Ideally, if listeners can construct an emotional experience from what they hear, so should viewers.

From the literature reviewed in Chapter 2, it appears that music communicates complex emotional signals and that, at least in Western cultures, it has specific conventions designed to manipulate emotions. What is not clear in the literature is whether this can be translated into other modalities.

The results discussed in Chapter 6 show that, in the case of the visualizations used, the emotional component of the music is not fully and reliably communicated. For example, participants interpreted songs that were angry or fearful as happy.

The results discussed in Chapter 8 suggest that different visualizations might be better suited to different genres. This would suggest that different visualizations might also be better suited to convey different moods. However, the results in Chapter 6, where respondents could indicate the intended emotion of a song on the PAD scale but not necessarily interpret the emotion of the song using the emotion scale, suggests that, for the set of visualizations explored, emotions can be approximately conveyed but may not be obvious. Based on the responses of the participants, for the set of visualizations explored, it is not likely that different visualizations will convey different moods.

Thus this question remains partially answered.

\section{Is it possible to create a $\mathrm{D} / \mathrm{HH}$ music experience similar to hearing audiences?}

The literature reviewed in Chapter 2 suggests that music is a highly complex container of shared information and knowledge presented through sound. In addition to the social experience, there is a personal experience. The song on its own is not the full source of the entertainment experience. The memories associated with past exposure to the song and the emotions those memories evoke influences the experience of the song. For hearing people, music is not just a cultural element, it impacts their lives. What is not clear in the literature is whether music impacts $\mathrm{D} / \mathrm{HH}$ communities in similar ways.

A further review of the literature in Chapter 4 showed that signing Deaf communities have indigenous forms of lyrical music. While these may not be melodic in the Western expectation, they still conform to the idea of music as metrical lyrics accompanied by instrumentation.

Chapter 4 showed anecdotally that members of the Deaf community do express an appreciation for music. They engage in this for various reasons including understanding their hearing friend's appreciation for music. Chapter 4 also suggests, on an anecdotal level, that there is evidence of members of the Deaf community who engage in the performance of Western music.

As noted in Chapter 6, for various reasons, no baseline PAD measures of the selected songs was developed to compare a hearing person's emotional experience with that of the $\mathrm{D} / \mathrm{HH}$ participants. Thus, it is not possible to determine if $\mathrm{D} / \mathrm{HH}$ participants experienced a similar emotional response to that of hearing people to the same song. While the results reported in Chapter 6 clearly showed that participants trended towards an accurate indication of the intended emotion of the song, we cannot determine if the experience is 'similar' to that of a hearing listener. 


\begin{tabular}{rrrrrrrr}
\hline & \multicolumn{3}{c}{ Frequency of listening to music } & Total \\
\hline Identity & $\begin{array}{c}\text { All the time } \\
\text { (daily) }\end{array}$ & $\begin{array}{c}\text { Frequently } \\
\text { (weekly) }\end{array}$ & Sometimes & Rarely & Never & $\begin{array}{c}\text { No } \\
\text { response }\end{array}$ \\
\cline { 2 - 6 } HH & 19 & 11 & 8 & 6 & 2 & 0 & 46 \\
& $41.30 \%$ & $23.91 \%$ & $17.39 \%$ & $13.04 \%$ & $4.35 \%$ & $0.00 \%$ & $100.00 \%$ \\
Deaf & 6 & 9 & 9 & 7 & 6 & 1 & 38 \\
& $15.79 \%$ & $23.68 \%$ & $23.68 \%$ & $18.42 \%$ & $15.79 \%$ & $2.63 \%$ & $100.00 \%$ \\
Deafened & 0 & 1 & 1 & 0 & 0 & 0 & 2 \\
& $0.00 \%$ & $50.00 \%$ & $50.00 \%$ & $0.00 \%$ & $0.00 \%$ & $0.00 \%$ & $100.00 \%$ \\
CI & 2 & 2 & 4 & 0 & 0 & 0 & 8 \\
Total & $25.00 \%$ & $25.00 \%$ & $50.00 \%$ & $0.00 \%$ & $0.00 \%$ & $0.00 \%$ & $100.00 \%$ \\
& 27 & 23 & 22 & 13 & 8 & 1 & 94 \\
& $28.72 \%$ & $24.47 \%$ & $23.40 \%$ & $13.83 \%$ & $8.51 \%$ & $1.06 \%$ & $100.00 \%$ \\
\hline
\end{tabular}

Table 9.1: How often do you listen to music? (by hearing identity)

Over the span of the experiments reported in Chapters 4 through 8, various common questions were asked of all participants. Table 9.1 shows the response to the frequency in which respondents listened to music across all experiments.

As shown in Table 9.1, over half of all respondents (50 or 53.2\%) indicated that they listen to music frequently, if not daily. Of the 37 participants who identified themselves as deaf, 15 (40.5\%) indicated that they listen to music frequently, if not daily. This suggests that whether the $\mathrm{D} / \mathrm{HH}$ participants of this project have a music experience similar to hearing audiences or not, they are active consumers of music.

Table 9.2 on the next page shows the degree in which respondents reported liking music across all experiments.

As shown in Table 9.2, over half of all respondents (76 or $82.6 \%$ ) indicated that they "like" or "really like" listening to music. Of the 37 participants who identified themselves as deaf, $26(70.3 \%)$ indicated that they "like" or "really like" listening to music. Again, this suggests that whether the D/HH participants of this project have a music experience similar to hearing audiences or not, they are active consumers of music.

Table 9.3 on page 163 reviews the data in Table 9.2 in terms of respondents who really enjoy or like music. Given the number of cells with counts less than one, a $2 \times 2$ contingency table was used to perform a chi-square test for independence to determine if there is any relationship between the participant's hearing identity (deaf or HH) and their report of how much they liked music.

$H_{0}$ : Deaf or HH identity and the Participant's report of how much they liked music are independent.

$H_{a}$ : Deaf or HH identity and the Participant's report of how much they liked music are not independent.

Fisher's exact test, a statistical test used to determine if there are nonrandom associations between two categorical variables, was also performed to test these hypotheses. 


\begin{tabular}{|c|c|c|c|c|c|c|c|}
\hline \multirow[b]{2}{*}{ Identity } & \multicolumn{6}{|c|}{ Statement that describes you best } & \multirow[t]{2}{*}{ Total } \\
\hline & $\begin{array}{l}\text { I really like } \\
\text { listening } \\
\text { to music. }\end{array}$ & $\begin{array}{l}\text { I like } \\
\text { listening } \\
\text { to music. }\end{array}$ & $\begin{array}{l}\text { I do not } \\
\text { like or } \\
\text { dislike } \\
\text { music. }\end{array}$ & $\begin{array}{l}\text { I do not } \\
\text { like } \\
\text { listening } \\
\text { to music. }\end{array}$ & $\begin{array}{l}\text { I do not } \\
\text { see } \\
\text { the point } \\
\text { of music. }\end{array}$ & $\begin{array}{l}\text { No } \\
\text { response. }\end{array}$ & \\
\hline \multirow[t]{2}{*}{$\mathrm{HH}$} & 22 & 18 & 2 & 2 & 1 & 1 & 46 \\
\hline & $47.83 \%$ & $39.13 \%$ & $4.35 \%$ & $4.35 \%$ & $2.17 \%$ & $2.17 \%$ & $100.00 \%$ \\
\hline \multirow[t]{2}{*}{ Deaf } & 13 & 13 & 8 & 1 & 2 & 1 & 38 \\
\hline & $34.21 \%$ & $34.21 \%$ & $21.05 \%$ & $2.63 \%$ & $5.26 \%$ & $2.63 \%$ & $100.00 \%$ \\
\hline \multirow[t]{2}{*}{ Deafened } & 2 & 0 & 0 & 0 & 0 & 0 & 2 \\
\hline & $100.00 \%$ & $0.00 \%$ & $0.00 \%$ & $0.00 \%$ & $0.00 \%$ & $0.00 \%$ & $100.00 \%$ \\
\hline \multirow[t]{2}{*}{$\mathrm{CI}$} & 5 & 3 & 0 & 0 & 0 & 0 & 8 \\
\hline & $62.50 \%$ & $37.50 \%$ & $0.00 \%$ & $0.00 \%$ & $0.00 \%$ & $0.00 \%$ & $100.00 \%$ \\
\hline \multirow[t]{2}{*}{ Total } & 42 & 34 & 10 & 3 & 3 & 2 & 94 \\
\hline & $44.68 \%$ & $36.17 \%$ & $10.64 \%$ & $3.19 \%$ & $3.19 \%$ & $2.13 \%$ & $100.00 \%$ \\
\hline
\end{tabular}

Table 9.2: What statement describes you best? (by hearing identity)

There is a statistically significant relationship between whether one likes music and their identity, $\mathcal{X}^{2}(1, \mathrm{~N}=82)=4.482, p=0.034$, two-tailed Fisher's exact test $p=0.049$. Since the $p$-values are less than the significance level (0.05), we cannot accept the null hypothesis. Thus, participants were more likely to report that they liked music if they were $\mathrm{HH}$.

While these results provide evidence that among the $\mathrm{D} / \mathrm{HH}$ participants of this project there appears to be great interest in music, care must be taken when generalizing these results to the larger $\mathrm{D} / \mathrm{HH}$ community. As noted in previous chapters, with the exception of the study presented in Chapter 4, it is likely that the participants of these studies are self-selected for high interest in music. In other words, it is unlikely, given the nature of the recruiting materials used, that a D/HH community member who might not like music would have participated in these studies. The study in Chapter 4 is an exception because participants were not informed of the musical nature of the stimuli until after they became involved in the experiment. This is a weakness of the interpretability of the data because it does not allow generalization of the results over all possible $\mathrm{D} / \mathrm{HH}$ consumers, only those who have any interest in music.

The above results would certainly suggest that D/HH people have some sort of music experience. Is it a "similar" experience to that of hearing audiences? Should D/HH people even expect an experience "similar to hearing audiences"? If so, what is a "similar" experience?

Of the principles of universal design, Principle One "Equitable use", is particularly relevant here. It requires the design of a system or product to be useful and marketable to people with diverse abilities, be appealing to all users, and provide the same or equivalent means of use for all users. This might provide us with some direction toward defining a "similar" experience because Principle One implies that the music itself, not just the visualization, should be equitable across users regardless of ability.

However, care must be taken that the focus remains, not on the user or the product (i.e., music), but, as suggested in Chapter 1, on the interaction between them. We cannot fix the user or the prod- 


\begin{tabular}{|c|c|c|c|c|c|}
\hline & & & \multicolumn{2}{|c|}{ Likes music } & Total \\
\hline & & & Yes & No & \\
\hline \multirow[t]{8}{*}{ Identity } & $\mathrm{HH}$ & Count & 40 & 5 & 45 \\
\hline & & $\%$ within Identity & $88.89 \%$ & $11.11 \%$ & $100.00 \%$ \\
\hline & Deaf & Count & 26 & 11 & 37 \\
\hline & & $\%$ within Identity & $70.27 \%$ & $29.73 \%$ & $100.00 \%$ \\
\hline & Deafened & Count & 2 & 0 & 2 \\
\hline & & $\%$ within Identity & $100.00 \%$ & $00.00 \%$ & $100.00 \%$ \\
\hline & CI & Count & 8 & 0 & 8 \\
\hline & & $\%$ within Identity & $100.00 \%$ & $00.00 \%$ & $100.00 \%$ \\
\hline \multirow[t]{2}{*}{ Total } & & Count & 76 & 16 & 92 \\
\hline & & $\%$ within Identity & $82.61 \%$ & $17.39 \%$ & $100.00 \%$ \\
\hline
\end{tabular}

Table 9.3: Tends to like music? (by hearing identity)

uct, only the interaction. Thus, the interaction is the space of the "similar" experience, if it exists.

The results in Chapter 8, shows that existing ALDs are not able to assist all HH music consumers. CI users, in particular, report not hearing music at all, a result consistent with other literature (e.g., He \& Limb, 2014). HA users report issues with distortion, environmental noise, and so on, a result also consistent with the literature (e.g., Chasin, 2003). Thus while ALDs can provide an experience (possibly negative) of music, they cannot provide a "similar" experience to hearing audiences.

Having access to lyrics and, in particular, lyrics synchronized to the music is one area that would improve the interaction. The results of Chapter 7, where HH music consumers reported wanting access to the lyrics above all else, would suggest that this sole intervention may meet the needs of many $\mathrm{HH}$ music consumers enough to create a "similar" experience. The issue of lyrics will be further discussed below.

What is not clear is what other invisible aspects of music would provide that "similar" experience for those who need more of music to be visible than just the lyrics.

Thus, the question of whether it is possible to create a $\mathrm{D} / \mathrm{HH}$ music experience similar to hearing audiences cannot be fully answered in this Dissertation and needs more exploration. While this question remains only partially answered, at least one intervention exists, the provision of lyrics, that may sufficiently meet the needs of some members of the $\mathrm{D} / \mathrm{HH}$ communities to have a music experience similar to hearing audiences.

\subsubsection{The Issue of Lyrics}

The results of Chapter 7 show that there is a strong desire (30 of 95 responses or $31.6 \%$ ) among $\mathrm{HH}$ music consumers for access to written (visible) lyrics. When asked about access to any other invisible music information, lyrics is still most important. Lyrics have become widely available and can usually be downloaded from the Internet and stored within one's own music library. Applications such as SongGenie have been developed to find lyrics based on one's iTunes library information and will update a song's music metadata (i.e., identifying information of the song such as the title, artist, album, 
track number, etc. stored in the MP3 file itself) to include lyrics.

In addition to music players themselves, various mobile applications also exist for presenting lyrics. For example, SoundHound provides users with access to a search mechanism that can identify a song being played and then present the lyrics of that song in real time. Using this application to play a file from one's own music library enables the user to access the lyrics, in real time, from SoundHound's own lyrics library.

While this appears to solve the problem of getting lyrics, it actually does not. First, since most online lyrics libraries are crowd sourced, D/HH music consumers cannot completely trust the accuracy or completeness of the lyrics that are available. Second, how to present a meaningful presentation of lyrics, in particular for D/HH users, has not been sufficiently researched.

As discussed in Chapter 2, the captioning of dialogue has a long history of research and has several well known usability issues, the issues related to the captioning of music is not well understood. Music has properties that make it very different from dialogue, yet the few studies that have explored the problem tend to start from the perspective that the issues are the same.

These studies "put the cart before the horse" in that they assumed the usability issues involved with captioning dialogue are the same as those involved in the captioning of lyrics without first conducting the necessary research to determine if they are indeed the same.

Music typically breaks the rules of dialogue even when being used as dialogue. For example, in musical theatre, music is commonly used to support dialogue. A common method is to have multiple performers sing the same song as if engaged in a conversation. When captioned, such songs look like dialogue except that additional characters (e.g., eighth notes) are appended to indicate the presence of music/ singing.

Occasionally performers of musical theatre sing different lines of the same song at the same time. Captioning this, using current approaches, in a manner that is understandable to $\mathrm{D} / \mathrm{HH}$ audiences is extremely difficult (e.g., it requires reading two lines of text at the same time, understanding that they are distinct voices and, in violation of existing captioning conventions, understanding they are being sung simultaneously rather than consecutively). As discussed in Chapter 2, there are no recommended approaches for presenting such lyrics.

Worse, musical theatre composers will sometimes combine multiple songs into the same "conversation". This involves different performers singing different melodies, sometimes in different keys, and sometimes overtop of each other all at the same time. An example of this can be seen in the English adaptation of the musical Les Misérables (Schönberg \& Kretzmer, 1986) where the song "One Day More" at the end of Act I includes at least three overlapping songs: the main song being sung by Valjean, Marius, and others; an overlapping verse of "Master of the House" performed by the Thenardiers; and another overlapping verse with a different melody and metre from the main song being sung by Javert. Accurately captioning this, using current approaches is impossible. Given that it is text-based, captioning provides no ability to quickly denote the different changes in melody or metre without adding to the audience member's already strained reading skills. Even if the audience member is sufficiently $\mathrm{HH}$ to understand generally what is going on, the cognitive load is significant and much will be missed. 
While there are some conventions used to display lyrics for HH people using current closed captioning technology, there is no research to substantiate if they constitute best practice. Further, not all captioning providers follow the same conventions (Clark, 2004). A better music captioning technique is desired (Jordan et al., 2003). Research on whether synchronized presentations of the lyrics (e.g., as SoundHound provides) or static presentations of the lyrics (e.g., like iTunes provides) would be preferred by D/HH consumers does not exist.

These issues provide ample opportunities for future directions of the research developed in this Dissertation.

\subsection{Some Design Guidelines and Constraints}

While the DRM was used in this Dissertation to organize the reporting of the experiments and the presentation of the results rather than being used during the execution of the experiments, some design constraints / guidelines were learned in the process:

1. Visualizations of music are largely deficient without sound (Chapter 5). Visualizations need to include the audio presentation of sound and should be used only as sensory supplementation.

2. Visualizations should not be too visually busy (Chapter 5 ).

3. Visualizations should include aesthetic / entertaining elements (Chapter 5). While users want to be entertained, simple representations that communicate a sense of what is happening in the music might still prove useful, especially if combined with entertaining views.

4. Visualizations intended for $\mathrm{D} / \mathrm{HH}$ audiences should not require the viewer to move their eyes left and right to see obvious information. The "important stuff" needs to be in one place (Chapter 5$)$.

5. Visualizations of music need to clearly highlight the melody (Chapter 5).

6. Visualizations intended for $\mathrm{D} / \mathrm{HH}$ audiences should be designed to not have information elements that too easily distract the viewer from more "important" elements. For example, the MPM visualization presented in Chapter 5 used a large, centrally located, constantly in motion, information element to display percussion information that drew viewer's attention away from other information elements. Large distractors can make an informative visualization less useful.

7. Colours need to be carefully selected. When visualizations use white backgrounds, the information they present is very difficult to see. Darker backgrounds make coloured information elements appear sharper (Chapters 5 and 8 ).

8. Any proposed colour palette should be user tested with elderly people because they may not be able to easily see the visualization information if the palette is not sufficiently bright (Chapter 8$)$. 
9. Rather than seeking a specific design for all music types, different visualizations could be used with different music genres. This would provide viewers a further means of assistance in interpreting the information presented and providing more information (Chapter 8).

10. Expect that any form of music information presentation (including visualizations) may need frequent, repeated exposure and training to be understandable or usable. This may be especially true for those $\mathrm{D} / \mathrm{HH}$ users with little musical experience or a limited psychological reality of music (Chapter 8).

These guidelines are consistent with several Principles of Universal Design, for example:

- Principle One, "Equitable use" suggests that a music visualization should should also be appealing while still enabling users to listen to the music, if desired.

- Principle Two, "Flexibility in use", implies that any solution should be adaptable; designed with as many user options as necessary. For example, options such as colour choice, size on screen, or amount of information presented should be possible.

- Principle Four, "Perceptible Information", requires the design to effectively communicate necessary information to the user, regardless of ambient conditions or the user's sensory abilities and it encourages using different modalities (i.e., auditory, visual, tactile) to redundantly present essential information. It is this principle that guides the need for visual representations of auditory information, such as music, to support $\mathrm{D} / \mathrm{HH}$ people.

\subsection{Contribution}

The research presented in this Dissertation impacts several areas of scholarship:

- In the field of information presentation, the expressed preferences of d/Deaf and HH participants contribute to new innovative design guidelines for the visualization of auditory information:

- the need for visualizations to not be too visually busy,

- to focus the core information space in specific areas of the visualization so users do not have to move their eyes around too much, and

- to attend carefully to aesthetics and entertainment while still visually presenting auditory information.

- In the area of accessibility, the research brings new perspectives to our understanding of aging and the impact of hearing loss.

- interview participants spoke of loss of connection not just socially but also to their past (e.g., no longer hearing the songs they once loved). 
- Within usability, this research tells us that $\mathrm{D} / \mathrm{HH}$ music consumers exist and what they say their needs are. One novel finding of this Dissertation was the expressed need for lyrics. This finding reveals an ongoing need of $\mathrm{D} / \mathrm{HH}$ captioning consumers for improved captioning solutions.

- In the field of Deaf Studies this research adds to the understanding of music and its uses in the cultures of the Deaf and HH. Chapter 4 discussed the various ways these communities engage with their own music as well as the music of the larger hearing community.

- the focus groups and surveys showed that there is a strong interest in these communities to explore new ways to engage with music information.

ISO has joint committees with the IEC. Joint Technical Committee 1 (JTC 1) is responsible for the development, and maintenance of IT standards. Recently a working group of ISO/IEC JTC 1/SC35 accepted a proposal to begin development on an International Standard related to captioning to be numbered ISO/IEC 20071-23. This timely research adds to the existing knowledge base used to develop standards for captioning:

- Without this research, the need to address issues relating to the presentation of music information and the kinds of information on which to focus would have been unknown. The standard under development could have ignored this area of concern and focused more on dialog captioning instead because that area represents the bulk of current captioning research.

In the same way, this research will contribute not just to the development of ISO/IEC 20071-23, but might also advance the progress of other international Internet accessibility projects.

\subsection{Future Directions}

There are several possible future projects from the research presented in this Dissertation:

- Designing simple representations intended to communicate a sense of what is happening in the music combined with entertaining views. As seen, for example, in Chapter 5, participants found the MAM Part Motion view enjoyable but really liked the Magnetosphere visualization of iTunes. There is a need to find a way to combine both ideas.

- At times, participants found that they, "could not really connect" to the different visualizations. As one person pointed out, the "music was not conveyed well." This suggests that something was missing in the visual representation. Beyond the need to identify the properties of music which people find entertaining, there is a need to capture that part of music which people internalize.

- There is a need to explore combinations of different forms of music information representation to visualize specific music information. The findings in Chapter 4 suggest high individual difference. While many participants in that study showed a clear interest in lyrics, song information, instruments, and rhythm / beat, others showed an interest in notes or the speed of music. 
- While it appears that viewers of music visualizations are able to have some emotional experience of the visualization, this experience is not necessarily the same as that of the hearing listener of the music or even the same as that intended by the creator / performer of the music. There is a need to better understand the conveyance of emotion via visualizations of music, determine if there is a need for $\mathrm{D} / \mathrm{HH}$ audiences to have an "equivalent" emotional experience, and explore ways to create greater and/or equivalent emotional experiences.

- No one develops an understanding of music without a lifetime of exposure. Given that hearing children develop a knowledge of music as a result of repeated exposure starting at a very young age, it would be useful to understand whether $\mathrm{D} / \mathrm{HH}$ people, adults in particular, would benefit from frequent, repeated exposure and training to music information presentations (including visualizations).

- Design and test viable solutions that can be applied to screened devices of all sizes when and where visualization / captions are desired. As shown in Chapter 7, D/HH music consumers do use small devices (e.g., mobile phones, iPods, etc.) to listen to music but they are faced with barriers such as the display of captions, if the device even supports such captions for music.

- Develop new ways for D/HH users to control the display of captions. The comments collected for the study in Chapter 4 suggest that, with respect to music, there is a need to explore appropriate ways for users to have more control over the presence and more options to control the presentation of captions.

- Given the strong expressed desire for lyrics in the results of Chapters 7 and 8 as well as the clear sense that D/HH adults do not feel that their needs are met by current music captioning approaches as shown in the results of Chapter 4, a usability study on the presentation of lyrics is needed.

- Design a means to support tasks that involve music without forcing one's entire attention to the music. The approaches to music visualization explored in this Dissertation would require a person to only watch the visualization when it is in use. If the music in question is being presented as part of another entertainment experience (e.g., while watching a movie), this approach to visualization would be inappropriate because it would not allow the user to attend to other information (e.g., the moving picture, dialogue captions, etc.). Thus there is a need to develop ways to blend different visual presentations of information.

\subsubsection{Example Future Study}

A future project from the research presented in this Dissertation would be a usability study on the presentation of lyrics. Lyrics are currently presented either via televised captioned commercial music videos or online videos of various captioning quality. A set of commercial captioned music videos would be compared to other ways of presenting lyrics to determine what is missing in the HH experience. The lyric presentation styles might include a notepad-like window that presents scrollable static 


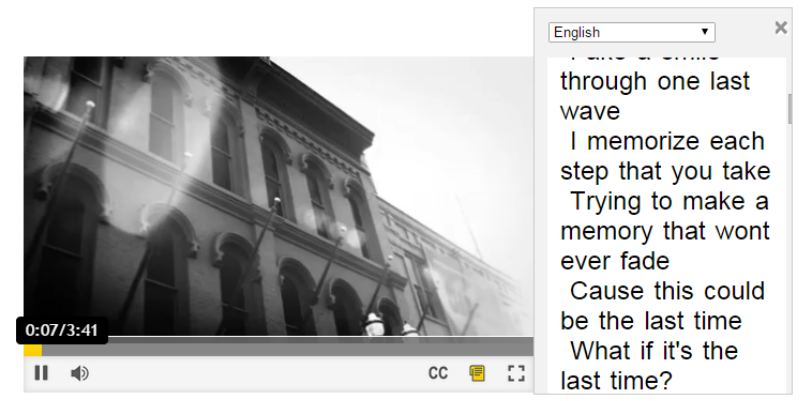

Figure 9.1: FLOE Project Video Player showing transcript view of lyrics.

lyrics (e.g., iTunes style presentation), or a video with embedded synchronized captioned lyrics. Information could be collected from participants to understand the types of options they might want / need to control the presentation of captions as well as develop an understanding of the various usability issues each presentation style raises.

One option for presenting synchronized captioned lyrics is to use a web browser with an HTML5 compatible video player. One current standard for encoding captions is Web Video Text Tracks (WebVTT) (Pfeiffer, Jägenstedt, \& Hickson, 2015). HTML5 compatible video players are able to accept multiple audio or timed text tracks (e.g., captions, subtitles, etc.) for the same video content. Using WebVTT, it is possible to caption a video and set different font attributes and behaviours throughout the timed text track with a Cascading Style Sheet (CSS).

An option for presenting scrollable static lyrics might be the HTML5 video player developed for the FLOE Project by the Inclusive Design Research Centre at The Ontario College of Art and Design University. Shown in Figure 9.1, this video player offers a transcript viewer alongside the video. Using this scrollable transcript viewer, one can watch a music video and follow the lyrics.

Open-ended interviews with some questionnaires could be used to collect information on responses to each presentation approach. Adding an eye-tracker may also help to understand the degree viewers attend to the text vs. the video. Sound would be presented for all experiments.

As part of the research for this Dissertation, an attempt was made over a two year period to conduct this very usability study.

After collecting the lyrics for various songs and having them checked for their correctness, the music videos for these songs were captioned. At first, this captioning was attempted using the proprietary scripting scheme of the EnACT tool, however this was not successful because the tool, which is based on Flash, was no longer stable. Captioning was instead completed using MovieCaptioner (SynchriMedia, 2015), which supports multiple formats including open standards such as WebVTT. The transcript view was created using the FLOE Project video player mentioned above. The corrected lyrics were adapted and reformatted for use in this tool.

In addition to the captioned video stimuli, questionnaires and interview scripts were developed for use in this study. The NASA TLX, a standardized survey (Hart, 2006), was to be used to assess the participant's subjective workload when viewing the videos. Because this questionnaire uses a very 
small font, it was adapted to a larger font to support the potential of elderly participants attending the usability study (recall that 17 people, or $18.1 \%$ of those who participated in the various experiments reported in Chapters 4, 5, 6, 7 and 8, were over 65).

A significant challenge was experienced when attempting to source an eye-tracking system. A portable system was found. It used a laptop that was poorly underpowered for the software and peripherals it was required to support. As a result, the eye-tracking software was unable to record any data until a new computer could be found to support it. Eventually, the time spent on this project exceeded what was available.

\subsection{Conclusion}

Music is a widely shared form of expression. It is found in all human cultures. Music is enjoyed on its own and for its own sake as well as in groups as part of a social experience. The $\mathrm{D} / \mathrm{HH}$ communities have formed their own musical traditions and interests. Thus, while the social bonds formed by music do not exclude the $\mathrm{D} / \mathrm{HH}$ communities, the barriers they experience to access the music of the larger hearing community do.

As mentioned in Section 2.7.4 on page 49, using music visualizations has many advantages for $\mathrm{D} / \mathrm{HH}$ people. However, the visualization of music as a means to support the understanding and entertainment of D/HH music consumers needs more development. Across all of the experiments completed for this Dissertation, only six participants indicated that they did not like music or did not see the point of music. Large numbers of members of the $\mathrm{D} / \mathrm{HH}$ communities repeatedly expressed a strong interest in the further development of this area of musical expression.

Design is iterative. Providing lyrics is an important area of information for this population. New visualizations that explore types of invisible music information not addressed in this Dissertation, or take a different approach to visibly presenting the types of music information that have been explored in this Dissertation, will need to be developed and feedback from the D/HH communities obtained as this process continues.

The road to making the invisible visible still has many yet to be seen obstacles. 
Appendices 



\section{Appendix A}

\section{Web-based Survey}

Below, in the form of code snippets, are the instructions given and questions asked of all participants during the web-based survey described in Chapter 4. The Music Questionnaire was included in the Post-study Questions.

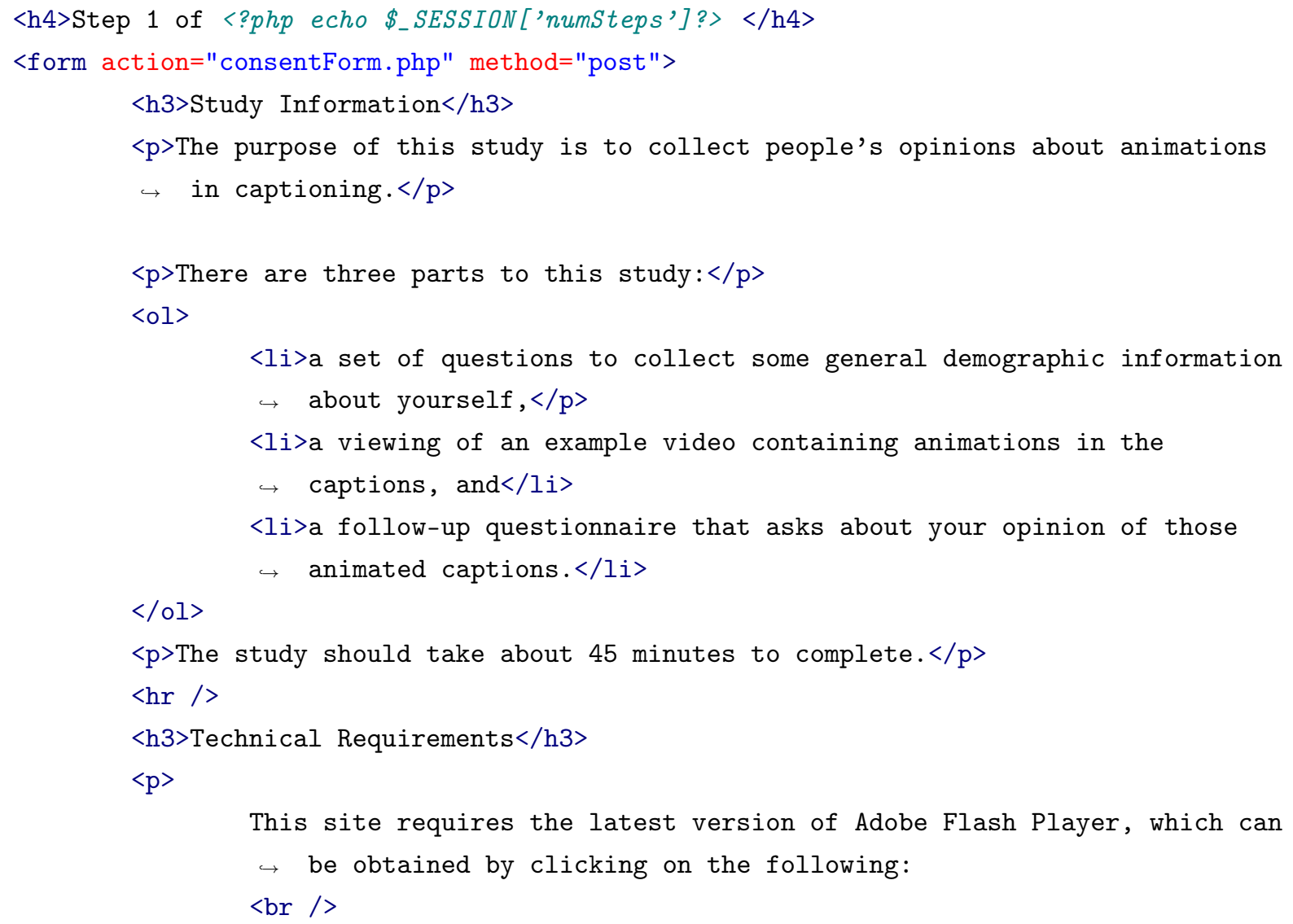




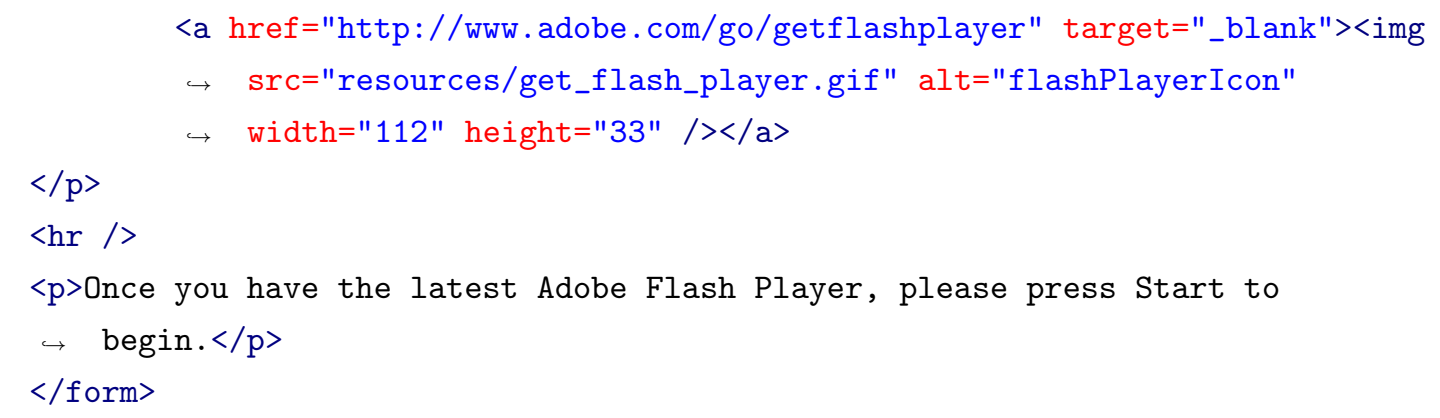

Listing 1: Study information and invitation to participate.

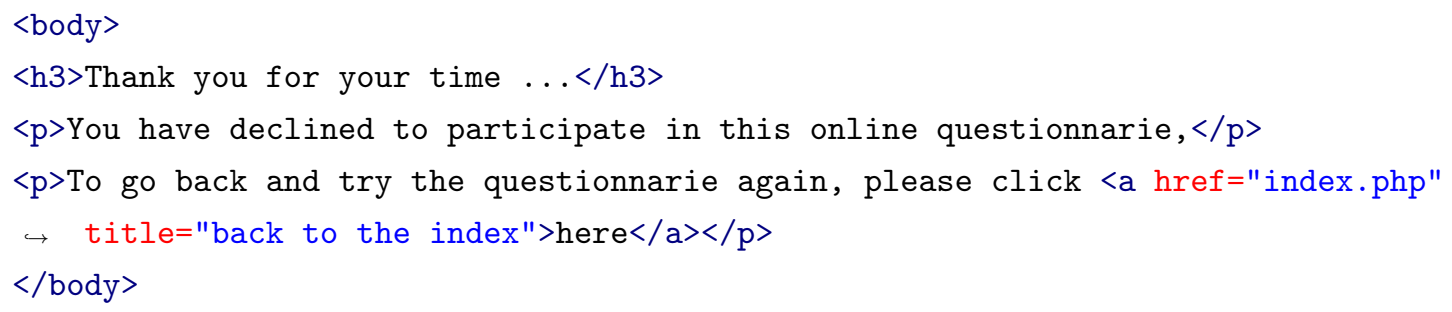

Listing 2: Person declines to participate.

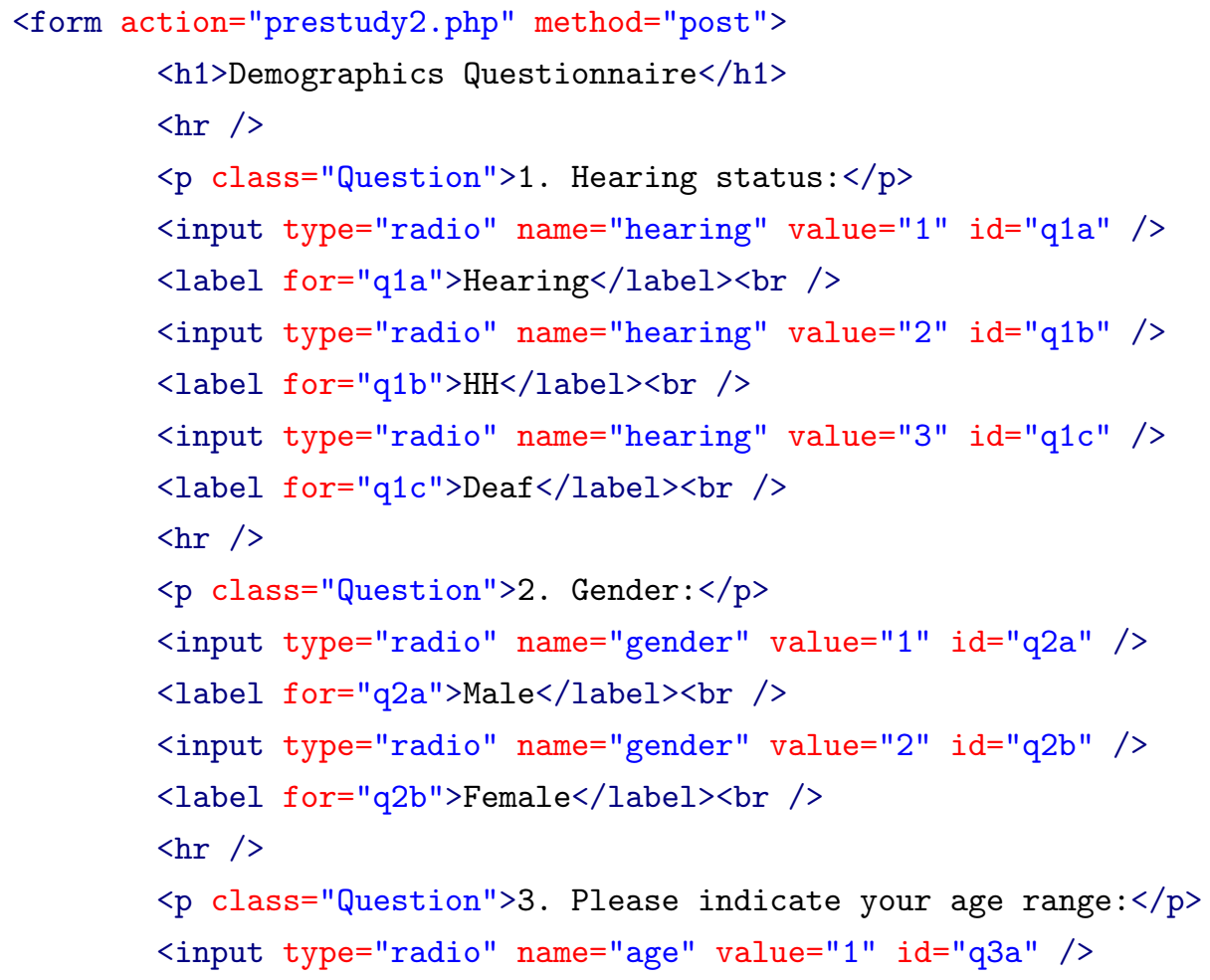


<label for="q3a">Under $18</$ label $><$ br $/>$

<input type="radio" name="age" value="2" id="q3b" />

$<$ label for="q3b">19 - 24</label ><br / >

<input type="radio" name="age" value="3" id="q3c" />

$<$ label for $=" q 3 c ">25-34</$ label $><$ br $/>$

<input type="radio" name="age" value="4" id="q3d" />

$<$ label for="q3d">35 - 44</label $><$ br $/>$

<input type="radio" name="age" value="5" id="q3e" />

$<$ label for $=" q 3 e ">45-54</$ label $><$ br $/>$

<input type="radio" name="age" value="6" id="q3f" />

$<$ label for="q3f">55 - 64</label ><br / >

<input type="radio" name="age" value="7" id="q3g" />

$<$ label for="q3g">0ver $65</$ label $><$ br $/>$

$<$ hr / >

$<\mathrm{p}$ class="Question" $>4$. What is your highest level of education completed? $</ \mathrm{p}>$

<input type="radio" name="education" value="1" id="q4a" />

$<$ label for="q4a" $>$ No formal education $</$ label $><$ br $/>$

<input type="radio" name="education" value="2" id="q4b" />

$<$ label for="q4b" $>$ Elementary school $</$ label $><$ br $/>$

<input type="radio" name="education" value="3" id="q4c" />

$<$ label for="q4c" >High school $</$ label $><$ br $/>$

<input type="radio" name="education" value="4" id="q4d" />

$<$ label for="q4d" $>$ Technical college $</$ label $><$ br $/>$

<input type="radio" name="education" value="5" id="q4e" />

<label for="q4e" $>$ University $</$ label $><$ br $/>$

<input type="radio" name="education" value="6" id="q4f" />

$<$ label for="q4f" $>$ Graduate school $</$ label $><$ br $/>$

$<\mathrm{hr} />$

$<\mathrm{p}$ class="Question" $>5$. How often do you use closed captions when watching

$\hookrightarrow$ television? $\langle/ \mathrm{p}>$

<input type="radio" name="frequencia" value="1" id="q5a" />

$<$ label for="q5a" >Always $</$ label $><$ br $/>$

<input type="radio" name="frequencia" value="2" id="q5b" />

$<$ label for="q5b">0ccasionally</label $><$ br $/>$

<input type="radio" name="frequencia" value="3" id="q5c" />

$<$ label for="q5c" $>$ Never $</$ label $><$ br $/>$

$<\mathrm{hr} />$

$<\mathrm{p}>\mathrm{Please}$ press Next to continue or Clear Answers to clear your answers. $</ \mathrm{p}>$ <input name="nextStep" type="hidden" value="<?php echo \$_POST['nextStep']+1?>" $\hookrightarrow \quad />$ 


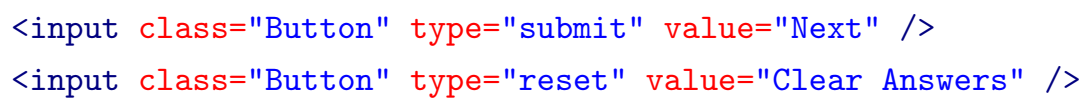

Listing 3: Prestudy Questions Page 1.

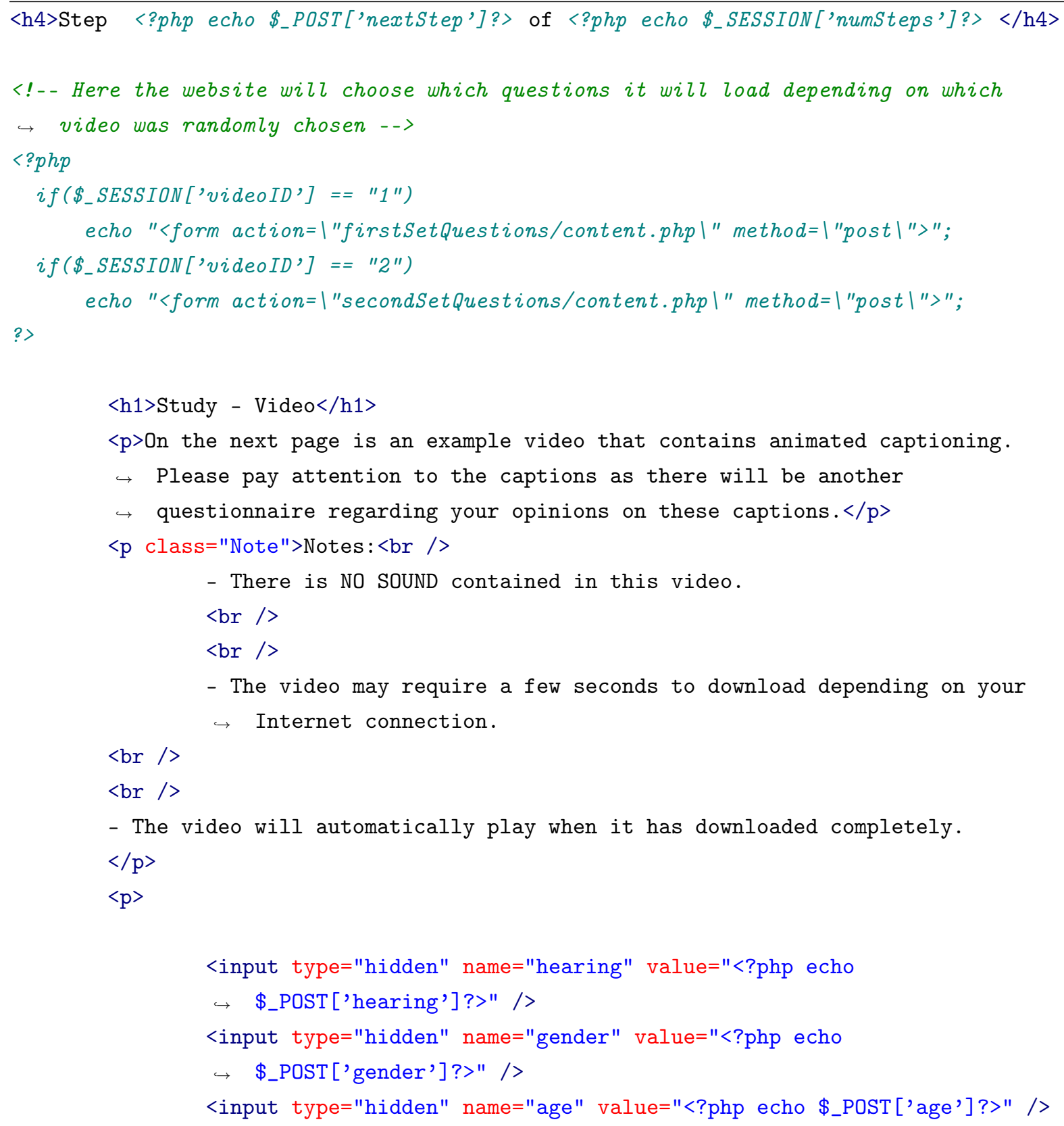




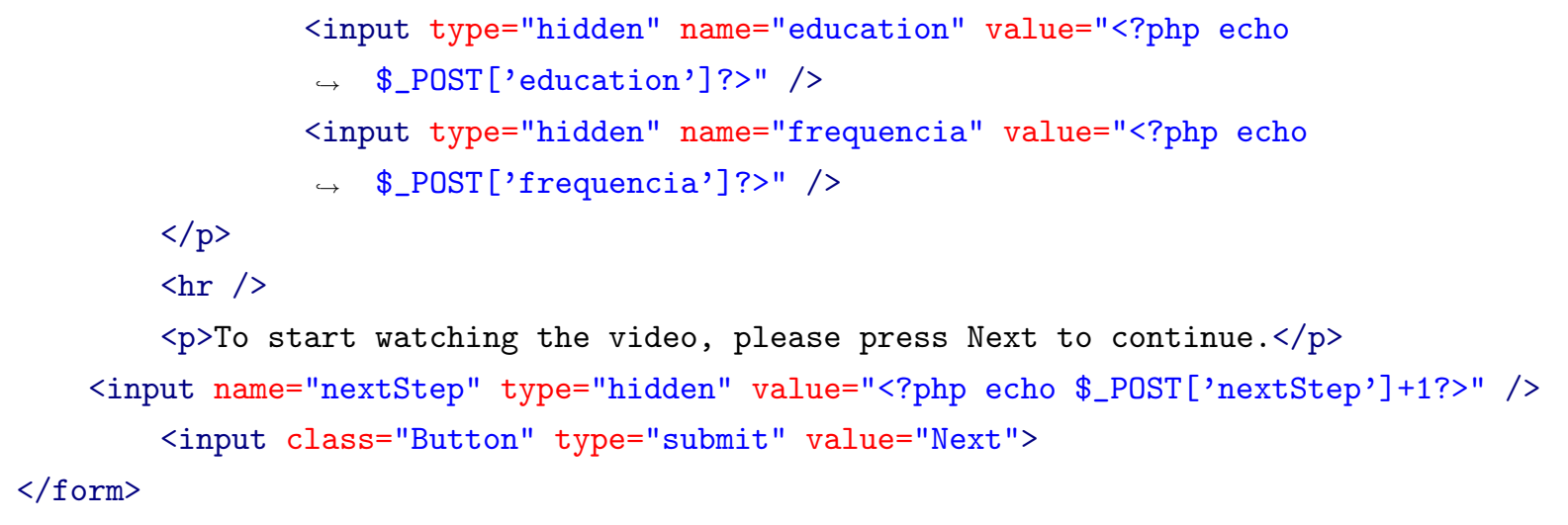

Listing 4: Prestudy Questions Page 2.

<h4>Step <?php echo \$_POST['nextStep']?> of <?php echo \$_SESSION ['numSteps'] ?> </h4> $<$ form action="study2.php" method="post">

$<$ h1 $>$ Study Questionnaire</h1>

$<\mathrm{p}$ class="Question" $>1$. In a few sentences (or less), please describe what the $\hookrightarrow$ video was about. $\langle/ \mathrm{p}\rangle$

$<$ textarea name="q1" cols="80" rows="8" $></$ textarea $>$

$<\mathrm{p}$ class="Question" $>2$. Please rate each of the following aspects of the

$\hookrightarrow \quad\langle\mathrm{u}>$ captioning of the larger size text. $</ \mathrm{u}>$ Select the option that best

$\hookrightarrow$ fits your rating. $</ p>$

<table cellpadding="0" cellspacing="0">

$<$ tr $>$

$<$ th $></$ th $>$

$<$ th $>$ Liked very much $</$ th $>$

$<$ th $>$ Liked $</$ th $>$

$<$ th $>$ Neither liked nor disliked $</$ th $>$

$<$ th $>$ Disliked $</$ th $>$

$<$ th $>$ Disliked very much $</$ th $>$

$</ \operatorname{tr}>$

$\langle$ tr $>$

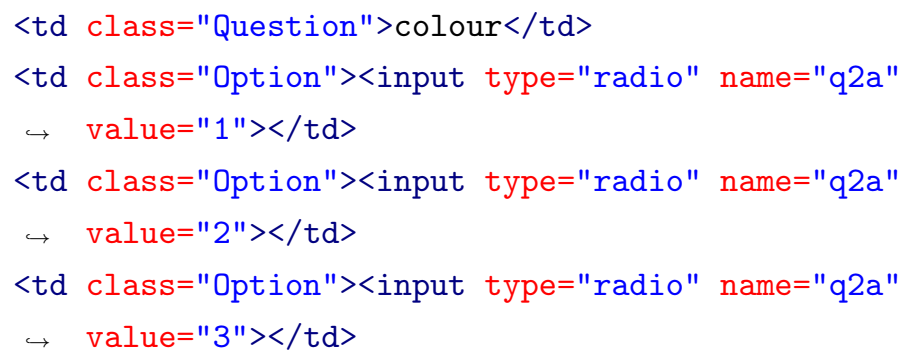




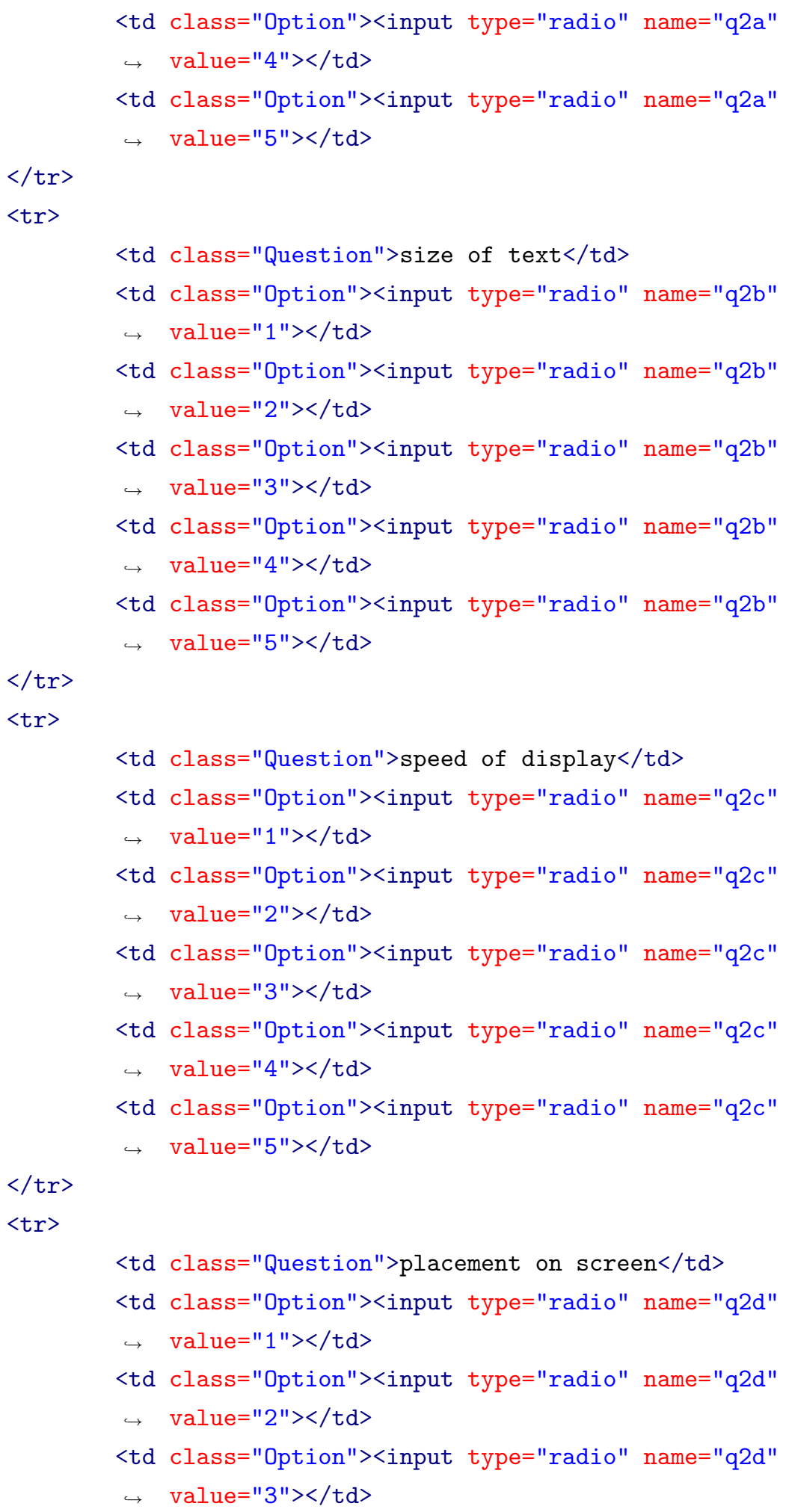




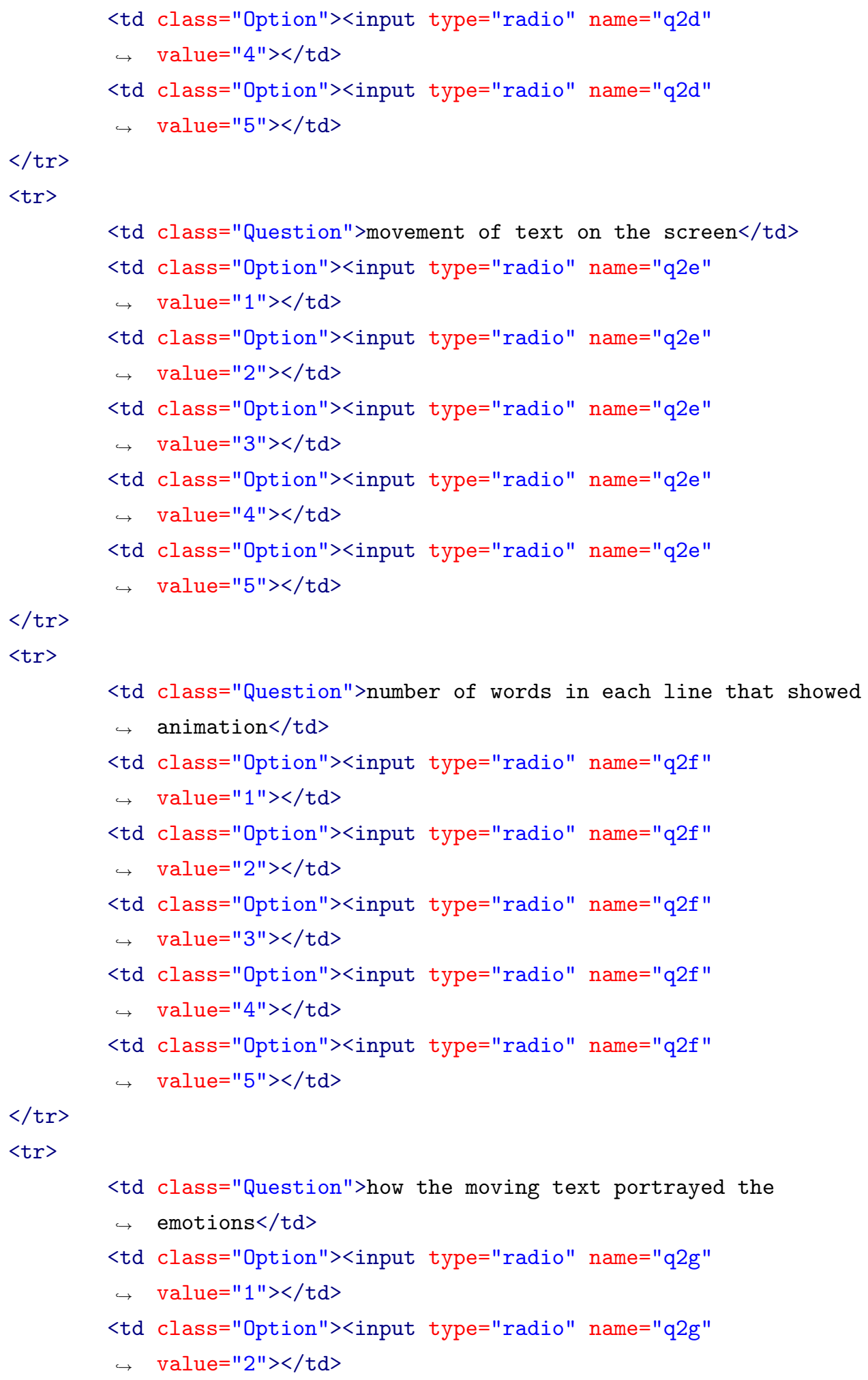




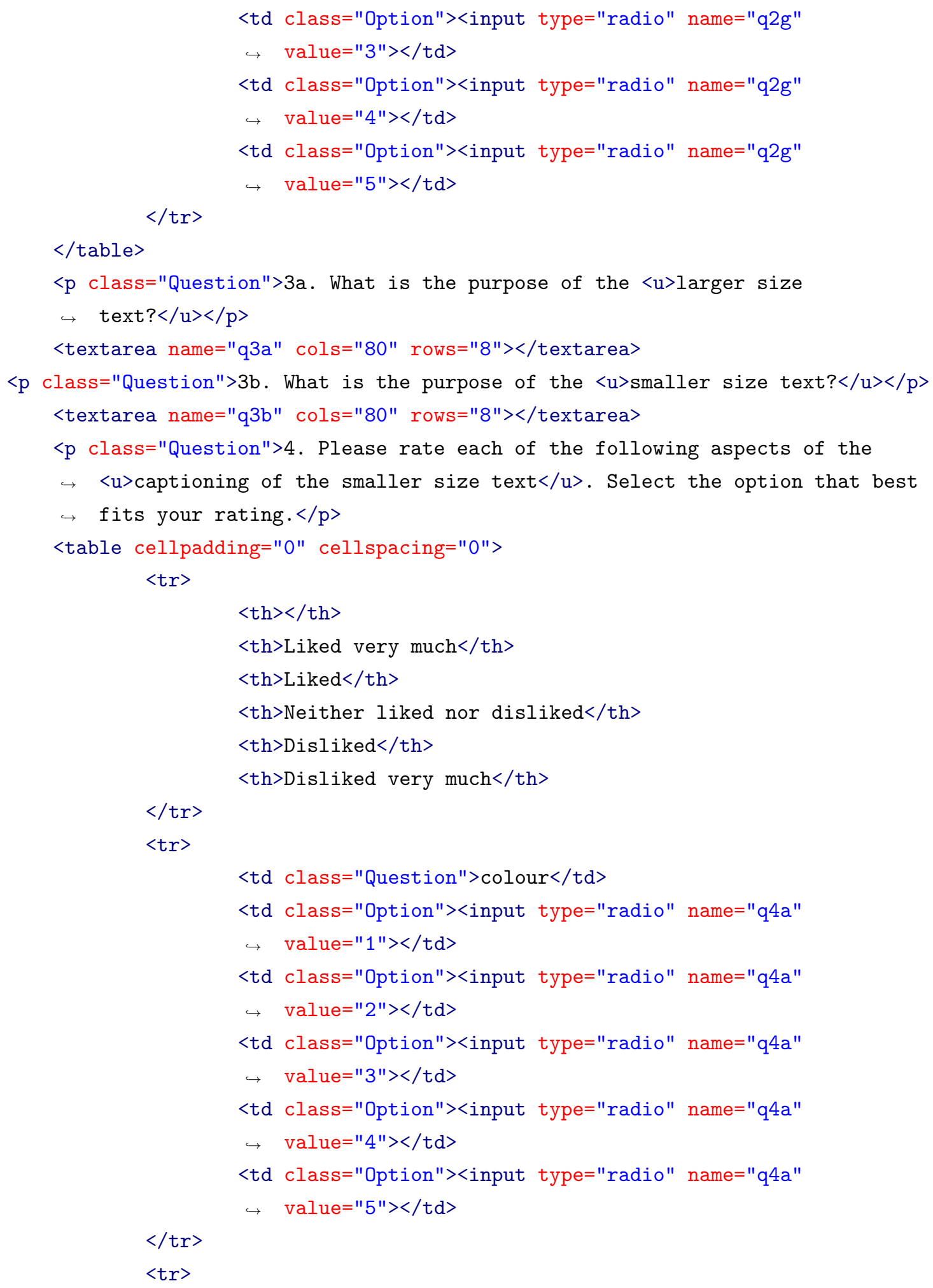




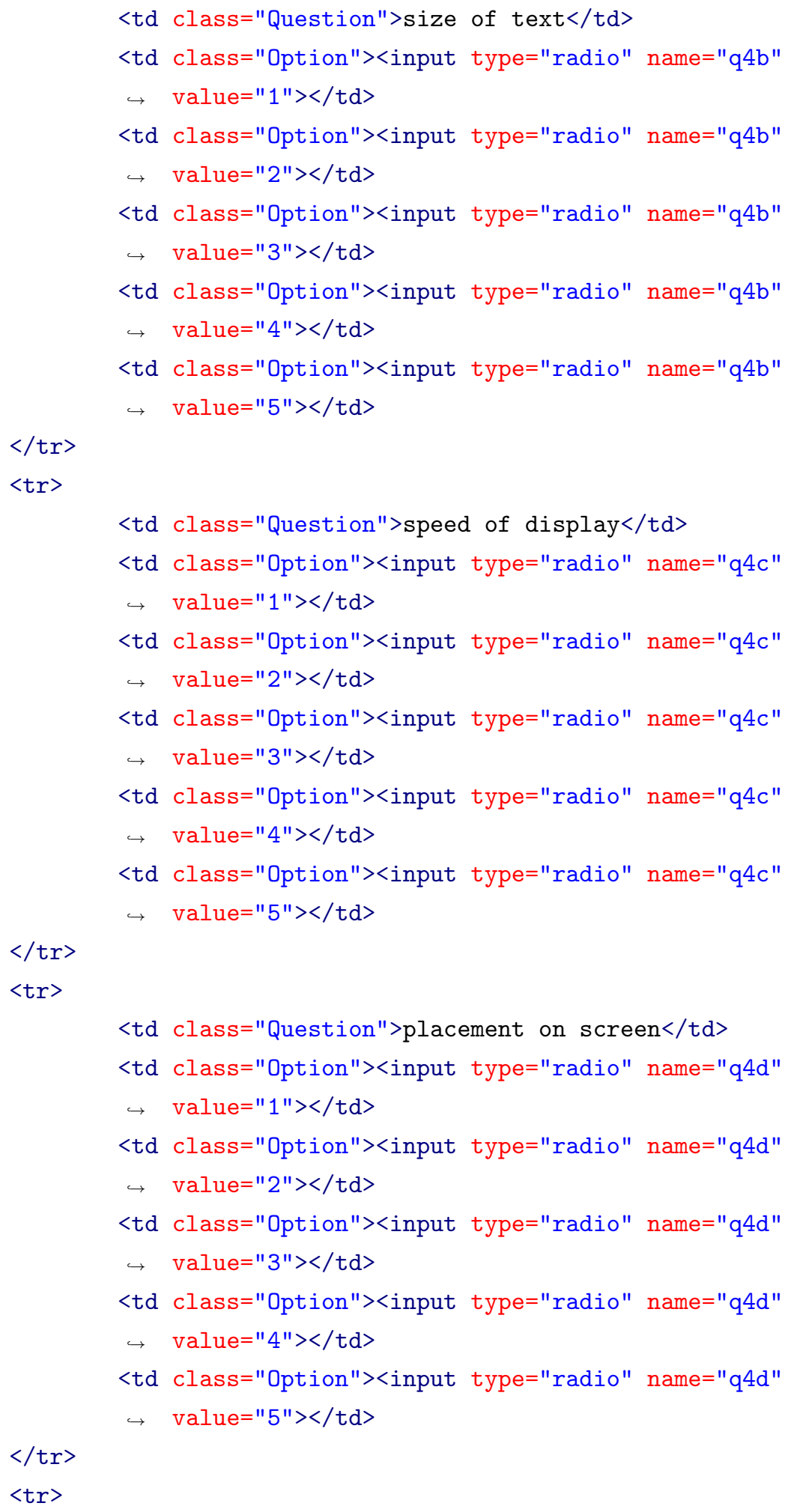


$<$ td class="Question" $>$ movement of text on the screen $</$ td $>$

<td class="Option"><input type="radio" name="q4e"

$\hookrightarrow$ value $=" 1 "></$ td $>$

$<$ td class="Option" ><input type="radio" name="q4e"

$\hookrightarrow$ value $=" 2 "></$ td $>$

$<$ td class="Option"><input type="radio" name="q4e"

$\hookrightarrow$ value $=" 3 "></$ td $>$

$<$ td class="Option" $><$ input type="radio" name="q4e"

$\hookrightarrow$ value $=" 4 ">\langle/$ td $\rangle$

$<$ td class="Option" $><$ input type="radio" name="q4e"

$\hookrightarrow$ value $=" 5 "></$ td $>$

$</ \operatorname{tr}>$

$<\operatorname{tr}>$

<td class="Question">number of words in each line that showed

$\hookrightarrow$ animation $</$ td $>$

$<$ td class="Option" ><input type="radio" name="q4f"

$\hookrightarrow$ value $=" 1 "></$ td $>$

$<$ td class="Option" ><input type="radio" name="q4f"

$\hookrightarrow$ value $=" 2 ">\langle/$ td $\rangle$

$<$ td class="Option"><input type="radio" name="q4f"

$\hookrightarrow$ value $=" 3 "></$ td $>$

<td class="Option" $><$ input type="radio" name="q4f"

$\hookrightarrow$ value $=" 4 "></$ td $>$

<td class="Option"><input type="radio" name="q4f"

$\hookrightarrow$ value $=" 5 ">\langle/$ td $\rangle$

$</ \operatorname{tr}>$

$<$ tr $>$

<td class="Question" $>$ how the moving text portrayed the

$\hookrightarrow$ emotions $</$ td $>$

<td class="Option" $><$ input type="radio" name="q4g"

$\hookrightarrow$ value $=" 1 "></$ td $>$

$<$ td class="Option"><input type="radio" name="q4g"

$\hookrightarrow$ value $=" 2 ">\langle/$ td $>$

$<$ td class="Option"><input type="radio" name="q4g"

$\hookrightarrow$ value="3" $></$ td $>$

$<$ td class="Option"><input type="radio" name="q4g"

$\hookrightarrow$ value $=" 4 "></$ td $>$

$<$ td class="Option" $><$ input type="radio" name="q4g"

$\hookrightarrow$ value $=" 5 ">\langle/$ td $\rangle$ 


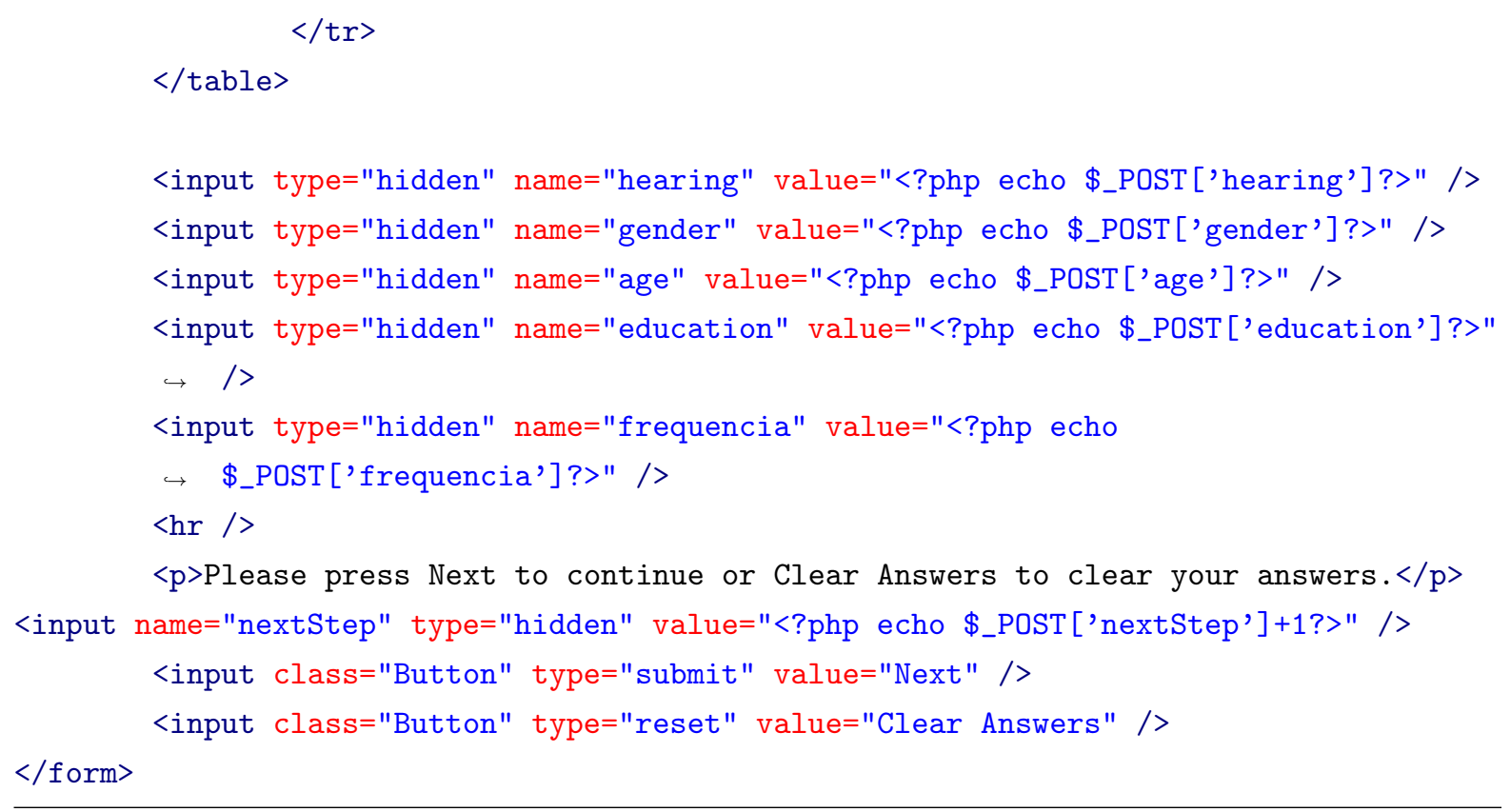

Listing 5: Forgive Me Questions Page 1.

<h4>Step <?php echo \$_POST ['nextStep']?> of <?php echo \$_SESSION['numSteps']?> </h4> $<$ form action="study3.php" method="post">

$<$ h1 $>$ Study Questionnaire - Cont'd $</$ h1 $>$

$<\mathrm{p}$ class="Question" $>5$. What effect did having two different sets of captions

$\hookrightarrow$ appearing on the screen at the same time have for you? Check all that

$\hookrightarrow$ apply. $\langle/ \mathrm{p}\rangle$

<input type="checkbox" name="q5a" value="1" id="q5a">

$<$ label for="q5a">Confused me.</label>

$<$ br $/>$

<input type="checkbox" name="q5b" value="2" id="q5b">

$<$ label for="q5b">Helped me understand the story.</label>

$<$ br $/>$

<input type="checkbox" name="q5c" value="3" id="q5c">

$<$ label for="q5c">It was going too fast. $</$ label $>$

$<$ br / >

<input type="checkbox" name="q5d" value="4" id="q5d">

$<$ label for="q5d">I didn't know where to look.</label $>$

$<$ br $/>$

<input type="checkbox" name="q5e" value="5" id="q5e">

$<$ label for="q5e" $>$ Helped me focus on reading the captions.</label> 
$<\mathrm{br} />$

<input type="checkbox" name="q5f" value="6" id="q5f">

$<$ label for="q5f">There was too much happening at once. $</$ label $>$

$<$ br / >

<input type="checkbox" name="q5g" value="7" id="q5g">

<label for="q5g">It was entertaining.</label>

$<$ p class="Question" $>0$ ther, please suggest: $\langle/ p>$

<textarea name="q5_comment" cols="80" rows="8"></textarea $>$

$<$ br $/>$

$<$ p class="Question" $>6$. Sometimes the captions appeared at the same time when

$\hookrightarrow$ two people were speaking at the same time. Other times, the captions

$\hookrightarrow$ appeared one after the other but both stayed on the screen.

$<\mathrm{br} />$

$<\operatorname{br} />$

When two people are speaking at the same time, or when there is some overlap, $\hookrightarrow$ how would you prefer those captions be displayed? $</ p>$

<input type="radio" name="q6" value="1" id="q6a">

<label for="q6a">0nly one caption on the screen at a time, no matter

$\hookrightarrow$ what. $</$ label $>$

$<\operatorname{br} />$

<input type="radio" name="q6" value="2" id="q6b">

$<$ label for="q6b">Both captions should appear at the same time regardless of $\hookrightarrow$ when spoken.</label>

<br />

<input type="radio" name="q6" value="3" id="q6c">

$<$ label for="q6c">Captions should appear when spoken.</label>

<br />

$<\mathrm{p}$ class="Question" $>$ Other, please suggest: $\langle/ \mathrm{p}\rangle$

<textarea name="q6a" cols="80" rows="8" ></textarea $>$

$<$ p class="Question" $>7$. Please rate how difficult it was for you to read the

$\hookrightarrow$ larger-size text. $</ p>$

<input type="radio" name="q7" value="1" id="q7a">

$<$ label for="q7a">Very difficult.</label>

$<$ br / >

<input type="radio" name="q7" value="2" id="q7b">

$<$ label for="q7b">Difficult. $</$ label $>$

<br />

<input type="radio" name="q7" value="3" id="q7c"> 


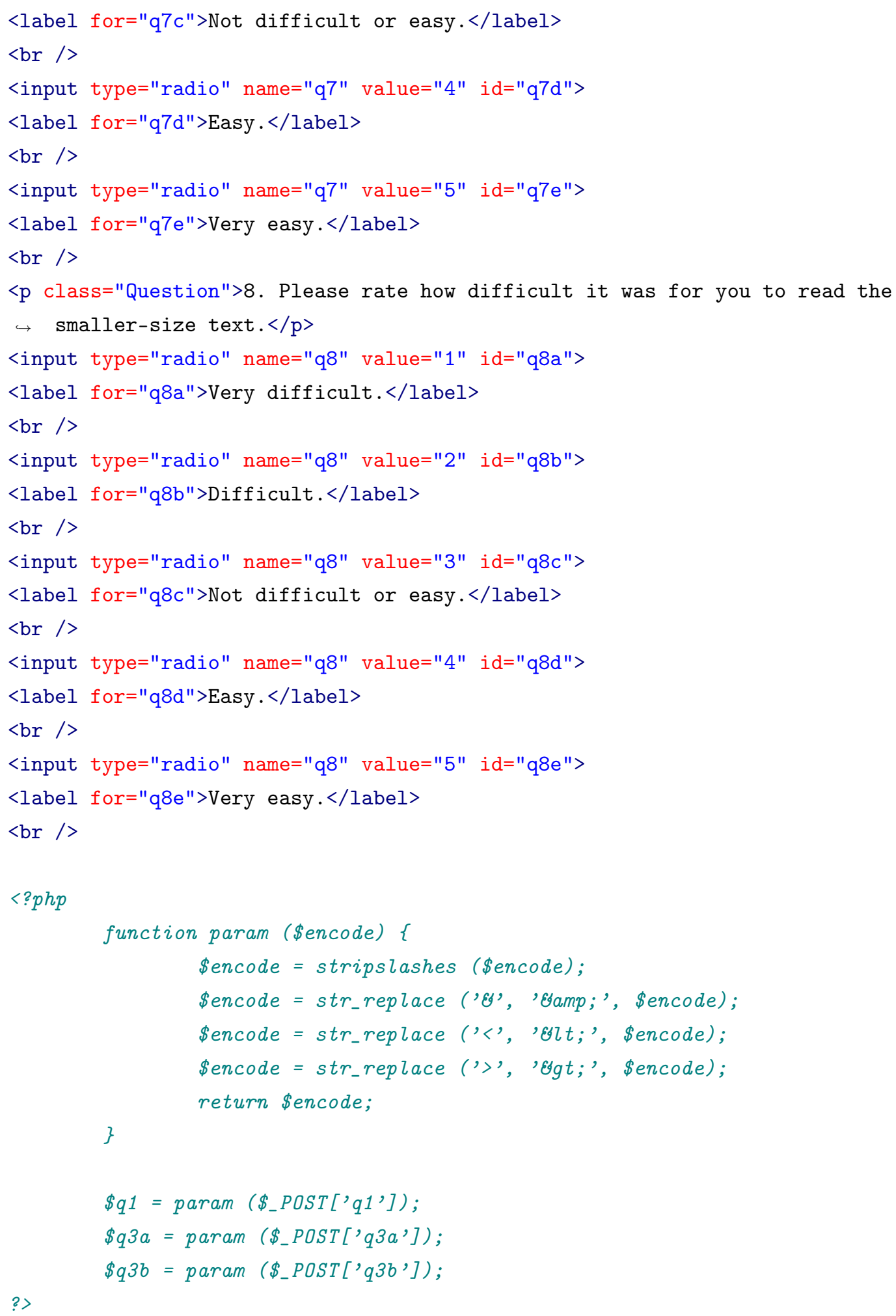




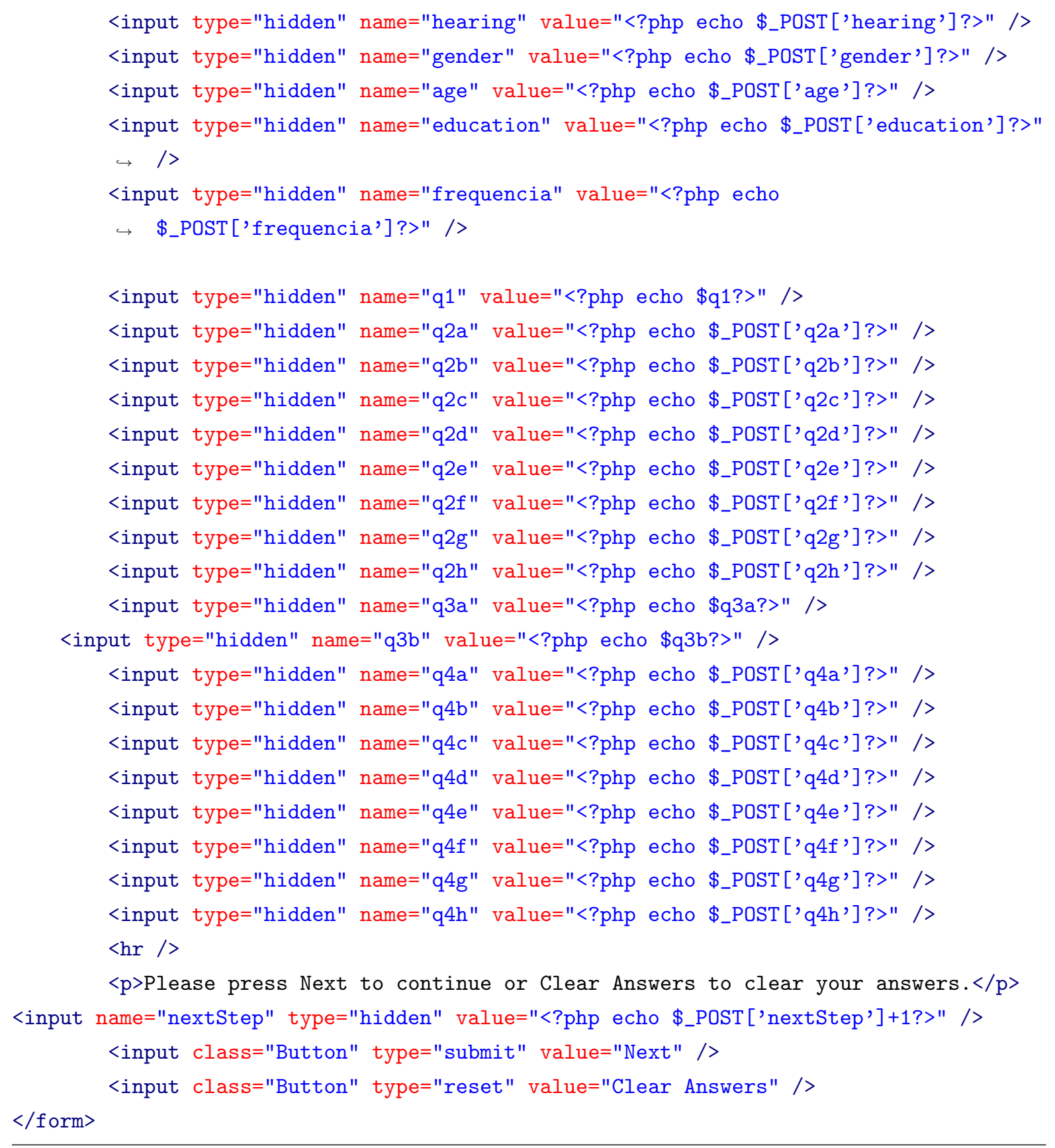

Listing 6: Forgive Me Questions Page 2.

<h4>Step <?php echo \$_POST ['nextStep']?> of <?php echo \$_SESSION ['numSteps']? ></h4> $<$ form action="study4.php" method="post"> 


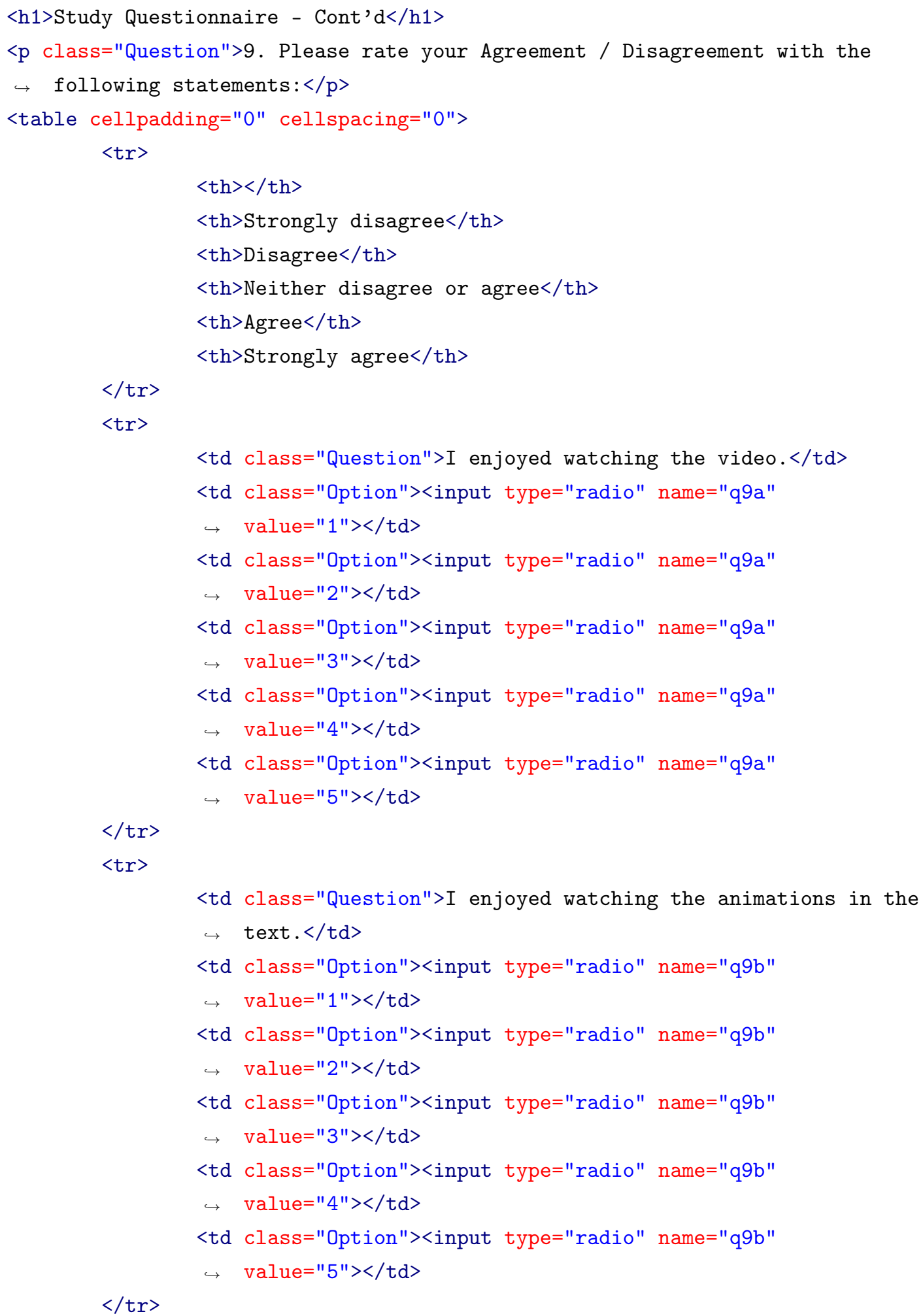


$<$ tr $>$

$<$ td class="Question" $>I$ was confused with watching the

$\rightarrow$ video. $\langle/$ td $>$

$<$ td class="Option" $><$ input type="radio" name="q9c"

$\hookrightarrow$ value $=" 1 ">\langle/$ td $\rangle$

$<$ td class="Option" $><$ input type="radio" name="q9c"

$\hookrightarrow$ value $=" 2 ">\langle/$ td $\rangle$

$<$ td class="Option" $><$ input type="radio" name="q9c"

$\hookrightarrow$ value $=" 3 ">\langle/$ td $\rangle$

$<$ td class="Option" $><$ input type="radio" name="q9c"

$\hookrightarrow$ value $=" 4 ">\langle/$ td $\rangle$

$<$ td class="Option" $><$ input type="radio" name="q9c"

$\hookrightarrow$ value $=" 5 ">\langle/$ td $>$

$</ \operatorname{tr}\rangle$

$<\operatorname{tr}>$

$<$ td class="Question" $>$ I concentrated on watching the text for

$\hookrightarrow$ the whole time. $\langle/$ td $>$

$<$ td class="Option" $><$ input type="radio" name="q9d"

$\hookrightarrow$ value $=" 1 ">\langle/$ td $>$

$<$ td class="Option" $><$ input type="radio" name="q9d"

$\hookrightarrow$ value $=" 2 ">\langle/$ td $\rangle$

$<$ td class="Option" $><$ input type="radio" name="q9d"

$\hookrightarrow$ value $=" 3 ">\langle/$ td $\rangle$

$<$ td class="Option" $><$ input type="radio" name="q9d"

$\hookrightarrow$ value $=" 4 ">\langle/$ td $\rangle$

$<$ td class="Option" $><$ input type="radio" name="q9d"

$\hookrightarrow$ value $=" 5 ">\langle/$ td $>$

$\langle/ \operatorname{tr}\rangle$

$<\operatorname{tr}>$

$<$ td class="Question" $>$ The animations hindered my understanding

$\hookrightarrow$ of the video. $\langle/ t d\rangle$

$<$ td class="Option" $><$ input type="radio" name="q9e"

$\hookrightarrow$ value $=" 1 ">\langle/$ td $>$

$<$ td class="Option" $><$ input type="radio" name="q9e"

$\hookrightarrow$ value $=" 2 ">\langle/$ td $>$

$<$ td class $=" O p t i o n "><$ input type="radio" name="q9e"

$\hookrightarrow$ value $=" 3 ">\langle/$ td $\rangle$

$<$ td class="Option" $><$ input type="radio" name="q9e"

$\hookrightarrow \quad$ value $=" 4 ">\langle/$ td $>$ 
<td class="Option" $><$ input type="radio" name="q9e"

$\hookrightarrow \quad$ value $=" 5 ">\langle/$ td $>$

$</ \operatorname{tr}>$

$<\operatorname{tr}>$

<td class="Question" $>$ The animations distracted me. $</$ td $>$

<td class="Option" ><input type="radio" name="q9f"

$\hookrightarrow \quad$ value $=" 1 ">\langle/$ td $\rangle$

$<$ td class="Option" $><$ input type="radio" name="q9f"

$\hookrightarrow$ value $=" 2 "\rangle\langle/$ td $\rangle$

$<$ td class="Option" $><$ input type="radio" name="q9f"

$\hookrightarrow$ value $=" 3 ">\langle/$ td $\rangle$

$<$ td class="Option" $><$ input type="radio" name="q9f"

$\hookrightarrow$ value $=" 4 ">\langle/$ td $\rangle$

$<$ td class="Option" $><$ input type="radio" name="q9f"

$\hookrightarrow$ value $=" 5 ">\langle/$ td $\rangle$

$\langle/ \operatorname{tr}\rangle$

$<$ tr $>$

<td class="Question">The animations helped me understand what

$\hookrightarrow$ was going on in the video. $\langle/$ td $>$

$<$ td class="Option" $><$ input type="radio" name="q9g"

$\hookrightarrow$ value $=" 1 "\rangle\langle/$ td $\rangle$

$<$ td class="Option"><input type="radio" name="q9g"

$\hookrightarrow$ value="2" $></$ td $>$

<td class="Option"><input type="radio" name="q9g"

$\hookrightarrow$ value $=" 3 ">\langle/$ td $\rangle$

$<$ td class="Option" $><$ input type="radio" name="q9g"

$\hookrightarrow$ value $=" 4 "\rangle\langle/$ td $\rangle$

$<$ td class="Option" $><$ input type="radio" name="q9g"

$\hookrightarrow$ value $=" 5 ">\langle/$ td $\rangle$

$</$ tr $>$

$\langle$ tr $>$

<td class="Question" $>$ I was bored. $\langle/$ td $>$

$<$ td class="Option" $><$ input type="radio" name="q9h"

$\hookrightarrow$ value="1" $>\langle/$ td $\rangle$

$<$ td class="Option" $><$ input type="radio" name="q9h"

$\hookrightarrow$ value $=" 2 "\rangle\langle/$ td $\rangle$

$<$ td class="Option" $><$ input type="radio" name="q9h"

$\hookrightarrow$ value="3" $></$ td $\rangle$

$<$ td class="Option" $><$ input type="radio" name="q9h"

$\hookrightarrow$ value $=" 4 "\rangle\langle$ td $\rangle$ 


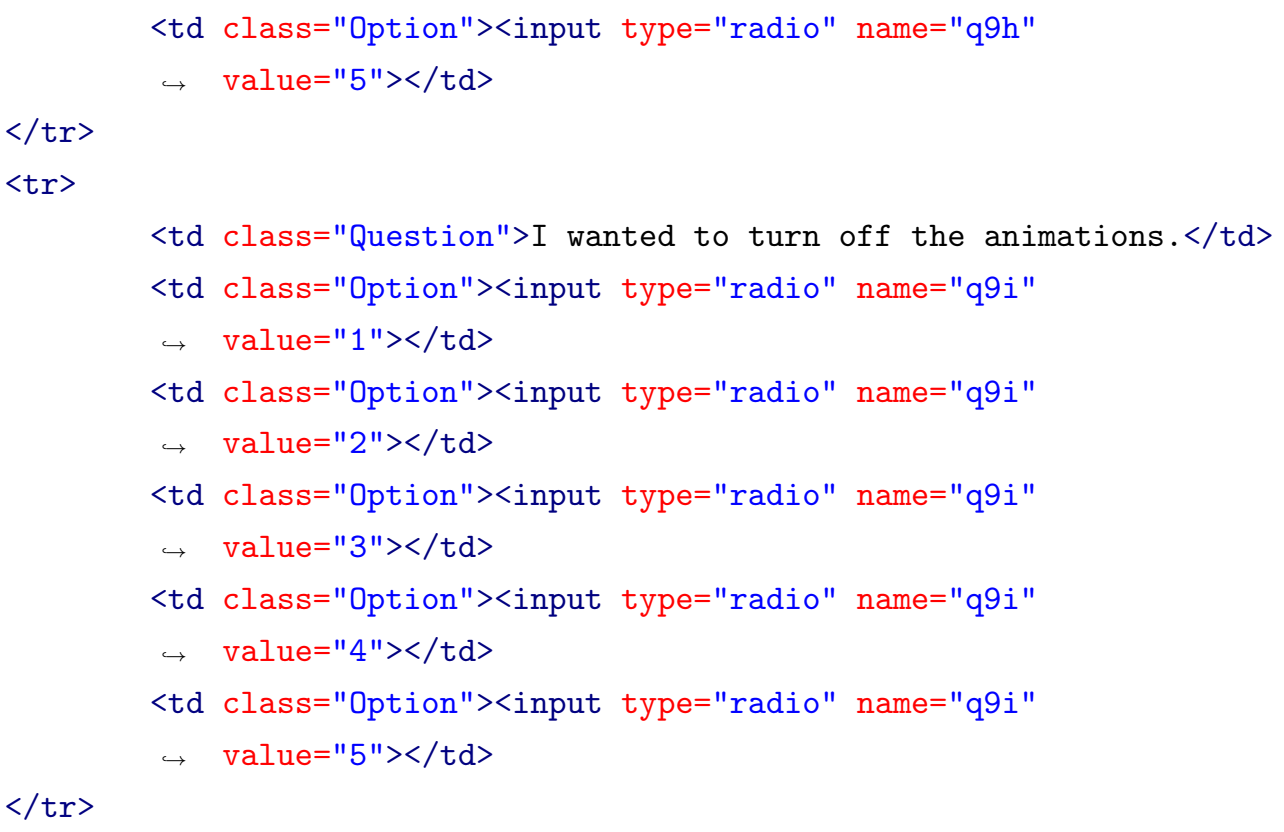

$</$ table $>$

$<$ p class="Question" $>10$. How much do you think the animated captions increased $\hookrightarrow$ your overall understanding of the video?</p $>$

<input type="radio" name="q10" value="1" id="q10a">

$<$ label for="q10a">Greatly decreased my level of understanding. $</$ label $>$

$<$ br / >

<input type="radio" name="q10" value="2" id="q10b">

$<$ label for="q10b">Somewhat decreased my level of understanding. $</$ label>

$<\mathrm{br} />$

<input type="radio" name="q10" value="3" id="q10c">

$<$ label for="q10c">No difference.</label>

$<\mathrm{br} />$

<input type="radio" name="q10" value="4" id="q10d">

$<$ label for="q10d">Somewhat increased my level of understanding. $</$ label>

$<\mathrm{br} />$

<input type="radio" name="q10" value="5" id="q10e">

$<$ label for="q10e">Greatly increased my level of understanding. $</$ label $>$

$<\mathrm{br} />$

$<$ p class="Question" $>11$. How much do you think the animated captions increased

$\hookrightarrow$ your overall understanding of the emotions that were being expressed? $</ p>$ <input type="radio" name="q11" value="1" id="q11a">

$<$ label for="q11a">Greatly decreased my level of understanding. $</$ label $>$

$<$ br $/>$ 


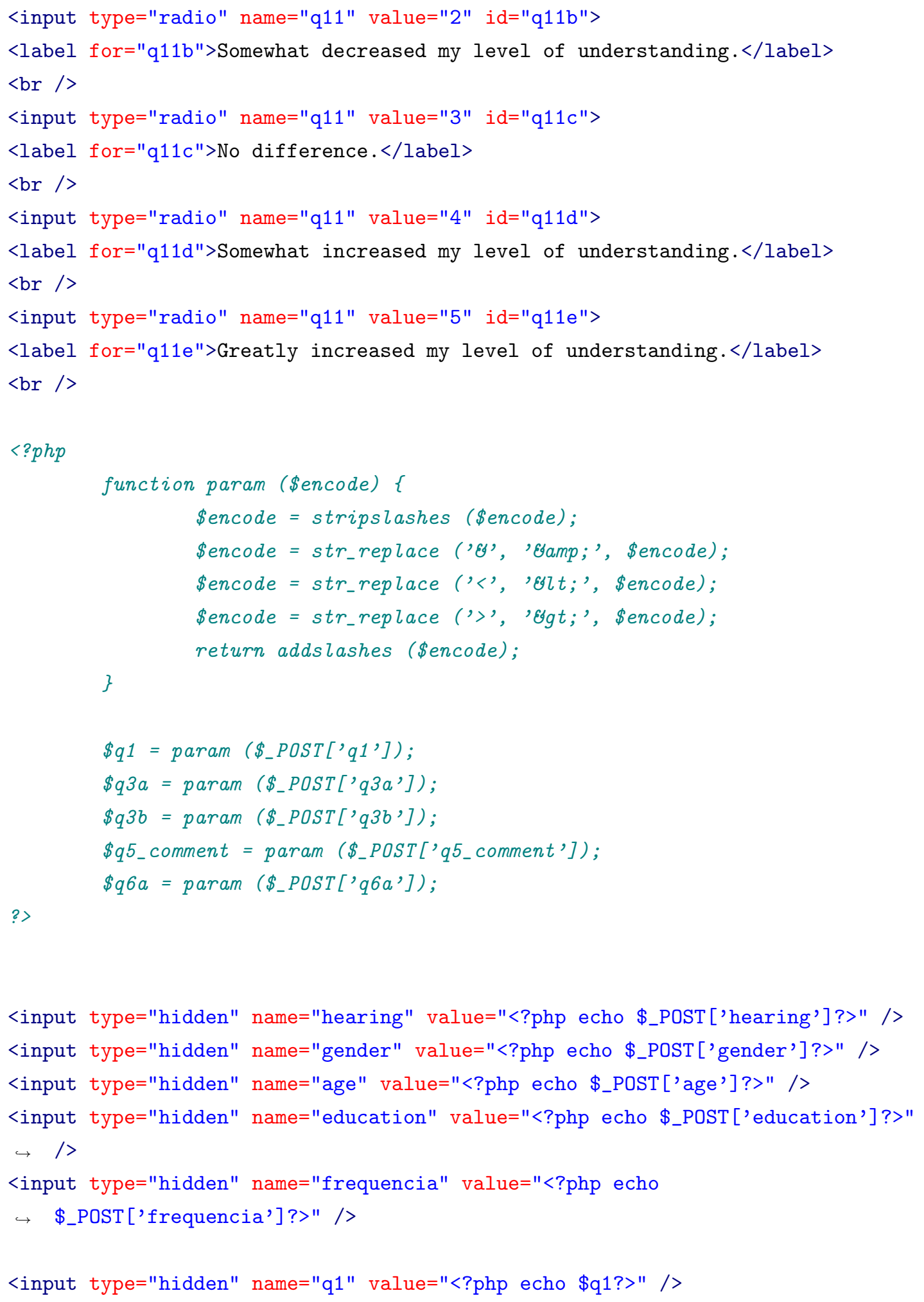




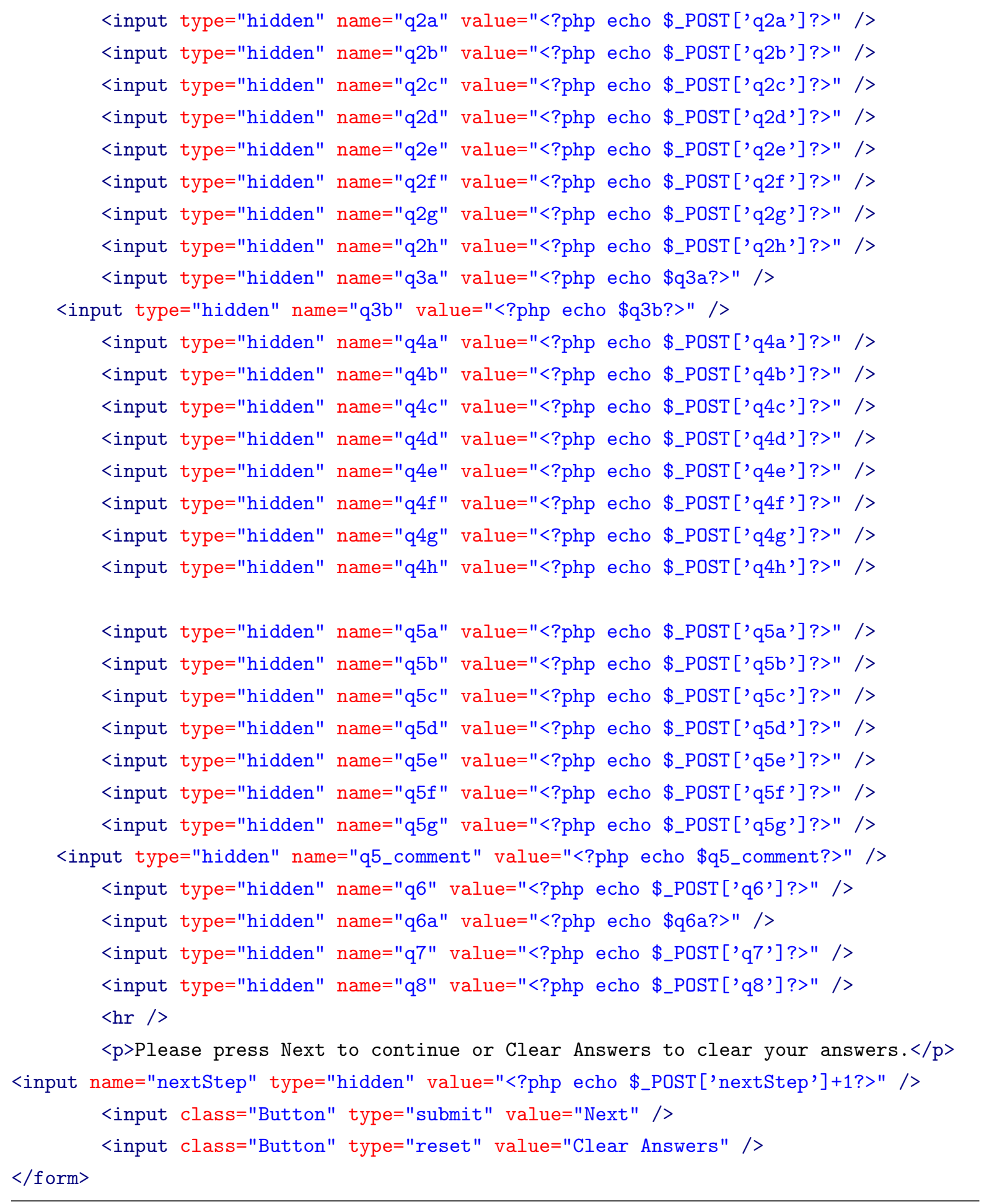

Listing 7: Forgive Me Questions Page 3. 
<h4>Step <?php echo \$_POST['nextStep']?> of <?php echo \$_SESSION['numSteps']? ></h4> <form action="../poststudy.php" method="post">

$<$ h1 $>$ Study Questionnaire - Cont'd $</$ h1 $>$

$<\mathrm{p}$ class="Question" $>12$. How much do you think the animated text distracted you

$\hookrightarrow$ from understanding the video?</p $>$

<input type="radio" name="q12" value="1" id="q12a">

$<$ label for="q12a">Did not distract me.</label>

$<$ br / >

<input type="radio" name="q12" value="2" id="q12b">

$<$ label for="q12b">Slightly distracted me.</label>

$<\mathrm{br} />$

<input type="radio" name="q12" value="3" id="q12c">

$<$ label for="q12c">Did not notice.</label>

$<$ br / >

<input type="radio" name="q12" value="4" id="q12d">

$<$ label for="q12d">Somewhat distracted m.e</label>

$<$ br / >

<input type="radio" name="q12" value="5" id="q12e">

$<$ label for="q12e">Greatly distracted me.</label>

$<\mathrm{br} />$

$<$ p class="Question">13. Suppose you had just watched the video with friends

$\hookrightarrow$ who were hearing, rate your willingness to engage in conversation with

$\hookrightarrow$ them about the video: $\langle/ \mathrm{p}\rangle$

<input type="radio" name="q13" value="1" id="q13a">

$<$ label for="q13a">Not willing to discuss the video at all.</label>

$<$ br / >

<input type="radio" name="q13" value="2" id="q13b">

$<$ label for="q13b">Not really willing to discuss the video.</label>

$<\mathrm{br} />$

<input type="radio" name="q13" value="3" id="q13c">

$<$ label for="q13c">Don't care.</label>

$<$ br / >

<input type="radio" name="q13" value="4" id="q13d">

$<$ label for="q13d">Somewhat willing to discuss the video.</label>

$<$ br / >

<input type="radio" name="q13" value="5" id="q13e">

$<$ label for="q13e">Very willing to discuss the video.</label>

$<$ br / >

$<$ p class="Question" $>$ Please indicate why you selected your particular

$\hookrightarrow$ rating: $</ \mathrm{p}>$ 


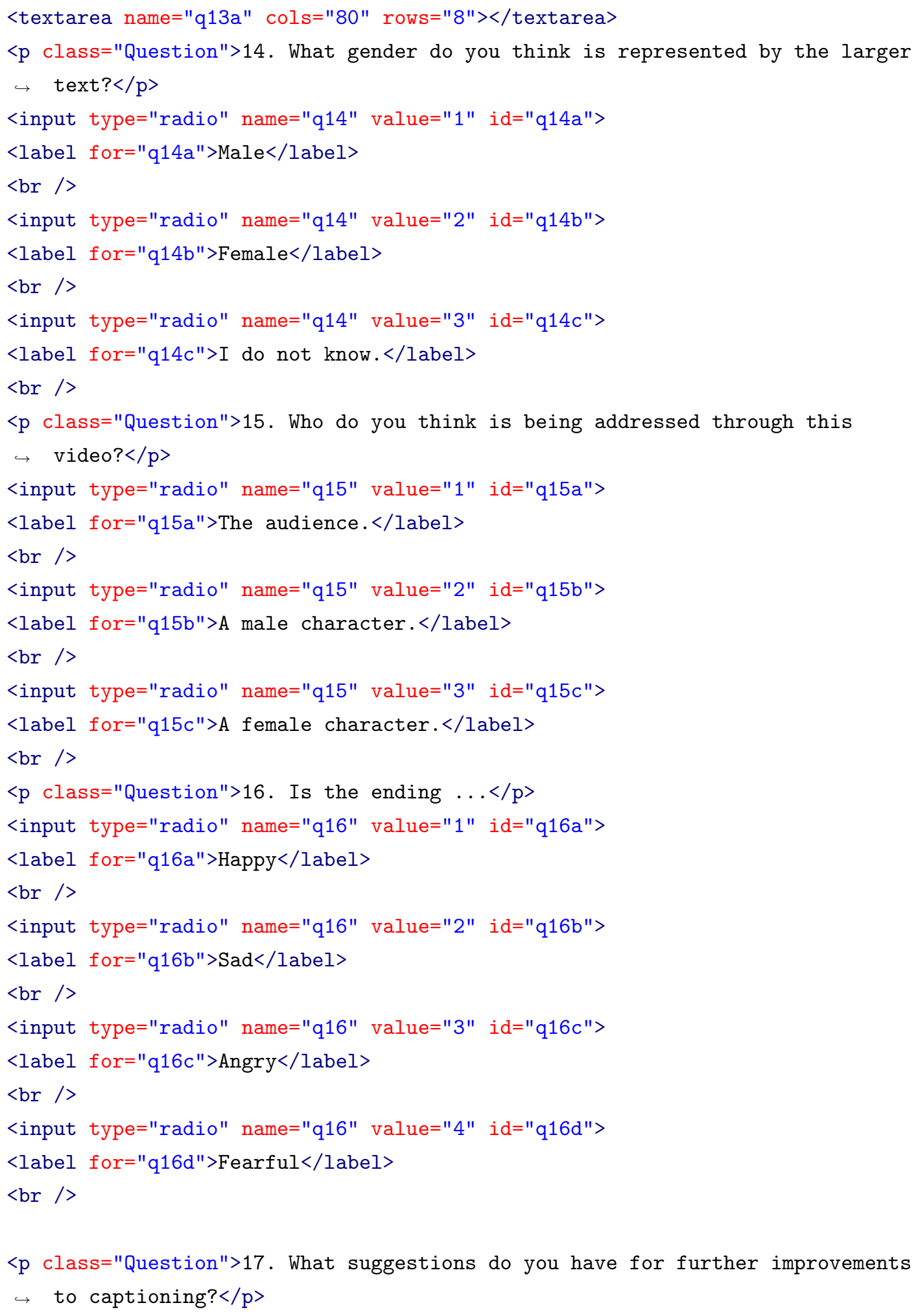




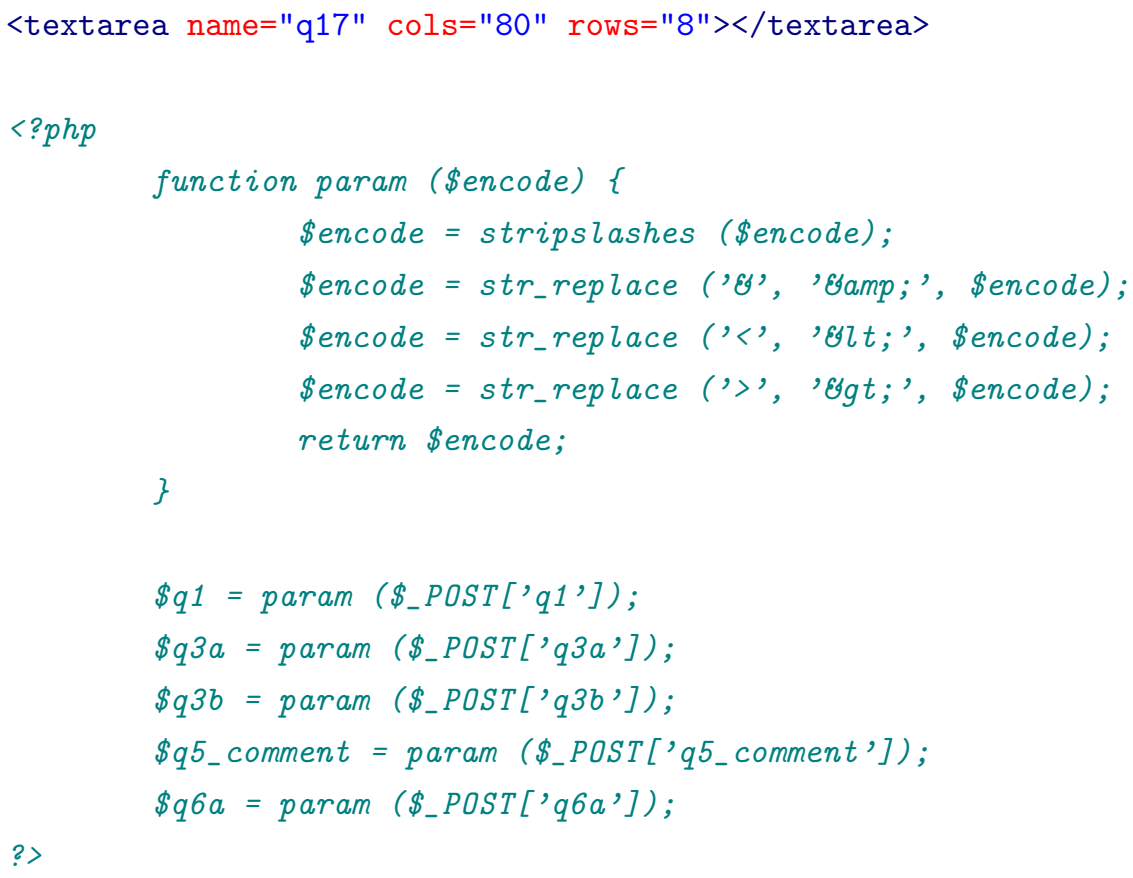




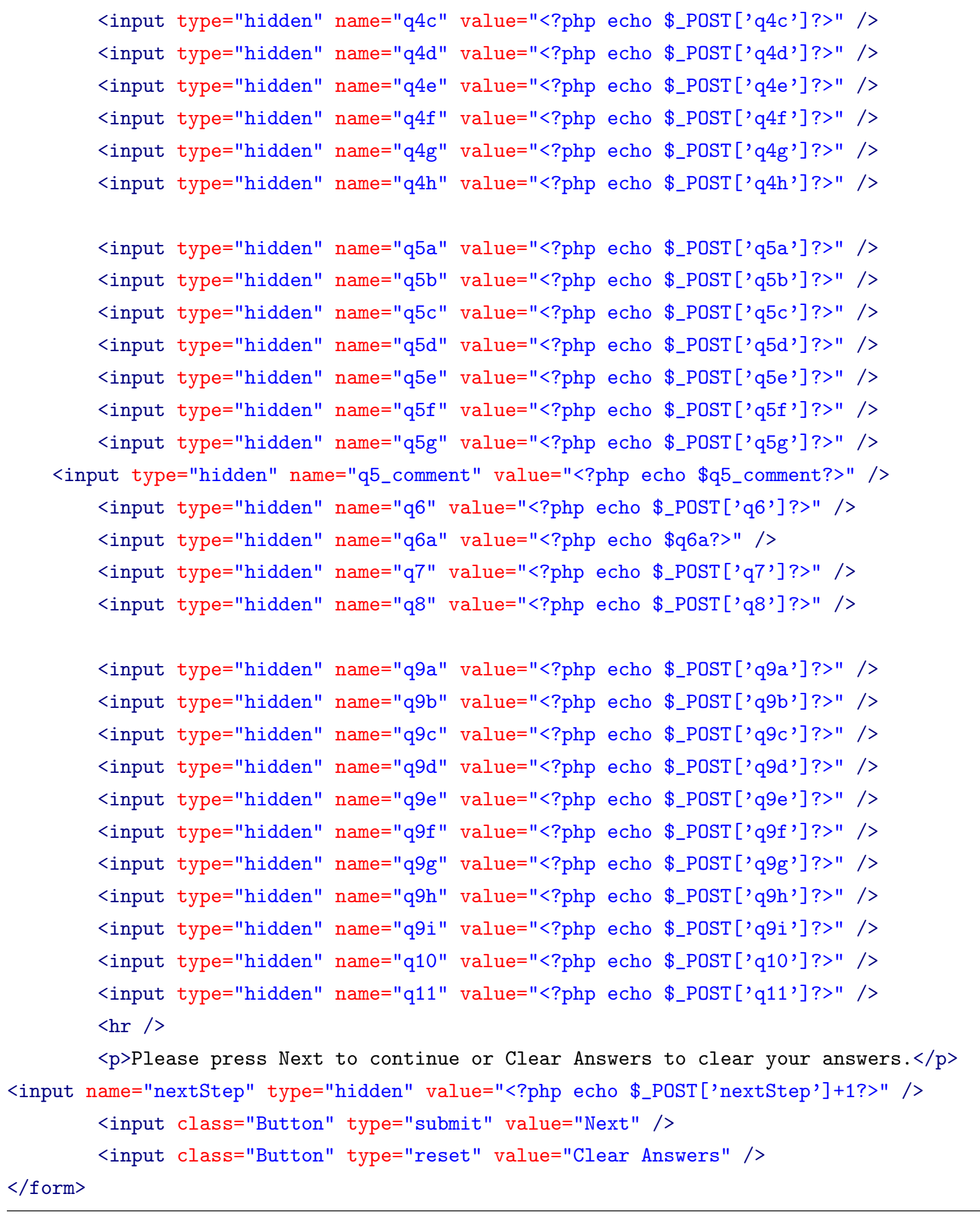

Listing 8: Forgive Me Questions Page 4. 
<h4>Step <?php echo \$_POST['nextStep']?> of <?php echo \$_SESSION['numSteps']? ></h4> <form action="../poststudy.php" method="post">

<h1>Study Questionnaire - Cont'd $</$ h1 $>$

$<\mathrm{p}$ class="Question" $>17$. What suggestions do you have for further improvements $\hookrightarrow$ to captioning? $</ \mathrm{p}>$

$<$ textarea name="q17" cols="80" rows="8" ></textarea $>$

$<? p h p$

function param (\$encode) \{

\$encode = stripslashes (\$encode);

\$encode = str_replace ('\&', 'Eamp;', \$encode);

\$encode = str_replace ('<', 'Glt;', \$encode);

\$encode = str_replace ('>', 'Egt; ', \$encode);

return \$encode;

\}

$\$ q 1=\operatorname{param}\left(\$+P O S T\left[{ }^{\prime} q 1 '\right]\right) ;$

$\$ q 3 a=\operatorname{param}\left(\$_{-} P O S T\left[{ }^{\prime} q 3 a,\right]\right)$;

$\$ q 3 b=\operatorname{param}\left(\$ \_P O S T[' q 3 b ']\right)$;

$\$ q 5_{-}$comment $=$param $\left(\$_{-} P O S T\left[' q 5_{-}\right.\right.$comment']);

$\$ q 6 a=\operatorname{param}\left(\$ \_P O S T\left[' q 6 a^{\prime}\right]\right)$;

$\$ q 13 a=\operatorname{param}\left(\$_{-} P O S T\left[{ }^{\prime} q 13 a^{\prime}\right]\right)$;

?>

<input type="hidden" name="hearing" value="<?php echo \$_POST['hearing']?>" /> <input type="hidden" name="gender" value="<?php echo \$_POST['gender'] ?>" />

<input type="hidden" name="age" value="<?php echo \$_POST['age']?>" />

<input type="hidden" name="education" value="<?php echo \$_POST['education'] ?>" $\hookrightarrow \quad />$

<input type="hidden" name="frequencia" value="<?php echo

$\hookrightarrow$ \$_POST['frequencia'] ?>" />

<input type="hidden" name="q1" value="<?php echo \$q1?>" />

<input type="hidden" name="q2a" value="<?php echo \$_POST['q2a']?>" />

<input type="hidden" name="q2b" value="<?php echo \$_POST['q2b']?>" />

<input type="hidden" name="q2c" value="<?php echo \$_POST['q2c']?>" />

<input type="hidden" name="q2d" value="<?php echo \$_POST['q2d']?>" />

<input type="hidden" name="q2e" value="<?php echo \$_POST['q2e']?>" />

<input type="hidden" name="q2f" value="<?php echo \$_POST['q2f']?>" /> 
<input type="hidden" name="q2g" value="<?php echo \$_POST['q2g']?>" /> <input type="hidden" name="q2h" value="<?php echo \$_POST['q2h']?>" /> <input type="hidden" name="q3a" value="<?php echo \$q3a?>" />

<input type="hidden" name="q3b" value="<?php echo \$q3b?>" /> <input type="hidden" name="q4a" value="<?php echo \$_POST['q4a']?>" /> <input type="hidden" name="q4b" value="<?php echo \$_POST['q4b']?>" /> <input type="hidden" name="q4c" value="<?php echo \$_POST['q4c']?>" /> <input type="hidden" name="q4d" value="<?php echo \$_POST['q4d']?>" /> <input type="hidden" name="q4e" value="<?php echo \$_POST['q4e']?>" /> <input type="hidden" name="q4f" value="<?php echo \$_POST['q4f']?>" /> <input type="hidden" name="q4g" value="<?php echo \$_POST['q4g']?>" /> <input type="hidden" name="q4h" value="<?php echo \$_POST['q4h']?>" />

<input type="hidden" name="q5a" value="<?php echo \$_POST['q5a']?>" /> <input type="hidden" name="q5b" value="<?php echo \$_POST['q5b']?>" /> <input type="hidden" name="q5c" value="<?php echo \$_POST['q5c'] ?>" /> <input type="hidden" name="q5d" value="<?php echo \$_POST['q5d']?>" /> <input type="hidden" name="q5e" value="<?php echo \$_POST['q5e']?>" /> <input type="hidden" name="q5f" value="<?php echo \$_POST['q5f']?>" /> <input type="hidden" name="q5g" value="<?php echo \$_POST['q5g']?>" />

<input type="hidden" name="q5_comment" value="<?php echo \$q5_comment?>" /> <input type="hidden" name="q6" value="<?php echo \$_POST['q6'] ?>" /> <input type="hidden" name="q6a" value="<?php echo \$q6a?>" /> <input type="hidden" name="q7" value="<?php echo \$_POST['q7']?>" /> <input type="hidden" name="q8" value="<?php echo \$_POST['q8']?>" />

<input type="hidden" name="q9a" value="<?php echo \$_POST['q9a']?>" /> <input type="hidden" name="q9b" value="<?php echo \$_POST['q9b']?>" /> <input type="hidden" name="q9c" value="<?php echo \$_POST['q9c']?>" /> <input type="hidden" name="q9d" value="<?php echo \$_POST['q9d']?>" /> <input type="hidden" name="q9e" value="<?php echo \$_POST['q9e']?>" /> <input type="hidden" name="q9f" value="<?php echo \$_POST['q9f']?>" /> <input type="hidden" name="q9g" value="<?php echo \$_POST['q9g']?>" /> <input type="hidden" name="q9h" value="<?php echo \$_POST['q9h']?>" /> <input type="hidden" name="q9i" value="<?php echo \$_POST['q9i']?>" /> <input type="hidden" name="q10" value="<?php echo \$_POST['q10']?>" /> <input type="hidden" name="q11" value="<?php echo \$_POST['q11']?>" />

<input type="hidden" name="q12" value="<?php echo \$_POST['q12']?>" /> 


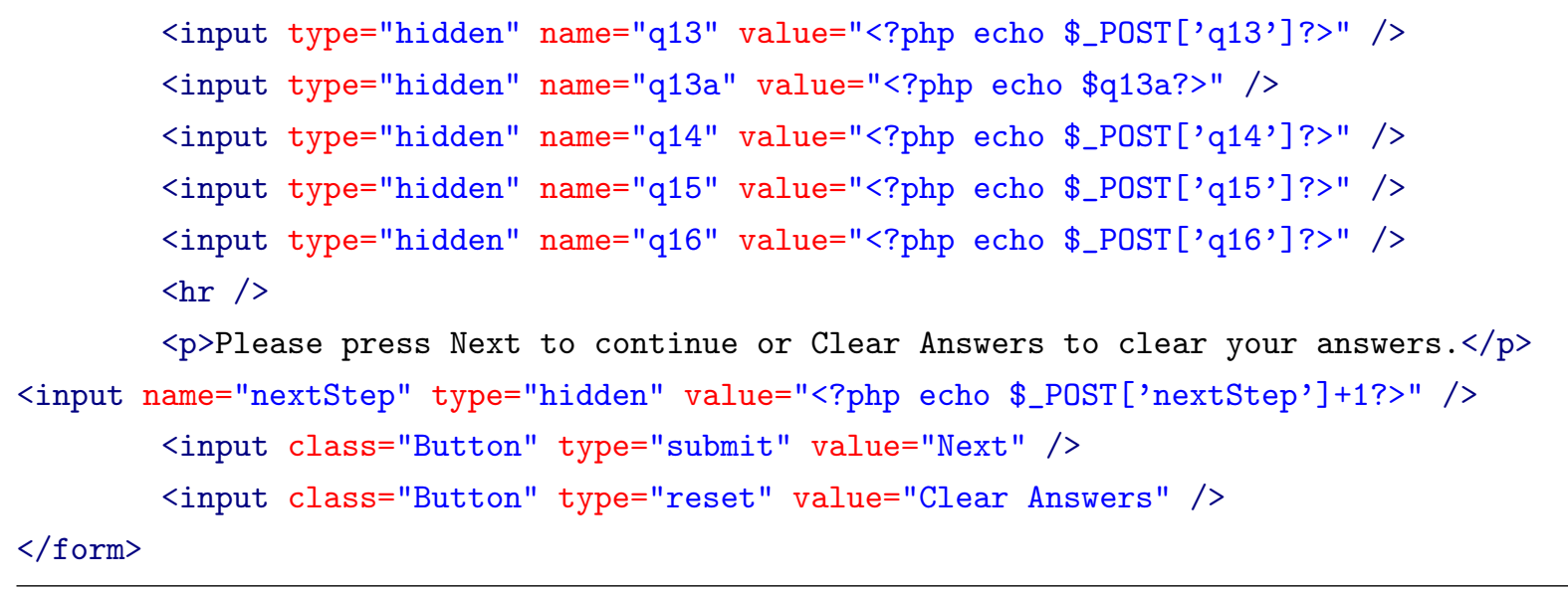

Listing 9: Forgive Me Questions Page 5.

<h4>Step <?php echo \$_POST['nextStep']?> of <?php echo \$_SESSION ['numSteps']? > </h4> <form action="study2.php" method="post"> <!--skipping poststudy2 since its empty --> $<$ h1 $>$ Study Questionnaire</h1 > $<$ p class="Question" $>1$. In a few sentences (or less), please describe what the $\hookrightarrow$ video was about. $\langle/ \mathrm{p}\rangle$ <textarea name="q1" cols="80" rows="8" ></textarea> $<$ p class="Question" $>2$. Please rate each of the following aspects of the text. $\hookrightarrow$ Select the option that best fits your rating. $</ p>$ <table cellpadding="0" cellspacing="0">

$<$ tr $>$

$<$ th $></$ th $>$

$<$ th $>$ Liked very much $</$ th $>$

$<$ th $>$ Liked $</$ th $>$

$<$ th $>$ Neither liked nor disliked $</$ th $>$

$<$ th $>$ Disliked $</$ th $>$

$<$ th $>$ Disliked very much $</$ th $>$

$</ \operatorname{tr}>$

$\langle$ tr $>$

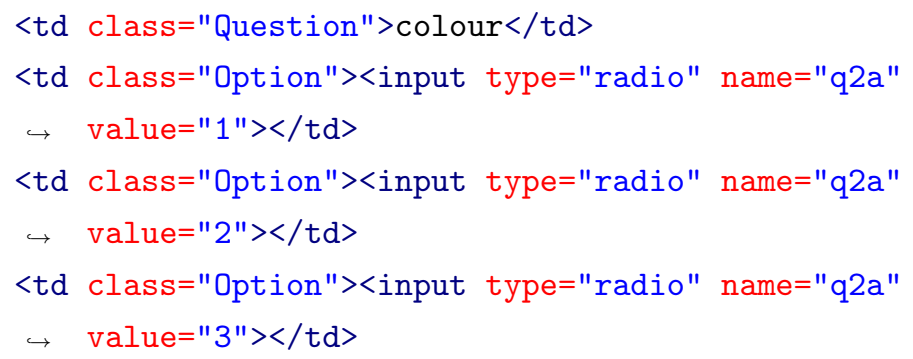




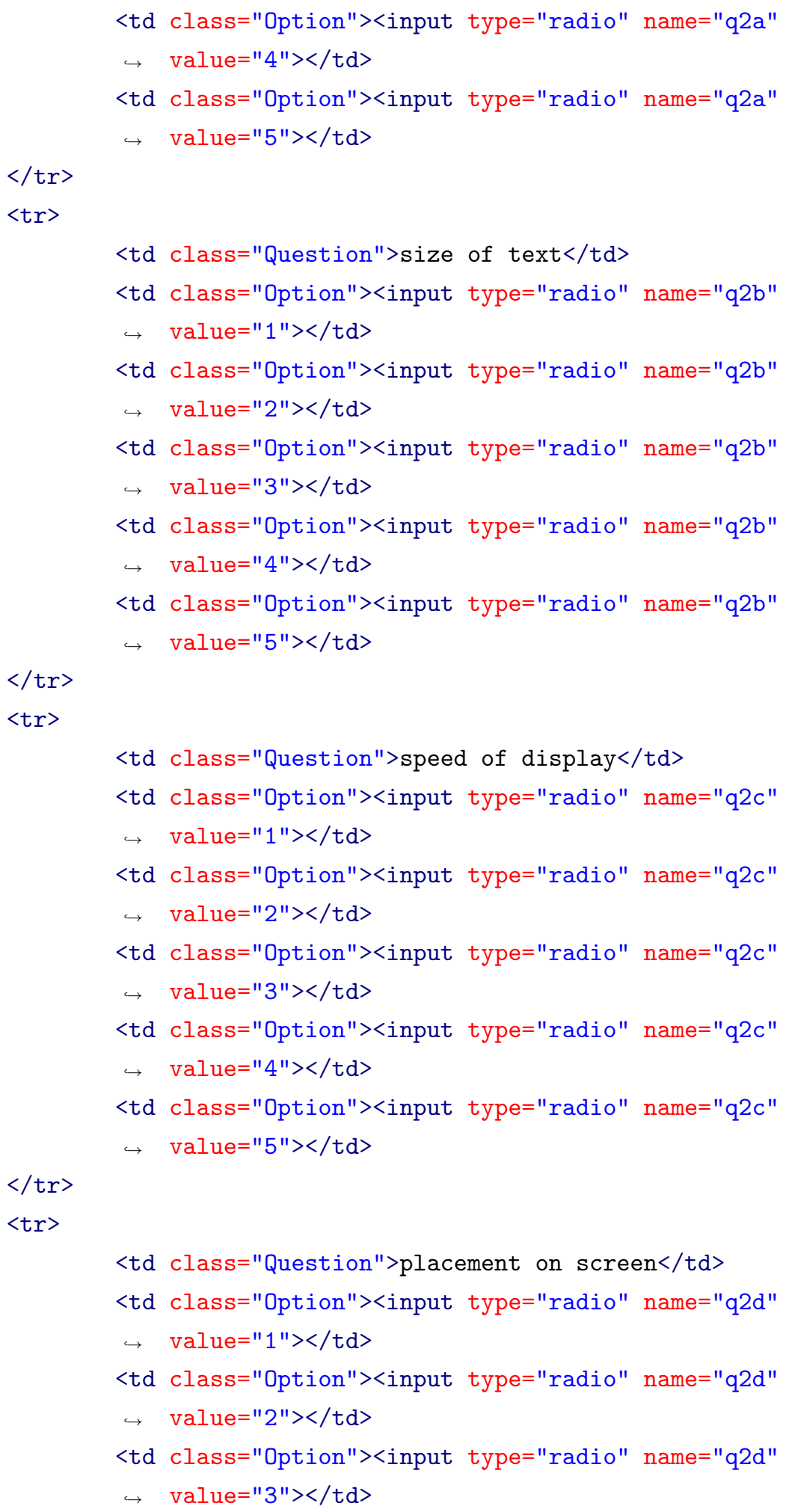




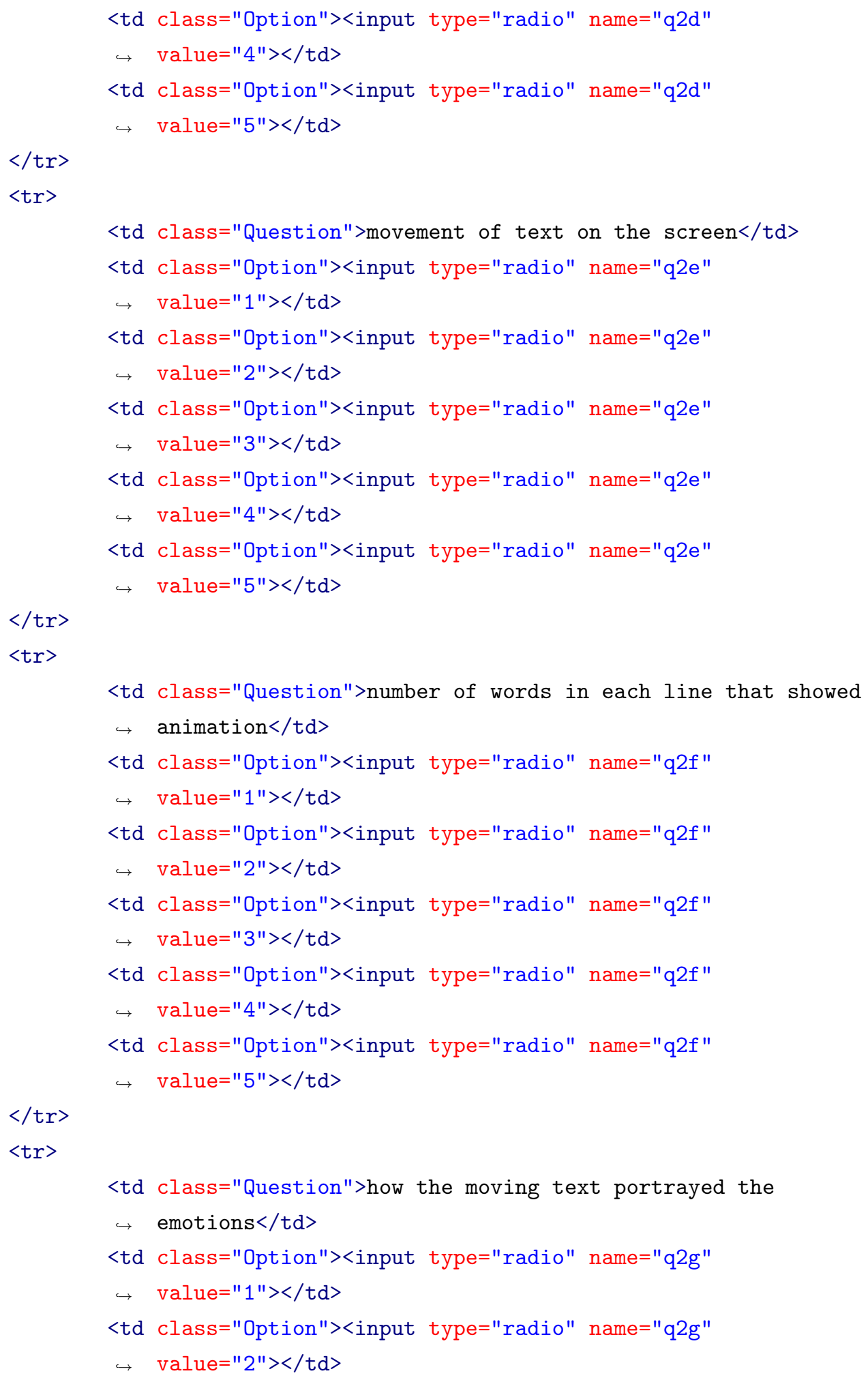




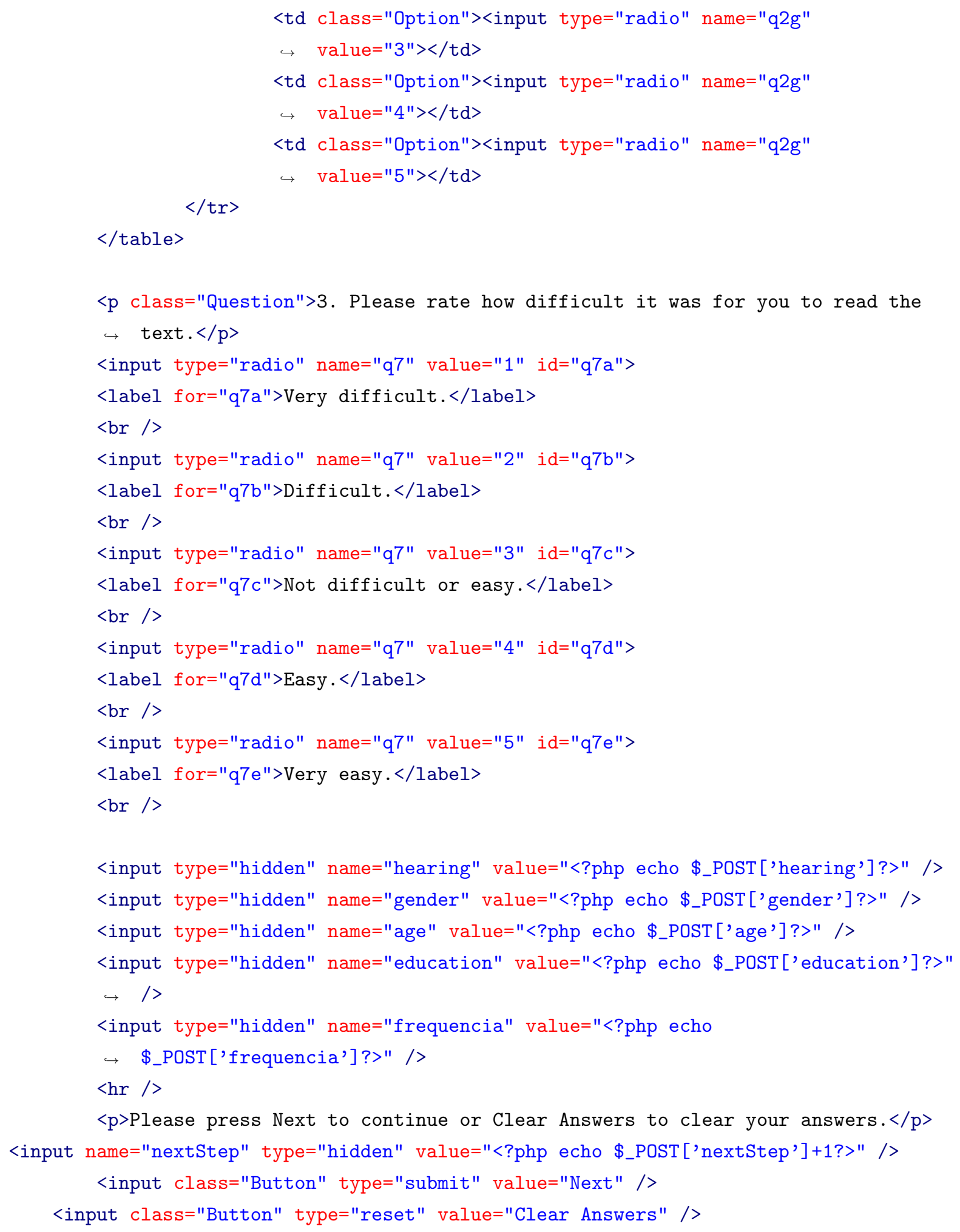


$</$ form $>$

Listing 10: Family Snapshot Questions Page 1.

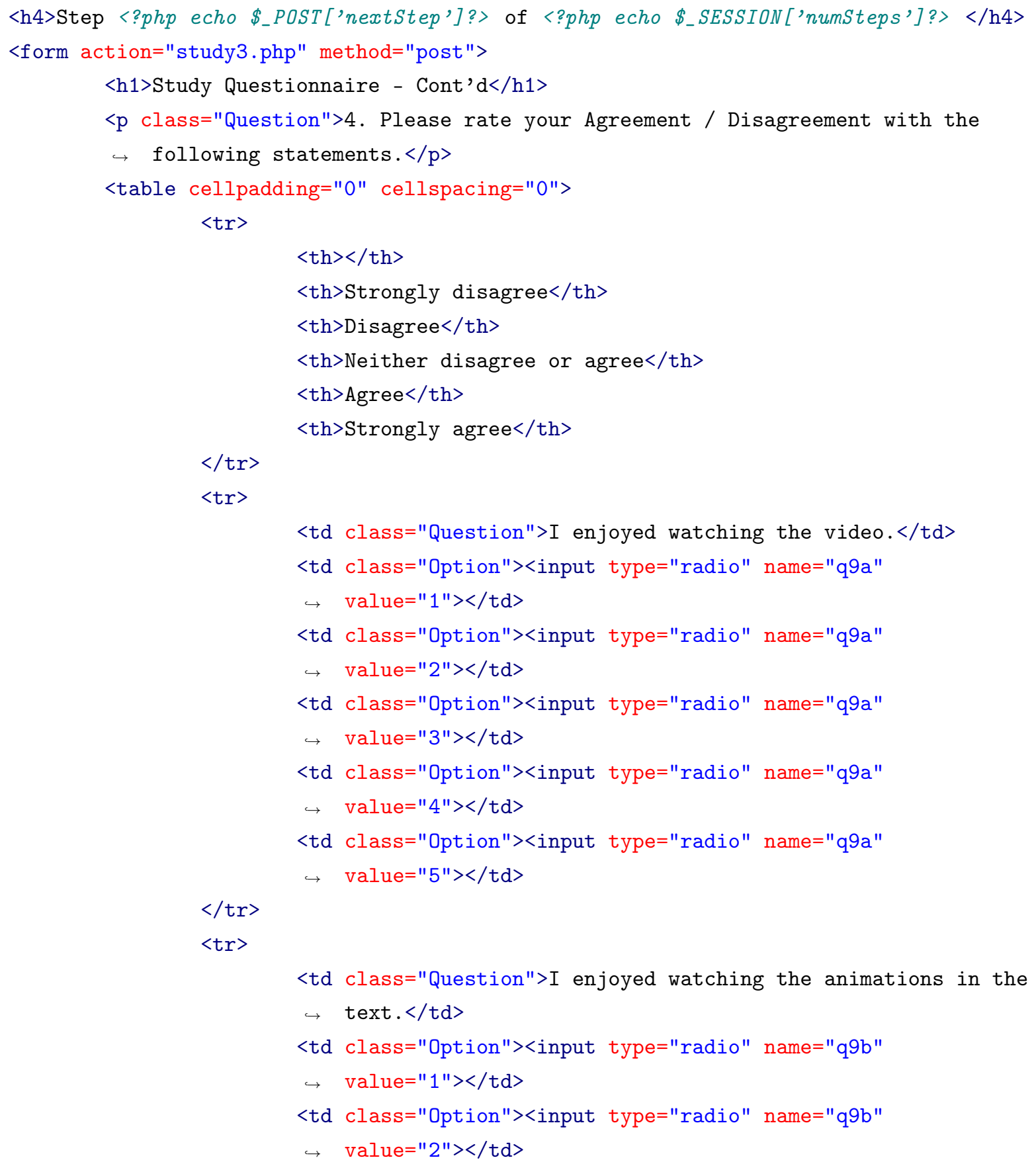




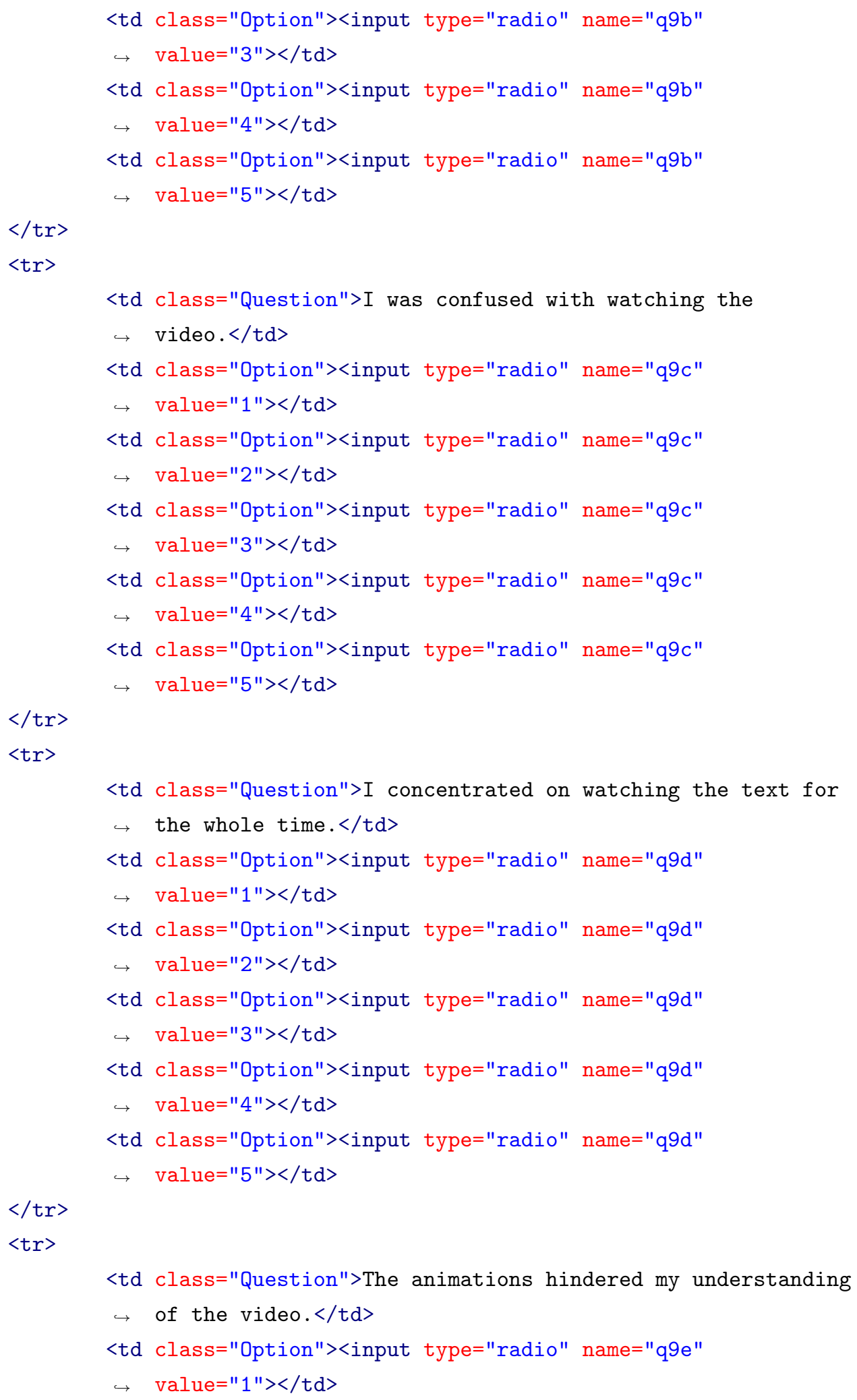




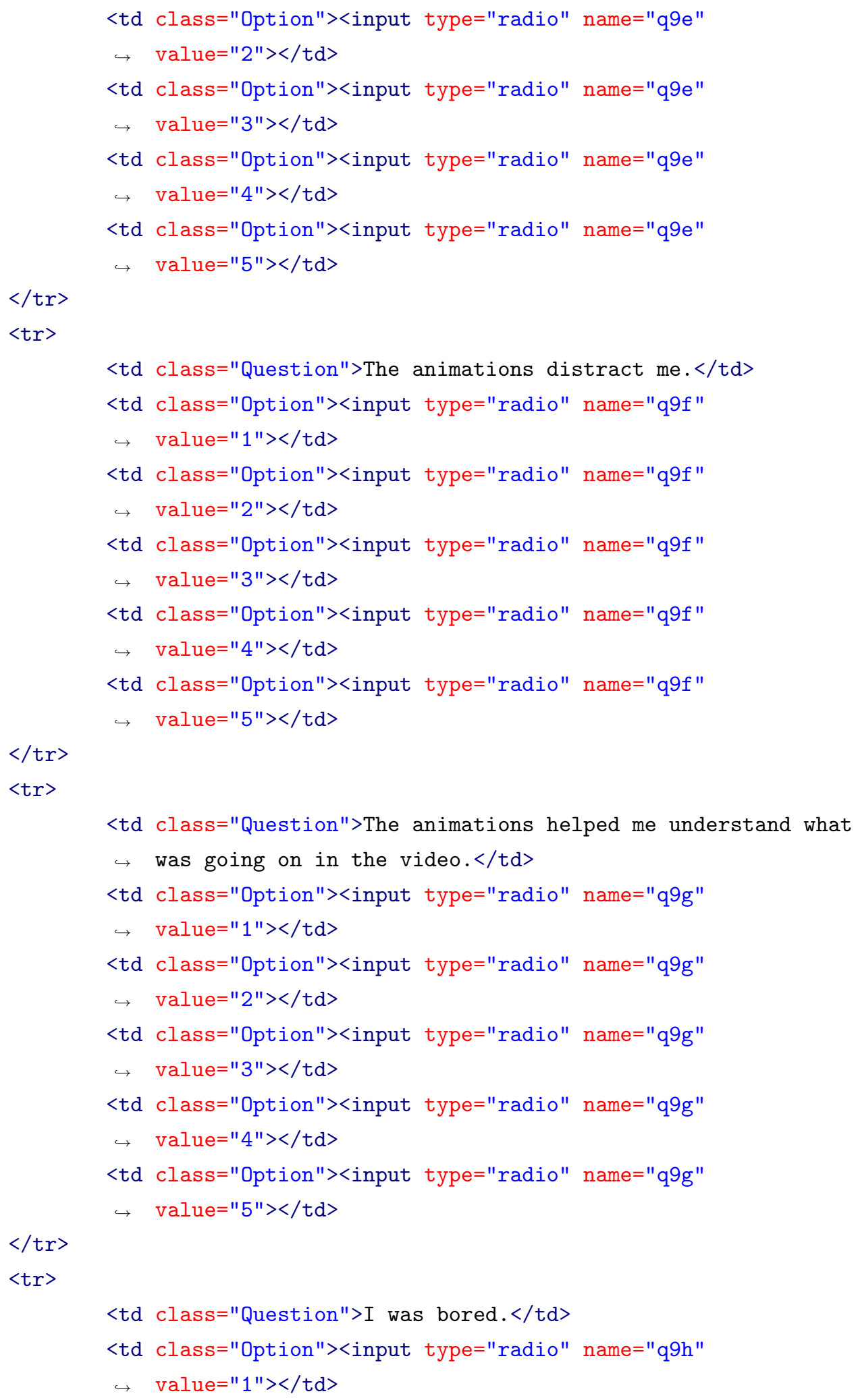




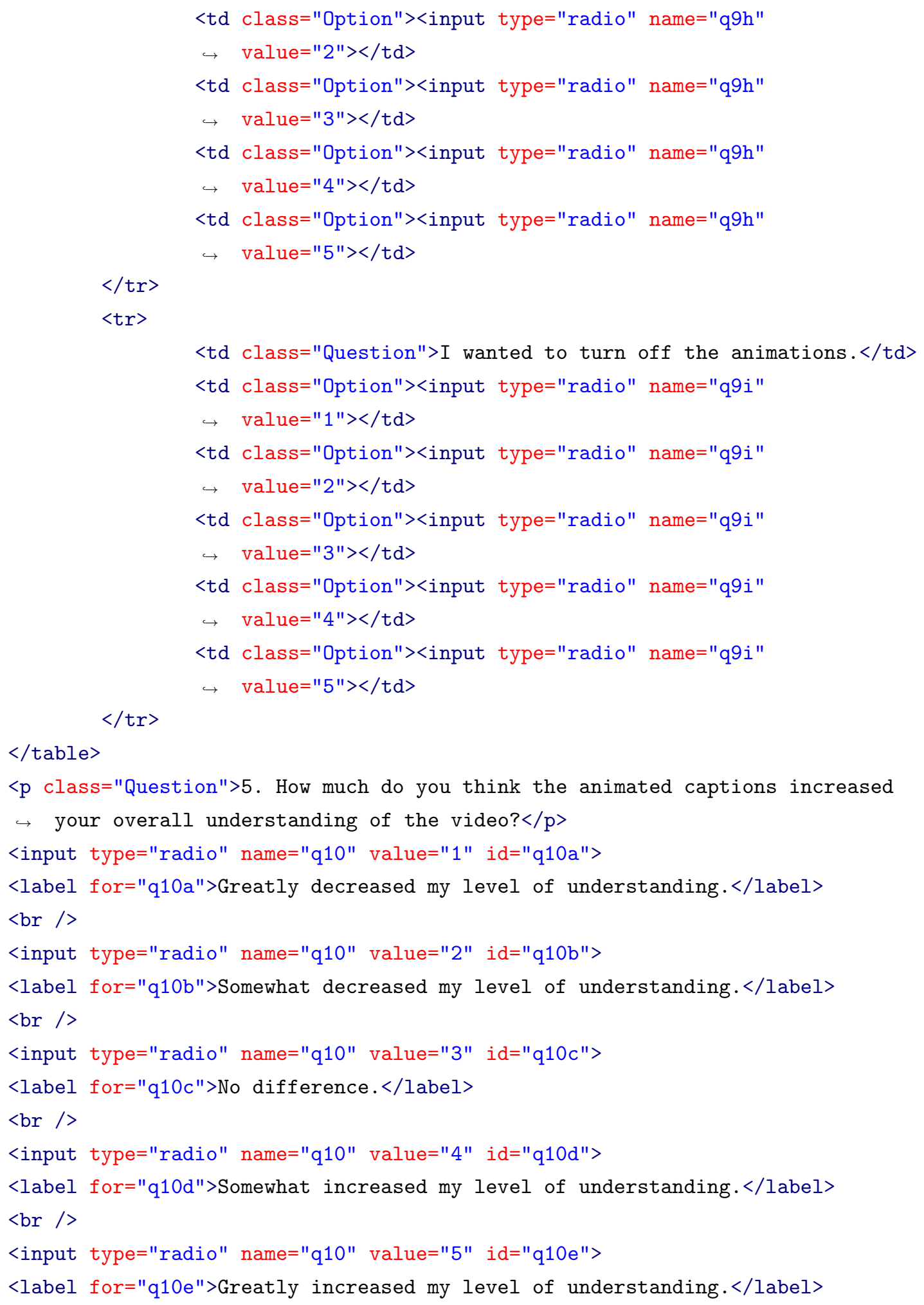


$<$ br $/>$

$<\mathrm{p}$ class="Question" $>6$. How much do you think the animated captions increased

$\hookrightarrow$ your overall understanding of the emotions that were being expressed? $</ p>$ <input type="radio" name="q11" value="1" id="q11a">

$<$ label for="q11a">Greatly decreased my level of understanding. $</$ label $>$

$<$ br $/>$

<input type="radio" name="q11" value="2" id="q11b">

$<$ label for="q11b" >Somewhat decreased my level of understanding. $</$ label $>$

$<$ br $/>$

<input type="radio" name="q11" value="3" id="q11c">

$<$ label for="q11c" $>$ No difference. $</$ label $>$

$<$ br $/>$

<input type="radio" name="q11" value="4" id="q11d">

$<$ label for="q11d">Somewhat increased my level of understanding. </label>

$<$ br $/>$

<input type="radio" name="q11" value="5" id="q11e">

$<$ label for="q11e">Greatly increased my level of understanding. $</$ label $>$

$<$ br $/>$

$<? p h p$

function param (\$encode) \{

\$encode $=$ stripslashes $(\$$ encode $)$;

\$encode = str_replace ('\&', 'Eamp;', \$encode);

\$encode = str_replace ('<', 'Glt;', \$encode);

\$encode = str_replace ('>', 'Egt;', \$encode);

return addslashes (\$encode);

\}

$\$ q 1=\operatorname{param}\left(\$ \_\right.$POST [' $\left.\left.q 1^{\prime}\right]\right) ;$

$\$ q 3=\operatorname{param}\left(\$ \_\right.$POST ['q3']);

$\$ q 6 a=\operatorname{param}\left(\$ \_P O S T\left[{ }^{\prime} q 6 a^{\prime}\right]\right)$;

?>

<input type="hidden" name="hearing" value="<?php echo \$_POST['hearing'] ?>" />

<input type="hidden" name="gender" value="<?php echo \$_POST['gender'] ?>" />

<input type="hidden" name="age" value="<?php echo \$_POST['age']?>" />

<input type="hidden" name="education" value="<?php echo \$_POST['education'] ?>"

$\rightarrow \quad />$

<input type="hidden" name="frequencia" value="<?php echo

$\rightarrow$ \$_POST ['frequencia'] ?>" /> 


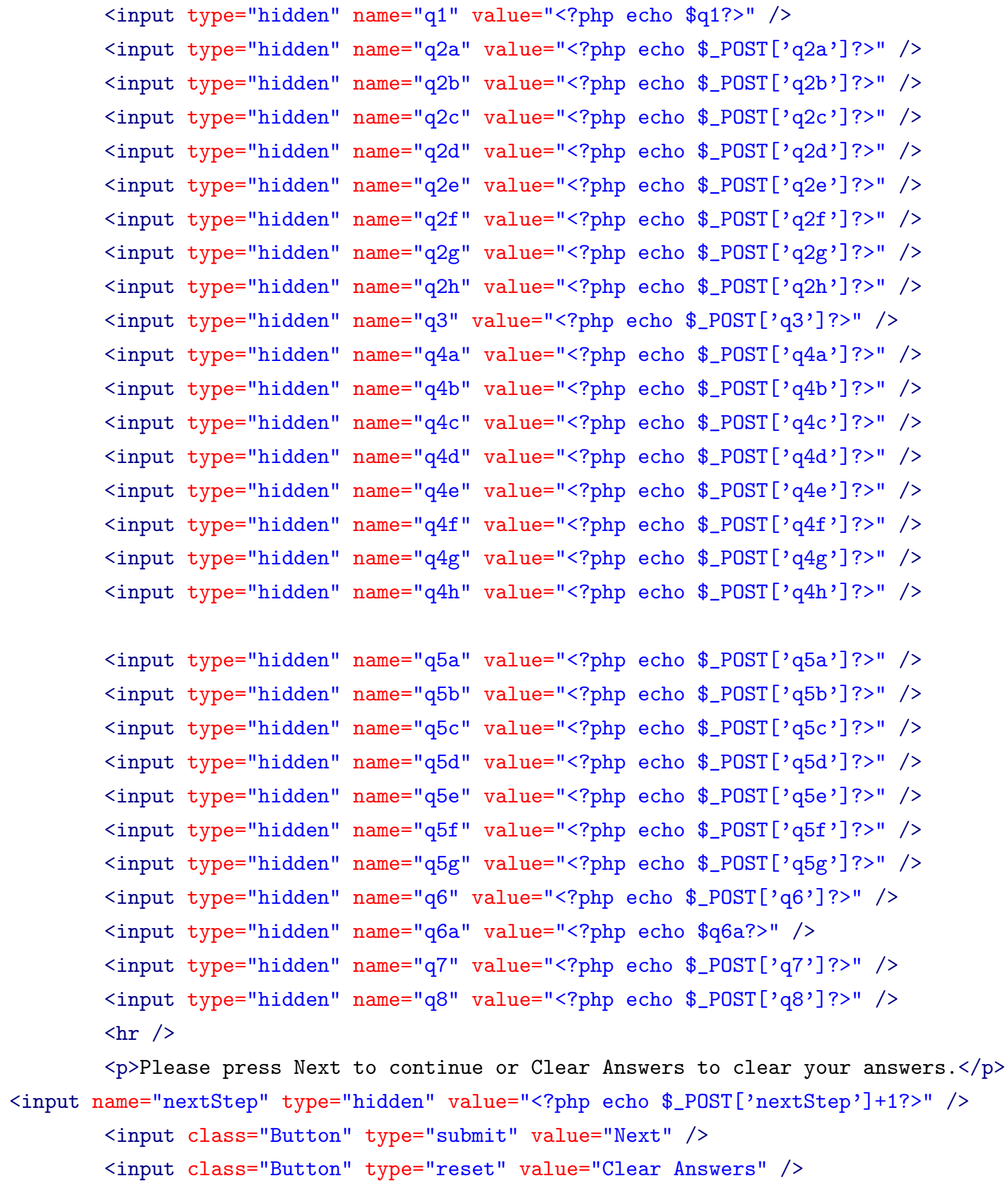

Listing 11: Family Snapshot Questions Page 2. 
<h4>Step <?php echo \$_POST['nextStep']?> of <?php echo \$_SESSION['numSteps']? </h4> <form action="study4.php" method="post">

$<$ h1 Study Questionnaire - Cont'd $</$ h1 $>$

$<\mathrm{p}$ class="Question" $>7$. How much do you think the animated text distracted you

$\hookrightarrow$ from understanding the video?</p $>$

<input type="radio" name="q12" value="1" id="q12a">

$<$ label for="q12a">Did not distract me.</label>

$<$ br / >

<input type="radio" name="q12" value="2" id="q12b">

$<$ label for="q12b">Slightly distracted me.</label>

$<\mathrm{br} />$

<input type="radio" name="q12" value="3" id="q12c">

$<$ label for="q12c">Did not notice.</label>

$<\mathrm{br} />$

<input type="radio" name="q12" value="4" id="q12d">

$<$ label for="q12d">Somewhat distracted me.</label>

$<$ br / >

<input type="radio" name="q12" value="5" id="q12e">

$<$ label for="q12e">Greatly distracted me.</label>

$<\mathrm{br} />$

$<\mathrm{p}$ class="Question">8. Suppose you had just watched the video with friends who $\hookrightarrow$ were hearing, rate your willingness to engage in conversation with them $\hookrightarrow$ about the video. $\langle/ \mathrm{p}\rangle$

<input type="radio" name="q13" value="1" id="q13a">

$<$ label for="q13a">Not willing to discuss the video at all.</label>

$<$ br / >

<input type="radio" name="q13" value="2" id="q13b">

$<$ label for="q13b">Not really willing to discuss the video.</label>

$<\mathrm{br} />$

<input type="radio" name="q13" value="3" id="q13c">

$<$ label for="q13c">Don't care.</label>

$<$ br / >

<input type="radio" name="q13" value="4" id="q13d">

$<$ label for="q13d">Somewhat willing to discuss the video.</label>

$<$ br / >

<input type="radio" name="q13" value="5" id="q13e">

$<$ label for="q13e">Very willing to discuss the video.</label>

$<$ br / >

$<$ p class="Question" $>$ Please indicate why you selected your particular

$\hookrightarrow \quad$ rating. $</$ p $>$ 


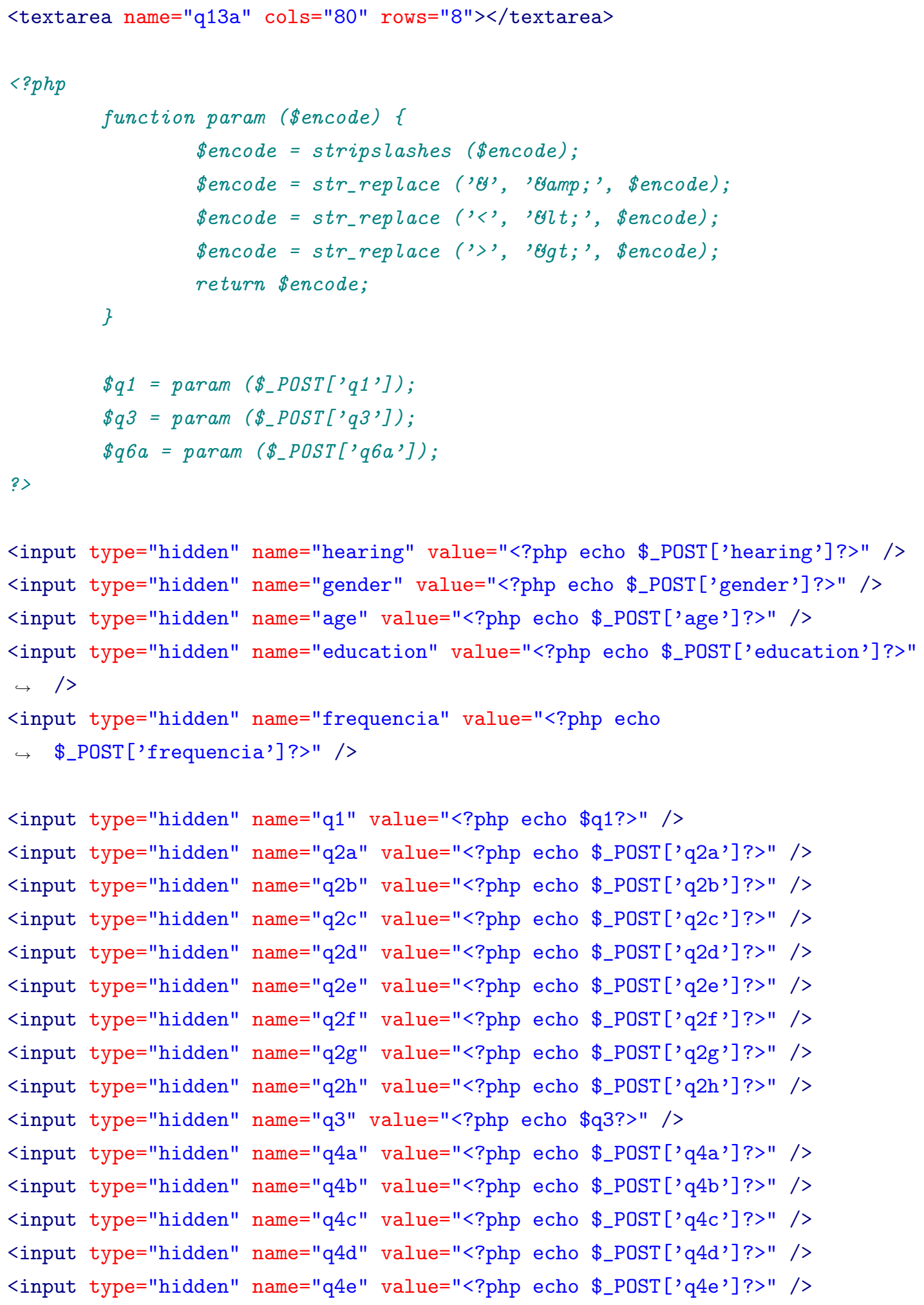




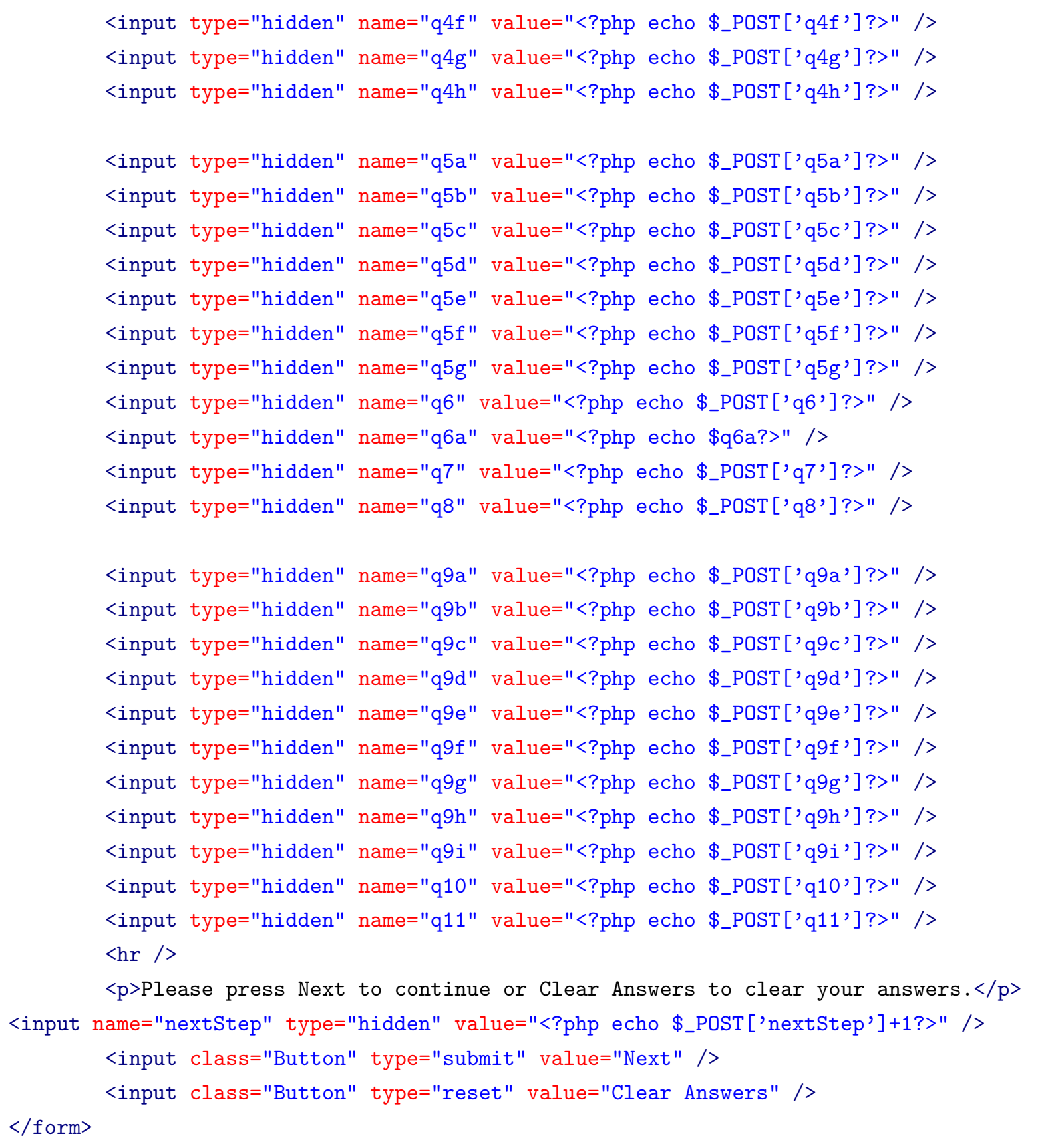

Listing 12: Family Snapshot Questions Page 3.

<h4>Step <?php echo \$_POST['nextStep']?> of <?php echo \$_SESSION ['numSteps']? > </h4> <form action="../poststudy.php" method="post">

$<$ h1 $>$ Study Questionnaire - Cont'd $</$ h1 $>$ 
$<\mathrm{p}$ class="Question" $>9$. What gender do you think is represented by the text? $</ \mathrm{p}>$ <input type="radio" name="q14" value="1" id="q14a">

$<$ label for="q14a" $>$ Male $</$ label $>$

$<$ br / >

<input type="radio" name="q14" value="2" id="q14b">

$<$ label for="q14b" $>$ Female $</$ label $>$

$<$ br / >

<input type="radio" name="q14" value="3" id="q14c">

$<$ label for="q14c">I do not know</label>

$<\mathrm{br} />$

$<$ p class="Question" $>10$. Who do you think is being addressed through this

$\hookrightarrow$ video?</p $>$

<input type="radio" name="q15" value="1" id="q15a">

$<$ label for="q15a" $>$ The audience</label>

$<$ br />

<input type="radio" name="q15" value="2" id="q15b">

$<$ label for="q15b">A male character</label>

$<$ br / >

<input type="radio" name="q15" value="3" id="q15c">

$<$ label for="q15c" $>$ A female character $</$ label $>$

$<$ br / >

$<$ p class="Question" $>11$. Is the ending ...</p $>$

<input type="radio" name="q16" value="1" id="q16a">

$<$ label for="q16a" $>$ Happy $</$ label $>$

$<$ br / >

<input type="radio" name="q16" value="2" id="q16b">

$<$ label for="q16b" $>$ Sad $</$ label $>$

$<\mathrm{br} />$

<input type="radio" name="q16" value="3" id="q16c">

$<$ label for="q16c" $>$ Angry $</$ label $>$

$<$ br / >

<input type="radio" name="q16" value="4" id="q16d">

$<$ label for="q16d">Fearful</label>

$<\mathrm{br} />$

$<$ p class="Question" $>12$. What suggestions do you have for further improvements

$\hookrightarrow$ to captioning? $</ \mathrm{p}>$

<textarea name="q17" cols="80" rows="8" ></textarea $>$ 


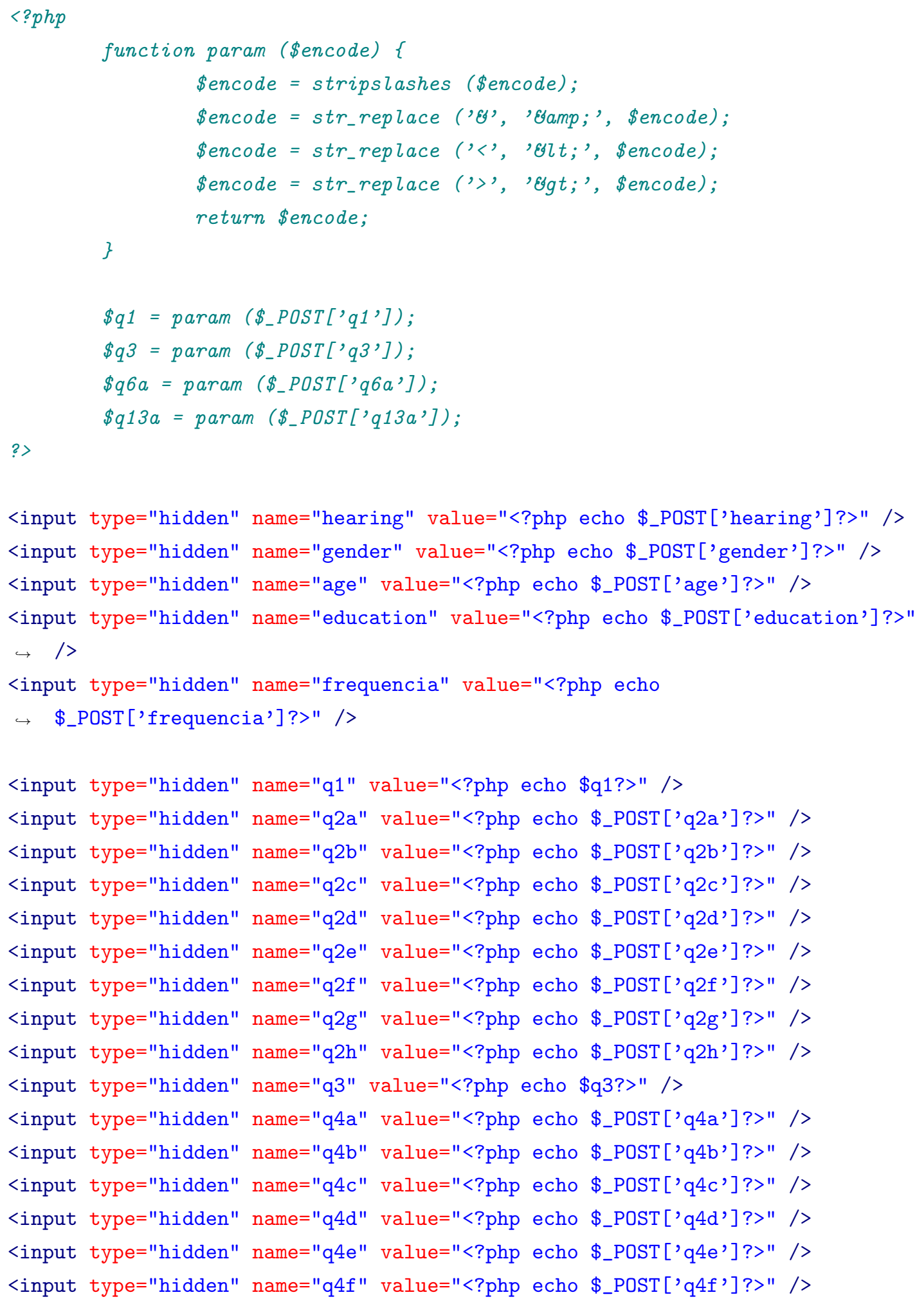




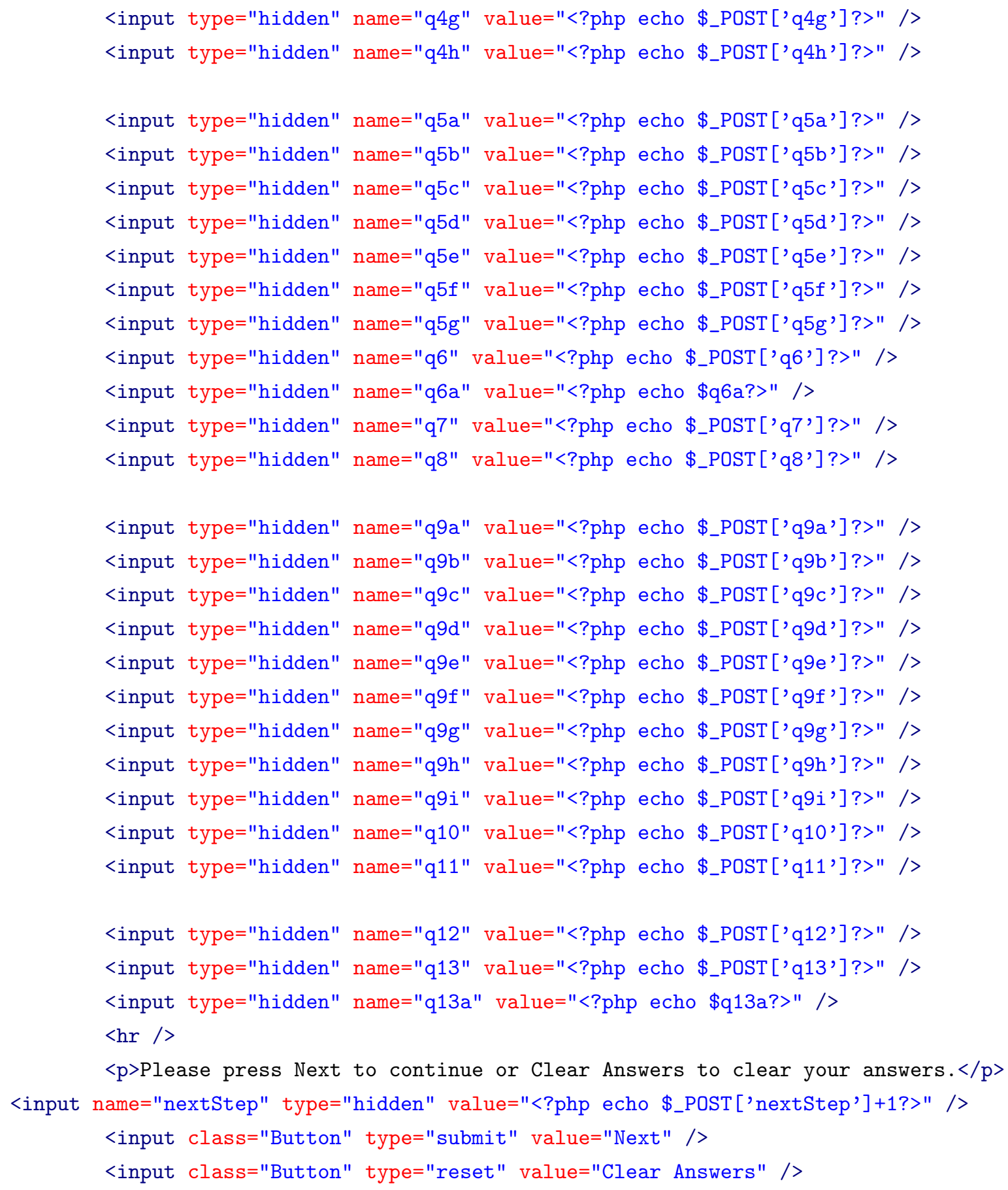

Listing 13: Family Snapshot Questions Page 4. 
<h4>Step <?php echo \$_POST['nextStep']?> of <?php echo \$_SESSION['numSteps']? ></h4> <form action="poststudy2.php" method="post">

$<$ h1 $>$ Music Questionnaire</h1>

$<$ p $>$ Because we used the lyrics of a song in this study, we are interested in $\hookrightarrow$ your perspective

and experience with music. $\langle/ p>$

$<\mathrm{p}$ class="Question" $>1$. How often do you listen to music? $</ \mathrm{p}>$

<input type="radio" name="frequency" value="1" id="frequency_a">

$<$ label for="frequency_a" $>$ All the time $</$ label $>$

$<$ br / >

<input type="radio" name="frequency" value="2" id="frequency_b">

$<$ label for="frequency_b" $>$ Frequently $</$ label $>$

$<$ br / >

<input type="radio" name="frequency" value="3" id="frequency_c">

$<$ label for="frequency_c" $>$ Sometimes $</$ label $>$

$<\mathrm{br} />$

<input type="radio" name="frequency" value="4" id="frequency_d">

$<$ label for="frequency_d" $>$ Rarely</label>

$<$ br / >

<input type="radio" name="frequency" value="5" id="frequency_e">

$<$ label for="frequency_e" $>$ Never $</$ label $>$

$<\mathrm{br} />$

$<\mathrm{p}$ class="Question" $>$ Comments $:\langle/ \mathrm{p}\rangle$

<textarea name="frequency_comment" cols="80" rows="8" $></$ textarea $>$

$<\mathrm{p}$ class="Question" $>2$. Which of the following statements describes you

$\hookrightarrow$ best?</p>

<input type="radio" name="description" value="1" id="description_a">

<label for="description_a">I really enjoy listening to music.</label>

$<$ br / >

<input type="radio" name="description" value="2" id="description_b">

<label for="description_b">I like listening to music.</label>

$<$ br / >

<input type="radio" name="description" value="3" id="description_c">

$<$ label for="description_c" $>$ I do not like or dislike music.</label>

$<$ br / >

<input type="radio" name="description" value="4" id="description_d"> 
$<$ label for="description_d">I do not like listening to music.</label> $<\mathrm{br} />$

<input type="radio" name="description" value="5" id="description_e">

<label for="description_e">I do not see the point of music.</label>

$<$ br / >

$\langle$ p class="Question" $>$ Comments $:\langle/ p\rangle$

<textarea name="description_comment" cols="80" rows="8" $></$ textarea $>$

$<$ p class="Question" $>3$. How do you most often listen to music (check all that

$\hookrightarrow$ apply) $?</ \mathrm{p}>$

<input type="checkbox" name="listen_a" value="1" id="listen_a">

$<$ label for="listena" >0n radio</label>

$<$ br / >

<input type="checkbox" name="listen_b" value="2" id="listen_b">

<label for="listen_b">On mobile music player $</$ label $>$

$<\mathrm{br} />$

<input type="checkbox" name="listen_c" value="3" id="listen_c">

$<$ label for="listen_c" $>$ Stereo at home $</$ label $>$

$<$ br / $>$

<input type="checkbox" name="listen_d" value="4" id="listen_d">

$<$ label for="listen_d" $>$ Internet $</$ label $>$

$<$ br / >

<input type="checkbox" name="listen_e" value="5" id="listen_e">

<label for="listen_e" $>$ Television</label>

$<\mathrm{br} />$

$\langle$ p class="Question" $>$ Comments $:\langle/ p\rangle$

<textarea name="listen_comment" cols="80" rows="8" ></textarea $>$

$<$ p class="Question" $>4$. If information about music from film, television, and

$\hookrightarrow$ radio could be displayed, what would

be useful to have and

$\hookrightarrow$ what form would be best? $\langle/ p>$

<table cellpadding="0" cellspacing="0">

$\langle$ tr $>$

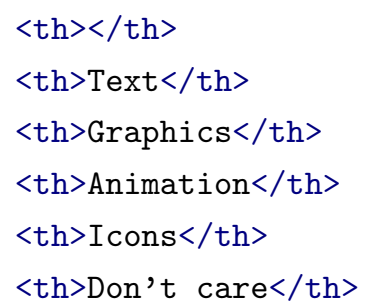


$</ \operatorname{tr}>$

$<\operatorname{tr}>$

<td class="Question" $>$ Lyrics/Words $</$ td $>$

<td class="Option" $><$ input type="radio" name="information_a"

$\hookrightarrow$ value="1" $\rangle\langle/$ td $\rangle$

<td class="Option" $><$ input type="radio" name="information_a"

$\hookrightarrow$ value="2" $>\langle/$ td $>$

<td class="Option" $><$ input type="radio" name="information_a"

$\hookrightarrow$ value $=" 3 "\rangle\langle/$ td $\rangle$

$<$ td class="Option" $><$ input type="radio" name="information_a"

$\hookrightarrow$ value $=" 4 ">\langle/$ td $\rangle$

<td class="Option" $><$ input type="radio" name="information_a"

$\hookrightarrow$ value $=" 5 ">\langle/$ td $\rangle$

$</ \operatorname{tr}>$

$\langle\operatorname{tr}>$

<td class="Question" $>$ Music Notes $</$ td $>$

$<$ td class="Option"><input type="radio" name="information_b"

$\hookrightarrow$ value $=" 1 ">\langle/$ td $\rangle$

<td class="Option"><input type="radio" name="information_b"

$\hookrightarrow$ value="2" $></$ td $>$

$<$ td class="Option" $><$ input type="radio" name="information_b"

$\hookrightarrow$ value $=" 3 "\rangle\langle/$ td $\rangle$

$<$ td class="Option" $><$ input type="radio" name="information_b"

$\hookrightarrow$ value $=" 4 "\rangle\langle$ td $\rangle$

$<$ td class="Option" $><$ input type="radio" name="information_b"

$\hookrightarrow$ value $=" 5 ">\langle/$ td $\rangle$

$</$ tr $>$

$<\operatorname{tr}>$

<td class="Question" $>$ Instruments $</$ td $>$

<td class="Option" $><$ input type="radio" name="information_c"

$\hookrightarrow$ value $=" 1 ">\langle/$ td $\rangle$

<td class="Option" $><$ input type="radio" name="information_c"

$\hookrightarrow$ value $=" 2 ">\langle/$ td $\rangle$

<td class="Option" $><$ input type="radio" name="information_c"

$\hookrightarrow$ value="3" $></$ td $>$

<td class="Option" $><$ input type="radio" name="information_c"

$\hookrightarrow$ value $=" 4 "\rangle\langle/$ td $\rangle$

$<$ td class="Option" $><$ input type="radio" name="information_c"

$\hookrightarrow$ value $=" 5 "\rangle\langle$ td $\rangle$ 
$\langle/ \operatorname{tr}\rangle$

$<\operatorname{tr}>$

$<$ td class="Question" $>$ Beat $/$ rhythm $</$ td $>$

$<$ td class="Option" $><$ input type="radio" name="information_d"

$\hookrightarrow$ value $=" 1 "></$ td $>$

<td class="Option" $><$ input type="radio" name="information_d"

$\hookrightarrow$ value $=" 2 "></$ td $>$

<td class="Option"><input type="radio" name="information_d"

$\hookrightarrow$ value $=" 3 ">\langle/$ td $\rangle$

<td class="Option" $><$ input type="radio" name="information_d"

$\hookrightarrow$ value $=" 4 "\rangle\langle$ td $\rangle$

<td class="Option"><input type="radio" name="information_d"

$\hookrightarrow$ value $=" 5 "\rangle\langle/$ td $\rangle$

$</ \operatorname{tr}\rangle$

$<\mathrm{tr}>$

$<$ td class="Question" $>$ Speed of music playing $</$ td $>$

<td class="Option"><input type="radio" name="information_e"

$\hookrightarrow$ value $=" 1 "></$ td $>$

<td class="Option"><input type="radio" name="information_e"

$\hookrightarrow$ value $=" 2 "></$ td $>$

<td class="Option" $><$ input type="radio" name="information_e"

$\hookrightarrow$ value $=" 3 ">\langle/$ td $\rangle$

<td class="Option"><input type="radio" name="information_e"

$\hookrightarrow$ value $=" 4 "\rangle\langle/$ td $\rangle$

$<$ td class="Option" $><$ input type="radio" name="information_e"

$\hookrightarrow$ value $=" 5 "\rangle\langle/$ td $\rangle$

$</ \operatorname{tr}\rangle$

$<$ tr $>$

<td class="Question" $>$ Emotion conveyed through the music $</$ td $>$

<td class="Option" $><$ input type="radio" name="information_f"

$\hookrightarrow$ value $=" 1 ">\langle/$ td $\rangle$

<td class="Option" $><$ input type="radio" name="information_f"

$\hookrightarrow$ value $=" 2 ">\langle/$ td $>$

<td class="Option" ><input type="radio" name="information_f"

$\hookrightarrow$ value="3" $></$ td $>$

$<$ td class="Option" $><$ input type="radio" name="information_f"

$\hookrightarrow$ value $=" 4 "></$ td $>$

$<$ td class="Option"><input type="radio" name="information_f"

$\hookrightarrow$ value $=" 5 ">\langle/$ td $>$ 


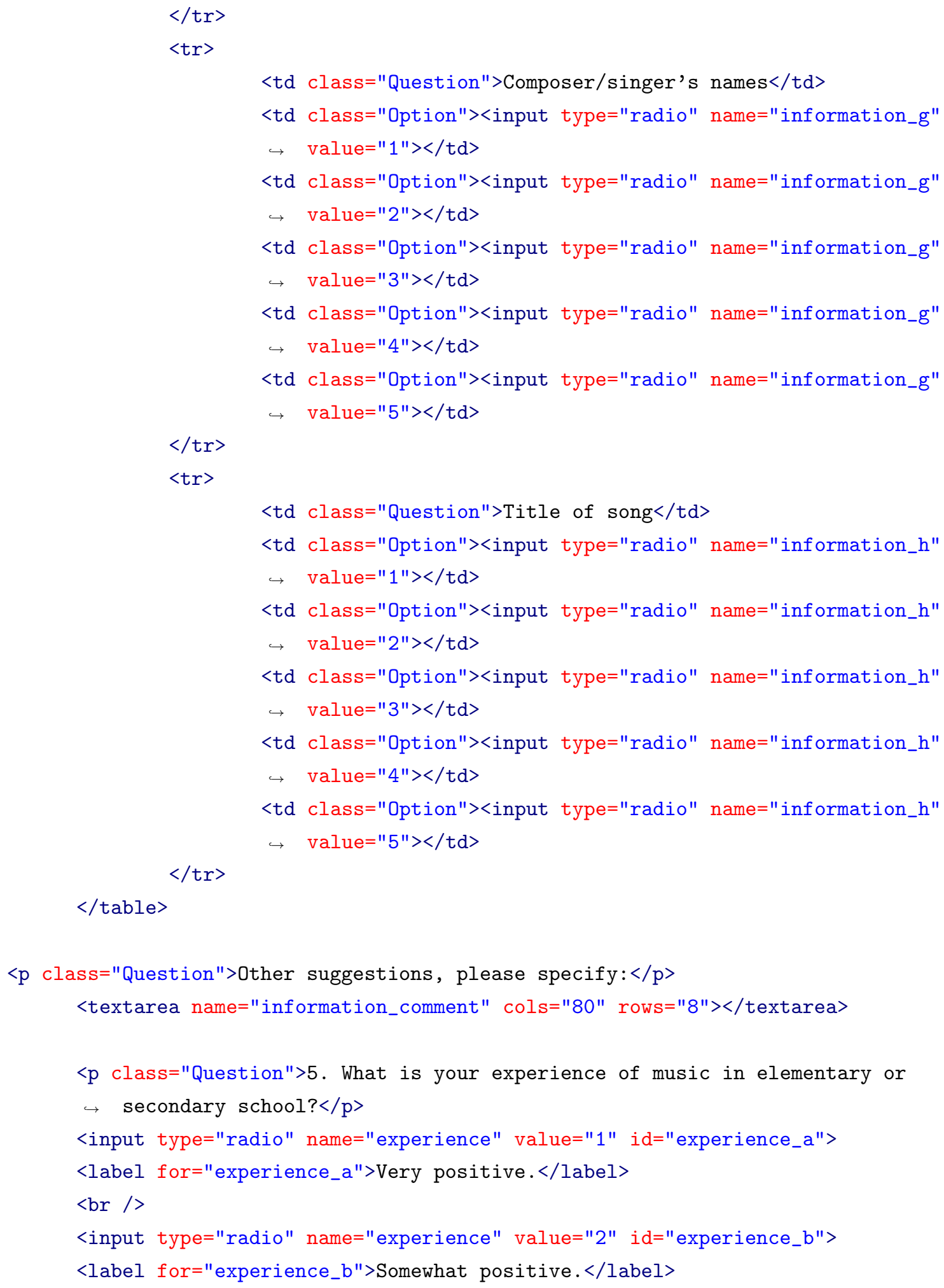




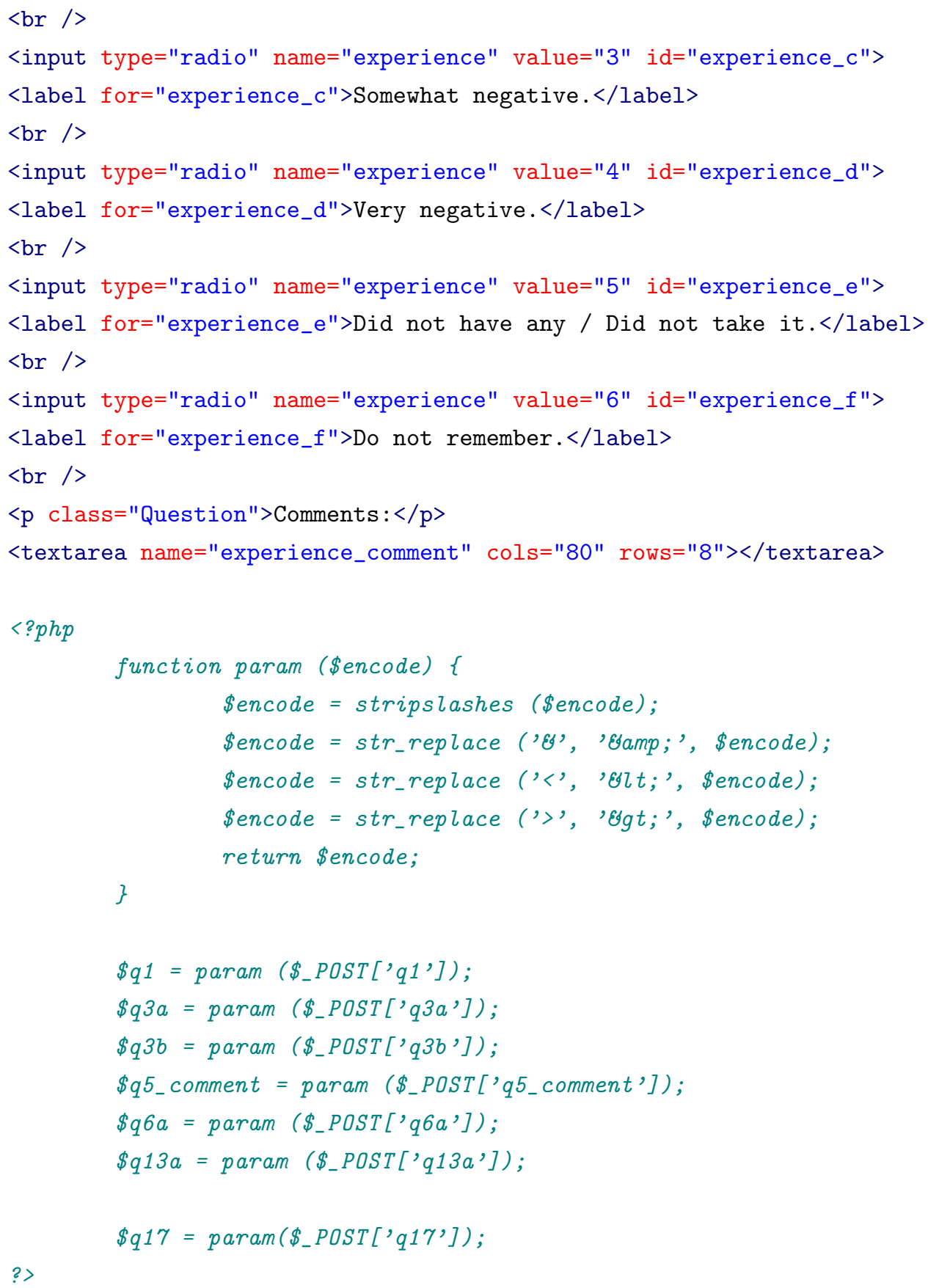




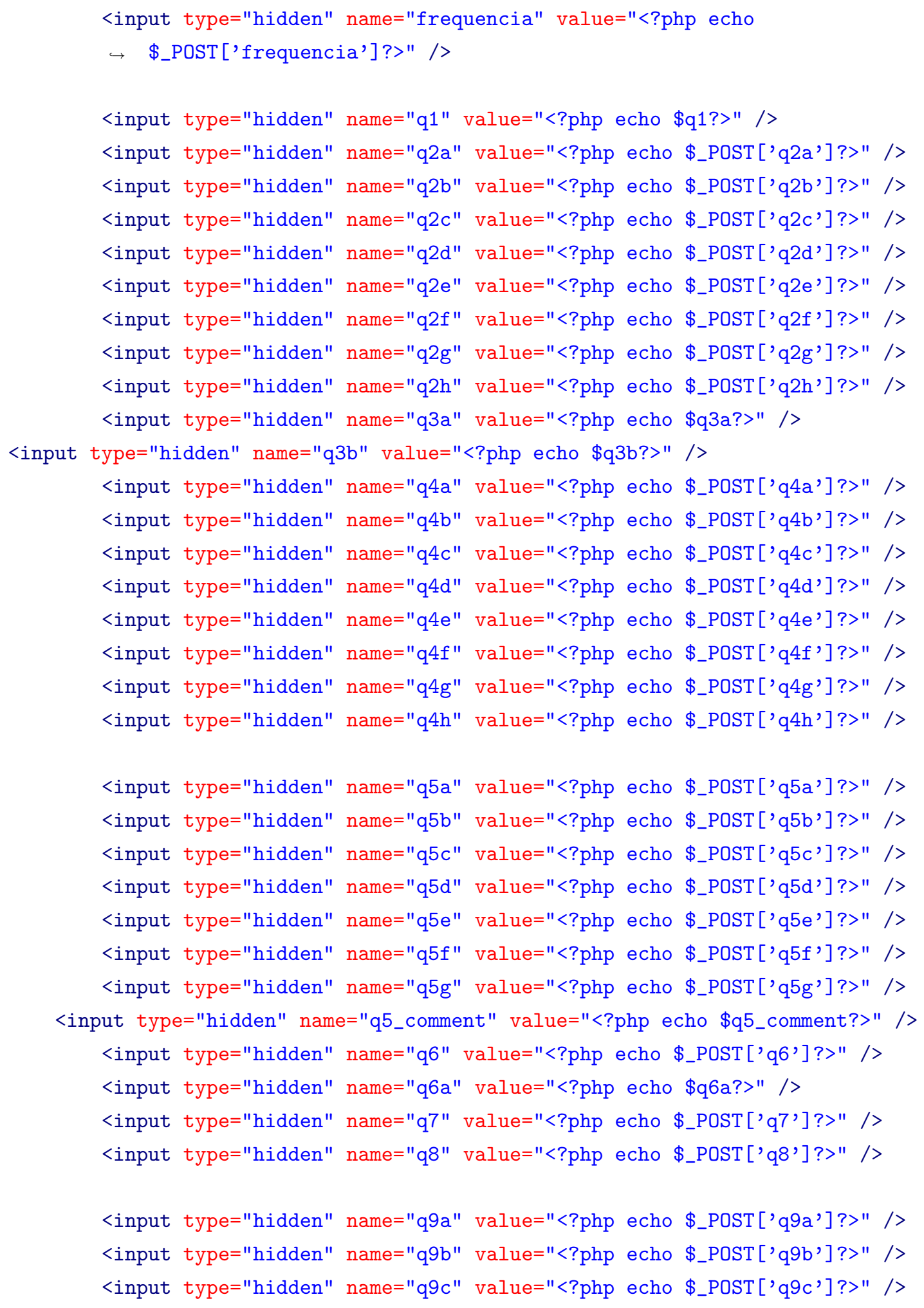




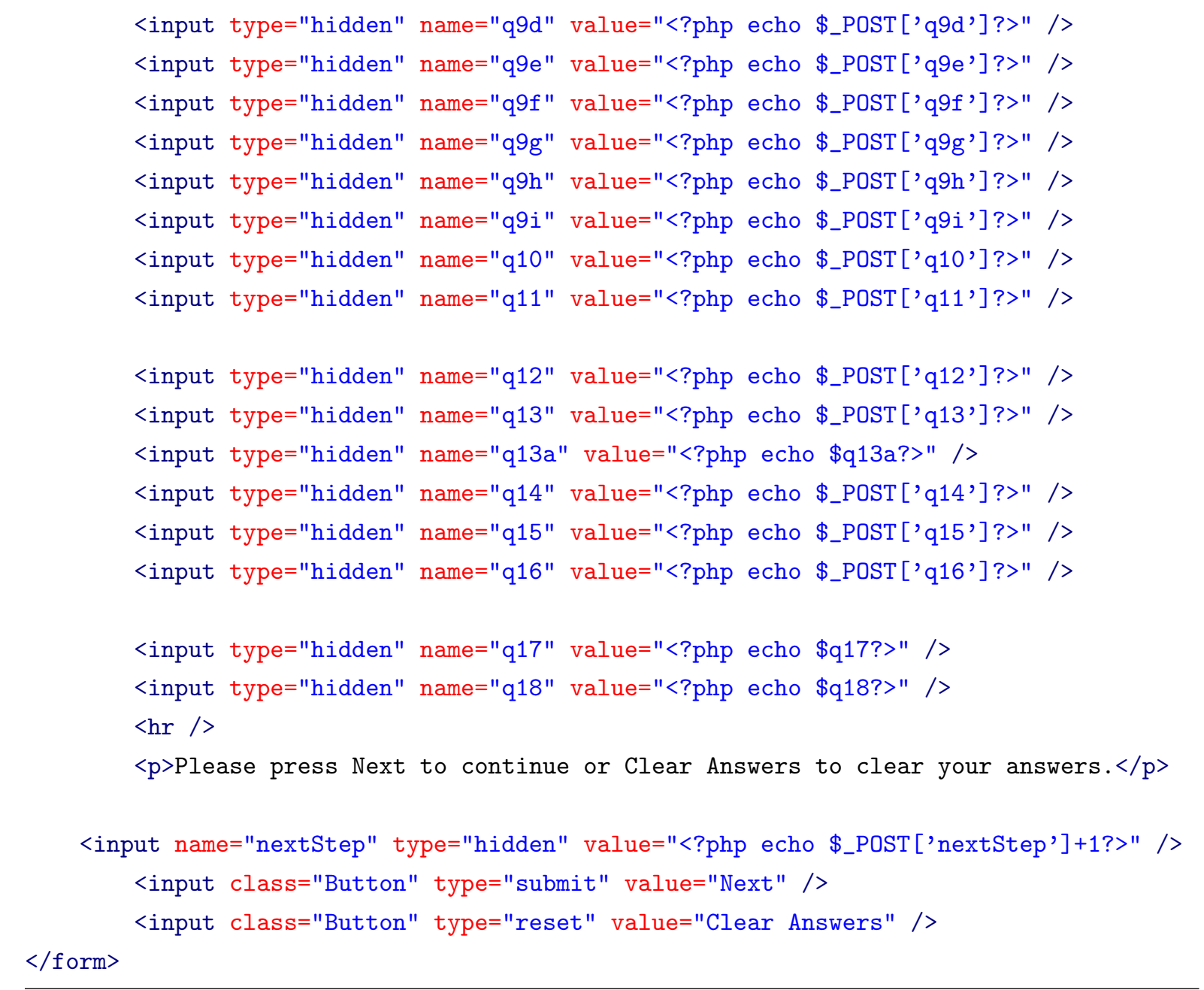

Listing 14: Post-study Questions Page 1.

<h4>Step <?php echo \$_POST ['nextStep']?> of <?php echo \$_SESSION ['numSteps']? ></h4> $<$ form action="thankyou.php" method="post">

$<$ h1 $>$ Music Questionnaire - Cont'd $</$ h1 $>$

$<\mathrm{p}$ class="Question" $>6$. We would greatly appreciate any additional comments. $\langle/ \mathrm{p}>$ <textarea name="general_comments" cols="80" rows="8" $></$ textarea $>$

$<? p h p$

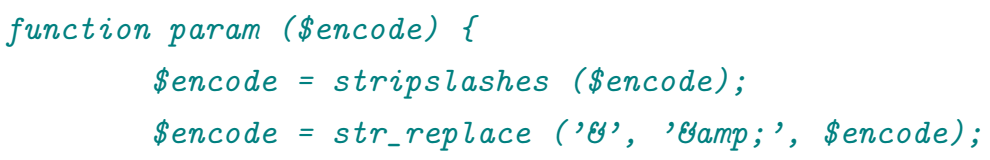




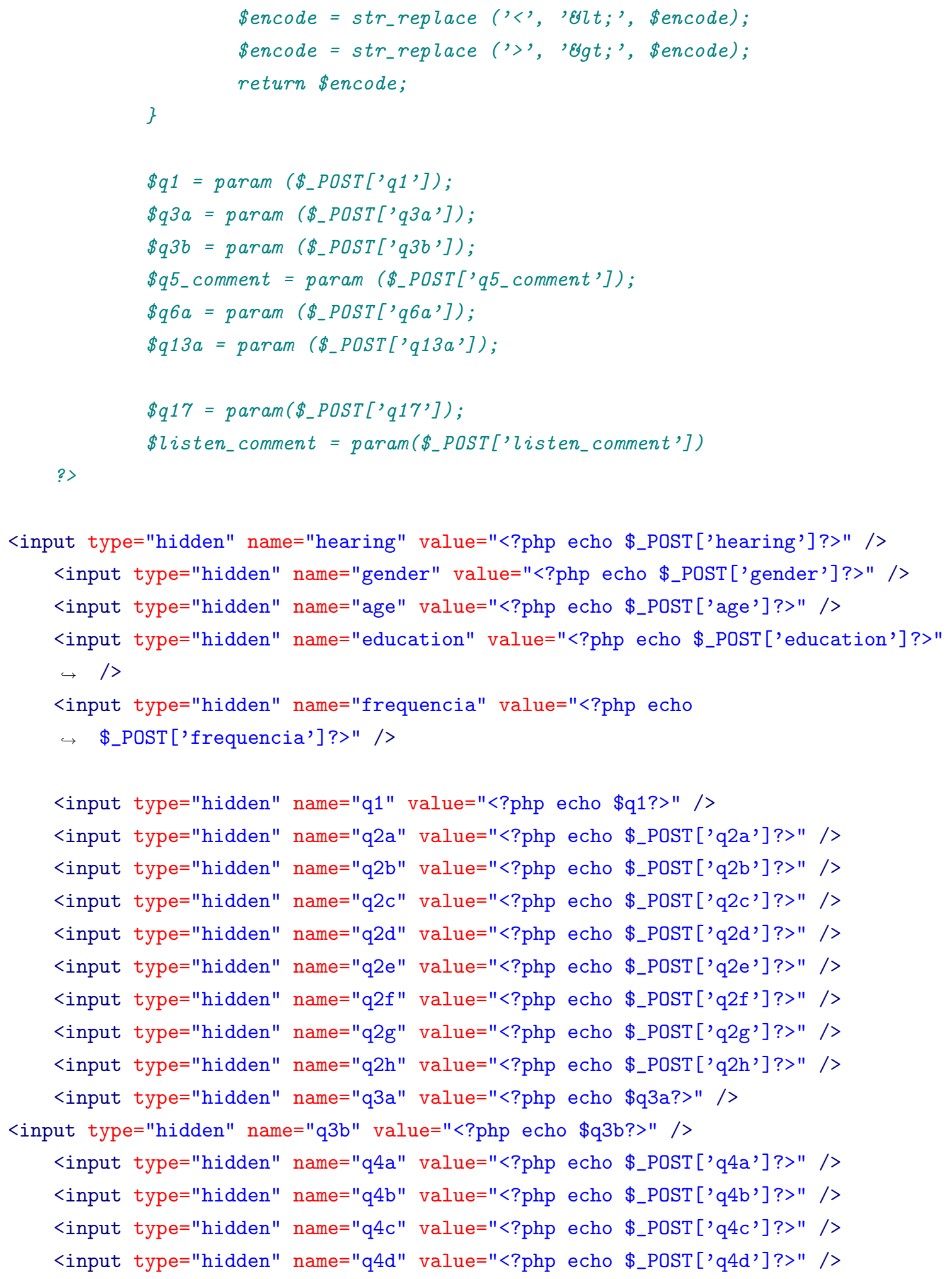


<input type="hidden" name="q4e" value="<?php echo \$_POST['q4e']?>" /> <input type="hidden" name="q4f" value="<?php echo \$_POST['q4f']?>" /> <input type="hidden" name="q4g" value="<?php echo \$_POST['q4g']?>" /> <input type="hidden" name="q4h" value="<?php echo \$_POST['q4h']?>" />

<input type="hidden" name="q5a" value="<?php echo \$_POST['q5a']?>" /> <input type="hidden" name="q5b" value="<?php echo \$_POST['q5b']?>" /> <input type="hidden" name="q5c" value="<?php echo \$_POST['q5c']?>" /> <input type="hidden" name="q5d" value="<?php echo \$_POST['q5d']?>" /> <input type="hidden" name="q5e" value="<?php echo \$_POST['q5e']?>" /> <input type="hidden" name="q5f" value="<?php echo \$_POST['q5f']?>" /> <input type="hidden" name="q5g" value="<?php echo \$_POST['q5g']?>" /> <input type="hidden" name="q5_comment" value="<?php echo \$q5_comment?>" /> <input type="hidden" name="q6" value="<?php echo \$_POST['q6']?>" /> <input type="hidden" name="q6a" value="<?php echo \$q6a?>" /> <input type="hidden" name="q7" value="<?php echo \$_POST['q7']?>" /> <input type="hidden" name="q8" value="<?php echo \$_POST['q8']?>" />

<input type="hidden" name="q9a" value="<?php echo \$_POST['q9a']?>" /> <input type="hidden" name="q9b" value="<?php echo \$_POST['q9b']?>" /> <input type="hidden" name="q9c" value="<?php echo \$_POST['q9c']?>" /> <input type="hidden" name="q9d" value="<?php echo \$_POST['q9d']?>" /> <input type="hidden" name="q9e" value="<?php echo \$_POST['q9e']?>" /> <input type="hidden" name="q9f" value="<?php echo \$_POST['q9f']?>" /> <input type="hidden" name="q9g" value="<?php echo \$_POST['q9g']?>" /> <input type="hidden" name="q9h" value="<?php echo \$_POST['q9h']?>" /> <input type="hidden" name="q9i" value="<?php echo \$_POST['q9i']?>" /> <input type="hidden" name="q10" value="<?php echo \$_POST['q10']?>" /> <input type="hidden" name="q11" value="<?php echo \$_POST['q11']?>" />

<input type="hidden" name="q12" value="<?php echo \$_POST['q12']?>" /> <input type="hidden" name="q13" value="<?php echo \$_POST['q13']?>" /> <input type="hidden" name="q13a" value="<?php echo \$q13a?>" /> <input type="hidden" name="q14" value="<?php echo \$_POST['q14']?>" /> <input type="hidden" name="q15" value="<?php echo \$_POST['q15']?>" /> <input type="hidden" name="q16" value="<?php echo \$_POST['q16']?>" /> <input type="hidden" name="q17" value="<?php echo \$q17?>" /> <input type="hidden" name="q18" value="<?php echo \$q18?>" /> 
<input type="hidden" name="frequency" value="<?php echo \$_POST['frequency']?>" />

<input type="hidden" name="frequency_comment" value="<?php echo

$\hookrightarrow$ \$_POST ['frequency_comment'] ?>" />

<input type="hidden" name="description" value="<?php echo \$_POST['description']?>"

$\hookrightarrow \quad />$

<input type="hidden" name="description_comment" value="<?php echo

$\hookrightarrow$ \$_POST['description_comment']?>" />

<input type="hidden" name="listen_a" value="<?php echo \$_POST['listen_a']?>" />

<input type="hidden" name="listen_b" value="<?php echo \$_POST['listen_b']?>" />

<input type="hidden" name="listen_c" value="<?php echo \$_POST['listen_c'] ?>" />

<input type="hidden" name="listen_d" value="<?php echo \$_POST['listen_d'] ?>" />

<input type="hidden" name="listen_e" value="<?php echo \$_POST['listen_e'] ?>" />

<input type="hidden" name="listen_comment" value="<?php echo \$listen_comment?>" />

<input type="hidden" name="information_a" value="<?php echo

$\hookrightarrow$ \$_POST['information_a'] ?>" />

<input type="hidden" name="information_b" value="<?php echo

$\hookrightarrow$ \$_POST['information_b']?>" />

$<$ input type="hidden" name="information_c" value="<?php echo

$\hookrightarrow$ \$_POST['information_c']?>" />

<input type="hidden" name="information_d" value="<?php echo

$\hookrightarrow$ \$_POST['information_d']?>" />

<input type="hidden" name="information_e" value="<?php echo

$\hookrightarrow$ \$_POST['information_e'] ?>" />

<input type="hidden" name="information_f" value="<?php echo

$\hookrightarrow$ \$_POST['information_f'] ?>" />

<input type="hidden" name="information_g" value="<?php echo

$\hookrightarrow$ \$_POST['information_g'] ?>" />

$<$ input type="hidden" name="information_h" value="<?php echo

$\hookrightarrow$ \$_POST['information_h'] ?>" />

<input type="hidden" name="information_i" value="<?php echo

$\hookrightarrow$ \$_POST['information_i']?>" />

<input type="hidden" name="information_comment" value="<?php echo

$\hookrightarrow$ \$_POST['information_comment']?>" />

<input type="hidden" name="experience" value="<?php echo \$_POST['experience']?>"

$\hookrightarrow \quad />$

<input type="hidden" name="experience_comment" value="<?php echo

$\hookrightarrow$ \$_POST ['experience_comment'] ?>" />

$<$ hr / >

$<\mathrm{p}>$ The questionnaire is now complete, please click Submit to submit the

$\hookrightarrow$ questionnaire or Clear Answers to clear your comments above. $</ p>$ 


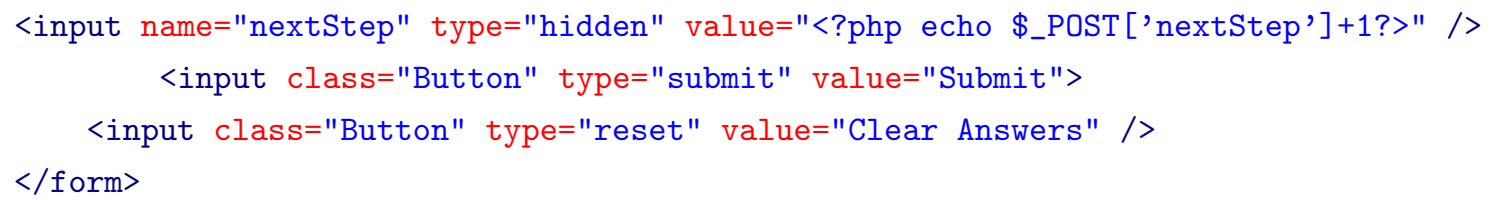

Listing 15: Post-study Questions Page 2.

The following tables present the data collected during the Music Questionnaire portion of the webbased survey described in Chapter 4. 


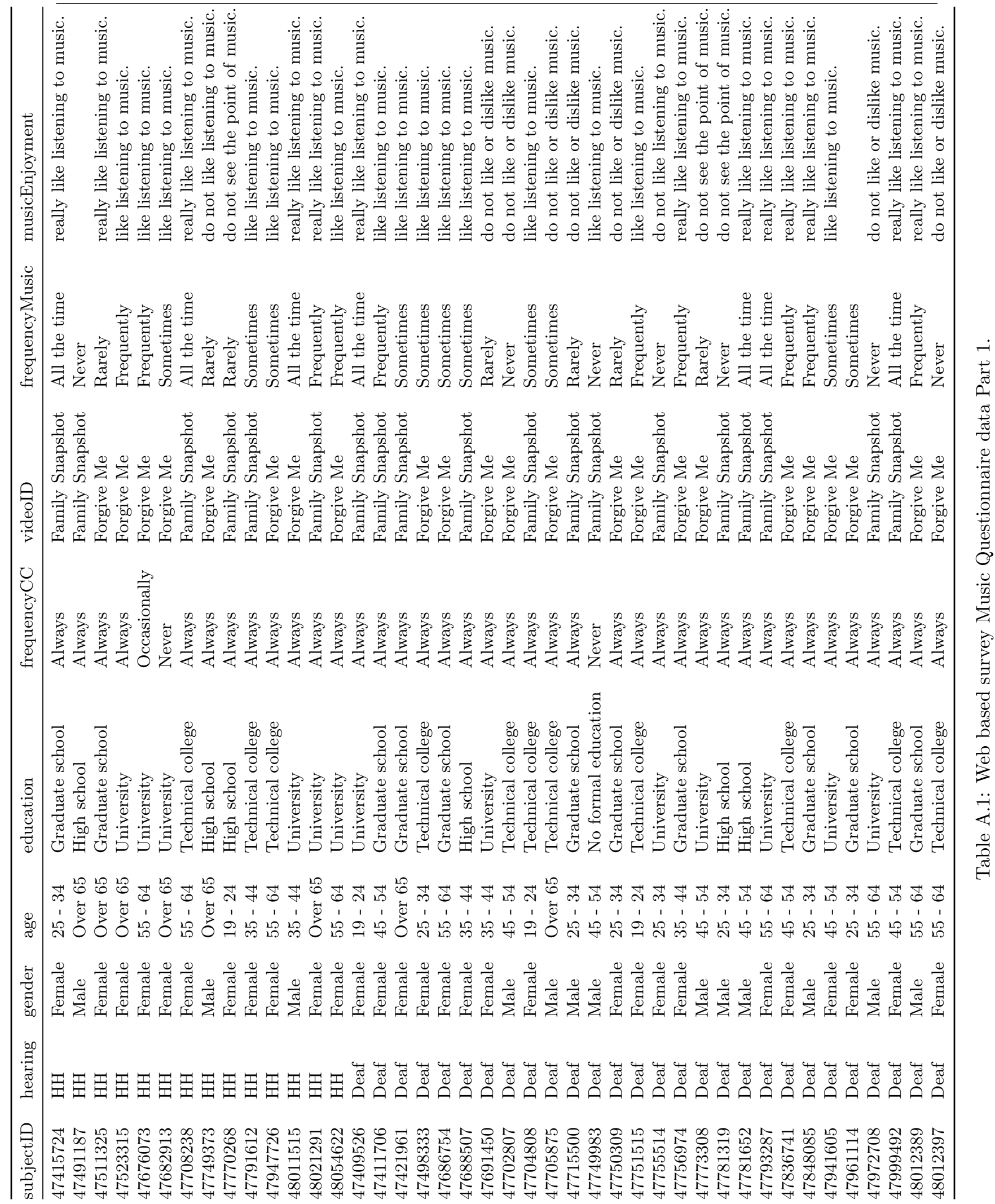




\begin{tabular}{|c|c|c|c|c|c|c|c|c|}
\hline subjectID & listen_radio & listen_player & listen_stereo & listen_internet & listen_tv & info_lyrics & info_notes & info_instruments \\
\hline 47415724 & & 2 & 3 & 4 & 5 & Text & Graphics/icons & Text \\
\hline 47491187 & & & & & & Text & Graphics/icons & Graphics/icons \\
\hline 47511325 & 1 & & & & & Text & & \\
\hline 47523315 & & & 3 & & & Text & & Text \\
\hline 47676073 & 1 & & 3 & & & Text & Text & Text \\
\hline 47682913 & 1 & & 3 & & & & & Graphics/icons \\
\hline 47708238 & 1 & & 3 & 4 & 5 & Text & Animation & Graphics/icons \\
\hline 47749373 & & & & & 5 & Text & Don't care & Don't care \\
\hline 47770268 & 1 & & & & 5 & Text & Animation & Graphics/icons \\
\hline 47791612 & & 2 & & & & Text & Don't care & Text \\
\hline 47947726 & & & 3 & & & Text & Don't care & Graphics/icons \\
\hline 48011515 & 1 & 2 & 3 & 4 & 5 & Text & Animation & Graphics/icons \\
\hline 48021291 & 1 & 2 & & & & Text & Don't care & Don't care \\
\hline 48054622 & & & 3 & & 5 & Text & & Graphics/icons \\
\hline 47409526 & 1 & 2 & 3 & 4 & 5 & Text & Text & Graphics/icons \\
\hline 47411706 & & 2 & & & 5 & Text & & Text \\
\hline 47421961 & 1 & 2 & 3 & 4 & 5 & Text & & Text \\
\hline 47498333 & & & 3 & & & Text & Don't care & Animation \\
\hline 47686754 & & & & & 5 & Animation & Don't care & Don't care \\
\hline 47688507 & & & 3 & 4 & & Text & Text & Graphics/icons \\
\hline 47691450 & & & & & 5 & Text & Graphics/icons & Graphics/icons \\
\hline 47702807 & & & & & & Don't care & Don't care & Don't care \\
\hline 47704808 & 1 & 2 & & 4 & 5 & Text & Graphics/icons & Graphics/icons \\
\hline 47705875 & & & & & 5 & Don't care & Animation & Don't care \\
\hline 47715500 & & & & & 5 & Text & Graphics/icons & Graphics/icons \\
\hline 47749983 & & & & & 5 & Text & Graphics/icons & Text \\
\hline 47750309 & & & 3 & & 5 & Text & Text & Text \\
\hline 47751515 & 1 & & 3 & 4 & 5 & Text & Don't care & Graphics/icons \\
\hline 47755514 & & & & & & Text & & Graphics/icons \\
\hline 47756974 & & & & & 5 & Text & Don't care & Don't care \\
\hline
\end{tabular}




\begin{tabular}{|c|c|c|c|c|c|c|c|c|}
\hline subjectID & listen_radio & listen_player & listen_stereo & listen_internet & listen_tv & info_lyrics & info_notes & info_instruments \\
\hline 47773308 & & & & & 5 & Don't care & Don't care & Don't care \\
\hline 47781652 & & & & & 5 & & & \\
\hline 47793287 & & & & & 5 & Text & Graphics/icons & Graphics/icons \\
\hline 47836741 & & & 3 & & & & Text & \\
\hline 47848085 & & & 3 & 4 & 5 & Animation & Animation & Animation \\
\hline 47941605 & & & 3 & & 5 & Animation & Don't care & Don't care \\
\hline 47961114 & & & & & 5 & Text & Text & Text \\
\hline 47972708 & & & & & & Text & Don't care & Don't care \\
\hline 47999492 & 1 & & 3 & & 5 & Text & Graphics/icons & Animation \\
\hline 48012389 & 1 & & & 4 & 5 & Text & Graphics/icons & Graphics/icons \\
\hline 48012397 & & & & & 5 & Text & & \\
\hline
\end{tabular}

Table A.2: Web based survey Music Questionnaire data Part 2.

\begin{tabular}{|c|c|c|c|c|c|c|}
\hline subjectID & info_beat & info_speed & info_emotion & info_artistInfo & info_songName & schoolExperience \\
\hline 47415724 & Text & Text & Text & Text & Text & Somewhat positive. \\
\hline 47491187 & Animation & & Animation & Text & Text & Very positive. \\
\hline 47511325 & & & & Text & Text & Somewhat positive. \\
\hline 47523315 & & & & & Text & Somewhat positive. \\
\hline 47676073 & Text & Text & Text & Text & Text & Somewhat negative. \\
\hline 47682913 & Animation & & & Text & Text & Somewhat positive. \\
\hline 47708238 & Graphics/icons & Animation & Text & Text & Text & Somewhat positive. \\
\hline 47749373 & Don't care & Don't care & Don't care & Don't care & Don't care & Did not have any / Did not take it. \\
\hline 47770268 & Animation & Animation & Graphics/icons & Text & Text & Somewhat positive. \\
\hline 47791612 & Graphics/icons & Graphics/icons & Don’t care & Text & Text & Very negative. \\
\hline 47947726 & Animation & Animation & Animation & Text & Text & Did not have any / Did not take it. \\
\hline 48011515 & Graphics/icons & Animation & & Text & Text & Do not remember. \\
\hline 48021291 & Animation & Don't care & Text & Text & Graphics & Somewhat positive. \\
\hline 48054622 & Text & & Text & Text & Text & Somewhat positive. \\
\hline
\end{tabular}




\begin{tabular}{|c|c|c|c|c|c|c|}
\hline subjectID & info_beat & info_speed & info_emotion & info_artistInfo & info_songName & schoolExperience \\
\hline 47409526 & Text & Text & Don't care & Text & Text & Very positive. \\
\hline 47411706 & Graphics/icons & Graphics/icons & Text & Text & Text & Somewhat negative. \\
\hline 47421961 & Text & Text & Text & Text & Text & Very negative. \\
\hline 47498333 & Animation & Animation & Animation & Text & Text & Somewhat positive. \\
\hline 47686754 & Animation & Text & Text & Graphics/icons & Text & Somewhat positive. \\
\hline 47688507 & Graphics/icons & Graphics/icons & Graphics/icons & Text & Text & Do not remember. \\
\hline 47691450 & Animation & Animation & Animation & Text & Text & Very positive. \\
\hline 47702807 & Don't care & Don't care & Don't care & Don't care & Don't care & Did not have any / Did not take it. \\
\hline 47704808 & Animation & Graphics/icons & Don't care & Graphics/icons & Text & Did not have any / Did not take it. \\
\hline 47705875 & Animation & Graphics/icons & Animation & Graphics/icons & Graphics & Somewhat positive. \\
\hline 47715500 & Graphics/icons & Graphics/icons & Animation & Text & Text & Somewhat negative. \\
\hline 47749983 & Graphics/icons & Text & Graphics/icons & Text & Graphics & Somewhat negative. \\
\hline 47750309 & Animation & Graphics/icons & Graphics/icons & Don't care & Text & Do not remember. \\
\hline 47751515 & Animation & Animation & Animation & Text & Text & Somewhat negative. \\
\hline 47755514 & Text & Text & Don't care & Text & Text & Somewhat positive. \\
\hline 47756974 & Animation & Animation & Animation & Text & Text & Very positive. \\
\hline 47773308 & Don't care & Don't care & Don't care & Don't care & Don't care & Somewhat positive. \\
\hline \multicolumn{7}{|l|}{47781319} \\
\hline 47781652 & & & & & & Somewhat positive. \\
\hline 47793287 & Graphics/icons & Graphics/icons & Graphics/icons & Text & Text & Did not have any / Did not take it. \\
\hline 47836741 & Animation & & Text & & Graphics & Somewhat positive. \\
\hline 47848085 & Animation & Animation & Animation & Animation & Animation & Somewhat positive. \\
\hline 47941605 & Animation & Animation & Animation & Text & Text & Did not have any / Did not take it. \\
\hline 47961114 & Text & & Graphics/icons & Don't care & Animation & Very positive. \\
\hline 47972708 & Don't care & Don't care & Don't care & Don't care & Don't care & Did not have any / Did not take it. \\
\hline 47999492 & Graphics/icons & Graphics/icons & Graphics/icons & Text & Text & Somewhat positive. \\
\hline 48012389 & Graphics/icons & Animation & Graphics/icons & Text & Text & Did not have any / Did not take it. \\
\hline 48012397 & & & & & Text & Somewhat negative. \\
\hline
\end{tabular}

Table A.3: Web based survey Music Questionnaire data Part 3. 


\begin{tabular}{|c|c|}
\hline subjectID & frequency_comment \\
\hline \multicolumn{2}{|l|}{47356056} \\
\hline \multicolumn{2}{|l|}{47396725} \\
\hline \multicolumn{2}{|l|}{47409526} \\
\hline \multicolumn{2}{|l|}{47411706} \\
\hline 47415724 & $\begin{array}{l}\text { I do not often know what the lyrics are, but I love the beats and rhythm. I am a skat- } \\
\text { ing coach and only listen to music with no lyrics, so I do love that. }\end{array}$ \\
\hline 47421961 & $\begin{array}{l}\text { When I first became able to hear ( via a cochlear implant ) I was elated. I felt part of } \\
\text { the world ! I am still amazingly grateful. Yet I still cannot "overhear" as I would love } \\
\text { to do, I cannot understand lyrics or plays or the phone,enough to pick up messages or } \\
\text { pick it up every time it rings. But I do love music, even though I know I am getting } \\
\text { but a shadow of the real thing. }\end{array}$ \\
\hline \multicolumn{2}{|l|}{47487254} \\
\hline 47491187 & $\begin{array}{l}\text { I played the piano proficiently, classical and jazz, at one time. Can no longer under- } \\
\text { stand the racket. } \\
\text { My "frequency detectors" do not allow for "music" and it is not what I knew for the } \\
\text { former } 60 \text { plus years. } \\
\text { Five years ago I was a candidate for a CI. I refused it. } \\
\text { I could hear nothing but garbage trucks (sometimes) going past me on the street, or } \\
\text { the odd loud bang or thump. This for seven months. I was offered a fast track CI in } \\
\text { Vancouver and turned it down considering my efforts at hearing exercises a potential. } \\
\text { Less than three weeks later, I was hearing again. Slowly at first, then better until I } \\
\text { had regained } 70-80 \% \text { of my former disability. I really must continue that "exercise" } \\
\text { program. } \\
\text { It was spawned by a Dr. Weil of the U.S., who believe "concentration of the mind" } \\
\text { had the power to heal. I used it for my cancer and it may have helped the healing pro- } \\
\text { cess. I decided to use the methods, somewhat object derived, to attempt to correct my } \\
\text { hearing loss. } \\
\text { Something worked after seven long months of speech-reading that actually became } \\
\text { quite proficient. Unfortunately, hearing has partially destroyed or weakened that ca- } \\
\text { pacity. Another exercise I must continue. } \\
\text { Life has its challenges, but where would we be without them? } \\
\text { No music for me - I will skip some groups here, though I do see below you could possi- } \\
\text { bly find ways to "recall" some "memories" that could prove enjoyable for some, painful } \\
\text { for others, especially those who may have played instruments and now can no longer } \\
\text { distinguish their "voice" or those who were close the musician. }\end{array}$ \\
\hline 47494188 & \\
\hline 47498333 & \\
\hline 47511325 & Although I play piano $\backslash$ \\
\hline 47523315 & \\
\hline 47676073 & But classical not pop \\
\hline
\end{tabular}




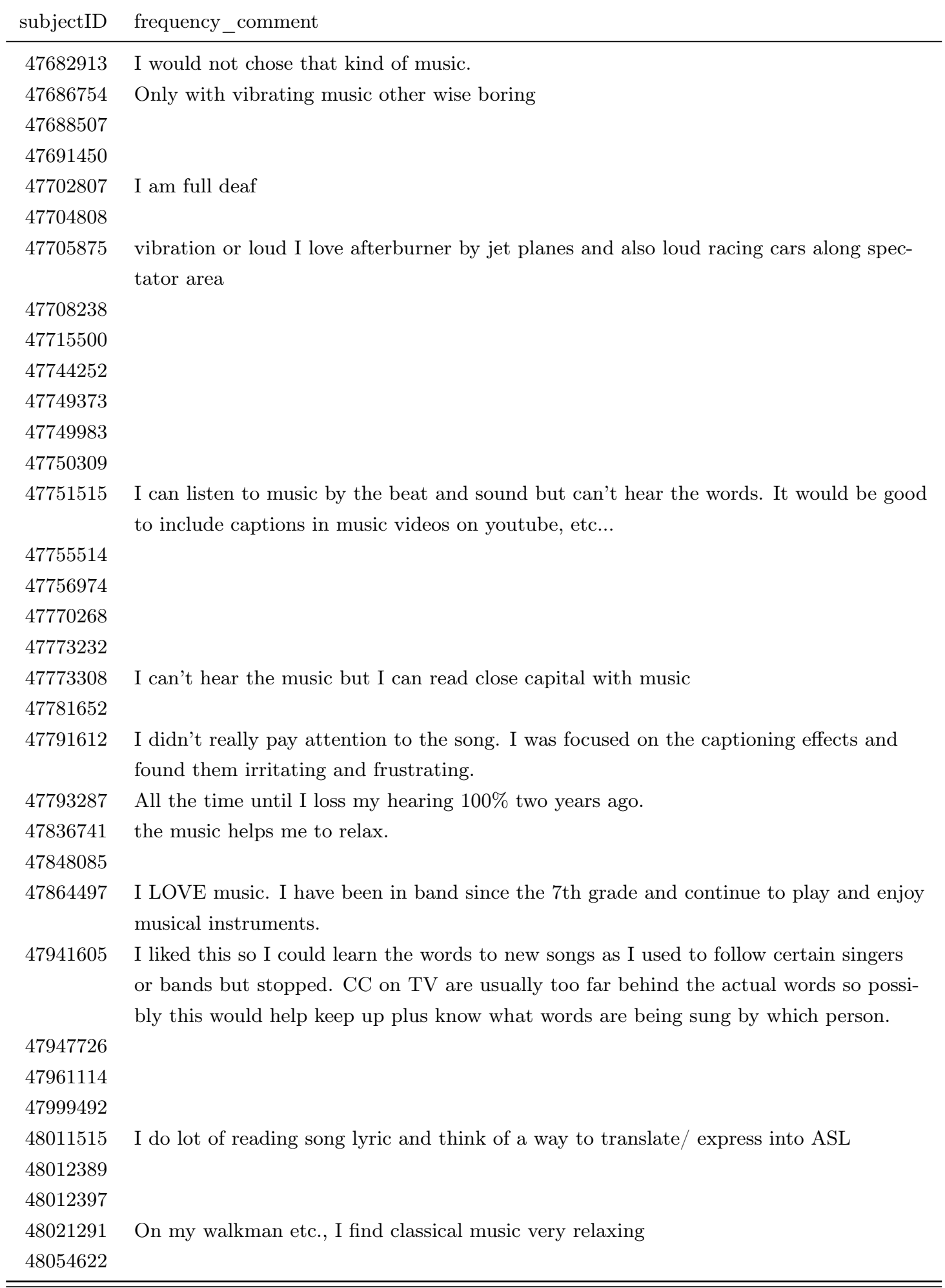


subjectID frequency_comment

Table A.4: Web based survey Music Questionnaire data Part 4.

\begin{tabular}{|c|c|}
\hline subjectID & description_comment \\
\hline \multicolumn{2}{|l|}{47356056} \\
\hline \multicolumn{2}{|l|}{47396725} \\
\hline \multicolumn{2}{|l|}{47409526} \\
\hline \multicolumn{2}{|l|}{47411706} \\
\hline 47415724 & LOVE IT! \\
\hline 47421961 & $\begin{array}{l}\text { I wish very much I could understand music. I wish I had a person who could talk } \\
\text { to me about music and explain and answer my questions. Some voices are literally } \\
\text { thrilling to listen to. I wish I could identify the instruments. I wish I knew what peo- } \\
\text { ple talk about when they discuss music. I wish I could sing, or play an instrument } \\
\text { myself. I like to watch captioned movies that talk about composers and their works. } \\
\text { I have met people who are able to tell which CONDUCTOR is playing a piece ! also } \\
\text { many who can identify who is singing a piece on the radio, or what the music is etc. I } \\
\text { would love to see more TV programs that have captions to tell, who it is, what they } \\
\text { are playing etc etc and more history about the piece. }\end{array}$ \\
\hline \multicolumn{2}{|l|}{47487254} \\
\hline \multicolumn{2}{|l|}{47491187} \\
\hline \multicolumn{2}{|l|}{47494188} \\
\hline \multicolumn{2}{|l|}{47498333} \\
\hline 47511325 & $\begin{array}{l}\text { Please see above - I miss the full experience of music as I knew it before my hearing } \\
\text { loss. }\end{array}$ \\
\hline \multicolumn{2}{|l|}{47523315} \\
\hline 47676073 & But again, usually classical not pop \\
\hline \multicolumn{2}{|l|}{47682913} \\
\hline \multicolumn{2}{|r|}{ only with high vibrations or rhythms } \\
\hline \multicolumn{2}{|l|}{47688507} \\
\hline \multicolumn{2}{|l|}{47691450} \\
\hline 47702807 & I should hear that music..uh? ...ready? \\
\hline \multicolumn{2}{|l|}{47704808} \\
\hline 47705875 & $\begin{array}{l}\text { I like to watch music by dancers or ice skating or ballets not choirs from church or fat } \\
\text { woman. }\end{array}$ \\
\hline \multicolumn{2}{|l|}{47708238} \\
\hline \multicolumn{2}{|l|}{47715500} \\
\hline \multicolumn{2}{|l|}{47744252} \\
\hline \multicolumn{2}{|l|}{47749373} \\
\hline \multicolumn{2}{|l|}{47749983} \\
\hline 47750309 & \\
\hline
\end{tabular}




\begin{tabular}{|c|c|}
\hline subjectID & description_comment \\
\hline 47751515 & \\
\hline 47755514 & \\
\hline 47756974 & \\
\hline 47770268 & \\
\hline 47773232 & \\
\hline 47773308 & I am deaf \\
\hline 47781319 & \\
\hline 47781652 & \\
\hline 47791612 & \\
\hline 47793287 & $\begin{array}{l}\text { I used to love listening to music. Wish they would add captioning to all music videos } \\
\text { on DVD all the time. }\end{array}$ \\
\hline 47836741 & \\
\hline 47848085 & \\
\hline 47864497 & A day without music is like a day without sunshine to me! \\
\hline 47941605 & \\
\hline 47947726 & \\
\hline 47961114 & no comment. I can't hear. :) \\
\hline 47972708 & \\
\hline 47999492 & \\
\hline 48011515 & \\
\hline 48012389 & \\
\hline 48012397 & \\
\hline 48021291 & See above \\
\hline 48054622 & \\
\hline
\end{tabular}

Table A.5: Web based survey Music Questionnaire data Part 5.

\begin{tabular}{ll}
\hline subjectID & listen_comment \\
\hline 47356056 & \\
47396725 & \\
47409526 & \\
47411706 & \\
47415724 & Music tunes out my tinnitus. \\
47421961 & $\begin{array}{l}\text { I borrow quite a lot of musical CD's and DVD's from the library. I am always disap- } \\
\text { pointed if the lyrics are not included in the package, because I like to try and follow }\end{array}$ \\
& $\begin{array}{l}\text { along. I think this should be mandatory! In the past, I've tried to get the lyrics from } \\
\text { the Internet, but this is time consuming, looking up each song. I have a CD (Frosty } \\
\end{array}$ \\
& the Snowman) which is apparently superb, but I still cannot get the lyrics. I don't \\
&
\end{tabular}




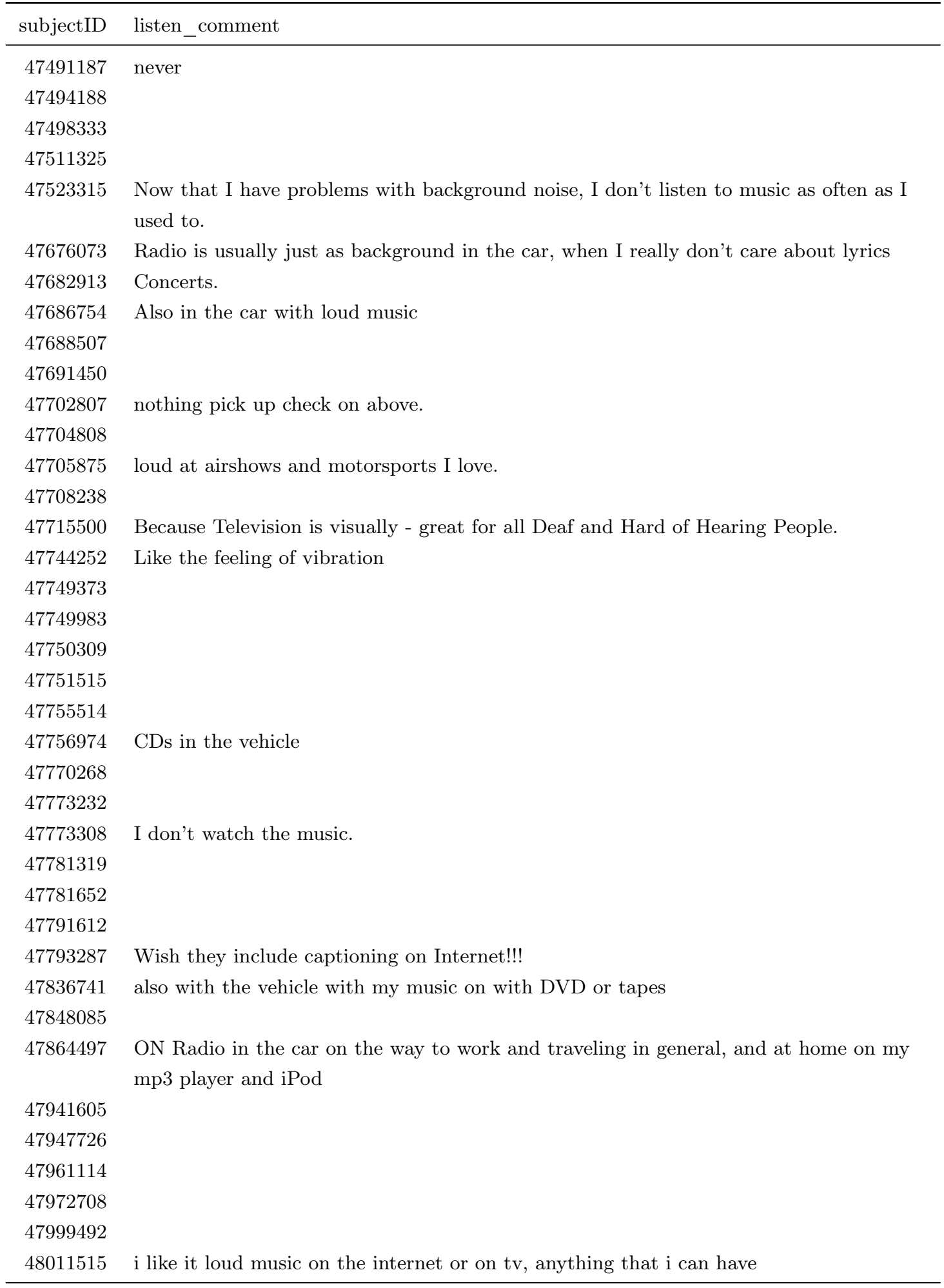




\begin{tabular}{ll}
\hline subjectID & listen_comment \\
\hline 48012389 & \\
48012397 & \\
48021291 & See above \\
48054622 & \\
\hline
\end{tabular}

Table A.6: Web based survey Music Questionnaire data Part 6 .

\begin{tabular}{|c|c|}
\hline subjectID & information_comment \\
\hline 47356056 & A question - In this case, what is the difference between a graphic and an icon? \\
\hline 47396725 & \\
\hline 47409526 & \\
\hline 47411706 & \\
\hline 47415724 & $\begin{array}{l}\text { I like when songs are captioned in text in italic writing with the music notes. However, } \\
\text { I wish there was sometimes a little more info on the beats and rhythm. }\end{array}$ \\
\hline 47421961 & $\begin{array}{l}\text { Radio input would be nice. I don't know how to find programs that I could benefit } \\
\text { from. I would need a synopsis and what to listen for. The one type of program I detest } \\
\text { is one with a laugh track. I hate not knowing the punch lines and I dislike the sound of } \\
\text { hysterical laughter ha ha! }\end{array}$ \\
\hline \multicolumn{2}{|l|}{47487254} \\
\hline \multicolumn{2}{|l|}{47491187} \\
\hline \multicolumn{2}{|l|}{47494188} \\
\hline \multicolumn{2}{|l|}{47498333} \\
\hline \multicolumn{2}{|l|}{47511325} \\
\hline \multicolumn{2}{|l|}{47523315} \\
\hline 47676073 & $\begin{array}{l}\text { It really depends on the purpose of the music. Are you talking about music as back- } \\
\text { ground to a movie, or for its own sake? If background to a movie, it's usually hard to } \\
\text { make out the words even if one is hearing - just a few phrases of text are probably } \\
\text { enough. }\end{array}$ \\
\hline \multicolumn{2}{|l|}{47682913} \\
\hline 47686754 & Not sure \\
\hline \multicolumn{2}{|l|}{47688507} \\
\hline \multicolumn{2}{|l|}{47691450} \\
\hline \multicolumn{2}{|r|}{ most deafers don't care on music name/songs } \\
\hline \multicolumn{2}{|l|}{47704808} \\
\hline 47705875 & $\begin{array}{l}\text { You miss any loud like bomb or racing car or battle combats or listen any loud or bird } \\
\text { singing or sea wave or wind or any sound. }\end{array}$ \\
\hline \multicolumn{2}{|l|}{47708238} \\
\hline \multicolumn{2}{|l|}{47715500} \\
\hline \multicolumn{2}{|l|}{47744252} \\
\hline 47749373 & \\
\hline
\end{tabular}




\begin{tabular}{|c|c|}
\hline subjectID & information_comment \\
\hline \multicolumn{2}{|l|}{47749983} \\
\hline \multicolumn{2}{|l|}{47750309} \\
\hline \multicolumn{2}{|l|}{47751515} \\
\hline \multicolumn{2}{|l|}{47755514} \\
\hline \multicolumn{2}{|l|}{47756974} \\
\hline \multicolumn{2}{|l|}{47770268} \\
\hline \multicolumn{2}{|l|}{47773232} \\
\hline 47773308 & Deaf people like loud bass than high pitch because it is feeling vibration. \\
\hline \multicolumn{2}{|l|}{47781319} \\
\hline \multicolumn{2}{|l|}{47781652} \\
\hline \multicolumn{2}{|l|}{47791612} \\
\hline 47793287 & $\begin{array}{l}\text { Do not use scroll format!! Use pop up, left to right, right to left, do not cover the sub- } \\
\text { title, do not use on top covering faces or half of faces! If there are subtitles (for names } \\
\text { for a few seconds), use captioning on bottom and put subtitles (for names for a few } \\
\text { second) on top as it is there for a few seconds. We ARE USED TO seeing captioning } \\
\text { on the bottom until someone has the stupid idea putting captioning on the top all } \\
\text { the time even subtitles were there for a few seconds. Put captioning on bottom, put } \\
\text { subtitles on top!! Hearing has to learn to accept it or tough luck!! }\end{array}$ \\
\hline \multicolumn{2}{|l|}{47836741} \\
\hline \multicolumn{2}{|l|}{47848085} \\
\hline \multicolumn{2}{|l|}{47864497} \\
\hline \multicolumn{2}{|l|}{47941605} \\
\hline \multicolumn{2}{|l|}{47947726} \\
\hline \multicolumn{2}{|l|}{47961114} \\
\hline 47972708 & $\begin{array}{l}\text { It's annoying when a song that is TWENTY YEARS OLD - just for an example, isn't } \\
\text { fully captioned. How hard can it be to look up lyrics THAT OLD? Also annoying is } \\
\text { when instead of lyrics, the captions just says }\end{array}$ \\
\hline \multicolumn{2}{|l|}{47999492} \\
\hline \multicolumn{2}{|l|}{48011515} \\
\hline \multicolumn{2}{|l|}{48012389} \\
\hline \multicolumn{2}{|l|}{48012397} \\
\hline 48021291 & I am not knowledgeable about music, just find it relaxing. \\
\hline 48054622 & \\
\hline
\end{tabular}

Table A.7: Web based survey Music Questionnaire data Part 7.

\begin{tabular}{ll}
\hline subjectID & school_experience_comment \\
\hline 47356056 & \\
47396725 & \\
47409526 & \\
\hline
\end{tabular}




\begin{tabular}{|c|c|}
\hline subjectID & school_experience_comment \\
\hline \multicolumn{2}{|l|}{47411706} \\
\hline \multicolumn{2}{|l|}{47415724} \\
\hline 47421961 & $\begin{array}{l}\text { I was told to stop singing. I sensed that my music teacher really hated me as she gave } \\
\text { so much body language! }\end{array}$ \\
\hline \multicolumn{2}{|l|}{47487254} \\
\hline \multicolumn{2}{|l|}{47491187} \\
\hline \multicolumn{2}{|l|}{47494188} \\
\hline \multicolumn{2}{|l|}{47498333} \\
\hline \multicolumn{2}{|l|}{47511325} \\
\hline \multicolumn{2}{|l|}{47523315} \\
\hline \multicolumn{2}{|l|}{47676073} \\
\hline 47682913 & We learned to read music and sang a great deal. \\
\hline 47686754 & rhythm class was fun sometimes \\
\hline \multicolumn{2}{|l|}{47688507} \\
\hline \multicolumn{2}{|l|}{47691450} \\
\hline 47702807 & HaHa! should deafer take music course at school uh? \\
\hline \multicolumn{2}{|l|}{47704808} \\
\hline \multicolumn{2}{|c|}{47705875 bands parade and cheerleaders sport events.. } \\
\hline \multicolumn{2}{|c|}{47708238} \\
\hline \multicolumn{2}{|l|}{47715500} \\
\hline \multicolumn{2}{|l|}{47744252} \\
\hline \multicolumn{2}{|l|}{47749373} \\
\hline \multicolumn{2}{|l|}{47749983} \\
\hline \multicolumn{2}{|l|}{47750309} \\
\hline 47751515 & $\begin{array}{l}\text { I did not take any music classes because I was not able to hear the notes or the sound } \\
\text { of music. Although I wear two hearing aids and I can hear well but I can't listen to the } \\
\text { radio because all i can hear is mumbling. I can communicate by reading lips and with } \\
\text { help of my hearing aids. }\end{array}$ \\
\hline \multicolumn{2}{|l|}{47755514} \\
\hline \multicolumn{2}{|l|}{47756974} \\
\hline \multicolumn{2}{|l|}{47770268} \\
\hline \multicolumn{2}{|l|}{47773232} \\
\hline 47773308 & Hearing people love to listen music. \\
\hline \multicolumn{2}{|l|}{47781319} \\
\hline \multicolumn{2}{|l|}{47781652} \\
\hline 47791612 & $\begin{array}{l}\text { As a hard of hearing person, it was very difficult and awkward for me to participate in } \\
\text { mandatory music classes. I wasn't able to hear the words or follow what was going on. }\end{array}$ \\
\hline \multicolumn{2}{|r|}{ ( } \\
\hline \multicolumn{2}{|l|}{47836741} \\
\hline 47848085 & \\
\hline
\end{tabular}




\begin{tabular}{ll}
\hline subjectID & school_experience_comment \\
\hline 47864497 & I have no further comments to make. \\
47941605 & \\
47947726 & \\
47961114 & They used American Sign Language and facial expressive while they song. \\
47972708 & \\
47999492 & \\
48011515 & \\
48012389 & \\
48012397 & \\
48021291 & Loved the Beatles \\
48054622 & \\
\hline
\end{tabular}

Table A.8: Web based survey Music Questionnaire data Part 8 .

\begin{tabular}{|c|c|}
\hline subjectID & general_comments \\
\hline \multicolumn{2}{|l|}{47356056} \\
\hline \multicolumn{2}{|l|}{47396725} \\
\hline \multicolumn{2}{|l|}{47409526} \\
\hline \multicolumn{2}{|l|}{47411706} \\
\hline \multicolumn{2}{|l|}{47415724} \\
\hline 47421961 & $\begin{array}{l}\text { Some computer software programs show a musical instrument followed by the sound } \\
\text { it makes, which can be played and replayed over again, so this is a graphic way of ex- } \\
\text { plaining a sound which is fun to see. I am always grateful if I am told about similar } \\
\text { music oriented sites to give one an identifiable music experience. I am chair of a group } \\
\text { of people who have had cochlear implants. Many of them are very interested in ex- } \\
\text { tending their musical experience with their new hearing, which is really a sound that } \\
\text { they must retrain their brains to understand with differing degrees of success. Any } \\
\text { URLS of Internet websites which you know of, or think might be useful to us, I would } \\
\text { appreciate. } \\
\text { Also, with this questionnaire, the very first question gave me trouble. Since I have } \\
\text { absolutely no hearing whatsoever ( any remaining is destroyed by the CI operation), I } \\
\text { ticked 'Deaf' but that is not right, because, with the CI I have, I do have about } 70 \% \\
\text { hearing and can respond to a question from my husband when I am in the basement } \\
\text { and he is upstairs... }\end{array}$ \\
\hline 47487254 & \\
\hline
\end{tabular}




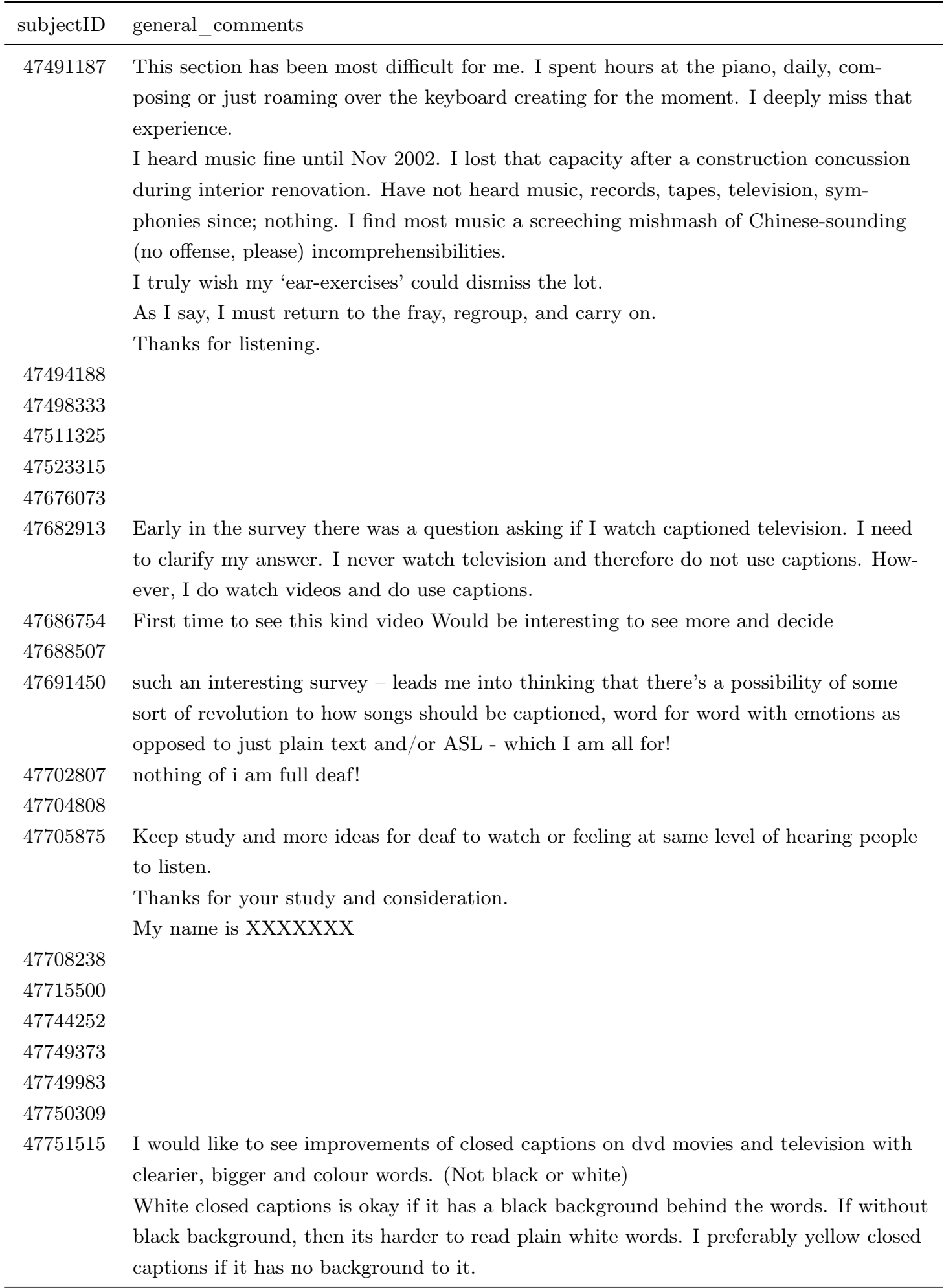

Continued on next page 


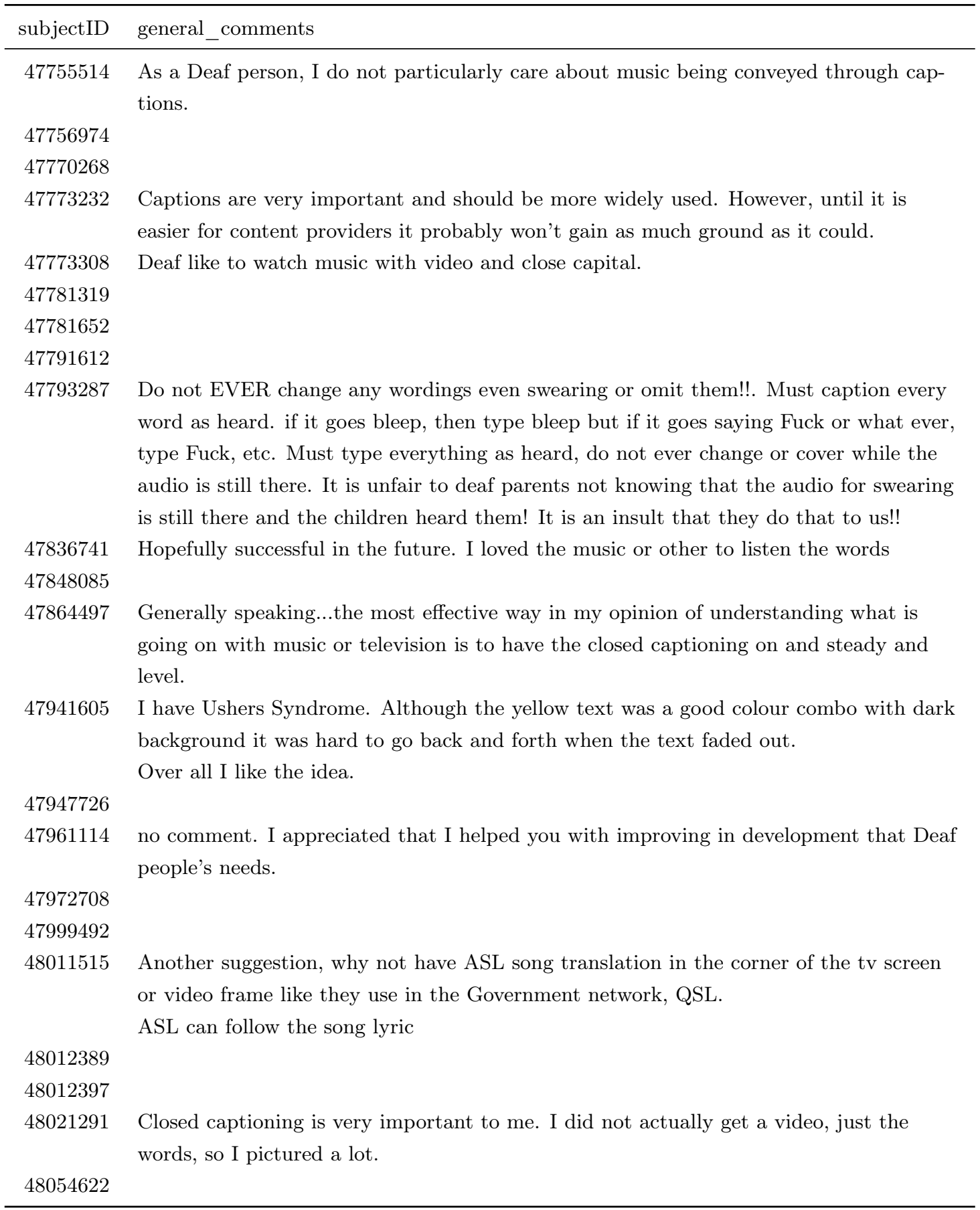

Table A.9: Web based survey Music Questionnaire data Part 9. 



\section{Appendix B}

\section{Focus Group I}

This Appendix presents the forms used and data collected in Focus Group I, described in Chapter 5. 


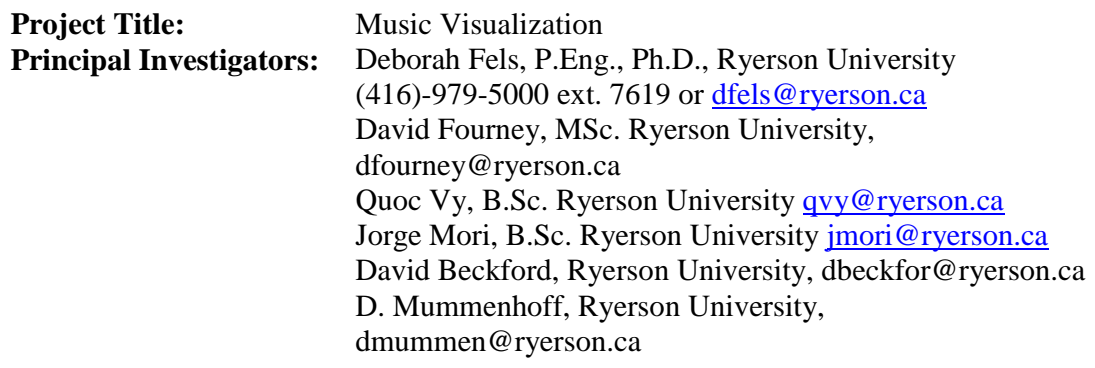

\section{Consent to Participate in Study from Subject}

\section{Information Form}

The purpose of this study is to evaluate different way of presenting music visually. In order to do this, we will ask you to watch eight different examples of these visualizations and provide us with your opinion about them. Each example runs between one and two minutes. You are also asked to complete two additional surveys, one prior to the study and one at the conclusion of the study.

\section{Confidentiality}

All raw data will be kept strictly confidential; however a summary of the data will be published in academic venues but no individual details will be identified in this summary. The information gathered from surveys will be strictly used for research and academic purposes with only the principal investigator and his supervisor having access to it

\section{Risks and Discomforts}

The risks associated with participating in this study are minimal. You may experience some fatigue with watching the visualizations. However, you are able to take breaks at any time or even stop participation in the study without penalty. You may also experience some discomfort with being filmed or audio recorded. In this case, you may choose not to participate in the study or record your opinions in writing and remain off camera.

\section{Expected Benefits}

Individual participants will not receive any direct benefits; however, this study will benefit the general community of audio description users. This study will test different styles of audio description that will assist in developing new practises for audio description. We hope that this information may lead to improvements in audio description technologies and techniques.

\section{Voluntary Nature of Participation:}

Participation in this study is entirely voluntary. If you do not wish to participate in this study it will not affect current or future relations with Ryerson University or The Centre for Learning Technologies. If you choose to participate, you have the ability to leave the study at any time and for any reason without penalty. In addition, you may refuse to answer any questions or participate in any task at any point of the study without penalty. 


\section{Questions about the Study:}

If you have any questions or concerns, about this study please feel free to contact John Deborah Fels at 416.979.5000 ext. 7619. If you have any concerns or complaints about this study in regards to its ethical nature please contact the Research Ethics Board, c/o

Office of the Vice President, Research and Innovation, Ryerson University, 350 Victoria St., Toronto, ON M5B 2K3, Tel: 416-979-5042 


\section{Project Title: $\quad$ Music Visualization \\ Principal Investigators: Deborah Fels, P.Eng., Ph.D., Ryerson University \\ (416)-979-5000 ext. 7619 or dfels@ ryerson.ca \\ David Fourney, MSc. Ryerson University, \\ dfourney@ryerson.ca \\ Quoc Vy, B.Sc. Ryerson University qvy@ryerson.ca \\ Jorge Mori, B.Sc. Ryerson University jmori@ryerson.ca \\ David Beckford, Ryerson University \\ Doug Mummel}

\section{Consent Form to Participate in Study}

I acknowledge that the research procedures described above have been explained to me and that any questions that $\mathrm{I}$ have asked have been answered to my satisfaction. I have been informed of that there may be a possible risk of psychological discomfort from being filmed or watching so many videos, however, strategies are in place to reduce this risk.

I have been informed of the alternatives to participation in this study, including my right not to participate and the right to withdraw without penalty. I hereby consent to participate in the study and to be video or audio recorded during the study. I have received a copy of the information sheet.

Signature of Participant:

Name of Participant (please print):

Date:

The details of this study were explained to me by:

Name of Investigator:

Date: 


\section{Music Visualization: Pre-study questionnaire}

This purpose of this questionnaire is to gather information about you and your experience with music and closed captioning. There are eight questions and it should take you about five minutes to complete this questionnaire. Thank you in advance for your time and assistance.

1. Do you identify yourself as: (please check one)

$$
\begin{array}{ll}
\square & \text { Hearing } \\
\square & \text { Hard of hearing } \\
\square & \text { Deaf } \\
\square & \text { Deafened } \\
\square & \text { Cochlear implant }
\end{array}
$$

\section{Are you}

$$
\begin{array}{ll}
\square & \text { Male } \\
\text { 口 Female }
\end{array}
$$

3. Please indicate your age
口 $19-24$
口 $25-34$
口 $35-44$
$45-54$
( $55-64$
$65+$

\section{What is your highest level of education?}

No formal education
Elementary school
G High School
a Technical college
University
$\square$ Graduate school

5. How often do you use closed captioning when watching television?
Always
- Sometimes
Never

6. How often do you listen to music?
All the time (daily).
Frequently (weekly)
Sometimes
ㅁ Rarely
Never

7. Which of the following statements best describes you?

I really enjoy listening to music.
I like listening to music.
I do not like or dislike music.
$\square$ I do not like listening to music
$\square$ I do not see the point of music.

8. What is your experience of music in elementary or secondary school?

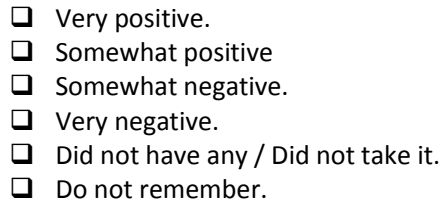


Music Questions

1. Was the music visualization enjoyable (circle one)?

$\begin{array}{lll}\text { Not enjoyable at Not that enjoyable Neutral } & \begin{array}{l}\text { Somewhat } \\ \text { enjoyable }\end{array} & \text { Enjoyable } \\ \text { all } & \end{array}$

2. What did the visualization mean to you?

3. On a scale from 1 to 7 , was the music

\section{Positive}

1
3 enjoyable

4. In your opinion, what was the energy level of that music (circle one)

Sleepy

1
2

5

Awake

Negative

6

7

5. Identify which emotion you thought was expressed and rate its strength from $\mathbf{1}$ to $\mathbf{7}$ (circle one)

Weak

Strong

Happy

1

\section{2}

3

4

4

4

Angry

1

2

3

1

2

3

4

6. What did you like best of the visualization style?

7. What did you like least of the visualization style? 
Music Visualization Post-Study Questionnaire

This purpose of this questionnaire is to gather information about you and your opinions about the visual representations that you just saw. There five questions and it should take you about ten minutes to complete this questionnaire. Thank you in advance for your time and assistance.

1. Which style did you enjoy most?

2. Which style gave you the best sense of what was happening in the music

3. Which style gave you the best sense of:

\begin{tabular}{|c|c|c|c|c|c|c|c|c|}
\hline & $\begin{array}{c}\text { Color } \\
\text { Going Out }\end{array}$ & $\begin{array}{c}\text { Color } \\
\text { Going In }\end{array}$ & $\begin{array}{l}\text { Lots of } \\
\text { Fans }\end{array}$ & $\begin{array}{l}\text { A Few } \\
\text { Fans }\end{array}$ & Psychedelic & $\begin{array}{l}\text { Flashing } \\
\text { Sun }\end{array}$ & $\begin{array}{l}\text { Moving } \\
\text { Shapes }\end{array}$ & None \\
\hline Drums/p & & & & & & & & \\
\hline $\begin{array}{l}\text { Instrume } \\
\text { playing }\end{array}$ & & & & & & & & \\
\hline Melody & & & & & & & & \\
\hline Rhythm & & & & & & & & \\
\hline
\end{tabular}

4. What was the most enjoyable about your experience with music visualization

5. What was the least enjoyable about your experience with music visualization 
DAVID: ARE YOU SET? ARE YOU READY? OKAY. WELCOME, EVERYBODY. THANK YOU VERY MUCH FOR COMING. OKAY. THANK YOU VERY MUCH FOR COMING. MY NAME IS DAVID, AND THIS IS MY SIGN NAME. I SIGN, BUT UNFORTUNATELY I CAN'T THINK AND SIGN AT THE SAME TIME, SO I'M GOING TO ASK TALA (PH.) AND JENNIFER TO BE OUR INTERPRETERS TODAY. NOW, WHAT I'LL DO IS JUST QUICKLY INTRODUCE YOU TO THE OTHER RESEARCHERS IN THE ROOM. IN THE BACK CORNER OVER HERE IS JORGE. WAVE. OKAY. THAT'S JORGE. THE WOMAN OVER HERE IS DEB FELS WHO IS THE PRINCIPAL RESEARCHER IN THE STUDY. THE GENTLEMAN HERE IS DOUG, AND HE WILL BE RUNNING OUR MOVIES TODAY. THIS GENTLEMAN IS QUOC, AND THAT WOMAN OVER THERE IS LIDA. NOW, LET ME SORT OF EXPLAIN WHAT IS GOING TO HAPPEN TODAY, AND THEN WE WILL FIGURE IT OUT FROM THERE. SO WHAT WE'RE GOING TO DO TODAY IS WE'RE GOING TO SHOW YOU A BUNCH OF VIDEOS -- COME IN, COME IN. OKAY. DO YOU NEED A PEN? OKAY. SO AS I WAS SAYING EARLIER, WHAT WE'RE DOING TODAY IS WE'RE GOING TO DO RESEARCH ON MUSIC, BUT WE'RE NOT GOING TO ACTUALLY PLAY MUSIC. YOU'RE NOT GOING TO HEAR ANYTHING. WHAT WE'RE GOING TO DO IS TRY DIFFERENT WAYS OF VISUALIZING MUSIC. WE'RE GOING TO PUT A VIDEO ON THIS SCREEN OVER HERE TO MY RIGHT, AND WE'RE GOING TO HAVE YOU WATCH IT AND THINK ABOUT IT, AND THEN WHAT YOU'RE GOING TO DO IS FILL OUT A QUICK QUESTIONNAIRE, AND LIDA, OVER HERE WILL PICK THEM UP, AND THEN WE WILL GO TO THE NEXT VIDEO. AND THAT'S THE IDEA. WHAT WE NEED IS WE ARE TRYING TO FIGURE OUT WAYS OF MAKING MUSIC MORE ACCESSIBLE FOR PEOPLE LIKE ME, WHO ARE HARD OF HEARING, OR LIKE YOU WHO ARE DEAF, AND WE'RE HOPING THAT ONE OF THE WAYS TO DO IT IS TO MAKE IT VISUAL,

DEB (COULD BE SOMEONE ELSE. COULD NOT SEE SPEAKER): CAN I JUST DO AN ACCESS CHECK?

DAVID: OKAY. CAN EVERYBODY SEE THE SCREEN?

(JUST DISCUSSING SEATING).

DAVID: NOW, FOR EVERY ONE OF OUR FORMS, YOU DON'T HAVE TO FILL IT OUT IF YOU DON'T WANT TO. IF YOU WANT TO USE SIGN TO FILL OUT THE FORM, JUST TELL ONE OF THE INTERPRETERS, OKAY, AND WHAT WE'LL DO IS WE WILL HAVE QUOC OR JORGE WILL FILM YOU, AND THEY WILL RECORD WHAT YOU SAID, AND THEN GO FROM THERE. SO YOU DON'T HAVE TO FILL OUT EVERYTHING IF YOU DON'T WANT TO. THE OTHER THING I WANT TO SAY IS WE'RE GOING TO HAVE A DISCUSSION, AND SO WHAT WE'RE GOING TO ASK YOU TO DO IS, YOU KNOW, RAISE YOUR HAND AND GO ONE AT A TIME. AND THAT'S FOR OBVIOUS REASONS. THE CAPTIONER CAN ONLY REALLY WORK WITH ONE PERSON AT A TIME, AND THE INTERPRETER CAN ONLY REALLY WORK WITH ONE PERSON AT A TIME, AND WE DON'T WANT THEM TO, YOU KNOW, GO INSANE ON US, SO, RIGHT? SO BEFORE I 
BEGIN, BEFORE I GET YOU INTO YOUR PACKAGES, DOES ANYBODY HAVE ANY QUESTIONS BEFORE I GET STARTED?

DAVID: NO? YOU'RE GOOD? OKAY. SO IN YOUR PACKAGE, WHAT YOU'LL FIND -AND I'M SORRY, THEY ARE THICK. FIRST YOU WILL HAVE FOUND THIS. HOPEFULLY YOU HAVE ALL READ IT. THIS LITTLE TWO-PAGE THING, YOU GET TO TAKE THIS HOME WITH YOU, OKAY. SO YOU CAN TAKE THIS HOME WITH YOU. THIS IS INFORMATION ABOUT WHO WE ARE AND WHAT WE'RE DOING, OKAY. IT IS TO HELP YOU UNDERSTAND WHAT THE STUDY IS ALL ABOUT FOR FUTURE INFORMATION. NOW, THIS IS THE CONSENT FORM. IF YOU HAVE SIGNED IT ALREADY, LIDA WILL GO AROUND AND SHE WILL PICK IT UP. IF YOU DON'T WANT TO SIGN THIS, IF YOU WANT TO VIDEO RECORD A SIGNED CONSENT -- A CONSENT IN ASL, JUST RAISE YOUR HAND AND QUOC WILL VIDEO RECORD YOUR CONSENT IN ASL. SO IF YOU CAN PLEASE SIGN THIS FORM OR RAISE YOUR HAND AND ASK FOR QUOC, AND THEN LIDA WILL COME AROUND AND PICK THEM UP. SO THAT'S THE FIRST THING WE NEED TO DO.

PARTICIPANT 1: SHOULD WE GIVE IT TO LIDA NOW, OR ARE WE WAITING?

DAVID: YEAH, LIDA. SHE'S GOING TO COME AROUND RIGHT NOW.

DAVID: AND THEN THE OTHER THING WE NEED YOU TO FILL OUT -- NO, I'M GOING TO WAIT. I'LL WAIT.

DAVID: IF YOU HAVEN'T DONE SO ALREADY, CAN YOU PLEASE FILL OUT OUR PRESTUDY QUESTIONNAIRE. THIS IS THE QUESTIONNAIRE THAT JUST TELLS US -$O H, I^{\prime} M$ SORRY.

DAVID: OKAY. IF YOU HAVEN'T DONE SO ALREADY PLEASE FILL OUT THE PRESTUDY QUESTIONNAIRE. IT LOOKS LIKE THIS. YOU HAVE DONE IT? OKAY. SO LIDA WILL BE PICKING UP THOSE.

(DISCUSSING CAMERA SETTINGS)

QUOC: SO WE'RE READY.

DAVID: OKAY. WE'RE GOOD. SO IN YOUR PACKAGE, YOU HAVE A STACK OF QUESTIONNAIRES THAT LOOK LIKE THIS. YOU HAVE ABOUT 30 OF THEM. WE'RE ONLY GOING TO USE 28, BUT YOU HAVE SPARES, SO THAT'S GOOD. NOW, LET ME EXPLAIN WHAT IS GOING TO HAPPEN. WHAT YOU'RE GOING TO SEE IN A MOMENT IS YOU'RE GOING TO SEE A VISUALIZATION OF A SONG. IT WILL BE ABOUT 30 SECONDS LONG, OKAY. IT'S GOING TO BE A -- IT WILL BE A WEIRD LOOKING PICTURE AND IT'S ONLY A FEW SECONDS AND THEN IT WILL BE DONE. WHEN IT'S DONE, WHAT WE WOULD LIKE YOU TO DO IS FILL OUT THIS FORM. NOW, AS I SAID BEFORE, YOU DON'T HAVE TO DO THIS, YOU CAN DO IT IN SIGN INSTEAD. JUST 
RAISE YOUR HAND, AND QUOC WILL COME AROUND WITH EITHER JENNIFER OR TALA AND TAKE YOUR COMMENTS. OKAY. DO YOU HAVE ANY QUESTIONS?

PARTICIPANT 5 (MIGHT HAVE BEEN SOMEONE ELSE. THE PERSON WAS BLOCKED): HOW OFTEN WILL WE BE SHOWING THE VISUALIZATION?

PARTICIPANT 5: (NOT VOICED).

DAVID: WHAT WERE GOING TO DO IS WE'RE GOING TO SHOW YOU A VISUALIZATION ABOUT 30 SECONDS LONG AND THEN WE WILL FILL OUT THIS, AND THEN WE WILL DO IT AGAIN AND FILL OUT THIS AND THEN DO IT AGAIN AND FILL OUT THIS. AFTER WE HAVE DONE 7 OF THESE, THEN WE ARE GOING TO STOP FOR A LITTLE BIT, TAKE A BIT OF A BREATHER, AND THEN TALK ABOUT WHAT WE HAVE SEEN. WE WILL DO SOME DISCUSSIONS.

PARTICIPANT 6: THERE'S A WARNING OF A PSYCHOLOGICAL NATURE, ARE WE GOING TO BE SENT OFF TO A HOSPITAL AFTER THIS? I'M WONDERING WHAT THIS WARNING IS ALL ABOUT.

DAVID: I'M VERY GLAD YOU ASKED. SO THE UNIVERSITY FORCES US TO MAKE SURE WE SAY CERTAIN THINGS IN OUR INFORMATION FORMS ALL THE TIME. ONE OF THEM IS A WARNING THAT YOU MAY BE PSYCHOLOGICALLY HARMED. WE DON'T EXPECT THAT YOU WILL ACTUALLY BE PSYCHOLOGICALLY HARMED. YOU MAY FEEL A LITTLE BIT TIRED AFTER SEEING, YOU KNOW, 20 OF THESE BEFORE WE GET TO THE LAST ONE. THAT I'M EXPECTING, BUT WE'RE HOPING WE'RE NOT SENDING YOU TO ST. MIKES WHEN WE'RE DONE. YES.

PARTICIPANT 4: SO THERE'S NO DANGER OF ME WANTING TO SMOKE UP AFTERWARDS.

DAVID: I'M NOT SAYING THAT, AND I'M NOT RESPONSIBLE FOR CRIMINAL BEHAVIOUR.

PARTICIPANT 4: OH, OKAY. NOT THAT I'M IN THE HABIT, NO. I DON'T INHALE.

DAVID: OH, I'M GOING TO HAVE FUN TODAY.

DAVID: OKAY. ANY OTHER QUESTIONS?

DEB: JUST A REMINDER THAT IT IS VOLUNTARY AND YOU CAN STOP AT ANY TIME

DAVID: OH, YES. AND AS YOU READ ON THE CONSENT FORM, JUST TO REMIND YOU, THIS IS VOLUNTARY. YOU CAN STOP AT ANY TIME. WE ARE NOT FORCING YOU TO GO THROUGH ALL 28 VIDEOS, THOUGH WE WOULD REALLY LIKE YOU TO.

PARTICIPANT 8: (CAN'T HEAR QUESTION) 
DAVID: I HOPING TO BE DONE BETWEEN 5:30 AND 6, YES.

DEB: SORRY

PARTICIPANT 8: I HAVE A CLASS

DAVID: SHE WAS ASKING IF WE WERE GOING TO BE DONE AT 5:30.

PARTICIPANT 8: YEAH, IT JUST THAT I HAVE A CLASS.

DEB: DAVID, DAVID?

DAVID: YUP, WE'RE GOING TO TRY. WE'RE GOING TO TRY FOR 5:30.

DAVID: SO LET'S GO. DOUG. THAT'S YOUR CUE.

(BLOCK 1, SONG 1).

DAVID: OKAY.

CANT SEE SPEAKER: THAT'S ALL? THAT'S IT.

DAVID: YEAH, THAT'S IT. JUST ABOUT 30 SECONDS. THAT'S IT. SO FILL OUT THE

FORM, AND WE WILL SET UP FOR THE NEXT ONE.

PARTICIPANT 1: THAT WAS THE FIRST ONE, CORRECT?

DAVID: YEAH, THAT WAS THE FIRST SONG, YES.

PARTICIPANT 4: IT SAID, WHAT WAS THE ENERGY LEVEL OF THE MUSIC, THERE'S NO MUSIC, RIGHT?

DAVID: THERE'S NO SOUND. SO IT'S VISUALIZING MUSIC BUT THERE'S NO SOUND.

PARTICIPANT 4: OH, OKAY. OKAY.

PARTICPANT 9: THIS IS A PROBLEM FOR ME BECAUSE MUSIC, MY UNDERSTANDING IS YOU HEAR. IT JUST SEEMS TO ME THAT I DON'T KNOW HOW TO ANSWER THAT. THAT'S NOT REALLY MUSIC TO ME.

DAVID: OK, THAT'S FINE. IT'S VISUAL MUSIC.

UNKNOWN: DAVID, I AM HAVING A HARD TIME HEARING THE QUESTION. CAN YOU PLEASE REPEAT THE QUESTION?

DAVID: OKAY, SURE SO YOUR QUESTION WAS WHETHER OR NOT YOU NEEDED TO FILL OUT THE WHOLE FORM, IS THAT CORRECT?

PARTICIPANT 9: I HAVE TO? 
DAVID: NO.

PARTICIPANT 9: I DON'T HAVE TO. OKAY.

DAVID: NO. ONLY AS MUCH AS YOU LIKE.

DAVID: OKAY. SO HAS EVERYBODY GIVEN THEIR FORM TO LIDA. THAT'S THE IMPORTANT THING. WE NEED TO MAKE SURE ALL YOUR FORMS GO TO LIDA JUST BEFORE WE START. UNFORTUNATELY, I KNOW THAT REALLY SLOWS IT DOWN.

DAVID: OKAY. SO LET ME EXPLAIN THIS ONE VERY QUICKLY. WHAT YOU'RE SEEING HERE IS YOU'RE SEEING -- LET ME EXPLAIN WHAT YOU'RE SEEING. WHAT YOU'RE SEEING HERE ARE FANS. THIS IS WHAT WE CALL FANS. EACH OF THESE GRAPHICS REPRESENTS A DIFFERENT INSTRUMENT, OR IN THIS CASE, A SINGER IF THERE IS ONE. AND WHAT YOU'RE GOING TO SEE IS YOU'RE GOING TO SEE THE -- WE'RE GOING TO HAVE COLOURS RUN THROUGH THIS. OKAY. MY FIRST QUESTION FOR YOU IS WHICH OF THE FOLLOWING BACKGROUNDS WORK BEST FOR YOU THIS ONE? THIS ONE? THIS ONE? OR --

PARTICIPANT 1, 2, 7, 8 AND MAYBE MORE: THE FIRST ONE, THE FIRST ONE.

PARTICIPANT 7: ACTUALLY THE BLACK ONE WAS BETTER.

DAVID: OK, SO THE SECOND ONE

PARTICPANT 1: NO, I LIKED THE FIRST ONE BETTER.

PARTICIPANT 7: COME ON! THE BLACK ONE IS BETTER.

PARTICIPANT 1: THE FIRST ONE.

DAVID: THIS ONE? SO IS THIS OKAY? OKAY. SO WE WILL GO FOR THIS ONE. WHAT YOU'RE GOING TO SEE IS THE COLOURS MOVE FROM HERE IN TOWARDS THE CENTRE. OKAY?

( BLOCK 1, SONG 2)

DAVID: OKAY. SO FILL OUT THE FORM. TELL US WHAT YOU THINK. THE COLOUR -I SHOULD SAY -- I WILL WAIT.

DAVID: OKAY. SO IF YOU ARE DONE, PLEASE HAND IT OFF TO LIDA.

DAVID: SO THE NEXT VISUALIZATION WE'RE GOING TO SHOW YOU IS WHAT WE -SORRY. WE REFER TO THIS AS A FLASHING SUN. SO BASICALLY IT'S AN OVAL THAT'S GOING TO PULSE. OKAY. READY?

(BLOCK 1, SONG 3). 
DAVID: OK READY? SO AGAIN, WE'RE GOING TO USE THE FANS. OKAY. LETS GO.

(BLOCK 1, SONG 4)

DAVID: OKAY.

PARTICIPANT 4: POKAROO (LAUGHS).

PARTICIPANT 6: IS THAT THE SAME AS BEFORE OR IS IT DIFFERENT?

DAVID: IT WAS DIFFERENT.

PARTICIPANT 6: IT KINDOF LOOKED THE SAME.

DAVID: THAT'S FINE. OKAY.

DAVID: SO OUR NEXT ONE -- OH. OKAY. SO OUR NEXT ONE, WHAT WE'RE GOING TO USE IS WE'RE GOING TO USE SORT OF A FIREWORKS EFFECT. SO EACH OF THE BURSTS ARE GOING TO, I GUESS, GOING TO BE MEANINGFUL TO MUSIC. I SHOULD SAY, BY THE WAY, IN THE ONE WE WERE JUST SEEING, THE ONE WE JUST FINISHED SEEING, YOU WERE ASKING WHETHER OR NOT THEY WERE THE SAME OR DIFFERENT. THEY ARE DIFFERENT. BUT IF YOU NOTICED THE COLOURED CIRCLES, THAT'S INTENDED TO BE SORT OF A BEAT EFFECT. UNFORTUNATELY, I COULDN'T TELL YOU THAT BEFORE PLAYING IT. AND THEN THE FANS, THE WAY THE COLOUR THAT IS SHOOTING OUT OR IN, IT IS SUPPOSED TO TELL YOU WHAT THE -- WHAT NOTE IS BEING PLAYED, HOW LONG THAT NOTE IS, THAT KIND OF THING. BUT THAT'S WHAT IS BEING COMMUNICATED. ARE WE READY?

PARTICIPANT 1: I THINK YOU HAVE TO DO IT AGAIN, BECAUSE YOU WERE TALKING AT THE SAME TIME AS IT WAS BEING SHOWN.

DAVID: SORRY, I DIDN'T REALIZE THAT. OKAY. YOU HAVE TO WARN ME

UNKNOWN: SORRY ABOUT THAT.

(BLOCK 1, SONG 5)

DAVID: OKAY. SORRY ABOUT THE DELAY. WE HAD A SOFTWARE ISSUE. BUT DOUG TELLS ME WE'RE GOOD TO GO. OKAY. OKAY. SO IS EVERYONE READY? EVERYONE IS READY?

(BLOCK 1, SONG 6).

DAVID: OKAY.

(DISCUSSING CAMERA SETTINGS) 
DAVID: OKAY SO THIS IS THE LAST VISUALIZATION FOR THIS FIRST BLOCK OF 7. AND I'LL JUST QUICKLY EXPLAIN. AS YOU CAN SEE HERE, EACH OF THESE GROUPS OF LINES REPRESENTS A DIFFERENT INSTRUMENT.

DAVID: OKAY. IS EVERYONE READY?

(BLOCK 1, SONG 7).

DAVID: THERE.

PARTICIPANT 1: (SIGNS)

TALA: (WHISPER PARTICIPANT 1'S QUESTION TO DAVID)

DAVID: SHE'S TRYING TO FIGURE OUT THE BEST WAY TO EXPRESS THE MUSIC GOING UP AND DOWN. IS THAT FREQUENCY, PITCH, WHAT IS THAT? AND SO WHATEVER WORD WORKS FOR YOU.

PARTICIPANT 1: OK THANKS.

PARTICIPANT 4: I UNDERSTAND THE LAST ONE, BUT ME, I HAVE NOTICED SINCE THE FIRST ONE, ONE QUESTION. WHAT DOES THE VISUALIZATION MEAN TO YOU? I'M ALWAYS ANSWERING WITH ONE OR TWO WORDS, YOU KNOW WHAT I MEAN? FOR EXAMPLE, THE LAST ONE REMINDED ME OF STAR WARS 3. I DON'T KNOW IF THAT'S GOING TO HELP YOUR RESEARCH OR NOT. I DON'T KNOW.

DAVID: I DON'T KNOW. YOU NEVER KNOW. YOU NEVER KNOW. SO THAT'S A GOOD QUESTION. HOW IS THIS WORKING FOR YOU GUYS? WAS THERE A PARTICULAR VISUALIZATION THAT YOU REALLY LIKED SO FAR?

PARTICIPANT 1: SOME WERE NOT BAD. SOME I LIKED THEM.

DAVID: ARE THERE SOME THAT YOU REALLY DON'T LIKE?

PARTICIPANT 2: THE LAST ONE.

UNKNOWN: YEAH, I AGREE.

DAVID: AND SO WHY DO YOU NOT LIKE IT?

PARTICIPANT 2: IT'S LIKE GOING TO THE ORCHESTRA OR SOMETHING?

PARTICIPANT 1: YEAH YOU KNOW, THE LEVELS. I CAN RECOGNIZE THERE ARE LEVELS GOING UP AND DOWN, BUT IN TERMS OF IT --IT'S STILL SLOW AND IS STILL A LITTLE FLAT. THE COLOURS ARE VERY CONFUSING. IF IT WAS THE SAME COLOUR, PERHAPS SHOWING LIKE AN EQUALIZER, YOU CAN MAYBE SEE IT. BUT THE DIFFERENT LINES, THERE WAS A DISCONNECT FOR ME. 
PARTICIPANT 8: IT LOOKS LIKE THE LINES ON AN EKG MACHINE, THE LAST ONE. THE HEART MONITOR, LIKE THE UP AND DOWN OF A HEART MONITOR.

DAVID: I WAS TOLD ACTUALLY THE MODEL WAS MORE LIKE A PIANO ROLL, LIKE AN OLD ANTIQUE PIANO YOU JUST TAKE A ROLL OF PAPER WITH THE PUNCHES IN IT...YEAH I WAS TOLD THAT IS WHAT THE MODEL IS.

PARTICIPANT 10: I WAS GOING TO MAKE A COMMENT. I DON'T KNOW IF IT WAS THE FIRST OR THE SECOND ONE THAT WAS SO COLOURFUL THAT WAS LIKE STAR WARS. I LIKED LOOKING AT IT, BUT BECAUSE THE CONCEPT IS SO DIFFERENT, I DON'T KNOW IF I COULD RELATE IT TO MUSIC, ACTUALLY. BUT IT WAS VERY VISUALLY APPEALING.

DAVID: SO, SO FAR, IF WE WERE TO TALK ABOUT MUSIC, DO THESE VISUALIZATIONS GIVE YOU A SENSE OF THE INFORMATION THAT YOU NEED?

UNKNOWN: NO.

DAVID: NO?

PARTICIPANT 1: IN TERMS OF THE ONES WITH THE DOTS WITH THE COLOUR DOTS, YOU GET A SENSE MORE OF -- SORT OF REMINDS ME OF LIKE DRUMS, A BEAT THING.

PARTICIPANT 4: I FIND THAT REALLY HAVE ENOUGH TIME TO ANSWER THE QUESTION, TO GIVE IT SOME THOUGHT AND THINK ABOUT IT MORE, YOU KNOW WHAT I MEAN? WE HAVE TO DO IT FAST, FAST, FAST. I WISH WE HAD A LITTLE MORE TIME.

DAVID: WELL, IS THERE ANYTHING THAT YOU WOULD LIKE TO SAY AT THIS POINT THAT SORT OF JUST STRIKES YOU AS YOU'RE --

PARTICIPANT 9: IT'S STILL NOT MUSIC TO ME. TO ME I CANNOT CONNECT THIS TO MUSIC. EVEN IF YOU DON'T HEAR, YOU STILL HAVE A SENSE OF THE VIBRATION RINGING. BUT THIS IS JUST PICTURES HERE. THERE'S NO VIBRATION.

PARTICIPANT 1: YEAH, IT'S INTERESTING. SOME OF IT I FEEL LIKE, OH, YEAH, I GUESS THAT'S WHAT MUSIC MIGHT LOOK LIKE. BUT IN TERMS OF -- I AGREE, I AGREE WITH WHAT SHE'S SAYING, THAT IF THERE'S SOME SORT OF VIBRATION OR SOMETHING WHILE WE HAD THE VISUALIZATION, YOU WOULD HAVE A DIFFERENT TACTILE RESPONSE. SO MAYBE THERE'S A CONNECT BETWEEN THAT BUT AS YOU WATCH THE VIDEO, I CAN MAYBE ENVISION WHAT THE MUSIC MIGHT BE. NOT COMPLETELY, BUT I GET A SENSE. SO MAYBE IF THERE'S MORE OF A STIMULUS, THEN YOU COULD PUT TWO AND TWO TOGETHER.

DAVID: YOU'RE SAYING ABOUT HALF AND HALF. 
PARTICIPANT 1: YES.

PARTICIPANT 9: LIKE WHAT SHE'S SAYING. I HAVE TO IMAGINE WHAT THIS IS LIKE MYSELF. THIS IS WORK. IT'S NOT ENJOYMENT. IT'S NOT COMING TO ME. I HAVE TO MAKE AN EFFORT TO VISUALIZE AND DEVELOP SOME UNDERSTANDING OF WHAT I SEE. IT IS LIKE WORK.

PARTICIPANT 10: I UNDERSTAND WHAT YOU'RE SAYING, BUT I HAVE TO MENTION THAT FOR ME IT IS LIKE WORK TO TAKE PART IN A CONVERSATION AS WELL. THAT'S HARD WORK, LISTENING AND TRYING TO HEAR, SO.

DAVID: I WAS SORT OF THINKING THE SAME THING. FOR HARD OF HEARING PEOPLE, THERE'S A LOT OF WORK INVOLVED JUST TRYING TO FOCUS ON A CONVERSATION.

DAVID: ANY OTHER THOUGHTS? ANY OTHER QUESTIONS? OKAY. SO WE'RE GOING TO MOVE INTO THE NEXT SET OF VIDEOS.

UNKNOWN: ARE YOU GOING TO DESCRIBE IT?

DAVID: NO.

(BLOCK 2. SONG 1).

DAVID: OKAY.

DAVID: SO THIS IS OUR FLASHING SUN VIEW AGAIN.

(BLOCK 2. SONG 2)

DAVID: OKAY.

DAVID: IS EVERYONE READY? IS EVERYONE READY? OKAY. SO ONCE AGAIN, WE'RE BACK TO THE FANS. I JUST WANTED TO POINT OUT, SOMEBODY ASKED WHAT SOME OF THE DIFFERENCES WERE. A LOT OF THE DIFFERENCES YOU MAY SEE IS AS THE COLOURS GO OUT, OKAY, ONE OF THE DIFFERENCES IS HOW THE COLOUR DECAYS. SOMETIMES WHAT HAPPENS IS IT WILL DECAY FROM THE MIDDLE OUTWARD, AND SOMETIMES IT WILL DECAY FROM THE OUTSIDE IN. ALSO, SOMETIMES WHAT YOU'LL SEE IS YOU'LL SEE WE HAVE A LOT MORE OF THESE. SOMETIMES WE ONLY HAVE 3 OR 4. SOMETIMES WE HAVE 5 OR 6. SO THOSE ARE SOME OF THE DIFFERENCES. AND, OF COURSE, EACH OF THESE REPRESENTS THE NUMBER OF INSTRUMENTS IN THE SONG.

PARTICIPANT 4: YEAH, I HAVE NOTICED FOR MYSELF, EVEN IF YOU HAVE THE DIFFERENT COLOURS, WHEN I SEE THE PICTURES OF THE FANS, I'M JUST GOING BORING, YOU KNOW. 
DAVID: THAT'S A FAIR COMMENT.

PARTICIPANT 4: NOT LIKE THE FIRST ONE, YOU KNOW WHAT I MEAN, WHICH WAS MORE COLOURFUL.

DAVID: ARE YOU READY? LET'S GET STARTED.

(BLOCK 2. SONG 3).

DAVID: OKAY. SO THIS ONE IS GOING TO BE -- THIS ONE WILL BE ANOTHER MOVING PICTURE AGAIN. ARE YOU ALL SET?

(BLOCK 2, SONG 4).

PARTICIPANT 4: NOW, THAT'S BETTER.

(WATCHING VIDEO)

DAVID: OKAY.

PARTICIPANT 4: IF I HAD A LIGHTER, I WOULD BE FLICKING IT RIGHT NOW.

DAVID: ACTUALLY, MY CELL PHONE HAS A LITTLE LIGHTER APPLICATION ON IT, SO WHEN I GO TO A CONCERT AND I'M JUST HOLDING MY CELL PHONE, TURN ON THE LIGHTER AND -- IT'S TRUE.

PARTICIPANT 4: COOL.

PARTICIPANT 3: I ALSO LIKE IT, BUT I DO NOT LIKE FANS. FANS ARE...

PARTICIPANT 7: YOU GETTING TIRED OF WALKING AROUND YET?

DAVID: OKAY. IS EVERYONE READY? OH, YOU'RE STILL COLLECTING. SORRY.

DAVID: OKAY, LETS GO.

(BLOCK 2, SONG 5).

DAVID: OKAY.

PARTICIPANT 4: CAN I HAND IN A BLANK PAPER?

DAVID: IT'S YOUR RIGHT. YOU CAN TELL US AS MUCH AS OR AS LITTLE AS YOU LIKE.

PARTICIPANT 4: THANK YOU. I'M CONCERNED. I WANT TO HELP YOU WITH YOUR RESEARCH, YOU KNOW WHAT I MEAN. BUT THAT PART, HOW AM I GOING TO EXPLAIN THAT? YOU KNOW, I DIDN'T LIKE IT. 
DAVID: THAT'S FINE.

DAVID: OKAY. SO I WILL JUST QUICKLY EXPLAIN THIS. SO IN THIS PARTICULAR VISUALIZATION, WHAT WE HAVE IS WE HAVE A LINE HERE IN THE MIDDLE, WHICH IS THE TIME POINT IN THE SONG. SO THAT'S YOUR POINT OF REFERENCE IN TERMS OF WHERE WE ARE. THE LINE GOING ACROSS THE THING IS TO SHOW YOU THE DIFFERENT VOLUMES OF THE NOTE. EACH OF THESE LINES REPRESENTS A DIFFERENT INSTRUMENT, AND YOU'RE GOING TO SEE THE CIRCLES HERE, THEY ARE LIKE BALLS, THEY PULSE TO GIVE YOU A SENSE OF HOW THE MUSIC IS SHOWN. IS EVERYONE GOOD TO GO?

(BLOCK 2, SONG 6).

DAVID: OKAY.

PARTICIPANT 4: CAN I BE EXCUSED FOR A FEW MINUTES? I FEEL LIKE HIGH SCHOOL.

DAVID: WE'LL TAKE A SHORT BREAK RIGHT NOW. WE WILL TAKE A SHORT BREAK RIGHT NOW. JUST TWO OR THREE MINUTES TO LET HIM GO TO THE WASHROOM AND COME BACK. DOES ANYONE ELSE WANT TO GO TO THE WASHROOM?

PARTICIPANT 7: I JUST WANTED TO LET YOU KNOW MY PARKING METRE IS UP UNTIL 5:30. I WONDER IF WE WILL BE DONE SOON.

DAVID: YEAH, I'M HOPING TO GET THROUGH THE NEXT -- WE'RE GOING TO GO THROUGH THIS VISUALIZATION, THEN WE'RE GOING TO HAVE A QUICK DISCUSSION, AND THEN WE WILL DO ONE MORE BLOCK OF 7 WHERE WE ARE ACTUALLY WE JUST HAD A DISCUSSION WHERE WE'RE GOING TO CUT THIS THING SHORT BECAUSE WE ARE FINDING THIS IS GOING TOO LONG TOO FOR US.

PARTICIPANT 6: (NOT VOICED)

TALA: IS IT A BREAK RIGHT NOW?

DAVID: WASHROOM, YOU GO OUT THE DOOR, YOU GO STRAIGHT DOWN, OR YOU GO UP AND THEN TURN RIGHT AND STRAIGHT DOWN.

TALA: THANKS.

DAVID: WHILE WE ARE WAITING FOR THE GENTLEMAN TO COME BACK, HOW ARE WE DOING SO FAR? WHAT DO YOU THINK? ARE THERE ANY VISUALIZATION IN PARTICULAR THAT YOU REALLY LIKED SO FAR?

PARTICIPANT 7: I PREFER THE COLOUR. I'M KIND OF COLOUR SENSITIVE TO STUFF. IF I SEE NOTHING WITH ANY COLOUR IN IT, THEN I FEEL THAT IT'S NOT VERY INTERESTING. I PREFER THE DESIGN. YOU KNOW, SOMETHING THAT'S 
KIND OF OLD FASHION DOESN'T REALLY APPEAL TO ME. I DON'T KNOW HOW EVERYBODY ELSE FEELS?

DAVID: IS THAT KIND OF DESIGN, THAT COLOURFUL EFFECT, IS THAT ACTUALLY MEANINGFUL TO YOU? DO YOU GET AN EMOTIONAL EXPERIENCE OUT OF THAT?

PARTICIPANT 7: NO, I'M NOT SURE. IT'S JUST, YOU KNOW, LIKE STUFF LIKE HOT PINK. I MEAN, I DON'T REALLY WEAR CLOTHES THAT ARE HOT PINK, SO I DON'T REALLY ENJOY LOOKING AT THOSE KINDS OF COLOURS EITHER.

PARTIICPANT 8: FOR ME DIFFERENT COLOURS REPRESENT DIFFERENT EMOTIONS. SO THE MORE COLOURFUL, THE MORE I GET OUT OF IT, BUT I ALSO FEEL MORE OF A CONNECTION WITH THE DIFFERENT COLOURS IN THE MUSIC, SO.

PARTICIPANT 10: I WAIT FOR IT TO COME UP AND THEN I CAN READ IT. I'M TOO LAZY TO MOVE TO ANOTHER SEAT.

DAVID: SO THEN WITH THE STUFF THAT DOESN'T WORK FOR YOU, WHY DOESN'T IT WORK? WHAT MAKES IT SORT OF NOT WORK? I'M SORRY, DID YOU HAVE SOMETHING TO SAY.

PARTICIPANT 5: NO.

PARTICIPANT 9: THIS ONE, YOU KEEP SHOWING IT. IT'S VERY SMALL, AND YOU KEEP SHOWING IT AGAIN AND AGAIN. I KNOW THIS IS A GUITAR, BUT WHAT IS THAT ONE? IS IT A PIANO?

DAVID: YEAH, THIS ONE IS LIKE AN ORGAN. THIS IS ONE IS I BELIEVE LIKE A GUITAR AND THIS ONE OVER HERE IS A BASS.

UKNOWN: YEAH, IT IS LIKE AN ELECTRIC AND THE OTHER ONE IS LIKE AN ACOUSTIC.

PARTICIPANT 9: THIS IRRITATES ME BECAUSE I HAVE TO FIGURE OUT WHAT'S GOING ON THERE. IT HAS NO MEANING TO ME. YOU KEEP SHOWING IT AGAIN AND AGAIN AND I DON'T KNOW WHAT IT MEANS.

DAVID: DOES ANYBODY -- UHM-HMM.

PARTICIPANT 3: WE CANNOT CONCENTRATE ON ONE. IT IS MOVING SO FAST, THE COLOURS WITH THE FANS. IT IS DIFFICULT TO MAKE SENSE. VERY DIFFICULT. THE FANS.

DAVID: IT'S TOO FAST. IT'S ALMOST TOO BUSY? LIKE, HOW WOULD YOU EXPLAIN IT? 
PARTICIPANT 3: YES. IT'S FAST. IT'S JUST CONFUSING.

PARTICIPANT 10: I THINK ESPECIALLY WHEN YOU HAVE THE 6 OF THEM, IT'S KIND OF DISTRACTING BECAUSE YOU'RE LOOKING AT THIS AND YOU'RE LOOKING AT THE COLOURS AND YOU'RE LOOKING BACK AND FORTH, AND I THINK THAT THE MEANING IS LOST. MY FAVOURITE IS THE BRIGHT COLOUR. I DO FIND THAT IT'S KIND OF RELATED TO COLOUR. WHEN YOU SEE SOMETHING LIKE THAT, IT'S INTRIGUING, UPLIFTING, IT MAKES YOU FEEL HAPPIER. IT IS MORE VISUALLY APPEALING, IN MY OPINION, TO WATCH. I HAVE TO AGREE WITH THAT GENTLEMAN OVER THERE. THIS IS A LITTLE BORING.

DAVID: YES.

PARTICIPANT 2: YOU SEE THE PROBLEM IS WE ARE SEEING IT, BUT WE'RE NOT FEELING ANYTHING, SO IT'S HARD TO...

PARTICIPANT 1: IT'S THE SAME IDEA WHEN YOU HAD THE 6 FANS BUT THEN YOU HAD THE COLOURED DOTS, LIKE, THEY SEEMED TO BE MORE CONNECTED. I LIKED THAT. BUT IF THEY ARE ALL GOING OFF AT DIFFERENT TIME AND KIND OF SEPARATELY. THEN IS NOT GOING OFF VERY SMOOTH.

PARTICIPANT 9: MY COMMENT IS RELATED TO HERS AND HERS. THERE'S TOO MANY THINGS GOING ON, YOU KNOW, IN ALL SORTS OF DIRECTION. AND THERE WAS ANOTHER ONE THAT YOU SHOWED US TO THAT ME REMINDED ME OF THE BIG BANG THEORY. IT'S LIKE MY VISION WAS BEING GUIDED FROM LEFT TO RIGHT IN A WAY THAT WAS MORE RELAXING TO ME. SO IT WASN'T LIKE I WAS BEING PULLED IN DIFFERENT DIRECTIONS.

DAVID: ANY OTHER COMMENTS? I'M GOING TO SORT OF NOT WAIT FOR HIM ANYMORE. WE'RE GOING TO KEEP GOING. SO DOUG.

(DAVID TALKING TO DOUG)

DAVID: SORRY. WE HAVE TO FIX A SOFTWARE ISSUE.

DAVID: A QUICK QUESTION FOR YOU. SO WHEN YOU SEE EACH OF THESE VISUALIZATIONS, ARE YOU PERCEIVING THAT YOU ARE SEEING A DIFFERENT SONG EVERYTIME? DO YOU THINK YOU ARE SEEING THE SAME SONG EVERY TIME? WHAT DO YOU FEEL ABOUT THAT?

PARTICIPANT 8: I JUST, I FIND I'M SO DISTRACTED BY WHAT'S GOING ON THAT I'M TRYING SO HARD FOR THE DURATION OF THE CLIP TO MAKE SENSE OF IT. SO I'M NOT REALLY FOCUSSED ON GETTING MORE OF A MESSAGE ON IT THAN JUST TO TRY TO FIGURE OUT WHAT'S GOING ON. 
PARTICIPANT 1: LIKE I SAID BEFORE, IT WOULD BE NICE IF WE HAD SOME SORT OF VIBRATIONS, LIKE IF WE WERE ACTUALLY FEELING MUSIC BECAUSE THERE'S NO CONNECTION TO WHAT IT IS SUPPOSED TO BE LIKE. YOU KNOW, ESPECIALLY FOR HARD OF HEARING PEOPLE, IT IS DIFFERENT, BUT I'M PROFOUNDLY DEAF AND I WOULD LIKE TO HAVE SOME KIND OF VIBRATION SENSATION TO GO ALONG WITH THE VISUALIZATION.

PARTICIPANT 8: I TOTALLY AGREE. I ALSO THINK THAT, AND NOT MAYBE FOR TODAY BUT SOMEWHERE DOWN THE ROAD, IF YOU COULD ALMOST STANDARDIZE THE COLOURS IF YOU ARE GOING TO USE THE COLOURS. SO JUST STANDARDIZE THE COLOURS JUST TO MEAN ONE THING. THEN YOU WOULDN'T HAVE TO WORRY ABOUT WHAT IT MEANS. THAT WOULD BE THE NEXT LOGICAL STEP TO ME, AND I WOULD PROBABLY GET MORE ENJOYMENT OUT OF IT.

DAVID: ALL SET? SO HERE'S THE NEXT ONE.

(BLOCK 3, SONG 1).

DAVID: OKAY.

PARTICIPANT 4: OH, I MISSED THE CLASSICS. I ONLY SAW THE LAST COUPLE SECONDS.

DAVID: WE ACTUALLY JUST STARTED AGAIN WHEN YOU WALKED IN THE DOOR.

PARTICIPANT 4: OH, OKAY. FINE. THANK YOU.

DAVID: OKAY. SO WE WILL MOVE TO THIS ONE NOW. IS EVERYONE READY?

(BLOCK 3, SONG 2).

DAVID: OKAY.

DAVID: OKAY, SO LET ME EXPLAIN THIS ONE VERY BRIEFLY. WHAT YOU ARE GOING TO SEE IN THIS ONE IS YOU'RE GOING TO SEE -- ONE MINUTE. WHAT YOU'RE GOING TO SEE IN THIS ONE IS YOU'RE GOING TO SEE A DOT APPEAR IN THE MIDDLE OF THE SCREEN AND YOU'RE GOING TO SEE LINES COME OUT OF THAT DOT, AND THEN YOU WILL SEE COLOURED CIRCLES. THE CIRCLES WILL REPRESENT DIFFERENT INSTRUMENTS, AND DIFFERENT FREQUENCIES, DIFFERENT NOTES, AND THEN IT WILL PULSE TO SHOW YOU HOW THE INSTRUMENT IS BEING PLAYED.

(BLOCK 3, SONG 5)

DAVID: OKAY. THAT'SIT.

DAVID: OKAY. SO WHAT DID YOU THINK OF THAT ONE? WAS THAT AN IMPROVEMENT? OR DID YOU LIKE IT? NO. OKAY. 
DAVID: OKAY. SO OUR NEXT VISUALIZATION. THIS IS, AGAIN, THE FANS. YOU WILL NOTE THE NUMBER OF INSTRUMENTS WE HAVE IN THIS ONE. SO LET'S SEE WHAT THIS LOOKS LIKE.

(BLOCK 4. SONG 1).

DAVID: OKAY.

DAVID: OKAY. SO JUST A FEW MORE, I PROMISE. WE ARE ALMOST DONE.

PARTICIPANT 5: IF IT'S PAST 6 O'CLOCK, I HAVE TO LET YOU KNOW YOU HAVE TO PROVIDE US WITH DINNER, OKAY.

DAVID: UHM-HMM. WELL, JUST TWO MORE, THAT'S IT.

PARTICIPANT 10: I JUST WANT TO MENTION THAT DINNER IS NO GOOD FOR ME BECAUSE IF I DON'T CATCH THE TRAIN AND GET HOME IN TIME, I'M GOING TO MISS “THE BACHELOR”.

DAVID: I HAVE THE SAME ISSUE. I HAVE TO GET HOME AT A CERTAIN TIME OR I MISS “HEROS”. SO WE ALL HAVE THE SAME PROBLEM. SO WE'RE GOOD TO GO.

(BLOCK 4, SONG 4).

DAVID: THAT'S IT.

DAVID: SO WHAT DID YOU FOLKS THINK OF THE LAST ONE YOU JUST SAW. SORRY. WHAT DID YOU FOLKS THINK OF THE LAST ONE YOU JUST SAW? BETTER? NO?

PARTICIPANT 6: I FEEL LIKE IT'S SIMILAR TO AN OLD AVC TEST WHERE YOU FLIP IT AND IT'S JUST CHARTED. NO MEANING FOR ME

PARTICIPANT 7: IT'S THE SAME SORT OF THING AS THE ONE -- I JUST FEEL LIKE IT'S SIMILAR TO ALL THE ONES BEFORE IT. IT'S JUST LIKE DIFFERENT BUT THE SAME SORT OF FEELING FOR EACH.

PARTICIPANT 2: THE MUSIC WAS PLAYING AT A VERY COMFORTABLE LEVEL.

PARTICIPANT 1: FOR ME, I LIKED THE LINE. IT SHOWS THE DIFFERENT SOUNDS. I LIKED THAT. THE DOTS, A LITTLE BIT CONFUSING FOR ME. SO THAT WOULD BE MY FEEDBACK.

PARTICIPANT 4: WHEN YOU HAVE THE ONES WITH THE FAN AND THE CIRCLES, THE CIRCLES DISTRACT. DO YOU KNOW WHAT I MEAN?

DAVID: SO YOU'RE TRYING TO INTERPRET WHAT INSTRUMENT YOU'RE LOOKING AT WITH THE CIRCLES, IS THAT WHAT YOU MEAN? CAN YOU EXPLAIN THAT A LITTLE? 
PARTICIPANT 4: I'M NOT TRYING TO INTERPRET THE INSTRUMENT, WHAT INSTRUMENT, WHATEVER IT IS. I'M JUST LOOKING YOU SAID IT'S SUPPOSED TO REPRESENT MUSIC. HOW DO I FEEL, RIGHT? BUT THE CIRCLES JUST KEEP PULSING. THE FAN, I UNDERSTAND THEY ARE CHANGING COLOUR, AND I NOTICE A BIT. BUT BECAUSE OF THE PULSING CIRCLES, I JUST FIND MYSELF FOCUSSED ON THE PULSING CIRCLES, YOU KNOW. YOU MAY NOTICE WITH DEAF, LIKE, IF TWO DEAF ARE TALKING, IF A PERSON, THEY SEE, THEY EASILY GET DISTRACTED. SO SAME WITH THE CIRCLE THING.

DAVID: THAT'S INTERESTING. ANY OTHER COMMENTS? NO? SO THIS IS OUR PULSING SUN AGAIN, AND THEN AFTER THIS WE HAVE ONE MORE.

DAVID: SO THE TONE IS SO LOW. THE LINE IS ACTUALLY THERE.

PARTICIPANT 1: I CAN'T SEE ANYTHING.

DAVID: THE LINE IS ACTUALLY RIGHT THERE, BUT IT'S JUST THAT THERE'S NOT VERY MUCH MUSIC IN THIS PARTICULAR PIECE. WE CAN BARELY SEE IT ON THIS SCREEN TOO.

DAVID: IT WAS THERE. AND THEN THIS IS THE LAST ONE. WE WILL BRING UP THE LAST ONE NOW. SO BEFORE WE GET TOO FAR, I WILL JUST MENTION --

PARITICIPANT 1: DO WE NEED TO FILL OUT FOR THAT ONE?

DAVID: NO. THERE WAS NOTHING THERE. IT WAS BLANK. EVEN I COULDN'T SEE IT, AND I WAS STANDING NEXT TO IT. I WILL JUST COMMENT THAT WHEN WE ARE DONE, IF YOU COULD PULL OUT THIS QUESTIONNAIRE, WE WOULD LIKE YOU TO FILL IT OUT BEFORE YOU LEAVE. SO THIS WILL BE THE LAST QUESTIONNAIRE AFTER THIS SONG. AND THEN ALSO, LIDA WILL BE COMING AROUND SOON AND SHE WILL PRESENT YOU WITH THIS WONDERFUL POSTER. YOU PROBABLY HAVE HEARD ABOUT THE CONCERT. WE ARE THE ONES THAT ARE RUNNING THE CONCERT. SO THIS IS A CONCERT WITH EMOTI-CHAIRS. YOU WERE TALKING EARLIER ABOUT VIBRATIONS AND STUFF. WELL THIS CONCERT IS YOU SIT IN A CHAIR AND FEEL THE VIBRATIONS THROUGHOUT YOUR BODY WHILE THE MUSIC PLAYING.

UNKNOWN: (CAN'T HEAR QUESTION)

DAVID: I BELIEVE IT'S 5.

PARTICIAPANT 2: I SAW IT ON TV.

DAVID: YEAH, YOU SAW IT ON TV. 
PARTICIPANT 4: I SAW THE ADVERTISEMENT. I THINK IT'S GOOD, BUT THE PROBLEM IS IT'S NO GOOD IF THERE'S NO LYRICS.

DAVID: OH, BUT THERE WILL BE LYRICS BECAUSE GEMME OR ONE OF HER FRIENDS WILL BE THERE TO CAPTION THE LYRICS. WE WILL CAPTION THE LYRICS. WE WILL DO A LITTLE BIT OF VISUALIZATION, AND WE'RE ALSO GOING TO USE THE CHAIRS. SO LIDA WILL HAND THESE OUT IN A MOMENT. AND THE LAST THING LIDA WILL BE HANDING OUT TO YOU IS AN ENVELOPE THAT LOOKS LIKE THIS.

PARTICIPANT 1: I SAW THAT ON THE NEWS LAST WEEK. IT'S RELATED TO THE CHAIRS, RIGHT?

DAVID: YEAH, WE'RE THE SAME BUNCH. AND FINALLY WHAT LIDA WILL BE HANDING OUT IS THIS WONDERFUL ENVELOPE HERE. AND WHAT WE WOULD LIKE YOU TO DO IS SIGN AND DATE IT, AND THEN TEAR THIS AWAY AND GIVE IT BACK TO LIDA. AND IF YOU OPEN THE WONDERFUL ENVELOPE, WHAT YOU WILL FIND INSIDE -- OOOH, GOODY! \$30. SO LAST SONG. HIT IT DOUG.

(BLOCK 4, SONG 7)

DAVID: WAS THAT BETTER TO SEE?

PARTICIPANT 1: I NOW SORT OF AGREE WITH HER ABOUT THE BLACK BACKGROUND. IT SEEMS LIKE IT IS BETTER THAN THE WHITE.

DAVID: I WAS WONDERING IF WE SHOULD FLIP THEM AS WE WERE GOING.

PARTICIPANT 1: YEAH MAYBE, THAT WOULD BE GOOD. IT WOULD BE GOOD TO SEE BOTH. IT'S GOOD FOR THE COLOUR. THE BLACK SEEMS TO BE BETTER. IT SEEMS TO BRING OUT THE COLOURS.

DAVID: THE REST, JUST LEAVE IT THERE, AND WE WILL COLLECT THE EXTRAS WHEN WE ARE DONE THE DAY.

PARTICIPANT 7: WANT TO MAKE A COMMENT. IN TERMS OF MUSIC AND THE EMOTIONS FOR THESE VISUALIZATIONS, THERE HAS TO BE A SORT OF HUMAN CONNECT. LIKE SAY FOR EXAMPLE MAYBE SOME DANCING OR TACTILE CONNECTION. TECHNICALLY THOSE THINGS CAN REPRESENT EMOTION, BUT IT'S DIFFICULT. BUT IF YOU SEE A PERSON SIGNING ASL IT CAN BE POETIC OR DANCING, THEN YOU CAN SEE THE CONNECTION. BUT THIS IS HARD TO SEE.

PARTICIPANT 1: OR EVEN SIMPLY HAVING THE VIBRATIONS. I THINK YOU WERE WRITING AT THE TIME, BUT I WAS TELLING HIM THE BLACK BACKGROUND IS NICER. IT'S JUST MORE VISUALLY APPEALING. SO YOU'RE RIGHT ABOUT THE BLACK. 
DAVID: IF THERE'S MORE COMMENTS, THANK YOU. THAT'S FINE, WE WELCOME YOUR COMMENTS. BUT, YOU KNOW, THANK YOU FOR COMING.

PARTICIPANT 1: JUST THINKING, THIS LAST ONE IS DIFFICULT FOR ME TO WRITE BECAUSE I CAN'T REMEMBER ALL OF THEM. FOR EXAMPLE, THE THIRD ONE, I DON'T KNOW HOW TO RESPOND. IF THERE WAS SOME SOUND MAYBE I WOULD HAVE AN EASIER TIME REMEMBERING.

(DAVID TALKING TO PARTICIPANT 1)

DAVID: ANY LAST COMMENTS OR QUESTIONS? WOULD ANYONE LIKE TO GIVE A LAST COMMENT OR QUESTION? NO. OTHERWISE, THANK YOU FOR COMING. WE REALLY APPRECIATE YOUR TIME TODAY.

TRANSLATOR SPEAKING ON BEHALF OF PARTICIPANT 10: IT WAS VERY REPITITIOUS. SHE SAW THE SAME THING AGAIN AND AGAIN AND AGAIN.

PARTICIPANT 10: IT WAS VERY REPITITIOUS, ESPICIALLY THE FANS. 


\begin{tabular}{|c|c|c|c|c|c|c|c|}
\hline $\begin{array}{l}\text { Subject } \\
\text { Number }\end{array}$ & Identity & Gender & Age & Education & $\begin{array}{c}\text { Use of } \\
\text { CC }\end{array}$ & $\begin{array}{c}\text { Music } \\
\text { Listening } \\
\text { Frequency }\end{array}$ & $\begin{array}{c}\text { Self } \\
\text { Description }\end{array}$ \\
\hline 16 & Deaf & Male & $45-54$ & University & Always & All the time & really enjoy listening to music \\
\hline 17 & $\mathrm{HH}$ & Female & $45-54$ & University & Always & Rarely & do not like or dislike music \\
\hline 18 & $\mathrm{HH}$ & Female & 65 plus & Graduate School & Always & Frequently & really like listening to music \\
\hline 19 & $\mathrm{HH}$ & Female & $35-44$ & University & Always & Sometimes & like listening to music \\
\hline 20 & Deafened & Female & $55-64$ & High School & Always & Sometimes & really like listening to music \\
\hline 21 & Deaf & Male & $55-64$ & Graduate School & Always & All the time & really like listening to music \\
\hline 22 & Deaf & Male & $25-34$ & Technical college & Always & Rarely & like listening to music \\
\hline 23 & Deaf & Female & $25-34$ & Graduate School & Always & Frequently & really like listening to music \\
\hline 24 & Deaf & Female & $25-34$ & High School & Always & Sometimes & like listening to music \\
\hline 25 & Hard of hearing & Female & $25-34$ & University & Sometimes & Frequently & like listening to music \\
\hline
\end{tabular}

Table B.1: Focus Group I demographics.

\begin{tabular}{lccc}
\hline $\begin{array}{l}\text { Subject } \\
\text { Number }\end{array}$ & $\begin{array}{c}\text { Music } \\
\text { Experience }\end{array}$ & $\begin{array}{c}\text { Most } \\
\text { Enjoyed }\end{array}$ & $\begin{array}{c}\text { Sense of } \\
\text { Music }\end{array}$ \\
\hline 16 & Somewhat positive & iTunes & iTunes \\
17 & Very positive & iTunes & iTunes \\
18 & Very positive & Balls & Compass \\
19 & Somewhat positive & iTunes & iTunes \\
20 & Very positive & iTunes & Balls \\
21 & Somewhat positive & Unknown & Unknown \\
22 & Very negative & iTunes & iTunes \\
23 & Somewhat positive & iTunes & iTunes \\
24 & Somewhat positive & iTunes & iTunes \\
25 & Somewhat positive & Balls & Balls \\
\hline
\end{tabular}

Table B.2: Most enjoyed visualization. 


\begin{tabular}{|c|c|c|c|c|c|c|c|c|}
\hline $\begin{array}{l}\text { Subject } \\
\text { Number }\end{array}$ & $\begin{array}{c}\text { Colours } \\
\text { Going } \\
\text { Out } \\
\text { Instruments } \\
\text { Playing }\end{array}$ & $\begin{array}{c}\text { Colours } \\
\text { Going } \\
\text { In } \\
\text { Instruments } \\
\text { Playing }\end{array}$ & $\begin{array}{c}\text { Lots } \\
\text { of } \\
\text { Fans } \\
\text { Instruments } \\
\text { Playing }\end{array}$ & $\begin{array}{c}\text { Few } \\
\text { Fans } \\
\text { Instruments } \\
\text { Playing }\end{array}$ & $\begin{array}{c}\text { Psychedelic } \\
\text { Instruments } \\
\text { Playing }\end{array}$ & $\begin{array}{c}\text { Flashing } \\
\text { Sun } \\
\text { Instruments } \\
\text { Playing }\end{array}$ & $\begin{array}{c}\text { Moving } \\
\text { Shapes } \\
\text { Instruments } \\
\text { Playing }\end{array}$ & $\begin{array}{c}\text { None } \\
\text { Instruments } \\
\text { Playing }\end{array}$ \\
\hline 16 & & & & & 1 & & & \\
\hline 17 & 1 & & & & & & & \\
\hline 18 & & 1 & & 1 & & & 1 & \\
\hline 19 & & & & & & 1 & & \\
\hline 20 & & & & & 1 & & & \\
\hline 21 & 1 & & & 1 & & & & \\
\hline 22 & & & & & & 1 & & \\
\hline 23 & & & & & & & 1 & \\
\hline 24 & & & & 1 & 1 & & & \\
\hline 25 & & & 1 & & & & & \\
\hline
\end{tabular}

Table B.3: Participant's selection of visualizations best showing instrument information.

\begin{tabular}{|c|c|c|c|c|c|c|c|c|}
\hline $\begin{array}{l}\text { Subject } \\
\text { Number }\end{array}$ & $\begin{array}{c}\text { Colours } \\
\text { Going } \\
\text { Out } \\
\text { Drums }\end{array}$ & $\begin{array}{c}\text { Colours } \\
\text { Going } \\
\text { In } \\
\text { Drums }\end{array}$ & $\begin{array}{c}\text { Lots } \\
\text { of } \\
\text { Fans } \\
\text { Drums }\end{array}$ & $\begin{array}{l}\text { Few } \\
\text { Fans } \\
\text { Drums }\end{array}$ & $\begin{array}{l}\text { Psychedelic } \\
\text { Drums }\end{array}$ & $\begin{array}{l}\text { Flashing } \\
\text { Sun } \\
\text { Drums }\end{array}$ & $\begin{array}{l}\text { Moving } \\
\text { Shapes } \\
\text { Drums }\end{array}$ & $\begin{array}{l}\text { None } \\
\text { Drums }\end{array}$ \\
\hline 16 & & & & & 1 & & & \\
\hline 17 & 1 & & & & & & & \\
\hline 18 & & & & & & & & 1 \\
\hline 19 & 1 & & & & & & & \\
\hline 20 & & & & & 1 & & & \\
\hline 21 & 1 & & & 1 & & & & \\
\hline 22 & & & & & & 1 & & 1 \\
\hline 23 & 1 & & & & & 1 & & \\
\hline 24 & 1 & 1 & & & & & & \\
\hline 25 & & & & & & 1 & & \\
\hline
\end{tabular}

Table B.4: Participant's selection of visualizations best showing percussion information. 


\begin{tabular}{|c|c|c|c|c|c|c|c|c|}
\hline $\begin{array}{l}\text { Subject } \\
\text { Number }\end{array}$ & $\begin{array}{l}\text { Colours } \\
\text { Going } \\
\text { Out } \\
\text { Melody }\end{array}$ & $\begin{array}{l}\text { Colours } \\
\text { Going } \\
\text { In } \\
\text { Melody }\end{array}$ & $\begin{array}{l}\text { Lots } \\
\text { of } \\
\text { Fans } \\
\text { Melody }\end{array}$ & $\begin{array}{c}\text { Few } \\
\text { Fans } \\
\text { Melody }\end{array}$ & $\begin{array}{l}\text { Psychedelic } \\
\text { Melody }\end{array}$ & $\begin{array}{l}\text { Flashing } \\
\text { Sun } \\
\text { Melody }\end{array}$ & $\begin{array}{l}\text { Moving } \\
\text { Shapes } \\
\text { Melody }\end{array}$ & $\begin{array}{l}\text { None } \\
\text { Melody }\end{array}$ \\
\hline 16 & & & & & 1 & & & \\
\hline 17 & 1 & & & & & & & \\
\hline 18 & & 1 & & 1 & & & & 1 \\
\hline 19 & & & & & & & 1 & \\
\hline \multicolumn{9}{|l|}{20} \\
\hline 21 & 1 & & & 1 & & & & \\
\hline 22 & & & & & & 1 & & \\
\hline 23 & & & & & & & 1 & \\
\hline 24 & & & & & & & 1 & \\
\hline 25 & & & & & & & 1 & \\
\hline
\end{tabular}

Table B.5: Participant's selection of visualizations best showing melody information.

\begin{tabular}{|c|c|c|c|c|c|c|c|c|}
\hline $\begin{array}{l}\text { Subject } \\
\text { Number }\end{array}$ & $\begin{array}{l}\text { Colours } \\
\text { Going } \\
\text { Out } \\
\text { Rhythm }\end{array}$ & $\begin{array}{l}\text { Colours } \\
\text { Going } \\
\text { In } \\
\text { Rhythm }\end{array}$ & $\begin{array}{c}\text { Lots } \\
\text { of } \\
\text { Fans } \\
\text { Rhythm }\end{array}$ & $\begin{array}{c}\text { Few } \\
\text { Fans } \\
\text { Rhythm }\end{array}$ & $\begin{array}{c}\text { Psychedelic } \\
\text { Rhythm }\end{array}$ & $\begin{array}{l}\text { Flashing } \\
\text { Sun } \\
\text { Rhythm }\end{array}$ & $\begin{array}{c}\text { Moving } \\
\text { Shapes } \\
\text { Rhythm }\end{array}$ & $\begin{array}{c}\text { None } \\
\text { Rhythm }\end{array}$ \\
\hline 16 & & & & & 1 & & & \\
\hline 17 & 1 & & & & & & & \\
\hline 18 & & 1 & & 1 & & & 1 & \\
\hline 19 & & & & & 1 & & & \\
\hline 20 & & & & & & & & \\
\hline 21 & 1 & & & 1 & & & & \\
\hline 22 & & & & & & 1 & & \\
\hline 23 & & & & & & & 1 & \\
\hline 24 & 1 & 1 & & & & 1 & 1 & \\
\hline 25 & & & & & 1 & & & \\
\hline
\end{tabular}

Table B.6: Participant's selection of visualizations best showing rhythm information. 


\begin{tabular}{|c|c|c|}
\hline $\begin{array}{l}\text { Subject } \\
\text { Number }\end{array}$ & $\begin{array}{c}\text { What Was } \\
\text { Most Enjoyable? }\end{array}$ & $\begin{array}{c}\text { What Was } \\
\text { Least Enjoyable? }\end{array}$ \\
\hline \multirow{8}{*}{$\stackrel{\Xi}{U}$} & $\begin{array}{l}\text { Feeling positive of the visualized beats } \\
\text { colors and instrument with melody } \\
\text { colors }\end{array}$ & $\begin{array}{l}\text { Not enough time to "digest" and write what we saw } \\
\text { lots of fans }\end{array}$ \\
\hline & looking at the changing colors & no accompanying sounds/vibrations \\
\hline & $\begin{array}{l}\text { the fact that this is being } \\
\text { experimental or will eventually }\end{array}$ & $\begin{array}{l}\text { the fans were boring and the flashing orange rectangle } \\
\text { was rather annoying. I feel that music was not really conveyed } \\
\text { well, perhaps vibrations would help }\end{array}$ \\
\hline & some of them are good & no music! \\
\hline & lots of color & dull color \\
\hline & $\begin{array}{l}\text { nothing really - need to connect music } \\
\text { to the human experience (dancing, hands, etc) }\end{array}$ & repetition and discomfort with opposing designs (hurts my eyes) \\
\hline & same as $\# 2$ & the orange flashing (oval) \\
\hline & $\begin{array}{l}\text { interesting concept - had never thought } \\
\text { about presenting music in this way before }\end{array}$ & couldn't really connect with any of the formats that were presented \\
\hline
\end{tabular}

Table B.7: Participant's post-test written responses on most / least enjoyable part of the focus group. 


\begin{tabular}{|c|c|c|c|c|c|c|c|c|c|c|}
\hline $\begin{array}{l}\text { Subject } \\
\text { Number }\end{array}$ & $\begin{array}{c}\text { Music } \\
\text { Enjoyment } \\
11\end{array}$ & $\begin{array}{l}\text { Meaning } \\
\quad 11\end{array}$ & $\begin{array}{c}\text { Positive } \\
\text { Negative } \\
11\end{array}$ & $\begin{array}{l}\text { Energy } \\
\quad 11\end{array}$ & $\begin{array}{l}\text { Happy } \\
11\end{array}$ & $\begin{array}{c}\text { Sad } \\
11\end{array}$ & $\begin{array}{l}\text { Angry } \\
11\end{array}$ & $\begin{array}{c}\text { Fear } \\
11\end{array}$ & $\begin{array}{c}\text { Most } \\
\text { Enjoyed } \\
11\end{array}$ & $\begin{array}{c}\text { Least } \\
\text { Enjoyed } \\
11\end{array}$ \\
\hline 16 & $\begin{array}{l}\text { Somewhat } \\
\text { enjoyable }\end{array}$ & Beatles, Alice Cooper & 4 & 5 & 6 & & & & Color & \\
\hline 17 & Enjoyable & & 4 & 7 & 4 & 3 & 2 & 3 & color & \\
\hline 18 & $\begin{array}{l}\text { Not that } \\
\text { enjoyable }\end{array}$ & $\begin{array}{l}\text { Just the beautiful } \\
\text { colors on the screen }\end{array}$ & 4 & 2 & 1 & & & & & \\
\hline 19 & $\begin{array}{l}\text { Not that } \\
\text { enjoyable }\end{array}$ & Just some pictures, rotating & & 1 & 3 & & & & the colors & $\begin{array}{l}\text { jagged } \\
\text { shapes }\end{array}$ \\
\hline 20 & Enjoyable & growth perhaps, change & 2 & 4 & 5 & 1 & 1 & 1 & $\begin{array}{l}\text { I liked where it } \\
\text { changed and } \\
\text { evolved to a } \\
\text { starburst and different } \\
\text { colors }\end{array}$ & not sure \\
\hline 21 & Neutral & $\begin{array}{l}\text { different colors going } \\
\text { around creative }\end{array}$ & 7 & 2 & 4 & 3 & 2 & 3 & change colors & no music \\
\hline 22 & Neutral & was I stoned? & 4 & 1 & 6 & 1 & 1 & 1 & feels relaxing & $\begin{array}{l}\text { color and } \\
\text { movement } \\
\text { of color }\end{array}$ \\
\hline 23 & $\begin{array}{l}\text { Not enjoyable } \\
\text { at all }\end{array}$ & $\begin{array}{l}\text { nothing really - reminded } \\
\text { me of the } 70 \mathrm{~s}\end{array}$ & 3 & 3 & 2 & 5 & 5 & 6 & & dated? \\
\hline 24 & Neutral & $\begin{array}{l}\text { nothing. Maybe just } \\
\text { first one. Will see a few more }\end{array}$ & 5 & 5 & 4 & 4 & 4 & 4 & not sure & not sure \\
\hline 25 & $\begin{array}{l}\text { Not that } \\
\text { enjoyable }\end{array}$ & $\begin{array}{l}\text { strange,- connected sound } \\
\text { more than "music" }\end{array}$ & 2 & 4 & 1 & 1 & 5 & 4 & color & speed \\
\hline
\end{tabular}

Table B.8: Participant's response to Block 1 Song 1 visualization. 


\begin{tabular}{|c|c|c|c|c|c|c|c|c|c|c|}
\hline $\begin{array}{l}\text { Subject } \\
\text { Number }\end{array}$ & $\begin{array}{c}\text { Music } \\
\text { Enjoyment } \\
12\end{array}$ & $\begin{array}{c}\text { Meaning } \\
12\end{array}$ & $\begin{array}{c}\text { Positive } \\
\text { Negative } \\
12\end{array}$ & $\begin{array}{c}\text { Energy } \\
12\end{array}$ & $\begin{array}{c}\text { Happy } \\
12\end{array}$ & $\begin{array}{c}\text { Sad } \\
12\end{array}$ & $\begin{array}{c}\text { Angry } \\
12\end{array}$ & $\begin{array}{c}\text { Fear } \\
12\end{array}$ & $\begin{array}{c}\text { Most } \\
\text { Enjoyed } \\
12\end{array}$ & $\begin{array}{c}\text { Least } \\
\text { Enjoyed } \\
12\end{array}$ \\
\hline 16 & Enjoyable & yellow submarine & 2 & 6 & 7 & 1 & 1 & 1 & $\begin{array}{l}\text { reminds me } \\
\text { of } 70 \text { s style cartoons }\end{array}$ & \\
\hline 17 & $\begin{array}{l}\text { Somewhat } \\
\text { enjoyable }\end{array}$ & confusing & 4 & 4 & 3 & 2 & 1 & 2 & & fast movement \\
\hline 18 & Neutral & $\begin{array}{l}\text { playing musical } \\
\text { instruments }\end{array}$ & 6 & 4 & 1 & & & & $\begin{array}{l}\text { colors going } \\
\text { to play instruments }\end{array}$ & \\
\hline 19 & $\begin{array}{l}\text { Somewhat } \\
\text { enjoyable }\end{array}$ & & & 2 & 3 & & & & $\begin{array}{l}\text { there seems to be } \\
\text { some sort of beat } \\
\text { / rhythm associated } \\
\text { with the circles }\end{array}$ & too sparse \\
\hline 20 & $\begin{array}{l}\text { Not that } \\
\text { enjoyable }\end{array}$ & $\begin{array}{l}\text { I guess it was } \\
\text { emphasizing different } \\
\text { instruments playing }\end{array}$ & 5 & 5 & 4 & 3 & 2 & 1 & $\begin{array}{l}\text { I didn'?t really } \\
\text { care for it }\end{array}$ & $\begin{array}{l}\text { I found it } \\
\text { distracting } \\
\text { with the } \\
\text { colors and } \\
\text { different } \\
\text { fans changing }\end{array}$ \\
\hline 21 & $\begin{array}{l}\text { Not that } \\
\text { enjoyable }\end{array}$ & $\begin{array}{l}\text { spot colors shows } \\
\text { the instruments playing }\end{array}$ & 6 & 4 & 4 & 3 & 2 & 2 & $\begin{array}{l}\text { shows different } \\
\text { instruments playing } \\
\text { with different } \\
\text { color dots }\end{array}$ & $?$ \\
\hline 22 & Enjoyable & $\begin{array}{l}\text { show the rhythms } \\
\text { of music }\end{array}$ & 1 & 4 & 7 & 1 & 1 & 1 & $\begin{array}{l}\text { visualizes the } \\
\text { beat and rhythms } \\
\text { of music. } \\
\text { Very stimulating } \\
\text { the rhythm - I was }\end{array}$ & $\begin{array}{l}\text { very addictive and } \\
\text { caused my eyes to } \\
\text { focus on the circles } \\
\text { popping }\end{array}$ \\
\hline 23 & Neutral & $\begin{array}{l}\text { reminded me of } \\
\text { polka dot door :) }\end{array}$ & 5 & 6 & 6 & 2 & 2 & 4 & $\begin{array}{l}\text { the rhythm - I was } \\
\text { able to detect } \\
\text { the sequence }\end{array}$ & color \\
\hline 24 & $\begin{array}{l}\text { Somewhat } \\
\text { enjoyable }\end{array}$ & $\begin{array}{l}\text { sounds with rhythms } \\
\text { (like drum, etc) }\end{array}$ & 2 & 5 & 6 & 3 & 1 & 1 & $\begin{array}{l}\text { showing which one } \\
\text { is happening with } \\
\text { sounds }\end{array}$ & none so far \\
\hline 25 & Neutral & $\begin{array}{l}\text { pleasant image, but } \\
\text { music doesn't come } \\
\text { to mind looking at it }\end{array}$ & 4 & 3 & 5 & 2 & 1 & 1 & $\begin{array}{l}\text { interesting shapes, } \\
\text { could represent } \\
\text { lots of different } \\
\text { things }\end{array}$ & ambiguity \\
\hline
\end{tabular}

Table B.9: Participant's response to Block 1 Song 2 visualization. 


\begin{tabular}{|c|c|c|c|c|c|c|c|c|c|c|}
\hline $\begin{array}{l}\text { Subject } \\
\text { Number }\end{array}$ & $\begin{array}{c}\text { Music } \\
\text { Enjoyment } \\
13\end{array}$ & $\begin{array}{c}\text { Meaning } \\
13\end{array}$ & $\begin{array}{c}\text { Positive } \\
\text { Negative } \\
13\end{array}$ & $\begin{array}{c}\text { Energy } \\
13\end{array}$ & $\begin{array}{l}\text { Happy } \\
13\end{array}$ & $\begin{array}{c}\text { Sad } \\
13\end{array}$ & $\begin{array}{c}\text { Angry } \\
13\end{array}$ & $\begin{array}{c}\text { Fear } \\
13\end{array}$ & $\begin{array}{c}\text { Most } \\
\text { Enjoyed } \\
13\end{array}$ & $\begin{array}{c}\text { Least } \\
\text { Enjoyed } \\
13\end{array}$ \\
\hline 16 & Enjoyable & disco & 1 & 7 & 7 & 1 & 1 & 1 & the pulse & \\
\hline 17 & Enjoyable & synchronize & 2 & 7 & 6 & 2 & 1 & 1 & movement & \\
\hline 18 & $\begin{array}{l}\text { Not enjoyable } \\
\text { at all }\end{array}$ & $\begin{array}{l}\text { music going } \\
\text { too fast } \\
\text { blank neon }\end{array}$ & 7 & 2 & & & & 3 & not at all & \\
\hline 19 & $\begin{array}{l}\text { Not enjoyable } \\
\text { at all }\end{array}$ & $\begin{array}{l}\text { signboard } \\
\text { - lights need } \\
\text { changing! }\end{array}$ & & 4 & & & & 3 & $\begin{array}{l}\text { the orange } \\
\text { color }\end{array}$ & $\begin{array}{l}\text { the flashing } \\
\text { / blinking }\end{array}$ \\
\hline 20 & $\begin{array}{l}\text { Not enjoyable } \\
\text { at all }\end{array}$ & $\begin{array}{l}\text { appears to } \\
\text { be loud } \\
\text { - maybe blasting } \\
\text { sound }\end{array}$ & 6 & 7 & 2 & 1 & 7 & 5 & $\begin{array}{l}\text { well, it gets } \\
\text { your attention }\end{array}$ & $\begin{array}{l}\text { if possible, I would } \\
\text { say it would give } \\
\text { me a headache! }\end{array}$ \\
\hline 21 & $\begin{array}{l}\text { Not that } \\
\text { enjoyable }\end{array}$ & $\begin{array}{l}\text { shows the beat } \\
\text { or rhythm of } \\
\text { music }\end{array}$ & 7 & 2 & 2 & 1 & 1 & & useless & $\begin{array}{l}\text { cannot tell } \\
\text { which instrument } \\
\text { is playing! }\end{array}$ \\
\hline 22 & $\begin{array}{l}\text { Somewhat } \\
\text { enjoyable }\end{array}$ & $\begin{array}{l}\text { felt like I } \\
\text { was in a } \\
\text { nightclub! } \\
\text { annoying }\end{array}$ & 4 & 2 & 1 & 4 & 1 & 1 & $\begin{array}{l}\text { the pulsing } \\
\text { of the oval } \\
\text { shape }\end{array}$ & too dull, plain \\
\hline 23 & $\begin{array}{l}\text { Not enjoyable } \\
\text { at all }\end{array}$ & $\begin{array}{l}\text { - reminded } \\
\text { me of a billboard } \\
\text { ad }\end{array}$ & 7 & 2 & 1 & 3 & 5 & 5 & nothing & $\begin{array}{l}\text { colors and lack } \\
\text { of movement }\end{array}$ \\
\hline 24 & $\begin{array}{l}\text { Not enjoyable } \\
\text { at all }\end{array}$ & nothing & 6 & 1 & 1 & 5 & 1 & 1 & nothing & too boring \\
\hline 25 & $\begin{array}{l}\text { Not enjoyable } \\
\text { at all }\end{array}$ & basic & 2 & 4 & 2 & 2 & 6 & 5 & & $\begin{array}{l}\text { very annoying } \\
\text { - seemed throbbing } \\
\text { (if Iooked at it for } \\
\text { too long, I suspect } \\
\text { I would get a headache) }\end{array}$ \\
\hline
\end{tabular}

Table B.10: Participant's response to Block 1 Song 3 visualization. 


\begin{tabular}{|c|c|c|c|c|c|c|c|c|c|c|}
\hline $\begin{array}{l}\text { Subject } \\
\text { Number }\end{array}$ & $\begin{array}{c}\text { Music } \\
\text { Enjoyment } \\
14\end{array}$ & $\begin{array}{c}\text { Meaning } \\
14\end{array}$ & $\begin{array}{l}\text { Positive } \\
\text { Negative } \\
14\end{array}$ & $\begin{array}{l}\text { Energy } \\
\quad 14\end{array}$ & $\begin{array}{l}\text { Happy } \\
14\end{array}$ & $\begin{array}{c}\mathrm{Sad} \\
14\end{array}$ & $\begin{array}{c}\text { Angry } \\
14\end{array}$ & $\begin{array}{c}\text { Fear } \\
14\end{array}$ & $\begin{array}{c}\text { Most } \\
\text { Enjoyed } \\
14\end{array}$ & $\begin{array}{l}\text { Least } \\
\text { Enjoyed } \\
14\end{array}$ \\
\hline 16 & $\begin{array}{c}\text { Somewhat } \\
\text { enjoyable }\end{array}$ & pokaroo & 1 & 5 & 7 & 1 & 1 & 1 & nice colors & \\
\hline 17 & $\begin{array}{l}\text { Somewhat } \\
\text { enjoyable }\end{array}$ & good & 2 & 6 & 5 & 3 & 2 & 2 & slow movement & \\
\hline 18 & Neutral & $\begin{array}{l}\text { one instrument at } \\
\text { a time playing } \\
\text { and finally got } \\
\text { the high pitch }\end{array}$ & 6 & 1 & & & & 4 & & \\
\hline 19 & $\begin{array}{l}\text { Not that } \\
\text { enjoyable }\end{array}$ & $\begin{array}{l}\text { different instruments } \\
\text { being played }\end{array}$ & & 3 & 3 & & & & the rhythm/beat & $\begin{array}{l}\text { even more } \\
\text { sparse than \#2 }\end{array}$ \\
\hline 20 & $\begin{array}{l}\text { Not that } \\
\text { enjoyable }\end{array}$ & $\begin{array}{l}\text { I guess emphasis } \\
\text { on different } \\
\text { instruments }\end{array}$ & 3 & 5 & 4 & 2 & 2 & 1 & the colors were okay & $\begin{array}{l}\text { again I find it } \\
\text { distracting }\end{array}$ \\
\hline 21 & $\begin{array}{l}\text { Somewhat } \\
\text { enjoyable }\end{array}$ & $\begin{array}{l}\text { can tell the } \\
\text { difference how } \\
\text { instruments play }\end{array}$ & 3 & 5 & 4 & & & & $\begin{array}{l}\text { shows little better than } \\
\text { the first one that } \\
\text { similar can see better } \\
\text { specific instruments } \\
\text { playing (dots and bars) }\end{array}$ & \\
\hline 22 & $\begin{array}{l}\text { Somewhat } \\
\text { enjoyable }\end{array}$ & polka dot music & 3 & 2 & 5 & 1 & 1 & 1 & $\begin{array}{l}\text { softer polka dot } \\
\text { music pulsing from } \\
\text { the first one }\end{array}$ & none \\
\hline 23 & $\begin{array}{l}\text { Not that } \\
\text { enjoyable }\end{array}$ & $\begin{array}{l}\text { a little bit painful } \\
\text { - made me feel } \\
\text { dizzy } \\
\text { showing which }\end{array}$ & 3 & 3 & 1 & 6 & 6 & 6 & the rhythm & the colors \\
\hline 24 & $\begin{array}{l}\text { Somewhat } \\
\text { enjoyable }\end{array}$ & $\begin{array}{l}\text { colors going on with } \\
\text { rhythms, I noticed } \\
\text { a bit more slower } \\
\text { than the first one }\end{array}$ & 4 & 5 & 5 & 2 & 1 & 1 & $\begin{array}{l}\text { showing the } \\
\text { difference in speed }\end{array}$ & none so far \\
\hline 25 & Neutral & $\begin{array}{l}\text { colors and actions } \\
\text { seemed to compliment } \\
\text { each other }\end{array}$ & 5 & 3 & 5 & 2 & 2 & 2 & symmetry & $\begin{array}{l}\text { needed more } \\
\text { vibrant colors }\end{array}$ \\
\hline
\end{tabular}

Table B.11: Participant's response to Block 1 Song 4 visualization. 


\begin{tabular}{|c|c|c|c|c|c|c|c|c|c|c|}
\hline $\begin{array}{l}\text { Subject } \\
\text { Number }\end{array}$ & $\begin{array}{c}\text { Music } \\
\text { Enjoyment } \\
15\end{array}$ & $\begin{array}{l}\text { Meaning } \\
15\end{array}$ & $\begin{array}{l}\text { Positive } \\
\text { Negative } \\
15\end{array}$ & $\begin{array}{l}\text { Energy } \\
\quad 15\end{array}$ & $\begin{array}{l}\text { Happy } \\
15\end{array}$ & $\begin{array}{l}\text { Sad } \\
15\end{array}$ & $\begin{array}{l}\text { Angry } \\
15\end{array}$ & $\begin{array}{c}\text { Fear } \\
15\end{array}$ & $\begin{array}{l}\text { Most } \\
\text { Enjoyed } \\
15\end{array}$ & $\begin{array}{l}\text { Least } \\
\text { Enjoyed } \\
15\end{array}$ \\
\hline 16 & Enjoyable & space, startrek & 1 & 7 & 7 & & & & felt surreal & \\
\hline 17 & Enjoyable & interaction & 1 & 6 & 6 & 3 & 2 & 2 & colors & fast movement \\
\hline 18 & $\begin{array}{l}\text { Somewhat } \\
\text { enjoyable }\end{array}$ & $\begin{array}{l}\text { musical note shooting } \\
\text { too loud and playing } \\
\text { too hard }\end{array}$ & 2 & 5 & 4 & & & & $\begin{array}{l}\text { fast and sound } \\
\text { music }\end{array}$ & \\
\hline 19 & $\begin{array}{l}\text { Somewhat } \\
\text { enjoyable }\end{array}$ & $\begin{array}{l}\text { looked like something } \\
\text { from outer space }\end{array}$ & & 5 & 3 & & & & $\begin{array}{l}\text { when the colors } \\
\text { were changing } \\
\text { gradually }\end{array}$ & $\begin{array}{l}\text { no accompanying } \\
\text { sound }\end{array}$ \\
\hline 20 & Enjoyable & $\begin{array}{l}\text { it was creative but } \\
\text { not sure if I relate it } \\
\text { to music }\end{array}$ & 1 & 6 & 6 & 2 & 1 & 1 & $\begin{array}{l}\text { it captured my } \\
\text { attention more } \\
\text { than the fans }\end{array}$ & \\
\hline 21 & $\begin{array}{l}\text { Not that } \\
\text { enjoyable }\end{array}$ & $\begin{array}{l}\text { fireworks with dots } \\
\text { moving around with } \\
\text { black background } \\
\text { wow! Felt like I was }\end{array}$ & 6 & 3 & & 5 & 3 & 3 & symphony & \\
\hline 22 & Enjoyable & $\begin{array}{l}\text { dying happily and } \\
\text { entering afterlife }\end{array}$ & 1 & 7 & 7 & & & & the sun & \\
\hline 23 & Neutral & $\begin{array}{l}\text { space wars } \\
\text { sounds from loud to } \\
\text { louder and lower volumes }\end{array}$ & 3 & 4 & 4 & 5 & 6 & 6 & variety & colors \\
\hline 24 & Enjoyable & $\begin{array}{l}\text { down and up with circles } \\
\text { hitting each other and small } \\
\text { green dots hitting circles } \\
\text { showing short loud sound }\end{array}$ & 1 & 7 & 6 & 1 & 1 & 1 & everything & none so far \\
\hline 25 & $\begin{array}{l}\text { Somewhat } \\
\text { enjoyable }\end{array}$ & outer space & 5 & 3 & 6 & 5 & 1 & 1 & $\begin{array}{l}\text { bright, lots } \\
\text { of colors, } \\
\text { explosions }\end{array}$ & $\begin{array}{l}\text { had to watch little } \\
\text { bright lights for } \\
\text { too long }\end{array}$ \\
\hline
\end{tabular}

Table B.12: Participant's response to Block 1 Song 5 visualization. 


\begin{tabular}{|c|c|c|c|c|c|c|c|c|c|c|}
\hline $\begin{array}{l}\text { Subject } \\
\text { Number }\end{array}$ & $\begin{array}{c}\text { Music } \\
\text { Enjoyment } \\
16\end{array}$ & $\begin{array}{l}\text { Meaning } \\
16\end{array}$ & $\begin{array}{c}\text { Positive } \\
\text { Negative } \\
16\end{array}$ & $\begin{array}{l}\text { Energy } \\
\quad 16\end{array}$ & $\begin{array}{l}\text { Happy } \\
\quad 16\end{array}$ & $\begin{array}{l}\text { Sad } \\
16\end{array}$ & $\begin{array}{l}\text { Angry } \\
16\end{array}$ & $\begin{array}{c}\text { Fear } \\
16\end{array}$ & $\begin{array}{l}\text { Most } \\
\text { Enjoyed } \\
16 \\
\end{array}$ & $\begin{array}{l}\text { Least } \\
\text { Enjoyed } \\
\quad 16 \\
\end{array}$ \\
\hline 16 & $\begin{array}{l}\text { Not that } \\
\text { enjoyable } \\
\text { at all }\end{array}$ & zzZZz & 4 & 1 & 5 & & & & & same old boring \\
\hline 17 & $\begin{array}{l}\text { Somewhat } \\
\text { enjoyable }\end{array}$ & & 5 & 6 & 6 & 3 & 2 & 2 & $\begin{array}{l}\text { slow and } \\
\text { simultaneous }\end{array}$ & \\
\hline 18 & Neutral & $\begin{array}{l}\text { all visual music } \\
\text { appears the same with } \\
\text { the fans and colors }\end{array}$ & 5 & 1 & 2 & & & & & \\
\hline 19 & Neutral & $\begin{array}{l}\text { different instruments } \\
\text { being played with a } \\
\text { rhythm / beat }\end{array}$ & & 5 & 5 & & & & $\begin{array}{l}\text { the color, } \\
\text { pulsing circles }\end{array}$ & $\begin{array}{l}\text { no music (sound) } \\
\text { or vibrations }\end{array}$ \\
\hline 20 & Neutral & $\begin{array}{l}\text { I find it difficult to } \\
\text { relate to music } \\
\text { actually }\end{array}$ & 2 & 5 & 5 & 2 & 2 & 1 & $\begin{array}{l}\text { the colors } \\
\text { were okay }\end{array}$ & $\begin{array}{l}\text { I don't care for the fans, } \\
\text { there are too many to } \\
\text { keep track of along with } \\
\text { the colors }\end{array}$ \\
\hline 21 & $\begin{array}{l}\text { Somewhat } \\
\text { enjoyable }\end{array}$ & $\begin{array}{l}\text { I like the instruments } \\
\text { shown, how they play } \\
\text { and also shows the } \\
\text { speed of the fan and } \\
\text { that match with the } \\
\text { white background }\end{array}$ & 3 & 6 & 5 & & & & $\begin{array}{l}\text { more message } \\
\text { to capture }\end{array}$ & $\begin{array}{l}\text { need more dots for } \\
\text { each instrument }\end{array}$ \\
\hline 22 & $\begin{array}{l}\text { Somewhat } \\
\text { enjoyable }\end{array}$ & & 3 & 4 & 5 & 1 & 1 & 1 & $\begin{array}{l}\text { relaxing and } \\
\text { smoothing / } \\
\text { soft beat }\end{array}$ & too many fans \\
\hline 23 & $\begin{array}{l}\text { Somewhat } \\
\text { enjoyable }\end{array}$ & drumming solo & 3 & 3 & 6 & 2 & 2 & 2 & strong beat & blue/aqua \\
\hline 24 & $\begin{array}{l}\text { Somewhat } \\
\text { enjoyable }\end{array}$ & $\begin{array}{l}\text { same as } 2 \text { previous } \\
\text { comments with fans } \\
\text { music and colored } \\
\text { circles }\end{array}$ & 3 & 5 & 4 & 2 & 1 & 1 & $\begin{array}{l}\text { same as previous, } \\
\text { like to show with } \\
\text { sound }\end{array}$ & $\begin{array}{l}\text { now I'm starting to } \\
\text { feel that the fans } \\
\text { should be a little bit } \\
\text { bigger to show which } \\
\text { music instrument is } \\
\text { coming from with } \\
\text { the colored circles }\end{array}$ \\
\hline 25 & $\begin{array}{l}\text { Somewhat } \\
\text { enjoyable }\end{array}$ & $\begin{array}{l}\text { different instruments } \\
\text { playing }\end{array}$ & 5 & 5 & 6 & 2 & 3 & 3 & $\begin{array}{l}\text { better use of } \\
\text { color coordination }\end{array}$ & \\
\hline
\end{tabular}

Table B.13: Participant's response to Block 1 Song 6 visualization. 


\begin{tabular}{|c|c|c|c|c|c|c|c|c|c|c|}
\hline $\begin{array}{l}\text { Subject } \\
\text { Number }\end{array}$ & $\begin{array}{c}\text { Music } \\
\text { Enjoyment } \\
17\end{array}$ & $\begin{array}{l}\text { Meaning } \\
17\end{array}$ & $\begin{array}{c}\text { Positive } \\
\text { Negative } \\
\quad 17\end{array}$ & $\begin{array}{c}\text { Energy } \\
17\end{array}$ & $\begin{array}{c}\text { Happy } \\
17\end{array}$ & $\begin{array}{c}\text { Sad } \\
17\end{array}$ & $\begin{array}{c}\text { Angry } \\
17\end{array}$ & $\begin{array}{c}\text { Fear } \\
17\end{array}$ & $\begin{array}{c}\text { Most } \\
\text { Enjoyed } \\
17\end{array}$ & $\begin{array}{c}\text { Least } \\
\text { Enjoyed } \\
17\end{array}$ \\
\hline 16 & Enjoyable & a starship & 1 & 6 & 7 & & & & \multicolumn{2}{|l|}{$\begin{array}{l}\text { remind me of } \\
\text { star wars } 3\end{array}$} \\
\hline 17 & Enjoyable & beat of instruments & 7 & 6 & 6 & 2 & 1 & 1 & & \\
\hline 18 & $\begin{array}{l}\text { Somewhat } \\
\text { enjoyable }\end{array}$ & Its like orchestra & 2 & 5 & 4 & & & & \multicolumn{2}{|l|}{$\begin{array}{l}\text { instrument playing } \\
\text { was well done }\end{array}$} \\
\hline 19 & $\begin{array}{l}\text { Not that } \\
\text { enjoyable } \\
\text { at all }\end{array}$ & $\begin{array}{l}\text { reminds me of } \\
\text { what I like to see } \\
\text { on my monitor when } \\
\text { the computer acts up } \\
\text { if I hadn't been told }\end{array}$ & & 2 & & 4 & & & nothing & $\begin{array}{l}\text { too dull - colors } \\
\text { and movement }\end{array}$ \\
\hline 20 & $\begin{array}{l}\text { Not that } \\
\text { enjoyable }\end{array}$ & $\begin{array}{l}\text { I'm not sure I would } \\
\text { have realized they } \\
\text { presented different } \\
\text { instruments }\end{array}$ & 4 & 3 & 3 & 5 & 1 & 1 & the vibrant green & $\begin{array}{l}\text { confusing - too } \\
\text { much going on }\end{array}$ \\
\hline 21 & $\begin{array}{l}\text { Somewhat } \\
\text { enjoyable }\end{array}$ & $\begin{array}{l}\text { I like the group of } \\
\text { lines but not sure } \\
\text { which instruments } \\
\text { playing. Good match! }\end{array}$ & 3 & & 5 & & & & $\begin{array}{l}\text { easily to follow } \\
\text { how the different } \\
\text { color lines playing } \\
\text { at the same }\end{array}$ & $\begin{array}{l}\text { need to show the } \\
\text { instruments that } \\
\text { belong to the color } \\
\text { lines } \\
\text { felt like I was }\end{array}$ \\
\hline 22 & & $\begin{array}{l}\text { is the computer } \\
\text { broken?! }\end{array}$ & 7 & 1 & 1 & 7 & 1 & 1 & nothing! & $\begin{array}{l}\text { reading garbled } \\
\text { (broken) typewriter } \\
\text { receipts }\end{array}$ \\
\hline \multicolumn{11}{|r|}{-1} \\
\hline 24 & & $\begin{array}{l}\text { frequency of sound } \\
\text { (hard to explain) }\end{array}$ & 4 & 3 & 3 & 3 & 1 & 1 & colorful & $\begin{array}{l}\text { too slow: confusion } \\
\text { with colors a bit }\end{array}$ \\
\hline 25 & Neutral & tetris video game & 4 & 2 & 2 & 5 & 5 & 4 & $\begin{array}{l}\text { colors on a black } \\
\text { background made it } \\
\text { easier to follow }\end{array}$ & $\begin{array}{l}\text { seemed a little } \\
\text { boring, slow }\end{array}$ \\
\hline
\end{tabular}

Table B.14: Participant's response to Block 1 Song 7 visualization. 


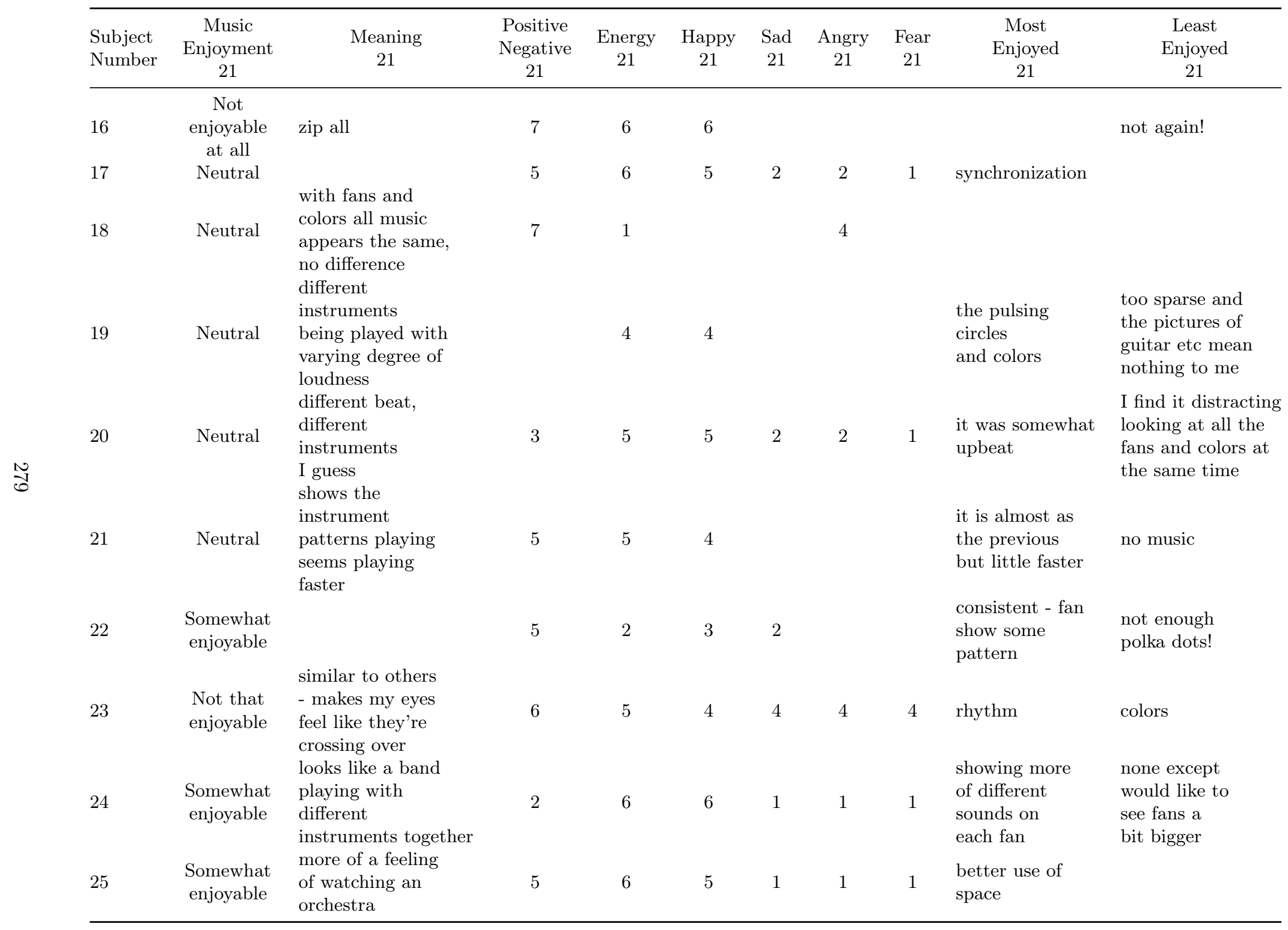

Table B.15: Participant's response to Block 2 Song 1 visualization. 


\begin{tabular}{|c|c|c|c|c|c|c|c|c|c|c|}
\hline $\begin{array}{l}\text { Subject } \\
\text { Number }\end{array}$ & $\begin{array}{c}\text { Music } \\
\text { Enjoyment } \\
22\end{array}$ & $\begin{array}{l}\text { Meaning } \\
\quad 22\end{array}$ & $\begin{array}{c}\text { Positive } \\
\text { Negative } \\
22\end{array}$ & $\begin{array}{c}\text { Energy } \\
22\end{array}$ & $\begin{array}{l}\text { Happy } \\
22\end{array}$ & $\begin{array}{c}\text { Sad } \\
22\end{array}$ & $\begin{array}{l}\text { Angry } \\
22\end{array}$ & $\begin{array}{l}\text { Fear } \\
22\end{array}$ & $\begin{array}{l}\text { Most } \\
\text { Enjoyed } \\
22\end{array}$ & $\begin{array}{l}\text { Least } \\
\text { Enjoyed } \\
22\end{array}$ \\
\hline 16 & Not enjoyable at all & zZZZZZ & 7 & 6 & & 6 & & & & \multirow[t]{3}{*}{ boring rectangle } \\
\hline 17 & Somewhat enjoyable & happy drumming & 6 & 6 & 6 & 3 & 1 & 1 & $\begin{array}{l}\text { center to one } \\
\text { point }\end{array}$ & \\
\hline 18 & Neutral & $\begin{array}{l}\text { sun view is the } \\
\text { same as before. } \\
\text { Its not too fast }\end{array}$ & 6 & 3 & & & & 2 & & \\
\hline 19 & Not that enjoyable & $\begin{array}{l}\text { blank flashing } \\
\text { neon sign }\end{array}$ & & 3 & & & & 3 & $\begin{array}{l}\text { the orange } \\
\text { color }\end{array}$ & \multirow{3}{*}{$\begin{array}{l}\text { too sparse and } \\
\text { meaningless to } \\
\text { look at } \\
\text { too strong }\end{array}$} \\
\hline 20 & Not enjoyable at all & \multirow{2}{*}{$\begin{array}{l}\text { loudness, anger } \\
\text { same old thing } \\
\text { as before } \\
\text { is this }\end{array}$} & 6 & 7 & 1 & 1 & 7 & 1 & it simple & \\
\hline 21 & Not enjoyable at all & & 6 & 2 & & & & & & \\
\hline 22 & Not enjoyable at all & $\begin{array}{l}\text { brainwashing! } \\
\text { Boring }\end{array}$ & 6 & 1 & & 7 & & & nothing & $\begin{array}{l}\text { repeated constant } \\
\text { pulsing }\end{array}$ \\
\hline 23 & Not enjoyable at all & billboard flashing & 2 & 2 & 1 & 3 & 6 & 7 & nothing & $\begin{array}{l}\text { beat and colors } \\
\text { too boring - no }\end{array}$ \\
\hline 24 & Not enjoyable at all & nothing & 7 & 1 & 1 & 7 & 1 & 1 & none & $\begin{array}{l}\text { connection because } \\
\text { no vibration with that }\end{array}$ \\
\hline 25 & Not enjoyable at all & annoyance! & 6 & 6 & 1 & 1 & 6 & 6 & & very annoying \\
\hline
\end{tabular}

Table B.16: Participant's response to Block 2 Song 2 visualization. 


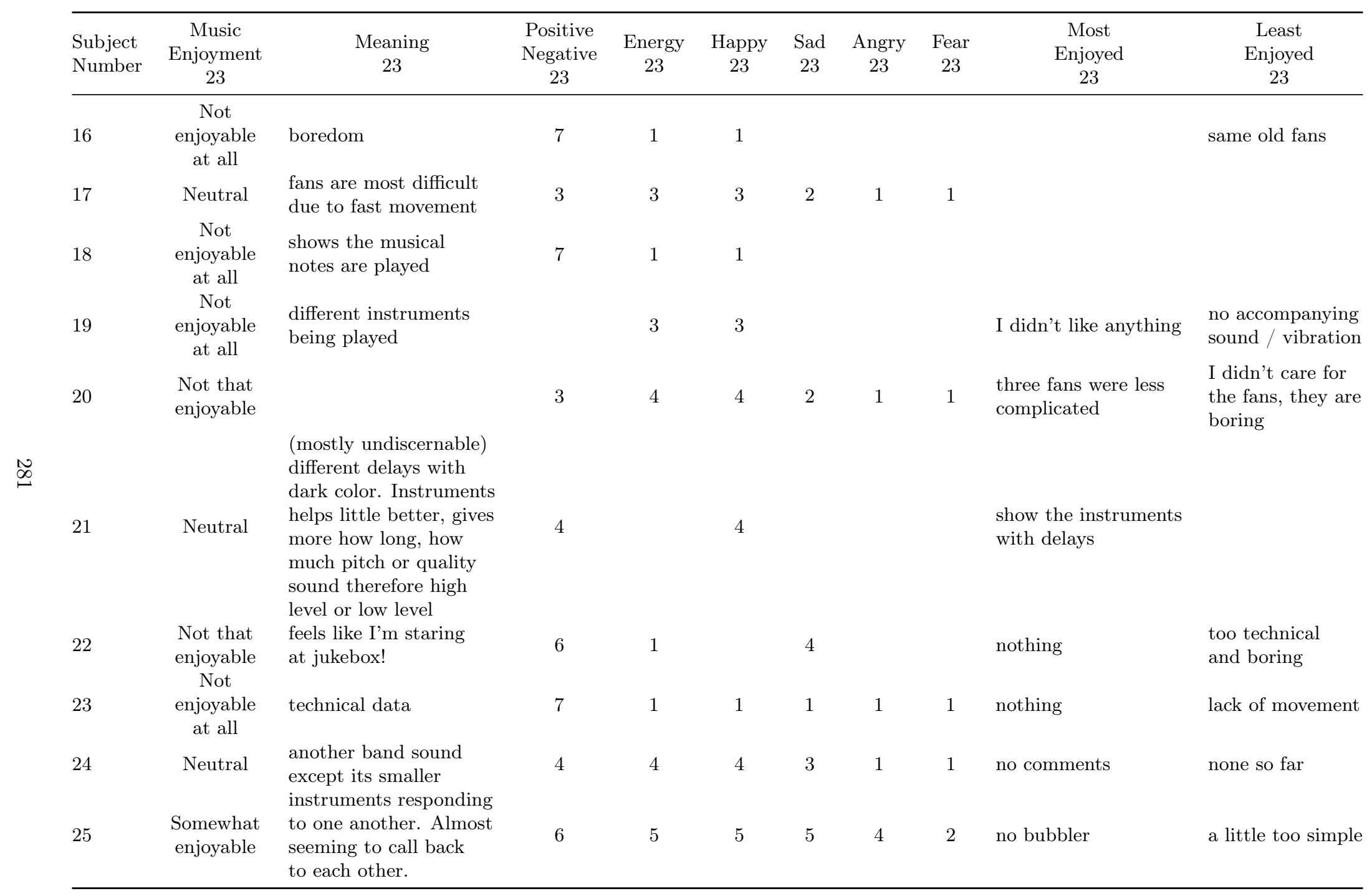

Table B.17: Participant's response to Block 2 Song 3 visualization. 


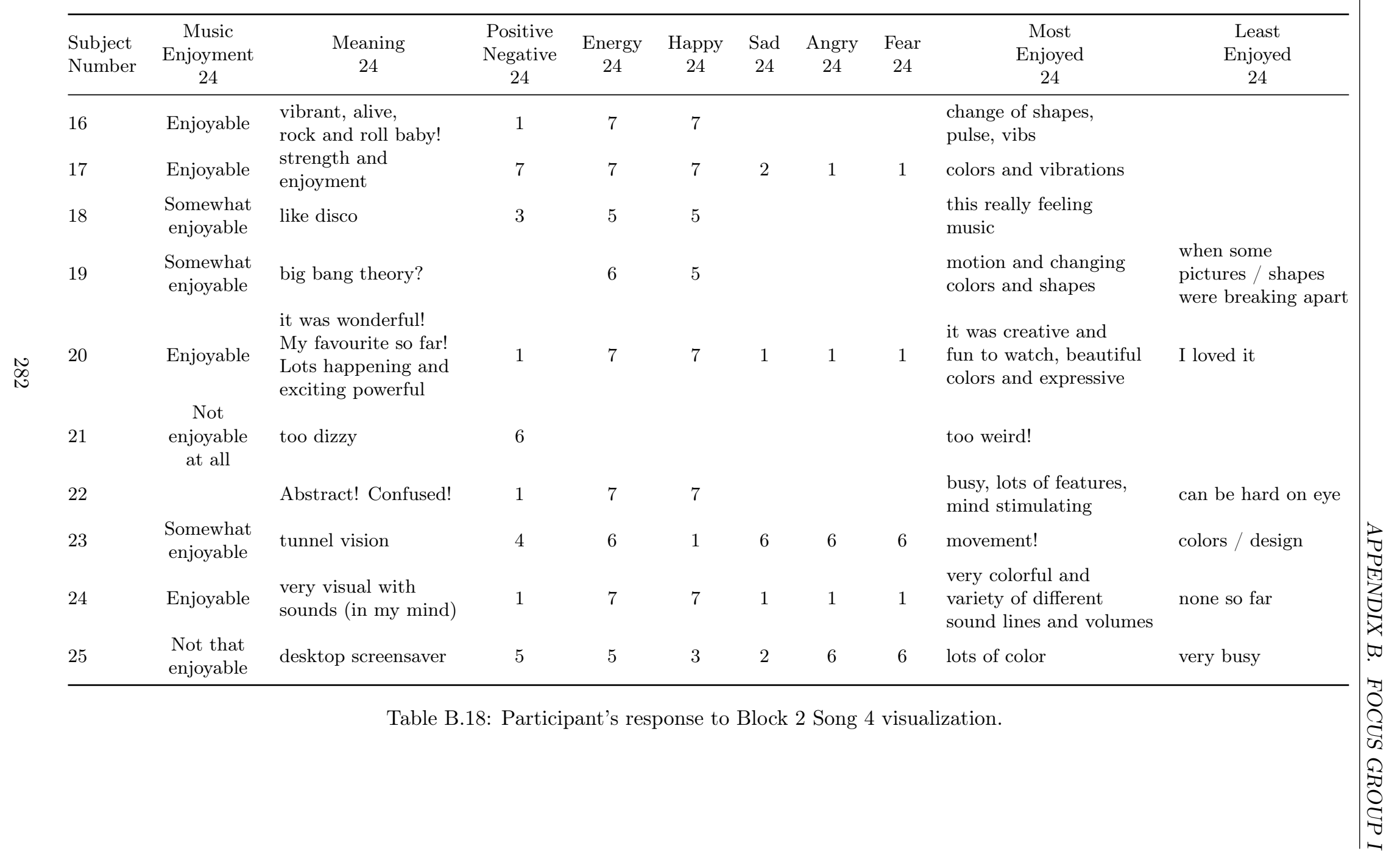




\begin{tabular}{|c|c|c|c|c|c|c|c|c|c|c|}
\hline $\begin{array}{l}\text { Subject } \\
\text { Number }\end{array}$ & $\begin{array}{c}\text { Music } \\
\text { Enjoyment } \\
25\end{array}$ & $\begin{array}{c}\text { Meaning } \\
25\end{array}$ & $\begin{array}{c}\text { Positive } \\
\text { Negative } \\
25\end{array}$ & $\begin{array}{c}\text { Energy } \\
25\end{array}$ & $\begin{array}{c}\text { Happy } \\
25\end{array}$ & $\begin{array}{c}\text { Sad } \\
25\end{array}$ & $\begin{array}{c}\text { Angry } \\
25\end{array}$ & $\begin{array}{c}\text { Fear } \\
25\end{array}$ & $\begin{array}{c}\text { Most } \\
\text { Enjoyed } \\
25\end{array}$ & $\begin{array}{l}\text { Least } \\
\text { Enjoyed } \\
25\end{array}$ \\
\hline 16 & & & & & & & & & & \\
\hline 17 & Neutral & & 6 & 2 & 3 & 2 & 2 & 1 & & \\
\hline 18 & Neutral & $\begin{array}{l}\text { same as before } \\
\text { with the fans }\end{array}$ & 7 & 1 & 1 & & & & & \\
\hline 19 & $\begin{array}{l}\text { Not that } \\
\text { enjoyable }\end{array}$ & $\begin{array}{l}\text { different instruments } \\
\text { being played with } \\
\text { accompanying beat }\end{array}$ & & 4 & 4 & & & & pulsing circles & too sparse \\
\hline 20 & $\begin{array}{l}\text { Not that } \\
\text { enjoyable }\end{array}$ & & 4 & 4 & 3 & 2 & 2 & 1 & & $\begin{array}{l}\text { too many } \\
\text { distractions } \\
\text { - I don't think } \\
\text { it conveys } \\
\text { music well }\end{array}$ \\
\hline 21 & Neutral & $\begin{array}{l}\text { shows dots the beat } \\
\text { or rhythm with } \\
\text { playing instruments. } \\
\text { Similar as before }\end{array}$ & 5 & & & & & & same as before & \\
\hline 22 & Neutral & $\begin{array}{l}\text { remind me of heart } \\
\text { beating }\end{array}$ & 4 & 3 & 3 & & & & & kind of boring \\
\hline 23 & $\begin{array}{l}\text { Not that } \\
\text { enjoyable } \\
\text { at all }\end{array}$ & nothing changed & 7 & 1 & 4 & 4 & 4 & 4 & nothing & again - colors \\
\hline 24 & $\begin{array}{l}\text { Somewhat } \\
\text { enjoyable }\end{array}$ & $\begin{array}{l}\text { same comments as } \\
\text { previously but I like } \\
\text { three fans on both sides } \\
\text { with three color circles. } \\
\text { Shows some sounds } \\
\text { in my mind }\end{array}$ & 2 & 6 & 5 & 1 & 1 & 1 & see number two & none \\
\hline 25 & Neutral & $\begin{array}{l}\text { orchestra, symphony, } \\
\text { coordination }\end{array}$ & 3 & 5 & 5 & 3 & 2 & 2 & color coordination & $\begin{array}{l}\text { not } 100 \% \text { sure } \\
\text { what is being } \\
\text { represented }\end{array}$ \\
\hline
\end{tabular}

Table B.19: Participant's response to Block 2 Song 5 visualization. 


\begin{tabular}{|c|c|c|c|c|c|c|c|c|c|c|}
\hline $\begin{array}{l}\text { Subject } \\
\text { Number }\end{array}$ & $\begin{array}{c}\text { Music } \\
\text { Enjoyment } \\
26\end{array}$ & $\begin{array}{c}\text { Meaning } \\
26\end{array}$ & $\begin{array}{c}\text { Positive } \\
\text { Negative } \\
26\end{array}$ & $\begin{array}{l}\text { Energy } \\
26\end{array}$ & $\begin{array}{l}\text { Happy } \\
26\end{array}$ & $\begin{array}{l}\text { Sad } \\
26\end{array}$ & $\begin{array}{c}\text { Angry } \\
26\end{array}$ & $\begin{array}{c}\text { Fear } \\
26\end{array}$ & $\begin{array}{l}\text { Most } \\
\text { Enjoyed } \\
26\end{array}$ & $\begin{array}{c}\text { Least } \\
\text { Enjoyed } \\
26\end{array}$ \\
\hline 16 & $\begin{array}{c}\text { Somewhat } \\
\text { enjoyable }\end{array}$ & & 2 & 5 & 7 & & & & $\begin{array}{l}\text { the schematic } \\
\text { reminds me of } \\
\text { a motherboard }\end{array}$ & $\begin{array}{l}\text { not vibrant } \\
\text { enough }\end{array}$ \\
\hline 17 & $\begin{array}{c}\text { Somewhat } \\
\text { enjoyable }\end{array}$ & variability & 2 & 6 & 6 & 2 & 1 & 1 & different vibration & \\
\hline 18 & $\begin{array}{c}\text { Somewhat } \\
\text { enjoyable }\end{array}$ & pitch of the notes & 3 & 5 & 3 & & & & & \\
\hline 19 & $\begin{array}{l}\text { Not that } \\
\text { enjoyable }\end{array}$ & $\begin{array}{l}\text { looked like a } \\
\text { videogame }\end{array}$ & & 2 & 1 & & & & nothing & $\begin{array}{l}\text { could not relate it } \\
\text { to any musical } \\
\text { instrument }\end{array}$ \\
\hline 20 & Neutral & $\begin{array}{l}\text { different } \\
\text { instruments at } \\
\text { different levels } \\
\text { but not sure it } \\
\text { actually conveys } \\
\text { music }\end{array}$ & 4 & 3 & 3 & 2 & 1 & 1 & $\begin{array}{l}\text { well it was } \\
\text { interesting } \\
\text { to watch }\end{array}$ & $\begin{array}{l}\text { I don't get } \\
\text { a sense of } \\
\text { music from } \\
\text { it really }\end{array}$ \\
\hline 21 & $\begin{array}{l}\text { Somewhat } \\
\text { enjoyable }\end{array}$ & $\begin{array}{l}\text { shows better on } \\
\text { different lines playing }\end{array}$ & 4 & 5 & 5 & & & & $\begin{array}{l}\text { shows lines where } \\
\text { they play. Soft lines } \\
\text { to bright lines to } \\
\text { soft lines when they } \\
\text { play }\end{array}$ & $\begin{array}{l}\text { getting rid } \\
\text { of balls }\end{array}$ \\
\hline 22 & & $\begin{array}{l}\text { what the hell is this! } \\
\text { EKG machine. Also } \\
\text { look like the schematic } \\
\text { drawing }\end{array}$ & 6 & 1 & & 5 & & & none & $\begin{array}{l}\text { plain and } \\
\text { repeated pattern }\end{array}$ \\
\hline 23 & $\begin{array}{l}\text { Not that } \\
\text { enjoyable }\end{array}$ & xray of music & 3 & 3 & 7 & 1 & 1 & 1 & $\begin{array}{l}\text { the movement } \\
\text { was smooth }\end{array}$ & $\begin{array}{l}\text { colors! Tacky } \\
\text { and dated (old) } \\
\text { use photoshop? }\end{array}$ \\
\hline
\end{tabular}




\begin{tabular}{|c|c|c|c|c|c|c|c|c|c|c|}
\hline $\begin{array}{l}\text { Subject } \\
\text { Number }\end{array}$ & $\begin{array}{c}\text { Music } \\
\text { Enjoyment } \\
26\end{array}$ & $\begin{array}{l}\text { Meaning } \\
26\end{array}$ & $\begin{array}{c}\text { Positive } \\
\text { Negative } \\
26\end{array}$ & $\begin{array}{c}\text { Energy } \\
26\end{array}$ & $\begin{array}{c}\text { Happy } \\
26\end{array}$ & $\begin{array}{c}\text { Sad } \\
26\end{array}$ & $\begin{array}{c}\text { Angry } \\
26\end{array}$ & $\begin{array}{c}\text { Fear } \\
26\end{array}$ & $\begin{array}{c}\text { Most } \\
\text { Enjoyed } \\
26\end{array}$ & $\begin{array}{c}\text { Least } \\
\text { Enjoyed } \\
26\end{array}$ \\
\hline 24 & Enjoyable & $\begin{array}{l}\text { shows each colored } \\
\text { line for sound } \\
\text { frequency and colored } \\
\text { circles showing } \\
\text { different sounds }\end{array}$ & 3 & 5 & 5 & 1 & 1 & 1 & $\begin{array}{l}\text { very visual } \\
\text { especially with } \\
\text { the colors }\end{array}$ & none \\
\hline 25 & $\begin{array}{l}\text { Somewhat } \\
\text { enjoyable }\end{array}$ & $\begin{array}{l}\text { strings on a } \\
\text { musical instrument }\end{array}$ & 3 & 3 & 3 & 4 & 3 & 3 & $\begin{array}{l}\text { seemed like there } \\
\text { was a good } \\
\text { connection between } \\
\text { different parts of the } \\
\text { music (easier to see } \\
\text { what was going on in } \\
\text { different sections of the } \\
\text { piece) }\end{array}$ & $\begin{array}{l}\text { not a big fan } \\
\text { of black } \\
\text { backgrounds }\end{array}$ \\
\hline
\end{tabular}

Table B.20: Participant's response to Block 2 Song 6 visualization. 


\begin{tabular}{|c|c|c|c|c|c|c|c|c|c|c|}
\hline $\begin{array}{l}\text { Subject } \\
\text { Number }\end{array}$ & $\begin{array}{c}\text { Music } \\
\text { Enjoyment } \\
31\end{array}$ & $\begin{array}{l}\text { Meaning } \\
\quad 31\end{array}$ & $\begin{array}{l}\text { Positive } \\
\text { Negative } \\
31\end{array}$ & $\begin{array}{l}\text { Energy } \\
\quad 31\end{array}$ & $\begin{array}{l}\text { Happy } \\
31\end{array}$ & $\begin{array}{c}\mathrm{Sad} \\
31\end{array}$ & $\begin{array}{l}\text { Angry } \\
31\end{array}$ & $\begin{array}{c}\text { Fear } \\
31\end{array}$ & $\begin{array}{l}\text { Most } \\
\text { Enjoyed } \\
31\end{array}$ & $\begin{array}{l}\text { Least } \\
\text { Enjoyed } \\
31\end{array}$ \\
\hline \multicolumn{11}{|l|}{16} \\
\hline 17 & $\begin{array}{l}\text { Somewhat } \\
\text { enjoyable }\end{array}$ & & 2 & 5 & 5 & 3 & 1 & 2 & colors & \\
\hline 18 & $\begin{array}{l}\text { Somewhat } \\
\text { enjoyable }\end{array}$ & & 5 & 5 & 3 & & & & & \\
\hline 19 & $\begin{array}{l}\text { Somewhat } \\
\text { enjoyable }\end{array}$ & & & 5 & 5 & & & & $\begin{array}{l}\text { the green color } \\
\text { - lively }\end{array}$ & $\begin{array}{l}\text { when the colors } \\
\text { turned to grey - too dull }\end{array}$ \\
\hline 20 & Enjoyable & $\begin{array}{l}\text { unfortunately I am } \\
\text { not really relating } \\
\text { even the best ones } \\
\text { to music }\end{array}$ & 1 & 6 & 6 & 2 & 1 & 1 & $\begin{array}{l}\text { colors were great, } \\
\text { was visually } \\
\text { appealing }\end{array}$ & \\
\hline 21 & $\begin{array}{l}\text { Not that } \\
\text { enjoyable }\end{array}$ & $\begin{array}{l}\text { nice color pattern, } \\
\text { no sense of music. }\end{array}$ & 6 & 2 & 4 & & & & $\begin{array}{l}\text { color pattern } \\
\text { is nice }\end{array}$ & no pattern of music \\
\hline 22 & Enjoyable & $\begin{array}{l}\text { stimulating, could } \\
\text { visualize music }\end{array}$ & 1 & 7 & 7 & & & & lots of variety & \\
\hline 23 & $\begin{array}{l}\text { Somewhat } \\
\text { enjoyable }\end{array}$ & fireworks & 2 & 6 & 6 & 1 & 1 & 1 & better colors & lack of emotions in all \\
\hline 24 & Neutral & $\begin{array}{l}\text { very visual but } \\
\text { a bit too much }\end{array}$ & 5 & 3 & 1 & 2 & 1 & 1 & $\begin{array}{l}\text { some of them, } \\
\text { I feel the } \\
\text { connections to the } \\
\text { sounds but not } \\
\text { all of them }\end{array}$ & $\begin{array}{l}\text { some - no connections } \\
\text { and (a bit) too much } \\
\text { of visuals }\end{array}$ \\
\hline 25 & $\begin{array}{l}\text { Not that } \\
\text { enjoyable }\end{array}$ & $\begin{array}{l}\text { desktop screensaver, } \\
\text { very electric }\end{array}$ & 5 & 6 & 3 & 2 & 5 & 6 & & $\begin{array}{l}\text { no consistency in } \\
\text { shapes, colors used. } \\
\text { Seemed very arbitrary } \\
\text { and random }\end{array}$ \\
\hline
\end{tabular}

Table B.21: Participant's response to Block 3 Song 1 visualization. 


\begin{tabular}{|c|c|c|c|c|c|c|c|c|c|c|}
\hline $\begin{array}{l}\text { Subject } \\
\text { Number }\end{array}$ & $\begin{array}{c}\text { Music } \\
\text { Enjoyment } \\
32\end{array}$ & $\begin{array}{c}\text { Meaning } \\
\quad 32\end{array}$ & $\begin{array}{c}\text { Positive } \\
\text { Negative } \\
32\end{array}$ & $\begin{array}{l}\text { Energy } \\
\quad 32\end{array}$ & $\begin{array}{l}\text { Happy } \\
32\end{array}$ & $\begin{array}{c}\text { Sad } \\
32\end{array}$ & $\begin{array}{l}\text { Angry } \\
32\end{array}$ & $\begin{array}{c}\text { Fear } \\
32\end{array}$ & $\begin{array}{c}\text { Most } \\
\text { Enjoyed } \\
32\end{array}$ & $\begin{array}{c}\text { Least } \\
\text { Enjoyed } \\
32\end{array}$ \\
\hline 16 & $\begin{array}{c}\text { Somewhat } \\
\text { enjoyable }\end{array}$ & the blues & 1 & 7 & 7 & & & & $\begin{array}{l}\text { the beat of the } \\
\text { circles }\end{array}$ & "fans, again!" \\
\hline 17 & Neutral & distracted & 5 & 4 & 5 & 1 & 1 & 1 & & \\
\hline 18 & Neutral & beating the drum & 6 & 2 & 1 & & & & & \\
\hline 19 & $\begin{array}{l}\text { Not } \\
\text { enjoyable } \\
\text { at all }\end{array}$ & $\begin{array}{l}\text { different instruments } \\
\text { being played } \\
\text { - slow music? }\end{array}$ & & 1 & & 6 & & & nothing & $\begin{array}{l}\text { very low energy } \\
\text { and makes no sense } \\
\text { (can't hear instruments) }\end{array}$ \\
\hline 20 & Neutral & nothing appealing & 3 & 5 & 4 & 2 & 1 & 1 & $\begin{array}{l}\text { color circles } \\
\text { were okay } \\
\text { shows color dots }\end{array}$ & don't like the fans \\
\hline 21 & $\begin{array}{l}\text { Somewhat } \\
\text { enjoyable }\end{array}$ & $\begin{array}{l}\text { shows some color } \\
\text { along with rhythm }\end{array}$ & 5 & 5 & & & & & $\begin{array}{l}\text { helps brighten up } \\
\text { along with color } \\
\text { fan }\end{array}$ & \\
\hline 22 & $\begin{array}{l}\text { Not } \\
\text { enjoyable } \\
\text { at all } \\
\text { Not }\end{array}$ & $\begin{array}{l}\text { enough of annoying } \\
\text { polka dots! }\end{array}$ & 7 & 1 & & & 1 & & & \\
\hline 23 & $\begin{array}{l}\text { enjoyable } \\
\text { at all }\end{array}$ & nothing & 7 & 1 & 4 & 4 & 4 & 4 & nothing & "again, colors" \\
\hline 24 & Neutral & $\begin{array}{l}\text { same as previously } \\
\text { commented but too } \\
\text { slow and boring } \\
\text { seemed like one fan }\end{array}$ & 5 & 1 & 1 & 3 & 1 & 1 & none & too slow and boring \\
\hline 25 & $\begin{array}{l}\text { Not that } \\
\text { enjoyable }\end{array}$ & $\begin{array}{l}\text { did nothing at all. } \\
\text { Very distracting } \\
\text { - why bother having it } \\
\text { at all }\end{array}$ & 3 & 2 & 3 & 4 & 2 & 2 & use of color & seemed really slow \\
\hline
\end{tabular}

Table B.22: Participant's response to Block 3 Song 2 visualization. 


\begin{tabular}{|c|c|c|c|c|c|c|c|c|c|c|}
\hline $\begin{array}{l}\text { Subject } \\
\text { Number }\end{array}$ & $\begin{array}{c}\text { Music } \\
\text { Enjoyment } \\
35\end{array}$ & $\begin{array}{c}\text { Meaning } \\
35\end{array}$ & $\begin{array}{l}\text { Positive } \\
\text { Negative } \\
35\end{array}$ & $\begin{array}{c}\text { Energy } \\
35\end{array}$ & $\begin{array}{c}\text { Happy } \\
35\end{array}$ & $\begin{array}{c}\mathrm{Sad} \\
35\end{array}$ & $\begin{array}{c}\text { Angry } \\
35\end{array}$ & $\begin{array}{c}\text { Fear } \\
35\end{array}$ & $\begin{array}{l}\text { Most } \\
\text { Enjoyed } \\
35\end{array}$ & $\begin{array}{l}\text { Least } \\
\text { Enjoyed } \\
35\end{array}$ \\
\hline 16 & Enjoyable & hard to say & 1 & 7 & 7 & & & & \multirow{3}{*}{$\begin{array}{l}\text { the style of visual } \\
\text { colors of } \\
\text { synchronization }\end{array}$} & \multirow{5}{*}{ not very high energy } \\
\hline 17 & Enjoyable & happy & 2 & 6 & 6 & 2 & 1 & 1 & & \\
\hline 18 & $\begin{array}{c}\text { Somewhat } \\
\text { enjoyable }\end{array}$ & music well played & 4 & 2 & 2 & & & & & \\
\hline 19 & Neutral & drums being played & & 6 & 5 & & & & \multirow{2}{*}{$\begin{array}{l}\text { motion and } \\
\text { changing colors } \\
\text { there was more } \\
\text { of a sense of } \\
\text { rhythm to it }\end{array}$} & \\
\hline 20 & $\begin{array}{c}\text { Somewhat } \\
\text { enjoyable }\end{array}$ & $\begin{array}{l}\text { it presented different } \\
\text { instruments or tones } \\
\text { better }\end{array}$ & 2 & 6 & 6 & 2 & 2 & 1 & & \\
\hline 21 & $\begin{array}{l}\text { Somewhat } \\
\text { enjoyable }\end{array}$ & $\begin{array}{l}\text { color dots are showing } \\
\text { that help it understand } \\
\text { the colors of } \\
\text { instruments }\end{array}$ & 4 & & 5 & & & & $\begin{array}{l}\text { color dots } \\
\text { brightness }\end{array}$ & $\begin{array}{l}\text { wish to the } \\
\text { instruments shown in } \\
\text { the dots can be helpful }\end{array}$ \\
\hline 22 & $\begin{array}{l}\text { Not that } \\
\text { enjoyable } \\
\text { at all }\end{array}$ & can't define & 6 & 1 & & 1 & & & none & brainwashing boring \\
\hline 23 & $\begin{array}{c}\text { Somewhat } \\
\text { enjoyable }\end{array}$ & pendulum swing & 5 & 2 & 2 & 1 & 1 & 1 & background & \multirow{3}{*}{$\begin{array}{l}\text { depending on the } \\
\text { music, I don'??t like } \\
\text { too fast movement }\end{array}$} \\
\hline 24 & $\begin{array}{l}\text { Somewhat } \\
\text { enjoyable }\end{array}$ & $\begin{array}{l}\text { shows different } \\
\text { colored circles and } \\
\text { the movement of } \\
\text { sounds }\end{array}$ & 3 & 5 & 4 & 1 & 1 & 1 & $\begin{array}{l}\text { I like different } \\
\text { colored circles } \\
\text { and the } \\
\text { movement } \\
\text { lots of color }\end{array}$ & \\
\hline 25 & Enjoyable & connect the dots & 2 & 6 & 5 & 2 & 4 & 3 & $\begin{array}{l}\text { symmetry, very } \\
\text { easy to follow } \\
\text { the beat }\end{array}$ & \\
\hline
\end{tabular}

Table B.23: Participant's response to Block 3 Song 5 visualization. 


\begin{tabular}{|c|c|c|c|c|c|c|c|c|c|c|}
\hline $\begin{array}{l}\text { Subject } \\
\text { Number }\end{array}$ & $\begin{array}{c}\text { Music } \\
\text { Enjoyment } \\
41\end{array}$ & $\begin{array}{l}\text { Meaning } \\
\quad 41\end{array}$ & $\begin{array}{c}\text { Positive } \\
\text { Negative } \\
41\end{array}$ & $\begin{array}{c}\text { Energy } \\
\quad 41\end{array}$ & $\begin{array}{c}\text { Happy } \\
41\end{array}$ & $\begin{array}{c}\mathrm{Sad} \\
41\end{array}$ & $\begin{array}{c}\text { Angry } \\
41\end{array}$ & $\begin{array}{c}\text { Fear } \\
41\end{array}$ & $\begin{array}{l}\text { Most } \\
\text { Enjoyed } \\
\quad 41\end{array}$ & $\begin{array}{l}\text { Least } \\
\text { Enjoyed } \\
41\end{array}$ \\
\hline 16 & & & & & & & & & & $\begin{array}{l}\text { I get distracted } \\
\text { by the circles }\end{array}$ \\
\hline 17 & $\begin{array}{l}\text { Not that } \\
\text { enjoyable } \\
\text { at all }\end{array}$ & distracted & 5 & 2 & 3 & 2 & 2 & 2 & & \\
\hline 18 & Neutral & & 6 & 1 & & 1 & & & & \\
\hline 19 & $\begin{array}{l}\text { Not that } \\
\text { enjoyable } \\
\text { at all }\end{array}$ & $\begin{array}{l}\text { different instruments } \\
\text { being played }\end{array}$ & & 2 & & 5 & & & nothing & $\begin{array}{l}\text { makes no sense } \\
\text { to me - no } \\
\text { accompanying sounds }\end{array}$ \\
\hline 20 & Neutral & & 3 & 4 & 2 & 4 & 1 & 1 & $\begin{array}{l}\text { the colors of the } \\
\text { circles and only two } \\
\text { of them }\end{array}$ & too many fans \\
\hline 21 & $\begin{array}{l}\text { Not that } \\
\text { enjoyable }\end{array}$ & $\begin{array}{l}\text { not enough dots } \\
\text { that match the } \\
\text { instruments }\end{array}$ & 5 & & & & & & $\begin{array}{l}\text { need more dots that } \\
\text { match the instruments }\end{array}$ & \\
\hline 22 & $\begin{array}{l}\text { Not that } \\
\text { enjoyable } \\
\text { at all }\end{array}$ & $\begin{array}{l}\text { nothing, just a } \\
\text { repeat of another } \\
\text { boring polka dots }\end{array}$ & 7 & 1 & & 7 & & & none & slow and boring \\
\hline 23 & $\begin{array}{l}\text { Not that } \\
\text { enjoyable } \\
\text { at all }\end{array}$ & & 7 & 1 & 1 & 1 & 1 & 1 & & hard to focus \\
\hline 24 & $\begin{array}{l}\text { Not that } \\
\text { enjoyable } \\
\text { at all }\end{array}$ & $\begin{array}{l}\text { same as all } \\
\text { previous comments }\end{array}$ & 7 & 1 & 1 & 7 & 1 & 1 & none & too boring and slow \\
\hline 25 & Neutral & orchestra, symphony & 4 & 2 & 3 & 5 & 2 & 5 & $\begin{array}{l}\text { colored dots }=\text { less } \\
\text { distracting than on } \\
\text { previous occasions }\end{array}$ & $\begin{array}{l}\text { not a big fan of } \\
\text { the "fan" style }\end{array}$ \\
\hline
\end{tabular}

Table B.24: Participant's response to Block 4 Song 1 visualization. 


\begin{tabular}{|c|c|c|c|c|c|c|c|c|c|c|}
\hline $\begin{array}{l}\text { Subject } \\
\text { Number }\end{array}$ & $\begin{array}{c}\text { Music } \\
\text { Enjoyment } \\
44 \\
\end{array}$ & $\begin{array}{c}\text { Meaning } \\
44\end{array}$ & $\begin{array}{c}\text { Positive } \\
\text { Negative } \\
\quad 44\end{array}$ & $\begin{array}{l}\text { Energy } \\
\quad 44\end{array}$ & $\begin{array}{l}\text { Happy } \\
\quad 44\end{array}$ & $\begin{array}{c}\mathrm{Sad} \\
44\end{array}$ & $\begin{array}{c}\text { Angry } \\
44\end{array}$ & $\begin{array}{c}\text { Fear } \\
44\end{array}$ & $\begin{array}{c}\text { Most } \\
\text { Enjoyed } \\
44 \\
\end{array}$ & $\begin{array}{c}\text { Least } \\
\text { Enjoyed } \\
44\end{array}$ \\
\hline 16 & $\begin{array}{l}\text { Not that } \\
\text { enjoyable } \\
\text { at all }\end{array}$ & not much, sorry & 5 & 4 & 6 & & & & see \#1 & \\
\hline 17 & $\begin{array}{l}\text { Somewhat } \\
\text { enjoyable }\end{array}$ & & 2 & 6 & 5 & 2 & 1 & 1 & & \\
\hline 18 & $\begin{array}{l}\text { Somewhat } \\
\text { enjoyable }\end{array}$ & $\begin{array}{l}\text { it appears too } \\
\text { many people playing } \\
\text { the instruments }\end{array}$ & 3 & 4 & 3 & & & & & \\
\hline 19 & $\begin{array}{l}\text { Not that } \\
\text { enjoyable }\end{array}$ & nothing really & & 3 & 1 & & & & movement & meaningless to me \\
\hline 20 & Neutral & $\begin{array}{l}\text { different instruments } \\
\text { and tones }\end{array}$ & 5 & 5 & 4 & 2 & 2 & 1 & $\begin{array}{l}\text { it was different } \\
\text { and more interesting }\end{array}$ & $\begin{array}{l}\text { not sure if it really } \\
\text { depicts music }\end{array}$ \\
\hline 21 & $\begin{array}{l}\text { Somewhat } \\
\text { enjoyable }\end{array}$ & $\begin{array}{l}\text { not bad, with } \\
\text { color lines }\end{array}$ & 6 & 5 & & & & & $\begin{array}{l}\text { more color lines and } \\
\text { balls get the eye catch! }\end{array}$ & \\
\hline 22 & $\begin{array}{l}\text { Not that } \\
\text { enjoyable } \\
\text { at all }\end{array}$ & $\begin{array}{l}\text { remind me of } \\
\text { chad punch card }\end{array}$ & 7 & 1 & & 1 & & & none & boring and plain \\
\hline 23 & $\begin{array}{l}\text { Not that } \\
\text { enjoyable }\end{array}$ & nothing & 5 & 2 & 1 & 1 & 1 & 1 & more variety & shapes \\
\hline 24 & Neutral & $\begin{array}{l}\text { different frequency } \\
\text { of sounds on } \\
\text { each line }\end{array}$ & 5 & 3 & 1 & 2 & 1 & 1 & $\begin{array}{l}\text { different lines and } \\
\text { different colored } \\
\text { balls and different } \\
\text { lines of sounds }\end{array}$ & $\begin{array}{l}\text { but different colors } \\
\text { while moving around } \\
\text { on different "volume" } \\
\text { of sounds } \\
\text { - too confusing with } \\
\text { the colors }\end{array}$ \\
\hline 25 & $\begin{array}{l}\text { Not that } \\
\text { enjoyable } \\
\text { at all }\end{array}$ & $\begin{array}{l}\text { driving down a } \\
\text { boring road }\end{array}$ & 2 & 2 & 2 & 2 & 5 & 4 & colors! & plain \\
\hline
\end{tabular}




\begin{tabular}{|c|c|c|c|c|c|c|c|c|c|c|}
\hline $\begin{array}{l}\text { Subject } \\
\text { Number }\end{array}$ & $\begin{array}{c}\text { Music } \\
\text { Enjoyment } \\
47\end{array}$ & $\begin{array}{l}\text { Meaning } \\
\quad 47\end{array}$ & $\begin{array}{l}\text { Positive } \\
\text { Negative } \\
\quad 47\end{array}$ & $\begin{array}{l}\text { Energy } \\
\quad 47\end{array}$ & $\begin{array}{l}\text { Happy } \\
47\end{array}$ & $\begin{array}{c}\mathrm{Sad} \\
47\end{array}$ & $\begin{array}{l}\text { Angry } \\
47\end{array}$ & $\begin{array}{c}\text { Fear } \\
47\end{array}$ & $\begin{array}{c}\text { Most } \\
\text { Enjoyed } \\
47\end{array}$ & $\begin{array}{c}\text { Least } \\
\text { Enjoyed } \\
47\end{array}$ \\
\hline \multicolumn{11}{|l|}{16} \\
\hline 17 & Neutral & & 3 & 6 & 6 & 2 & 1 & 1 & & \\
\hline 18 & $\begin{array}{l}\text { Somewhat } \\
\text { enjoyable }\end{array}$ & piano playing & 3 & 4 & 3 & & & & & \\
\hline 19 & $\begin{array}{l}\text { Somewhat } \\
\text { enjoyable }\end{array}$ & $\begin{array}{l}\text { different instruments } \\
\text { being played }\end{array}$ & & 4 & 4 & & & & $\begin{array}{l}\text { colors were more } \\
\text { vibrant with black } \\
\text { background }\end{array}$ & $\begin{array}{l}\text { still meaningless } \\
\text { because I don't hear } \\
\text { the instruments } \\
\text { being played }\end{array}$ \\
\hline 20 & $\begin{array}{l}\text { Somewhat } \\
\text { enjoyable }\end{array}$ & $\begin{array}{l}\text { different instruments } \\
\text { playing but still } \\
\text { confusing }\end{array}$ & 3 & 5 & 5 & 2 & 1 & 1 & $\begin{array}{l}\text { I liked the black } \\
\text { background, it was } \\
\text { better visually and } \\
\text { I liked the colored } \\
\text { circles better }\end{array}$ & too many fans! \\
\hline \multicolumn{11}{|c|}{ (1) } \\
\hline 23 & & & & & & & & & & \\
\hline 24 & Enjoyable & same previously :) & 1 & 6 & 2 & 1 & 1 & 1 & $\begin{array}{l}\text { black background with } \\
\text { colorful instruments } \\
\text { and colorful circles } \\
\text { better outline of the }\end{array}$ & none \\
\hline 25 & Enjoyable & technicolor & 3 & 5 & 5 & 3 & 2 & 2 & $\begin{array}{l}\text { better outline of the } \\
\text { musical shapes } \\
\text { and use of color }\end{array}$ & \\
\hline
\end{tabular}

Table B.26: Participant's response to Block 4 Song 7 visualization. 



\section{Appendix $\mathrm{C}$}

\section{Focus Group II}

This Appendix presents the forms used and data collected in Focus Group II, described in Chapter 6. 
Project Title: Principal Investigators:
Music Visualization

Deborah Fels, P.Eng., Ph.D., Ryerson University dfels@ryerson.ca

David Fourney, MSc. Ryerson University dfourney@ryerson.ca

Quoc Vy, B.Sc. Ryerson University qvy@ryerson.ca

Jorge Mori, B.Sc. Ryerson University jmori@ryerson.ca

Michael Pouris, Ryerson University, mpouris@ryerson.ca

Hong Tai Lee, Ryerson University, h35lee@ryerson.ca

\section{Consent to Participate in Study from Subject}

Information Form

The purpose of this study is to evaluate different ways of presenting music visually. To do this, we will ask you to watch twelve (12) different examples of visualizations and provide us with your opinion about them. Each example runs less than one minute. You are also asked to complete two additional surveys, one prior to the study and one at the conclusion of the study.

\section{Confidentiality}

All raw data will be kept strictly confidential. A summary of the data will be published in academic venues but no individual details will be identified in this summary. The information gathered from surveys will be strictly used for research and academic purposes with only the principal investigator and his supervisor having access to it

\section{Risks and Discomforts}

The risks associated with participating in this study are minimal. You may experience some fatigue with watching the visualizations. However, you are able to take breaks at any time or even stop participation in the study without penalty. You may also experience some discomfort with being video or audio recorded. In this case, you may choose not to participate in the study or record your opinions in writing and remain off camera.

\section{Expected Benefits}

Individual participants will not receive any direct benefits; however, this study will benefit the general community of deaf or hard of hearing users. This study will test different styles of music visualization that will assist in developing new practises for music visualization. We hope that this information may lead to improvements in music visualization technologies and techniques.

\section{Voluntary Nature of Participation:}

Participation in this study is entirely voluntary. If you do not wish to participate in this study it will not affect current or future relations with Ryerson University or The Centre for Learning Technologies. If you choose to participate, you have the ability to leave the study at any time and for any reason without penalty. In addition, you may refuse to answer any questions or participate in any task at any point of the study without penalty. 
TED

MANACOLOF RYERSON

TED ROGERS SCHOOL OF INFORMATION TECHNOLOGY MANAGEMENT

Project Title:

Principal Investigators:

\section{Music Visualization}

Deborah Fels, P.Eng., Ph.D., Ryerson University

dfels@ryerson.ca

David Fourney, MSc. Ryerson University dfourney@ryerson.ca

Quoc Vy, B.Sc. Ryerson University qvy@ryerson.ca

Jorge Mori, B.Sc. Ryerson University jmori@ryerson.ca

Michael Pouris, Ryerson University, mpouris@ryerson.ca

Hong Tai Lee, Ryerson University, h35lee@ryerson.ca

\section{Questions about the Study:}

If you have any questions or concerns, about this study please feel free to contact Deborah Fels at dfels@ryerson.ca. If you have any concerns or complaints about this study in regards to its ethical nature please contact the Research Ethics Board, c/o Office of the Vice President, Research and Innovation, Ryerson University, 350 Victoria St., Toronto, ON M5B 2K3, Tel: 416-979-5042 
TED

ROGERS

SCHOOLOF RYERSON

MANAGEMENT UNIVERSITY

TED ROGERS SCHOOL OF INFORMATION TECHNOLOGY MANAGEMENT

Project Title:

Principal Investigators:

\section{Music Visualization}

Deborah Fels, P.Eng., Ph.D., Ryerson University

(416)-979-5000 ext. 7619 or dfels@ryerson.ca

David Fourney, MSc. Ryerson University dfourney@ryerson.ca

Quoc Vy, B.Sc. Ryerson University qvy@ryerson.ca

Jorge Mori, B.Sc. Ryerson University jmori@ryerson.ca

Michael Pouris, Ryerson University, mpouris@ryerson.ca

Hong Tai Lee, Ryerson University, h35lee@ryerson.ca

\section{Consent Form to Participate in Study}

I acknowledge that the research procedures described above have been explained to me and that any questions that I have asked have been answered to my satisfaction. I have been informed that there may be a possible risk of psychological discomfort from being filmed or watching so many videos; however, strategies are in place to reduce this risk.

I have been informed of the alternatives to participation in this study, including my right not to participate and the right to withdraw without penalty. I hereby consent to participate in the study and to be video or audio recorded during the study. I have received a copy of the information sheet.

Signature of Participant:

Name of Participant (please print):

Date:

The details of this study were explained to me by:

Name of Investigator:

Date:

ADDRESS: 575 Bay Street (entrance at 55 Dundas St. West) ROOM: 2-004 | TE-: 416-979-5316 MAUUNG ADDRESS: 350 Victoria Street, Toronto, Ontario, Canada M5B 2K3| FAX: 416-979-524

WEB: www.ryerson.caltedrogersschoolitit 
Music Visualization Pre-study Questionnaire

The purpose of this questionnaire is to gather information about you and your experience with music and closed captioning. There are eight questions and it should take you about five minutes to complete this questionnaire. Thank you in advance for your time and assistance.

1. Do you identify yourself as: (please check one)

$$
\begin{aligned}
& \square \text { Hearing } \\
& \square \text { Hard of hearing } \\
& \square \text { Deaf } \\
& \square \text { Deafened } \\
& \square \text { Cochlear implant }
\end{aligned}
$$

\section{Please indicate your gender}

$$
\begin{array}{ll}
\square & \text { Male } \\
\square & \text { Female }
\end{array}
$$

3. Please indicate your age
ㄷ $19-24$
口 $25-34$
口 $35-44$
प $45-54$
D 55- 64
口 $65+$

\section{What is your highest level of education?}

No formal education
Elementary school
aigh School
a Technical college
University
$\square$ Masters / Ph.D.

5. How often do you use closed captioning when watching television?
Always
- Sometimes
$\square$ Never

6. How often do you listen to music?
All the time (daily).
- Frequently (weekly)
- Sometimes
Rarely
a Never

7. Which of the following statements best describes you?
I really enjoy listening to music.
I like listening to music.
- I do not like or dislike music.
I I do not like listening to music
- I do not see the point of music.

8. What is your experience of music in elementary or secondary school?

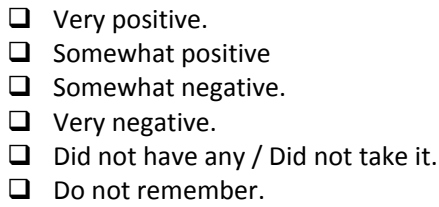


Music Questions

1. Was the music visualization enjoyable (circle one)?
Not enjoyable at all
Not that enjoyable
Neutral
Somewhat enjoyable
Enjoyable

2. What did the visualization mean to you?

3. Was the music (mark one for each):

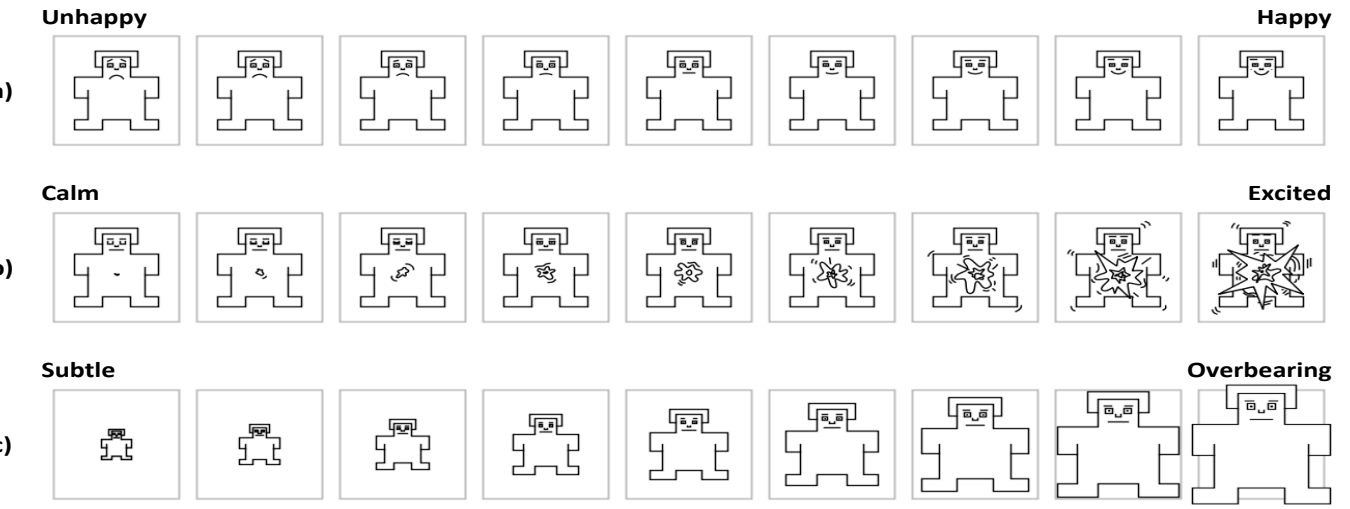

4. Identify which emotion you thought was expressed and rate its strength from 1 to 7 (circle one):

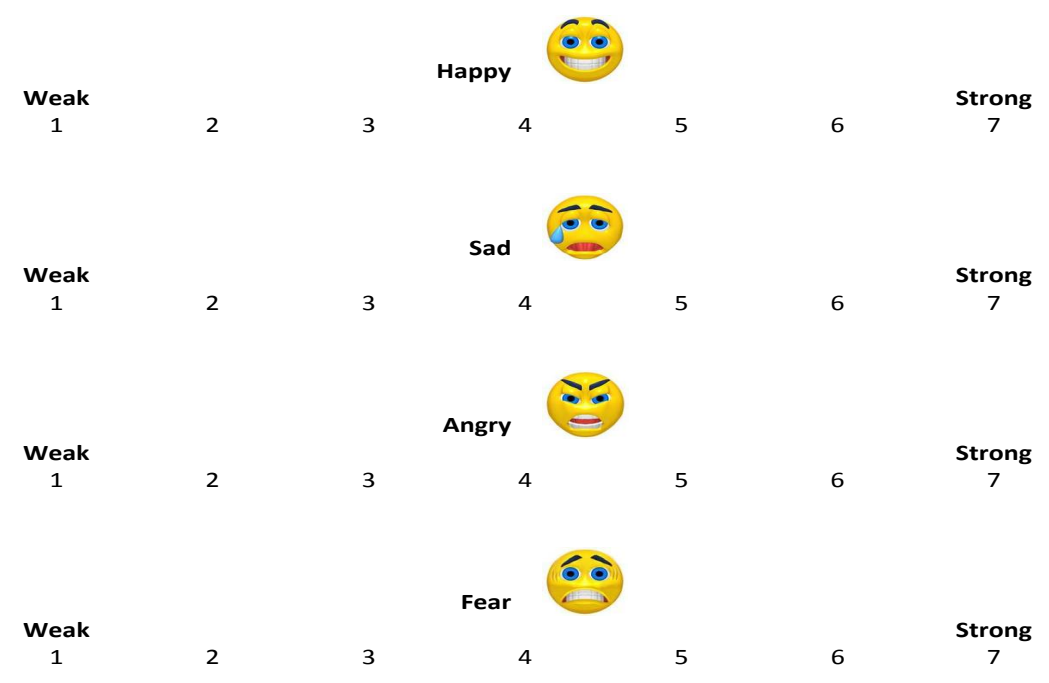


Music Visualization Post-Study Questionnaire

The purpose of this questionnaire is to gather information about you and your opinions about the visual representations that you just saw. There five questions and it should take you about ten minutes to complete this questionnaire. Thank you in advance for your time and assistance.

1. Which style did you enjoy most?

2. Which style gave you the best sense of what was happening in the music?

3. Which style gave you the best sense of:

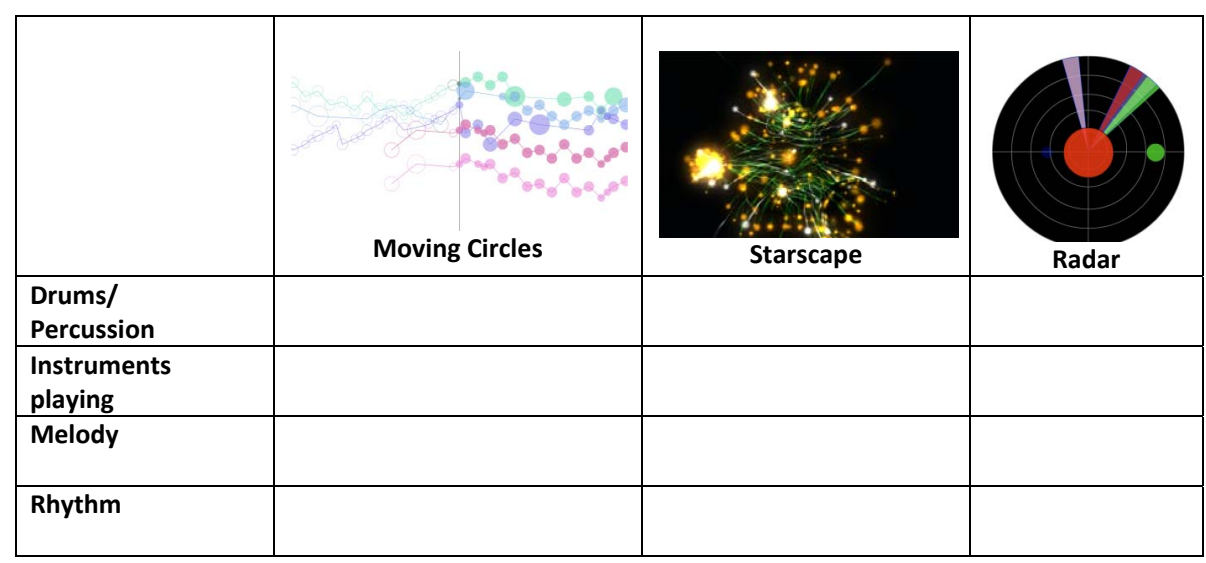

4. What was most enjoyable about your experience with music visualization?

5. What was least enjoyable about your experience with music visualization? 
David: First of all I apologize for the late start. I was hoping to get started about 10 minutes sooner, but whatever. The technology broke down, and these things happen. So just to start, My name is David. And I am a PhD candidate here at Ryerson. I am studying Mechanical Engineering, and my area is how to make certain kinds of information more available to deaf and hard of hearing people. I am hard of hearing myself, and I am a signer, but I'm going to rely on Tala tonight to make sure that, you know, my brain and my signing doesn't get confused. I appreciate that. Tonight we also have Tanya who will be providing captioning services for those of us who don't sign. Those of us who do may also follow on the screen - whatever. Let me just quickly introduce the team so you know [who] we all are. In the green shirt we have Mike. Say hi.

Mike: Hi.

David: Over here with the camera and the camera we have Jorge.

Jorge: Hey.

David: In the dark blue shirt we have Quoc. Today what will happen is Mike will be running the technology, Jorge will be running the camera, and Quoc will be picking up all the paper. This will be complex.

David: So, I handed out to you this nice package. What I will just do is if you take the paperclip off, and take the forms out, you will see inside the envelope a few pieces of paper. Some of you already got your consent forms. If you didn't bring one, we have one here for you to sign. The information form is for you to take home. If you have not already done so, we would appreciate if you sign the consent form. I will quickly explain what we are asking you to do. So just let me briefly explain what we are going to be doing today, what we are going to do 
today is run some music in the room, and we're going to put visualization on the screen over there next to Jorge. We will ask you to watch these visualizations and give us feedback. And just to let you know, your participation here today is completely voluntary. You can choose to leave at any time. Upon request we can remove you from the videotape and remove your information from the data centre. If you don't want to be videotaped, please tell us now too so Jorge can adjust the camera.

Participant 5: What is the purpose of the filming? What will it be used for just so I know and I can decide if I should be filmed or not.

David: Excellent question. Thank you very much for asking. The point of the video is primarily for me to have a record on top of the transcripts of what was done today so that if I'm not entirely clear what the transcript is telling me I can look at the signed version of your comment and get a sense of what you actually said compared to what Tala says you said. That's the primary purpose. The video cassette will be protected in a locked cabinet, no one else will have access to it except me and the three other gentlemen in this room. It will only be used for research purposes.

Participant 5: Sure.

David: So, please feel free to sign the consent forms and Quoc will come around and pick them up. Then I will explain the rest.

(Quoc is collecting the forms)

David: The next thing I will ask you to do is you'll find in the envelope a Pre-Study Questionnaire. This is just a quick questionnaire to give me a little bit of information about you. Fill out what you feel comfortable. 
David: So this is the next step. This is a pre study questionnaire. I will ask each of you to fill out the pre study questionnaire. This information will only help me get a sense of who you are.

Participant 3: (cannot hear question)

David: I am signing it later. That is go on file for myself.

Participant 2: What do we do with this after?

David: You fill it out. Quoc will be coming around to pick up the pre study questionnaire.

Participant 4: (cannot hear question)

David: Did you fill it out?

Participant 4: (shakes head)

David: If you could fill it out now that would be...

Participant 1: (cannot hear question)

David: The question is primarily designed to ask what your general experience was. If you want to...

Participant 1: (cannot hear question)

David: ... you have lots of options

Participant 1: (cannot hear question)

David: That is true. I have learned overtime there are a lot of schools that provide a music curriculum and there are a lot of school of the deaf that don't. It's an odd attitude. Deaf people have rhythm. They have a sense of rhythm.

Participant 1: (cannot hear question)

David: Exactly.

David: I'm just waiting for one more. 
David: Okay, so this form here [showing] I want you to hold on to it. You will fill this out at the end. Let me explain this before we get started. What we are going to do is I am just going to give a quick example of what we are going to be doing in the study. What we are going to do is Mike will start up a nice piece of music [music playing]. In case you don't know, that's "Happy Birthday". Okay kill it Mike. Thank you Mike. The idea is you would see some nice visualization like you just saw. It won't look like that, that's just a throw away we wanted to show you. And what you're going to do is you're going to take out one of these sheets [showing].

Participant 5: I was wondering is it possible that we...We have predominantly deaf participants on this side, so can we flip the screens? So that the captions are on the far side and the music visualization is on this screen? I don't know if that is possible.

David: Nope, you're already set up right?

Tanya (Captionist): Yup, I would have to move this whole thing. Can we can move the people, not the projector?

David: We can move the people, not the projector.

Participant 5: That's okay, it would just be better to have good sightlines when you have a deaf audience. If the deaf participant knows where the video will be, then we will adjust it accordingly.

David: I wanted it to be originally on this screen, but we had difficulty getting the computer to talk to the right screen. We're lucky it's talking to one of them and not both. But I appreciate your comment. So let me go back to my example. The visualization you just watched - then you will fill out this (shows form). The first question asks whether or not you enjoyed it. And I asking you to circle your preference. Then you are asked what did the visualization mean, and what did you get out of it, if anything? Then I have these three nice lines of cartoon characters here; what I am going to ask you to do is I am going to ask you to mark off one on 
each line to tell me in your opinion whether the music was happy, calm, excited, over-bearing or what subtle? How you feel? What did the music get across? Then finally I want you to rate the emotion as happy, sad, angry, cheerful, etc. That's essentially what this is. So what we are going to do is, at the end of each visualization, you will all get about 2 minutes to fill this out. Once we collect them all, then we'll go on to the next visualization. We will do this for all 12. If any of you at any time need a break, just ask and we will take a quick break. Okay, all set? This is the fun part, really.

[Visualization \#1: Day Tripper]

David: Okay? So please fill out the form.

Participant 4: I want that for my Windows Media Player [laughs].

Participant 1: Circle?

David: You can circle, or mark with an X. You can put it in pencil if your pen is not working.

Participant 1: Do we have to answer everything?

David: It's all voluntary; you can answer as much or as little as you like.

Participant 1: But what's all this for?

David: I want to get a sense of what you thought was expressed in the music.

Participant 3: (signs)

David: There is one form for each visualization, that's correct.

David: So, how does that feel so far? Is that working? Do you have any questions with regard to the forms?

Participant 4: I kind of like it. It made me feel upbeat, good.

Participant 3: It was neat. Very cool. 
Participant 5: The form is good, but where it says "weak and strong" perhaps one suggestion is to have the happy, sad, and the fear, perhaps all of them have pictures. In the middle it is not quite clear what does that mean, is its neutral form? Is that an extremity? Maybe from weak to strong. Each number with a matching picture.

David: What worked with this visualization?

Participant 2: When you listen to the music, and you get a different feel from the music when you watch it versus just listening it.

David: Are you hearing?

Participant 2: I am hearing.

David: So you're hearing it at the same time?

Participant 2: Yeah.

Participant 2: Am I supposed to be thinking about what the music tells me or what the visualization tells me? Do I look just at the visualization or do you want me to listen?

David: The whole experience.

Participant 5: I know what you mean. For example if you are sitting in the motor chair and you actually feel the vibration and listen to the music, it's tough to know what to focus on, the music or the chair.

Participant 2: Maybe when you do this again if you have music separately from the visualizations and see if they give the same feelings. There are those who are totally deaf and they will get more from the visualizations, and the others who have some hearing would get more from the music. Compare to see if you get the same feedback. 
Participant 5: Maybe it was the interpretation, or the captioning. It's not that it's tough to focus on, I mean more so if you're sitting in the motor chair and you have the music on, you can make the connection between the sound and the sight and the feelings.

Participant 4: Just adding to what she said, I'm thinking maybe next time you try the musical chairs with the visualization and or using headphones. Even though some deaf can't hear, with the headphones when you up the bass you can feel the vibrations. Somehow they can feel connected to what's happening there. I think that might give you a better, clearer answer than right now.

David: Mike, are we set for the next visualization? Well Mike, hit it; let's see what we get this time around.

[Visualization \#2]

David: Okay?

David: So what were you thinking about this. Was there something that worked here or didn't work here?

Participant 1: For me, I couldn't hear anything but solely relying on the visualization it was great. It's similar to a video game. It was nice and kind of neat. Participant 6: I can hear the music, but I liked when you reached the end of the piece there was a disconnect between what I could hear and what I could see.

David: Each piece is about 20-30 seconds long; so we're intentionally cutting it off at a certain point. I agree, you will get a disconnect.

Participant 2: I found that this one was more kind of came at me... I really liked it. David: Well Mike, are you ready to go again?

Mike: Yes I am.

Participant 3: Should we be putting down the number for what was what? 
David: We'll handle that. I just want you to worry about what you're seeing and what it is.

[Visualization \#3]

Mike: Sorry.

Participant 1: Don't worry about it. Just take your time.

Participant 3: Is it broken?

David: Oh, no it's not broken.

(Visualization starts again)

David: Okay, so what did you think of that? It's a little different.

Participant 5: I didn't feel any sort of connection at all with that piece.

Participant 4: I don't know why but when I was watching the visualization I was thinking back in my mind to "Ode to Joy" - I was thinking in the back of my mind with the way they moved and pulsated and then broke off into smaller pieces moving around. That just popped up.

David: Interesting. Okay, so we'll hit the next one. Is everyone ready?

[Visualization \#4]

David: Any comments?

Participant 1: Looking at it, it just looks like light. It doesn't feel like there's a connection to sound or music. Just things floating around on the screen. Maybe it does connect, but I cannot imagine what kind of music it is after seeing something like that. I don't hear anything though, so I guess that's just me.

Participant 5: I felt that one was a bit better. Not bad. Better than the first one, the one with the spots. I thought this one was a little bit better. 
David: Okay, so we'll move right along.

[Visualization \#5]

David: Any thoughts before we move on?

Participant 1, 3 and 5: I think we should just move on.

David: Okay, the next one.

[Visualization \#6: Beautiful People]

David: Any thoughts?

Participant 2: I think the music - there needs to be more connection between the music and the type of visualization. Circles and a black background make me think of night and that the music should be calm, and it's not calm.

David: Interesting.

Participant 3: Just in terms of the... you think of the moving spots as perhaps having a story, something negative and interesting. When they collide perhaps it's supposed to show turmoil. Perhaps there could be some kind of theatre to it, some kind of drama, in my opinion.

Participant 6: The background is very confusing. I think it leads to preconceived notions about what the music is supposed to be. If it's something unrelated it's confusing.

Participant 1: Lucky for me I can't hear anything. I focus on the visuals so I hear nothing. For me it's more like if you're driving at night and it's raining - it's the fluttering lights of the other drivers and that hazy look. It reminds me of driving at night and other people's headlights.

Quoc: Before we continue does anyone need a short break? 
David: You're alright? Remember if at any time you need a break, tell us and we'll take a break.

Quoc: We're just halfway through.

David: Thank you Quoc.

[Visualization \#7]

Participant 5: (Signing)

David: Yeah, I know. And the challenging thing for me is when I am dealing with music is trying to figure how, you know, how would English speaking hearing people talk about this stuff? What do they mean when they talk about loud or soft? What do they mean when they talk about...?

Participant 4: Watching it, that reminded me of the Walt Disney movie Fantasiathe part called "The Sorcerer's Apprentice." In that part, if I remember correctly, I remember reading that Walt Disney made that movie to somehow show pictures of how music affects people. It shows how music touches them, and moves them. Are you trying to do something similar?

David: Not quite.

Participant 4: Not quite.

David: The interpretation is entirely yours. The software is only taking the information of the music file and then presenting it according to the logic of the software. The interpretation is all yours, not the software's.

David: Any other comments? Okay, Mike?

[Visualization \#8]

David: Okay? So there are 4 more songs left.

Participant 4: We should just keep going. 
David: Yep, we're going to just keep going.

David: Okay? So we'll do the next one.

[Visualization \#9]

David: Okay.

David: Everyone ready? Okay, we'll start the next one.

[Visualization \#10]

Participant 5: Maybe next time, say it's for 2 hours, so in case you lose time.

David: Normally we do, but the person that did the booking said we started at 6, but we said to come at 6 . But also we were having technology problems that started us off really late, as well. This is going to be the last one.

Participant 5: I thought we had two more.

David: We can go for two more, if you want to go for two more. This is the eleventh.

[Visualization \#11]

David: Okay.

David: Okay, so what we're going to do now is play the very last visualization. After you fill out this form [for the visualization], I am going to get you to fill out this one. I will quickly explain what it asks. It's going to ask you first what did you like, what did you enjoy the most? Which of these visualizations gave you the best sense of what was going on with the music? What aspect of the music did it give you the best sense of-drums, instruments, and melodies? We have screen shots of the different visuals to help you out. Also we will ask you out what was the most enjoyable and least enjoyable aspect of this. Feel free to use the back of the page. Then we will have a wrap up discussion for any other comments.

[Visualization \#12] 
David: So what do you folks think? What do you think this last visualization, or the visualizations as a whole?

Participant 4: I enjoyed the stars much more. They give me an interpretation of the music, I'm not sure if that's the right word. I thought the radar was good, but I noticed I appreciated the stars more, and how they showed music to me. I feel positive about it.

Participant 1: I agree. It's the same experience for me. The stars are visually beautiful. I found myself mesmerized and it's really neat.

Participant 3: The starscape made me feel nauseous. There was too much movement and it was overwhelming. It made me feel sick.

Participant 5: Positive thing for me, before there was a white background and now a black background. It's easier on the eyes with the black. The white it was really difficult; I couldn't stare at the screen, but the black was better. I'm impressed.

Participant 2: There would need to be a lot of human interpretation as well, if you can't interpret it with the ears, it's going to be hard for someone to pick up on the tones and melodies of the music. The moving circles helped me see the rhythm better than the starscape, although the starscape told a better story. You'd have to pick which one went better with a different sound. If you left it up to the computer it wouldn't tell a story.

Participant 5: Just to clarify your comment, what do you mean in terms of interpreting the sound?

Participant 2: Because I can hear and see it, I found that hearing the music gives me a different feeling than what I'm seeing. There needs to be more connection between hearing and seeing. Different types of pictures showed the sound better than others. The hearing interpretation made it easier to pick out which one did a better job of representing what you're hearing. I would use the circle one that shows the rhythm. If you're going to a concert and want to see what gives a better interpretation of what is going on in terms of drums and instruments. The 
other one didn't do that as well. It's complicated and I can't really explain it as well.

Participant 4: I hope you continue with this study. What I would like to see next time is that we use the chairs. I've heard stories about them. I would like to hear what the chairs do for the experience. Or next thing is, when I listen to music I have headphones, and to understand the song I have to read the lyrics. But, even if there were no lyrics I know the lyrics have an effect if I don't know the words. I want to match the chairs with the visualization to see if it's different.

David: None of the music had lyrics. Most of it was standard, popular music. The last piece was the jazz piece "At Last". Two of the pieces are from video games one from Doom and one from Final Fantasy.

Participant 4: Still, even with the music itself, l'd like to see how my reaction would be for the music plus the visualization at the same time. I'm sure it would be totally different from how I feel now.

Participant 5; It's the same idea in terms of levels, someone with a cochlear implant, hearing, hard of hearing, deaf, or profoundly deaf person - it depends on the level of hearing loss. We would link it to our ability or inability to hear. You would have a better image of what's going on. As a deaf person you don't have that, you can't hear it so it's a different experience.

David: I'm deaf on one half of my head and moderate on the other. There are different interpretations and experiences. When I'm wearing a headset-

Participant 3: I need to go out. Puppy needs a bathroom break.

David: I will quickly explain the last thing you have to do. This thing [showing] is an envelope with money. I want you to sign it and date it. Tear the slip off the envelope and give it to Quoc.

David: Any last comments before our interpreter disappears? 
Participant 4: I just hope next time you have the chairs. As she said, I can't hear the words but if we had the chairs plus the visualization - wow it boggles my mind just at the thought of it.

David: Tonight the movie Cast Away is being shown at Dundas Square starting at 8:45. XXXXXX promises this time we will be using the chairs - as long as the weather holds up and stays dry, he'll use the chairs. You're more than welcome to come watch the movie and sit in one of the chairs.

Participant 4: When's the movie?

David: It's at 8:45 pm tonight.

Participant 4: Just in time to go get a snack and then see the chairs. Do I have to book the chairs?

David: There will be a free-flow chair that people can switch in and out of. It depends on how many show up, but there will be others you can sit in for the whole 2 hour movie. So there's going to be lots of sharing. It will be weather dependent. They can't have the electronics in the rain, so if it rains we can't be there.

Participant 4: That would be a shocking experience. [laughs]

[End of Focus Group Meeting] 


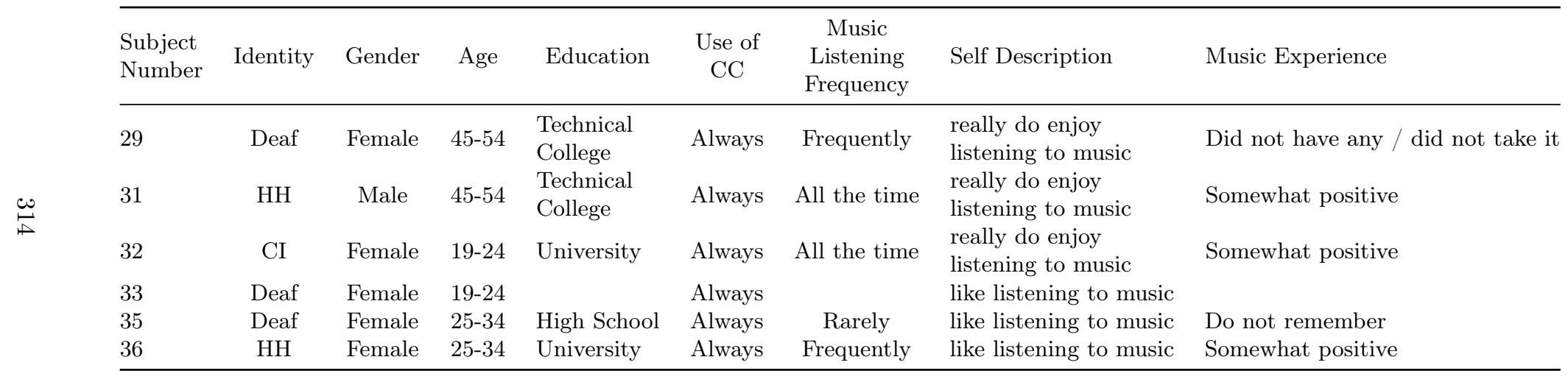

Table C.1: PreTest responses. 


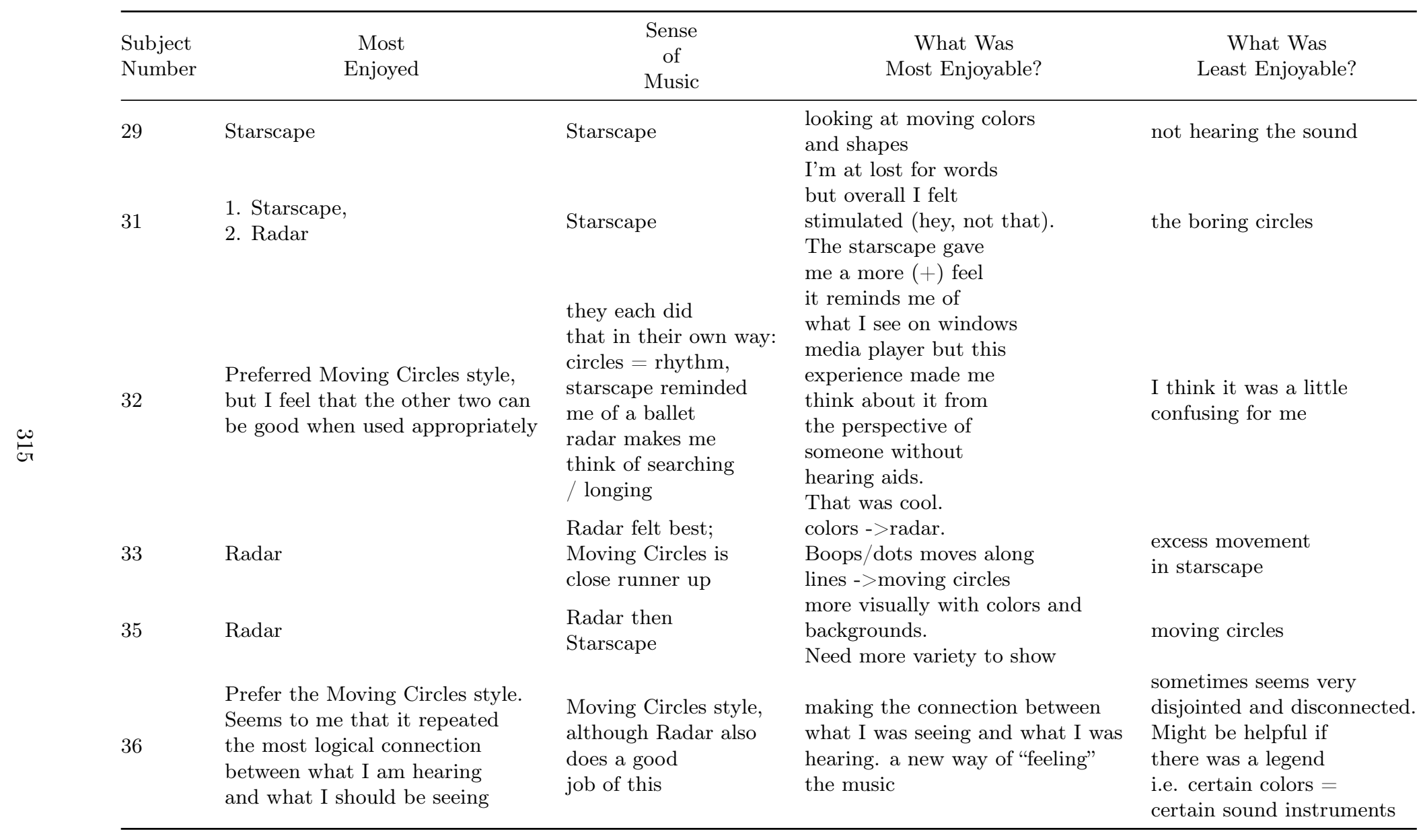

Table C.2: PostTest written responses. 


\begin{tabular}{|c|c|c|c|c|c|c|c|c|c|c|c|c|}
\hline \multirow{3}{*}{$\begin{array}{l}\text { Subject } \\
\text { Number }\end{array}$} & \multicolumn{12}{|c|}{ Provided the best sense of ... } \\
\hline & \multicolumn{4}{|c|}{ Moving Circles } & \multicolumn{4}{|c|}{ Starscape } & \multicolumn{4}{|c|}{ Radar } \\
\hline & Drums & Instruments & Melody & Rhythm & Drums & Instruments & Melody & Rhythm & Drums & Instruments & Melody & Rhythm \\
\hline 29 & & & & & & & & 1 & & & & \\
\hline 31 & & & & & & 1 & 1 & 1 & 1 & & & \\
\hline 32 & 1 & 1 & 1 & 1 & & & 1 & & & & & \\
\hline 33 & 1 & 1 & 1 & 1 & & & 1 & & 1 & & 1 & 1 \\
\hline 35 & & 1 & 1 & 1 & 1 & 1 & & 1 & 1 & 1 & & 1 \\
\hline 36 & & 1 & 1 & & & & & & 1 & & & 1 \\
\hline
\end{tabular}

Table C.3: PostTest responses.

官

\begin{tabular}{|c|c|c|c|c|c|c|c|c|c|}
\hline $\begin{array}{l}\text { Subject } \\
\text { Number }\end{array}$ & $\begin{array}{c}\text { V1 } \\
\text { Music } \\
\text { Enjoyment }\end{array}$ & $\begin{array}{c}\text { V1 } \\
\text { Meaning }\end{array}$ & $\begin{array}{c}\text { V1 } \\
\text { Happy } \\
\text { Unhappy }\end{array}$ & $\begin{array}{c}\text { V1 } \\
\text { Calm } \\
\text { Excited }\end{array}$ & $\begin{array}{c}\text { V1 } \\
\text { Subtle } \\
\text { Overwhelming }\end{array}$ & $\begin{array}{c}\text { V1 } \\
\text { Happiness }\end{array}$ & $\begin{array}{c}\text { V1 } \\
\text { Sadness }\end{array}$ & $\begin{array}{c}\text { V1 } \\
\text { Anger }\end{array}$ & $\begin{array}{l}\text { V1 } \\
\text { Fear }\end{array}$ \\
\hline 29 & & & 9 & 5 & 5 & 5 & 1 & 1 & 1 \\
\hline 31 & Enjoyable & Good, happy, vibrant & 9 & 9 & & 7 & & & \\
\hline 32 & Neutral & $\begin{array}{l}\text { I actually prefer to } \\
\text { just listen to the music :) }\end{array}$ & 7 & 7 & 7 & 6 & & & \\
\hline 33 & Enjoyable & $\begin{array}{l}\text { Happy but knows there is } \\
\text { stuff going on. Overall content } \\
\text { with life, has life beat, like life's } \\
\text { ups and downs }\end{array}$ & 7 & 6 & 5 & 5 & 4 & 1 & 1 \\
\hline 35 & $\begin{array}{l}\text { Somewhat } \\
\text { enjoyable }\end{array}$ & Rhythms & 6 & 4 & 4 & 3 & 1 & 1 & 1 \\
\hline 36 & $\begin{array}{l}\text { Somewhat } \\
\text { enjoyable }\end{array}$ & catchy music. Good beat. & 7 & 4 & 4 & 5 & 2 & 3 & 1 \\
\hline
\end{tabular}

Table C.4: Responses to Visualization 1. 


\begin{tabular}{|c|c|c|c|c|c|c|c|c|c|}
\hline $\begin{array}{l}\text { Subject } \\
\text { Number }\end{array}$ & $\begin{array}{c}\text { V2 } \\
\text { Music } \\
\text { Enjoyment }\end{array}$ & $\begin{array}{c}\mathrm{V} 2 \\
\text { Meaning }\end{array}$ & $\begin{array}{c}\text { V2 } \\
\text { Unhappy } \\
\text { Happy }\end{array}$ & $\begin{array}{c}\mathrm{V} 2 \\
\text { Calm } \\
\text { Excited }\end{array}$ & $\begin{array}{c}\text { V2 } \\
\text { Subtle } \\
\text { Overwhelming }\end{array}$ & $\begin{array}{c}\mathrm{V} 2 \\
\text { Happiness }\end{array}$ & $\begin{array}{c}\text { V2 } \\
\text { Sadness }\end{array}$ & $\begin{array}{c}\text { V2 } \\
\text { Anger }\end{array}$ & $\begin{array}{c}\text { V2 } \\
\text { Fear }\end{array}$ \\
\hline 29 & & & 6 & 4 & 5 & 4 & 2 & & \\
\hline 31 & Neutral & & & 1 & & 2 & & & \\
\hline 32 & Enjoyable & & 7 & 3 & 3 & 3 & & & \\
\hline 33 & Enjoyable & & 4 & 2 & 3 & 3 & 6 & 1 & 1 \\
\hline 35 & Not that enjoyable & & 4 & 1 & 4 & 1 & 5 & 1 & 1 \\
\hline 36 & Somewhat enjoyable & & 6 & 2 & 3 & 4 & 3 & 2 & 2 \\
\hline
\end{tabular}

Table C.5: Responses to Visualization 2.

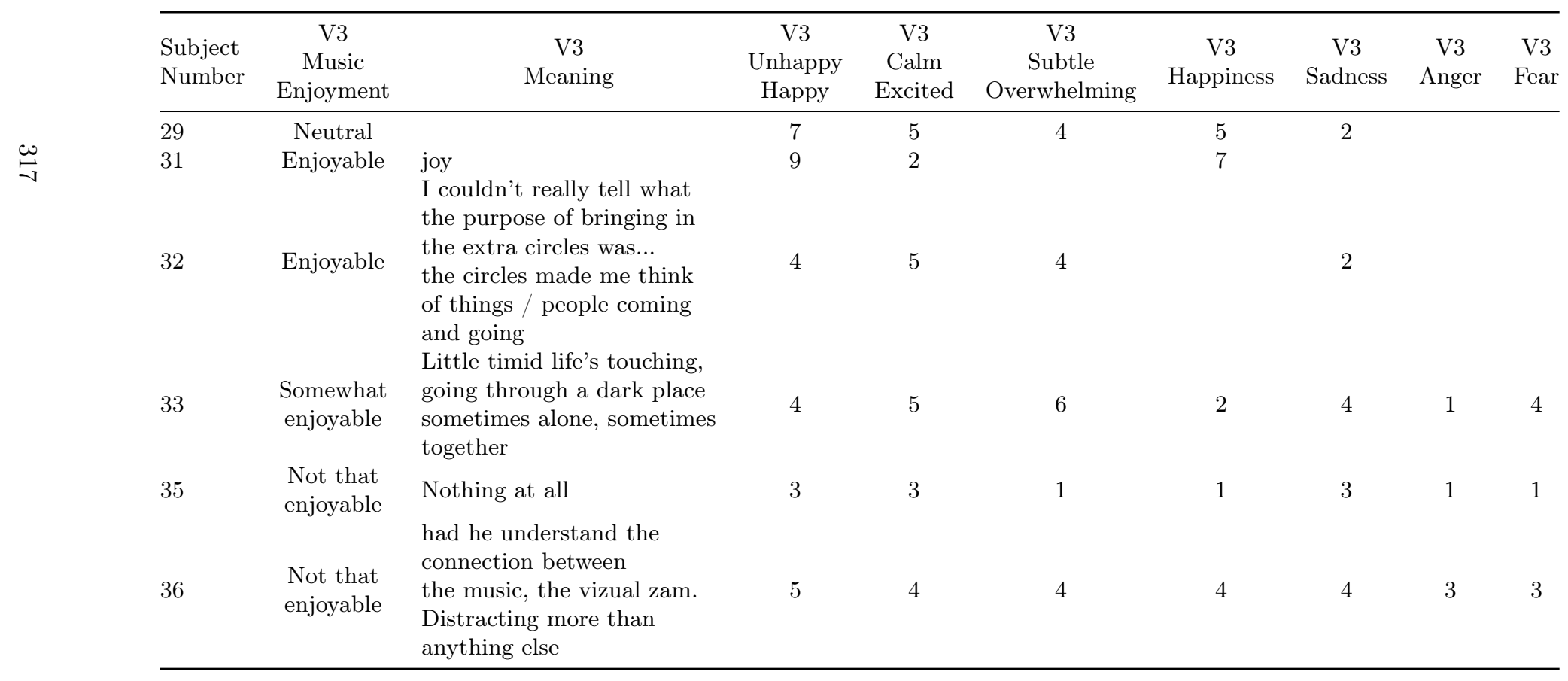

Table C.6: Responses to Visualization 3. 


\begin{tabular}{|c|c|c|c|c|c|c|c|c|c|}
\hline $\begin{array}{l}\text { Subject } \\
\text { Number }\end{array}$ & $\begin{array}{c}\text { V4 } \\
\text { Music } \\
\text { Enjoyment }\end{array}$ & $\begin{array}{c}\text { V4 } \\
\text { Meaning }\end{array}$ & $\begin{array}{l}\text { V4 } \\
\text { Unhappy } \\
\text { Happy }\end{array}$ & $\begin{array}{c}\mathrm{V} 4 \\
\text { Calm } \\
\text { Excited }\end{array}$ & $\begin{array}{c}\text { V4 } \\
\text { Subtle } \\
\text { Overwhelming }\end{array}$ & $\begin{array}{c}\text { V4 } \\
\text { Happiness }\end{array}$ & $\begin{array}{c}\text { V4 } \\
\text { Sadness }\end{array}$ & $\begin{array}{l}\text { V4 } \\
\text { Anger }\end{array}$ & $\begin{array}{c}\text { V4 } \\
\text { Fear }\end{array}$ \\
\hline 29 & Neutral & $\begin{array}{l}\text { Movement of lights not } \\
\text { related to any music }\end{array}$ & 6 & 2 & 3 & 3 & 1 & & \\
\hline 31 & Neutral & & & & & & & & \\
\hline 32 & $\begin{array}{l}\text { Somewhat } \\
\text { enjoyable }\end{array}$ & $\begin{array}{l}\text { I preferred the second one. } \\
\text { I got the feeling that there } \\
\text { was rapid "outbursts" towards } \\
\text { the end }\end{array}$ & 4 & 5 & 5 & & & 1 & \\
\hline 33 & Enjoyable & $\begin{array}{l}\text { rapid person mothing through } \\
\text { a city, bit tough }\end{array}$ & 3 & 8 & 6 & 2 & 2 & 6 & 2 \\
\hline 35 & $\begin{array}{l}\text { Somewhat } \\
\text { enjoyable }\end{array}$ & different sounds of beats & 6 & 5 & 5 & 5 & 1 & 1 & 1 \\
\hline 36 & Enjoyable & $\begin{array}{l}\text { Enjoyed the color coding } \\
\text { here re: seemed like each } \\
\text { pt / component of the song } \\
\text { was reflected by a particular } \\
\text { color that helped somogenize } \\
\text { the piece / listening experience }\end{array}$ & 3 & 3 & 3 & 3 & 4 & 3 & 2 \\
\hline
\end{tabular}

Table C.7: Responses to Visualization 4. 


\begin{tabular}{|c|c|c|c|c|c|c|c|c|c|}
\hline $\begin{array}{l}\text { Subject } \\
\text { Number }\end{array}$ & $\begin{array}{c}\text { V5 } \\
\text { Music } \\
\text { Enjoyment }\end{array}$ & $\begin{array}{c}\text { V5 } \\
\text { Meaning }\end{array}$ & $\begin{array}{l}\text { V5 } \\
\text { Unhappy } \\
\text { Happy }\end{array}$ & $\begin{array}{c}\text { V5 } \\
\text { Calm } \\
\text { Excited }\end{array}$ & $\begin{array}{c}\text { V5 } \\
\text { Subtle } \\
\text { Overwhelming } \\
\end{array}$ & $\begin{array}{c}\text { V5 } \\
\text { Happiness }\end{array}$ & $\begin{array}{c}\text { V5 } \\
\text { Sadness }\end{array}$ & $\begin{array}{c}\text { V5 } \\
\text { Anger }\end{array}$ & $\begin{array}{l}\text { V5 } \\
\text { Fear }\end{array}$ \\
\hline 29 & \multirow{2}{*}{$\begin{array}{l}\text { Enjoyable } \\
\text { Somewhat } \\
\text { enjoyable }\end{array}$} & \multirow[t]{2}{*}{ More colors to enjoy looking at } & \multirow[t]{2}{*}{8} & 4 & \multirow[t]{2}{*}{4} & \multirow[t]{2}{*}{5} & \multirow[t]{2}{*}{1} & & \\
\hline 31 & & & & 4 & & & & & \\
\hline 32 & Neutral & $\begin{array}{l}\text { Better than } \# 1 \text { - I felt like the } \\
\text { person was on a stage, running } \\
\text { from something or in a ballet. } \\
\text { Like hiding, coming out, running } \\
\text { to a new place, more hiding. Don't }\end{array}$ & 4 & 9 & 9 & & & & 5 \\
\hline 33 & Enjoyable & $\begin{array}{l}\text { want caught. Innocent? } \\
\text { Relief moments when } \\
\text { hid well }\end{array}$ & 2 & 9 & 6 & 2 & 1 & 1 & 6 \\
\hline 35 & $\begin{array}{c}\text { Somewhat } \\
\text { enjoyable }\end{array}$ & Different beats with sound waves & 5 & 6 & 5 & 4 & 1 & 1 & 1 \\
\hline 36 & $\begin{array}{l}\text { Not that } \\
\text { enjoyable }\end{array}$ & $\begin{array}{l}\text { Not my favourite format. Could be } \\
\text { any piece of music. Don't like the } \\
\text { background. Makes me think / feel } \\
\text { that I'm watching a target / } \\
\text { target practice }\end{array}$ & 3 & 6 & 7 & 3 & 4 & 6 & 5 \\
\hline
\end{tabular}

Table C.8: Responses to Visualization 5. 


\begin{tabular}{|c|c|c|c|c|c|c|c|c|c|}
\hline $\begin{array}{l}\text { Subject } \\
\text { Number }\end{array}$ & $\begin{array}{c}\text { V6 } \\
\text { Music } \\
\text { Enjoyment }\end{array}$ & $\begin{array}{c}\text { V6 } \\
\text { Meaning }\end{array}$ & $\begin{array}{l}\text { V6 } \\
\text { Unhappy } \\
\text { Happy }\end{array}$ & $\begin{array}{c}\text { V6 } \\
\text { Calm } \\
\text { Excited }\end{array}$ & $\begin{array}{c}\text { V6 } \\
\text { Subtle } \\
\text { Overwhelming }\end{array}$ & $\begin{array}{c}\text { V6 } \\
\text { Happiness }\end{array}$ & $\begin{array}{c}\text { V6 } \\
\text { Sadness }\end{array}$ & $\begin{array}{c}\text { V6 } \\
\text { Anger }\end{array}$ & $\begin{array}{l}\text { V6 } \\
\text { Fear }\end{array}$ \\
\hline 29 & Neutral & $\begin{array}{l}\text { looking at lights on a rainy } \\
\text { night on the highway }\end{array}$ & 8 & 4 & 4 & 4 & 1 & & \\
\hline 31 & Enjoyable & like a dance, dancing & & & & 6 & & & \\
\hline 32 & Enjoyable & $\begin{array}{l}\text { I think that care has to be } \\
\text { taken when selecting type } \\
\text { of visualization to use with } \\
\text { each style of music }\end{array}$ & 3 & 6 & 6 & & & 5 & \\
\hline 33 & $\begin{array}{l}\text { Somewhat } \\
\text { enjoyable }\end{array}$ & $\begin{array}{l}\text { Sort of fight/argue, group } \\
\text { branch off, hang out one } \\
\text { group, tough times }\end{array}$ & 5 & 6 & 7 & 2 & 1 & 4 & 4 \\
\hline 35 & $\begin{array}{l}\text { Not } \\
\text { enjoyable } \\
\text { at all }\end{array}$ & Nothing at all & 5 & 1 & 1 & 1 & 3 & 1 & 1 \\
\hline 36 & $\begin{array}{l}\text { Not that } \\
\text { enjoyable }\end{array}$ & $\begin{array}{l}\text { just a jumble of lights, } \\
\text { no connection to the music, } \\
\text { very distracting }\end{array}$ & 3 & 5 & 5 & 2 & 4 & 6 & 6 \\
\hline
\end{tabular}

Table C.9: Responses to Visualization 6. 


\begin{tabular}{|c|c|c|c|c|c|c|c|c|c|}
\hline $\begin{array}{l}\text { Subject } \\
\text { Number }\end{array}$ & $\begin{array}{c}\text { V7 } \\
\text { Music } \\
\text { Enjoyment }\end{array}$ & $\begin{array}{c}\mathrm{V} 7 \\
\text { Meaning }\end{array}$ & $\begin{array}{l}\text { V7 } \\
\text { Unhappy } \\
\text { Happy }\end{array}$ & $\begin{array}{c}\mathrm{V} 7 \\
\text { Calm } \\
\text { Excited }\end{array}$ & $\begin{array}{c}\mathrm{V} 7 \\
\text { Subtle } \\
\text { Overwhelming }\end{array}$ & $\begin{array}{c}\text { V7 } \\
\text { Happiness }\end{array}$ & $\begin{array}{c}\text { V7 } \\
\text { Sadness }\end{array}$ & $\begin{array}{c}\text { V7 } \\
\text { Anger }\end{array}$ & $\begin{array}{l}\text { V7 } \\
\text { Feal }\end{array}$ \\
\hline 29 & Enjoyable & $\begin{array}{l}\text { going into space, } \\
\text { looking at universe }\end{array}$ & 9 & 7 & 5 & 6 & 1 & & \\
\hline 31 & Enjoyable & $\begin{array}{l}\text { reminds me of Walt Disney's } \\
\text { "Fantasia" movie }\end{array}$ & & & & 7 & & & \\
\hline 32 & Enjoyable & $\begin{array}{l}\text { LOVE it. I think it did a good } \\
\text { job of telling the "story" }\end{array}$ & 6 & 7 & 4 & 3 & & & \\
\hline 33 & $\begin{array}{c}\text { Somewhat } \\
\text { enjoyable }\end{array}$ & $\begin{array}{l}\text { Somewhat lost traveller of space, } \\
\text { admiring new places but sometimes } \\
\text { gets bumped around. Still feels } \\
\text { a little confused in surroundings } \\
\text { Booming sounds with different }\end{array}$ & 6 & 7 & 8 & 4 & 1 & 1 & 1 \\
\hline 35 & Enjoyable & $\begin{array}{l}\text { Booming sounds with different } \\
\text { volumes going up and down, } \\
\text { almost like rhythms }\end{array}$ & 8 & 7 & 5 & 5 & 1 & 1 & 1 \\
\hline 36 & Enjoyable & $\begin{array}{l}\text { I liked this one - I wonder if } \\
\text { it would work in a slower, more } \\
\text { calm piece though }\end{array}$ & 6 & 8 & 6 & 4 & 4 & 3 & 5 \\
\hline
\end{tabular}

Table C.10: Responses to Visualization 7. 


\begin{tabular}{|c|c|c|c|c|c|c|c|c|c|}
\hline $\begin{array}{l}\text { Subject } \\
\text { Number }\end{array}$ & $\begin{array}{l}\text { V8 } \\
\text { Music } \\
\text { Enjoyment }\end{array}$ & $\begin{array}{l}\text { V8 } \\
\text { Meaning }\end{array}$ & $\begin{array}{l}\text { V8 } \\
\text { Unhappy } \\
\text { Happy }\end{array}$ & $\begin{array}{l}\text { V8 } \\
\text { Calm } \\
\text { Excited }\end{array}$ & $\begin{array}{l}\text { V8 } \\
\text { Subtle } \\
\text { Overwhelming }\end{array}$ & $\begin{array}{l}\text { V8 } \\
\text { Happiness }\end{array}$ & $\begin{array}{l}\text { V8 } \\
\text { Sadness }\end{array}$ & $\begin{array}{l}\text { V8 } \\
\text { Anger }\end{array}$ & $\begin{array}{l}\text { V8 } \\
\text { Fear }\end{array}$ \\
\hline 29 & Enjoyable & $\begin{array}{l}\text { A parade of nice and beautiful } \\
\text { dancing lights }\end{array}$ & 9 & 6 & 3 & 6 & 1 & & \\
\hline 31 & Neutral & & & & & & & & \\
\hline 32 & Enjoyable & $\begin{array}{l}\text { It gave a really good visualization } \\
\text { of the rhythm! } \\
\text { Happy-go-lucky falls into trouble, } \\
\text { almost burst free but sucked right }\end{array}$ & 7 & 9 & 6 & 6 & & & \\
\hline 33 & & $\begin{array}{l}\text { back then faces series of fearful } \\
\text { situations. Is sick of it, frustrated } \\
\text { by end }\end{array}$ & 3 & 7 & 6 & 2 & 1 & 3 & 7 \\
\hline 35 & $\begin{array}{l}\text { Somewhat } \\
\text { enjoyable }\end{array}$ & $\begin{array}{l}\text { Sound rhythms like boom-boom } \\
\text { (like drum) then bang. Not a big bang } \\
\text { - sounds almost the same level but the } \\
\text { pink part on the top seems distraction } \\
\text { for me }\end{array}$ & 5 & 6 & 6 & 3 & 1 & 1 & 1 \\
\hline 36 & $\begin{array}{l}\text { Somewhat } \\
\text { enjoyable }\end{array}$ & $\begin{array}{l}\text { I prefer this format. I like that the } \\
\text { format helps you anticipate in the } \\
\text { piece, re: what's coming next as well } \\
\text { as showing a discernible history of } \\
\text { what's already happened }\end{array}$ & 2 & 3 & 3 & 2 & 5 & 6 & 5 \\
\hline
\end{tabular}

Table C.11: Responses to Visualization 8. 


\begin{tabular}{|c|c|c|c|c|c|c|c|c|c|}
\hline $\begin{array}{l}\text { Subject } \\
\text { Number }\end{array}$ & $\begin{array}{c}\text { V9 } \\
\text { Music } \\
\text { Enjoyment }\end{array}$ & $\begin{array}{c}\text { V9 } \\
\text { Meaning }\end{array}$ & $\begin{array}{c}\text { V9 } \\
\text { Happy } \\
\text { Unhappy }\end{array}$ & $\begin{array}{c}\text { V9 } \\
\text { Calm } \\
\text { Excited }\end{array}$ & $\begin{array}{c}\text { V9 } \\
\text { Subtle } \\
\text { Overwhelming }\end{array}$ & $\begin{array}{c}\text { V9 } \\
\text { Happiness }\end{array}$ & $\begin{array}{c}\text { V9 } \\
\text { Sadness }\end{array}$ & $\begin{array}{c}\text { V9 } \\
\text { Anger }\end{array}$ & $\begin{array}{l}\text { V9 } \\
\text { Fear }\end{array}$ \\
\hline 29 & $\begin{array}{l}\text { Somewhat } \\
\text { enjoyable }\end{array}$ & concert of colors and lights & 5 & 2 & 1 & 3 & 1 & & \\
\hline 31 & Enjoyable & not sure & & & & 6 & & & \\
\hline 32 & Neutral & $\begin{array}{l}\text { I feel like this visualization } \\
\text { requires too much to be explained } \\
\text { in the sense that I don't understand } \\
\text { what all the circles mean, versus } \\
\text { the "lines" "spreading" }\end{array}$ & 3 & 6 & 6 & & & & 5 \\
\hline 33 & Enjoyable & $\begin{array}{l}\text { Enjoyable life, also has } \\
\text { mystery and intrigue }\end{array}$ & 7 & 5 & & 5 & 1 & 1 & 2 \\
\hline 35 & $\begin{array}{l}\text { Somewhat } \\
\text { enjoyable }\end{array}$ & $\begin{array}{l}\text { Sounds with rhythm - with } \\
\text { a bit of higher sounds }\end{array}$ & 5 & 6 & 4 & 4 & 1 & 1 & 1 \\
\hline 36 & $\begin{array}{l}\text { Somewhat } \\
\text { enjoyable }\end{array}$ & lots of color, brightness, beat & 5 & 5 & 5 & 3 & 4 & 6 & 6 \\
\hline
\end{tabular}

Table C.12: Responses to Visualization 9.

\begin{tabular}{|c|c|c|c|c|c|c|c|c|c|}
\hline $\begin{array}{l}\text { Subject } \\
\text { Number }\end{array}$ & $\begin{array}{c}\text { V10 } \\
\text { Music } \\
\text { Enjoyment }\end{array}$ & $\begin{array}{c}\text { V10 } \\
\text { Meaning }\end{array}$ & $\begin{array}{l}\text { V10 } \\
\text { Unhappy } \\
\text { Happy }\end{array}$ & $\begin{array}{c}\text { V10 } \\
\text { Calm } \\
\text { Excited }\end{array}$ & $\begin{array}{c}\text { V10 } \\
\text { Subtle } \\
\text { Overwhelming }\end{array}$ & $\begin{array}{c}\text { V10 } \\
\text { Happiness }\end{array}$ & $\begin{array}{c}\text { V10 } \\
\text { Sadness }\end{array}$ & $\begin{array}{l}\text { V10 } \\
\text { Anger }\end{array}$ & $\begin{array}{l}\text { V10 } \\
\text { Fear }\end{array}$ \\
\hline 29 & Neutral & dancing colors in the distance & 4 & 3 & 2 & 4 & 2 & & \\
\hline 31 & Enjoyable & $\begin{array}{l}\text { comfort, soothing, nice } \\
\text { This one does a good job }\end{array}$ & & & & 7 & & & \\
\hline 32 & Enjoyable & $\begin{array}{l}\text { of representing a "searching" } \\
\text { or a "longing" }\end{array}$ & 2 & 2 & 2 & & 5 & & \\
\hline 33 & Enjoyable & sorrow in autumn & 1 & 2 & 4 & 1 & 7 & 1 & 1 \\
\hline 35 & $\begin{array}{l}\text { Somewhat } \\
\text { enjoyable }\end{array}$ & seems slow relaxing music & 6 & 1 & 4 & 4 & 1 & 1 & 1 \\
\hline 36 & $\begin{array}{l}\text { Somewhat } \\
\text { enjoyable }\end{array}$ & $\begin{array}{l}\text { liked that the color seemed toned } \\
\text { down to match the mood of the } \\
\text { piece (?). Good tempo re: bars } \\
\text { of colors }\end{array}$ & 2 & 3 & 3 & 2 & 5 & 3 & 3 \\
\hline
\end{tabular}

Table C.13: Responses to Visualization 10. 


\begin{tabular}{|c|c|c|c|c|c|c|c|c|c|}
\hline $\begin{array}{l}\text { Subject } \\
\text { Number }\end{array}$ & $\begin{array}{c}\text { V11 } \\
\text { Music } \\
\text { Enjoyment }\end{array}$ & $\begin{array}{c}\text { V11 } \\
\text { Meaning }\end{array}$ & $\begin{array}{l}\text { V11 } \\
\text { Unhappy } \\
\text { Happy }\end{array}$ & $\begin{array}{c}\text { V11 } \\
\text { Calm } \\
\text { Excited }\end{array}$ & $\begin{array}{c}\text { V11 } \\
\text { Subtle } \\
\text { Overwhelming }\end{array}$ & $\begin{array}{c}\text { V11 } \\
\text { Happiness }\end{array}$ & $\begin{array}{c}\text { V11 } \\
\text { Sadness }\end{array}$ & $\begin{array}{l}\text { V11 } \\
\text { Anger }\end{array}$ & $\begin{array}{l}\text { V11 } \\
\text { Fear }\end{array}$ \\
\hline 29 & Neutral & $\begin{array}{l}\text { dancing in the universe with all } \\
\text { planets and stars }\end{array}$ & 9 & 6 & 5 & 7 & 1 & & \\
\hline 31 & Enjoyable & $\begin{array}{l}\text { excitement, adventure } \\
\text { I like the way it fades off at the end. }\end{array}$ & & & & 7 & & & \\
\hline 32 & $\begin{array}{l}\text { Somewhat } \\
\text { enjoyable }\end{array}$ & $\begin{array}{l}\text { It reminded me of a song telling the } \\
\text { story of someone's life, slowing down } \\
\text { in "old age" }\end{array}$ & 3 & 1 & 2 & & 2 & & \\
\hline 33 & $\begin{array}{l}\text { Not that } \\
\text { enjoyable }\end{array}$ & wonders, fazed, slightly lost & 5 & & 8 & & & 1 & 1 \\
\hline 35 & Neutral & $\begin{array}{l}\text { seems electric music nowadays. } \\
\text { Seems ok but not everything fitting } \\
\text { for me }\end{array}$ & 4 & 5 & 6 & 3 & 1 & 1 & 1 \\
\hline 36 & $\begin{array}{l}\text { Not that } \\
\text { enjoyable }\end{array}$ & $\begin{array}{l}\text { the format really doesn't work for } \\
\text { me when it's a slow piece. Too busy }\end{array}$ & 4 & 3 & 3 & 3 & 5 & 3 & 3 \\
\hline
\end{tabular}

Table C.14: Responses to Visualization 11.

\begin{tabular}{|c|c|c|c|c|c|c|c|c|c|}
\hline $\begin{array}{l}\text { Subject } \\
\text { Number }\end{array}$ & $\begin{array}{c}\text { V12 } \\
\text { Music } \\
\text { Enjoyment }\end{array}$ & $\begin{array}{l}\text { V12 } \\
\text { Meaning }\end{array}$ & $\begin{array}{l}\text { V12 } \\
\text { Unhappy } \\
\text { Happy }\end{array}$ & $\begin{array}{c}\text { V12 } \\
\text { Calm } \\
\text { Excited }\end{array}$ & $\begin{array}{c}\text { V12 } \\
\text { Subtle } \\
\text { Overwhelming }\end{array}$ & $\begin{array}{l}\text { V12 } \\
\text { Happiness }\end{array}$ & $\begin{array}{c}\text { V12 } \\
\text { Sadness }\end{array}$ & $\begin{array}{l}\text { V12 } \\
\text { Anger }\end{array}$ & $\begin{array}{l}\text { V12 } \\
\text { Fear }\end{array}$ \\
\hline 29 & Neutral & $\begin{array}{l}\text { cannot identify the music from } \\
\text { the dancing balloons }\end{array}$ & 5 & 4 & 2 & 4 & 1 & & \\
\hline 31 & Neutral & & & & & & & & \\
\hline 32 & $\begin{array}{l}\text { Somewhat } \\
\text { enjoyable }\end{array}$ & $\begin{array}{l}\text { it's picking upon different things } \\
\text { each time I see this type of visualization }\end{array}$ & 2 & 2 & 3 & & 3 & & \\
\hline \multicolumn{10}{|c|}{ 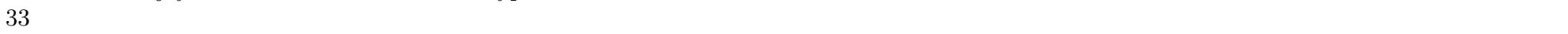 } \\
\hline 35 & $\begin{array}{l}\text { Not that } \\
\text { enjoyable }\end{array}$ & $\begin{array}{l}\text { nothing at all. Too confusing with } \\
\text { big circles (and colored as well) }\end{array}$ & 4 & 5 & 7 & 1 & 2 & 1 & 1 \\
\hline 36 & $\begin{array}{l}\text { Somewhat } \\
\text { enjoyable }\end{array}$ & $\begin{array}{l}\text { very nb that the viewer knows he watch } \\
\text { the circles in the center - otherwise } \\
\text { the background can be very distracting. } \\
\text { Visual "noise" }\end{array}$ & 4 & 3 & 2 & 3 & 5 & 3 & 3 \\
\hline
\end{tabular}

Table C.15: Responses to Visualization 12. 


\section{Appendix D}

\section{Paper Survey}

This Appendix presents the forms used and data collected in the paper survey, described in Chapter 7. 


\section{Music Visualization Questionnaire}

The purpose of this questionnaire is to gather information about you and your experience with music. There are fourteen questions and it should take you about five to ten minutes to complete this questionnaire. Thank you in advance for your time and assistance.

1. Do you identify yourself as: (please check one)
$\square$ Hearing
$\square$ Deaf
$\square$ Cochlear implant
- Hard of hearing
$\square$ Deafened

2. Please indicate your gender: (please check one)

a Male

$\square$ Female

3. Please indicate your age: (please check one)
口 18-24
口 $35-44$
口 $55-64$
口 $25-34$
口 $45-54$
口 $65+$

4. What is your highest level of education completed? (please check one)
$\square$ No formal education
High School
$\square$ University
Elementary school
$\square$ Community college
Graduate school (e.g., Master's)

5. How often do you use closed captioning when watching television? (please check one)
$\square$ Always
$\square$ Sometimes
口 Never

6. How often do you listen to music? (please check one)
$\square$ Every day
$\square$ Weekly
$\square$ Sometimes
$\square$ Rarely
$\square$ Never

7. What technology do you use to listen to music? (check all that apply)
$\square$ Radio
$\square$ Music player
$\square$ Home stereo
Music video
$\square$ Other

8. Which of the following statements best describes you? (check all that apply)

$\square$ I really enjoy listening to music

$\square$ I do not like listening to music

$\square$ I like listening to music

I do not see the point of music

$\square$ I do not like nor dislike music

9. If you wanted to use a computer to help you to better understand music, what would be the three most important pieces of information you would want to know? (please circle your three choices)

The name of the song

Artist information (e.g., band name)

Lyrics

Instrument currently being played

Beat

Other

Please complete reverse side.

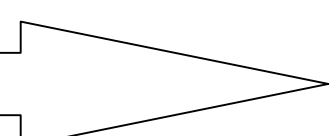


10. What types of solutions have you tried to access music: (check all that apply).

- Turn volume up higher

P Place hands/body close to speakers

u Use a balloon

$\square$ Set stereo equalizer

Adjust the balance, bass, or treble settings

$\square$ Other $\square$ Use ear phones

Only listen to music with a strong beat

U Use music playing software with visualizations

(e.g., iTunes, Windows Media Player)

$\square$ Wear hearing aids / Cl / FM system

11. Are you satisfied with this solution(s)? (please check one)

$\square$ Satisfied $\square$ Somewhat satisfied $\square$ Neutral $\square$ Somewhat dissatisfied $\square$ Dissatisfied

12. Why or why not:

13. Please comment on any specific frustrations that you have had with trying to access music and what would improve your experience with music. (Use extra paper if needed.)

14. Please comment on what positive experiences you have had with music. (Use extra paper if needed.)

As this is an ongoing study, should you wish to participate further or at a later time /stage please provide contact information below (optional). Your contact information will not be shared with anyone.

Name: Phone: Email:

\section{Please drop off your completed questionnaire at the Registration Desk.}

\section{THANK YOU!}




\begin{tabular}{|c|c|c|c|c|c|c|c|}
\hline Subject Number & Identity & Gender & Age & Education & Uses CC & Listens to Music & Music Enjoyment \\
\hline 301 & CI & Female & $55-64$ & Community College & Always & Sometimes & I really like listening to music \\
\hline 302 & $\mathrm{CI}$ & Female & $65+$ & Community College & Always & Weekly & I like listening to music \\
\hline 303 & $\mathrm{HH}$ & Female & $55-64$ & University & Sometimes & Weekly & I really like listening to music \\
\hline 304 & $\mathrm{HH}$ & Female & $18-24$ & Grad School & Always & Every day & I like listening to music \\
\hline 305 & $\mathrm{HH}$ & Male & $55-64$ & High School & Sometimes & Every day & I really like listening to music \\
\hline 306 & $\mathrm{HH}$ & Male & $18-24$ & Elementary School & Always & Sometimes & I really like listening to music \\
\hline 307 & Deafened & Female & $45-54$ & Grad School & Always & Weekly & I really like listening to music \\
\hline 308 & $\mathrm{CI}$ & Female & $55-64$ & Grad School & Always & Sometimes & I like listening to music \\
\hline 309 & $\mathrm{HH}$ & Female & $45-54$ & Community College & Sometimes & Every day & I really like listening to music \\
\hline 310 & $\mathrm{HH}$ & Female & $55-64$ & Grad School & Always & Rarely & I like listening to music \\
\hline 311 & $\mathrm{HH}$ & Female & $25-34$ & Grad School & Always & Every day & I really like listening to music \\
\hline 312 & $\mathrm{HH}$ & Male & $25-34$ & University & Always & Every day & I really like listening to music \\
\hline 313 & $\mathrm{HH}$ & Female & $45-54$ & Community College & Always & Every day & I like listening to music \\
\hline 314 & $\mathrm{HH}$ & Female & $65+$ & University & Sometimes & Every day & I really like listening to music \\
\hline 315 & $\mathrm{HH}$ & Female & $65+$ & Community College & Sometimes & Weekly & I really like listening to music \\
\hline 316 & $\mathrm{HH}$ & Female & $55-64$ & University & Sometimes & Every day & I really like listening to music \\
\hline 317 & $\mathrm{HH}$ & Male & $45-54$ & Grad School & Always & Weekly & I like listening to music \\
\hline 318 & Deaf & Female & $35-44$ & Grad School & Always & Rarely & I do not like nor dislike music \\
\hline 319 & $\mathrm{HH}$ & Female & $65+$ & University & Never & Every day & I really like listening to music \\
\hline 320 & $\mathrm{HH}$ & Male & $55-64$ & Community College & Always & Every day & I really like listening to music \\
\hline 321 & CI & Female & $45-54$ & Community College & Always & Every day & I really like listening to music \\
\hline 322 & $\mathrm{CI}$ & Female & $45-54$ & Community College & Always & Weekly & I really like listening to music \\
\hline 323 & $\mathrm{HH}$ & Female & $55-64$ & University & Always & Every day & I like listening to music \\
\hline 324 & $\mathrm{HH}$ & Female & $65+$ & University & Always & Sometimes & I like listening to music \\
\hline 325 & $\mathrm{HH}$ & Female & $18-24$ & High School & Always & Every day & I really like listening to music \\
\hline 326 & $\mathrm{CI}$ & Female & $45-54$ & University & Always & Sometimes & I really like listening to music \\
\hline 327 & CI & Female & $65+$ & Community College & Always & Sometimes & I like listening to music \\
\hline 328 & $\mathrm{HH}$ & Female & $65+$ & Community College & Always & Never & I do not like listening to music \\
\hline 329 & $\mathrm{HH}$ & Male & $55-64$ & Grad School & Sometimes & Every day & I really like listening to music \\
\hline 330 & $\mathrm{HH}$ & Male & $55-64$ & Community College & Always & Rarely & I do not like nor dislike music \\
\hline 331 & $\mathrm{HH}$ & Female & $18-24$ & Grad School & Always & Sometimes & I like listening to music \\
\hline 332 & Deaf & Male & $55-64$ & Community College & Sometimes & Weekly & I really like listening to music \\
\hline 333 & $\mathrm{HH}$ & Male & $55-64$ & Community College & Never & Weekly & I like listening to music \\
\hline 335 & $\mathrm{HH}$ & Female & $18-24$ & Community College & Always & Sometimes & I like listening to music \\
\hline 336 & $\mathrm{HH}$ & Male & $65+$ & University & Never & Every day & I really like listening to music \\
\hline 337 & $\mathrm{HH}$ & Male & $55-64$ & University & Always & Every day & I really like listening to music \\
\hline
\end{tabular}

Table D.1: Paper survey demographic responses. 


\begin{tabular}{|c|c|c|c|c|c|c|}
\hline \multirow{2}{*}{ Subject Number } & \multicolumn{5}{|c|}{ Listens to music using ... } & \multirow{2}{*}{ Comments } \\
\hline & Radio & MP3 Player & Stereo & Video & Other & \\
\hline \multicolumn{7}{|l|}{301} \\
\hline 302 & & & & & 1 & Wireless headphones \& stereo \\
\hline 303 & 1 & & & & 1 & "laptop, car" \\
\hline 304 & & & & & 1 & Computer and car \\
\hline 305 & 1 & 1 & 1 & & & \\
\hline 306 & & 1 & 1 & & & \\
\hline 307 & & & & & 1 & Computer w/CD's \& ALD device \\
\hline 308 & 1 & & 1 & & & \\
\hline 309 & 1 & 1 & 1 & 1 & 1 & Ipod \\
\hline 310 & 1 & & & & & \\
\hline 311 & & 1 & & & & \\
\hline 312 & & & & 1 & & \\
\hline 313 & & 1 & & & & \\
\hline 314 & 1 & & 1 & & & \\
\hline 315 & 1 & 1 & 1 & & & \\
\hline 316 & 1 & & 1 & & 1 & Ipod computer \\
\hline 317 & 1 & & & & 1 & $\mathrm{TV} \mathrm{w} /$ captioning \\
\hline 318 & 1 & & & & & \\
\hline 319 & 1 & 1 & & & 1 & concert goer, sing in 2 choirs \\
\hline 320 & 1 & & 1 & & & \\
\hline 321 & & & & & 1 & It varies \\
\hline 322 & & & 1 & & & \\
\hline 323 & & & & & 1 & earphones \\
\hline 324 & & & 1 & & & \\
\hline 325 & & 1 & & & & \\
\hline 326 & & & & & 1 & laptop \\
\hline 327 & & & 1 & 1 & & \\
\hline \multicolumn{7}{|l|}{328} \\
\hline 329 & 1 & & & & & \\
\hline 330 & 1 & 1 & & & & \\
\hline 331 & & 1 & 1 & & & \\
\hline 332 & 1 & & & & & \\
\hline 333 & 1 & 1 & & 1 & & \\
\hline 335 & 1 & & & & & \\
\hline 336 & & & 1 & & & \\
\hline 337 & 1 & & & & & \\
\hline
\end{tabular}

Table D.2: What technologies do you use to listen to music? 


\begin{tabular}{|c|c|c|c|c|c|c|c|}
\hline \multirow{2}{*}{$\begin{array}{l}\text { Subject } \\
\text { Number }\end{array}$} & \multicolumn{6}{|c|}{ Want a computer to provide information on ... } & \multirow{2}{*}{ Comments } \\
\hline & Song Name & Artist & Lyrics & Instrument being played & Beat & Other & \\
\hline 301 & 1 & & 1 & & 1 & & \\
\hline 302 & 1 & 1 & 1 & & & & \\
\hline 303 & & & 1 & 1 & 1 & & \\
\hline 304 & & & 1 & & & & $\begin{array}{l}\text { [Lyrics was indicated as "1 } 23 \text { " - first, second, } \\
\text { and third most important?] }\end{array}$ \\
\hline 305 & & & & & & & \\
\hline 306 & 1 & 1 & 1 & & & & \\
\hline 307 & & 1 & 1 & 1 & & & \\
\hline 308 & 1 & 1 & 1 & & & & \\
\hline 309 & & & 1 & 1 & 1 & & \\
\hline 310 & 1 & & 1 & 1 & & 1 & $\begin{array}{l}\text { ["Lyrics" circled and "\#1" put beside it. } \\
\text { "Name of the song" circled and "2" added beside it. } \\
\text { A " } 3 \text { " was put beside "Instrument"] } \\
\text { if the music is produced and played at a very high } \\
\text { quality, then the only other thing I want is the lyrics. }\end{array}$ \\
\hline 311 & & & 1 & & & & \\
\hline 312 & 1 & 1 & 1 & & & & \\
\hline 313 & 1 & 1 & 1 & & & & \\
\hline 314 & & 1 & 1 & 1 & & 1 & I prefer classical (or semiclassical music) \\
\hline 315 & 1 & 1 & 1 & & & & \\
\hline 316 & 1 & 1 & 1 & 1 & & & ["Important, also" written beside 'Instrument'] \\
\hline 317 & 1 & 1 & 1 & & & 1 & captioning \\
\hline 318 & 1 & & 1 & 1 & 1 & & \\
\hline 319 & & & 1 & 1 & 1 & & \\
\hline 320 & 1 & 1 & 1 & & & & \\
\hline 321 & 1 & 1 & 1 & & 1 & & $\begin{array}{l}\text { ["It depends" with arrows pointing to } \\
\text { both 'artist' and 'beat' options] }\end{array}$ \\
\hline 322 & & 1 & 1 & 1 & & & \\
\hline
\end{tabular}




\begin{tabular}{|c|c|c|c|c|c|c|c|}
\hline \multirow{2}{*}{$\begin{array}{l}\text { Subject } \\
\text { Number }\end{array}$} & \multicolumn{6}{|c|}{ Want a computer to provide information on ... } & \multirow{2}{*}{ Comments } \\
\hline & Song Name & Artist & Lyrics & Instrument being played & Beat & Other & \\
\hline 323 & 1 & & 1 & 1 & & & \\
\hline 324 & & & 1 & 1 & 1 & & \\
\hline 325 & 1 & 1 & 1 & & & & \\
\hline 326 & 1 & 1 & 1 & & & & \\
\hline 327 & & & & & & & not very computer literate yet. \\
\hline 328 & 1 & 1 & 1 & & & & \\
\hline 329 & 1 & & & & & & \\
\hline 330 & 1 & & 1 & & 1 & & \\
\hline 331 & 1 & & 1 & & 1 & & ['lyrics' indicated as "\#1 choice"] \\
\hline 332 & 1 & & 1 & & 1 & & \\
\hline 333 & 1 & 1 & & & & & \\
\hline 335 & & & & & & & \\
\hline 336 & & & & & & & \\
\hline 337 & 1 & 1 & 1 & & & & ["words comprehension" written next to 'lyrics'] \\
\hline
\end{tabular}

Table D.3: What are the most important pieces of information you would want a computer to help you know?

\begin{tabular}{|c|c|c|c|c|c|c|c|c|c|c|c|}
\hline \multirow{2}{*}{$\begin{array}{l}\text { Subj } \\
\#\end{array}$} & \multicolumn{10}{|c|}{ Tried using ... } & \multirow{2}{*}{$\begin{array}{l}\text { Satisfied } \\
\text { with } \\
\text { solution? }\end{array}$} \\
\hline & Volume & Body & Balloon & Equalizer & $\begin{array}{c}\text { Stereo } \\
\text { Settings }\end{array}$ & Earphones & $\begin{array}{l}\text { Music } \\
\text { Choice }\end{array}$ & Viz. & ALDs & Other & \\
\hline 301 & & 1 & 1 & 1 & 1 & & & & & & $\begin{array}{l}\text { Somewhat } \\
\text { dissatisfied }\end{array}$ \\
\hline 302 & & & & 1 & & 1 & & & & & $\begin{array}{c}\text { Somewhat } \\
\text { satisfied }\end{array}$ \\
\hline 303 & 1 & 1 & & & 1 & 1 & & 1 & & 1 & $\begin{array}{c}\text { Somewhat } \\
\text { satisfied }\end{array}$ \\
\hline 304 & 1 & 1 & & & 1 & & & & 1 & 1 & Satisfied \\
\hline
\end{tabular}




\begin{tabular}{|c|c|c|c|c|c|c|c|c|c|c|c|}
\hline Subj. \# & Volume & Body & Balloon & Equalizer & Settings & Earphones & Choice & Viz. & ALDs & Other & Satisfied \\
\hline 305 & 1 & & & & 1 & 1 & & & & & $\begin{array}{c}\text { Somewhat } \\
\text { satisfied }\end{array}$ \\
\hline 306 & 1 & & & & & 1 & & 1 & 1 & 1 & $\begin{array}{c}\text { Somewhat } \\
\text { satisfied }\end{array}$ \\
\hline 307 & 1 & & & & & 1 & & & 1 & & $\begin{array}{c}\text { Somewhat } \\
\text { satisfied }\end{array}$ \\
\hline 308 & 1 & & & & & & & & 1 & & Dissatisfied \\
\hline 309 & 1 & 1 & & 1 & 1 & 1 & & & 1 & & $\begin{array}{l}\text { Somewhat } \\
\text { dissatisfied }\end{array}$ \\
\hline 310 & 1 & & & & & & & & 1 & & Dissatisfied \\
\hline 311 & & & & & & 1 & & & & & $\begin{array}{c}\text { Somewhat } \\
\text { satisfied }\end{array}$ \\
\hline 312 & & & & & & & & & 1 & 1 & Satisfied \\
\hline 313 & 1 & & & & 1 & & & & 1 & 1 & $\begin{array}{c}\text { Somewhat } \\
\text { satisfied }\end{array}$ \\
\hline 314 & 1 & & & 1 & 1 & 1 & & & & & $\begin{array}{l}\text { Somewhat } \\
\text { dissatisfied }\end{array}$ \\
\hline 315 & 1 & 1 & & & 1 & & & & 1 & 1 & Dissatisfied \\
\hline 316 & 1 & & & & 1 & 1 & & 1 & 1 & & $\begin{array}{c}\text { Somewhat } \\
\text { satisfied }\end{array}$ \\
\hline 317 & 1 & & & & & & & & 1 & 1 & $\begin{array}{c}\text { Somewhat } \\
\text { satisfied }\end{array}$ \\
\hline 318 & & & & & 1 & & 1 & & 1 & & Dissatisfied \\
\hline 319 & 1 & & & & 1 & & & & 1 & & $\begin{array}{l}\text { Somewhat } \\
\text { dissatisfied }\end{array}$ \\
\hline 320 & 1 & & & & 1 & & & & 1 & 1 & $\begin{array}{c}\text { Somewhat } \\
\text { satisfied }\end{array}$ \\
\hline 321 & 1 & & & & & & 1 & & 1 & & $\begin{array}{l}\text { Somewhat } \\
\text { dissatisfied }\end{array}$ \\
\hline
\end{tabular}




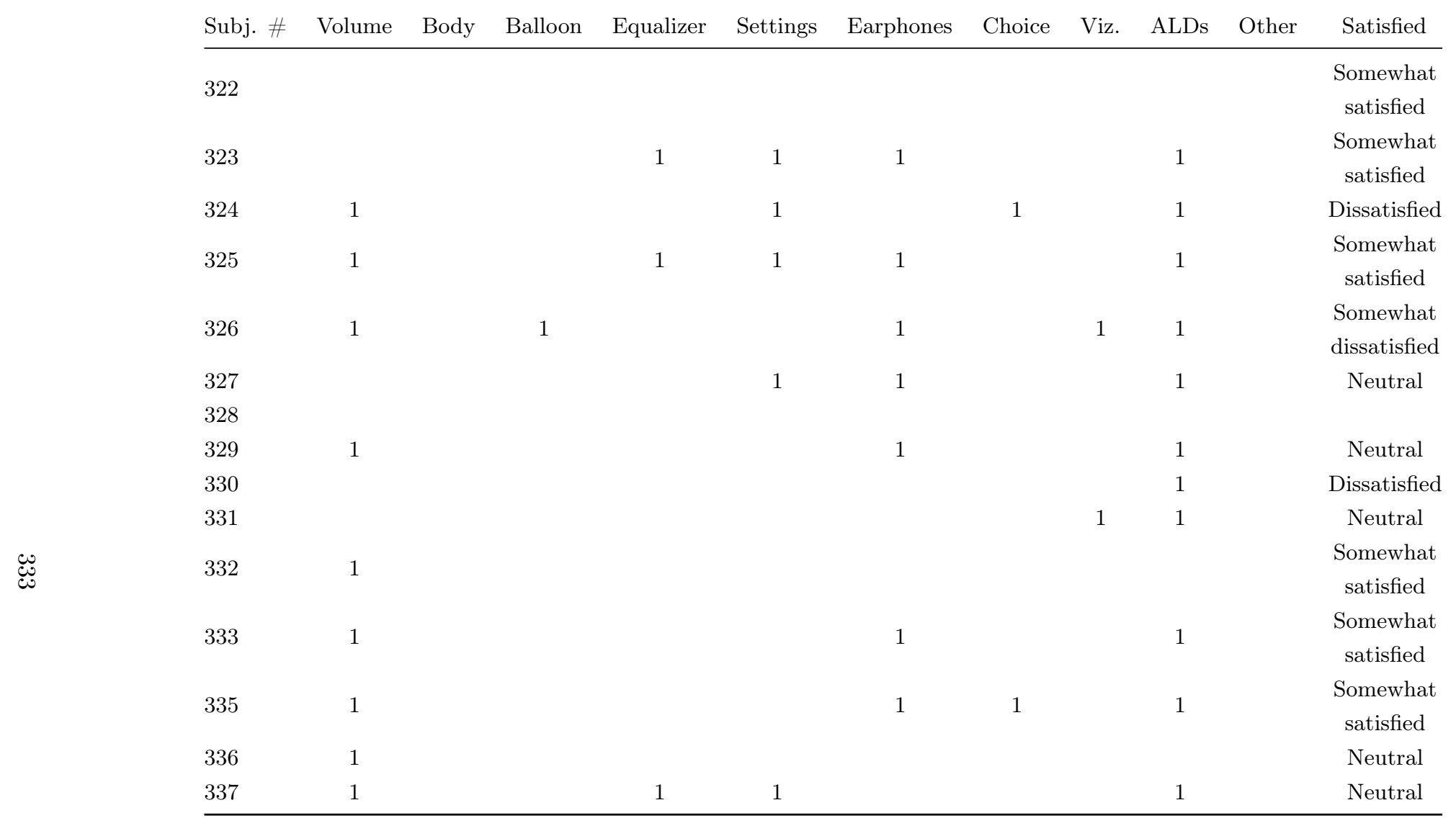

Table D.4: What types of solutions have you tried to access music? 


\begin{tabular}{|c|c|c|c|}
\hline $\begin{array}{l}\text { Subject } \\
\text { Number }\end{array}$ & Solutions Satisfaction & Music Frustrations & Music Positive Experiences \\
\hline 301 & $\begin{array}{l}\text { It just does not sound good. I } \\
\text { cannot make sense of a song. Old } \\
\text { songs are not always recogniz- } \\
\text { able[.] I wondered if I was missing } \\
\text { some clarity of individual instru- } \\
\text { ments but am now second guessing } \\
\text { myself when I heard David Shipp } \\
\text { mention a } 4 \text { note difference. }\end{array}$ & $\begin{array}{l}\text { I listen to old songs I once knew } \\
\text { and wish I had that back again. } \\
\text { Am getting Clear Voice with } \\
\text { Advanced Bionics in the Fall (I } \\
\text { think) and am wondering if this } \\
\text { will help at all. }\end{array}$ & $\begin{array}{l}\text { I can still get the beat so try to be } \\
\text { happy with that. I have been to a } \\
\text { few concerts to make the best of } \\
\text { my situation. I can hear the music } \\
\text { now with my CI - I just cannot } \\
\text { make any sense out of it. }\end{array}$ \\
\hline 302 & $\begin{array}{l}\text { Not very. Difficult to follow } \\
\text { melody, but often can identify } \\
\text { instruments }\end{array}$ & $\begin{array}{l}\text { I just try to find some enjoyment } \\
\text { in what I hear Music I am familiar } \\
\text { with from the past is best for me } \\
\text { to listen to }\end{array}$ & \\
\hline 303 & $\begin{array}{l}\text { I still miss many of the words if } \\
\text { I can't see them or get close to } \\
\text { the music. Sometimes I miss the } \\
\text { full sound and, hence, the full } \\
\text { message. }\end{array}$ & $\begin{array}{l}\text { Visuals help me - words, colour. } \\
\text { Also, strong rhythms and swelling } \\
\text { sounds are easier to hear. }\end{array}$ & $\begin{array}{l}\text { Oh, so many! Music stirs me emo- } \\
\text { tionally, spiritually \& physically. } \\
\text { I find it to be soothing, exciting, } \\
\text { invigorating, fun, satisfying. Very } \\
\text { important to a full life for me. }\end{array}$ \\
\hline 304 & $\begin{array}{l}\text { I don't know any other ways, } \\
\text { don't know what/if I miss. }\end{array}$ & $\begin{array}{l}\text { My hearing aids dampen sound } \\
\text { above (I think) about } 90 \mathrm{~dB}\end{array}$ & $\begin{array}{l}\text { Lyrics are a lifesaver! The only } \\
\text { reason I enjoy music is because I } \\
\text { memorize lyrics. }\end{array}$ \\
\hline 305 & & $\begin{array}{l}\text { NECK LOOP \& CONTGEGA } \\
\text { SYSTEM [,] NOT CATCHING } \\
\text { ALL WORDS }\end{array}$ & \\
\hline 306 & $\begin{array}{l}\text { I like the beat and noise, but I } \\
\text { couldn't hear the lyrics that well. }\end{array}$ & $\begin{array}{l}\text { Hard time understanding the } \\
\text { lyrics sometimes. }\end{array}$ & $\begin{array}{l}\text { I enjoyed the beat and background } \\
\text { noise }\end{array}$ \\
\hline
\end{tabular}

Continued on next page 
miss specific instruments - but using ALD - FM system directly attached to sound/music source is an improvement - realized some sounds \& music I thought I knew, I have missed a lot! (or now with new digital aids \& FM system Phonak) *just got new system Jan '10

308

I would like to be able to hear the lyrics. I can only catch a few words here and there and therefore miss the full message trying to be delivered.

Can't understand lyrics. Not accessible enough would like to know lyrics while I listen - then I can make out words. Cannot concentrate/hear sounds/music from stereo in a room - too much of the bkgrd noise- need to listen by myself headphones - or even in my car. ['car' was circled]

After CI music was difficult to listen to - sounded very harsh [np] Now, 6 yrs. Later, music is pleasant but familiar songs sound different. I often recognize song lyrics rather than the melody which is somewhat monotonous Inability to hear all the words (lyrics) or identify instruments being used. Not sure what would help - FM system.

[arrow indicating same answer as in previous response

Cannot understand lyrics [right arrow] a way to access the words
I love music, like to sing - used to sing in a choir as a child - voice changed from soprano to alto but I gave up singing [.] Did not lose my hearing until about 25 yrs old.

I can now enjoy listening to music, especially familiar songs.

Opera! The Flying Dutchman in new Toronto Opera/Ballet house - their telecoil interface with my CI was perfect \& captioning was provided for all.

Music is positive - I play piano \& it's a wonderful way to get a sense of achievement 
Hearing music via my hearing aids is the most enjoyable. Listening through my FM system or silhouette isn't the same, but I use them to respect other people's wish for peace and quiet.

As long as I know the words usually ok. If not then do not enjoy. often the sound of the music is not as I feel it should be - using earphones- \& adjusting balance

Nothing's changed. I can't make out the melody.

316

Many songs I remember the music but not the words. Captioning helps me enjoy it. For new music, I need the words to enjoy it or figure out if I like it at all.
It'd be great if there was an app for iPhones and it would automatically upload lyrics that are being played in the concert or on the radio.

[arrow indicating same as previous response]

Trying different hearing aid settings.

- many videos/cds of musicians from my generation 50's 60's 70's have CD's/video uncaptioned.
Enjoyment. Great memories. Positive Flashbacks. Keeps me awake while driving. Helps me to relax. Euphoria. Share stories about music. Adds to my enjoyment of tv shows \& movies.

On my computer, I have heard, using cd's/videos of choirs that are wonderful, Mormon Tabernacle choir- and Jacqui Lawson on musical cards that can be emailed [np] I am not good with electronic equipment, but get help from my grown (early 20's) grandchildren. I've been involved in music my whole life, playing the piano, singing in a choir, singing solos for weddings / funerals, and now I can't do any of it

T.V. music shows captioned but only show new songs. Rarely do they show standards. 
Subject

Number

318

don't know software I can use to

follow music - too busy as mom to explore these solutions

319

AM 1ST SOPRANO - CAN GET

VOLUME INCREASE WITH

AIDS BUT CLARITY DEPARTS.

DESCANT(S) ENTRIES PROB-

LEMATIC [np] STATIC AFTER

HI C UNBEARABLE, CONFUS-

ING, I NOT SURE CORRECT

TONE - RUINS JOY

320
If I had more time to analyze it, I -need visual cues to follow

words/lyrics of songs

\section{see above $[\mathrm{q}]$ " 12 "}

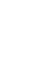

321

\begin{abstract}
can probably make it better.
\end{abstract}

\section{I r} I really want to be able to learn
and follow the words to the music
and/song.
- like loud bass/drums - easier to

follow music

I AM 73 - always a choirster, opera buff and attend concerts, book study \& author readings - all now difficult - have to go early and sit front row where and when possible. TALKY PLAYS FRUSTRATING!

\section{MUSIC VIDEOS THAT ARE} CAPTIONED ARE A GREAT JOY.

I can recognized [sic] certain songs on radio only [-] "You drive me crazy" by Fine Young Cannibals[,] "For the longest time" by Billy Joel[,] "Everything I do, I do for you" by Bryan Adams

Continued on next page 


\begin{tabular}{|c|c|c|c|}
\hline $\begin{array}{l}\text { Subject } \\
\text { Number }\end{array}$ & Solutions Satisfaction & Music Frustrations & Music Positive Experiences \\
\hline 322 & $\begin{array}{l}\text { AM USUALLY LISTENING TO } \\
\text { MUSIC MY PARTNER HAS ON. } \\
\text { LOTS OF JAZZ AND I ENJOY } \\
\text { WHAT I AM HEARING THO } \\
\text { I KNOW I DON'T "GET" IT } \\
\text { ALL. FOR ME, IF I HAVE TIME } \\
\text { TO LISTEN SPECIFICALLY I } \\
\text { PUT A CD IN THE COMPUTER } \\
\text { WHILE I WORK - MOSTLY } \\
\text { OLDIES 50'S-80'S ROCK THAT I } \\
\text { KNEW WELL BEFORE I LOST } \\
\text { MY HEARING[;] MY MEMORY } \\
\text { OF WHAT THEY "SHOULD" } \\
\text { SOUND LIKE HELPS ME TO } \\
\text { HEAR THEM. }\end{array}$ & $\begin{array}{l}\text { HAVING LYRICS IS A HELP } \\
\text { TO DECIPHER WORDS BEING } \\
\text { SUNG. "BRAND NEW" MUSIC } \\
\text { IS FRUSTRATING AS I CAN- } \\
\text { NOT DIFFERENTIATE TONES } \\
\text { TO "GET" THE MELODY[.] } \\
\text { THE PLUS HERE IS BEING } \\
\text { SPARED THINGS LIKE BRI- } \\
\text { TANEY SPEAR![sic] }\end{array}$ & $\begin{array}{l}\text { LOVE MUSIC - THE C.I. HAS } \\
\text { MADE IT MORE ACCESSIBLE } \\
\text { FOR ME I TRY TO ENJOY } \\
\text { WHATEVER I AM ABLE TO } \\
\text { DECIPHER! }\end{array}$ \\
\hline $\begin{array}{l}323 \\
324\end{array}$ & $\begin{array}{l}\text { Even with my hearing aids, mu- } \\
\text { sic is distorted and I do not hear } \\
\text { the bass notes well. It sounds as } \\
\text { tho there are two different[sic] } \\
\text { sep tunes playing very often. The } \\
\text { lyrics are not clear and I have al- } \\
\text { ways really "tuned into" the lyrics } \\
\text { of music. }\end{array}$ & $\begin{array}{l}\text { hearing words during a concert } \\
\text { As my hearing loss is the common } \\
\text { type - that the lower tones have } \\
\text { gone and the very high "notes" } \\
\text { remain- hearing aids amplify but } \\
\text { distort and do not completely } \\
\text { capture the lower tones. }\end{array}$ & $\begin{array}{l}\text { I hear music well when is is live, in } \\
\text { small venues with good acoustics } \\
\text { - it is such a treat but rarely ac- } \\
\text { cessible where we live. The choir } \\
\text { on opening night of this concert } \\
\text { was so enjoyable. Occasionally } \\
\text { when one of the kitchen cupboards } \\
\text { closes - it sounds like a drum beat } \\
\text { in a Caribbean band - I love it! I } \\
\text { also drum with a small native[?] } \\
\text { drum - sometimes in a small } \\
\text { group. }\end{array}$ \\
\hline
\end{tabular}

Continued on next page 
For the most part, I can hear the

beat and rhythm of the music.

The problem for me is understanding and hearing the lyrics of the song. For this I look up the lyrics online and remember them for future listening. By adjusting the volume, bass and equalizer, I can listen better, but the main problem with that is I usually have to adjust it for every song, since one may be softer and the next very loud.

CI, it's either the

beat / music or words, but never the clarification of both being blended together. I heard better when used to wear a hearing aid.
Like mentioned above, hearing and understanding lyrics and having

to constantly change the equalizer. Although there is nothing more that can be changed about the lyrics, it would be nice if there was a system that automatically adjusted the settings to fit the song.

I missed going to concerts where as no closed or real-time captioning is being used. Not all concerts are set up for those with FM systems.
Music, both instrumental / classical and lyrical is wonderful.as long as I can hear, I will always appreciate music.

No positive experience with the exception of the Rita McNeil concert last night. First time to actually "follow" Lyrics.

I really don't enjoy listening to music unless I am by myself without any background interference

Continued on next page 


\begin{tabular}{|c|c|c|c|}
\hline $\begin{array}{l}\text { Subject } \\
\text { Number }\end{array}$ & Solutions Satisfaction & Music Frustrations & Music Positive Experiences \\
\hline 328 & & $\begin{array}{l}\text { 1) I never hear / understand the } \\
\text { words of the lyrics like last night } \\
\text { at Rita McNeil's concert I hear } \\
\text { the music (loudly) but seldom the } \\
\text { words } 2 \text { ) if I listen to music I can- } \\
\text { not focus on my surroundings[-] } \\
\text { don't hear telephone ring, front } \\
\text { door knock, Husband calling or } \\
\text { (in the past) my babies crying [np] } \\
\text { I prefer to be connected to the } \\
\text { world rather than to music Music } \\
\text { is mostly background noise }\end{array}$ & dancing to music would be great \\
\hline 329 & $\begin{array}{l}\text { I'm using what I have at my } \\
\text { disposition[-] would love to have } \\
\text { better equipment but then if I buy } \\
\text { something will I hear better? I'm } \\
\text { not convinced. }\end{array}$ & $\begin{array}{l}\text { For some strange reason I seem to } \\
\text { be more frustrated to access music } \\
\text { at home rather than my vehicle. } \\
\text { At home even if it is loud I can } \\
\text { hear but can't understand. In the } \\
\text { car it will depend at what station } \\
\text { I'm listening too. Soft music is } \\
\text { normally what I will seek. }\end{array}$ & $\begin{array}{l}\text { If the music is instrumental I will } \\
\text { be able to hear. If their is words } \\
\text { such as OPERA (no way) I'm able } \\
\text { however to detect beat as we have } \\
\text { now started taking ballroom danc- } \\
\text { ing and I have no problem hearing } \\
\text { the beat as long as the acoustic is } \\
\text { good. I used to sing in a choir had } \\
\text { to stop! this was one of my major } \\
\text { disappointed. I lost my hearing } \\
\text { overnight } 6 \text { yrs ago }\end{array}$ \\
\hline 330 & $\begin{array}{l}\text { LOSING MORE INTEREST. } \\
\text { FAMILIAR MUSIC DOES NOT } \\
\text { NOW SOUND THE SAME }\end{array}$ & $\begin{array}{l}\text { SURPRISED AT LYRICS. WHEN } \\
\text { I HEARD MUSIC I LIKED THE } \\
\text { SOUND BETTER }\end{array}$ & NIL \\
\hline
\end{tabular}

Continued on next page 
Subject

Music has a distorted sound and with a CI. It is hard to discriminate vocals/lyrics. For me, I enjoy 'understanding' what music means to the artist and why it was composed.

\section{I do not or can not pick up the}

lyrics or what the singer is singing.

333

335

336
I usually listen to books on tapes

I feel [comment incomplete]

all my life I have been HOH. I have always listened to music but never for the most part understood words made out through music. I just listen to beats etc
- Too garbled! I like classical music because its 'softer' (and country too!) I don't like Rock music because it sounds like drums

beating that make no sense.

As above

attending live concerts, performances

That the volume might be [remainder of comment illegible] not understanding words or knowing the name of song or who is singing even though like the songs
I used to be a good singer but I am unable to carry a tune (whether it is age or my deafness I don't know)

I have been able to find music where lyrics are available - this REALLY helps!

I really enjoy my CD's and the concerts I go to.

listen to music does give relaxation even though I don't understand the words

Table D.5: Written responses. 



\section{Appendix E}

\section{One-on-One Interviews}

This Appendix presents the forms used and data collected in the interviews described in Chapter 8 . Note: All participants also completed the questionnaire presented in the previous Appendix. 


\title{
Are you looking for better, more enjoyable ways to listen to music?
}

\section{$\underline{\text { Research Participants Wanted }}$}

\begin{abstract}
I am a hard of hearing student at Ryerson University pursuing a Ph.D. degree. I am studying ways to use technology to express music visually to support music listening by Deaf, Deafened, and Hard of Hearing people. I am exploring new ways to access music and I am seeking help and input from the community.
\end{abstract}

My aim is to gather people's opinions and thoughts on this issue to assist in the completion of my studies. I would like to talk with people at the Conference about their experiences with listening to music in hopes of learning what they feel is missing and what works. Lastly, I am interested in people's ideas on how to use technology and music visualization to improve their music experience.

\section{Your feedback is invaluable!}

If you would like to participate, I will be available at the $\square$ Conference for one-on-one interviews. During the interview, I will ask you about your music experience and I will show you at least three short video examples of existing ways to visualize music that use changing colors and shapes to describe music. After each example I will ask for your feedback. Each interview will take approximately 30 minutes of your time to complete. If you are able to participate in an interview, your participation is entirely voluntary and you may stop the interview at any time.

If you are over 18, are Deaf, Deafened, Hard of Hearing, or use a Cl and are interested in participating, contact me any time during the conference.

David Fourney
Phone/Text:
Email: dfourney@ryerson.ca
Or if you see me, flag me down.)

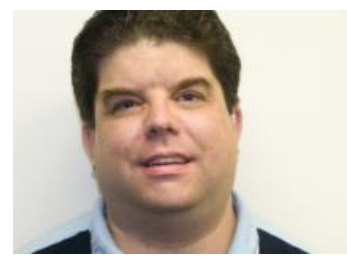

OR

If you are interested in providing your input but are unable to participate in an interview, please complete the attached questionnaire and return to:

\section{Registration Desk (located in the foyer)}




\author{
Project Title: $\quad$ Music Visualization \\ Principal Investigators: Deborah Fels, P.Eng., Ph.D., Ryerson University \\ dfels@ryerson.ca \\ David Fourney, MSc. Ryerson University \\ dfourney@ryerson.ca
}

\title{
Consent to Participate in Study from Subject \\ Information Form
}

The purpose of this study is to evaluate different ways of presenting music visually. To do this, we will ask you to watch at least three (3) different examples of visualizations and provide us with your opinion about them. Each example runs about one minute. You are also asked to complete two additional surveys, one prior to the study and one at the conclusion of the study to gather your opinion about music and music visualization.

\section{Confidentiality}

All raw data will be kept strictly confidential. A summary of the data will be published in academic venues but no individual details will be identified in this summary. The information gathered from surveys will be strictly used for research and academic purposes with only the principal investigator and his supervisor having access to it

\section{Risks and Discomforts}

The risks associated with participating in this study are minimal. You may experience some fatigue with watching the visualizations. However, you are able to take breaks at any time or even stop participation in the study without penalty. You may also experience some discomfort with being video or audio recorded. In this case, you may choose not to participate in the study or record your opinions in writing and remain off camera.

\section{Expected Benefits}

Individual participants will not receive any direct benefits; however, this study will benefit the general community of deaf or hard of hearing users. This study will test different styles of music visualization that will assist in developing new practises for music visualization. We hope that this information may lead to improvements in music visualization technologies and techniques.

\section{Voluntary Nature of Participation:}

Participation in this study is entirely voluntary. If you do not wish to participate in this study it will not affect current or future relations with Ryerson University or The Centre for Learning Technologies. If you choose to participate, you have the ability to leave the study at any time and for any reason without penalty. In addition, you may refuse to answer any questions or participate in any task at any point of the study without penalty.

\section{Questions about the Study:}

If you have any questions or concerns, about this study please feel free to contact Deborah Fels at dfels@ryerson.ca. If you have any concerns or complaints about this study in regards to its ethical nature please contact the Research Ethics Board, c/o Office of the Vice President, Research and Innovation, Ryerson University, 350 Victoria St., Toronto, ON M5B 2K3, Tel: 416-979-5042 


\section{Project Title: $\quad$ Music Visualization}

Principal Investigators: Deborah Fels, P.Eng., Ph.D., Ryerson University

dfels@ryerson.ca

David Fourney, MSc. Ryerson University

dfourney@ryerson.ca

\section{Consent Form to Participate in Study}

I acknowledge that the research procedures described above have been explained to me and that any questions that I have asked have been answered to my satisfaction. I have been informed that there may be a possible risk of psychological discomfort from being filmed or watching so many videos; however, strategies are in place to reduce this risk.

I have been informed of the alternatives to participation in this study, including my right not to participate and the right to withdraw without penalty. I hereby consent to participate in the study and to be video or audio recorded during the study. I have received a copy of the information sheet.

I have received a copy of the information form.

I hereby consent to participate in the study.

Signature of Participant:

I hereby consent to be video-taped during the study.

Signature of Participant:

Name of Participant (please print):

Date:

The details of this study were explained to me by:

Name of Investigator:

Date: 
Music Visualization Pre-study Questionnaire

The purpose of this questionnaire is to gather information about you and your experience with music and closed captioning. There are eight questions and it should take you about five minutes to complete this questionnaire. Thank you in advance for your time and assistance.

1. Do you identify yourself as: (please check one)

$$
\begin{array}{ll}
\square & \text { Hearing } \\
\square & \text { Hard of hearing } \\
\square & \text { Deaf } \\
\square & \text { Deafened } \\
\square & \text { Cochlear implant }
\end{array}
$$

\section{Please indicate your gender}

$$
\begin{array}{ll}
\text { a } & \text { Male } \\
\text { a } & \text { Female }
\end{array}
$$

3. Please indicate your age
ㅁ $19-24$
口 $25-34$
ㄷ $35-44$
$45-54$
D 55- 64
$65+$

\section{What is your highest level of education?}

$\begin{array}{ll}\square & \text { No formal education } \\ \square & \text { Elementary school } \\ \square & \text { High School } \\ \square & \text { Technical college } \\ \square & \text { University } \\ \square & \text { Masters / Ph.D. }\end{array}$

5. How often do you use closed captioning when watching television?
always
- Sometimes
a Never

6. How often do you listen to music?
A All the time (daily).
a Frequently (weekly)
- Sometimes
R Rarely
a Never

7. Which of the following statements best describes you?
口 I really enjoy listening to music.
I I like listening to music.
a I do not like or dislike music.
I I do not like listening to music
- I do not see the point of music.

8. What is your experience of music in elementary or secondary school?

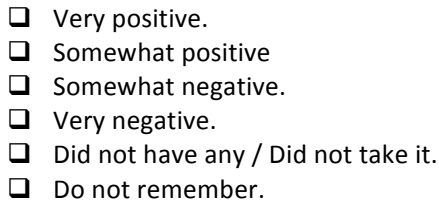


Music Questions

1. Was the music visualization enjoyable (circle one)?

$$
\text { Not enjoyable at all Not that enjoyable Neutral Somewhat enjoyable Enjoyable }
$$

2. What did the visualization mean to you?

3. Was the music (mark one for each):

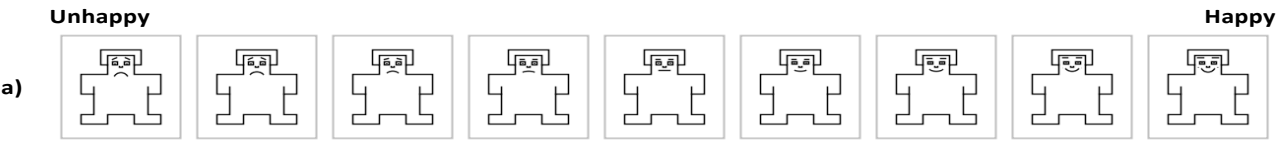

b)

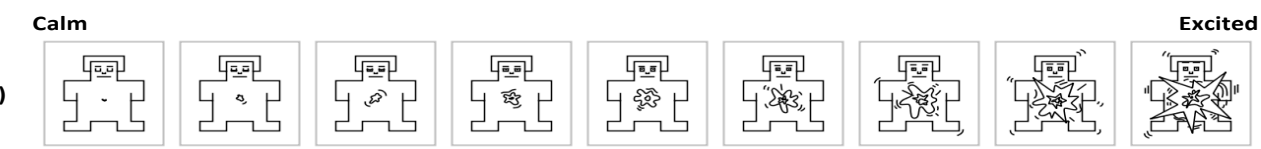

c)

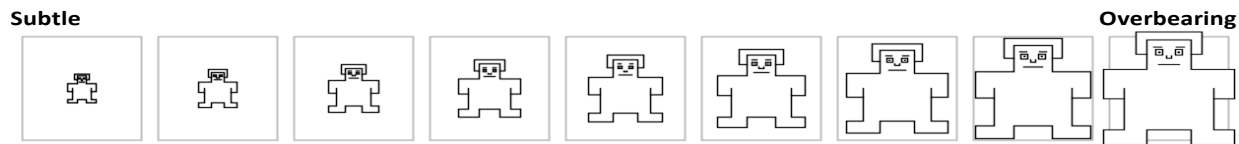

4. Identify which emotion you thought was expressed and rate its strength from 1 to 7 (circle one):

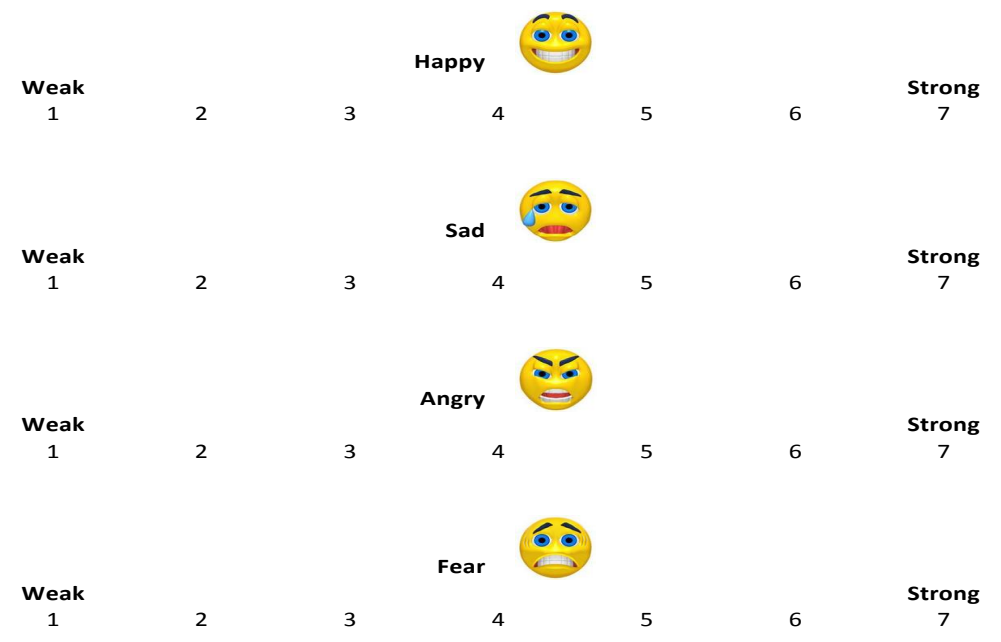


Music Visualization Post-Study Questionnaire

The purpose of this questionnaire is to gather information about your opinions on the visual representations that you just saw. There are three questions and it should take you about five minutes to complete this questionnaire. Thank you for your time and assistance.

1. Which style gave you the best sense of:

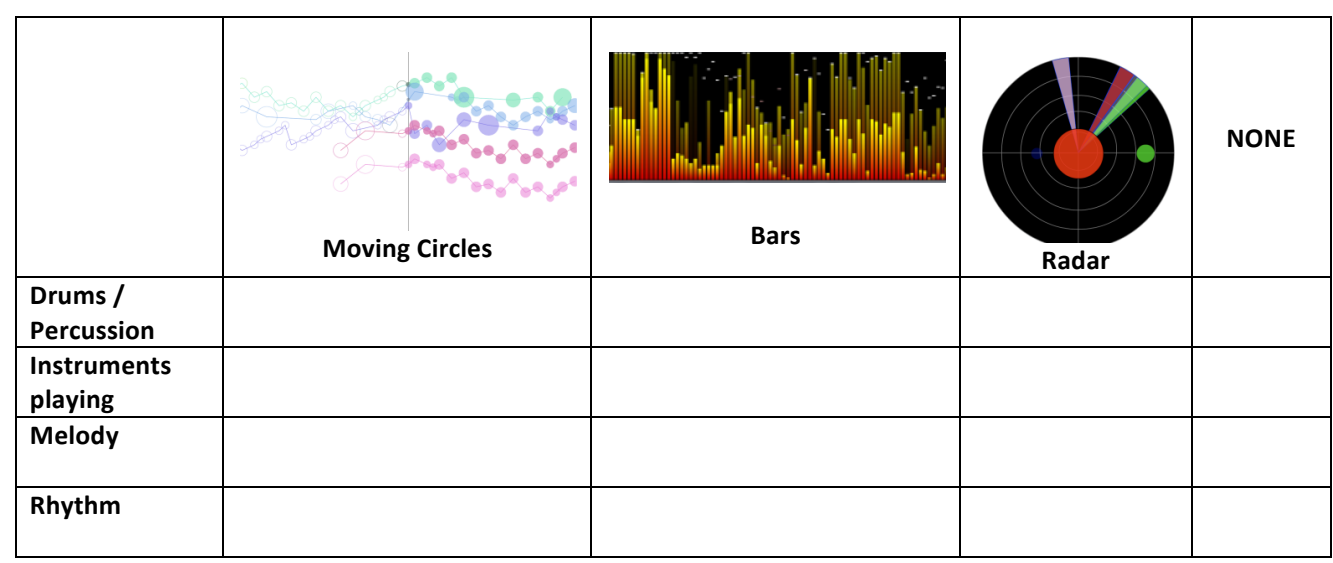

2. Which style did you enjoy most? Why?

3. Which style gave you the best sense of what was happening in the music? Why? 


\section{Music Visualization Interview Questions}

Gender: $M \quad F$

Identifies self as: Hearing, Hard of hearing, Deaf, Deafened, Cochlear implantee, Other, None

Age: $\quad 18-24,25-34,35-44,45-54,55-64,65+$

1. Please tell me a little about yourself. Were you born deaf/hard of hearing or did you experience a hearing loss later in your life?

2. If you experienced a hearing loss, how old were you?

a. What memories do you have of music prior to your hearing loss?

3. Is there a difference in your experience of music today compared to the past? Do the same recordings still sound the same? What, if anything is missing today?

4. Do you think your music preferences are shaped by what you can or cannot hear? How does your hearing influence your choices of music you want to listen to?

5. When you listen to music, what strategies or solutions do you use to help you enjoy, understand, etc. the experience?

a. How well does strategy $X$ work for you?

b. Why do you use it?

c. Does it work all of the time, some of the time?

6. (optional) Do you wear hearing aids while listening to music? How well does this work for you? 
7. (optional) When you want to listen to music, what do you do (e.g., alone in a quiet room, concerts, etc.)?

8. How do you listen to music (e.g., Radio, Music player, Home stereo, Music videos, etc.)?

a. How well does strategy $X$ work for you?

b. Why do you use it?

c. Does it work all of the time, some of the time?

9. What specific frustrations have you experienced when trying to listen to music? a. What do you think would improve your experience with music?

10. If you wanted to use a computer to help you to better understand music, what would be the important pieces of information you would really want to know? (e.g., Song title, Artist name, Lyrics, etc.)

Now I am going to show you some short videos of music visualizations that have been developed at Ryerson and elsewhere. I will not play the sound. As you watch it please keep in mind how you would interpret this. 
A

1. Does this video say anything to you?

a. What worked for you?

b. What did not work for you?

2. What did you think of the colours? Do the colours have any meaning for you?

a. If yes, what did they mean to you?

b. If not, did you want the colours to have any meaning for you?

3. What did you think of the speed of the visualization?

a. Was it too fast/slow?

b. What do you think should happen to the visualization if the song is fast? 
B

1. Does this video say anything to you?

a. What worked for you?

b. What did not work for you?

2. What did you think of the colours? Do the colours have any meaning for you?

a. If yes, what did they mean to you?

b. If not, did you want the colours to have any meaning for you?

3. What did you think of the speed of the visualization?

a. Was it too fast/slow?

b. What do you think should happen to the visualization if the song is fast? 
C

1. Does this video say anything to you?

a. What worked for you?

b. What did not work for you?

2. What did you think of the colours? Do the colours have any meaning for you?

a. If yes, what did they mean to you?

b. If not, did you want the colours to have any meaning for you?

3. What did you think of the speed of the visualization?

a. Was it too fast/slow?

b. What do you think should happen to the visualization if the song is fast?

That was the last video; I will ask you to fill out a final questionnaire.

Thank you again for your time and assistance with my research. 


\section{E.1 Interview Coding}

\begin{tabular}{|c|c|c|}
\hline Name & Sources & References \\
\hline Age hearing was lost & 8 & 15 \\
\hline Adulthood & 4 & 9 \\
\hline Birth or shortly after & 3 & 5 \\
\hline Childhood & 1 & 1 \\
\hline Bars & 8 & 8 \\
\hline Bars reaction & 8 & 42 \\
\hline Busy & 3 & 4 \\
\hline Colours & 6 & 10 \\
\hline Did not work & 2 & 2 \\
\hline Related visualization to something & 6 & 9 \\
\hline Sees music & 6 & 15 \\
\hline Speed & 7 & 11 \\
\hline Desired solution & 7 & 11 \\
\hline Favourite genre & 7 & 21 \\
\hline Hard of hearing education & 3 & 6 \\
\hline Hearing history & 8 & 22 \\
\hline Hearing influences music choice & 6 & 13 \\
\hline No & 1 & 2 \\
\hline Yes & 5 & 11 \\
\hline Hearing loss still changing & 2 & 3 \\
\hline It's mostly the same. If not maybe a little harder with some of the words. & 1 & 1 \\
\hline Live music & 5 & 12 \\
\hline Memory of hearing & 2 & 2 \\
\hline Memory of music & 6 & 9 \\
\hline Missing in music & 8 & 22 \\
\hline Beat & 1 & 1 \\
\hline Entire song & 2 & 3 \\
\hline Instruments & 1 & 2 \\
\hline Lyrics & 6 & 12 \\
\hline Melody & 1 & 1 \\
\hline Other & 1 & 1 \\
\hline Pitch & 2 & 2 \\
\hline Rhythm & 1 & 1 \\
\hline Mourning hearing loss & 3 & 4 \\
\hline Moving circles & 8 & 8 \\
\hline Moving circles reaction & 8 & 71 \\
\hline Colour & 8 & 18 \\
\hline Did not work & 7 & 12 \\
\hline
\end{tabular}




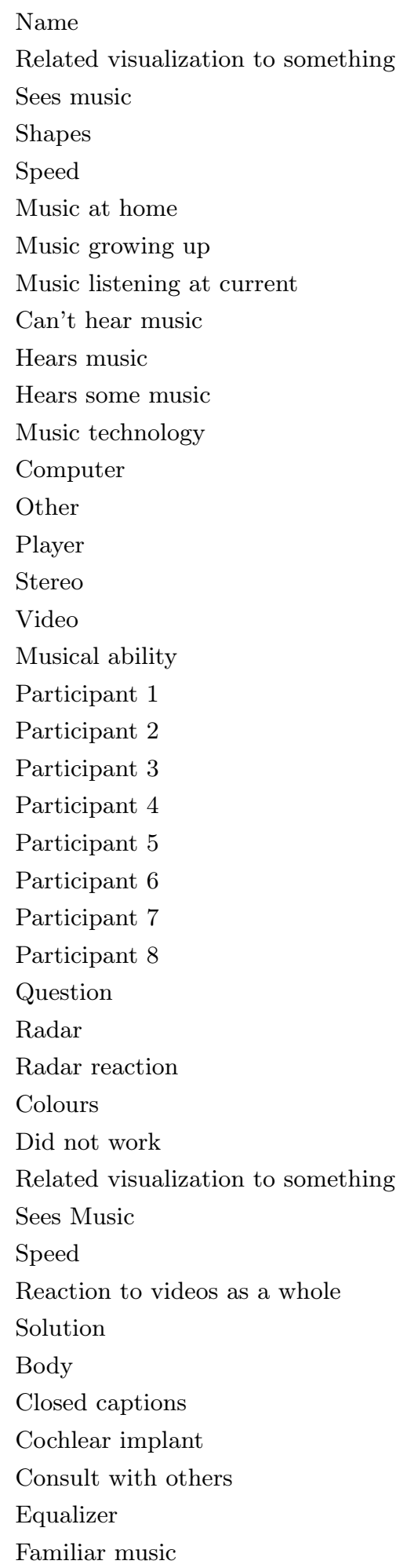

\begin{tabular}{|c|c|}
\hline Sources & References \\
\hline 3 & 4 \\
\hline 7 & 19 \\
\hline 4 & 7 \\
\hline 8 & 11 \\
\hline 5 & 18 \\
\hline 4 & 5 \\
\hline 8 & 27 \\
\hline 5 & 18 \\
\hline 4 & 7 \\
\hline 2 & 2 \\
\hline 7 & 37 \\
\hline 4 & 9 \\
\hline 3 & 6 \\
\hline 4 & 5 \\
\hline 4 & 10 \\
\hline 5 & 9 \\
\hline 5 & 14 \\
\hline 2 & 2 \\
\hline 2 & 2 \\
\hline 2 & 104 \\
\hline 2 & 2 \\
\hline 2 & 2 \\
\hline 2 & 2 \\
\hline 2 & 2 \\
\hline 2 & 190 \\
\hline 8 & 389 \\
\hline 8 & 10 \\
\hline 8 & 57 \\
\hline 7 & 14 \\
\hline 4 & 4 \\
\hline 5 & 5 \\
\hline 6 & 17 \\
\hline 7 & 8 \\
\hline 1 & 1 \\
\hline 8 & 58 \\
\hline 2 & 6 \\
\hline 4 & 9 \\
\hline 2 & 8 \\
\hline 3 & 3 \\
\hline 2 & 3 \\
\hline 5 & 7 \\
\hline
\end{tabular}

Continued on next page 
Name

Guessing

Headphones

Hearing aids

Listen more than once

Listen with lyrics

Listening environment

Live Music

Other

Visualization

Volume

$\begin{array}{ll}\text { Sources } & \text { References } \\ 1 & 1 \\ 4 & 10 \\ 8 & 24 \\ 3 & 4 \\ 4 & 7 \\ 2 & 3 \\ 2 & 4 \\ 2 & 2 \\ 2 & 5 \\ 4 & 6\end{array}$

Table E.1: Codes used in data analysis. 


\section{E.2 Interview Transcripts}

\section{E.2.1 Participant 1}

Do you consider yourself hard of hearing, deaf, deafened?

Hard of hearing.

Hard of hearing?

Yes.

I'd like to know a little bit more about who you are before I go forward very much. Were you born hard of hearing?

No, I mean, we were unsure what hard of hearing is. I think I was kind of normal hearing until the age of 7 and then my hearing started to go down.

$\mathrm{Oh}, \mathrm{OK}$.

Do you have much memory of when you were hearing?

Not very much.

Not very much.

No.

Do you remember listening to music at all when you were a small child, perhaps hearing music in your day care or kindergarten classes or maybe at home?

I heard my parents singling a lot...a lot...just stuff on the radio. I can't remember too much because now I am more use to the sounds I hear now.

Right

And your hearing, did it just suddenly change overnight or was it more of a gradual decline?

I think it was more a constant, like, constant drop.

Right.

Not immediately, just more slowly.

Overtime.

Yup.

And, did you notice that change?

Not...really.

Not particularly. 
And, do you think your hearing is more steady now or is it still changing?

It's still changing.

Downward or all over?

Down.

Yeah?

Yeah, I can tell it's getting worse...gradually overtime.

Oh, OK.

Yes.

So, the reason I am asking this is because I want to get a sense of whether you noticed changes to the music recordings that you listened to over your teenage years. So when you were, seven, for example, you don't remember very much is what you are saying, right?

Not too much. I do remember it was much easier to listen though.

Right.

And when you got older, when you were in grade 7,8 , do you remember listening to like radio and playing music and stuff like that?

I remember listening to music, but I had to, I had to listen to it a constant amount of times to actually understand what the words were saying.

Wow, ok.

And has that changed for you now, when you listen to those same songs is it harder or...?

It's mostly the same. If not maybe a little harder with some of the words. All I have to do is end up with the lyrics with the songs to actually understand what they are saying.

So when you listen to music then what do you find is missing, just the lyrics or what else do you think is missing?

Mostly the lyrics are just blurred up. Other times it's maybe the beats with the rhythm or you know not catching the full amount. I get the, the higher pitch sounds, not so much lower pitch ones

Oh ok. OK, well that's that is interesting 
Do you think that the way you hear influences the stuff you choose to listen to?

Quite possibly actually.

Do you think it's more peer pressure or do you think it's more your personal preferences?

I am sorry?

Well, do you listen to what your friends tend to listen to or do you find that you have your own preferences more because of what you yourself can hear and what you your self can enjoy?

I think it's a bit of both because I don't explore music much so I ask my friends to...I listen to some of their music and sometimes I find stuff I enjoy listening [to], too, so.

OK, interesting.

So you were talking a little bit earlier about um about lyrics and trying to understand lyrics

Yup.

What do you do to help you understand lyrics? Do you listen more closely? Do you look them up on the Internet? What, how do you do this?

I try to listen more closely the first few times and try to work out what I think they are saying, and at some point I just look them up, and see oh ok well I got this right but this phrase is completely wrong because I am doing something different, so

Yeah, I think that is a familiar experience.

What other strategies do you find that you use when you are listening to music?

Changing the volume past sometimes for certain songs. The equalizer. That's pretty much all I use when I am listening to music.

When you shift the base, when you shift the equalizer, how well does that work for you? Does it really improve it or is it touch and go?

It improves it to a certain degree. It makes it easier to pick up more stuff.

Why do you use it? Do you just use it to pick up more stuff or do you use it to shift more into your range? Why do you play with the equalizer?

Maybe so I can hear and understand music better.

Do you wear hearing aids? 
Yes.

Do you wear hearing aids when you listen to music?

Well, if I am listening to my iPod then I have to take them out. But I use in-the-ear headphones-so those are buds that go inside your ear-because it's supposed to sound closer to your ears. But when I am just listening to the computer without my headphones, then yes, I need to keep my hearing aids in because otherwise I will catch even less.

Oh OK.

So do you find that the hearing aids help you when you're listening to music or...?

Yes

Do you believe the hearing aids distort your experience of music or improves it or...?

It helps me but probably...I think it distorts the music a little bit. I am not sure how but it sounds different.

It sounds different when you're wearing hearing aids then when you're not wearing hearing aids?

Yes, when I am listening to my headphones, and when I am listening to my hearing aids, there's a bit of difference. I am not sure what it is though. But there is a difference in the sound.

Yeah. Sure. OK.

When you listen to music-now I am sure Mom is a bit of an influence in this-what do you do when you listen to music? Do you try to get out of the room? Get away from other people? What do you do when you want to listen to music? Let me put it that way.

If I want to listen to music I just...

I know it's a weird question. And I realize that because you live at home with your parents it influences the listening of music in the kitchen or living room opposed to the bedroom or whatever else.

Participant's Mother: I am not particularly bossy about music. I encourage music anywhere and everywhere. I think music should always be heard at all times.

Right.

Yes, but you don't like some of my music. So I am not encouraged about that. 
And that is what I am trying to get at.

Participant's Mother: You'd be surprised about the music I don't like though (laughing).

You don't like that Japanese song (addressing Mother)

Participant's Mother: I am not crazy about the Japanese. She likes the Japanese style music.

I like Japanese. I like French music.

Very eclectic. You're not the Mariah Carey, Britney Spears, Boy band type.

I don't have a favorite kind of music. I listen to anything and everything.

Do you go to concerts at all?

Not often.

Not often.

What kinds of concerts do you go to? You live in XXXXXX so do you go to something like the Air Canada Centre, the ACC, or do you go to something smaller?

Well, if you go to concerts, then that's the place to go. The most concerts I have ever been to is for classical music but that is because I have taken music class.

Tell me a little bit about that. You take music class within school, or outside of school?

In the school.

When you take music at school? I am assuming you're talking about high school.

Yup.

Is that Grade 9 music? Grade 12 music? Or is that Band? What are you doing?

I have taken it for the past three years and it's a band. There is the grade 10 band and then when you get to grade 11 and 12, it's a combination of grade 11 and 12 . It's just a band on its own

What instrument did you play?

I play the Alto saxophone

Alto sax.

Yes. It's quite fun. 
Yes, my little brother played bass clarinet.

What strategies did you use to work in the band? Because obviously as Jennifer Rowland commented on earlier this morning, she has to get feedback from her colleagues in the band, compared to when she is playing alone. So what did you do to I guess to help you with that?

I talk to my music teacher and to my band mates about if I miss something with what we're playing. I just get their help with any issues I have.

In high school did you use an interpreter, FM? How did you support yourself?

For my classes I used an FM and I used note taking. For music, I did not use anything because especially in class it's less people, so it's easier to just listen with hearing aids.

When you do listen to music at home, you were talking about using your computer; you were talking about using your iPod. Do you use a home stereo or anything like that or just a computer and an iPod?

Just my computer and iPod mainly.

And what about music videos? Do you watch MTV or Much or anything like that?

Sometimes.

Does that help you or not?

It does. I can put closed captioning on TV, whenever the music video is playing. Some of them have closed captioning, some of them don't, but the ones that do, it really helps me to actually see words. It helps me enjoy the song.

When you are watching it with captioning, do you prefer the synchronized pop up on captioning that goes with the song? Or the roll up style captions? What's your preference? When you watch captioned music, what do you like to see?

Coordination with the song. If they just write the last lyric that is already gone, it kind of messes you up with the beat.

So you were talking about some of the stuff that you have of music. That your frustration can be around lyrics. What do you think would help improve your experience of music? You have experienced a change in your hearing over your teenage years, right? So you know what you were hearing in grade 9 and you know what you are hearing now. You sort of have a memory of that, right?

Yup. 
So what do you think would help you have a better experience with music?

That is a hard thing to explain because what helps me better appreciate music and enjoy music more is by adjusting everything and obviously I can't ask the bands or companies to just to do that just for me because all hard of hearing people are on different levels and so it's more that you have to figure it out for your personal level.

If you wanted to have a computer to help you better understand music you're listening to, give you a better experience, what would be the really important piece of information that you would want the computer to give you?

Like what? The song title or...?

If the computer was doing something automatic, OK? Just really you just wanted to play a particular piece and it were to help you better enjoy that piece, what kind of things would you want the computer to be doing?

I know, it's an awkward question to ask.

Obviously the title, the artist. That's already a given for computers especially.

Right.

I don't know lyrics, I suppose.

So what I am going to do is I am going to show you now some short videos. The videos are based on work that is going on at Ryerson and as well as other places. And what I am going to ask you to do as you're watching each of these videos is I want you to sort of to keep in mind how you would interpret what it is you're watching. And I recognize some of these videos might be more confusing or whatever. But just sort of try and get a sense of what you think of it.

OK.

So as I said earlier I am not going to play sound.

OK.

(RADAR STARTS)

(RADAR ENDS)

So what did that video say to you, if anything?

It was a little confusing to understand because there was no volume to help me with understanding why, for example, the circles in the middle were... I forgot the word, the lines that's were going around and stuff 
What did those circles then mean to you?

After a while it kind of reminded me of like a rock band kind of thing. It was the drums or the percussion system of it. That's the only idea it gave me because it kept a constant beat.

And those lines and shades that were moving up and down?

I am still trying to figure out what that was supposed to be. At one point when it went all the way around, I figured it must be something to do with the vocal system.

$O K$.

It was interesting to look at it.

Was there anything in there that just did not work you? You were like, you know...

It was just at the beginning when you just see the lines and just like...

Now what.

What's happening.

OK.

It was a little weird.

What did you think of the colours?

They were general. Nothing too flashy. That is just my preference. It wasn't too bright. They mostly kept constant. It was fine.

Did the colours mean anything to you?

I didn't give them any kind of thought

Did you want the colours to be meaningful to you? Did you want the colours to mean something?

If the colours could maybe represent something while the music was playing then it might help me understand so like I could coordinate the red with louder sound or something that just tells me...I just colour coordinate the sounds together.

What did you think of the speed of this video? Was it too fast, too slow? What did you think of the speed?

It seemed moderate. I don't know what to say. I wouldn't say it was slow or fast. 
When a song is fast, do you think the visualization should be or do you think something else should happen?

If it coordinates with a song even a fast song, I think it should keep up with that. I don't know. I don't know how to explain it.

Nope, I fully understand that.

So what I am going to show you now is another visualization. Oddly enough it's the same song.

(MOVING CIRCLES START)

(MOVING CIRCLES ENDS)

So what do you think? What do you think of that one?

I thought that was more understandable than the other one.

More understandable than the other one?

Yeah

It was almost like reading composition. You know for me I play classical music so you have the sheet music. It reminded me of that just without actually seeing the notes or words or anything but you could understand the beat...you could tell where things were. That was pretty cool.

As were watching this video what actually worked for you in getting the understanding of music?

The visuals in the lines definately helped, especially when it hit the circles it put emphasis on it. So even if you can't hear the song know that something...

Has changed?

Yes

What didn't work for you? What do you find confusing about this?

At first it was just a few lines and all these more lines start coming in. So it's like I think I know what this is, I think I know what this is, but what is this supposed to be? So have to try and figured it out

$A h, O K$

But other than that I thought it was really good. I could follow right through it I think. 
Did you like this visualization more than the first one?

This one made a little more sense to me than the first one did

Did it look more like music or less like music than the first one?

I guess since I play classical music it actually made more sense to me

What did you think of the colours?

Good actually. It didn't have the black back...

Background?

Yeah, black backdrop and each one was a different colour so there was a plain constant colour and they weren't too bad actually.

Did colour actually mean anything to you?

Yeah it did. Again it's like categorization. You can tell difference. You can tell that there are different sounds happening throughout the whole song.

As you were watching it, did you want different colours to mean something to you?

Again probably, but you know, the way it works it doesn't need to be colourized. You can just follow through without colour.

Did you find this visualization too fast? too slow?

No, it was actually pretty good. It was really good.

Now what I am going to do is show you one more video.

Alright.

And again I am not going to play the sound and again it's going to be the same song.

(BARS STARTS)

(BARS ENDS)

So what did you think?

I should mention-because it will probably come up again - but it was very fast, like extremely fast. But I suppose if the song is fast that would make sense. But it was just like up and down so fast you just had to try and keep up with it.

Yeah, I was watching your eyes follow it. Your eyes were jumping. 
Did you find as you been watching each of these videos, did you find any one of these videos more busy than the others?

More...?

Like more visually busy, like there was just too much there.

I think the last one had too much there. You had the whole screen. You had all of them to watch at the same time. And they were going really fast. So, trying to keep up with it.

Did it mean anything to you? Did it say anything to you? Was it meaningful at all?

The music was very fast and very loud. That's what it told me.

What did you think of the colours?

Good. Every time it kept going up, it reminded me, especially with all them being at different levels, it reminded me of the wall of fire kept going up. So it was kind of cool.

Did you think that the colours meant anything or did you want them to mean something?

I don't think they meant anything really. And they don't have to because with that kind of layout it's pretty basic. If you go on ITunes they have the same thing

It's a basic equalizer view.

What we're going to do is I am going to ask you to fill out a quick questionnaire. 


\section{E.2.2 Participant 2}

Do you identify yourself as hard of hearing, deaf, deafened?

I like deafened.

About how old are you?

I will be 47.

Can you please tell me a bit about yourself? Were you born hearing? Were you born deaf?

I was born premature. My mother was entering her $7^{\text {th }}$ month of pregnancy when I was born. They did not quite discover until I was five years old that I was born with profound deafness. I remember being fitted with a hearing aid at six years of agegrade 1 . The hearing aid didn't really do much for me. They figured I had what they called sensory neurological loss.

Yes, sensory neural loss.

Yes, sensory neural loss, profound deafness-that is what they classified it as. I wore a hearing aide up until 33 years of age. I became deafened for no known reason. I seen Dr. Julian Nedzelski, Sunny Brook hospital, to be a candidate for the cochlear in 1997 in December. He said to me from their discovery that my hair cell die and he don't know why and that the cochlear hair cell dying. So I became a candidate for the surgery. I had the surgery the week before Christmas and I was first hooked up at the end of January 1998.

So you have had a CI since 1998 ?

Yes

So from what you are telling me, at different stages of your life as you listen to music things sort of changed, right?

Yes.

So can you tell me a little bit about the differences between what you experience today when you listen to music compared to what you would experience in the past?

With the hearing aid in the past I was able to pick up music very well. I can hear the clarity of the words and the music combined very well. But when I was fitted with the CI I couldn't do that anymore. And I mourn the loss of music. In the past I was able to play the clarinet and was able to hear it well and also differentiate the music with my fingertips - the vibration. But with the CI I couldn't. And I was very, very frustrated that I couldn't tell where I was. Even with the vibration I couldn't hear that tone, that level of what that sound is. 
So today you can't hear music at all?

I can hear some music. For example, if it is music from the past, like for example, I am a Streisand fan. If I can hear the simple music of hers like "The Way We Were" or "Evergreen", I will pick it up because my mind is memorizing those words. And especially thank God for YouTube because YouTube allows me to visualize and see her sing. Without the visualization, I would not pick it up as much, even with the memory.

Do you think what you listen to in terms of your music preferences, what you go out and buy or whatever, is shaped by your hearing loss? Is shaped by your hearing?

That is a good question because if I go out and buy a video or music right now, I would not because I can't recognize it. I have a hard time recognizing. I might hear a little bit of a violin, or a bass playing or a guitar, but put the words and the music together, no. So to me its obsolete of purchasing music and I am wasting my time and my money.

So when you do listen to the older stuff that you know your mind will help you fill in, what strategies do you use to help you enjoy the music?

I have a laptop. So what I do is I go to YouTube and plug in my earphones. I put the big earphones over top of the mic of my CI and I listen really intently to it to see what is it I can pick up? What is it that my mind memorized and remembered to pick up? And then I follow through. Now most music have video captioning that I will try and match it. Like for an example last night with Rita McNeil I could capture maybe out of 7 words in a sentence, I picked up 3 or 4 maximum. But when her music over powers her voice, I can't. Even with the closed captioning, I had a hard time following it. Because when there is closed captioning it's not like following her right on cue.

Yeah, it was live caption so there was delay.

It was a little frustrating because I love her music and want to hear her and yet I could not.

Do you wear your CI when you listen to music?

Yes.

So obviously then that is better than without, right?

The $\mathrm{CI}$ is much better than the hearing aid, if I were to make comparison. If you were to ask me this 6 years ago, I would of [sic] said that the hearing aid is better, but as time went on, getting used to this, I can say the $\mathrm{CI}$ is better than the hearing aid. Because it is picking up what it is being trained to pick up. Even though it is 
trained for voice-voice recognition. I think the program she incorporated included music, so I am able to pick up music a bit more, but not as much as I'd like.

Do you play around with your stereo settings or anything like that?

No, I don't because I still can't tell where the bass is, where to put those tone areas.

When you want to listen to music, do you try and put yourself into a quiet room or something like that? Do you manipulate your space?

I try to put myself in a quiet room, especially if the room is textured like this. This is wall texture. And the music is not echoing, not bouncing off the wall, I will be able to pick it up slightly better.

You were telling me earlier that you mostly use YouTube, so you're not usually listening to an iPod or something like that. You have visual information while you are listening.

I go to YouTube all the time. It's the only area I know for music so far. Even when catching up with news, like CBC news on their website but they don't have close caption, so that is why I go to YouTube and I get a better visual on YouTube than I do with CBC.

If a computer could automatically do some things that would help you better understand music, what would be the really important pieces of information that you would want the computer to help you to know?

If it's closed captioning, so I can follow. Closed captioning is really important because it allows so I can follow word for word. And also at the same time, I am training myself to listen for the words and I can quickly glance at it to see if I am not missing anything. So it's helping me. It's training me. It's doing both. So its help me train and listening.

So now what I am going to do is I am going show three quick videos these are music visualizations that have been designed at Ryerson and elsewhere. They're not necessarily designed to help deaf people. I am not going to play the sound, it's just going to be straight video.

Straight video, $\mathrm{OK}$

While watching the videos, what I would like you to do is keep in mind how you would interpret what it is your looking at it.

OK, how am I going to interpret it. OK. By speech reading...?

How would you interpret it as... 
Interpret by how I am hearing it.

What it means to you.

What message is it sending to me?

What is it saying to you? Those kinds of things.

What kind of body language, facial expression...

Right.

(MOVING CIRCLES START)

(MOVING CIRCLES ENDS)

When it is going along, I notice there are a lot of circles. I notice that there is a rhythm. I am seeing rhythm in there. Now, the bright light that goes "ba-ding", "bading"-touching each ball-is the different level tones, I am assuming. So it`s following it. Because I study music in Grade 9, it's indicating to me that I am hearing different instruments in there, rather reading different the instruments in there. So in my mind I am thinking what kind of level tone am I going to be hearing if there were sound on. That is what I am interpreting from this.

What in this did not work for you?

As it runs by its that bar that goes "ba-ding", "ba-ding", "ba-ding", "ba-ding", "bading". It`s just like, what is that?

Oh this white bar?

Yes and you got that green light. It`s like a distraction. What is this trying to tell me? Ok that is interesting.

What did you think of the colours?

The first thing I noticed of the colours is it may represent the different tones of the music. It`s very attractive and it`s not harsh. It`s gentle and easy to follow.

What did you think of the speed? Was it too fast? Too slow?

I find that the speed is generally normal. It is what a hearing person would listen to.

Right.

Now what I am going to do now is play a different video, but the same song.

Same song? 


\section{Same song}

I wonder if I would recognize it.

(RADAR STARTS)

I swear there is a drum playing in there.

Pardon?

I swear there is a drum playing in there. When there is a feather like that might be the drum. The heartbeat. That is your bass.

I am looking at a clown.

This one is very attractive and easy to follow.

What do you think these are? What do you think these moving...?

I am thinking this is a background noise or something or gentle music. There is something in that background. That's what I am thinking it is. This is your bass, which is the foundation of the music. This is your background. The feather like thing is like a thimble. You know like... Like someone playing the piano. Going like this with the piano. That could be anything. The red could be your drum, it could be your guitar or anything else. And I find that very attractive.

\section{(RADAR ENDS)}

Did you find anything in that confusing? It just didn't work for you?

What I am finding very annoying is that purple dot. I think you should just have the feather like thing and maybe one or the two dots and you decide. That is acceptable. But if you try to add a bit more too it, it becomes very annoying. It's confusing and annoying.

\section{Did colours mean anything to you?}

Colours? The red does in this case. The number one. The heartbeat, as I mentioned earlier - the heartbeat. The colours again could be either the tone or it could be the words being sung...maybe it`s different words.

What about the speed? Was it too fast? Too slow?

It was easy for me to follow. I didn't find either. It was very comfortable.

I am going to show you one more video.

(BARS STARTS) 
(BARS ENDS)

To tell you the truth I really don't like that one. I really don't. I find it very, very annoying and upsetting. I find too many actions going on at once. Now, if I were a producer and recorder, I would probably understand it further. It would be similar to what they have at their studio. But no, it turned me off right off the bat.

Did you think the colours had any meaning?

No, it has no meaning to me whatsoever. It's like monotone. And because the colours are blending in...the yellow and the red blend in, it all becomes one. It has no meaning to me.

Did you find it too fast, too slow or just too much?

It's just a mixture. It's like a jumble.

So that's all the videos

OK.

What I am going to do now is just get you to fill out this final questionnaire.

OK. 


\section{E.2.3 Participant 3}

So what I am going to do is I am going to ask a few questions about you and who you are. And then I am going to show you a few quick videos and ask you a few questions about each of them. And then we will have a bit of a wrap up activity-a short questionnaire-and that will be it.

Do you identify yourself as hard of hearing, deafened, deaf? How do you label yourself, if you do?

Hard of hearing.

If I may, how old are you?

55.

So, were you born hard of hearing?

Industrial from working in industry. Working the mines and in surface plants-so mine is work related.

And about how old were you when you lost your hearing?

I started working in the industry at about 19 and it's been about over the last 20 years or so...approximately. I think I was losing it, but I didn't really realize it. Then it got to the point through testing that...

Then it became more obvious?

Then it became more obvious.

So you have been hard of hearing from about 30 or 35 ?

Probably between 35 and 40 years old.

So what kind of memories do you have of music, prior to when you lost your hearing?

Like things that I could hear, that I noticed?

Yeah.

Birds. I spend a lot of time outside in the bush so a lot of the nature things like birds, frogs. Catching words on TV, catching punch lines on movies and films and having to ask friends to repeat themselves quite often when we're together

With respect to music, when you were younger compared to now, do you really notice a difference in what you hear and what you remember?

Big time, yeah. 
So tell me a little about that, what is it that you have difficulty hearing today when you listen to music, compared when you were 19 or 25 ?

Distinguishing the words. Lots of times I have to be face to face if people talk fast or if the words bunch in too quickly together. It takes longer to distinguish or figure out what's being said and just trying to decipher things it's slower.

When you were younger, you had a record collection of some sort?

Yeah, the $45 \mathrm{~s}$ and stuff. And I could understand, I could listen to those quite well. I never had a problem with those.

Today when you listen to that same music-maybe you listen to it on a CD or something now-but today, when you listen to those same songs, do you hear them the same way or do you hear them differently?

I don't hear them the same. Lots of them I know what the words will be because you have listened to them for so long, so lots of the stuff is by memory. But some of the music on some of the CDs or records I would have had would be...-the more familiar songs I go better with. But the ones I didn't listen to quite as much you have to really pay attention to catch it all.

So when you hear the stuff you're really familiar with, do you think you're still hearing the same tune or are there big gaps?

I am not sure. I think I am anticipating. Like there are times you sort of anticipate what an answer is going to be, so it's locked in to your mind that's what the answer is going to be, so that's sort of what you hear. Sometimes I think that in conversations or when things are going on, I am actually guessing or as the word came up over the weekend, bluffing my way through sometimes. Sometimes you get lucky and sometimes you answer out in left field.

What strategies do you use today when you want to listen to music? What kind of things do you do to help you when you want to listen to music?

If I am alone I will turn the volume up.

Get it louder.

Yeah, if I am by myself. I just tried using [brand name] machine with a neck loop and the headphones and that made it a lot better.

Yeah, yeah.

If I am in a quiet room and I can get away from any background noises and stuff, that makes a big difference. If I can be sitting facing the speaker or facing wherever the sound is coming from, that makes a big difference. 
Do you try and do these as much as possible?

Yeah, pretty much. I try to set myself up where it's going to be to my benefit the most.

You were hearing aids, correct?

All the time, yeah.

Do you find the hearing aids help you?

Yes, very much. If I take them out, even in the kitchen, if I take them out, I don't hear the fridge running, I don't hear the furnace running, I don't hear a lot of those things.

So when you listen to music, you wear hearing aids as well?

Yeah, music, watching TV, just about everything.

When you want to listen to music, what do you usually do? Do you use a stereo, or a radio, or some sort of iPod? How do you do it? What do you do to listen to music?

Usually it's the CD player, the little portable one. If I am sitting in the living room, I will have the TV set on one of the Galaxy stations or something.

Do you find one or the other to be better for you?

It's all in positioning.

It's all the same.

If I were ever to build another house, I would probably have it with speakers in every room because if I go from one room to the next I will lose it, unless if I have the headphone set on, then I can wander around.

You were saying earlier that lyrics seem to be a problem for you now; you are not hearing the words very much. What other stuff do you find really frustrating when you're trying to listen to music? What else is really frustrating for you?

Sometimes the instruments block out the words.

The music is over bearing.

Sometimes I can hear the instruments more than the words, depending on the tones that are coming in.

What do you think would help you? What do you think would improve your experience with music? 
If I had one of those FM headphone sets or the neck loops seem to have helped when I used it for a week. Probably it's just the only thing that could really help is make myself in the right position and not have a bunch obstacles in front of me or anything blocking the speaker where the voices are coming from.

Is this your first CHHA conference?

Yeah

OK, so you're really beginning to experience some new ideas this weekend, eh?

Yeah, well actually what happened was when I took the Lip Reading and Speech 10 week course, I really got a lot of ideas through that, just through the Sudbury chapter here and with the instructor we had, he explained to us in the group that I was in a lot of the opportunities and a lot of the stuff that is out there. And when I went and seen them at the Sudbury Hard of Hearing office, they told me about the CHHA conference.

Conference.

Yeah and this has really been great. I have met a lot of good people and got a lot of good ideas and stuff. It's been very beneficial.

The more you explore what is out there, the more you experiment and try stuff out. You never know.

If you wanted to use a computer to help you better understand music, what would be the really important pieces of information, the really important stuff in the music, that you would want the computer to just automatically to do?

First of all, I don't touch the computer. I am not in the computer era yet, but if I was going to have a computer and stuff, I am not really sure because I am not too much into the technical end of things and stuff, one that you could almost see the words as its playing might help but then you'd have to sit down to do it. It's not something you could use as you're driving down the road or nothing.

No, exactly.

I think the biggest thing I have used besides the hearing aids was the stereo type heading phones that you can use with the TV or that you could plug into your machine.

With the big jack?

Yeah. 
So now what I am going to do is show you some short music videos. Sorry, some short videos of music visualizations. So what these things are, is they are software, computer software, that presents music information in a particular way. They are not all designed to support deaf or hard of hearing people and some of them have been developed at Ryerson and some have not. I don't want you to think that anything is necessarily my work. I will not play the sound. I will only play the video. And as you watch it, what I would like you do is keep in the back of your mind how you would interpret this thing. What it means to you. What it says to you.

\section{(MOVING CIRCLE STARTS)}

\section{(MOVING CIRCLE ENDS)}

So did this video say anything to you?

To me it just looks like something that is getting deleted as it goes across.

Everything is disappearing. I couldn't really hear anything on it.

No, there's no sound.

It just looked like something being deleted off of a...erasing something off a board or something.

So it didn't look like music to you?

Not really, no.

What did you think of the colours?

Sort of a pale blue. The colours don't really do much for me. Black.

Was this video too fast, too slow?

Going across it was a good speed. It wasn't really fast or very slow. It just seen the ball sort of thing going up and down and seemed to delete things as...

So what I am going to do now is show you a second video.

\section{(BARS STARTS)}

\section{(BARS ENDS)}

So did this video say anything to you?

It just brings back memories of the old days when they came out with speakers that had lights that flashed on and off, like back in the 70s I guess-when I was young and I had hair. 
(David laughs)

As far as if there was music or anything to it, the flashing up and down I find more of a distraction to me, then actually...I would be watching or concentrating more on the light movement more than the music.

Did you find the colours to have any meaning for you?

No, all I could visualize is a stereo system with a set of speakers with blue, purple and red lights that flashed on and off and stuff

What did you think of the speed? Was it too fast, too slow?

No, speed was a...

Wasn't a factor.

(RADAR STARTS)

(RADAR ENDS)

So did that video say anything to you?

No, if it's supposed to do anything to sort of make music or sound better or anything, it would be more of a distraction than help anything

Did you find any of it to give you any meaning?

No, just lights.

Just a lot of lights

Lights going on and off in circles and stuff. But as far as interpreting .... The only thing that flashed in my mind from that with the round circle is if they would of had something like that for Test Pattern when they had the Indian Chief on the old CBC it would give it some movement or something to it.

Right.

So what I am going to do is give you this last quick questionnaire and I am going to step off and get the next person. I will be right back.

I don't know if I was much help, but I found that none of that stuff actually made me think of any music or any beats or anything. It was just squiggly cut lines going up and down. I found if I was at a concert of something, I'd much rather look at a blank screen and listen to the music then try and listen to music with a bunch of lights going on and off. 


\section{E.2.4 Participant 4}

Can you please tell me how do you identify yourself-hard of hearing, deaf, deafened, none of those, all of those?

I am hard of hearing.

You call yourself hard of hearing?

Yeah.

Can you please advise about how old you are?

I am in my sixties, my late sixties.

Late sixties, $O K$

Were you born hard of hearing or did you experience a hearing loss?

No, I suffered acute hearing loss about a year ago.

Oh, $O K$.

I was driving down the street at home...everything went silent. It's called acute hearing loss.

Yeah, just immediate.

Given that hearing loss has been so recent you have a lot of memories of music that you've listened to for quite a while now.

Oh yeah, my whole life.

What is the difference today from what you were experiencing up until last year?

I can't get any melody in anything. Music to me now is just noise. I can't differentiate what song is being sung. At the Rita MacNeil concert last night, for example-I was very good fan of hers-I couldn't tell what song. Everyone would clap when they would hear the song being started. Well I couldn't get the melody. When I look at the words of course I know the words of the song, so I knew what she is singing. So music is just gone, it's lost. It's really weird because my two hearing aids-which I purchased about two months after I had the hearing loss-I am doing very well, functioning very well with conversation, but music is just gone. I was in choirs my whole life. In fact, I played the piano. I used to play the organ in Church. I can't even do that know because if I play the piano now or the organ what I am playing is right, it's perfectly the right keys, but to me it sounds like what am I playing? It's nothing like what it should be sounding. It's really weird.

The stuff that is familiar to you...the stuff that you know you should be hearing...you 're still able to hear some music, but you're not able to hear enough to make it meaningful? 
To make it meaningful or to enjoy it.

Right.

I just can't enjoy it. I used to love going to concerts. Particularly coming from

Newfoundland, we are into Irish music a lot.

Yes, I am sure.

I go to see a lot of Irish tenors.

A lot of Celtic stuff.

Now all my nice Irish tenors now when they come visit, there is not much point in me going because I am hearing them, but I am not getting the nice quality of their voice and I am not even getting enough to tell me what song they are singing.

Are you able to hear the lyrics at all?

No, not really. Like I say, the only way I'll know it is if you said to me oh that's-pick an Irish song-that's Galway Bay they are singing. Well, because I know Galway Bay, then I can get what he's singing, but it's my own memory. I think I am drawing on that tells me what he is singing.

I have you tried experimenting with coming up with strategies that might help you understand or might help you gain a better experience?

Well I have gone to every audiologist I know and I made a special appointment with Marshall Chasin, who was one of our speakers. I think you were in the same session.

Yup.

Well, I met with him the night before he spoke and he gave me a couple of techniques to try. He said when you go to Rita MacNeil tomorrow night put scotch tape-he mentioned it in his presentation-put scotch tape on your hearing aide.

And did that help?

It didn't help a damn bit. He said take out this hearing aide (points to right ear) because he said I have a high frequency loss in this ear and I don't know what I got in this ear (points to left ear) - probably a low frequency loss, but just take this one out (right ear) and it might help. I did that. I put the scotch tape on. I still couldn't tell what Rita MacNeil was singing.

And if you have taken them both off?

Well then, I wouldn't hear anything. 
$O K$.

Noise to me now is just...I mean music to me now is just noise. There is nothing pleasant. You know?

There's nothing pleasant.

When you're at home and you want to listen to the stereo or play your piano or your organ...

Yeah.

You're basically not even doing it anymore right now?

I am not even doing it because I sit down to play a song that I am quite familiar with because I play by ear as well as by note, well I'd say to my husband, "What am I playing? What am I playing?" He'd say, "You're playing that perfectly, perfectly right" Well, I'd say "It's not coming out like the song I know." You know?

My grandmother lost hearing fairly late in life as well. She also, same thing, this is a women who grew up in a Presbyterian Church all her life

Sang all the time.

Mostly played the organ, played the piano, that kind of stuff.

So one of the things she ended up doing was she used really high-powered headphones on her organ.

Under the organ?

She would plug it into the organ

Plug it in, yeah.

So instead of the organ giving out sound, all the sound would go into the headphones.

Oh, OK

That seemed to work for her, but she was not like you. She was more hard of hearing and then she lost that over time too. By the time she passed away, she was very severely hard of hearing.

Really?

But that was a strategy that seemed to work for her. So obviously one of things you're doing right now is looking for strategies that are going to help you.

I am, yeah. 
If you wanted a computer to sort of do this automatic kind of thing, to kind of help you with music and with the music experience, what would you want the computer to do?

Well, I guess if it could in some way allow me to hear notes again-to actually hear the music. Now I know when I went back north to do some of this...I think some of this is from visualization.

Yeah, that's right. I'll show you some visualizations.

I don't know. I can't imagine that being the same thing as sitting and hearing the song word. You know?

Right, yeah.

Well it's not going to be the same thing, but may be it might give you some thoughts on ways to support what you don't hear now.

OK, so what I am going to do is, as I said earlier, I am going to show you some short videos of music visualizations. Now, these visualization have been developed in various places-some at Ryerson and some at various other places. Some of the videos have not been developed specifically with deaf people in mind, so I just want to warn you about that. I am not going to play the sound; you won't hear anything because I am not running anything for sound. So if you're wondering why you're not hearing anything, I am not playing the sound. As you watch the videos, what I am going to ask you to do is I am going to ask you to sort of keep in the back of your mind how you would interpret it? What it might mean to you? What it says to you? Those kinds of things. OK?

OK.

So this is the first video.

(BARS STARTS)

\section{(BARS ENDS)}

Did that video say anything to you?

It reminded me of fountains spraying up in Vegas when I was there. You would go to some casinos and all the fountains would be spraying up. I could imagine that was being done to the beat of some fast jazz music and as the piece went on it intensified; the music intensified. I could almost visualize everybody getting into it, you know?

What in that did not work for you? What just went uh? You know?

I liked it. Nothing really jumped out at me to dislike it.

What did you think of the colours? 
They were OK. I liked the pink, red...yeah. I liked the bright colours.

Did the colours mean anything to you?

No.

Did you want the colours to mean anything to you?

I was just trying to get a sense what the colours all seem to be dancing together and I was just trying to get a sense of the beat of it all. The colours themselves didn't mean anything. I probably would of gotten the same feeling if it had been yellow colours. I don't know.

What about the speed of video? Did you find the video too fast? Too slow?

It got a little fast towards the end

OK.

Was it fast for you or fast in general?

No, it was fast. It seemed to have sped up. The dancing images seemed to have sped up.

$O K$

So, I am going to show you the next video now.

(RADAR STARTS)

(RADAR ENDS)

What did that video say to you?

I didn't get as much out of that one. I found that one more difficult to interpret. It just seemed to be a series of fans spreading out. And once again, as it got towards the end of it, they spread out farther. They are encompassing more space. But, that one wasn't speaking to me as well as the first one.

Those fans, did that mean anything to you?

No.

Did their movement say anything to you?

No.

What about the big circles? Did that say anything to you?

No, I just wondered what they had to do with it all when they appeared.

$O K$. 
What did you think about the colours?

I would of perhaps liked more bright colours. You see the odd black and white. I would of preferred more vibrant colours, although there was the odd blue in there and the odd colour in there. I didn't like the colours as well.

Did you feel the colours had any meaning to you at all?

No, they perhaps wouldn't make me as happy as the first one. I did not feel as happy watching that.

Did you want the colours to have meaning for you in some way?

No, not really. I just thought that they should. The purpose of this is they should have meaning, but I wasn't getting any...

Anything out of it?

Anything out of it.

What about the speed? Was it too fast for you? Too slow for you?

The last part once again was a little too fast. When it started spreading out it picked up speed.

So this is the last one.

(MOVING CIRCLES STARTS)

(MOVING CIRCLES ENDS)

So what did you think of that video?

That reminded me of the very, very olden days, the black and white pictures days, when there was no colour at all and then towards the end some colour came in and then I could see maybe some old fashioned music.

When you were watching that video did it say anything to you that would be music?

I kind of thought about Fred Astaire and Ginger Rogers swaying on to something like that. Now I know he was a tap dancer but you know some this ballroom stuff. I could somehow visualize some ballroom into that.

$O K$.

Ballroom dance I mean, you know?

Yeah, yeah, yeah. 
As you were watching this video was there anything about the video that really confused you?

Confused me?

Yeah.

I wondered what the large, towards the end once again, what the large surface came into, what they had to with it all. They didn't seem to belong with the flow that was going on. They seemed to be in congress with what was going on before.

$O K$.

What did you think of the colours?

Once again, not enough colour for me.

Not enough for you?

Yeah.

Not that the colour wasn't bright enough. The colour wasn't vibrant enough-the colours themselves.

Right.

I tend to like bright colours, you know reds and greens and...

$O K$.

Did you find this visualization too fast or did you find it too slow?

No, I didn't find...I would say the first part of it was perhaps a nice even pace. The last part would of probably been a little too fast.

OK.

What I am going to do is I am going to give you this quick questionnaire. So if I can get you to fill this out. And I will be right back I am just going to go get my next person, so they know I haven't forgotten them. 


\section{E.2.5 Participant 5}

So to start, what I am going to do is ask you a few questions about yourself and your experiences with music and that kind of stuff and then what I will do is switch to the videos. I will show you three short videos. After each video I will ask you a few questions. And then once we have finished all three videos, I will give you a wrap up questionnaire and we will just wrap up from there. OK?

OK.

So to start, how do you identify yourself-hearing, hard of hearing, deaf?

Hard of hearing.

And if you don't' mind, about how old are you?

58. Actually I am almost 59, so whichever you want.

Were you born hard of hearing?

No, I have been slowly losing my hearing over the last 10 years or so.

Oh OK.

I first started really noticing it 10 , maybe 12 years ago...admitting it 10 years ago.

OK (laughing).

Are you continuing to lose hearing?

Yeah, it's getting worse.

What memories do you have of listening to music before your hearing? Do you have a fairly good memory of listening to music?

Oh yeah, really good. I've listened to music since I was a young kid. We weren't a musical family, but I loved listening to music as a teenager. I sang in choirs all through public school and high school. I sang in an adult community choir for a number of years. I don't read music, but I catch on pretty quickly; I have a fairly good ear. So I try participating as well. I have continued to listen to music all my life as an adult. But definitely I've noticed since my hearing has started going, its tinged a little bit by the fact I cannot hear the lyrics if its vocal, if its music that has lyrics. I like instrumental music also, but I miss it when I am missing the words. It's not just quite the same.

So the recordings - I mean I assume you have a CD collection or an album collection of some sort-so the recording you have from 10 or 20 years ago, that you are quite familiar with, when you play them now do you really notice a difference?

Yeah, I am relieved often that I know the words. 
Right.

Because I know I am not really hearing what they're saying clearly anymore.

What about the melody and the music its self?

I can still follow that. My hearing is still alright that I can follow the music. But...I just became involved with [org.]. I took a speech reading class from _ recently, so I've just become more consciously aware, like the women that just spoke at the end of the last session there...how powerful it's been for her...it's been very similar for me. So it's made me more conscious of looking at ways my hearing loss has affected me. And one of them has been, at some level, of which I am now more aware, I am wondering how much I missing auditorily now in terms of listening to a classical piece or...I am not sure. I can definitely still follow it. I have a good sense of rhythm, which helps me. And I don't know really because I can't tell; I can still enjoy music.

Right.

But, yeah.

You were saying earlier that you have a tendency to listen to instrumental music. Do you think that your loss of hearing has shaped, I don't know, what it is you listen to?

That's interesting. I haven't thought of that. It might. I have sometimes thought it's just because I am getting older. Except, I still like Rock n Roll. I still like Rap. I still like the old stuff I use to listen to in the 70s, so I still like that stuff also.

Beatles, Elvis...

I still liked classical then as well. But...that's very interesting. Maybe, I am going to have to think about that one. That's very possible because it's easier. It's like how not going out is easier than going out sometimes. So that kind of strikes a chord with me. There is probably something to that for me.

When you listen to music, what kinds of things do you do? What kinds of strategies do you try to enjoy the experience more?

I definitely like listening to familiar music and again I started on your questionnaire earlier and that got me thinking too, and I think that's partly because it's easy. I definitely, especially in recent years, if I can have the J card. I don't know if they call that with CDs, but you know with audio tapes it's called the J card that has the words on it.

With the album it was the liner...the liner notes.

Now with the CDs whatever they call that then. If I can have that, especially the first few times I listen to the music because I do like to sing along with it, I enjoy the whole thing a lot more if I can be reading the words...because I simply can't catch a lot of it 
anymore...without that. I can still hear the music. All the rhythm, all that is fine. But again I don't know what I might be missing anymore...but I can still enjoy it. I like to follow the words along. I turn it up louder, which now I learned from $\square$ in our speech reading classes it was actually doing me harm...somewhat...could be doing me harm. But I also always liked it loud.

My brother sometimes says certain things were intended to be played loud.

Yeah, just blast it, you know?

Yeah.

I like the words. I like familiarity. I like it loud. I do find myself trying to get closer to the source of the music.

So you will sit next to the speaker or something?

Yeah, to try and be nearer. I find now, I don't have a stereo at the moment-but I want to get one-but one of my kids was home for a while and he had a really nice stereo, but I had to either blast it to hear it elsewhere in the house-we don't have a really big house, butor I had to be into the living to really feel like I was hearing. That I could tell, If I was in another room, moving around, doing whatever, even with it fairly loud, if I moved back into the living room I was getting a fuller sound, so I am clearly missing some of the sound...when I am not there.

You wear hearing aids, right?

No, I am in the process of...I'll be getting them.

Oh.

Just before I heard about the speech reading class, I thinking, "OK, it's time to get retested" because 10 years ago I was told hearing aids would not help me. And then I heard about the speech reading class...took that, went to the audiologist, got tested, but I was just about to get my knee replaced, so she said these are two big changes in your life, why don't you just deal with the knee and when that is settled, come back and we'll look at the hearing aide.

So you haven't been fitted for a hearing aid yet?

Not yet.

Interesting, interesting.

You were saying that when you listen to music, you were using your son's home stereo. What do you like to do? Do you use an iPod or a Walkman or what? 
I don't have one. Part of it was I was leery to get an iPod-this was again before I took the speech reading class-because I thought I can't imagine how that would be good for me, with already damaged hearing, I wasn't sure, so I've just always not. Until you know I thought one of these days I will look into it and then I took the class and she said it's not great.

You have to be aware...One of the nice things about an iPod is you can set it to max at a certain point. So it will never get louder than a certain point.

Yeah, she was telling us about that. Levelers and...

Where that doesn't exist with stuff like Walkmans. But what I am sort of trying to get at is what kind of stuff do you use when you want to listen to music?

My laptop or I listen in the car. I have an old stereo downstairs, so when I am downstairs I am playing something on there.

Your laptop, your car radio, your stereo in the basement-which of the three do you find really works best for you?

Sound wise. The sound of the music itself. I'd say the stereo in the basement because it has half decent speakers...for a fuller sound. You know the laptop has tiny little speakers; I don't have extra speakers for it.

So then you tend to enjoy music more when it's in your basic stereo?

Either that or the car. My car stereo is not bad.

Oh OK.

Currently, my CD player is broken, but that's just the last couple weeks and so that will get fixed. It's pretty nice in my car and then in my husband's car.

Do you listen to the radio in your car pretty much all the time?

The radio yes, but as often as not it's the CBC, so not always a lot of music...depending when I am driving somewhere, I'd be listening or I am putting in my own CDs. If I am driving a long distance, I usually stick in my own CDs.

Other than the lyrics, what other frustrations do you find you have when you listen to music?

I think just that thing I was mentioning before, that I realized that when my son was living at home for about 7 months, with a really nice stereo, and really realizing that when I wasn't in the living room, I was definitely missing some of the music, not just the lyrics.

So if you wanted to use a computer then, to help you better understand or help you have a better experience or something like that, what would be the most important pieces of 
information? What would be the most important things in the music that you would want this computer to show you?

The lyrics, if there are lyrics. I did do part of your questionnaire, I like the idea of it naming the instruments that are playing because I know music well enough that it might help me identify what I am hearing. I also really like the idea, because my rhythm is good, and I depend a lot on rhythm for enjoyment and I find even in speech reading it helps me. And I love colour and I use colour a lot in my life, so the idea-and I have no idea what you're thinking of doing with your research-kind of like the Windows music thing that has the colour, whatever that is. I don't like those in particular but I do notice I like the idea of colour and pattern being attached to music because I am pretty visual. But like I say, I don't necessarily always like what they do with it, but I like the idea.

What kinds of patterns, what kinds of colours, do you think would really work?

The colours would be tricky because colour means different things to so many different people. Different cultures, different ages, different genders-all that stuff.

Absolutely.

I think the rhythm of the patterns changing. What is the name of that? What can't I think of that? Windows...

You're thinking of Windows Media Player.

Thank you, that's the one.

You're welcome.

Yes.

I do like how it gets more intense, when the music gets more intense and it sort of simmer downs a bit when it is getting calmer and more relaxed. That kind of thing. I like that about it. I like the way it's reflecting the mood. I like the way it's reflecting the intensity of the music, the speed of the music - the pace. I do find if sometimes I just sit and listen to the music and if I watch that-again I don't always like what they do with it-it does sort of...These are all kind of interesting questions that I have never consciously thought of. It's kind of neat to think about them. I think it does kind of enrich my experience of listening to the music. Yeah, that is all I can think of.

$O K$

So what I am going to show you now is I am going to show you the videos. So these are videos of music visualizations. Now these visualizations have been developed at Ryerson and elsewhere, OK?

$O K$. 
So they're not necessarily made by me. The other thing is they are not necessarily developed specifically with hearing loss in mind.

OK.

$O K$

Some are just random visualizations. There will not be any sounds. If you're wondering why you're not hearing sound, it is because I am not playing sound. What I'm going to ask you to do as well, while watching these videos, I am going to ask you to keep in mind how you would interpret what it is you are watching? And, you know, what you get from it? OK?

OK.

So this is the first one.

I have one other thought about what enhances my enjoyment of music.

I am sorry?

Can I back up for a sec?

Sure.

One other thing is I don't generally watch music videos. I guess I am just the wrong generation. Although I still like Rock 'n Roll, so who knows. But you know the theatrics and the extremity of some them, I just really don't enjoy. But I love going to live music. I am not even a big fan of someone necessarily, but I just love being at a live performance. So something that would be showing me the performer, performing that music that would really enhance my enjoyment too.

Really?

Yup.

OK.

Do you go to concerts a lot?

Well I live two hours away from Sudbury and I am self-employed on a limited income, so the short answer is no. I'd go to more if I could.

But you find that with your hearing, do you find that you are able to get more when you are at a concert...

Absolutely.

Compared to a stereo or whatever? 
Absolutely.

What kind of "that" do you get?

Well, I think it's partly the actual physical vibration of actually being, especially if it's at a smaller place-there are some smaller concert venues, which are just fantastic. I can feel it. I can feel the bass.

The building literally rocks

Yeah.

Whether its blues or you know, folk or whatever it is, it's really, really great.

I also just love the energy of the people, the performers themselves. Like last night, Jennifer Rowland's, I am not a big Rita MacNeil fan, but I still enjoyed the whole experience of watching the band interact with one another and the kibitzing and the...You know all that part that allows me to enter into their experience of performing...doing what they love to do. That's a big thing for me. It really enhances my enjoyment, even if I missed a lot of the words and the typist wasn't always accurate. It looks great. It definitely helped. But just being a part of it...I always enjoy that.

$O K$.

So this is the first video.

OK.

(RADAR STARTS)

This reminds me of the old TV signal from when I was a kid.

$H m m ?$

This reminds me of the old TV signal from when I was a kid.

You are not the first person to say that.

Really, eh?

Do you want me commenting as I watch or just when I am done?

(RADAR ENDS)

As you were watching that what did you think? Did it say anything to you?

It felt like music. That was my overriding feeling. It was really kind of neat. Once the shafts of colour, and it reminded me of the early TV signals, after I got past that thought, I was liking the rhythm...the feeling of it being a happy, bouncy...it felt like music. It felt like 
a happy, bouncy song. I kind of thought of Jennifer Rowland's actually...that kind of you know lively, really lively. So then when circles came in that was like another section of the orchestra or the band coming in. Then the other thing, I forgot even what it was already, the part that came into the lower to quadrants that joined in, it was just having another part of the band, or orchestra-now this one-or the conductor or musicians...it just felt like music. It was really enjoyable actually.

Interesting.

What do you think the fan like blade things represented for you?

Blasts of sounds. Tones of like...Instruments playing whatever they would play. That is what it felt like.

What in this video didn't work for you? What was annoying?

The first part went on too long of the blasts of lights, the rays of lights. They went on a bit too long. As soon as those little circles came in, that just sort started to fill it out. It was kind of like the beginning of a song that goes on a bit too long. It was sort of like that kind of a feeling, "Ok, Ok, got it".

That was a beginning of a song.

Yeah? Oh OK.

What did you think of the colours?

They were fine. I had no negative reaction to them.

Did the colour mean anything for you? Any of the colouring mean anything to you?

There was the red and the bright orange that was repeating in the slices. That felt high energy to me. One of the circles I think was blue or was blue part of the time or something... the fact they were circles brought a depth and richness to it that was kind of a relief from all the blasts of (makes blasting sound) and kind of balanced it. I feel a little. The colours worked. Like I say I can't hardly remember what they were actually. I remember some of them being white, some of them being a variety of colours. I know that. And that was good because if it had been too much the same colour it would felt like a boring rhythm.

Did you want any of the colours to mean anything to you?

No, not particularly, no.

What did you think of the speed of the visualization? Was it too fast, too slow? 
Because it just seemed to me like a lively tune. It felt like a lively tune, so I was fine with it. But like I said, I still liked when the other parts of the band or the other parts of the orchestra or conductor jumped in. It seemed to provide some depth. But the pace was fine. I just thought it was a fast song.

So now I am going to show you another visualization.

You know one other thought. As that was playing, and I don't know if I would of thought this before this conference and being part of this, that felt like music and I didn't care that there wasn't anything music, which is kind of interesting.

That is interesting.

Yeah.

OK, so this is the second visualization.

(MOVING CIRCLES STARTS)

(MOVING CIRCLE ENDS)

So did this visualization say anything to you?

Change in good time and then it got really quite intriguing.

What did you think of the colours?

Again, at first they were all kind of hmmm boring, but the whole thing I think worked. I think the combo colours worked with the whole angles, and the peaks and valleys and the spiking in the circles. I think it worked with this.

Did the colours mean anything to you?

Well they are calm colours and then I guess at a certain level I'd say the piece was intriguing and the colours aren't intriguing.

Ok.

Interesting, interesting. That is definitely the first word that came to mind and stayed with me all the way through. It reminded me of bicycles and medical, you know, tracking machines...

Yeah.

Which was a bit odd.

Did it make you think of music at all? 
At first not. It just kind of felt medical and scientific and cell biology or something. But again, as the other parts began to come in, it again had that same feeling of now this section is joining in, this section is joining in and the piece is getting more complex. So I wouldn't say I had any strong like or dislike for it. But it was definitely interesting and by the end, I was wishing I could hear the music...to see what would go with that.

$O K$.

Or not to make music because I don't play any instruments. But it was intriguing, yeah intriguing.

Was there anything in this visualization that you just didn't like? It just didn't work for you?

No, because it changed soon enough. I was just thinking same kind of thing as the other one, "I hope this doesn't go on too long, I am bored", for the first few seconds, but it did. But the fact they were put together made the whole thing more intriguing.

$O K$

I think the colours ended up sort of adding because they are soft. The rest is, except for the little circles of course, all so angular and sort of mechanical looking and the softness of the colour there was sort of a sense of a balance there.

What about the speed of the visualization? Did you find it too fast, too slow?

No, actually I liked it.

So this is the last of the visualizations that I am going to show you?

(BARS STARTS)

(BARS ENDS)

So what did you think?

Interesting. I liked that one.

Did that say anything to you?

Well, cityscape was the first thing, nighttime cityscape, visually, was the first thing that popped into my mind. The second thing, quickly, was my ex-husband was a roadie and it reminded me of some of the soundboards and stuff (laughs). The colours were great. I liked the hot colours. It worked really well I thought. It had a lot of energy I thought. It reminded me of Rock ' $n$ Roll. That's what it made me think of. It varied enough that as I got used to kind of looking at it. I began understanding the pattern, or not pattern, but how it was working, so I started trying to anticipate where it might spike up higher, which was kind of fun. I don't know if I want to watch that for a very long time. It'd get kind of boring. 
Did it say anything to you in terms of music?

Rock 'n Roll or something like that.

$O K$

But I think that is partly combined with colours as well. The fire-y colours...

Yeah.

Definitely really worked with the spiking kind of pattern. And also the fact it looks like a nighttime cityscape just seemed like it could be that or maybe jazz.

OK.

Was there anything about this visualization that just didn't work for you?

No, I liked it. I thought it was really interesting. I liked the layers, the foreground or background kind of thing, the twinkling stars or whatever they are supposed to be.

Pardon?

The twinkling stars or whatever they are supposed to be was neat, yeah

What did you think of the speed? Was it too fast, too slow?

It worked for this, I think. It worked well with...again, what it made me think of was a very good jazzy beat or Rock 'n Roll or something that has a lot of energy. Has a fire-y kind of feeling to it and unexpected parts like jazz has. So I think the visuals, and the patterns, the colours and tempo and everything I think worked really well.

What I am going to do now is give you this questionnaire, you'll see each of these visualization types is given on here and I am just going to go and grab my next person and leave you to fill it out. You can use X's, checkmarks, in here and whatever it is, what you want to say,

comments, whatever. 


\section{E.2.6 Participant 6}

So as I was saying earlier today, basically what I am going to do today is I am going to ask you a few questions about yourself and your thoughts on music and then what I am going to do is show you some videos. After each video I am going to ask you a few questions about the videos and then at the end I will do a little bit of a wrap up, a short questionnaire, that kind of stuff.

OK.

$O K$

If at any time you need me to Sign, just ask and I will switch to Sign.

OK.

$O K$.

So to start, do you identify yourself as hard of hearing, deaf, deafened? How do you identify yourself?

I am hard of hearing.

You call yourself hard of hearing?

Yup.

Were you born hard of hearing?

No.

When did you lose your hearing?

Shortly after when I was born because I was born premature and my lungs collapsed so they had to give me medication and that's how I became deaf after that.

$O K$.

So then, do you have any memory of hearing?

Maybe, I am not exactly sure.

$O K$.

So do you listen to music?

Yes.

And you have a CD collection or music collection of some sort?

Yeah, I listen to my iPod and... 
Right.

Yeah

And do you believe that the music that you like to listen to...Do your choices in music reflect your friend's or do they reflect how you hear?

What do you mean by reflect?

So when you listen to a particular musician, do you listen to that musician because you hear that person better or you hear that song better than other songs or do you think that the music choices that you make are influenced very strongly by your friends?

I would say either of that. Some of my friends make me hear that music and I think it is cool, but I would have trouble with the lyrics most of the time.

Right.

I can mostly hear the background noise, the beat of it and how it sounds. Yeah.

$O K$.

And what kind of music do you tend to like to listen to?

All different kinds of music.

$O K$.

Do you have any particular favorite groups?

I think I like to listen to Rock n Roll. I like listening to The Who.

The Who? Interesting.

I like listening to that and the Rolling Stones a little bit. A little bit of David Bowie-a tiny bit not very much. Madonna. Some James Bond themes.

Interesting.

A little bit of everything.

Do you prefer music where the lyrics are more clear or do you prefer music with no lyrics at all?

I think I like both.

OK.

But, I just don't understand the lyrics much of the time. I can hear part of it. 
So when you listen to music what kinds of things do you do to help enjoy the experience?

It helps calm me down or if I have nothing really to do I listen to music. Do some work with it—if I do my homework, I listen to music.

Alright.

Yeah, that is what I do.

But when you're listening to the music, when you want to listen to music, when you want to only listen to music, do you do specific things, do you do anything, any sort of strategies perhaps, that you do just to help you understand the music and have a better experience with the music?

Maybe I would repeat the music again to understand the lyrics or my parents could explain what the lyrics say; what the music is saying. That kind of thing.

When you listen to music, do you use the stereo or an iPod or your computer? What do you do when you want to listen to music?

I could use my stereo, computer or iPod, either one of those would work.

Do you play with settings on your stereo at all? Just jack up the volume? Make it louder? Or do you do anything with bass?

No, I don't put it up loud...way to too loud. I don't think it really helps when it's up loud...just to hear the lyrics...no.

Do you wear hearing aids or a cochlear implant?

I wear one hearing aid and one cochlear implant.

OK.

Yeah.

When you're listening to music, do you use your hearing aid as well?

Yup, I would use my headphones...

Yeah.

...on top of it.

On top of the cochlear implant and the hearing aid?

Yup.

$O K, O K$. 
And whenever I travel, I don't use my headphones because it's too big. I use an audio cable wire to connect up to my cochlear implant.

Connect to your cochlear implant?

Yeah.

And which strategy do you think works better?

I think both.

You think both?

Yeah, I use my head phone to hear from both ears. If I hear from one ear, I don't like it that way. It makes it more balanced.

$O K$

And when you want to listen to music, do you try to isolate yourself? Go into a quiet room somewhere, so you can just focus on that or... What kinds of things do you do when you just want to listen to music?

Yeah, I'd go to a quiet room and listen to it. Or as the music goes on I'd try to go in front of it or go to a quiet room to listen to it

Do your parents like your music choices?

(nods)

Do they tend to tell you to turn it down or...?

If I turn on the stereo?

Yeah.

You live with your parents, right?

Yeah.

So that is going to influence what you can do with music, obviously

Right.

So I am just wondering, what those rules are, if you will?

No, they don't mind. I can listen to any music.

Now you were saying you use an iPod, you use a computer, sometimes you even use the home stereo, which one do you think works best for you? 
I think the computer because the computer makes a clearer sound to it. I think that's the best.

You think that's the best? Do you use the computer speakers or do you use the jack?

Yeah, I use the computer speakers. I put on my computer then I click on that then I can hear it.

\section{Interesting.}

Yeah, I think so. Stereo...yeah, maybe not. But I mean we have a computer connection that comes from the computer to the stereo. So I think they both work. I think they both work fine. But I think I prefer just working from a computer.

$O K$

You were mentioning earlier, that lyrics are a particularly big issue for you. What other things do you find really frustrating when you are trying to listen to music?

To understand what they are singing about.

$h m m ?$

To understand what they are singing about.

Do you hear the melody? Are you able to hear the melody?

I think so, yes.

Are you able to hear the instruments?

Yeah, the drums and...

You can hear the drums opposed to the bass or something or is that a little more... (makes hand gesture suggesting iffy)?

Sometimes I can. Sometimes I can't.

Is that something you want to be able to do?

To be able to hear the different instruments?

To be able to know what it is your hearing?

Yeah, I think so, yes.

Did I say the wrong thing?

Whatever you say there is no right or wrong answer. 
Right.

OK. So I am asking some questions to be a little bit more probing because in part I want to understand the nature of what you can hear and what you can't hear, OK?

OK.

I was born hard of hearing as well. So I know and I understand, I remember when I was ..., so I know and understand that there were things that really just frustrated me

$\mathrm{OK}$

And I know what I can hear, right? So I am trying to get a sense or a picture of what you can hear because I am not you.

Right, right.

There's no right answer. There's no wrong answer. Don't worry about what it is your saying. Just focus on what it is you think or what your experience is or how you feel.

OK.

$O K ?$

Yup.

Let's say in some future day, let's say a computer might actually be able to do something that would automatically present information about music to you in a way that is not just the sound, OK?

OK.

What would you want the computer to help you with? What would be the really important stuff that you would want the computer to present to you in order to make your experience with music more enjoyable?

$\mathrm{OK}$

It would be nice if they had some subtitles in the music, if I'm watching a music video. But I know some music right now has subtitles for YouTube videos, if I want to watch any kind of music. Maybe some sort of invention one day that will make it better, but I am not sure what it is. To make the sound quality better. Something like that might happen in the future.

Do you watch a lot of music videos?

Sometimes, yup 
What are some of the things that you really like about music videos that work for you?

Probably like the singers and dancers and that kind of stuff. Sometimes I can read lips when they are singing in the video...a little bit...not much. Yeah, stuff like that.

Right.

And of course you watch television with captions all the time too, right? You use captions all the time, right?

Yup.

So

I find it kind of frustrating when they don't have the captions. I just look at the video and I am like I like that sound, it's cool.

But yeah, without the captions it's like now what?

Yeah.

$O K$

So now what I am going to do is show you some short videos of music visualizations.

OK.

There's a few things I need to say: First of all, some of these have been developed at Ryerson, some of these have not, OK? So they're from all over the place.

OK.

The second thing is that the videos I am going to show you are not necessarily designed for deaf and hard of hearing people, OK?

OK.

So they may not help you at all, OK?

OK.

I am not going to play any sound, OK? So if you think you're missing something because you think you should be hearing something, don't worry, you're not.

OK.

I am not playing the sound.

OK. 
And as you watch the videos, what I want you to do is want you to sort of keep in the back of your mind, how you would interpret what you are seeing.

OK.

$O K$

What it says to you? What it means to you? If anything. If anything.

OK.

So this is the first one.

(MOVING CIRLCES STARTS)

It kind of reminds me of a band, a vibration.

Pardon?

It kind of reminds me of a band or a vibration. I kind of have a pattern going on in my mind.

Like a drummer.

(MOVING CIRCLES ENDS)

$\mathrm{Hmm}, \mathrm{OK}$.

That is what I got on my mind.

So does video sort of say anything to you music? Did it look like music to you?

A little bit, yes.

Yeah.

I don't know what else to say.

Yeah.

Was there anything in this video that you found just confusing?

I am not really sure what this is about at all. A bunch of...balls just flashing.

Right.

Yeah.

Yeah, $\mathrm{OK}, \mathrm{OK}$ 
But I know it's like a vibrator. That's what it's like in my mind. The balls are quite big. Kind of like a music background.

What did you think of the colours?

Difference? Different variety of colours?

Did the colours mean anything to you?

The different sounds maybe.

Did you want the colours to mean anything? Were you looking for something that maybe you didn't see?

Not really.

No.

What did you think of the speed of the video?

Some of them are slow. Some of them are fast.

Was it too slow for you? Was it too fast for you?

No, it's OK.

And what did you think of the shapes?

It's different, different order, different lines.

And what did the shapes mean to you?

Well, they're going in all different directions. Different patterns

So let me show you another one.

(RADAR STARTS)

Even though there is no music involved, I am just looking at the lighting, it kind of reminds of going into a concert with all these lights and music going on. And all these circles kind of reminds me of when I turn on the radio real loud-it vibrates really loudly.

These things (pointing at screen), these shapes do they mean anything to you? Do they say anything to you?

No?

Not really.

Do their movement up and down say anything to you? 
(nods)

What do they say to you?

I guess the music is going all different directions.

What about these circles do they say anything to you?

Yeah, some of them are big and small and they're all different sizes. The colours...

Do you see music in this at all?

No.

No, you don't think so?

OK.

Do you think the colours mean anything?

\section{(RADAR ENDS)}

No.

No?

No I don't think so.

$O K$

That's perfectly $\mathrm{OK}$.

Did you find this to be too fast or too slow?

No, it's OK. Yup.

So I am going to show you this. So this is the last video.

\section{(BARS STARTS)}

Kind of remind of bursting.

(laughs)

Kind of reminds me of the crowd maybe when they are cheering or something like they are happy or excited. Very noisy. Hard to hear the music. It could be a little frustrating.

(BARS ENDS)

Pardon? 
It could be a little frustrating if you can't hear the music that well because there is a lot of

That's what you are thinking of?

Do you see the music in there? Do you see music in there?

I think so.

You think so? How?

Movement and there's music going on.

OK

Yeah.

Did the colour mean anything?

Yes, the colour is darker at the bottom...orange and the red. The red shows more...I am not sure.

OK, that's fine.

Did you think this was really fast or really slow?

I think this is fast.

You thought it was fast?

Going in all different directions.

Did you want it faster or slower than it was? Did you want it faster? Did you want it slower?

Maybe a little slower.

A little slower?

(Nods).

So what I want to do is give you this questionnaire. So you will see these are each of the visualizations I showed you, right?

(agrees)

And you can fill it in however you like. You can check anything in here, write words, write comments, whatever. Entirely up to you and then you have each of these. I am going to leave you this and I will be back in a few minutes, OK?

Yup. 


\section{E.2.7 Participant 7}

Briefly, what I am going to do today is I am going to ask you a series of questions about yourself and about your experiences with music, OK?

OK.

Then I am going to show you a few short videos. After each video, I am going to ask you a few questions about the video-what you thought.

Alright.

And then after the last video, I will give you a quick wrap up questionnaire...

Alright.

...just to close it all up.

First of all, to start, do you identify yourself as deaf, hard of hearing, hearing, nothing at all? How do you label yourself?

Hard of hearing.

And if you don't mind my asking, about how old are you?

I will be 69 this week.

So 65 plus.

Were you born hard of hearing or...?

No, I started losing my hearing 10 years ago.

OK.

And it's 8 years, no, 7 years since I got my first hearing aid and 5 since I got the second.

So you wear 2 hearing aids?

2 hearing aids.

OK.

I have moderate to severe hearing loss. I am at the 60 line with one ear and the 70 line with the other. And I have the hearing loss of Meniere's disease, not the more traditional hearing loss where the higher tones are lost. So I have lost-we were talking about music-I have lost the bass tones. So I can hear extremely high tones somewhat over the normal speaking voice. I hear birds very well, violins...

A lot people actually think hearing birds is a bad part of the deal, but anyway. 
Do you remember...you were saying you lost your hearing about 10 years ago?

Started to.

Started to. Is it progressive? Are you continuing to lose hearing?

It's been two years since I had a hearing test, but it's been pretty consistent over that time frame-those 7,8 years.

Did you notice your hearing loss before that 10 year point?

No, it was just very gradual. Like when you get to 40 and you start needing glasses-it was a bit like that. Just a little bit, then a little bit more.

I have worn these since I was 12 (touches glasses), but OK.

(laughs)

So then you have a lot of memories of music from before 10 years ago?

Definitely. Definitely, yeah.

Do you have a music collection of some sort? A CD collection, record, albums, anything like that?

Yeah, we have a pretty extensive CD collection. Being Manitoban, we don't have access to a lot of places to go out for music. So we do have quite a bit and a very good stereo system at home.

So, if you think about the stuff you had before you lost your hearing and when you play it today, do you notice any difference between what you use to hear and what you hear now, when you listen to that piece?

Major difference.

What are the things that you really notice?

I have started an initial progression. At first what I notice is if I would be at a restaurant and there would be music playing, it would sound to me like two different systems were playing and one was playing at a different speed than the other.

Interesting.

Yeah, just not very pleasant, you know?

Right.

And then it gradually got to where other people might find music annoying and I didn't even know any music was playing, you know? 
$O K$.

So again that loss on the low end, right? Coming back...

Yeah.

Yeah.

So, are you going to skip on? Do I need to go ahead to where I am now or are you going to ask that?

Go right ahead. I probably will.

Alright.

The more you tell me, the less I need to ask.

So where I am now...Let's see, it's three years since we bought our current stereo system. And we went out and looked at a high end system and very, very good speakers. We tried lots of speakers and my husband was very happy to buy whichever set of speakers I could hear best with.

\section{Right.}

And we did that because he really enjoys music. I hardly listen to it all now because...You know, I have only learned part of what is happening this time at this conference: With digital hearing aids, I already hear high tones well but I think my hearing aids are dumbing out the part that I hear and I am not hearing...even with my hearing aids, I am not hearing the bass parts well, so. The music I use to recognize, I just don't recognize any longer.

Did you use to be a very avid listener of music before?

Avid? I wouldn't say avid, probably pretty average.

So you have noticed a drop in wanting to listen to music?

I hardly ever turn it on anymore.

$O K$.

And when you do listen to music, do you wear your hearing aids?

I wear my hearing aids, if my husband is there and we are listening to music together and then I just try to sort of ignore it. The only time I hear something that is sort of for my own pleasure is...what I do is I might be ironing or something and I take my hearing aids out and I put stereo up really loud. And then, I mean my husband can't stand that, but at least I have some semblance of what music use to sound like. 
So pumping up the volume does seem to help a little?

It helps some, yeah. And I fiddle around with the knobs a bit.

You play with the equalizer and stuff?

Yeah, and then I always need to remember to turn the volume back down.

Do you think that the music you tend to want to listen to today is influenced by your hearing, by what you can hear today?

Music that I want to hear? No, I don't think so.

Your music choices...you don't think your music choices or music preferences right now are influenced or shaped by what you can hear?

No, I still listen to what I like and often is what I do listen to are groups that I have gone to see in person because usually if I go to a live concert, if it is a small venue, I feel like I have a satisfactory experience listening to music. It doesn't happen very often in Manitoba, but once in a while...there is a venue where once a month or so during the winter a good small combo might come on in-somebody like Rick Fines or Suzie Vinnick or someone like that. And its small enough and the acoustics are good enough that I actually have a good experience there. And I try to listen...we buy the CDs and take them home or our kids give us music and their choice is pretty good; they kind of know what I'd like-stuff like Michael Buble.

Right.

It's always just current stuff that I listen to, you know? I am not a Classical person.

Right.

So then the concert experience, it's much more enjoyable to you, than before the music? Is that what you are basically saying? That you get more out of the concert experience?

Sorry, I am going to have to ask you to repeat that?

Do you feel that you get more out of the concert experience then recorded music?

Definitely, definitely, yeah. As long as it's not the really heavy "boom, boom" type of music. I can't go there. Even last night at the concert, I took my hearing aids out and I could have a pretty good experience last night. It was excellent when Jennifer played the first time. And when the choir was here...I have really been happy with sound systems and how they seem to work with my hearing aids-here in the building especially.

$A h, O K$.

How about you? Been talking about me. 
It's fine.

So you've been talking about how you guys bought a specialized home stereo for...

We tried to buy one to suit my hearing ability.

Right, to suit you. Do you use anything else? Do you try and listen to the radio? Do you try iPods-anything like that?

(Shakes head indicating no)

Have you tried music videos-anything like that?

No, no. I don't tend to listen to music, unless I am ironing. The only the other time I have it on is when I just want to sit down and listen to it.

Right.

That is a relaxing thing my husband and I will do during an evening. I don't tend to have it...I think I found out when I was relatively young-I was a nurse living in an apartment with some coworkers - and if the radio was on all day, the stereo or the TV, I had a much higher stress level, than if I just had it quiet. So that's my reason for not listening to music a lot.

So then what specific frustrations do you have when you try and listen to music?

The distortion.

The distortion?

It's really distorted and there isn't a proper balance between what should be the bass and what should be the high tones. It just all kind of gets garbled in there. It's just not enjoyable.

The...

Somebody hit it on the head at one of the sessions here that hearing aids are meant for speech and they don't work well with music. I guess that's what I've been struggling with and not really realizing the difference. It was happening to me, but I guess I wasn't really putting the blame in the right place.

If you wanted to use some piece of technology, like a computer or something or some other technology, to help you better understand music, what would be the most important pieces of information that you would really want the technology to be showing you or to be giving you from the music?

The most important pieces of information that I would want to know about the technology? 
To know about the music.

To know about the music?

What would be the most important pieces of information that you would want the technology to give you about the music?

I would need to...sort through some of the planning I am already doing. I am already realizing I need some different hearing aids that have an expanded band that fits into where the music plays. And I am very interested now after going to the blue tooth. Blue tooth? Do I have the right term for that?

Blue tooth.

Yeah, I guess

Yeah.

Presentation, you know, that Siemens have...I already have Siemens hearing aids. I would want to know that the hearing aids I would get would give me a truer picture of the music I listen to and I am really interested in that technology where I could tap into the television or the stereo with that system.

If you were to get something in a visual presentation...

Right.

...that is supposed to somehow be music...

Right.

...what aspects of music would you want the visual presentation to be showing you?

I think I am just, I think it's just my age, I think I am just more in tuned to just hearing music. I think younger people have got more interested in the music videos, you know, in music. I don't sit down a whole lot to watch television-I never have, you know. Watching music with something on a screen...I don't know when I would have time to do that actually.

So what I am doing to is ... what I am going to do is show you some short videos.

OK.

These videos will be videos of music visualizations.

Music video?

They are going to be music visualizations. 
Oh, music visualizations, $\mathrm{OK}$.

It's software that tries to show music in a visual way.

OK.

$O K$.

Is it something like...it was a revelation for me to feel the music in the balloon. So doing something like that visually?

Yeah.

OK.

So now, the software I am going to show you, the videos I am going to show you, they are not all from Ryerson, OK? They were developed all over the place.

OK.

They are not necessarily designed for a deaf or hard of hearing person to watch, OK?

(agrees)

I will not be playing sound; so you will not be hearing any sound.

Alright.

And as you are watching the videos, what I am going to ask you to do is sort of keep in the back of your mind, how you would interpret what you are watching?

OK.

OK.

This is probably something that is easier for younger people to do

You might be surprised actually. You might be pleasantly surprised.

So here is the first video.

(BARS STARTS)

(BARS ENDS)

Seems to me it's a symphony. But also what I found myself doing when I first started watching that was the fact...I have tinnitus and part of it for music, not specific songs per se...kind of a musical non-stop thing. Which I found was some of the peaks were fitting with some of the rhythms I hear in my head. 
Interesting. That's interesting.

When it became more of a crescendo at the end then it was getting out of my tinnitus rhythm...spiking more than it was...

Interesting.

If you don't have it...maybe I am not explaining it well.

Did this look like music to you?

Yeah, it looked like a symphony.

Was there any part of this video that you found confusing; that you just didn't understand?

No, it was kind of mesmerizing.

Interesting, $\mathrm{OK}$.

It's a bit like watching, something we took our kids to a long, long time ago-Laser light show, Dark Side of the Moon. We took our kids to when they were teens.

What did you think of the colours?

I like that colour combination. To me, it's a very relaxing nighttime sky and the shapes are high rises and the nice cheerful colours, the young lights, it's nice...the city sky line.

Do you think the colours meant anything?

Yeah, they were. Well I was hearing the movement of the colours as the kind of music that I always, always have.

Did you find this video too slow or too fast? What did you think of the speed of the video?

It was kind of faster at the end; that's probably when it wasn't too fitting with what I hear. Tinnitus adds a special dimension to what you hear.

I know. I have it too.

It's fun isn't. I sometimes learn that I change the tune.

I learned that sometimes that you can change the channel, you what I mean?

Yes.

But it takes it a bit. I also find for me that tinnitus kicks in more when I am tired.

More?

When I am tired. 
When I am not feeling all that up and energetic?

Yeah.

My favorite one is the sticks and triangle orchestra down the hall.

That will do it.

And my husband whistles allot and when I pick that up...

Did your husband come to the conference?

No, I wish he had. I truly wish he had.

There was a talk on the spouse experience.

I was there because I was wanting to hear what he would be experiencing, but there were very few spouses there and I really wish he had been there. He is a patient and loving guy.

The last time I went to a conference I brought my wife and I said the reason I am bringing you is because you are going to go to this talk about spouses.

Right.

And she wasn't happy with me because I said this is why I am taking you. But at the same time, for her it was an interesting experience because she got a lot out of it. She did-she got a lot out of it.

some issues.

so I think it helped her a lot understanding

I am thinking I might be able to get him to

To

Maybe.

Well if you can't do the one after that is

which might be easier.

Yeah,

Well OK, so this is the second video.

Alright.

(RADAR STARTS)

(RADAR ENDS) 
This is like Jennifer Rowland in the ticks and reels. You know it starts of kind of slow and then there is more depth to it and more speed to it and just when you think it can't go any faster, she speeds it up another bit. So I suppose I interpret that as something like that. That wouldn't fit in with my tinnitus at all

It did not fit in with your tinnitus?

Did watching that, did you feel that you were watching music?

Watching rhythm.

Watching rhythm?

Yeah.

When I think about rhythm, I think about drums, but it was more like her quick violin moving around there, not like drums.

Did this video say anything to you?

No, no, I don't think so.

No?

Was there anything about the video that you found confusing or frustrating?

No.

No.

What did you think of the things moving up and down (points at screen)?

Well, first they were getting me off in another tangent because I also quilt so I was seeing those things as pieces of fabric, looking at the colours and how they interacted. And that was kind of neat, but then I pulled myself back, which isn't unpleasant at all.

Yeah, yeah, I know.

If that's what you do.

Right.

What if anything, did those shapes moving up and down mean anything to you?

No?

I think I was just enjoying them for what they were. I don't think I'd try and fit them to anything. 
What about the circle? Did the circles mean anything to you?

Actually, the circles would have been more like drumming now that I think about it. The fact that it was, not round, not just that, but the pulsating part of it on a drum, kind of like a heart actually.

\section{Kind of like what?}

Heart. Now, the beat going there, I mean heart as to...

Did you find that the colours meant something to you?

No I was more caught up in their movement. When I was looking at the colours I got more distracted about fabric colours, but I was enjoying the movement of them.

Did you want the colours to mean something?

Did I want them to be that way?

Did you want them to mean something?

At one point I remember thinking this kind of reminds me of a cartoon.

Right.

Did you find this video too fast or did you find it too slow? What was the speed like?

No, I was enjoying the speed.

$O K$.

No it wasn't neither. I just went with what it was. It didn't bother me either way.

So we will bring up the last of these. So this is the last video.

(MOVING CIRCLES STARTS)

Here we have molecules.

This one is like a lullaby.

It's really quite mesmerizing isn't it?

Pardon?

Nice.

What did you say?

It's mesmerizing. 


\section{(MOVING CIRCLES ENDS)}

I felt it like a lullaby. I think maybe the visualization there for me was this is line is sort of dividing the space between sleep and awake. This being the wake side and this is gradually fading over there. It's kind of a gentle lyric maybe...lyrical-gently lyrical. It was very relaxing. Did you design that? Did somebody design that?

I can't tell you.

Oh well.

Did you find anything in this that you did not like? That you perhaps you found confusing or frustrating?

No.

No?

Maybe, the line in the centre, maybe because I think I was fitting the things into what I thought was a lullaby. Maybe it was going a little too fast.

Going fast how?

The little box going up and down. I think if it would just be a little slower it would fit my...they were doing their job.

What did you think of the shapes?

I like them.

What did the shapes say to you?

The shapes are busy to me. I don't see them as notes or anything. I guess if I got analytical I might see them as notes, but I don't want to think about it now. I like the movement of them-gently going side to side and changing them with a interface.

Does this look like music to you?

Yeah. Yeah, it did-very gentle music.

OK.

What if anything did you think about the colours?

I don't think I did.

The colours, did it mean anything to you?

Actually were they the same colours that were in the quarter of the circle? 
Yeah, a little bit yeah, similar.

Nothing particularly...they are colours I like so I found them very pleasant.

You were commenting that you found this stuff going on around this middle line really busy. But did you find the video itself fast or slow?

No, I found it was at a very nice pace going across.

$\mathrm{OK}$, I guess what I was thinking was the speed that was going across was a little bit faster than the speed that was going up and down. So maybe there was a little disharmony there.

I see. I see what you were thinking.

And if a song is a fast song, what do you think should happen to the video? When the song is a fast song? When a song is a slow song? Should the video be fast or slow?

I think it would speed up for a fast song.

So that was the last video. So what I am going to do now is ask you to fill out a questionnaire.

So how do you want them rated?

You can ...

So there's one of each?

You can mark it anyway you like, so if you want to add words, check marks, whatever go ahead. 


\section{E.2.8 Participant 8}

OK, so as I explained earlier, the procedure in this study is that I am going to ask a few questions about you and get a sense of who you are and your experiences with respect to music and that kind of thing.

OK.

And then I am going to show you a few videos.

OK.

At the end of each video I am going to ask you some questions.

OK.

At the end of all the videos I am going to give you a bit of a wrap up questionnaire...

OK.

... and that will basically be the end of the study.

OK.

So hopefully we should be done in about a half an hour or so.

Sounds good.

So to start, do you identify yourself as hard of hearing, deaf, deafened?

I identify as hard of hearing.

And about how old are you?

I am 26.

So if you could just tell me a little about yourself. Were you born hard of hearing or...?

Yes, I was born with bilateral hearing loss with poor speech discrimination. I wasn't diagnosed until I was four at which point I was outfitted with binaural hearing aids and I wore those until Grade 9 unwillingly. I chose to stop wearing hearing aids through my high school and early University. And then in the third year of my undergrad, my first undergrad, I got new digital hearing aids and I have sort of been working with that now and I am about to buy a second pair. And that's where I am at.

Do you remember much of what it is was like to hear music with the hearing aids you had in Grade 8 ? 
I didn't wear them to listen to music. I would frequently...Concerts are too loud to wear hearing aids.

Yeah, of course.

For my own personal music experience I would usually wear headphones.

And so you still wear headphones usually?

I do wear headphones. Not to say I don't ever listen to music without headphones because there's social situations. I listen to the radio in the car...that's fine. I usually prefer familiar music. When it comes to music with lyrics and I can't understand the lyrics, I have less interest in the song, so it depends. I started listening to much more purely instrumental music to avoid that.

So your hearing loss in some way has shaped the decisions you make when you buy music or when you go out and pursue?

Yes, I tend to choose music with lyrics that either I am familiar with or I can easily become familiar with or I choose music with no lyrics at all.

Something very clear or nothing...No?

Depends on the style of music. I listen to a lot of psychedelic trance and I wouldn't call that clear, but there is no lyrics, so...

Interesting. $\mathrm{OK}$.

You were saying you use the radio in your car, right?

(agrees)

Do you have an iPod, home stereo or anything like that?

I use an iPhone.

$O K$.

And you use headphones with your Ipod or...?

Yup.

Do you use any strategies or any particular tricks to help you enjoy music more?

When it comes to music with lyrics, I will often download an entire album, look up all the lyrics, learn them, and this allows me to actually hear the song better when I am listening to them. The same thing goes for when, for example, last night we were 
in the bar and there was music playing and it sounded sort of familiar and I saw someone else singing along to it and by reading the lips I realized it was "Wonderwall" by Oasis, which is a song I actually know very well. And once I knew what song it was, I could hear the song better. So I think it's memory, right? Of the music allows me to actually hear the song better, which allows me to enjoy the music much better at that point. It stopped being words and started being started being a song.

Interesting.

This is going to sound a little odd, but when you listen to music, are you able to hear the melody and the different instruments or...Tell me a little bit about what you think you hear when you listen to music.

I can distinguish between certain instruments. I was having this conversation last night at the Rita MacNeil concert. Somebody asked me if I could hear the symbols. And I could hear the symbols, but I don't consciously hear them. I need to focus on a particular sound to hear it, otherwise I get the overall effect. If I am concentrating, I hear it better. Background music doesn't really do it for me, so I have to be paying attention to the music to enjoy it.

And you were saying that through high school and the beginning of University, you did not tend to wear hearing aids. You are wearing digital aids now. Do you wear digitally aids normally when you listen to music now?

No.

You don't want to wear them at all.

I don't wear them as much as I should to begin with. I find that with the hearing aids on I am hearing things that are actually distracting me from the music. I guess I am not comfortable yet with the high frequencies. I will often hear things that sound like birds chirping, even though I am sure that it just part of the music. But it is distracting me a lot from the song, so I don't wear them.

You were talking about how you will listen to music in your and you talked little bit about going to concerts. Do you go to concerts fairly frequently?

I do.

You live in $\square$ so...

I live in during the school year, so yes. 
Yeah, so it makes it easier.

Do you have your own apartment?

Yes.

When you are at home, when you are at your parent's home, do you find that you have to go to a quiet space or something to listen to music? Do you...

My house is a quiet space.

Right, so I see.

So no, that is a quiet place.

OK.

So let's see. When you are trying to listen to music, what do you find particularly frustrating?

If I am focusing on the music, it is frustrating trying to hear the music over other sounds. So at a party, if there is a good song on and I want to listen to the song and everyone is talking at that the party, then I can't pay attention to the song. But I don't really think that is what you are asking. What do I find frustrating? Really, it's just understanding lyrics; It's understanding what is being sung. I like to sing; not that I do it particularly well. But it's part of the enjoyment of listening to music-the ability to participate in that. And my inability to participate in that, I find frustrating.

Ah, interesting.

Other than lyrics, is there anything else that you find particularly frustrating?

Often when there is maybe a specific instrument being played. As you said earlier can you distinguish between different instruments?

Right.

And there are some styles of music where they have a flute, for example or an instrument in a higher frequency and I can't really hear that. And I miss the bulk of what's really good about that song. And that can be frustrating because you can go like "you're missing it?" and you see it, but you can't hear it. And that can be a little bit frustrating, but I guess I have compensated by just choosing to not listen to that kind of music to begin with, so I don't really feel like I am at a loss. 
When you say you can see it, like when you are at a concert?

Yeah. I frequently watch music videos or concert videos. I actually prefer that to just listening to an album. But I think that is the student life-you are always on YouTube.

Well, yeah, yes and no.

In terms of music videos, or videoing in general, do you tend to watch captioned videos or not? Do you...

Captions?

Yeah.

YouTube doesn't really have that available.

Yeah, I know.

I don't have cable and I don't really watch...I don't really listen to mainstream music, so Much Music doesn't really do it for me. And the captioning on Much Music is not good.

No.

It's like the News.

I know.

It doesn't really help, when your trying to be in pace with music-so no, in that context. However, I do really enjoy buying movies of music. So the "Last Waltz" or Pink Floyd's "The Wall" and that has really good subtitles. And I will always have the subtitles on when I am watching those.

So this is going to be a weird question, but if you had access to a computer or some other kind of technology that had the job of supporting you or your enjoyment of music, what things, what information, would you want that technology to give you?

What would be the important stuffyou would really want to know?

I would like to know all about the music, the band and all the background. Often if I find a new band that I like I will Wikipedia them to death and learn more about them. It's just part of enjoying the music scene and learning about it. It would be great if...You know how some modern car radios...they have that rolling screen that says the song name, the artist name and the...and if they could continue that also 
give you lyrics and what instrument is being played and just having a rolling set of information that would be really good. I am skeptical about something like a computer. The reason I got my iPhone was because I could consolidate all the crap I had, right? So there, if there is an application on the iPhone that could recognize what song is being played and stream that information for you, that's cool. But I would never carry something outside of that because the whole point of me getting the consolidated piece of technology is to not have to have all this stuff to enjoy my music.

There's actually an application for Mac-I don't know if it's on the iPhone-where it takes a sampling of the song that you are hearing, like it will literally take a sampling off the radio, and it will go to the Internet and grab a compressed sample of an equivalence to that and then suddenly boom, this is what you are hearing.

It has all the information?

Yeah.

When I load a new album on to iTunes, it can actually get all the album information. And I do like that feature, but I want something you can take with you.

No.

I just don't sit at home and listen to music; that's not what I do. I don't sit at home to do anything really.

Do you try and avoid staying at home?

I am sorry?

Do you try and avoid staying at home? Is what you're saying?

I don't avoid staying at home. I like being at home. If I am not home and I am listening to music, I am studying at which point I am listening to classical because it doesn't distract me from my work, right? So I am not listening to the music, it's just on and I am working.

So you listen to music help you focus as well as entertain you?

Yeah.

Yeah. OK.

Or I will have the TV playing a movie that I am familiar with so that I am not feeling the need to watch it. But I like background noise when I study, yeah. 
OK.

And another problem is with technology is that I recently rediscovered that...I recently rediscovered vinyl and vinyl sounds much better than CDs, tapes, MP3's. The sound quality is extremely layered...

Yeah, it is.

...and rich.

I mean they have modern record players these days, but I am not going to go and buy a new record player. I have my dad's old record player. It works fine. I don't have the motivation to buy supplemental technology for vinyl.

Right.

Right.

So there's that as well. But I mean you can reintroduce vinyl. It's still good.

No I agree with you. I grew up on vinyl. I am old enough to say I grew up on vinyl. I actually own albums. I actually bought albums.

I still buy albums. Some of the best rock bands that around still release on vinyl.

Vinyl, yeah.

We go out of our way to buy vinyl.

Yeah, and actually they go out of their way to produce vinyl because they know that if they...

A better experience. And there are actually people who are Music aficionados who do know that, like myself and my partner, and that we seek of the vinyl. So they produce it for us.

So now what I am going to do is I am going to show you the videos at this point.

OK.

Now these are short videos of music visualizations.

I am sorry?

These videos are music visualizations.

OK. 
They are based on software that takes some piece of music, right, and tries to present it visually.

Like a visualizer on a music program or like sheet music?

Yeah, very similar. Yeah, very similar.

So the vibes.

Except you're not going to see Windows Media Player kind of thing, but it's very similar.

So what I am going to show you...the videos I am going to show you are not necessarily tools that were developed at Ryerson. They've been developed by various people.

They're just videos that you have?

That's right.

The other thing is they are not necessarily designed for people that are deaf or hard of hearing. They are not necessarily designed to support people that are deafor hard of hearing.

OK.

Also, as I play it, you're not going to hear anything. I won't play the sound.

So you want me to give you my impression of what it should sound like?

No, no.

So as you're...the reason I am telling you that I am not going to play the sound because some people that are deaf or hard of hearing aren't sure if they should be hearing the sound.

OK.

So sometimes in the absence of sound, you're thinking should I be hearing something?

Should I be hearing something?

Right.

You're probably more hard of hearing than some people, so you know the difference. For some people they can't quite tell, so I am telling you I am not playing the sound.

OK. 
So what I want you to do is as you're watching the video is I want you think about how you would interpret the information that you are seeing.

OK.

What it might mean to you - that kind of thing.

OK.

$O K ?$

Sounds good. Let's do it.

So this is the first one.

(MOVING CIRCLES STARTS)

Oh, I finally get that, $\mathrm{OK}$.

(MOVING CIRCLES ENDS)

What is it you finally got?

I was trying to figure out this line in the middle with the very bright dots moving up and down were very distracting and make it difficult to watch the rest of it and I just realize it's supposed to be the drum-the bass rhythm. Just basically in my head I can see the various melodies and harmonies depending on the line-the bass line, the treble line. And as it goes up and down-because I did play piano-and I am sort of thinking of the notes as they go up and down. And so you can sort of imagine what it should sound like. However, when it gets to this point, it is too much to watch. So at this point it's just like they are rocking out or it's just noise, you know? But that line in the middle was distracting, it was difficult to watch.

Which line are you talking about?

This with the very bright dots moving up and down.

Oh, this white line right here.

Yeah, so and all these bright dots and it making me want to just and look away.

The intention of that line actually is to tell you the time point in the video, right?

Yeah

So this is the point where the note starts. 
Yeah.

What did you think about the shapes? Did you get any...

You mean the squares and the circles or the actual...?

Yeah.

The circles I mean at first they didn't really mean anything to me. I was thinking if they are playing guitar, it might be where the strumming is.

Right.

But at that point I wasn't really watching them. If they were moving, I could maybe see how fast the music is going and whether it was going higher or lower based on the line direction. But all this here, it's too much it doesn't mean anything to me.

$O K$

Did you find that the colours meant anything to you at all?

You know, I like the blue, the green and the purple. It distinguishes the lines without making you focus too much on one line. The white is too harsh I can't look at it.

OK, that's fine.

What did you think of the speed of the shapes?

Average. Seemed like a country song. Average.

$O K$

Well what I am going to do now is I am going to switch you to another visualization.

(RADAR STARTS)

So what do you think of this video?

I actually like this one a lot better. It took me awhile to get it, but I think the longer you watch it, the more you can imagine how sound can relate to it. I would say that this up here is the main gnawing of the music. I am thinking higher ranges because it is in the upper quadrant. But as it goes down you can sort of hear how long the music holds out. It's playing quite fast up here, but then it goes longer and goes down. This is a really great representation of the music and the beat. Depending if you know what the colours mean, it could be bass drum, snare drum, cymbals even. 
It's giving you a better impression of how hard it's been hit or how loud it is. I really like that. It's much, much nicer to watch than the first one. It's kind of neat.

Do you think the colours mean anything?

I am sure they do, but I think that interpretation depends on the person. And I can't attribute the sound to it and that's why it was difficult for me to figure out at first what it was supposed to be.

$O K$.

What do you think of the speed of this thing?

I am thinking electronic. It's like...So it makes me think of electronic music with a bass driving beat throughout it. So you can tell the music is being played very fast but the actual drum is not being played very fast so there's a lot of different sounds going on.

One of things I find interesting about your responses is...Maybe I should ask you first. How much music training do you have?

\section{(RADAR ENDS)}

I played piano from the age of 5 to the age of 18. I played trumpet in high school band. I played flute for a year, but I was terrible. I get music.

Yeah, that is what I saying. You seem to have a lot of experience with music and thinking with a musical brain.

So I am going to show you my last video now. Now this might be a little bit more familiar to you, partly because of your generation.

\section{(BARS STARTS)}

Yeah, this is a rock song, if I am looking at it correctly.

\section{(BARS ENDS)}

What I am seeing is how red it is; I guess how loud and deep the sound is. When I say deep, I guess I mean low, but it depends on the spectrum. It's being played quite fast. The beat is a rock beat. And you can see the bridge come up as everything sort of raises a little bit. These dots make me think these are the notes that are being held a little bit longer. So even though the music is being played quite quickly, there are some notes being held. I think for an inexperienced watcher though it would be difficult to follow because it's a lot to look at all at once. I guess the colours can be 
important in that when there are different colours for different kinds of sounds, it's easier to distinguish what you're looking at. And here, I mean I can tell by how much at one end is going up but one who cannot see that might have a harder time distinguishing it.

So you think something like this...you can clearly...If I am hearing you correctly what you're saying is you can clearly see the music in this.

Yeah.

But for something like this it requires a fair amount of training to really see and understand. Well, they might. I don't really know, but it doesn't seem to be as clearcut as, for example, the second one where you could easily see where the beat was verses where some of the other music was. Here, it's all across the spectrum. And for someone who maybe can't quite hear the music as well and they don't know what the sounds are like and how they are distinguished, they might not be able to see that there.

OK.

So now what I am going to do is I am going to give you this-it's a final questionnaire. As you can see each of these are representations of what I showed you earlier. In this first question you can mark it however you want-X, check, you can even write.

Just fit it in the square?

You can even write words if you want-totally up to you.

Would you rather have me rate them or just pick one?

Just answer the question and go from there 


\section{References}

Abrahamian, S. (2003). EIA-608 and EIA-708 captioning. Retrieved from http://www.evertz.com/ resources/eia_608_708_cc.pdf

Altman, R. (2001). That most American of attractions, the illustrated song. In R. Abel \& R. Altman (Eds.), The Sounds of Early Cinema (pp. 143-155). Bloomington, IN: Indiana University Press.

American Statistical Association (ASA). (1997). ASA Series: What is a survey? How to conduct pretesting. Alexandria, VA. Retrieved from http://www.prm.nau.edu/prm447/asa\% 20 brochures/pretesting.pdf

Aussie Deaf Kids. (n.d.). Captions on your television. Retrieved from http://www. aussiedeafkids . org.au/captions-on-your-television.html

Bahan, B. (2006). Face-to-face tradition in the American Deaf community: Dynamics of the teller, the tale, and the audience. In H.-D. Bauman, H. Rose, \& J. Nelson (Eds.), Signing the body poetic: Essays on American Sign Language literature. Berkeley: University of California Press.

Balkwill, L.-L., \& Thompson, W. F. (1999). A cross-cultural investigation of the perception of emotion in music: Psychophysical and cultural cues. Music Perception, 17(1), 43-64.

Bavelier, D., Dye, M. W., \& Hauser, P. C. (2006). Do deaf individuals see better? Trends in Cognitive Sciences, 10(11), 512-518. doi: 10.1016/j.tics.2006.09.006

Beentjes, J. W., Koolstra, C. M., \& van der Voort, T. H. A. (1996). Combining background media with doing homework: Incidence of background media use and perceived effects. Communication Education, 45(1), 59-72. doi: 10.1080/03634529609379032

Bell, A. G. (1914). Discovery and invention. National Geographic, 25(6), 649-655.

Berardinelli, J. (2002). Jaws. Retrieved 2015-02-17, from http://www.reelviews.net/movies/j/ jaws.html

Bergman, E., \& Johnson, E. (1995). Towards accessible human-computer interaction. In J. Nielsen (Ed.), Advances in Human-Computer Interaction (Vol. 5, pp. 87-114). Norwood, New Jersey: Ablex Publishing Corporation. Retrieved from http://www.sun.com/access/developers/updt .HCI . advance. $\mathrm{html}$

Blessing, L. T. M., \& Chakrabarti, A. (2009). DRM, A design research methodology. London: Springer Science \& Business Media.

Bradley, M. M., \& Lang, P. J. (1994). Measuring emotion: The self-assessment manikin and the semantic differential. Journal of Behavior Therapy and Experimental Psychiatry, 25(1), 49-59. 
doi: 10.1016/0005-7916(94)90063-9

Branje, C., Maksimouski, M., Karam, M., Fels, D. I., \& Russo, F. (2010). Vibrotactile display of music on the human back. In ACHI '10 Proceedings of the 2010 Third International Conference on Advances in Computer-Human Interactions (pp. 154-159). Washington, DC, USA: Institute of Electrical \& Electronics Engineers (IEEE). doi: 10.1109/achi.2010.40

Brokaw, G. (1997). "Visual sound: Music videos for the deaf": Expanding on closed caption television (Unpublished master's thesis). Kent State University, Kent, OH.

Brown, R. J. (1978, March 28). Audio activated video display (No. 4081829). (US Patent)

Burns, L. (1965). Music in deaf education. Music Journal, 23(8), 42-44.

Busse, D., Kudella, P., Grüning, N.-M., Gisselmann, G., Ständer, S., Luger, T., ... Benecke, H. (2014). A synthetic sandalwood odorant induces wound-healing processes in human keratinocytes via the olfactory receptor OR2AT4. Journal of Investigative Dermatology, 134(11), 2823-2832. doi: 10.1038/jid.2014.273

Canadian Association of Broadcasters (CAB). (2012a). Closed captioning standards and protocol for Canadian English language television programming services. Retrieved from http://www.cab -acr.ca/english/social/captioning/cc_standards.pdf

Canadian Association of Broadcasters (CAB). (2012b). Normes universelles du sous-titrage codé à l'intention des télédiffuseurs canadiens de langue française /Closed captioning encoding standards for Canadian French language television broadcasters/. Retrieved from http://www.cab-acr.ca/ french/societal/captioning/normes_universelles.pdf

Canadian Association of the Deaf (CAD). (2007, May 26). Statistics on Deaf Canadians. Retrieved from http://www.cad.ca/en/issues/statistics_on_deaf_canadians.asp

Canadian Hard of Hearing Association (CHHA). (2006). Bylaws of the Canadian Hard of Hearing Association / L'Association des malentendants canadiens. Retrieved from http://www.chha .ca/documents/en/national_bylaws_2006.pdf

Canadian Hearing Society (CHS). (2002). Canadian Hearing Society Awareness survey.

Canadian Network for Inclusive Cultural Exchange (CNICE). (2005). Online enhanced captioning guidelines: A companion document to the cnice general guidelines for inclusive online cultural content. Retrieved from http://cnice.idrc.ocadu.ca/guidelines/caption.php

Canadian Radio-television and Telecommunications Commission (CRTC). (2010). MuchMusic - Licence amendments. Retrieved from http://crtc.gc.ca/eng/archive/2010/2010-875.pdf (CRTC 2010-875)

Carter, J., \& Fourney, D. (2004). Using a universal access reference model to identify further guidance that belongs in ISO 16071. Universal Access in the Information Society, 3(1), 17-29. doi: 10 $.1007 / \mathrm{s} 10209-003-0074-6$

Cavalcanti, A. (1939). Sound in films. Films, 1(2), 25-39.

Cawthon, N., \& Moere, A. V. (2007). The effect of aesthetic on the usability of data visualization. In 11th International Conference Information Visualization (IV'O'7) (pp. 637-648). Institute of Electrical \& Electronics Engineers (IEEE). doi: 10.1109/iv.2007.147

Center for Science Education (CSE). (2010). Solar wind sounds examples - sound of stereo. Retrieved 
from http://cse.ssl.berkeley.edu/stereo_solarwind/sounds_examples.html

Champlin, C. (1967, March 12). Sound and fury over film music. Los Angeles Times, C14.

Chasin, M. (2003). Music and hearing aids. The Hearing Journal, 56(7), 36-41.

Chasin, M. (2010). Six ways to improve listening to music through hearing aids. The Hearing Journal, 63(9), 27-30. doi: 10.1097/01.hj.0000388537.27405.77

Chasin, M., \& Hockley, N. S. (2014). Some characteristics of amplified music through hearing aids. Hearing Research, 308, 2-12. doi: 10.1016/j.heares.2013.07.003

Chen, C. (2005). Top 10 unsolved information visualization problems. IEEE Computer Graphics and Applications, 25(4), 12-16. doi: 10.1109/mcg.2005.91

CineWiki. (2009). Illustrated songs. Retrieved March 19, 2015, from http://cinewiki.wikispaces .com/Illustrated+Songs

Clark, J. (2004). How standardization solves problems in captioning and beyond. In Proceedings of the NAB Broadcast Engineering Conference. Retrieved from http://openandclosed.org/docs/ NAB2004.pdf

Coalition for Music Education in Canada (CMEC). (2005). Music education "State of the Nation" benchmark study. Retrieved from http://musicmakesus.ca/wp-content/uploads/2010/10/ EnglishMusicReport.pdf

Colburn, H. S., Shinn-Cunningham, B., Kidd, G., Jr, \& Durlach, N. (2006). The perceptual consequences of binaural hearing: Las consecuencias perceptuales de la audición binaural. International Journal of Audiology, 45(7), 34-44. doi: 10.1080/14992020600782642

CONNECTUS Consulting Inc. (2008). Closed captioning validation sessions - Findings and analysis. Retrieved from http://www.cab-acr.ca/english/research/08/sub_dec1508.pdf

Cooke, D. (1959). The language of music. London: Oxford University Press.

Corbin, J., \& Strauss, A. (1990). Basics of qualitative research: Techniques and procedures for developing grounded theory. Thousand Oaks: Sage publications.

Crider, S. (2009). Re-defining Deaf music through a Deaf lens (Unpublished master's thesis). Gallaudet University, Washington, DC.

Csíkszentmihályi, M. (1990). Flow: The psychology of optimal experience. New York: Harper Collins.

Csíkszentmihályi, M. (2004). Stalking a new world order. New Literary History, 35(2), 339-348.

Cytowic, R. E. (2002). Synesthesia: A union of the senses (2 $2^{\text {nd }}$ ed.). Cambridge, MA: MIT Press.

D-PAN [dpanvideos]. (2008, March 28). D-PAN ASL Music Video: "Beautiful" by Christina Aguilera. [Video file]. Retrieved 2015-03-21, from https://www . youtube.com/watch?v=C6zVFGpGNJQ

Dalgarno, G. (1989). Music for deaf and partially hearing people - Part 4 - Visual and tactile representation of music. Magazine of British Association of Teachers of the Deaf , 8-11.

Darrow, A.-A. (1992). The effect of vibrotactile stimuli via the SOMATRON ${ }^{\mathrm{TM}}$ on the identification of pitch change by hearing impaired children. Journal of Music Therapy, 29(2), 103-112.

Darrow, A.-A. (1993). The role of music in Deaf Culture: Implications for music educators. Journal of Research in Music Education, 41(2), 93. doi: 10.2307/3345402

Darrow, A.-A. (2006). The role of music in deaf culture: Deaf students' perception of emotion in music. Journal of Music Therapy, 43(1), 2-15. doi: 10.1093/jmt/43.1.2 
Darrow, A.-A., \& Gfeller, K. (1991). A study of public school music programs mainstreaming hearing impaired students. Journal of Music Therapy, 28(1), 23-39.

Darrow, A.-A., \& Goll, H. (1989). The effect of vibrotactile stimuli via the Somatron ${ }^{\text {TM }}$ on the identification of rhythmic concepts by hearing impaired children. Journal of Music Therapy, 26(3), $115-124$.

Darrow, A.-A., \& Heller, G. N. (1985). Early advocates of music education for the hearing impaired: William Wolcott Turner and David Ely Bartlett. Journal of Research in Music Education, 33(4), 269. doi: $10.2307 / 3345253$

Deaf Culture Centre. (n.d.). Deaf rap drum. Retrieved from http://www.deafculturecentre.ca/ Public/Place/Item_Details . aspx?ID=30

Deaf Performing Artists Network (D-PAN). (n.d.). D-PAN: Deaf Professional Arts Network Home. Retrieved from http://www.d-pan.com/

de Lespinois, P. (Director)., \& Doe, J. (Producer). (2010). And man created dog. [DVD]. Evergreen Films.

Diaz-Marino, R., Carpendale, S., \& Greenberg, S. (2005). Lyric text: An animated display of song lyrics (Tech. Rep.). Grouplab, Dept. Computer Science, University of Calgary. Retrieved from http://grouplab.cpsc.ucalgary.ca/grouplab/uploads/Publications/Publications/2005 -LyricText.VideoReport.pdf (Research report iLab-2005-1)

Disabled Peoples' International. (1993). Constitution of Disabled Peoples' International. Retrieved November 5, 2002, from http://www.dpi.org/en/about_us/constitution.htm

Dowling, W. J. (1999). The development of music perception and cognition. In D. Deutsch (Ed.), The Psychology of Music (2 ${ }^{\text {nd }}$ ed., pp. 603-626).

Editors of the American Heritage Dictionaries. (1992). American heritage dictionary of the English language ( $3^{\text {rd }}$ ed.). Boston: Houghton Mifflin.

Ekman, P. (1999). Basic emotions. In T. Dalgleish \& M. Power (Eds.), The handbook of cognition and emotion (pp. 45-60). Sussex, U.K.: John Wiley \& Sons, Ltd. Retrieved from https:// www. paulekman.com/wp-content/uploads/2013/07/Basic-Emotions.pdf

Ekman, P., \& Friesen, W. V. (1986). A new pan-cultural facial expression of emotion. Motivation and Emotion, 10(2), 159-168. doi: 10.1007/bf00992253

Fels, D. I., Lee, D. G., Branje, C., \& Hornburg, M. (2005). Emotive captioning and access to television. 11th Americas Conference in Information Systems (AMCIS) 2005 Proceedings, 2026-2033.

Ferguson, S., Vande Moere, A., \& Cabrera, D. (2005). Seeing sound: Real-time sound visualisation in visual feedback loops used for training musicians. In Ninth International Conference on Information Visualisation (IV 2005) (pp. 97-102). doi: 10.1109/IV.2005.114

Field, G. (2000). Implementing DTV closed captions and video description (Vol. 28). Retrieved from http://ncam.wgbh.org/invent_build/analog/dtv-access-a-resource-site-for/ implementing-dtv-cc-dvs

Filonik, D., \& Baur, D. (2009). Measuring aesthetics for information visualization. 13th International Conference Information Visualisation (IV 2009). doi: 10.1109/iv.2009.94

Fix, J. (2008). The use of music education in oral schools for children who are deaf and hard of hear- 
ing. Retrieved from http://digitalcommons.wustl.edu/pacs_capstones/354 (Independent Studies and Capstones. Paper 354. Program in Audiology and Communication Sciences, Washington University School of Medicine.)

Flexner, S. B., \& Hauck, L. C. (Eds.). (1987). Random House dictionary of the English language (2 ${ }^{\text {nd }}$ ed., unabridged.). New York: Random House.

Ford, J. (1992). Hard of Hearing people: Are we a community? Are we a culture? In Congress Report of the 4th International Congress of Hard of Hearing People (pp. 66-68). Port Coquitlam, BC: MDEnterprises.

Fourney, D. (2007). Using a common accessibility profile to improve accessibility (Unpublished master's thesis). University of Saskatchewan.

Fourney, D. (2012). Can computer representations of music enhance enjoyment for individuals who are hard of hearing? In K. Miesenberger, A. Karshmer, P. Penaz, \& W. Zagler (Eds.), Proceedings of Computers Helping People with Special Needs, $13^{\text {th }}$ International Conference, (ICCHP 2012), July 11-13, Linz, Austria (Vol. 7382, pp. 535-542). Springer Berlin Heidelberg. doi: 10.1007/ 978-3-642-31522-0_80

Fourney, D., \& Fels, D. (2008). "Thanks for pointing that out." Making sarcasm accessible for all. In Proceedings of the 52 ${ }^{\text {nd }}$ Annual Meeting of the Human Factors and Ergonomics Society (HFES 2008), September 22-26, New York, NY, USA (pp. 571-575). doi: 10.1177/ 154193120805200613

Fourney, D., \& Fels, D. (2009). Creating access to music through visualization. IEEE Toronto International Conference Science and Technology for Humanity (TIC-STH 2009). doi: 10.1109/ tic-sth.2009.5444364

Fox, K. (n.d.). The smell report. Oxford, UK. Retrieved from http://www.sirc.org/publik/smell .pdf

Fox, S. (2015). High-frequency hearing loss. Retrieved from http://www.noisehelp.com/high -frequency-hearing-loss.html

Frankfort-Nachmias, C., \& Leon-Guerrero, A. (2011). Social statistics for a diverse society $\left(6^{\text {th }}\right.$ ed.). London: Sage Publications.

Frenzel, H., Bohlender, J., Pinsker, K., Wohlleben, B., Tank, J., Lechner, S. G., .. Lewin, G. R. (2012). A genetic basis for mechanosensory traits in humans. PLoS Biology, 10(5), e1001318. doi: 10.1371/journal.pbio.1001318

Gault, R. H. (1927). "Hearing" through the sense organs of touch and vibration. Journal of the Franklin Institute, 204 (3), 329-358. doi: 10.1016/s0016-0032(27)92101-2

Gephart, R. P. (2004). Qualitative research and the Academy of Management Journal. Academy of Management Journal, 47(4), 454-462.

Geringer, J. M., \& Worthy, M. D. (1999). Effects of tone-quality changes on intonation and tonequality ratings of high school and college instrumentalists. Journal of Research in Music Education, 47(2), 135-149. Retrieved from http://www.jstor.org/stable/3345719

Glaser, B. (1978). Theoretical sensitivity: Advances in the methodology of grounded theory. Sociology Press. 
Glaser, B. (1995). A look at grounded theory: 1984 to 1994. In B. G. Glaser (Ed.), Grounded Theory: 1984-1994 (pp. 3-17). Sociology Press.

Glaser, B., \& Strauss, A. (1967). The discovery of grounded theory: Strategies for qualitative research. Chicago: Adline.

Glennie, E. (n.d.). Disability essay. Retrieved from http://www.evelyn.co.uk/Resources/Essays/ Disability\%20Essay.pdf

Gorbman, C. (1987). Unheard melodies: Narrative film music. Bloomington, Indiana: Indiana University Press.

Goulding, C. (1999). Grounded theory: some reflections on paradigm, procedures and misconceptions (Tech. Rep.). University of Wolverhampton. Retrieved from http://www.wlv.ac.uk/PDF/uwbs _WP006-99\%20Goulding.pdf

Gove, P. B., et al. (Eds.). (2002). Webster's third new international dictionary of the English language (unabridged). Springfield, MA: Merriam-Webster.

Graziplene, L. R. (2000). Teletext: Its promise and demise. Lehigh University Press.

Green, E. (2014). Atari Video Music. Retrieved from http://www.thelogbook.com/phosphor/1976/ atari-video-music/

Gregory, R. (1998). Snapshots from the decade of the brain: Brainy mind. BMJ, 317(7174), 16931695. doi: $10.1136 /$ bmj.317.7174.1693

Griffiths, R. (2008, sep 10). First look: iTunes 8.0. Macworld. Retrieved from http://www.macworld .com/article/1135463/itunes8firstlook.html

Groce, N. (1985). Everyone here spoke sign language: Hereditary deafness on Martha's Vineyard. Cambridge, MA: Harvard University Press.

Gutwin, C., \& Skopik, A. (2003). Fisheyes are good for large steering tasks. In Proceedings of the SIGCHI Conference on Human Factors in Computing Systems (pp. 201-208). doi: 10.1145/ 642611.642648

Hamm, C., Nettl, B., \& Byrnside, R. (1975). Contemporary music and music culture. Englewood Cliffs, NJ: Prentice-Hall, Inc.

Handzo, S. (1985). Appendix: A narrative glossary of film sound technology. In E. Weis \& J. Belton (Eds.), Film sound: Theory and practice (pp. 383-426). New York: Columbia University Press.

Harkins, J. E. (1995). Non-speech information in captioned video: A consumer opinion study with guidelines for the captioning industry. Gallaudet University, Gallaudet Research Institute, Technology Assessment Program.

Hart, S. G. (2006). NASA-Task Load Index (NASA-TLX): 20 Years Later., 904-908. doi: 10.1177/ 154193120605000909

Hawkins, J. E. (2014). Human ear. Encyclopæedia Britannica Online. Retrieved from http://www .britannica.com/EBchecked/topic/175622/human-ear

He, A., \& Limb, C. J. (2014). Music perception and enjoyment show little association in CI users. The Hearing Journal, 67(12), 16, 18. doi: 10.1097/01.HJ.0000459166.76675.80

Hearit. (2006). Evaluation of the social and economic costs of hearing impairment. Retrieved from http://www.hearit.org/multimedia/Hear_It_Report_October_2006.pdf 
von Helmholtz, H. L. F. (1867/1925). Von den Wahrnehmungen im Allgemeinen [Concerning the perceptions in general]. In J. P. C. Southall (Trans.), Handbuch der Physiologischen Optik [Treatise on Physiological Optics] (3 ${ }^{\text {rd }}$ ed., Vol. III, pp. 1-37). The Optical Society of America. Retrieved from http://poseidon.sunyopt.edu/BackusLab/Helmholtz/

Herman, N. (n.d.). Nancy Clearwater Herman. Retrieved from http://www. nancyherman.com Herman, N. (2009). If $C$ is red: A translation of music to color. New York: Blurb.

Hodgin, R. (n.d.). Magnetosphere, part 1. Retrieved from http://roberthodgin.com/ magnetosphere/

Holden, S. (1989, July 16). How rock is changing Hollywood's tune. New York Times, H1, H18-19.

Holmquist, L. E. (2004). Evaluating the comprehension of ambient displays. In CHI'04 Extended Abstracts on Human Factors in Computing Systems (pp. 1545-1545). doi: 10.1145/985921.986121

Hong Kong Equal Opportunities Commission. (1999, December). VII Summary of Proceedings: Cluster Three: Definitions of disability. In Report of the Interregional Seminar and Symposium on International Norms and Standards relating to Disability. Hong Kong, Special Administrative Region of China. Retrieved from http://www.worldenable.net/hongkong99/ hkreport9907.htm

IBM Corp. (2013). IBM SPSS Statistics for Windows (Version 22.0) [Software].

International Federation of the Phonographic Industry (IFPI). (2009). Recorded Music Sales 2008. Retrieved from http://www. ifpi.org/content/library/Recorded-music-sales-2008.pdf

International Organization for Standardization. (1998). ISO 9241-11:1998: Ergonomic requirements for office work with visual display terminals (VDTs) - Part 11: Guidance on usability. Geneva, Switzerland: International Organization for Standardization.

International Organization for Standardization. (2008). ISO 9241-171:2008 Ergonomics of human system interaction - Guidance on software accessibility. Geneva, Switzerland: International Organization for Standardization.

Isaacson, E. (2005). What you see is what you get: On visualizing music. In J. D. Reiss \& G. A. Wiggins (Eds.), Proceedings of the $6^{\text {th }}$ International Conference on Music Information Retrieval (ISMIR 2005) (pp. 389-395). Retrieved from http://ismir2005.ismir.net/proceedings/ 1129.pdf

Israelite, N., Ower, J., \& Goldstein, G. (2002). Hard-of-hearing adolescents and identity construction: Influences of school experiences, peers, and teachers. Journal of Deaf Studies and Deaf Education, 7(2), 134-148.

Jacobs, T. (2013, August 5). The sounds that bind: Why we evolved to love music. Pacific Standard. Retrieved from http://www.psmag.com/blogs/news-blog/the-sounds-that-bind-new -evidence-of-why-we-love-music-63907/

Janata, P., Tomic, S. T., \& Rakowski, S. K. (2007). Characterisation of music-evoked autobiographical memories. Memory, 15(8), 845-860. doi: 10.1080/09658210701734593

Jones, I. M. (1937, June 20). Emotional power of music revealed. Los Angeles Times, C9.

Jordan, A., Albright, A., Branner, A., \& Sullivan, J. (2003). The state of closed-captioning services in the United States: An assessment of quality, availability, and use. Philadelphia, PA: The 
Annenberg Public Policy Center of The University of Pennsylvania. A Report to The National Captioning Institute Foundation. Retrieved from http://www.dcmp.org/caai/nadh136.pdf Judkins, J. (1997). The Helfgott problem. Philosophy and Literature, 21(2), 363-367.

Kalinak, K. (1982). The Fallen Woman and the Virtuous Wife: Musical Stereotypes in The Informer, Laura and Gone with the Wind. Film Reader, 5, 76-82.

Kalinak, K. (2007). How the west was sung: Music in the westerns of John Ford. University of California Press.

Karam, M., Branje, C., Nespoli, G., Thompson, N., Russo, F. A., \& Fels, D. I. (2010). The Emotichair: An interactive tactile music exhibit. In CHI '10 Extended Abstracts on Human Factors in Computing Systems (pp. 3069-3074). New York, NY, USA: ACM. doi: 10.1145/1753846 .1753919

Karam, M., \& Fels, D. (2008). Designing a model human cochlea: Issues and challenges in crossmodal audio-haptic displays. In Proceedings of the 2008 Ambi-Sys Workshop on Haptic User Interfaces in Ambient Media Systems (p. 8). doi: 10.4108/ICST.AMBISYS2008.2837

Kassabian, A. (2001). Hearing film: Tracking identifications in contemporary Hollywood film music. New York: Routledge.

Kestner, T. (2001). History and uses of music education for children who are hearing impaired. Retrieved from http://digitalcommons. wustl.edu/pacs_capstones/79 (Independent Studies and Capstones. Paper 79. Program in Audiology and Communication Sciences, Washington University School of Medicine.)

Kim, Y. E., Schmidt, E. M., Migneco, R., Morton, B. G., Richardson, P., Scott, J., .. Turnbull, D. (2010). Music emotion recognition: A state of the art review. In Proceedings of the $11^{\text {th }}$ International Society for Music Information Retrieval Conference (ISMIR 2010) (pp. 255-266). Retrieved from http://ismir2010.ismir.net/proceedings/ismir2010-45.pdf

Kivy, P. (1980). The corded shell: Reflections on musical expression. Princeton, NJ: Princeton University Press.

Knight, W. E., \& Rickard, N. S. (2001). Relaxing music prevents stress-induced increases in subjective anxiety, systolic blood pressure, and heart rate in healthy males and females. Journal of Music Therapy, 38(4), 254-272.

Kochkin, S. (1990). Introducing MarkeTrak: A consumer tracking survey of the hearing instrument market. The Hearing Journal, 43(5), 17-27. Retrieved from http://old.betterhearing.org/ hia/publications/MR01.PDF

Kochkin, S. (2000). MarkeTrak V: Consumer satisfaction revisited. The Hearing Journal, 53(1), $38-40$.

Kochkin, S. (2005). MarkeTrak VII. The Hearing Journal, 58(9), 30. doi: 10.1097/01.hj.0000286545 .33961.e7

Kochkin, S. (2010). MarkeTrak VIII: Customer satisfaction with hearing aids is slowly increasing. The Hearing Journal, 63(1), 11-19. doi: 10.1097/01.HJ.0000366912.40173.76

Kohut, H., \& Levarie, S. (1950). On the enjoyment of listening to music. Psychoanalytic Quarterly, $19,64-87$. 
Kosara, R. (2007). Visualization criticism - The missing link between information visualization and art. In 11th International Conference Information Visualization (IV'O7) (pp. 631-636). Institute of Electrical \& Electronics Engineers (IEEE). doi: 10.1109/iv.2007.130

Kramer, G. (1994). An introduction to auditory display. In Auditory Display: Sonification, Audification and Auditory Interfaces, SFI Studies in the Sciences of Complexity, Proceedings (pp. 1-77). Reading, MA: Addison-Wesley.

Kramer, G., Walker, B., Bonebright, T., Cook, P., Flowers, J., Miner, N., \& Neuhoff, J. (1997). Sonification report: Status of the field and research agenda, prepared for the national science foundation by members of the international community for auditory display. Retrieved from http://www. icad.org/websiteV2.0/References/nsf.html

Kung, C. (2005). A possible unifying principle for mechanosensation. Nature, 436(7051), 647-654. doi: $10.1038 /$ nature 03896

Lane, H. L. (1992). The mask of benevolence: Disabling the Deaf community. Knopf New York.

Lang, P. J. (1985). The cognitive psychophysiology of emotion: Anxiety and the anxiety disorders. Lawrence Eribaum, Hillsdale.

Laszlo, C. (1994). Is there a hard-of-hearing identity? Journal of Speech Language Pathology and Audiology, 18(4), 248-252.

Lee, D. G., Fels, D. I., \& Udo, J. P. (2007). Emotive captioning. Computers in Entertainment, 5(2), 11. doi: $10.1145 / 1279540.1279551$

Levitin, D. J. (2007). This is your brain on music: The science of a human obsession. Plume, New York.

Levitin, D. J., \& Tirovolas, A. K. (2009). Current advances in the cognitive neuroscience of music. Annals of the New York Academy of Sciences, 1156(1), 211-231.

Lipscomb, S. D., \& Kim, E. M. (2004). Perceived match between visual parameters and auditory correlates: an experimental multimedia investigation. In Proceedings of the $8^{\text {th }}$ International Conference on Music Perception and Cognition (pp. 72-75).

Loeffler, D. (2006). Instrument timbres and pitch estimation in polyphonic music. Retrieved from https://smartech.gatech.edu/bitstream/handle/1853/10568/loeffler_dominik_b_200605 _mast.pdf

Loersch, C., \& Arbuckle, N. L. (2013). Unraveling the mystery of music: Music as an evolved group process. Journal of Personality and Social Psychology, 105(5), 777-798. doi: 10.1037/a0033691

Lottridge, D. (2008). Emotional response as a measure of human performance. In CHI'08 Extended Abstracts on Human Factors in Computing Systems (pp. 2617-2620).

Lübbers, D. (2005). Sonixplorer: Combining visualization and auralization for content-based exploration of music collections. In J. D. Reiss \& G. A. Wiggins (Eds.), Proceedings of the $6^{\text {th }}$ International Conference on Music Information Retrieval (ISMIR 2005) (pp. 590-593). Retrieved from http://ismir2005.ismir.net/proceedings/2071.pdf

Lynch, M. (2007). Classic Spectrum Analyzer - Accurate, detailed, customizable spectrum analyzer. Retrieved from https://web.archive.org/web/20080526113339/http://www. winamp.com/ plugins/details/165966 
Lynch, M. (2009). Mike's Winamp Plugins. Retrieved from https://web.archive.org/web/ 20091027055048/http://geocities.com/SiliconValley/Peaks/9546/

Maler, A. (2013). Songs for hands: Analyzing interactions of sign language and music. Society for Music Theory, 19(1), 1-15. Retrieved from http://www.mtosmt.org/issues/mto.13.19.1/ mto.13.19.1.maler.php

Malinowski, S. A. (n.d.). Music animation machine. Retrieved from http://www.musanim.com

Martin, J.-R., \& Le Corre, F. (2015). Sensory substitution is substitution. Mind $\mathscr{E}$ Language, 30(2), 209-233. doi: 10.1111/mila.12078

Matessino, M. (1999, Sept. 24). Letter in response to 'A Study of Jaws' Incisive overture to close off the century'. Retrieved from http://www.filmscoremonthly.com/articles/1999/24_Sep--Film_Score_Friday.asp

McGraw, P. (2008, Nov. 12). Dr. Phil uncensored: Hitman [video blog]. Retrieved from http:// drphil.com/shows/show/1164

McKellin, W. (1994). Hearing and listening: Audiology, hearing, and hearing impairment in everyday life. Journal of Speech-Language Pathology, 18(4), 212-219.

McKendrick, J. G. (1897). Sound and speech waves as revealed by the phonograph (Science Lecture for 1896). Proceedings of the Royal Philosophical Society of Glasgow, 28, 201-235. Retrieved from https://ia601407.us.archive.org/4/items/proceedingsroya04glasgoog/ proceedingsroya04glasgoog.pdf

McPherson, J. G. (1897, June). Can the deaf appreciate music? Knowledge: an Illustrated Magazine of Science, Literature, and Art, 20, 135-136.

Media Access. (n.d.). Frequently asked questions about TV/Media Access Australia. Retrieved from http://www.mediaaccess.org.au/tv-video/frequently-asked-questions/frequently -asked-questions-about-tv

Medugno, R. (2005). Deaf daughter, hearing father. Washington, DC: Gallaudet University Press.

Mehrabian, A. (1996). Pleasure-arousal-dominance: A general framework for describing and measuring individual differences in temperament. Current Psychology, 14 (4), 261-292.

Miles, M. B., \& Huberman, A. M. (1994). Qualitative data analysis: An expanded sourcebook. Thousand Oaks, CA: Sage.

Ministry of Education and Training. (1998). The Ontario Curriculum, Grades 1-8: The Arts, 1998. Toronto.

Ministry of Labour. (2007). Health and safety guidelines: Noise requirements in the regulations for industrial establishments and oil and gas offshore, guidelines. Retrieved from http:// www. labour.gov.on.ca/english/hs/pdf/gl_noise.pdf

Mitchell, R. E. (2006). How many deaf people are there in the United States? Estimates from the survey of income and program participation. Journal of Deaf Studies and Deaf Education, 11(1), $112-119$.

Mitroo, J. B., Herman, N., \& Badler, N. I. (1979). Movies from music: Visualizing musical compositions. ACM SIGGRAPH Computer Graphics, 13(2), 218-225. doi: 10.1145/800249.807447

Mori, J., \& Fels, D. I. (2009). Seeing the music: Can animated lyrics provide access to the emotional 
content in music for people who are deaf or hard of hearing? In IEEE Toronto International Conference Science and Technology for Humanity (TIC-STH 2009) (pp. 951-956). Institute of Electrical \& Electronics Engineers (IEEE). doi: 10.1109/TIC-STH.2009.5444362

Murphy, S. (2014). A pop music progression in recent popular movies and movie trailers. Music, Sound, and the Moving Image, 8(2), 141-162. doi: 10.3828/msmi.2014.9

Murray, R. (1978). Balloon captioning: A system for word emphasis. In B. Braverman \& B. Cronin (Eds.), Captioning: Shared perspectives: Proceedings of a National Captioning Conference. Rochester, NY: NTID.

Murray, R. (1979). Reinforcement of speech through the balloon captioning of song lyrics. American Annals of the Deaf, 124(5), 656-662.

National Institute for Occupational Safety and Health (NIOSH). (1998). Criteria for a recommended exposure limit: Noise. (Report 98-126). Retrieved from http://www.cdc.gov/niosh/docs/ 98-126/pdfs/98-126.pdf

Nielsen, J., \& Landauer, T. K. (1993). A mathematical model of the finding of usability problems. In Proceedings of the SIGCHI Conference on Human Factors in Computing Systems - CHI '93 (pp. 206-213). Association for Computing Machinery (ACM). doi: 10.1145/169059.169166

Norman, J. (2008, Dec. 12). Keynote address In "Deaf filmmakers: Take one!" An exhibit of the Deaf Culture Centre, Toronto, ON.

NVivo. (2012). NVivo qualitative data analysis software (Version 10) [Software]. (QSR International Pty Ltd.)

Oliva, G. (2004). Alone in the mainstream: A deaf woman remembers public school. Washington, DC: Gallaudet University Press.

Oregon Public Broadcasting. (2012). History Detectives: Frank Zappa collage. Retrieved from http:// www.pbs.org/opb/historydetectives/investigation/frank-zappa-collage/

Ortony, A., \& Turner, T. J. (1990). What's basic about basic emotions? Psychological Review, 97(3), 315-331. doi: 10.1037/0033-295x.97.3.315

Pace, S. (2004). A grounded theory of the flow experiences of web users. International Journal of Human-Computer Studies, 60(3), 327-363. Retrieved from http://www.nova.edu/ssss/QR/ QR2-4/pandit.html

Padden, C., \& Humphries, T. (1988). Deaf in America: Voices from a culture. Cambridge: Havard University Press.

Pandit, N. R. (1996). The creation of theory: A recent application of the grounded theory method. The Qualitative Report, 2(4), 1-14.

Percino, G., Klimek, P., \& Thurner, S. (2014). Instrumentational complexity of music genres and why simplicity sells. PLoS ONE, 9(12), e115255. doi: 10.1371/journal.pone.0115255

Peretz, I., \& Hyde, K. L. (2003). What is specific to music processing? Insights from congenital amusia. Trends in Cognitive Sciences, 7(8), 362-367. doi: 10.1016/s1364-6613(03)00150-5

Pfeiffer, S., Jägenstedt, P., \& Hickson, I. (2015). WebVTT: Living standard. Retrieved from http:// dev.w3.org/html5/webvtt/

Pierce, D. (2013). The survival of American silent feature films: 1912-1929. Washington, D.C.: 
Council on Library and Information Resources and The Library of Congress. Retrieved from http://www.loc.gov/film/pdfs/pub158.final_version_sept_2013.pdf

Plutchik, R. (1980). Emotion: A psychoevolutionary synthesis. New York: Harper \& Row.

Porter, N. (Ed.). (1913). Webster's revised unabridged dictionary of the English language. Springfield, MA: Merriam-Webster.

Powell, J. (2010). How music works: The science and psychology of beautiful sounds, from Beethoven to the Beatles and beyond. Little, Brown.

Puckette, M., \& Apel, D., Theodore Zicarelli. (1998). Real-time audio analysis tools for Pd and MSP. In Proceedings of the International Computer Music Conference. Retrieved from http://vud . org/max/icmc98.pdf

Richards, L. (2014). Handling qualitative data: A practical guide ( $3^{\text {rd }}$ ed.). Los Angeles: Sage.

Richardson, B. L., \& Frost, B. J. (1977). Sensory substitution and the design of an artificial ear. The Journal of Psychology, 96(2), 259-285.

Rose, T. (1994). Black noise: Rap music and black culture in contemporary America. Hanover, NH: Wesleyan University Press.

Russell, D. (1989). Closed captioning of clips increases. Billboard, 101(48), 48.

Russell, J. A., \& Mehrabian, A. (1977). Evidence for a three-factor theory of emotions. Journal of Research in Personality, 11 (3), 273-294. doi: 10.1016/0092-6566(77)90037-X

Sack, W. (2011). Aesthetics of information visualization. In M. Lovejoy, V. Vesna, \& C. Paul (Eds.), Context Providers: Conditions of Meaning in Media Arts (pp. 123-150). University of Minnesota Press. Retrieved from http://danm.ucsc.edu/ wsack/Writings/wsack-infoaesthetics.pdf

Sacks, O. (2007). Musicophilia: Tales of music and the brain. New York: Alfred A. Knopf.

Sarter, N. B. (2006). Multimodal information presentation: Design guidance and research challenges. International Journal of Industrial Ergonomics, 36(5), 439-445. doi: 10.1016/j.ergon.2006.01 .007

Sato, J., Ohtsu, K., Bannai, Y., \& Okada, K. (2009). Effective presentation technique of scent using small ejection quantities of odor. In IEEE Virtual Reality Conference (VR 2009) (pp. 151-158). Institute of Electrical \& Electronics Engineers (IEEE). doi: 10.1109/vr.2009.4811015

Sauter, D. A., Eisner, F., Ekman, P., \& Scott, S. K. (2010). Cross-cultural recognition of basic emotions through nonverbal emotional vocalizations. Proceedings of the National Academy of Sciences, 107(6), 2408-2412. doi: 10.1073/pnas.0908239106

Schmidt-Jones, C. (2013). Timbre: The color of music. OpenStax CNX. Retrieved from http:// cnx.org/contents/f306fb29-f034-4a34-99d5-a44adf8ae813015

Schönberg, C.-M. (Composer)., \& Kretzmer, H. (Lyricist). (1986). Full score Act 1 Part 1 Les Misérables. New York: Alain Boublil Music Ltd.

Schroeder, M. R. (1992). Concert halls: From magic to number theory. In D. Deutsch (Ed.), The Psychology of Music (pp. 25-46). San Diego: Academic Press.

Secundo, L., Snitz, K., \& Sobel, N. (2014). The perceptual logic of smell. Current Opinion in Neurobiology, 25, 107-115. doi: 10.1016/j.conb.2013.12.010

Shepard, R. N., \& Levitin, D. J. (2002). Cognitive psychology and music. In D. J. Levitin (Ed.), 
Foundations of cognitive psychology: Core readings (pp. 503-514). MIT Press.

Shneiderman, B. (1996). The eyes have it: A task by data type taxonomy for information visualizations. In Proceedings of IEEE Symposium on Visual Languages (pp. 336-343). doi: 10.1109/VL.1996.545307

Shneiderman, B. (2000). Universal usability. Communications of the ACM, 43(5), 84-91. doi: 10 $.1145 / 332833.332843$

Silverman, C., \& Fels, D. I. (2002). Emotive captioning in a digital world. In Computers Helping People with Special Needs (pp. 292-294). Springer. doi: 10.1007/3-540-45491-8_61

Smith, H., \& Schneider, A. (2009). Critiquing models of emotions. Sociological Methods \& Research, 37(4), 560-589. doi: 10.1177/0049124109335790

Smith, S. (1987). Videotex and teletext: A conference report. Serials Review, 13(2), 69-75. doi: 10.1016/s0098-7913(87)80026-6

Solomon, A. L. (1980). Music in special education before 1930: Hearing and speech development. Journal of Research in Music Education, 28(4), 236-242. doi: 10.2307/3345033

Spence, R. (2007). Information visualization: Design for interaction ( $2^{\text {nd }}$ ed.). Toronto: Pearson/Prentice Hall.

Stern, P. (1995). Grounded theory methodology: Its uses and processes. In B. Glaser (Ed.), Grounded Theory 1984-1994 (pp. 29-39). Mill Valley, CA: Sociology Press.

Story, M., Mueller, J., \& Mace, R. (1998). The universal design file: Designing for people of all ages and abilities. The Center for Universal Design, NC State University. Retrieved from http:// www.ncsu.edu/ncsu/design/cud/pubs_p/pudfiletoc.htm

Strauss, K. P. (2006). A new civil right: Telecommunications equality for deaf and hard of hearing Americans. Washington, DC: Gallaudet University Press.

Straw, W. (1988). Music video in its contexts: Popular music and post-modernism in the 1980s. Popular Music, 7(3), 247-266.

Suddaby, R. (2006). From the editors: What grounded theory is not. Academy of Management Journal, 49(4), 633-642.

SynchriMedia. (2015). MovieCaptioner (Version 4.97) [Software]. Retrieved from http://www .synchrimedia.com/

Tang, L., Fourney, D., Huang, F., \& Carter, J. (2008). Secondary encoding. In Proceedings of 52 ${ }^{\text {nd }}$ Annual Meeting of the Human Factors and Ergonomics Society (HFES 2008), September 22-26, New York, NY, USA (pp. 561-565). doi: 10.1177/154193120805200611

Taylor, R., Torres, D., \& Boulanger, P. (2005). Using music to interact with a virtual character. In Proceedings of the 2005 Conference on New Interfaces for Musical Expression (NIME05) (pp. 220-223).

Tierney, J., Zissman, M. A., \& Eddington, D. K. (1994). Digital signal processing applications in cochlear implant research. Lincoln Laboratory Journal, 7(1), 31-62. Retrieved from http:// www.ll.mit.edu/publications/journal/pdf/vol07_no1/7.1.2.cochlearimplant.pdf

Tipton, B. (1997). Deaf music. Retrieved from http://members.tripod.com/ obdc/music.html

Toland, M. (2001). What does music mean to you? High Bias. Retrieved from https://web.archive 
.org/web/20090318095048/http://highbias.com/features/musicmeans.html

Turton, L., \& Smith, P. (2013). Prevalence \& characteristics of severe and profound hearing loss in adults in a UK National Health Service clinic. International Journal of Audiology, 52(2), 92-97. doi: $10.3109 / 14992027.2012 .735376$

Tyler, R. (Ed.). (2006). Tinnitus treatment: Clinical protocols. New York: Thieme.

UN. (2011). World population prospects, The 2010 revision. Retrieved from http://esa.un.org/ unpd/wpp/Documentation/WPP\%202010\%20publications.htm

United Nations Commission for Social Development on Disability. (1993). United Nations Standard Rules on the Equalization of Opportunities for Persons with Disabilities $(A / R E S / 48 / 96)$. Retrieved from http://www.un.org/esa/socdev/enable/dissre00.htm

Vanderheiden, G. (1990). Thirty-something million: Should they be exceptions? Human Factors, 32(4), 383-396. doi: 10.1177/001872089003200402

Vitense, H. S., Jacko, J. A., \& Emery, V. K. (2002). Foundation for improved interaction by individuals with visual impairments through multimodal feedback. Universal Access in the Information Society, 2(1), 76-87. doi: 10.1007/s10209-002-0038-2

Vy, Q. V., Mori, J. A., Fourney, D. W., \& Fels, D. I. (2008). Enact: A software tool for creating animated text captions. In K. Miesenberger et al. (Eds.), Proceedings of $11^{\text {th }}$ International Conference on Computers Helping People with Special Needs (ICCHP 2008) (pp. 609-616). Springer. doi: 10.1007/978-3-540-70540-6_87

Weinberger, N. M. (1999). Music and the auditory system. In D. Deutsch (Ed.), The psychology of music ( $2^{\text {nd }}$ ed., pp. 47-87). San Diego: Academic Press.

Welsh, L. W., Welsh, J. J., Rosen, L. F., \& Dragonette, J. E. (2004). Functional impairments due to unilateral deafness. Annals of Otology, Rhinology and Laryngology, 113(12), 987-993. doi: $10.1177 / 000348940411301209$

Wierzbicki, J. (2009). Film music: A history. New York: Routledge.

Wikipedia. (2014a). Atari video music - Wikipedia, The Free Encyclopedia. Retrieved February 17, 2015, from http://en.wikipedia.org/w/index.php?title=Atari_Video_Music\&oldid= 605213149

Wikipedia. (2014b). EIA-608 - Wikipedia, The Free Encyclopedia. Retrieved February 19, 2015, from http://en.wikipedia.org/w/index .php?title=EIA-608\&oldid=635890460

Wikipedia. (2014c). Hit List (TV series) - Wikipedia, The Free Encyclopedia. Retrieved February 18, 2015, from http://en.wikipedia.org/w/index.php?title=Hit_List_(TV_series)\&oldid= 639168034

Wikipedia. (2014d). Illustrated song - Wikipedia, The Free Encyclopedia. Retrieved March 19, 2015, from http://en.wikipedia.org/w/index.php?title=Illustrated_song\&oldid=624069024

Wikipedia. (2015a). Ceefax - Wikipedia, The Free Encyclopedia. Retrieved February 19, 2015, from http://en.wikipedia.org/w/index.php?title=Ceefax\&oldid=641805312

Wikipedia. (2015b). I-V-vi-IV progression - Wikipedia, The Free Encyclopedia. Retrieved February 19, 2015, from http://en.wikipedia.org/w/index.php?title $=\mathrm{I} \% \mathrm{E} 2 \% 80 \% 93 \mathrm{~V} \% \mathrm{E} 2 \% 80 \% 93 \mathrm{vi} \% \mathrm{E} 2 \%$ 80\%93IV_progression\&oldid=647904909 
Wikipedia. (2015c). Ototoxicity - Wikipedia, The Free Encyclopedia. Retrieved April 7, 2015, from http://en.wikipedia.org/wiki/Ototoxicity

Williams, C. J. (Director). (1913). All on account of a transfer. [film]. Edison Company.

Winton, W. M., Putnam, L. E., \& Krauss, R. M. (1984). Facial and autonomic manifestations of the dimensional structure of emotion. Journal of Experimental Social Psychology, 20(3), 195-216.

Wolf, M. J., \& Perron, B. (2003). The video game theory reader. New York: Routledge.

Woodcock, K., \& Aguayo, M. (2000). Deafened people: Adjustment and support. Toronto: University of Toronto Press.

Woodcock, K., \& Pole, J. D. (2007). Health profile of deaf Canadians: Analysis of the Canada Community Health Survey. Canadian Family Physician, 53(12), 2140-2141. Retrieved from http://www.cfp.ca/cgi/content/abstract/53/12/2140

Woodward, J. C. (1972). Implications for sociolinguistic research among the deaf. Sign Language Studies, 1(1), 1-7. doi: 10.1353/sls.1972.0004

World Health Organization. (2013). Multi-country assessment of national capacity to provide hearing care (Tech. Rep.). Author. Retrieved from http://www.who.int/pbd/publications/ WHOReportHearingCare_Englishweb.pdf

World Health Organization, et al. (2001). Deafness and hearing impairment survey: Report of the consultative meeting of principal investigators (Tech. Rep.). World Health Organization. Retrieved from http://whqlibdoc.who.int/searo/2001/SEA_Deaf_4.pdf (WHO Project ICP DPR 001)

Yang, H.-J., Lay, Y.-L., Liou, Y.-C., Tsao, W.-Y., \& Lin, C.-K. (2007). Development and evaluation of computer-aided music-learning system for the hearing impaired. Journal of Computer Assisted Learning, 23(6), 466-476. doi: 10.1111/j.1365-2729.2007.00229.x

Yani-de Soriano, M. M., \& Foxall, G. R. (2006). The emotional power of place: The fall and rise of dominance in retail research. Journal of Retailing and Consumer Services, 13(6), 403-416. doi: 10.1016/j.jretconser.2006.02.007 



\title{
Acronyms
}

\author{
ALD Assistive Listening Device. \\ ASL American Sign Language. \\ CI Cochlear Implant. \\ D/HH Deaf, Deafened, and Hard of Hearing. \\ DRM Design Research Methodology. \\ GT Grounded Theory.
}

HA Hearing Aid.

HH Hard of Hearing.

IEC International Electrotechnical Commission.

IFPI International Federation of the Phonographic Industry.

ISO International Organization for Standardization.

PAD Pleasure / Arousal / Dominance.

SAM Self-Assessment Manikin. 